\title{
Parametrized Homotopy Theory
}

\author{
J. P. May \\ J. Sigurdsson
}

Department of Mathematics, The University of Chicago, Chicago, IL 60637

E-mail address: may@math.uchicago.edu

Department of Pure Mathematics, University of Sheffield, Sheffield S3 7RH, ENGLAND

E-mail address: J.Sigurdsson@sheffield.ac.uk 
2000 Mathematics Subject Classification. Primary 19D99, 55N20, 55P42; Secondary 19L99, 55N22, 55T25

Key words and phrases. ex-space, parametrized spectrum, parametrized homotopy theory, equivariant homotopy theory, parametrized stable homotopy theory, equivariant stable homotopy theory, transfer, twisted $K$-theory, Poincaré duality, Atiyah duality, Thom spectrum, bicategory, model category

May was partially supported by the NSF. Sigurdsson was partially supported by the EPSRC.

ABSTRACT. We develop rigorous foundations for parametrized homotopy theory. After preliminaries on point-set topology, base change functors, and proper actions of non-compact Lie groups, we develop the homotopy theory of equivariant ex-spaces (spaces over and under a given space) and of equivariant parametrized spectra. We emphasize several issues of independent interest and include a good deal of new material on the general theory of topologically enriched model categories. The essential point is to resolve problems in parametrized homotopy theory that have no nonparametrized counterparts. In contrast to previously encountered situations, model theoretic techniques are intrinsically insufficient. Instead, a rather intricate blend of model theory and classical homotopy theory is required. Stably, we work with equivariant orthogonal spectra, which are simpler for the purpose than alternative kinds of spectra that have highly structured smash products.

We give a fiberwise duality theorem that allows fiberwise recognition of dualizable and invertible parametrized spectra. This allows use of formal duality theory in closed symmetric monoidal categories to construct and analyze transfer maps. Surprisingly, the notion of duality relevant to the parametrized analogue of Spanier-Whitehead duality relating homology and cohomology is entirely different. It was discovered by Costenoble and Waner. We give it a conceptual treatment based on formal duality theory in "closed symmetric bicategories", which is a new theory of independent interest. Parametrized Atiyah duality is central to the theory and leads to a better conceptual understanding of classical Poincaré duality and to a fiberwise version of CostenobleWaner duality that gives a fiberwise version of Poincaré duality. Equivariantly, the Wirthmüller isomorphism is a very special case of parametrized Atiyah duality, and the Adams isomorphism is a very special case of fiberwise Costenoble-Waner duality. Fiberwise bundles of spectra, first studied rigorously here, are crucial to both forms of fiberwise duality theory.

We begin the study of parametrized homology and cohomology theories. We describe such theories both axiomatically and in represented form, showing that parametrized homology and cohomology theories are represented by parametrized spectra. Theories represented by bundles of spectra are of particular interest and include twisted $K$-theory as a very special case. The parametrized way of thinking sheds light on the construction of Thom spectra and leads to a plethora of curious new nonparametrized commutative algebra spectra. These are obtained by an entirely elementary general construction that uses only ex-spaces, not parametrized spectra. Among other examples, it gives iterated Thom spectra $M^{q} U$ for $q \geq 1$ such that $M^{q} U$ is a commutative $M^{q-1} U$-algebra. 


\section{Contents}

$\begin{array}{ll}\text { Prologue } & 1\end{array}$

Part I. Point-set topology, change functors, and proper actions 11

$\begin{array}{ll}\text { Introduction } & 13\end{array}$

$\begin{array}{lll}\text { Chapter 1. The point-set topology of parametrized spaces } & 15\end{array}$

Introduction $\quad 15$

1.1. Convenient categories of topological spaces $\quad 15$

1.2. Topologically bicomplete categories and ex-objects $\quad 16$

$\begin{array}{ll}\text { 1.3. Convenient categories of ex-spaces } & 19\end{array}$

1.4. Convenient categories of ex- $G$-spaces 22

1.5. Philosophical comments on the point-set topology 23

1.6. Technical point-set topological lemmas 24

1.7. Appendix: nonassociativity of smash products in $\mathscr{T} o p_{*} \quad 26$

$\begin{array}{lll}\text { Chapter 2. Change functors and compatibility relations } & 29\end{array}$

Introduction $\quad 29$

2.1. The base change functors $f_{!}, f^{*}$, and $f_{*} \quad 30$

2.2. Compatibility relations 32

2.3. Change of group and restriction to fibers 35

$\begin{array}{ll}\text { 2.4. Normal subgroups and quotient groups } & 37\end{array}$

2.5. The closed symmetric monoidal category of retracts 40

Chapter 3. Proper actions, equivariant bundles and fibrations 43 Introduction $\quad 43$

3.1. Proper actions of locally compact groups 43

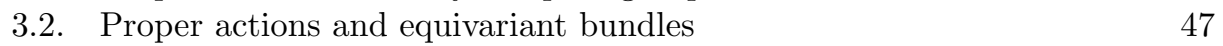

3.3. The bundle construction 48

3.4. Spaces of the homotopy types of $G$-CW complexes 51

3.5. Some classical theorems about fibrations 53

3.6. Quasifibrations 54

$\begin{array}{ll}\text { Part II. Model categories and parametrized spaces } & 57\end{array}$

$\begin{array}{ll}\text { Introduction } & 59\end{array}$

$\begin{array}{lll}\text { Chapter } 4 . & \text { Topologically bicomplete model categories } & 61\end{array}$ Introduction 61

4.1. Model theoretic philosophy: $h, q$, and $m$-model structures 62

4.2. Strong Hurewicz cofibrations and fibrations 63 
4.3. Towards classical model structures in topological categories $\quad 66$

4.4. Classical model structures in general and in $\mathscr{K}$ and $\mathscr{U} \quad 69$

4.5. Compactly generated $q$-type model structures $\quad 72$

$\begin{array}{lll}\text { Chapter 5. Well-grounded topological model categories } & 77\end{array}$ $\begin{array}{ll}\text { Introduction } & 77\end{array}$

5.1. Over and under model structures $\quad 78$

5.2. The specialization to over and under categories of spaces $\quad 82$

5.3. Well-grounded topologically bicomplete categories 85

$\begin{array}{ll}\text { 5.4. Well-grounded categories of weak equivalences } & 87\end{array}$

5.5. Well-grounded compactly generated model structures 90

5.6. Properties of well-grounded model categories 91

$\begin{array}{lll}\text { Chapter } 6 . & \text { The } q f \text {-model structure on } \mathscr{K}_{B} & 97\end{array}$ $\begin{array}{ll}\text { Introduction } & 97\end{array}$

6.1. Some of the dangers in the parametrized world 98

6.2. The $q f$ model structure on the category $\mathscr{K} / B \quad 100$

$\begin{array}{lll}\text { 6.3. Statements and proofs of the thickening lemmas } & 102\end{array}$

6.4. The compatibility condition for the $q f$-model structure 105

6.5. The quasifibration and right properness properties 107

$\begin{array}{lll}\text { Chapter 7. Equivariant } q f \text {-type model structures } & 109\end{array}$

$\begin{array}{ll}\text { Introduction } & 109\end{array}$

7.1. Families and non-compact Lie groups $\quad 110$

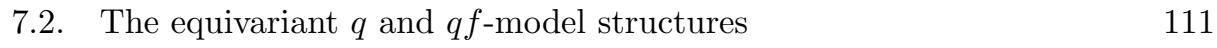

7.3. External smash product and base change adjunctions $\quad 115$

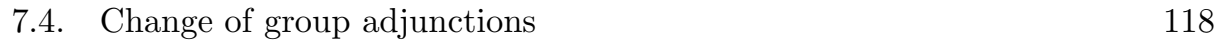

7.5. Fiber adjunctions and Brown representability 122

$\begin{array}{lll}\text { Chapter 8. Ex-fibrations and ex-quasifibrations } & 127\end{array}$

$\begin{array}{ll}\text { 8.1. Ex-fibrations } & 128\end{array}$

$\begin{array}{ll}\text { 8.2. Preservation properties of ex-fibrations } & 129\end{array}$

8.3. The ex-fibrant approximation functor 131

8.4. Preservation properties of ex-fibrant approximation 133

8.5. Quasifibrant ex-spaces and ex-quasifibrations 135

Chapter 9. The equivalence between $\mathrm{Ho} G \mathscr{K}_{B}$ and $h G \mathscr{W}_{B} \quad 137$ $\begin{array}{ll}\text { Introduction } & 137\end{array}$

9.1. The equivalence of $\mathrm{Ho} G \mathscr{K}_{B}$ and $h G \mathscr{W}_{B} \quad 138$

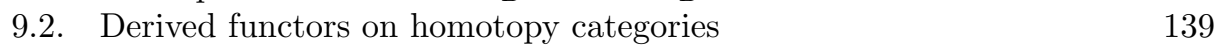

9.3. The functors $f_{*}$ and $F_{B}$ on homotopy categories 140

9.4. Compatibility relations for smash products and base change 142

$\begin{array}{lll}\text { Part III. Parametrized equivariant stable homotopy theory } & 147\end{array}$

$\begin{array}{ll}\text { Introduction } & 149\end{array}$

$\begin{array}{ll}\text { Chapter 10. Enriched categories and } G \text {-categories } & 151\end{array}$ $\begin{array}{ll}\text { Introduction } & 151\end{array}$

10.1. Parametrized enriched categories $\quad 151$

10.2. Equivariant parametrized enriched categories 153 
Chapter 11. The category of orthogonal $G$-spectra over $B \quad 159$ Introduction $\quad 159$

11.1. The category of $\mathscr{I}_{G}$-spaces over $B \quad 159$

11.2. The category of orthogonal $G$-spectra over $B \quad 163$

11.3. Orthogonal $G$-spectra as diagram ex- $G$-spaces $\quad 166$

11.4. The base change functors $f^{*}, f_{!}$, and $f_{*} \quad 167$

11.5. Change of groups and restriction to fibers 170

11.6. Some problems concerning non-compact Lie groups 172

Chapter 12. Model structures for parametrized $G$-spectra $\quad 175$

$\begin{array}{ll}\text { Introduction } & 175\end{array}$

12.1. The level model structure on $G \mathscr{S}_{B} \quad 176$

12.2. Some Quillen adjoint pairs relating level model structures $\quad 179$

12.3. The stable model structure on $G \mathscr{S}_{B} \quad 180$

12.4. Cofiber sequences and $\pi_{*}$-isomorphisms 183

12.5. Proofs of the model axioms 186

12.6. Some Quillen adjoint pairs relating stable model structures $\quad 190$

Chapter 13. Adjunctions and compatibility relations 195

$\begin{array}{ll}\text { Introduction } & 195\end{array}$

13.1. Brown representability and the functors $f_{*}$ and $F_{B} \quad 196$

13.2. The category $G \mathscr{E}_{B}$ of excellent prespectra over $B \quad 200$

13.3. The level ex-fibrant approximation functor $P$ on prespectra 202

13.4. The auxiliary approximation functors $K$ and $E \quad 205$

13.5. The equivalence between $\mathrm{Ho} G \mathscr{P}_{B}$ and $h G \mathscr{E}_{B} \quad 207$

13.6. Derived functors on homotopy categories 208

13.7. Compatibility relations for smash products and base change 209

Chapter 14. Module categories, change of universe, and change of groups 215 $\begin{array}{ll}\text { Introduction } & 215\end{array}$

14.1. Parametrized module $G$-spectra 215

14.2. Change of universe $\quad 219$

14.3. Restriction to subgroups 223

14.4. Normal subgroups and quotient groups 226

Part IV. Parametrized duality theory 229

Introduction $\quad 231$

Chapter 15. Fiberwise duality and transfer maps 233

Introduction 233

15.1. The fiberwise duality theorem 234

15.2. Duality and trace maps in symmetric monoidal categories 236

15.3. Transfer maps of Hurewicz fibrations 238

15.4. The bundle construction on parametrized spectra 240

15.5. П-free parametrized $\Gamma$-spectra 242

15.6. The fiberwise transfer for $(\Pi ; \Gamma)$-bundles 244

Chapter 16. Closed symmetric bicategories 247 
Introduction $\quad 247$

16.1. Recollections about bicategories 248

16.2. The definition of symmetric bicategories 249

16.3. The definition of closed symmetric bicategories 252

16.4. Duality in closed symmetric bicategories 255

16.5. Composites and naturality of dualities 259

16.6. A quick review of triangulated categories 261

16.7. Compatibly triangulated symmetric bicategories $\quad 262$

16.8. Duality in triangulated symmetric bicategories 266

Chapter 17. The closed symmetric bicategory of parametrized spectra 269 $\begin{array}{ll}\text { Introduction } & 269\end{array}$

17.1. The definition of the bicategory $\mathscr{E} x \quad 269$

17.2. Base change spectra 273

17.3. Duality of base change spectra 277

17.4. Using $\mathscr{E} x$ to encode relations between $\mathrm{HoG} \mathscr{S}_{B}$ and $\mathrm{HoG} \mathscr{S} \quad 278$

17.5. Sketch proofs of the compatible triangulation axioms 280

Chapter 18. Costenoble-Waner duality 285

Introduction $\quad 285$

18.1. The two notions of duality in $\mathrm{Ho} \mathscr{S}_{B} \quad 286$

18.2. Costenoble-Waner dualizability of finite cell spectra 288

18.3. Costenoble-Waner $V$-duality 290

18.4. Preliminaries on unreduced relative mapping cones 292

18.5. $V$-duality of $G$-ENRs 295

18.6. Parametrized Atiyah duality for closed manifolds 296

18.7. Parametrized Atiyah duality for manifolds with boundary 300

18.8. The proof of the Costenoble-Waner duality theorem 302

Chapter 19. Fiberwise Costenoble-Waner duality 311

Introduction 311

19.1. Costenoble-Waner duality and homotopical Poincaré duality 312

19.2. The bicategories $\mathscr{E} x_{B} \quad 314$

19.3. Comparisons of bicategories 316

19.4. The bundle construction pseudo-functor 319

19.5. The fiberwise Costenoble-Waner duality theorem 320

19.6. Fiberwise Poincaré duality 324

19.7. The Adams isomorphism 326

19.8. Some background and comparisons 328

Part V. Homology and cohomology, Thom spectra, and addenda 333

Introduction $\quad 335$

Chapter 20. Parametrized homology and cohomology theories $\quad 337$ Introduction $\quad 337$

20.1. Axioms for parametrized homology and cohomology theories 338

20.2. Represented homology and cohomology theories 341

20.3. Coefficient systems and restriction maps 343

20.4. The Serre spectral sequence 344 
20.5. Poincaré duality and the Thom isomorphism 347

20.6. Relative Poincaré duality 350

20.7. Products in parametrized homology and cohomology 350

20.8. The representability of homology theories 353

Chapter 21. Equivariant parametrized homology and cohomology 357 $\begin{array}{ll}\text { Introduction } & 357\end{array}$

21.1. Equivariant homology and cohomology theories 358

21.2. Represented equivariant theories $\quad 360$

21.3. Change of base and equivariant cofficient systems 361

21.4. Duality theorems and orientations 363

21.5. Products and the representability of homology 366

21.6. Fiberwise parametrized homology and cohomology 367

21.7. Fiberwise Poincaré duality and orientations 369

$\begin{array}{lll}\text { Chapter 22. Twisted theories and spectral sequences } & 373\end{array}$

Introduction $\quad 373$

22.1. Twisted homology and cohomology theories 374

22.2. Automorphism monoids of spectra and $G L_{1}(k) \quad 375$

22.3. Twisted $K$-theory $\quad 378$

22.4. The simplicial spectral sequence 380

22.5. Čech type spectral sequences 384

22.6. The twisted Rothenberg-Steenrod spectral sequence 386

22.7. The parametrized Künneth spectral sequence 388

Chapter 23. Parametrized FSP's and generalized Thom spectra 393

Introduction 393

23.1. $\mathscr{D}$-functors with products in symmetric monoidal categories $\quad 395$

23.2. The specialization of $\mathscr{D}$-FP's to spaces and ex-spaces 397

23.3. Group, monoid, and module FCP's; examples 399

23.4. The two-sided bar construction on FCP's 402

23.5. Examples: iterated Thom spectra 403

23.6. $\mathscr{I}_{c}$-FCP's and $\mathscr{L}$-spaces $\quad 405$

23.7. Universal spherical fibration spectra 407

23.8. Some historical background 408

Chapter 24. Epilogue: cellular philosophy and alternative approaches 411

Introduction $\quad 411$

24.1. CW spaces over $B \quad 412$

24.2. CW spectra and stable homotopy categories 415

24.3. Structured spectra and well-grounded model categories 418

24.4. The stable category of parametrized spectra 420

24.5. Towards parametrized $S_{G}$-modules 423

Bibliography 425

Index 433

Index of notation $\quad 439$ 



\section{Prologue}

What is this book about and why is it so long? Parametrized homotopy theory concerns systems of spaces and spectra that are parametrized as fibers over points of a given base space $B$. Parametrized spaces, or "ex-spaces", are just spaces over and under $B$, with a projection, often a fibration, and a section. Parametrized spectra are analogous but considerably more sophisticated objects. They provide a world in which one can apply the methods of stable homotopy theory without losing track of fundamental groups and other unstable information. Stable homotopy theory has tended to ignore such intrinsically unstable data. This has the effect of losing contact with more geometric branches of mathematics in which the fundamental group cannot be ignored.

Parametrized homotopy theory is a natural and important part of homotopy theory that is implicitly central to all of bundle and fibration theory. Results that make essential use of it are widely scattered throughout the literature. For classical examples, the theory of transfer maps is intrinsically about parametrized homotopy theory, and Eilenberg-Moore type spectral sequences are parametrized Künneth theorems. Several new and current directions, such as "twisted" cohomology theories and parametrized fixed point theory cry out for the rigorous foundations that we shall develop.

On the foundational level, homotopy theory, and especially stable homotopy theory, has undergone a thorough reanalysis in recent years. Systematic use of Quillen's theory of model categories has illuminated the structure of the subject and has done so in a way that makes the general methodology widely applicable to other branches of mathematics. The discovery of categories of spectra with associative and commutative smash products has revolutionized stable homotopy theory. The systematic study and application of equivariant algebraic topology has greatly enriched the subject.

There has not been a study of parametrized homotopy theory that takes these developments into account, and we shall provide one. We shall also give some direct applications, especially to equivariant stable homotopy theory where the new theory is particularly essential. However, we shall leave many interesting loose ends, and we shall end the book with just glimpses of several new directions that are only beginning to be mapped out.

One reason this study is so lengthy is that, rather unexpectedly, many seemingly trivial nonparametrized results fail to generalize, and many of the conceptual and technical obstacles to a rigorous treatment have no nonparametrized counterparts. Another reason is that new general concepts are required to understand the full structure present in the parametrized setting and, in particular, to understand 
parametrized duality theory. For these reasons, the resulting theory is considerably more subtle than its nonparametrized precursors. We indicate some of these problems and phenomena here.

How to read this book. Before getting to this, we offer some words of advice on reading this book. There is a lot of technical material that most readers will want to skip at a first reading. The first three parts comprise the lengthy justification of results that can be summarized quite briefly. Part I introduces the basic categories of spaces, spaces over spaces, and ex-spaces in which we shall work, describing the closed symmetric monoidal category of ex-spaces (in \$1.3), the basic triple of base change functors (in $\S \S 2.1$ and 2.2), and many other interrelated functors. Part II explains in careful detail how all structure in sight passes to homotopy categories unstably. Part III explains how to do all of this stably. Along the way, we also explain how to do everything equivariantly, at least for actions by compact Lie groups. In the end, everything works out as well as can be expected, despite the unexpected technicalities that we encounter. Accepting this, much of Parts IV and $\mathrm{V}$, which treat duality and homology and cohomology, should make sense without a careful reading of Parts I-III. We have tried to signpost where things are going with introductions to each of the five Parts and to each of the twenty-four Chapters. We urge the reader to peruse these introductions, as well as this Prologue, before plunging into the details.

Base change functors. A central conceptual subtlety in the theory enters when we try to prove that structure enjoyed by the point-set level categories of parametrized spaces descends to their homotopy categories. Many of our basic functors occur in Quillen adjoint pairs, and such structure descends directly to homotopy categories. Recall that an adjoint pair of functors $(T, U)$ between model categories is a Quillen adjoint pair, or a Quillen adjunction, if the left adjoint $T$ preserves cofibrations and acyclic cofibrations or, equivalently, the right adjoint $U$ preserves fibrations and acyclic fibrations. It is a Quillen equivalence if, further, the induced adjunction on homotopy categories is an adjoint equivalence. We originally hoped to find a model structure on parametrized spaces in which all of the relevant adjunctions are Quillen adjunctions. It eventually became clear that there can be no such model structure, for altogether trivial reasons. Therefore, it is intrinsically impossible to lay down the basic foundations of parametrized homotopy theory using only the standard methodology of model category theory.

The force of parametrized theory largely comes from base change functors associated to maps $f: A \longrightarrow B$. The existing literature on fiberwise homotopy theory says surprisingly little about such functors. This is especially strange since they are the most important feature that makes parametrized homotopy theory useful for the study of ordinary homotopy theory: such functors are used to transport information from the parametrized context to the nonparametrized context. One of the goals of our work is to fill this gap.

On the point-set level, there is a pullback functor $f^{*}$ from ex-spaces (or spectra) over $B$ to ex-spaces (or spectra) over $A$. That functor has a left adjoint $f !$ and a right adjoint $f_{*}$. We would like both of these to be Quillen adjunctions, but that is not possible unless the model structures lead to trivial homotopy categories. We mean literally trivial: one object and one morphism. We explain why. It will be 
clear that the explanation is generic and applies equally well to other situations where one encounters analogous base change functors.

Counterexample 0.0.1. Consider the following diagram.

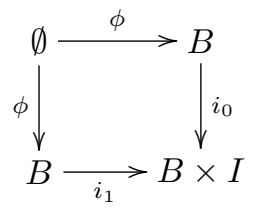

Here $\emptyset$ is the empty set and $\phi$ is the initial (empty) map into $B$. This diagram is a pullback since $B \times\{0\} \cap B \times\{1\}=\emptyset$. The category of ex-spaces over $\emptyset$ is the trivial category with one object, and it admits a unique model structure. Let $*_{B}$ denote the ex-space $B$ over $B$, with section and projection the identity map. Both $\left(\phi_{!}, \phi^{*}\right)$ and $\left(\phi^{*}, \phi_{*}\right)$ are Quillen adjoint pairs for any model structure on the category of ex-spaces over $B$. Indeed, $\phi_{!}$and $\phi_{*}$ preserve weak equivalences, fibrations, and cofibrations since both take $*_{\emptyset}$ to $*_{B}$. We have $\left(i_{0}\right)^{*} \circ\left(i_{1}\right) ! \cong \phi_{!} \circ \phi^{*}$ since both composites take any ex-space over $B$ to $*_{B}$. If $\left(i_{1}\right)$ ! and $\left(i_{0}\right)^{*}$ were both Quillen left adjoints, it would follow that this isomorphism descends to homotopy categories. If, further, the functors $\left(i_{1}\right)$ ! and $\left(i_{0}\right)^{*}$ on homotopy categories were equivalences of categories, this would imply that the homotopy category of ex-spaces over $B$ with respect to the given model structure is equivalent to the trivial category.

Information in ordinary homotopy theory is derived from results in parametrized homotopy theory by use of the base change functors $r_{\text {! }}$ and $r_{*}$ associated to the trivial map $r: B \longrightarrow *$, as we shall illustrate shortly. For this and other reasons, we choose our basic model structure to be one such that $\left(f_{!}, f^{*}\right)$ is a Quillen adjoint pair for every map $f: A \longrightarrow B$ and is a Quillen equivalence when $f$ is a homotopy equivalence. Then $\left(f^{*}, f_{*}\right)$ cannot be a Quillen adjoint pair in general. However, it is essential that we still have the adjunction $\left(f^{*}, f_{*}\right)$ after passage to homotopy categories. For example, taking $f$ to be the diagonal map on $B$, this adjunction is used to obtain the adjunction on homotopy categories that relates the fiberwise smash product functor $\wedge_{B}$ on ex-spaces over $B$ to the function ex-space functor $F_{B}$. To construct the homotopy category level right adjoints $f_{*}$, we shall have to revert to more classical methods, using Brown's representability theorem. However, it is not clear how to verify the hypotheses of Brown's theorem in the model theoretic framework.

Counterexample 0.0.1 also illustrates the familiar fact that a commutative diagram of functors on the point-set level need not induce a commutative diagram of functors on homotopy categories. When commuting left and right adjoints, this is a problem even when all functors in sight are parts of Quillen adjunctions. Therefore, proving that compatibility relations that hold on the point-set level descend to the homotopy category level is far from automatic. In fact, proving such "compatibility relations" is often a highly non-trivial problem, but one which is essential to the applications. We do not know how to prove the most interesting compatibility relations working only model theoretically. 
Poincaré duality. Before continuing our discussion of the foundations, we pause to whet the reader's appetite by pointing out how the parametrized theory sheds new light on even the most classical parts of algebraic topology. Nonequivariantly, we shall construct a good homotopy category of spectra over $B$ for any space $B$. A spectrum $k_{B}$ over $B$ represents (reduced) homology and cohomology theories, denoted $k_{*}^{B}$ and $k_{B}^{*}$, on ex-spaces over $B$ and, more generally, on spectra over $B$. We shall have a smash product that assigns a spectrum $k \wedge X$ over $B$ to an ordinary spectrum $k$ and an ex-space or spectrum $X$ over $B$. Taking $k_{*}$ to mean reduced homology and implicitly stabilizing by applying suspension spectrum functors, we shall have the following conceptual variant of the usual homotopical proof of the Poincaré duality theorem. See $\S 20.5$ for details. It illustrates the use of the functor $r$ ! from spectra over $B$ to spectra, which collapses sections to a point, and its proof features a comparison between $r_{\text {! }}$ and $r_{*}$; the latter is the "global sections" functor from spectra over $B$ to spectra.

Example 0.0.2 (Poincaré duality). Let $M$ be a smooth closed $n$-manifold and let $S^{\tau}$ denote the spherical fibration obtained from the tangent bundle $\tau$ of $M$ by fiberwise one-point compactification; it is an ex-space over $M$. Let $S_{M}^{n}$ denote the ex-space $S^{n} \times M$; it is a trivial spherical fibration, and $S_{M}^{0}=M \amalg M$. For any spectrum $k$, the "parametrized Atiyah duality theorem" implies an isomorphism

$$
k_{q}\left(M_{+}\right) \cong\left(k \wedge S^{\tau}\right)_{M}^{-q}\left(S_{M}^{0}\right) .
$$

Thus the parametrized theory implicitly gives a direct global homotopical construction of a version of "generalized cohomology with local coefficients" that gives Poincaré duality for any representing spectrum $k$, without orientation hypotheses. Now let $k$ be a commutative ring spectrum. The Thom space of $\tau$ is $T \tau=r_{!} S^{\tau}$, $r: M \longrightarrow *$, while $r ! S_{M}^{n}=\Sigma^{n}\left(M_{+}\right)$. By definition, a $k$-orientation of $M$ is a cohomology class $\mu \in k^{n}(T \tau)$ that restricts to a unit of $k^{n}\left(S^{n}\right) \cong k^{0}\left(S^{0}\right)$ on each fiber. We may view $\mu$ as a map

$$
\mu: r ! S^{\tau} \longrightarrow \Sigma^{n} k
$$

with adjoint

$$
\tilde{\mu}: S^{\tau} \longrightarrow r^{*}\left(\Sigma^{n} k\right) \cong k \wedge S_{M}^{n} .
$$

Smashing with $k$ and using the product $k \wedge k \longrightarrow k$, we find that $\tilde{\mu}$ induces a map

$$
\bar{\mu}: k \wedge S^{\tau} \longrightarrow k \wedge S_{M}^{n}
$$

of $k$-module spectra over $M$. The unit property of $\mu$ is exactly the statement that $\bar{\mu}$ restricts to an equivalence on each fiber, and this implies that $\bar{\mu}$ is an equivalence of spectra over $M$. This is a precise mathematical formulation of the intuition that the tangent bundle of a $k$-orientable manifold is stably trivial when viewed through the eyes of $k$-theory. The equivalence $\bar{\mu}$ induces an "untwisting" isomorphism

$$
\left(k \wedge S^{\tau}\right)_{M}^{-q}\left(S_{M}^{0}\right) \cong k^{n-q}\left(M_{+}\right)
$$

This completes a proof of the Poincaré duality theorem as a formal implication of parametrized Atiyah duality and the definition of an orientation. The proof does not use the Thom isomorphism directly, but the equivalence of spectra $r ! \bar{\mu}$ implies the isomorphism $k_{q}(T \tau) \cong k_{q-n}\left(M_{+}\right)$on passage to homotopy groups, and similarly for cohomology.

Here is another example of something that should be an old result and is intuitively very plausible, but seems to be new. Details are given in $§ 18.6$. 
EXAMPLE 0.0.3. As an application of a relative form of parametrized Atiyah duality, we prove that if $M$ is a smooth closed manifold embedded in $\mathbb{R}^{q}$ and $L$ is a smooth closed submanifold, then $M / L$ is $(q-1)$-dual to the cofiber of the Pontryagin-Thom map $T \nu_{M} \longrightarrow T \nu_{L}$ of Thom spaces, where $\nu_{M}$ and $\nu_{L}$ are the normal bundles of $M$ and $L$.

Model structures. Returning to our discussion of the foundations, we shall of course use model structures wherever we can. However, even in the part of the theory in which model theory works, it does not work as expected. There is an obvious naive model structure on ex-spaces over $B$ in which the weak equivalences, fibrations, and cofibrations are the ex-maps whose maps of total spaces are weak equivalences, fibrations, and cofibrations of spaces in the usual Quillen model structure. This " $q$-model structure" is the natural starting point for the theory, but it turns out to have severe drawbacks that limit its space level utility and bar it from serving as the starting point for the development of a useful spectrum level stable model structure. In fact, it has two opposite drawbacks. First, it has too many cofibrations. In particular, the model theoretic cofibrations need not be cofibrations in the intrinsic homotopical sense. That is, they fail to satisfy the fiberwise homotopy extension property (HEP) defined in terms of parametrized mapping cylinders. This already fails for the sections of cofibrant objects and for the inclusions of cofibrant objects in their cones. Therefore the classical theory of cofiber sequences fails to mesh with the model category structure.

Second, it also has too many fibrations. The fibrant ex-spaces are Serre fibrations, and Serre fibrations are not preserved by fiberwise colimits. Such colimits are preserved by a more restrictive class of fibrations, namely the well-sectioned Hurewicz fibrations, which we call ex-fibrations. Such preservation properties are crucial to resolving the problems with base change functors that we have indicated.

In model category theory, decreasing the number of cofibrations increases the number of fibrations, so that these two problems cannot admit a solution in common. Rather, we require two different equivalent descriptions of our homotopy categories of ex-spaces. First, we have another model structure, the " $q f$-model structure", which has the same weak equivalences as the $q$-model structure but has fewer cofibrations, all of which satisfy the fiberwise HEP. Second, we have a description in terms of the classical theory of ex-fibrations, which does not fit naturally into a model theoretic framework. The former is vital to the development of the stable model structure on parametrized spectra. The latter is vital to the solution of the intrinsic problems with base change functors.

Other foundational issues. Before getting to the issues just discussed, we shall have to resolve various others that also have no nonparametrized analogues. Even the point set topology requires care since function ex-spaces take us out of the category of compactly generated spaces. Equivariance raises further problems, although most of our new foundational work is already necessary nonequivariantly. Passage to the spectrum level raises more serious problems. One main source of difficulty is that the underlying total space functor is too poorly behaved, especially with respect to smash products and fibrations, to give good control of homotopy groups as one passes from parametrized spaces to parametrized spectra. Moreover, since the underlying total space functor does not commute with suspension, it does not give a forgetful functor from parametrized spectra to nonparametrized spectra. 
The resolution of base change problems requires a different set of details on the spectrum level than on the space level.

This theory gives perhaps the first worked example in which a model theoretic approach to derived homotopy categories is intrinsically insufficient and must be blended with a quite different approach even to establish the essential structural features of the derived category. Such a blending of techniques seems essential in analogous sheaf theoretic contexts that have not yet received a modern model theoretic treatment. Even nonequivariantly, the basic results on base change, smash products, and function ex-spaces that we obtain do not appear in the literature. Such results are essential to serious work in parametrized homotopy theory.

Much of our work should have applications beyond the new parametrized theory. The model theory of topological enriched categories has received much less attention in the literature than the model theory of simplicially enriched categories. Despite the seemingly equivalent nature of these variants, the topological situation is actually quite different from the simplicial one, as our applications make clear. In particular, the interweaving of $h$-type and $q$-type model structures that pervades our work seems to have no simplicial counterpart. Such interweaving does also appear in algebraic contexts of model categories enriched over chain complexes, where foundations analogous to ours can be developed. One of our goals is to give a thorough analysis and axiomatization of how this interweaving works in general in topologically enriched model categories.

The foundational issues that we have been discussing occupy the first three parts of this book. Part I gives basic preliminaries, Part II develops unstable parametrized homotopy theory, and Part III develops stable parametrized homotopy theory. The end result of this foundational work may seem intricate, but it gives a very powerful framework in which to study homotopy theory, as we illustrate in the last two parts.

Parametrized duality theory. In Part IV, we develop parametrized duality theory. This has three aspects. First, there is a fiberwise duality theory that leads to a smooth general treatment of transfer maps. There are two ways of thinking about transfer maps. For fibrations in general, they are best thought of as instances of generalized trace maps present in any closed symmetric monoidal category. For bundles, they are best thought of fiberwise, with transfer maps on fibers inserted fiberwise into bundles of spectra. It is not obvious that these give equivalent constructions when both apply, and our fiberwise duality theory makes that comparison transparent.

Second, there is a new kind of parametrized duality theory that was first discovered by Costenoble and Waner. It, rather than fiberwise duality, is the appropriate parametrized analogue of Spanier-Whitehead duality, and it is the kind of duality that is used in the proof of Poincaré duality described in Example 0.0.2. These two notions of duality are quite different. Parametrized sphere spectra are invertible and therefore fiberwise dualizable, but they are not Costenoble-Waner dualizable in general. Parametrized finite cell spectra are Costenoble-Waner dualizable, but they are not fiberwise dualizable in general. The previous sentence hides another subtlety. Finite cell objects in topological model categories such as ours are elusive structures because their fibrant approximations are no longer finite. In our triangulated stable categories, the parametrized finite cell spectra and their retracts do not 
seem to give the objects of a thick subcategory, which is contrary to all previously encountered situations.

More centrally, conceptual understanding of the new duality theory requires the new categorical notion of a closed symmmetric bicategory and a formal duality theory for 1-cells in such a bicategory. Bimodules over varying rings, their derived and brave new counterparts, and parametrized spectra over varying base spaces all give examples. Dual pairs of "base change bimodules" and "base change spectra" encode base change functors in terms of the bicategory operations. These categorical foundations promise to have significant applications in other fields and will be more fully developed elsewhere. The basic treatment here, in Chapter 16, can be read independently of everything else in the book.

Third, there is a way to insert parametrized Atiyah duality fiberwise into bundles of spectra to develop a fiberwise Costenoble-Waner duality theory. The basic change of groups isomorphisms of equivariant stable homotopy theory, namely the generalized Wirthmüller and Adams isomorphisms, are very special cases of our duality theorems, which are already of considerable interest nonequivariantly. These applications depend on a clear and precise definition of a bundle of spectra and an analysis of how such bundles behave homotopically. This notion has appeared sporadically in the literature, although without rigorous foundations. It seems certain to become important.

Parametrized homology and cohomology. The first three parts, and most of the fourth, give reasonably complete treatments of the topics they cover, but Part $\mathrm{V}$ has a different character. Its main focus is the definition of parametrized homology and cohomology theories and the beginning of their study. It seems to us that another book this length could well be written on this topic, which we believe will come to play an increasingly important role in algebraic topology and its applications. We just scratch the surface. In Chapters 20 and 21, we show how to axiomatize and represent parametrized homology and cohomology theories, and we say a little about duality, base change, coefficient systems, products, and the Serre and parametrized Atiyah-Hirzebruch spectral sequences. We give separate treatments of the nonequivariant and equivariant theory for the reader's convenience. While we describe various calculational tools, we do not turn to explicit computations here.

We observe in Chapter 22 that twisted $K$-theory is an example of a particular kind of parametrized cohomology theory, thereby making its associated homology theory precise and making all of the standard tools of algebraic topology readily available. We also explain a Čech local to global (or descent) spectral sequence, the twisted Rothenberg-Steenrod spectral sequence, and a construction of the Eilenberg-Moore spectral sequence, viewed as a parametrized Künneth spectral sequence in the stable homotopy category of spectra over $B$.

Generalizations of Thom spectra. Another topic in Part V is the construction of generalized analogues of Thom spectra. We explain how the parametrized way of thinking leads directly to the construction of new nonparametrized orthogonal ring spectra and thus $E_{\infty}$ ring spectra. Our iterated Thom spectra are examples. The construction is entirely elementary and only uses ex-spaces, not parametrized spectra. We urge the interested reader to turn directly to Chapter 23, since the construction is almost completely independent of everything else in the book. A more 
sophisticated application of parametrized methods constructs the Thom spectrum associated to a map $f: X \longrightarrow B$, where $B$ is the classifying space for some class of bundles or fibrations, by pulling back the universal sphere bundle or fibration spectrum $E$ over $B$ along $f$ and then pushing down along $r: X \longrightarrow *$ to obtain the ordinary spectrum $r_{!} f^{*} E$. We explain the idea briefly, but we do not pursue it here. It is the starting point of work in progress with Andrew Blumberg.

In fact, we have hardly begun the serious study of any of the topics in Part V. There are other areas, such as fixed point theory and Goodwillie calculus, where parametrized theory is expected to play an important role but has not yet been seriously applied due to the lack of firm foundations. It is time for this book to appear, but it is only a beginning. There is a great deal more work to be done in this emerging area of algebraic topology.

History. This project began with unpublished notes of the first author, dating from the summer of 2000 [111]. He put the project aside and returned to it in the fall of 2002, when he was joined by the second author. Some of Parts I and II was originally in a draft of the first author that was submitted and accepted for publication, but was later withdrawn. That draft was correct, but it did not include the " $q f$-model structure", which comes from the second author's $2004 \mathrm{PhD}$ thesis [152]. The first author's notes [111] claimed to construct the stable model structure on parametrized spectra starting from the $q$-model structure on ex-spaces. Following [111], the monograph [78] of $\mathrm{Po} \mathrm{Hu}$ also takes that starting point and makes that claim. The second author realized that, with the obvious definitions, the axioms for the stable model structure cannot be proven from that starting point and that any naive variant would be disconnected with cofiber sequences and other essential needs of a fully worked out theory. His $q f$-model structure is the crucial new ingredient that is used to solve this problem.

The new duality theory of Chapters 16, 17, and 18 was inspired by work of Costenoble and Waner [41]. The applications of Chapter 19 were inspired by Hu's work [78]. The implementation of her results as manifestations of fiberwise Costenoble-Waner duality came as a pleasant surprise.

Thanks. We thank the referee of the partial first version for several helpful suggestions. We thank Gaunce Lewis and Peter Booth for help with the point set topology, Mike Cole for sharing his remarkable insights about model categories, Mike Mandell for much technical help, and Brooke Shipley for an illuminating discussion of different types of generators. We thank Kathleen Lewis for working out the counterexample in Theorem 1.7.1, Victor Ginzburg for giving us the striking Counterexample 11.6.2, Amnon Neeman for giving us the useful Lemma 20.8.4, and Shmuel Weinberger for pointing out Proposition 21.7.4.

We are especially grateful to Kate Ponto for a meticulously careful reading that uncovered many obscurities and infelicities. Needless to say, she is not to blame for those that remain.

Some of the work on this book was done during the second author's visits to the University of Chicago and the Institut Mittag-Leffler and he gratefully acknowledges their hospitality and support. He would also like to thank the members of the homotopy group at the University of Sheffield for useful conversations and a very stimulating environment. 
Finally, we would like to thank the Editorial Committee of the Mathematical Surveys and Monographs series of the AMS for accepting this book for publication unconditionally, while gently suggesting that we add more motivation and "an attempt at more examples" and also suggesting a tentative deadline for us not to meet. The freedom and pressure their decision gave us led directly to our working out and writing up the new material that begins in Chapter 16, despite the preliminary nature of much of it. Nearly all of this work postdates the acceptance of the book on April 13, 2005.

Dedication. On May 17, 2006, Gaunce Lewis died after a lengthy bout with brain cancer. This book is in large part the culmination of a long development of the foundations of equivariant stable homotopy theory that began with Lewis's 1978 Chicago $\mathrm{PhD}$ thesis and his joint work with the first author that appeared in 1986 [98]. His influence on our work will be evident. With sadness, we dedicate this book to his memory.

July 11, 2006 



\section{Part I}

\section{Point-set topology, change functors, and proper actions}





\section{Introduction}

The point-set topology of parametrized spaces is surprisingly subtle. Parametrized mapping spaces are especially delicate, and to have them one must leave the most commonly accepted convenient category of topological spaces. Such issues are dealt with in Chapter 1. Rather than give complete proofs, we shall collate results from the extensive literature on the subject to arrive at the framework that we find most convenient.

While in Chapter 1 we focus on single categories, in Chapter 2 we study "change" functors between categories, focusing on change of base space and change of groups. There are myriads of such functors, and sorting out all of the relationships among them is a thankless task. In fact, from a categorical point of view, a full theory of coherence relating them is well beyond current reach. Analogous compatibility relations in algebraic geometry are well-known to be as important to the applications as they are tedious to prove. We are interested here in the point-set level, preparing the way for our later study of these relations in derived homotopy categories.

Chapter 3 gives foundations for the generalization of parts of our theory from compact Lie groups to general Lie groups. It was already observed by Palais [136] that many results in equivariant homotopy theory can be generalized to Lie groups, or even to locally compact groups, provided that one restricts to proper actions. In the parametrized world, the homotopy theory is captured on fibers. When we restrict to proper actions on base spaces, the fibers have actions by the compact isotropy groups of the base space. So even though our primary interest is in compact Lie groups of equivariance, proper actions on the base space seem to provide the right natural level of generality. We set the stage for such a theory by generalizing various classical results about equivariant homotopy types and equivariant bundles and fibrations to the setting of proper actions by Lie groups. In Part II, we develop foundations for space level parametrized homotopy theory in that generality, but we will not go on to the spectrum level analogue in this book. Little of the material in Chapter 3 is needed in the nonequivariant specialization of our work. 



\section{CHAPTER 1}

\section{The point-set topology of parametrized spaces}

\section{Introduction}

It is well understood that a convenient category of topological spaces should be complete and cocomplete and should be closed cartesian monoidal. That is, it should have all limits and colimits and it should be symmetric monoidal under cartesian products; "closed" means that it should have mapping spaces related in the expected way to cartesian products. With cartesian products replaced by smash products, we have the same criteria for a convenient category of based spaces, but here not even the associativity of the smash product is clear. The same criteria for convenience also apply in the parametrized context, but there are additional ones concerning the relationships between spaces and parametrized spaces. We develop the basic point-set level properties of the category of ex-spaces over a fixed base space $B$ in this chapter.

In $\S 1.1$, we fix our convenient categories of topological spaces. We defer discussion of our choices to $\S 1.5$, and we defer some technical results to $\S 1.6$. The usual category of compactly generated spaces is not adequate for our work, and we shall see later that the interplay between model structures and the relevant convenient categories is quite subtle. In $\S 1.2$, we give basic facts about based and unbased topologically bicomplete categories. This gives the language that is needed to describe the good formal properties of the various categories in which we shall work. We discuss convenient categories of ex-spaces in $\S 1.3$, and we discuss convenient categories of ex-G-spaces in $\S 1.4$.

As a matter of recovery of lost folklore, $\S 1.7$ is an appendix, the substance of which is due to Kathleen Lewis. It is only at her insistence that she is not named as its author. It documents the nonassociativity of the smash product in the ordinary category of based spaces, as opposed to the category of based $k$-spaces. When writing the historical paper [120], the first author came across several 1950's references to this phenomenon, including an explicit, but unproven, counterexample in a 1958 paper of Puppe [138]. However, we know of no reference that gives details, and we feel that this should be documented in the modern literature.

We are very grateful to Gaunce Lewis for an extended correspondence and many details about the material of this chapter, but he is not to be blamed for the point of view that we have taken. We are also much indebted to Peter Booth. He is the main pioneer of the theory of fibered mapping spaces and function ex-spaces (see [16-18]), and he sent us several detailed proofs about them.

\subsection{Convenient categories of topological spaces}

We recall the following by now standard definitions. 
Definition 1.1.1. Let $B$ be a space and $A$ a subset. Let $f: K \longrightarrow B$ run over all continuous maps from compact Hausdorff spaces $K$ into $B$.

(i) $A$ is compactly closed if each $f^{-1}(A)$ is closed.

(ii) $B$ is weak Hausdorff if each $f(K)$ is closed.

(iii) $B$ is a $k$-space if each compactly closed subset is closed.

(iv) $B$ is compactly generated if it is a weak Hausdorff $k$-space.

Let $\mathscr{T}_{o p}$ be the category of all topological spaces and let $\mathscr{K}, w \mathscr{H}$, and $\mathscr{U}=$ $\mathscr{K} \cap w \mathscr{H}$ be its full subcategories of $k$-spaces, weak Hausdorff spaces, and compactly generated spaces. The $k$-ification functor $k: \mathscr{T} o p \longrightarrow \mathscr{K}$ assigns to a space $X$ the same set with the finer topology that is obtained by requiring all compactly closed subsets to be closed. It is right adjoint to the inclusion $\mathscr{K} \longrightarrow \mathscr{T}$ op, and it restricts to a right adjoint to the inclusion $\mathscr{U} \longrightarrow w \mathscr{H}$. The weak Hausdorffication functor $w: \mathscr{T} o p \longrightarrow w \mathscr{H}$ assigns to a space $X$ its maximal weak Hausdorff quotient. It is left adjoint to the inclusion $w \mathscr{H} \longrightarrow \mathscr{T} o p$, and it restricts to a left adjoint to the inclusion $\mathscr{U} \longrightarrow \mathscr{K}$.

From now on, we work in $\mathscr{K}$, implicitly $k$-ifying any space that is not a $k$-space to begin with. In particular, products and function spaces are understood to be $k$-ified. With this convention, $B$ is weak Hausdorff if and only if the diagonal map embeds it as a closed subspace of $B \times B$. Like $\mathscr{U}$, the category $\mathscr{K}$ is closed cartesian monoidal. This means that it has function spaces $\operatorname{Map}(X, Y)$ with homeomorphisms

$$
\operatorname{Map}(X \times Y, Z) \cong \operatorname{Map}(X, \operatorname{Map}(Y, Z)) \text {. }
$$

This was proven by Vogt [166], who uses the term compactly generated for our $k$-spaces. See also [174]. (An early unpublished preprint by Clark [33] also showed this, and an exposition of ex-spaces based on [33] was given by Booth [17]).

We have concomitant categories $\mathscr{K}_{*}$ and $\mathscr{U}_{*}$ of based spaces in $\mathscr{K}$ and in $\mathscr{U}$. We generally write $\mathscr{T}$ for $\mathscr{U}_{*}$ to mesh with a number of relevant earlier papers. Using duplicative notations, we write $\operatorname{Map}(X, Y)$ for the space $\mathscr{K}(X, Y)$ of maps $X \longrightarrow Y$ and $F(X, Y)$ for the based space $\mathscr{K}_{*}(X, Y)$ of based maps $X \longrightarrow Y$ between based spaces. Both $\mathscr{K}_{*}$ and $\mathscr{T}$ are closed symmetric monoidal categories under $\wedge$ and $F[94,166,174]$. This means that the smash product is associative, commutative, and unital up to coherent natural isomorphism and that $\wedge$ and $F$ are related by the usual adjunction homeomorphism

$$
F(X \wedge Y, Z) \cong F(X, F(Y, Z)) .
$$

\subsection{Topologically bicomplete categories and ex-objects}

We need some standard and some not quite so standard categorical language. All of our categories $\mathscr{C}$ will be topologically enriched, with the enrichment given by a topology on the underlying set of morphisms. We therefore agree to write $\mathscr{C}(X, Y)$ for the space of morphisms $X \longrightarrow Y$ in $\mathscr{C}$. Enriched category theory would have us distinguish notationally between morphism spaces and morphism sets, but we shall not do that. A topological category $\mathscr{C}$ is said to be topologically bicomplete if, in addition to being bicomplete in the usual sense of having all limits and colimits, it is bitensored in the sense that it is tensored and cotensored over $\mathscr{K}$. We shall denote the tensors and cotensors by $X \times K$ and $\operatorname{Map}(K, X)$ for a space $K$ and an object $X$ of $\mathscr{C}$. The defining adjunction homeomorphisms are

$$
\mathscr{C}(X \times K, Y) \cong \mathscr{K}(K, \mathscr{C}(X, Y)) \cong \mathscr{C}(X, \operatorname{Map}(K, Y)) .
$$


By the Yoneda lemma, these have many standard implications. For example,

$$
X \times * \cong X \quad \text { and } \quad \operatorname{Map}(*, Y) \cong Y,
$$

(1.2.3) $X \times(K \times L) \cong(X \times K) \times L$ and $\operatorname{Map}(K, \operatorname{Map}(L, X)) \cong \operatorname{Map}(K \times L, X)$.

We say that a bicomplete topological category $\mathscr{C}$ is based if the unique map from the initial object $\emptyset$ to the terminal object $*$ is an isomorphism. In that case, $\mathscr{C}$ is enriched in the category $\mathscr{K}_{*}$ of based $k$-spaces, the basepoint of $\mathscr{C}(X, Y)$ being the unique map that factors through $*$. We then say that $\mathscr{C}$ is based topologically bicomplete if it is tensored and cotensored over $\mathscr{K}_{*}$. We denote the tensors and cotensors by $X \wedge K$ and $F(K, X)$ for a based space $K$ and an object $X$ of $\mathscr{C}$. The defining adjunction homeomorphisms are

$$
\mathscr{C}(X \wedge K, Y) \cong \mathscr{K}_{*}(K, \mathscr{C}(X, Y)) \cong \mathscr{C}(X, F(K, Y)) .
$$

The based versions of (1.2.2) and (1.2.3) are

$$
\begin{gathered}
X \wedge S^{0} \cong X \quad \text { and } F\left(S^{0}, Y\right) \cong Y, \\
X \wedge(K \wedge L) \cong(X \wedge K) \wedge L \text { and } F(K, F(L, X)) \cong F(K \wedge L, X) .
\end{gathered}
$$

Although not essential to our work, a formal comparison between the based and unbased notions of bicompleteness is illuminating. The following result allows us to interpret topologically bicomplete to mean based topologically bicomplete whenever $\mathscr{C}$ is based, a convention that we will follow throughout.

Proposition 1.2.7. Let $\mathscr{C}$ be a based and bicomplete topological category. Then $\mathscr{C}$ is topologically bicomplete if and only if it is based topologically bicomplete.

Proof. Suppose given tensors and cotensors for unbased spaces $K$ and write them as $X \ltimes K$ and $\operatorname{Map}(K, X)_{*}$ as a reminder that they take values in a based category. We obtain tensors and cotensors $X \wedge K$ and $F(K, X)$ for based spaces $K$ as the pushouts and pullbacks displayed in the respective diagrams
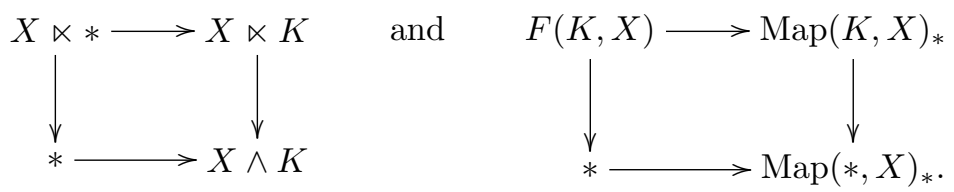

Conversely, given tensors and cotensors $X \wedge K$ and $F(K, X)$ for based spaces $K$, we obtain tensors and cotensors $X \ltimes K$ and $\operatorname{Map}(K, X)_{*}$ for unbased spaces $K$ by setting

$$
X \ltimes K=X \wedge K_{+} \quad \text { and } \operatorname{Map}(K, X)_{*}=F\left(K_{+}, X\right),
$$

where $K_{+}$is the union of $K$ and a disjoint basepoint.

As usual, for any category $\mathscr{C}$ and object $B$ in $\mathscr{C}$, we let $\mathscr{C} / B$ denote the category of objects over $B$. An object $X=(X, p)$ of $\mathscr{C} / B$ consists of a total object $X$ together with a projection map $p: X \longrightarrow B$ to the base object $B$. The morphisms of $\mathscr{C} / B$ are the maps of total objects that commute with the projections.

Proposition 1.2.8. If $\mathscr{C}$ is a topologically bicomplete category, then so is $\mathscr{C} / B$. 
Proof. The product of objects $Y_{i}$ over $B$, denoted $\times_{B} Y_{i}$, is constructed by taking the pullback of the product of the projections $Y_{i} \longrightarrow B$ along the diagonal $B \longrightarrow \times_{i} B$. Pullbacks and arbitrary colimits of objects over $B$ are constructed by taking pullbacks and colimits on total objects and giving them the induced projections. General limits are constructed as usual from products and pullbacks. If $X$ is an object over $B$ and $K$ is a space, then the tensor $X \times{ }_{B} K$ is just the tensor $X \times K$ in $\mathscr{C}$ together with the projection $X \times K \longrightarrow B \times * \cong B$ induced by the projection of $X$ and the projection of $K$ to a point. Note that this makes sense even though the tensor $\times$ in $\mathscr{C}$ need have nothing to do with cartesian products in general; see Remark 1.2.10 below. The cotensor $\operatorname{Map}_{B}(K, X)$ is the pullback of the diagram

$$
B \stackrel{\iota}{\longrightarrow} \operatorname{Map}(K, B)<\operatorname{Map}(K, X)
$$

where $\iota$ is the adjoint of $B \times K \longrightarrow B \times * \cong B$.

The terminal object in $\mathscr{C} / B$ is $\left(B\right.$,id). Let $\mathscr{C}_{B}$ denote the category of based objects in $\mathscr{C} / B$, that is, the category of objects under $(B$,id) in $\mathscr{C} / B$. An object $X=(X, p, s)$ in $\mathscr{C}_{B}$, which we call an ex-object over $B$, consists of on object $(X, p)$ over $B$ together with a section $s: B \longrightarrow X$. We can therefore think of the ex-objects as retract diagrams

$$
B \stackrel{s}{\longrightarrow} X \stackrel{p}{\longrightarrow} B .
$$

The terminal object in $\mathscr{C}_{B}$ is $\left(B\right.$,id, id), which we denote by $*_{B}$; it is also an initial object. The morphisms in $\mathscr{C}_{B}$ are the maps of total objects $X$ that commute with the projections and sections.

Proposition 1.2.9. If $\mathscr{C}$ is a topologically bicomplete category, then the category $\mathscr{C}_{B}$ is based topologically bicomplete.

Proof. The coproduct of objects $Y_{i} \in \mathscr{C}_{B}$, which we shall refer to as the "wedge over $B$ " of the $Y_{i}$ and denote by $\vee_{B} Y_{i}$, is the coequalizer of the maps $B \longrightarrow \amalg Y_{i}$ given by the sections of the $Y_{i}$, that is, the pushout of the coproduct $\amalg B \longrightarrow \amalg Y_{i}$ of the sections along the codiagonal $\amalg_{i} B \longrightarrow B$; it is given the evident induced projection. Dually, the product of the $Y_{i}$ is constructed in $\mathscr{C} / B$ and given the evident induced section. Pushouts and pullbacks in $\mathscr{C}_{B}$ are constructed by taking pushouts and pullbacks on total objects and giving them the evident induced sections and projections. The tensor $X \wedge_{B} K$ of $X=(X, p, s)$ and a based space $K$ is the pushout of the diagram

$$
B \longleftarrow(X \times *) \cup_{B}(B \times K) \longrightarrow X \times K,
$$

where the right map is induced by the basepoint of $K$ and the section of $X$. The cotensor $F_{B}(K, X)$ is the pullback of the diagram

$$
B \stackrel{s}{\longrightarrow} X<\varepsilon \operatorname{Map}_{B}(K, X),
$$

where $\varepsilon$ is evaluation at the basepoint of $K$, that is, the adjoint of the evident map $X \times K \longrightarrow X$ over $B$.

REMARK 1.2.10. Notationally, it may be misleading to write $X \times K$ and $X \wedge K$ for unbased and based tensors. It conjures up associations that are appropriate for the examples on hand but that are inappropriate in general. The tensors in a topologically bicomplete category $\mathscr{C}$ may bear very little relationship to cartesian 
products or smash products. The standard uniform notation would be $X \otimes K$. However, we have too many relevant examples to want a uniform notation. In particular, as in the proofs above, we generally use the notations $X \times_{B} K$ and $X \wedge_{B} K$ for tensors in the parametrized context, and there a notation such as $X \otimes_{B} K$ would conjure up its own misleading associations. Actually, we shall later use the notations $X \times K$ and $X \times{ }_{B} K$ interchangeably for tensors in $\mathscr{C} / B$, since $X \times_{B} K$ is just the tensor $X \times K$ in $\mathscr{C}$ equipped with a projection. The distinguishing notation $X \wedge_{B} K$ for tensors in $\mathscr{C}_{B}$ is more essential since it serves as a useful reminder of the fiberwise nature of the smash product.

\subsection{Convenient categories of ex-spaces}

We need a convenient topologically bicomplete category of ex-spaces ${ }^{1}$ over a space $B$, where "convenient" requires that we have smash product and function exspace functors $\wedge_{B}$ and $F_{B}$ under which our category is closed symmetric monoidal. Denoting the unit $B \times S^{0}$ of $\wedge_{B}$ by $S_{B}^{0}$, a formal argument shows that we will then have isomorphisms

$$
X \wedge_{B} K \cong X \wedge_{B}\left(S_{B}^{0} \wedge_{B} K\right) \text { and } F_{B}(K, Y) \cong F_{B}\left(S_{B}^{0} \wedge_{B} K, Y\right)
$$

relating tensors and cotensors to the smash product and function ex-space functors. In particular, $S_{B}^{0} \wedge_{B} K$ is just the product ex-space $B \times K$ with section determined by the basepoint of $K$.

The point-set topology leading to such a convenient category is delicate, and there are quite a few papers devoted to this subject. They do not give exactly what we need, but they come close enough that we shall content ourselves with a summary. It is based on the papers [16-18,21,22,96] of Booth, Booth and Brown, and Lewis; see also James [83,84].

We assume once and for all that our base spaces $B$ are in $\mathscr{U}$. We allow the total spaces $X$ of spaces over $B$ to be in $\mathscr{K}$. We let $\mathscr{K} / B$ and $\mathscr{U} / B$ denote the categories of spaces over $B$ with total spaces in $\mathscr{K}$ or $\mathscr{U}$. Similarly, we let $\mathscr{K}_{B}$ and $\mathscr{U}_{B}$ denote the respective categories of ex-spaces over $B$.

Both the separation property on $B$ and the lack of a separation property on

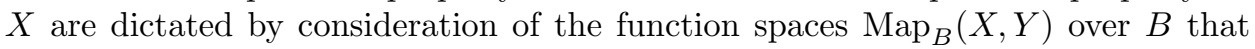
we shall define shortly. These are only known to exist when $B$ is weak Hausdorff. However, even when $B, X$ and $Y$ are weak Hausdorff, $\operatorname{Map}_{B}(X, Y)$ is generally not weak Hausdorff. This motivates us to drop the weak Hausdorff condition on total spaces and to focus on $\mathscr{K}_{B}$ as our preferred convenient category of ex-spaces over $B$. The cofibrant ex-spaces in our $q$-type model structures are weak Hausdorff, hence this separation property is recovered upon cofibrant approximation. Therefore, use of $\mathscr{K}$ can be viewed as scaffolding in the foundations that can be removed when doing homotopical work.

We topologize the set of ex-maps $X \longrightarrow Y$ as a subspace of the space $\mathscr{K}(X, Y)$ of maps of total spaces. It is based, with basepoint the unique map that factors through $*_{B}$. Therefore the category $\mathscr{K}_{B}$ is enriched over $\mathscr{K}_{*}$. It is based topologically bicomplete by Proposition 1.2.8. Recall that we write $\times_{B} Y_{i}$ and $\vee_{B} Y_{i}$ for products and wedges over $B$. We also write $Y /{ }_{B} X$ for quotients, which are

\footnotetext{
${ }^{1}$ Presumably the prefix "ex" stands for "cross", as in "cross section". The unlovely term "exspace" has been replaced in some recent literature by "fiberwise pointed space". Used repetitively, that is not much of an improvement. The term "retractive space" has also been used.
} 
understood to be pushouts of diagrams $*_{B} \longleftarrow X \longrightarrow Y$. We give a more concrete description of the tensors and cotensors in $\mathscr{K} / B$ and $\mathscr{K}_{B}$ specified in Propositions 1.2.8 and 1.2.9.

For a space $X$ over $B$, we let $X_{b}$ denote the fiber $p^{-1}(b)$. If $X$ is an ex-space, then $X_{b}$ has the basepoint $s(b)$.

Definition 1.3.2. Let $X$ be a space over $B$ and $K$ be a space. Define $X \times{ }_{B} K$ to be the space $X \times K$ with projection the product of the projections $X \longrightarrow B$ and $K \longrightarrow *$. Define $\operatorname{Map}_{B}(K, X)$ to be the subspace of $\operatorname{Map}(K, X)$ consisting of those maps $f: K \longrightarrow X$ that factor through some fiber $X_{b}$; the projection sends such a $\operatorname{map} f$ to $b$.

Definition 1.3.3. Let $X$ be an ex-space over $B$ and $K$ be a based space. Define $X \wedge_{B} K$ to be the quotient of $X \times_{B} K$ obtained by taking fiberwise smash products, so that $\left(X \wedge_{B} K\right)_{b}=X_{b} \wedge K$; the basepoints of fibers prescribe the section. Define $F_{B}(K, X)$ to be the subspace of $\operatorname{Map}_{B}(K, X)$ consisting of the based maps $K \longrightarrow X_{b} \subset X$ for some $b \in B$, so that $F_{B}(K, X)_{b}=F\left(K, X_{b}\right)$; the section sends $b$ to the constant map at $s(b)$.

The category $\mathscr{K} / B$ is closed cartesian monoidal under the fiberwise cartesian product $X \times_{B} Y$ and the function space $\operatorname{Map}_{B}(X, Y)$ over $B$. The category $\mathscr{K}_{B}$ is closed symmetric monoidal under the fiberwise smash product $X \wedge_{B} Y$ and the function ex-space $F_{B}(X, Y)$. We recall the relevant definitions.

Definition 1.3.4. For spaces $X$ and $Y$ over $B, X \times_{B} Y$ is the pullback of the projections $p: X \longrightarrow B$ and $q: Y \longrightarrow B$, with the evident projection $X \times{ }_{B} Y \longrightarrow B$. When $X$ and $Y$ have sections $s$ and $t$, their pushout $X \vee_{B} Y$ specifies the coproduct, or wedge, of $X$ and $Y$ in $\mathscr{K}_{B}$, and $s$ and $t$ induce a map $X \vee_{B} Y \longrightarrow X \times_{B} Y$ over $B$ that sends $x$ and $y$ to $(x, t p(x))$ and $(s q(y), y)$. Then $X \wedge_{B} Y$ is the pushout in $\mathscr{K} / B$ displayed in the diagram

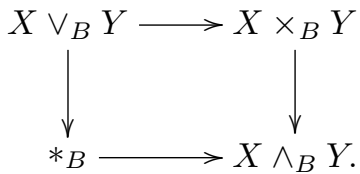

This arranges that $\left(X \wedge_{B} Y\right)_{b}=X_{b} \wedge Y_{b}$, and the section and projection are evident.

The following result is $[22,8.3]$.

Proposition 1.3.5. If $X$ and $Y$ are weak Hausdorff ex-spaces over $B$, then so is $X \wedge_{B} Y$. That is, $\mathscr{U}_{B}$ is closed under $\wedge_{B}$.

Function objects are considerably more subtle, and we need a preliminary definition in order to give the cleanest description.

Definition 1.3.6. For a space $Y \in \mathscr{K}$, define the partial map classifier $\tilde{Y}$ to be the union of $Y$ and a disjoint point $\omega$, with the topology whose closed subspaces are $\tilde{Y}$ and the closed subspaces of $Y$. The point $\omega$ is not a closed subset, and $\tilde{Y}$ is not weak Hausdorff. The name "partial map classifier" comes from the observation that, for any space $X$, pairs $(A, f)$ consisting of a closed subset $A$ of $X$ and a continuous map $f: A \longrightarrow Y$ are in bijective correspondence with continuous maps $\tilde{f}: X \longrightarrow \tilde{Y}$. Given $(A, f), \tilde{f}$ restricts to $f$ on $A$ and sends $X-A$ to $\omega$; given $\tilde{f}$, $(A, f)$ is $\tilde{f}^{-1}(Y)$ and the restriction of $\tilde{f}$. 
Definition 1.3.7. Let $p: X \longrightarrow B$ and $q: Y \longrightarrow B$ be spaces over $B$. Define $\operatorname{Map}_{B}(X, Y)$ to be the pullback displayed in the diagram

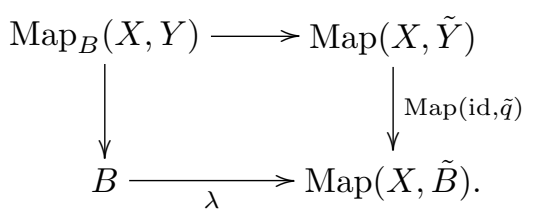

Here $\lambda$ is the adjoint of the map $X \times B \longrightarrow \tilde{B}$ that corresponds to the composite of the inclusion $\operatorname{Graph}(p) \subset X \times B$ and the projection $X \times B \longrightarrow B$ to the second coordinate. The graph of $p$ is the inverse image of the diagonal under $p \times$ id: $X \times B \longrightarrow B \times B$, and the assumption that $B$ is weak Hausdorff ensures that it is a closed subset of $X \times B$, as is needed for the definition to make sense. Explicitly, $\lambda(b)$ sends $X_{b}$ to $b$ and sends $X-X_{b}$ to the point $\omega \in \tilde{B}$.

This definition gives one reason that we require the base spaces of ex-spaces to be weak Hausdorff. On fibers, $\operatorname{Map}_{B}(X, Y)_{b}=\operatorname{Map}\left(X_{b}, Y_{b}\right)$. The space of sections of $\operatorname{Map}_{B}(X, Y)$ is $\mathscr{K} / B(X, Y)$. We have (categorically equivalent) adjunctions

$$
\begin{aligned}
& \operatorname{Map}_{B}\left(X \times_{B} Y, Z\right) \cong \operatorname{Map}_{B}\left(X, \operatorname{Map}_{B}(Y, Z)\right), \\
& \mathscr{K} / B\left(X \times_{B} Y, Z\right) \cong \mathscr{K} / B\left(X, \operatorname{Map}_{B}(Y, Z)\right) .
\end{aligned}
$$

These results are due to Booth [16-18], but we follow [21] and [22]; $\S 7$ of the first of these and $\S 8$ of the second explain details in the categories in which we are working; see also [83, II $\S 9]$, and [96].

As we have already mentioned, $\operatorname{Map}_{B}(X, Y)$ need not be weak Hausdorff even when $X$ and $Y$ are. Some examples are given in $[21,5.3]$ and $[96,1.7]$. The question of when $\operatorname{Map}_{B}(X, Y)$ is Hausdorff or weak Hausdorff was studied in [21, §5] and later in $[83,84]$, but the definitive criterion was given by Lewis $[96,1.5]$.

Proposition 1.3.10. Consider a fixed map $p: X \longrightarrow B$ and varying maps $q: Y \longrightarrow B$, where $X$ and the $Y$ are weak Hausdorff. The map $p$ is open if and only if the space $\operatorname{Map}_{B}(X, Y)$ is weak Hausdorff for all $q$.

Proposition 1.3.11. If $p: X \longrightarrow B$ and $q: Y \longrightarrow B$ are Hurewicz fibrations, then the projections $X \times{ }_{B} Y \longrightarrow B$ and $\operatorname{Map}_{B}(X, Y) \longrightarrow B$ are Hurewicz fibrations. The second statement is false with Hurewicz fibrations replaced by Serre fibrations.

Proof. The statement about $X \times_{B} Y$ is clear. The statements about $\operatorname{Map}_{B}(X, Y)$ are due to Booth $[16,6.1]$ or, in the present formulation [17, 3.4]; see also [83, 23.17].

Definition 1.3.12. For ex-spaces $X$ and $Y$ over $B$, define $F_{B}(X, Y)$ to be the subspace of $\operatorname{Map}_{B}(X, Y)$ that consists of the points that restrict to based maps $X_{b} \longrightarrow Y_{b}$ for each $b \in B$; the section sends $b$ to the constant map from $X_{b}$ to the basepoint of $Y_{b}$. Formally, $F_{B}(X, Y)$ is the pullback displayed in the diagram

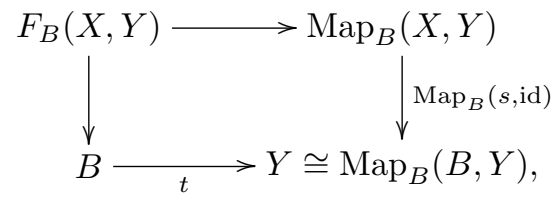

where $s$ and $t$ are the sections of $X$ and $Y$. 
The space of maps $S_{B}^{0} \longrightarrow F_{B}(X, Y)$ is $\mathscr{K}_{B}(X, Y)$, and we have adjunctions

$$
\begin{aligned}
& F_{B}\left(X \wedge_{B} Y, Z\right) \cong F_{B}\left(X, F_{B}(Y, Z)\right), \\
& \mathscr{K}_{B}\left(X \wedge_{B} Y, Z\right) \cong \mathscr{K}_{B}\left(X, F_{B}(Y, Z)\right) .
\end{aligned}
$$

Proposition 1.3.10 implies the following analogue of Proposition 1.3.5.

Proposition 1.3.15. If $X$ and $Y$ are weak Hausdorff ex-spaces over $B$ and $X \longrightarrow B$ is an open map, then $F_{B}(X, Y)$ is weak Hausdorff.

We record the following analogue of Proposition 1.3.11. The second part is again due to Booth, who sent us a detailed write-up. The argument is similar to his proofs in $[16,6.1(\mathrm{i})]$ or $[17,3.4]$, but a little more complicated, and a general result of the same form is given by Morgan [130].

Proposition 1.3.16. If $X$ and $Y$ are ex-spaces over $B$ whose sections are Hurewicz cofibrations and whose projections are Hurewicz fibrations, then the projections of $X \wedge_{B} Y$ and $F_{B}(X, Y)$ are Hurewicz fibrations.

\subsection{Convenient categories of ex- $G$-spaces}

The discussion just given generalizes readily to the equivariant context. Let $G$ be a compactly generated topological group. Subgroups of $G$ are understood to be closed. Let $B$ be a compactly generated $G$-space (with $G$ acting from the left). We consider $G$-spaces over $B$ and ex- $G$-spaces $(X, p, s)$. The total space $X$ is a $G$-space in $\mathscr{K}$, and the section and projection are $G$-maps. The fiber $X_{b}$ is a based $G_{b}$-space with $G_{b}$-fixed basepoint $s(b)$, where $G_{b}$ is the isotropy group of $b$.

Recall from [105, II $\S 1$ the distinction between the category $\mathscr{K}_{G}$ of $G$-spaces and nonequivariant maps and the category $G \mathscr{K}$ of $G$-spaces and equivariant maps; the former is enriched over $G \mathscr{K}$, the latter over $\mathscr{K}$. We have a similar dichotomy on the ex-space level. Here we have a conflict of notation with our notation for categories of ex-spaces, and we agree to let $\mathscr{K}_{G, B}$ denote the category whose objects are the ex- $G$-spaces over $B$ and whose morphisms are the maps of underlying ex-spaces over $B$, that is, the maps $f: X \longrightarrow Y$ such that $f \circ s=t$ and $q \circ f=p$. Henceforward, we call these maps "arrows" to distinguish them from $G$-maps, which we often abbreviate to maps. For $g \in G, g f$ is also an arrow of ex-spaces over $B$, so that $\mathscr{K}_{G, B}(X, Y)$ is a $G$-space. Moreover, composition is given by $G$-maps

$$
\mathscr{K}_{G, B}(Y, Z) \times \mathscr{K}_{G, B}(X, Y) \longrightarrow \mathscr{K}_{G, B}(X, Z) .
$$

We obtain the category $G \mathscr{K}_{B}$ by restricting to $G$-maps $f$, and we may view it as the $G$-fixed point category of $\mathscr{K}_{G, B}$. Of course, $G \mathscr{K}_{B}(X, Y)$ is a space and not a $G$-space. The pair $\left(\mathscr{K}_{G, B}, G \mathscr{K}_{B}\right)$ is an example of a $G$-category, a structure that we shall recall formally in $\S 10.2$.

Since $*_{B}$ is an initial and terminal object in both $\mathscr{K}_{G, B}$ and $G \mathscr{K}_{B}$, their morphism spaces are based. Thus $\mathscr{K}_{G, B}$ is enriched over the category $G \mathscr{K}_{*}$ of based $G$-spaces and $G \mathscr{K}_{B}$ is enriched over $\mathscr{K}_{*}$. As discussed in [105, II.1.3], if we were to think exclusively in enriched category terms, we would resolutely ignore the fact that the $G$-spaces $\mathscr{K}_{G, B}(X, Y)$ have elements (arrows), thinking of these $G$-spaces as enriched hom objects. From that point of view, $G \mathscr{K}_{B}$ is the "underlying category" of our enriched $G$-category. While we prefer to think of $\mathscr{K}_{G, B}$ as a category, it must be kept in mind that it is not a very well-behaved one. For example, because its arrows are not equivariant, it fails to have limits or colimits. 
In contrast, the category $G \mathscr{K}_{B}$ is bicomplete. Its limits and colimits are constructed in $\mathscr{K}_{B}$ and then given induced $G$-actions. The category $\mathscr{K}_{G, B}$, although not bicomplete, is tensored and cotensored over $\mathscr{K}_{G, *}$. The tensors $X \wedge_{B} K$ and cotensors $F_{B}(K, X)$ are constructed in $\mathscr{K}_{B}$ and then given induced $G$-actions. They satisfy the adjunctions

$$
\mathscr{K}_{G, B}\left(X \wedge_{B} K, Y\right) \cong \mathscr{K}_{G, *}\left(K, \mathscr{K}_{G, B}(X, Y)\right) \cong \mathscr{K}_{G, B}\left(X, F_{B}(K, Y)\right)
$$

and, by passage to fixed points,

$$
G \mathscr{K}_{B}\left(X \wedge_{B} K, Y\right) \cong G \mathscr{K}_{*}\left(K, \mathscr{K}_{G, B}(X, Y)\right) \cong G \mathscr{K}_{B}\left(X, F_{B}(K, Y)\right) .
$$

It follows that $G \mathscr{K}_{B}$ is tensored and cotensored over $G \mathscr{K}_{*}$ and, in particular, is topologically bicomplete.

The category $\mathscr{K}_{G, B}$ is closed symmetric monoidal via the fiberwise smash products $X \wedge_{B} Y$ and function objects $F_{B}(X, Y)$. Again, these are defined in $\mathscr{K}_{B}$ and then given induced $G$-actions. The unit is the ex-G-space $S_{B}^{0}=B \times S^{0}$. The category $G \mathscr{K}_{B}$ inherits a structure of closed symmetric monoidal category. We have homeomorphisms of based $G$-spaces

$$
\mathscr{K}_{G, B}\left(X \wedge_{B} Y, Z\right) \cong \mathscr{K}_{G, B}\left(X, F_{B}(Y, Z)\right)
$$

and, by passage to $G$-fixed points, homeomorphisms of based spaces

$$
G \mathscr{K}_{B}\left(X \wedge_{B} Y, Z\right) \cong G \mathscr{K}_{B}\left(X, F_{B}(Y, Z)\right) .
$$

The first of these implies an associated homeomorphism of ex- $G$-spaces

$$
F_{B}\left(X \wedge_{B} Y, Z\right) \cong F_{B}\left(X, F_{B}(Y, Z)\right)
$$

REMARK 1.4.6. There is an alternative parametrized view of equivariance that is important in torsor theory but that we shall not study. It focuses on "topological groups $G_{B}$ over $B$ " and " $G_{B}$-spaces $E$ over $B$ ", where $G_{B}$ is a space over a nonequivariant space $B$ with a product $G_{B} \times_{B} G_{B} \longrightarrow G_{B}$ that restricts on fibers to the products of topological groups $G_{b}$ and $E$ is a space over $B$ with an action $G_{B} \times_{B} E \longrightarrow E$ that restricts on fibers to actions $G_{b} \times E_{b} \longrightarrow E_{b}$. That theory intersects ours in the special case $G_{B}=G \times B$ for a topological group $G$. Since, at least implicitly, all of our homotopy theory is done fiberwise, our work adapts without essential difficulty to give a development of parametrized equivariant homotopy theory in that context.

\subsection{Philosophical comments on the point-set topology}

We would have preferred to work in $\mathscr{U}$ rather than $\mathscr{K}$, since there are many counterexamples which reveal the pitfalls of working without a separation property. However, as we explained in $§ 1.3$, several inescapable facts about ex-spaces forced us out of that convenient category.

Philosophically, we can justify a preference for $\mathscr{K}$ over $\mathscr{U}$ by remarking that the weak Hausdorffication functor $w$ is so poorly behaved that we prefer to minimize its use. In $\mathscr{U}$, colimits must be constructed by first constructing them in $\mathscr{K}$ and then applying the functor $w$, which changes the underlying point set and loses homotopical control. However, this justification would be more persuasive were it not that colimits in $\mathscr{K}$ that are not colimits in $\mathscr{U}$ can already be quite badly behaved topologically. For example, $w$ itself is a colimit construction in $\mathscr{K}$. We 
describe a relevant situation in which colimits behave better in $\mathscr{U}$ than in $\mathscr{K}$ in Remark 1.6.4 below.

More persuasively, $w$ is a formal construction that only retains formal control because both colimits and the functor $w$ are left adjoints. We have encountered right adjoints constructed in $\mathscr{K}$ that do not preserve the weak Hausdorff property when restricted to $\mathscr{U}$, and in such situations we cannot apply $w$ without losing the adjunction. In fact, when restricted to $\mathscr{U}$, the relevant left adjoints do not commute with colimits and so cannot be left adjoints there. An obvious advantage of $\mathscr{K}$ is that $\mathscr{U}$ sits inside it, so that we can use $\mathscr{K}$ when it is needed, but can restrict to the better behaved category $\mathscr{U}$ whenever possible. Actually, as we have seen, the situation is more subtle than a simple dichotomy. In order to have parametrized mapping spaces, it was essential to combine use of the two categories, requiring base spaces to be in $\mathscr{U}$ but allowing total spaces to be in $\mathscr{K}$.

Lewis proposed alternative convenient categories of ex-spaces to those that we have chosen. As we have seen, the cartesian monoidal category $\mathscr{U} / B$ is not closed cartesian monoidal since mapping spaces over $B$ need not be weak Hausdorff. Wishing to retain the separation property and motivated by Propositions 1.3 .10 and 1.3.15, Lewis [96] defined his preferred categories as follows.

DEFINITION 1.5.1. Let $\mathscr{O}(B)$ and $\mathscr{O}_{*}(B)$ be the categories of those compactly generated spaces and ex-spaces over $B$ whose projection maps are open.

REMARK 1.5.2. Bundle projections over $B$ are open maps. Hurewicz fibrations over $B$ are open maps if the diagonal $B \longrightarrow B \times B$ is a Hurewicz cofibration $[96,2.3]$; this holds, for example, if $B$ is a $\mathrm{CW}$ complex.

REMARK 1.5.3. If $p$ is an open map, then so are the projections of $X \wedge_{B} K$ and $F_{B}(K, Y)$, by $\left[96\right.$, p. 85]. Therefore $\mathscr{O}_{*}(B)$ is tensored and cotensored over $\mathscr{T}$.

However, the categories $\mathscr{O}(B)$ and $\mathscr{O}_{*}(B)$ are insufficient for our purposes. Working in these categories, we only have the base change adjunction $\left(f^{*}, f_{*}\right)$ of $\S 2.1$ for open maps $f: A \longrightarrow B$, which is unduly restrictive. For example, we need the adjunction $\left(\Delta^{*}, \Delta_{*}\right)$, where $\Delta: B \longrightarrow B \times B$ is the diagonal map. Even more importantly, the generating cofibrations of our $q$-type model structures cannot be restricted to have open projection maps.

We record a comparison between $\mathscr{K} / B$ and $\mathscr{U} / B$ and between $\mathscr{K}_{B}$ and $\mathscr{U}_{B}$. Since we have little homotopical control of the construction, we rarely use it.

Proposition 1.5.4. Weak Hausdorffication $w: \mathscr{K} \longrightarrow \mathscr{U}$ induces a retraction of $\mathscr{K} / B$ to $\mathscr{U} / B$ and of $\mathscr{K}_{B}$ to $\mathscr{U}_{B}$.

Proof. The functor $w$ comes with a natural quotient map $q: X \longrightarrow w X$ such that any map $X \longrightarrow B, B \in \mathscr{U}$, factors uniquely through a map $w X \longrightarrow B$. When $X$ is in $\mathscr{U}, q$ is the identity map. Therefore, for any space $X$ in $\mathscr{K} / B$, we have an induced space $w X$ in $\mathscr{U} / B$ and a natural map $q: X \longrightarrow w X$ in $\mathscr{K} / B$. If $X$ has a section $s$, so that $X \in \mathscr{K}_{B}$, then $q \circ s$ gives $w X$ a section, so that $w X \in \mathscr{U}_{B}$, and then $q: X \longrightarrow w X$ is a map in $\mathscr{K}_{B}$.

\subsection{Technical point-set topological lemmas}

We record several technical lemmas and observations that we shall need at various points in our work. 
Let $A \times{ }_{c} B$ denote the classical cartesian product in $\mathscr{T} o p$ and recall that $B$ is Hausdorff if and only if the diagonal embeds it as a closed subspace of $B \times{ }_{c} B$. The following result is proven in [94, App.§2].

Proposition 1.6.1. Let $A$ and $B$ be $k$-spaces. If one of them is locally compact or if both of them are first countable, then

$$
A \times B=A \times{ }_{c} B .
$$

Therefore, if $B$ is either locally compact or first countable, then $B$ is Hausdorff if and only if it is weak Hausdorff.

We need some observations about inclusions and colimits. Recall that a map is an inclusion if it is a homeomorphism onto its image. Of course, inclusions need not have closed image. As noted by Strøm [158], the simplest example of a non-closed inclusion in $\mathscr{K}$ is the inclusion $i:\{a\} \subset\{a, b\}$, where $\{a, b\}$ has the indiscrete topology. Here $i$ is both the inclusion of a retract and a Hurewicz cofibration (satisfies the homotopy extension property, or HEP). As is well-known, such pathology cannot occur in $\mathscr{U}$.

Lemma 1.6.2. Let $i: A \longrightarrow X$ be a map in $\mathscr{K}$.

(i) If there is a map $r: X \longrightarrow A$ such that $r \circ i=i d$, then $i$ is an inclusion. If, further, $X$ is in $\mathscr{U}$, then $i$ is a closed inclusion.

(ii) If $i$ is a Hurewicz cofibration, then $i$ is an inclusion. If, further, $X$ is in $\mathscr{U}$, then $i$ is a closed inclusion.

Proof. Inclusions $i: A \longrightarrow X$ are characterized by the property that a function $j: Y \longrightarrow A$ is continuous if and only if $i \circ j$ is continuous. This implies the first statement in (i). Alternatively, one can note that a map in $\mathscr{K}$ is an inclusion if and only if it is an equalizer in $\mathscr{K}$, and a map in $\mathscr{U}$ is a closed inclusion if and only if it is an equalizer in $\mathscr{U}[94,7.6]$. Since $i$ is the equalizer of $i \circ r$ and the identity map of $X$, this implies both statements in (i). For (ii), let $M i$ be the mapping cylinder of $i$. The canonical map $j: M i \longrightarrow X \times I$ has a left inverse $r$ and is thus an inclusion or closed inclusion in the respective cases. The evident closed inclusions $i_{1}: A \longrightarrow M i$ and $i_{1}: X \longrightarrow X \times I$ satisfy $j \circ i_{1}=i_{1} \circ i$, and the conclusions of (ii) follow.

REMARK 1.6.3. The section of an ex-space in $\mathscr{U}_{B}$ is closed, by Lemma 1.6.2. Quite reasonably, references such as $[42,83]$ make the blanket assumption that sections of ex-spaces must be closed. We have not done so since we have not checked that all constructions in sight preserve this property.

The following remark, which we learned from Mike Cole [35] and Gaunce Lewis, compares certain colimits in $\mathscr{K}$ and $\mathscr{U}$. It illuminates the difference between these categories and will be needed in our discussion of $h$-type model structures.

REMARK 1.6.4. Suppose given a sequence of inclusions $g_{n}: X_{n} \longrightarrow X_{n+1}$ and maps $f_{n}: X_{n} \longrightarrow Y$ in $\mathscr{K}$ such that $f_{n+1} g_{n}=f_{n}$. Let $X=\operatorname{colim} X_{n}$ and let $f: X \longrightarrow Y$ be obtained by passage to colimits. Fix a map $p: Z \longrightarrow Y$. The maps $Z \times_{Y} X_{n} \longrightarrow Z \times_{Y} X$ induce a map

$$
\alpha: \operatorname{colim}\left(Z \times_{Y} X_{n}\right) \longrightarrow Z \times_{Y} X .
$$

Lewis has provided counterexamples showing that $\alpha$ need not be a homeomorphism in general. However, if $Y \in \mathscr{U}$, then a result of his [94, App. 10.3] shows that $\alpha$ is a homeomorphism for any $p$ and any maps $g_{n}$. In fact, as in Proposition 2.1.3 
below, if $Y \in \mathscr{U}$, then the pullback functor $p^{*}: \mathscr{K} / Y \longrightarrow \mathscr{K} / Z$ is a left adjoint and therefore commutes with all colimits. To see what goes wrong when $Y$ is not in $\mathscr{U}$, consider the diagram

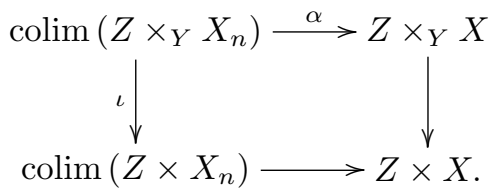

Products commute with colimits, so the bottom arrow is a homeomorphism, and the top arrow $\alpha$ is a continuous bijection. The right vertical arrow is an inclusion by the construction of pullbacks. If the left vertical arrow $\iota$ is an inclusion, then the diagram implies that $\alpha$ is a homeomorphism. The problem is that $\iota$ need not be an inclusion. One point is that the maps $Z \times_{Y} X_{n} \longrightarrow Z \times X_{n}$ are closed inclusions if $Y$ is weak Hausdorff, but not in general otherwise. Now assume that all spaces in sight are in $\mathscr{U}$. Since the $g_{n}$ are inclusions, the relevant colimits, when computed in $\mathscr{K}$, are weak Hausdorff and thus give colimits in $\mathscr{U}$. Therefore the commutation of $p^{*}$ with colimits (which is a result about colimits in $\mathscr{K}$ ) applies to these particular colimits in $\mathscr{U}$ to show that $\alpha$ is a homeomorphism.

The following related observation will be needed for applications of Quillen's small object argument to $q$-type model structures in $\S 4.5$ and elsewhere.

LEMMA 1.6.5. Let $X_{n} \longrightarrow X_{n+1}, n \geq 0$, be a sequence of inclusions in $\mathscr{K}$ with colimit $X$. Suppose that $X / X_{0}$ is in $\mathscr{U}$. Then, for a compact Hausdorff space $C$, the natural map

$$
\operatorname{colim} \mathscr{K}\left(C, X_{n}\right) \longrightarrow \mathscr{K}(C, X)
$$

is a bijection.

Proof. The point is that $X_{0}$ need not be in $\mathscr{U}$. Let $f: C \longrightarrow X$ be a map. Then the composite of $f$ with the quotient map $X \longrightarrow X / X_{0}$ takes image in some $X_{n} / X_{0}$, hence $f$ takes image in $X_{n}$. The conclusion follows.

SCHOLIUM 1.6.6. One might expect the conclusion to hold for colimits of sequences of closed inclusions $X_{n-1} \longrightarrow X_{n}$ such that $X_{n}-X_{n-1}$ is a $T_{1}$ space. This is stated as $[81,4.2]$, whose authors got the statement from May. However, Lewis has shown us a counterexample.

\subsection{Appendix: nonassociativity of smash products in $\mathscr{T} o p_{*}$}

In a 1958 paper [138], Puppe asserted the following result, but he did not give a proof. It was the subject of a series of e-mails among Mike Cole, Tony Elmendorf, Gaunce Lewis and the first author. Since we know of no published source that gives the details of this or any other counterexample to the associativity of the smash product in $\mathscr{T}_{o p_{*}}$, we include the following proof. It is due to Kathleen Lewis.

Let $\mathbb{Q}$ and $\mathbb{N}$ be the rational numbers and the nonnegative integers, topologized as subspaces of $\mathbb{R}$ and given the basepoint zero. Consider smash products as quotient spaces, without applying the $k$-ification functor. Then we have the following counterexample to associativity.

TheOREM 1.7.1. $(\mathbb{Q} \wedge \mathbb{Q}) \wedge \mathbb{N}$ is not homeomorphic to $\mathbb{Q} \wedge(\mathbb{Q} \wedge \mathbb{N})$. 
Proof. Consider the following diagram.

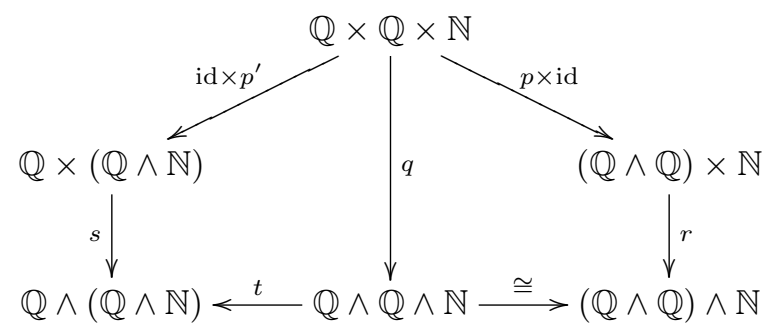

Here $\mathbb{Q} \wedge \mathbb{Q} \wedge \mathbb{N}$ denotes the evident quotient space of $\mathbb{Q} \times \mathbb{Q} \times \mathbb{N}$. The maps $p, p^{\prime}$, $q, r$, and $s$ are quotient maps. Since $\mathbb{N}$ is locally compact, $p \times$ id is also a quotient map, hence so is $r \circ(p \times$ id $)$. The universal property of quotient spaces then gives the bottom right homeomorphism. Since $\mathbb{Q}$ is not locally compact, id $\times p^{\prime}$ need not be a quotient map, and in fact it is not. The map $t$ is a continuous bijection given by the universal property of the quotient map $q$, and we claim that $t$ is not a homeomorphism. To show this, we display an open subset of $\mathbb{Q} \wedge \mathbb{Q} \wedge N$ whose image under $t$ is not open.

Let $\beta$ be an irrational number, $0<\beta<1$, and let $\gamma=(1-\beta) / 2$. Define $V^{\prime}(\beta)$ to be the open subset of $\mathbb{R} \times \mathbb{R}$ that is the union of the following four sets.

(1) The open ball of radius $\beta$ about the origin

(2) The tubes $[1, \infty) \times(-\gamma, \gamma),(-\infty,-1] \times(-\gamma, \gamma),(-\gamma, \gamma) \times[1, \infty)$, and $(-\gamma, \gamma) \times$ $(-\infty,-1]$ of width $2 \gamma$ about the axes.

(3) The open balls of radius $\gamma$ about the four points $( \pm 1,0),(0, \pm 1)$.

(4) For each $n \geq 1$, the open ball of radius $\gamma / 2^{n}$ about the four points $\left( \pm \gamma_{n}, 0\right)$, $\left(0, \pm \gamma_{n}\right)$, where $\gamma_{n}=1-\sum_{k=0}^{k=n-1} \gamma / 2^{k}$.

To visualize this set, it is best to draw a picture. It is symmetric with respect to 90 degree rotation. Consider the part lying along the positive $x$-axis. A tube of width $2 \gamma$ covers the part of the $x$-axis to the right of $(1,0)$. A ball of radius $\beta$ centers at the origin. A ball of radius $\gamma$ centers at $(1,0)$. Its vertical diagonal is the edge of the tube going off to the right. On the left, by the choice of $\gamma$, this ball reaches halfway from its center $(1,0)$ to the point $(\beta, 0)$ at the right edge of the ball centered at the origin. The point $(1-\gamma, 0)$ at the left edge of the ball centered at $(1,0)$ is the center of another ball, which reaches half the distance from $(1-\gamma, 0)$ to $(\beta, 0)$. And so on: the point where the left edge of the $n$th ball crosses the $x$-axis is the center point of the $(n+1)$ st ball, which reaches half the distance from its center to the edge of the ball centered at the origin.

Define $V(\beta)=V^{\prime}(\beta) \cap(\mathbb{Q} \times \mathbb{Q})$. Note that the only points of the coordinate axes of $\mathbb{R} \times \mathbb{R}$ that are not in $V^{\prime}(\beta)$ are $( \pm \beta, 0)$ and $(0, \pm \beta)$. Since $\beta$ is irrational, $V(\beta)$ contains the coordinate axes of $\mathbb{Q} \times \mathbb{Q}$. Because the radii of the balls in the sequence are decreasing, for each $\varepsilon>\beta$, there is no $\delta>0$ such that $((-\varepsilon, \varepsilon) \times(-\delta, \delta)) \cap(\mathbb{Q} \times \mathbb{Q})$ is contained in $V(\beta)$.

Now let $\alpha$ be an irrational number, $0<\alpha<1$. Let $\bullet$ be the basepoint of $\mathbb{Q} \wedge \mathbb{N}$ and $*$ be the basepoint of $\mathbb{Q} \wedge \mathbb{Q} \wedge \mathbb{N}$. Let $U$ be the union of $\{*\}$ and the image under $q$ of $\cup_{n \geq 1} V(\alpha / n) \times\{n\}$. This is an open subspace of $\mathbb{Q} \wedge \mathbb{Q} \wedge \mathbb{N}$ since

$$
q^{-1}(U)=\mathbb{Q} \times \mathbb{Q} \times\{0\} \cup\left(\cup_{n \geq 1} V(\alpha / n) \times\{n\}\right)
$$


is an open subset of $\mathbb{Q} \times \mathbb{Q} \times \mathbb{N}$. We claim that $t(U)$ is not open in $\mathbb{Q} \wedge(\mathbb{Q} \wedge \mathbb{N})$. Assume that $t(U)$ is open. Then

$$
s^{-1}(t(U))=\left(\mathrm{id} \times p^{\prime}\right)\left(q^{-1}(U)\right)
$$

is an open subset of $\mathbb{Q} \times(\mathbb{Q} \wedge \mathbb{N})$, hence it contains an open neighborhood $V$ of $(0, \bullet)$. Now $V$ must contain $((-\varepsilon, \varepsilon) \cap \mathbb{Q}) \times W$ for some $\varepsilon>0$ and some open neighborhood $W$ of $\bullet$ in $\mathbb{Q} \wedge \mathbb{N}$. Since $\mathbb{Q} \wedge \mathbb{N}$ is homeomorphic to the wedge over $n \geq 1$ of the spaces $\mathbb{Q} \times\{n\}, W$ must contain the wedge over $n \geq 1$ of subsets $\left(\left(-\delta_{n}, \delta_{n}\right) \cap \mathbb{Q}\right) \times\{n\}$, where $\delta_{n}>0$. By the definition of $U$, this implies that

$$
\left((-\varepsilon, \varepsilon) \times\left(-\delta_{n}, \delta_{n}\right)\right) \cap(\mathbb{Q} \times \mathbb{Q}) \subset V(\alpha / n) .
$$

However, for $n$ large enough that $\varepsilon>\alpha / n$, there is no $\delta_{n}$ for which this holds. 


\section{CHAPTER 2}

\section{Change functors and compatibility relations}

\section{Introduction}

In the previous chapter, we developed the internal properties of the category $G \mathscr{K}_{B}$ of ex- $G$-spaces over $B$. As $B$ and $G$ vary, these categories are related by various functors, such as base change functors, change of groups functors, orbit and fixed point functors, external smash product and function space functors, and so forth. We define these functors and discuss various compatibility relations among them in this chapter.

We particularly emphasize base change functors. We give a general categorical discussion of such functors in $\$ 2.1$, illustrating the general constructions with topological examples. In $\S 2.2$, we discuss various compatibility relations that relate these functors to smash products and function objects.

In $\S 2.3$ and $\S 2.4$ we turn to equivariant phenomena and study restriction of group actions along homomorphisms. As usual, we break this into the study of restriction along inclusions and pullback along quotient homomorphisms.

In $\S 2.3$, we discuss restrictions of group actions to subgroups, together with the associated induction and coinduction functors. We also consider their compatibilities with base change functors. In particular, this gives us a convenient way of thinking about passage to fibers and allows us to reinterpret restriction to subgroups in terms of base change and coinduction. That is the starting point of our generalization of the Wirthmüller isomorphism in $\S 19.1$.

In $\S 2.4$, we consider pullbacks of group actions from a quotient group $G / N$ to $G$, together with the associated quotient and fixed point functors. Again, we also consider compatibilities with base change functors. For an $N$-free base space $E$, we find a relation between the quotient functor $(-) / N$ and the fixed point functor $(-)^{N}$ that involves base change along the quotient map $E \longrightarrow E / N$. The good properties of the bundle construction in $\S 15.4$ can be traced back to this relation, and it is at the heart of the proof of the Adams isomorphism in equivariant stable homotopy theory given in $\S 19.7$.

In $\S 2.5$, we describe a different categorical framework, one appropriate to exspaces with varying base spaces. We show that the relevant category of retracts over varying base spaces is closed symmetric monoidal under external smash product and function ex-space functors. The internal smash product and function ex-space functors are obtained from these by use of base change along diagonal maps. The external smash products are much better behaved homotopically than the internal ones, and homotopical analysis of base change functors will therefore play a central role in the homotopical analysis of smash products. 
In much of this chapter, we work in a general categorical framework. In some places where we restrict to spaces, more general categorical formulations are undoubtedly possible. When we talk about group actions, all groups are assumed to be compactly generated spaces but are otherwise unrestricted.

\subsection{The base change functors $f_{!}, f^{*}$, and $f_{*}$}

Let $f: A \longrightarrow B$ be a map in a bicomplete subcategory $\mathscr{B}$ of a bicomplete category $\mathscr{C}$. We are thinking of $\mathscr{U} \subset \mathscr{K}$ or $G \mathscr{U} \subset G \mathscr{K}$. We wish to define functors

$$
f_{!}: \mathscr{C}_{A} \longrightarrow \mathscr{C}_{B}, \quad f^{*}: \mathscr{C}_{B} \longrightarrow \mathscr{C}_{A}, \quad f_{*}: \mathscr{C}_{A} \longrightarrow \mathscr{C}_{B}
$$

such that $f_{!}$is left adjoint and $f_{*}$ is right adjoint to $f^{*}$. The definitions of $f^{*}$ and $f_{\text {! }}$ are dual and require no further hypotheses. The definition of $f_{*}$ does not work in full generality, but it only requires the further hypothesis that $\mathscr{C} / B$ be cartesian closed. Thus we assume given internal hom objects $\operatorname{Map}_{B}(Y, Z)$ in $\mathscr{C} / B$ that satisfy the usual adjunction, as in (1.3.9). One reason to work in this generality is to emphasize that no further point-set topology is needed to construct these base change functors in the context of ex-spaces. This point is not clear from the literature, where the functor $f_{*}$ is often given an apparently different, but naturally isomorphic, description. We work with generic ex-objects

$$
A \stackrel{s}{\longrightarrow} X \stackrel{p}{\longrightarrow} A \quad \text { and } \quad B \stackrel{t}{\longrightarrow} Y \stackrel{q}{\longrightarrow} B
$$

in this section.

Definition 2.1.1. Define $f_{!} X$ and its structure maps $q$ and $t$ by means of the map of retracts in the following diagram on the left, where the top square is a pushout and the bottom square is defined by the universal property of pushouts and the requirement that $q \circ t=\mathrm{id}$. Define $f^{*} Y$ and its structure maps $p$ and $s$ by means of the map of retracts in the following middle diagram, where the bottom square is a pullback and the top square is defined by the universal property of pullbacks and the requirement that $p \circ s=$ id.
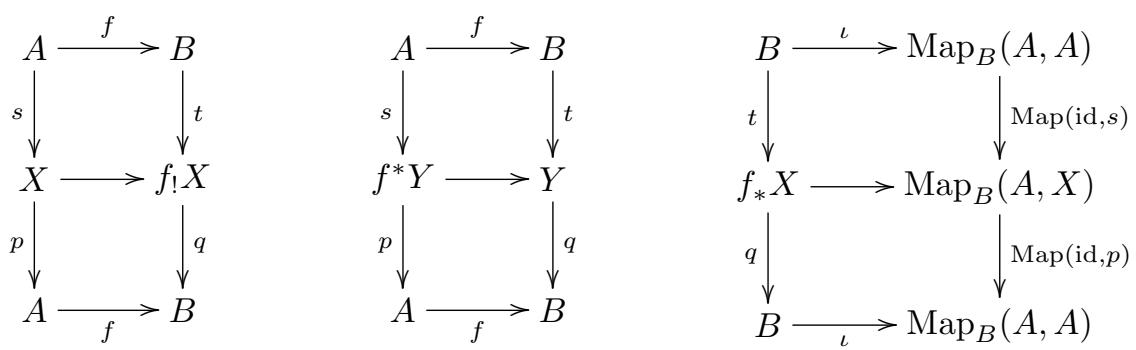

Thinking of $X$ and $A$ as objects over $B$ via $f \circ p$ and $f$ and observing that the adjoint of the identity map of $A$ gives a map $\iota: B \longrightarrow \operatorname{Map}_{B}(A, A)$, define $f_{*} X$ and its structure maps $q$ and $t$ by means of the map of retracts in the above diagram on the right, where the bottom square is a pullback and the top square is defined by the universal property of pullbacks and the requirement that $q \circ t=\mathrm{id}$.

Proposition 2.1.2. $\left(f_{!}, f^{*}\right)$ is an adjoint pair of functors:

$$
\mathscr{C}_{B}\left(f_{!} X, Y\right) \cong \mathscr{C}_{A}\left(X, f^{*} Y\right)
$$


Proof. Maps in both hom sets are specified by maps $k: X \longrightarrow Y$ in $\mathscr{C}$ such that $q \circ k=f \circ p$ and $k \circ s=t \circ f$.

Proposition 2.1.3. $\left(f^{*}, f_{*}\right)$ is an adjoint pair of functors:

$$
\mathscr{C}_{A}\left(f^{*} Y, X\right) \cong \mathscr{C}_{B}\left(Y, f_{*} X\right)
$$

Proof. A map $k: f^{*} Y=Y \times{ }_{B} A \longrightarrow X$ such that $p \circ k=p$ and $k \circ s=s$ has adjoint $\tilde{k}: Y \longrightarrow \operatorname{Map}_{B}(A, X)$ such that $\operatorname{Map}(\mathrm{id}, p) \circ \tilde{k}=\iota \circ q$ and $\tilde{k} \circ t=$ Map(id, $s) \circ \iota$. The conclusion follows directly.

REMARK 2.1.4. Writing these proofs diagrammatically, we see that the adjunction isomorphisms are given by homeomorphisms in our context of topological categories.

We specialize to ex-spaces (or ex- $G$-spaces), in the rest of the section. Observe that the fiber $\left(f_{*} X\right)_{b}$ is the space of sections $A_{b} \longrightarrow X_{b}$ of $p: X_{b} \longrightarrow A_{b}$.

REMARK 2.1.5. If $f: A \longrightarrow B$ is an open map and $X$ is in $\mathscr{U}$, then $f_{*} X$ is in $\mathscr{U}$ and $\mathscr{U}_{A}\left(f^{*} Y, X\right) \cong \mathscr{U}_{B}\left(Y, f_{*} X\right)$ for $Y \in \mathscr{U}$, by $[96,1.5]$.

ExAmple 2.1.6. Let $f: A \longrightarrow B$ be an inclusion. Then $f^{*} Y$ is the restriction of $Y$ to $A$ and $f_{!} X=B \cup_{A} X$ is obtained by expanding the section from $A$ to $B$ and composing the projection $X \longrightarrow A$ with $f$. The ex-space $f_{*} X$ over $B$ is analogous to the prolongation by zero of a sheaf over $A$. The fiber $\left(f_{*} X\right)_{b}$ is $X_{a}$ if $a \in A$ and a point $\{b\}$ otherwise. To see this from the definition, recall that $\operatorname{Map}(\emptyset, K)$ is a point for any space $K$ and that $\operatorname{Map}_{B}(A, X)_{b}=\operatorname{Map}\left(A_{b}, X_{b}\right)$. As a set, $f_{*} X \cong B \cup_{A} X$, but the topology is quite different. It is devised so that the map $Y \longrightarrow f_{*} f^{*} Y$ that restricts to the identity on $Y_{a}$ for $a \in A$ but sends $Y_{b}$ to $\{b\}$ for $b \notin A$ is continuous.

ExAmple 2.1.7. For $b \in B$, let $b$ also denote the map $* \longrightarrow B$ that sends $*$ to $B$; we may view this as the case $A=\{b\}$ of the previous example. Then $b^{*} Y$ is the fiber $Y_{b}$, and we write $K^{b}$ and ${ }^{b} K$ for the spaces $b_{!} K$ and $b_{*} K$ over $B$ associated to a based space $K$. In the equivariant case, we must take the isotropy group $G_{b}$ into account. Let $\tilde{b}: G / G_{b} \longrightarrow B$ be the induced map of orbits, so that $\tilde{b}$ maps $G / G_{b}$ onto the orbit $G b$. We shall reinterpret the functors $\tilde{b}^{*}, \tilde{b}_{\text {! }}$ and $\tilde{b}_{*}$ as an equivariant fiber functor and its left and right adjoints in Example 2.3.12 below.

EXAMPLE 2.1.8. Let $r: B \longrightarrow *$ be the unique map. We shall use the letter $r$ with this meaning throughout the book. For a based space $X$ and an ex-space $E=(E, p, s)$ over $B$, we have

$$
r^{*} X=B \times X, \quad r_{!} E=E / s(B), \quad \text { and } \quad r_{*} E=\operatorname{Sec}(B, E),
$$

where $\operatorname{Sec}(B, E)$ is the space of maps $t: B \longrightarrow E$ such that $p \circ t=$ id, with basepoint the section $s$. These elementary base change functors are the key to using parametrized homotopy theory to obtain information in ordinary homotopy theory. Let $\varepsilon: r_{!} r^{*} \longrightarrow$ id and $\eta$ : id $\longrightarrow r^{*} r_{\text {! }}$ be the counit and unit of the adjunction $\left(r_{!}, r^{*}\right)$. Then $r_{!} r^{*} X \cong B_{+} \wedge X$ and $\varepsilon$ is $r_{+} \wedge$ id, while $r^{*} r_{!} E=B \times E / s(B)$ and $\eta$ has coordinates $p$ and the quotient map $E \longrightarrow E / s(B)$. Thus $r_{!} r^{*} r_{!} E \cong B_{+} \wedge E / s(B)$ and $r_{!} \eta: r_{!} E \longrightarrow r_{!} r^{*} r_{!} E$ is the "Thom diagonal" $E / s(B) \longrightarrow B_{+} \wedge E / s(B)$. If $p: E \longrightarrow B$ is a spherical fibration with section, such as the fiberwise one-point compactification $S^{\nu}$ of a vector bundle $\nu$, then $r_{!} E$ is the Thom complex of $p$. 
REMARK 2.1.9. Although our main interest will be in the sectioned theory, we shall occasionally use the analogous base change functors on categories of parametrized objects without sections, especially in Chapter 18. The definitions are similar but a bit simpler. For a map $f: A \longrightarrow B$, we can always define the first two of the functors

$$
f_{!}: \mathscr{C} / A \longrightarrow \mathscr{C} / B, \quad f^{*}: \mathscr{C} / B \longrightarrow \mathscr{C} / A, \quad f_{*}: \mathscr{C} / A \longrightarrow \mathscr{C} / B,
$$

and we can define the third whenever $\mathscr{C} / B$ is cartesian closed. The first is given by composing the projection of an object in $\mathscr{C} / A$ with the map $f$ to obtain a projection to $B$, and the other two are defined by the same pullbacks as the corresponding functors in the sectioned case. We again get two pairs of adjoint functors, $\left(f_{!}, f^{*}\right)$ and $\left(f^{*}, f_{*}\right)$. The results of the following section go through just as well in this simpler setting.

\subsection{Compatibility relations}

The term "compatibility relation" has been used in algebraic geometry in the context of Grothendieck's six functor formalism in sheaf theory that relates base change functors to tensor product and internal hom functors. We describe how the analogous, but simpler, formalism appears in our categories of ex-objects.

We recall some language. We are especially interested in the behavior of base change functors with respect to closed symmetric monoidal structures that, in our topological context, are given by smash products and function objects. Relevant categorical observations are given in [62]. We say that a functor $T: \mathscr{B} \longrightarrow \mathscr{A}$ between closed symmetric monoidal categories is closed symmetric monoidal if $(2.2 .1)$

$T S_{\mathscr{B}} \cong S_{\mathscr{A}}, \quad T\left(X \wedge_{\mathscr{B}} Y\right) \cong T X \wedge_{\mathscr{A}} T Y, \quad$ and $\quad T F_{\mathscr{B}}(X, Y) \cong F_{\mathscr{A}}(T X, T Y)$,

where $S_{\mathscr{B}}, \wedge_{\mathscr{B}}$ and $F_{\mathscr{B}}$ denote the unit object, product, and internal hom of $\mathscr{B}$, and similarly for $\mathscr{A}$. These isomorphisms must satisfy appropriate coherence conditions relating to the unit, associativity and commutativity isomorphisms in $\mathscr{A}$ and $\mathscr{B}$. In the language of [62], the following result states that any map $f$ of base spaces gives rise to a "Wirthmüller context", which means that the functor $f^{*}$ is closed symmetric monoidal and has both a left adjoint and a right adjoint.

Proposition 2.2.2. If $f: A \longrightarrow B$ is a map of base $G$-spaces, then the functor $f^{*}: G \mathscr{K}_{B} \longrightarrow G \mathscr{K}_{A}$ is closed symmetric monoidal. Therefore, by definition and implication, $f^{*} S_{B}^{0} \cong S_{A}^{0}$ and there are natural isomorphisms

$$
\begin{gathered}
f^{*}\left(Y \wedge_{B} Z\right) \cong f^{*} Y \wedge_{A} f^{*} Z, \\
F_{B}\left(Y, f_{*} X\right) \cong f_{*} F_{A}\left(f^{*} Y, X\right), \\
f^{*} F_{B}(Y, Z) \cong F_{A}\left(f^{*} Y, f^{*} Z\right), \\
f_{!}\left(f^{*} Y \wedge_{A} X\right) \cong Y \wedge_{B} f_{!} X, \\
F_{B}\left(f_{!} X, Y\right) \cong f_{*} F_{A}\left(X, f^{*} Y\right),
\end{gathered}
$$

where $X$ is an ex-G-space over $A$ and $Y$ and $Z$ are ex-G-spaces over $B$.

Proof. The isomorphism $f^{*} S_{B}^{0} \cong S_{A}^{0}$ is evident since $f^{*}(B \times K) \cong A \times K$ for based $G$-spaces $K$. The isomorphism (2.2.3) is obtained by passage to quotients 
from the evident homeomorphism

$$
\left(Y \times_{B} A\right) \times_{A}\left(Z \times_{B} A\right) \cong\left(Y \times_{B} Z\right) \times_{B} A
$$

As explained in $[62, \S \S 2,3]$, the isomorphism (2.2.3) is equivalent to the isomorphism (2.2.4), and it determines natural maps from left to right in (2.2.5), (2.2.6), and (2.2.7) such that all three are isomorphisms if any one is. By a comparison of definitions, we see that the categorically defined map in (2.2.5), which is denoted $\alpha$ in $[62,3.3]$, coincides in the present situation with the map, also denoted $\alpha$, on [22, p. 167]. As explained on [22, p. 178], in the point-set topological framework that we have adopted, that map $\alpha$ is a homeomorphism.

REMARK 2.2.8. Only the very last statement refers to topology. The categorically defined map $\alpha$ should quite generally be an isomorphism in analogous contexts, but we have not pursued this question in detail. An alternative self-contained proof of the previous proposition is given in Remark 2.5.8 below by using Proposition 2.2.11 to prove (2.2.6) instead of (2.2.5). In that argument, the only non-formal ingredient is the fact that the functor $D \times{ }_{B}(-)$ commutes with pushouts.

The categorical situation here entails a vast variety of coherence diagrams relating the given data. We shall much later need two examples, and it is convenient to insert them here. The following observation will play a minor role in $\S 20.3$, but is of independent conceptual interest.

REMARK 2.2.9. Applying (2.2.3) and (2.2.6), we obtain isomorphisms

$$
Y \wedge_{B} f_{!} f^{*} Z \cong f_{!}\left(f^{*} Y \wedge_{A} f^{*} Z\right) \cong f_{!} f^{*}\left(Y \wedge_{B} Z\right)
$$

The counit $f_{!} f^{*} \longrightarrow$ id of the adjunction $\left(f_{!}, f^{*}\right)$ induces maps from the left and right terms to $Y \wedge_{B} Z$, and the evident diagram commutes, so that these induced maps agree under the isomorphism. Similarly, applying (2.2.4) and (2.2.7), we obtain isomorphisms

$$
F_{B}\left(Y, f_{*} f^{*} Z\right) \cong f_{*} F_{A}\left(f^{*} Y, f^{*} Z\right) \cong F_{B}\left(f_{!} f^{*} Y, Z\right) .
$$

The unit id $\longrightarrow f_{*} f^{*}$ and counit $f_{!} f^{*} \longrightarrow$ id of our adjunctions induce maps from $F_{B}(Y, Z)$ to the left and right terms, and the evident diagram commutes, so that these induced maps agree under the isomorphism.

The following more technical observation about the categorically defined isomorphism $\alpha$ in (2.2.5) will play a key role in the proof of the fiberwise duality theorem of $\S 15.1$.

REMARK 2.2.10. Let $f^{*}: \mathscr{B} \longrightarrow \mathscr{A}$ be any symmetric monoidal functor. We are thinking of a base change functor, but the observation is general. The map

$$
\alpha: f^{*} F_{\mathscr{B}}(X, Y) \longrightarrow F_{\mathscr{A}}\left(f^{*} X, f^{*} Y\right)
$$

is defined to be the adjoint of

$$
f^{*} F_{\mathscr{B}}(X, Y) \wedge_{\mathscr{A}} f^{*} X \cong f^{*}\left(F_{\mathscr{B}}(X, Y) \wedge_{\mathscr{B}} X\right) \stackrel{f^{*} \mathrm{ev}}{\longrightarrow} f^{*} Y
$$


The dual of $X$ is $D_{\mathscr{B}} X=F_{\mathscr{B}}\left(X, S_{\mathscr{B}}\right)$, where $S_{\mathscr{B}}$ is the unit of $\mathscr{B}$. Taking $Y=S_{\mathscr{B}}$, the definition of $\alpha$ implies that the top triangle commutes in the diagram

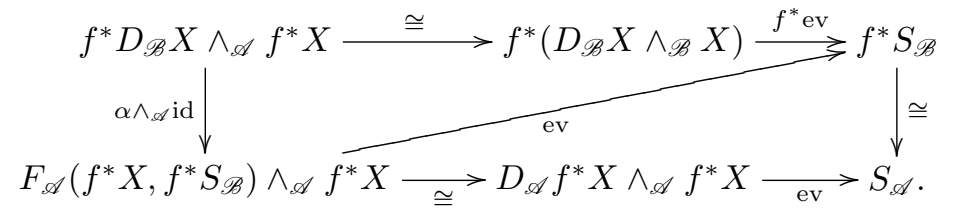

The bottom triangle is a naturality diagram. The outer rectangle is $[62,3.7]$, but its commutativity in general was not observed there. However, it was observed in $[62,3.8]$ that its commutativity implies the commutativity of the diagram

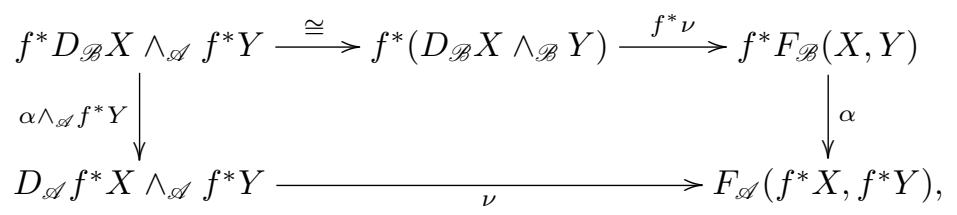

where $\nu: D_{\mathscr{B}} X \wedge_{\mathscr{B}} Y \longrightarrow F_{\mathscr{B}}(X, Y)$ is the adjoint of

$$
D_{\mathscr{B}} X \wedge_{\mathscr{B}} Y \wedge_{\mathscr{B}} X \cong D_{\mathscr{B}} X \wedge_{\mathscr{B}} X \wedge_{\mathscr{B}} Y \stackrel{\mathrm{ev} \wedge \mathrm{id}}{\longrightarrow} S_{\mathscr{B}} \wedge_{\mathscr{B}} Y \cong Y .
$$

In other contexts, the analogue of (2.2.6) is called the "projection formula", and we shall also use that term. The following base change commutation relations with respect to pullbacks are also familiar from other contexts. We state the result for spaces but, apart from use of the fact that the functor $D \times_{B}(-)$ commutes with pushouts, the proof is formal.

Proposition 2.2.11. Suppose given a pullback diagram of base spaces

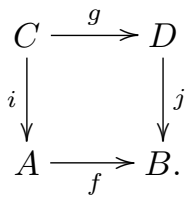

Then there are natural isomorphisms of functors

$$
j^{*} f_{!} \cong g_{!} i^{*}, \quad f^{*} j_{*} \cong i_{*} g^{*}, \quad f^{*} j_{!} \cong i_{!} g^{*}, \quad j^{*} f_{*} \cong g_{*} i^{*} .
$$

Proof. The first isomorphism is one of left adjoints, and the second is the corresponding "conjugate" isomorphism of right adjoints. Similarly for the third and fourth isomorphisms. By symmetry, it suffices to prove the first isomorphism. The commutativity of the square already implies a canonical map $g i^{*} \longrightarrow j^{*} f_{!}$, namely the composite

$$
g_{!} i^{*} \longrightarrow g_{!} i^{*} f^{*} f_{!} \cong g_{!} g^{*} j^{*} f_{!} \longrightarrow j^{*} f_{!}
$$

given by the unit of the adjunction $\left(f_{!}, f^{*}\right)$ and the counit of the adjunction $\left(g_{!}, g^{*}\right)$. When the diagram is a pullback, this map is an isomorphism because $j^{*}=D \times_{B}(-)$ commutes with pushouts. For a space $X$ over $A$ regarded by composition with $f$ as a space over $B, C \times_{A} X \cong D \times_{B} X$. This gives

$$
j^{*} f_{!} X=D \times_{B}\left(B \cup_{A} X\right) \cong D \cup_{C}\left(C \times_{A} X\right)=g ! i^{*} X .
$$




\subsection{Change of group and restriction to fibers}

This section begins the study of equivariant phenomena that have no nonequivariant counterparts. In particular, using a conceptual reinterpretation of the adjoints of the fiber functors $(-)_{b}$, we relate restriction to subgroups to restriction to fibers. Recall that subgroups of $G$ are understood to be closed and fix an inclusion $\iota: H \subset G$ throughout this section. Parametrized theory gives a convenient way of studying restriction along $\iota$ without changing the ambient group from $G$ to $H$.

Proposition 2.3.1. The category $G \mathscr{K}_{G / H}$ of ex-G-spaces over $G / H$ is equivalent to the category $H \mathscr{K}_{*}$ of based $H$-spaces.

Proof. The equivalence sends an ex- $G$-space $(Y, p, s)$ over $G / H$ to the $H$ space $p^{-1}(e H)$ with basepoint the $H$-fixed point $s(e H)$. Its inverse sends a based $H$-space $X$ to the induced $G$-space $G \times_{H} X$, with the evident structure maps.

More formally, recall that there are "induction" and "coinduction" functors $\iota$ ! and $\iota_{*}$ from $H$-spaces to $G$-spaces that are left and right adjoint to the forgetful functor $\iota^{*}$ that sends a $G$-space $Y$ to $Y$ regarded as an $H$-space. Explicitly, for an $H$-space $X$,

$$
\iota ! X=G \times_{H} X \quad \text { and } \quad \iota_{*} X=\operatorname{Map}_{H}(G, X) .
$$

The latter is the space of maps of (left) $H$-spaces, with (left) action of $G$ induced by the right action of $G$ on itself. Similarly, when $X$ is a based $H$-space, we have the based analogues

$$
\iota_{!} X=G_{+} \wedge_{H} X \quad \text { and } \quad \iota_{*} X=F_{H}\left(G_{+}, X\right) .
$$

With this notation, some familiar natural isomorphisms take the forms

$$
\iota_{!}\left(\iota^{*} Y \times X\right) \cong Y \times \iota_{!} X \quad \text { and } \quad \iota_{*} \operatorname{Map}\left(\iota^{*} Y, X\right) \cong \operatorname{Map}\left(Y, \iota_{*} X\right)
$$

and, in the based case,

$$
\iota_{!}\left(\iota^{*} Y \wedge X\right) \cong Y \wedge \iota_{!} X \quad \text { and } \quad \iota_{*} F\left(\iota^{*} Y, X\right) \cong F\left(Y, \iota_{*} X\right) .
$$

By the uniqueness of adjoints, or inspection of definitions, we see that these familiar change of groups functors are change of base functors along $r: G / H \longrightarrow *$.

COROLlaRY 2.3.6. The change of group and change of base functors associated to $\iota$ and $r$ agree under the equivalence of categories between $H \mathscr{K}_{*}$ and $G \mathscr{K}_{G / H}$ :

$$
\iota^{*} \cong r^{*}, \quad \iota ! \cong r_{!}, \quad \text { and } \quad \iota_{*} \cong r_{*} .
$$

We can generalize this equivalence of categories, using the following definitions. We have a forgetful functor $\iota^{*}: G \mathscr{K}_{B} \longrightarrow H \mathscr{K}_{\iota^{*} B}$. It doesn't have an obvious left or right adjoint, but we have obvious analogues of induction and coinduction that involve changes of base spaces. The first will lead to a description of $\iota^{*}$ as a base change functor and thus as a functor with a left and right adjoint.

Definition 2.3.7. Let $A$ be an $H$-space and $X$ be an $H$-space over $A$. Define $\iota !: H \mathscr{K}_{A} \longrightarrow G \mathscr{K}_{\iota !} A$ by letting $\iota ! X$ be the $G$-space $G \times_{H} X$ over $\iota ! A=G \times_{H} A$. Define $\iota_{*}: H \mathscr{K}_{A} \longrightarrow G \mathscr{K}_{\iota_{*} A}$ by letting $\iota_{*} X$ be the $G$-space $\operatorname{Map}_{H}(G, X)$ over $\iota_{*} A=\operatorname{Map}_{H}(G, A)$. 
For an $H$-space $A$ and a $G$-space $B$, let

$$
\mu: G \times{ }_{H} \iota^{*} B=\iota ! \iota^{*} B \longrightarrow B \text { and } \nu: A \longrightarrow \iota^{*} \iota ! A=\iota^{*}\left(G \times{ }_{H} A\right)
$$

be the counit and unit of the $\left(\iota !, \iota^{*}\right)$ adjunction. The following result says that ex- $H$-spaces over an $H$-space $A$ are equivalent to ex- $G$-spaces over the $G$-space $\iota$ ! $A$.

Proposition 2.3.9. The functor $\iota_{!}: H \mathscr{K}_{A} \longrightarrow G \mathscr{K}_{\iota !}$ is a closed symmetric monoidal equivalence of categories with inverse the composite

$$
G \mathscr{K}_{\iota ! A} \stackrel{\iota^{*}}{\longrightarrow} H \mathscr{K}_{\iota^{*} \iota !} A \stackrel{\nu^{*}}{\longrightarrow} H \mathscr{K}_{A} .
$$

Applied to $A=\iota^{*} B$, this equivalence leads to the promised description of $\iota^{*}: G \mathscr{K}_{B} \longrightarrow H \mathscr{K}_{\iota^{*} B}$ as a base change functor.

Proposition 2.3.10. The functor $\iota^{*}: G \mathscr{K}_{B} \longrightarrow H \mathscr{K}_{\iota^{*} B}$ is the composite

$$
G \mathscr{K}_{B} \stackrel{\mu^{*}}{\longrightarrow} G \mathscr{K}_{\iota ! \iota^{*} B} \cong H \mathscr{K}_{\iota^{*} B} .
$$

Change of base and change of groups are related by various further consistency relations. The following result gives two of them.

Proposition 2.3.11. Let $f: A \longrightarrow \iota^{*} B$ be a map of $H$-spaces and $\tilde{f}: \iota ! A \longrightarrow B$ be its adjoint map of $G$-spaces. Then the following diagrams commute up to natural isomorphism.
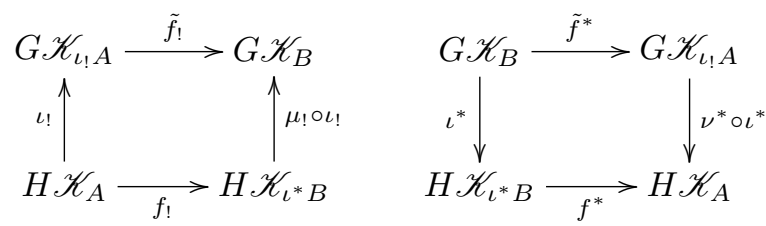

Proof. Since $\tilde{f}=\mu \circ \iota ! f$, we have

$$
\tilde{f}_{!} \circ \iota ! \cong(\mu \circ \iota ! f) ! \circ \iota ! \cong \mu ! \circ(\iota ! f) ! \circ \iota ! \cong \mu ! \circ \iota ! \circ f_{!}
$$

where the last isomorphism holds because $G \times{ }_{H}(-)$ commutes with pushouts. Since $f=\iota^{*} \tilde{f} \circ \nu$, we have

$$
f^{*} \circ \iota^{*} \cong\left(\iota^{*} \tilde{f} \circ \nu\right)^{*} \circ \iota^{*} \cong \nu^{*} \circ\left(\iota^{*} \tilde{f}\right)^{*} \circ \iota^{*} \cong \nu^{*} \circ \iota^{*} \circ \tilde{f}^{*},
$$

where the last isomorphism holds because pulling the $G$ action back to an $H$-action commutes with pullbacks.

The reader may find it illuminating to work out these isomorphisms in the context of Proposition 2.3.1. That result leads to the promised reinterpretations of the functors $\tilde{b}^{*}, \tilde{b}_{!}$and $\tilde{b}_{*}$ defined in Example 2.1.7.

ExAmple 2.3.12. Let $b \in B$. We have the $G_{b}$-map $b: * \longrightarrow B$ and the $G$-map $\tilde{b}: G / G_{b} \longrightarrow B$. Under the equivalence $G \mathscr{K}_{G / G_{b}} \cong G_{b} \mathscr{K}_{*}$ of Proposition 2.3.1, $\tilde{b}^{*}$ may be interpreted as the fiber functor $G \mathscr{K}_{B} \longrightarrow G_{b} \mathscr{K}_{*}$ that sends $X$ to $X_{b}$, $\tilde{b}_{\text {! }}$ may be interpreted as its left adjoint, which we denote by $K^{b}$, and $\tilde{b}_{*}$ may be interpreted as its right adjoint, which we denote by ${ }^{b} K$. With these notations, the 
isomorphisms of Proposition 2.2.2 specialize to the following natural isomorphisms, where $Y$ and $Z$ are in $G \mathscr{K}_{B}$ and $K$ is in $G_{b} \mathscr{K}_{*}$.

$$
\begin{gathered}
\left(Y \wedge_{B} Z\right)_{b} \cong Y_{b} \wedge Z_{b}, \\
F_{B}\left(Y,{ }^{b} K\right) \cong{ }^{b} F\left(Y_{b}, K\right), \\
F_{B}(Y, Z)_{b} \cong F\left(Y_{b}, Z_{b}\right), \\
\left(Y_{b} \wedge K\right)^{b} \cong Y \wedge_{B} K^{b}, \\
F_{B}\left(K^{b}, Y\right) \cong{ }^{b} F\left(K, Y_{b}\right) .
\end{gathered}
$$

ExAMPLE 2.3.13. Several earlier results come together in the following situation. Let $f: A \longrightarrow B$ be a $G$-map. For $b \in B$, let $b:\{*\} \longrightarrow B$ and $i_{b}: A_{b} \longrightarrow A$ denote the evident inclusions of $G_{b}$-spaces. We have the following compatible pullback squares, the first of $G_{b}$-spaces and the second of $G$-spaces.
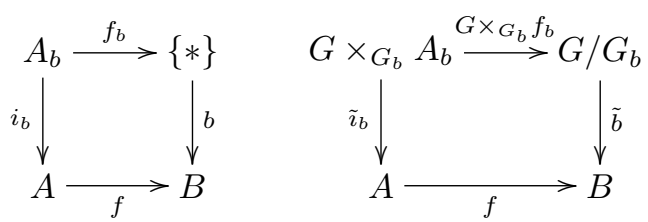

Applying Proposition 2.2.11 to the right-hand square and interpreting the conclusion in terms of fibers by Definition 2.3.7, we obtain canonical isomorphisms of $G_{b}$-spaces

$$
\left(f_{!} X\right)_{b} \cong f_{b !} i_{b}^{*} X \quad \text { and } \quad\left(f_{*} X\right)_{b} \cong f_{b *} i_{b}^{*} X,
$$

where $X$ is an ex- $G$-space over $A$, regarded on the right-hand sides as an ex- $G_{b^{-}}$ space over $A$ by pullback along $\iota: G_{b} \longrightarrow G$.

\subsection{Normal subgroups and quotient groups}

Observe that any homomorphism $\theta: G \longrightarrow G^{\prime}$ factors as the composite of a quotient homomorphism $\varepsilon$, an isomorphism, and an inclusion $\iota$. We studied change of groups along inclusions in the previous section. Here we consider a quotient homomorphism $\epsilon: G \longrightarrow J$ of $G$ by a normal subgroup $N$. We still have a restriction functor

$$
\epsilon^{*}: J \mathscr{K}_{A} \longrightarrow G \mathscr{K}_{\epsilon^{*} A}
$$

and we also have the functors

$$
(-) / N: G \mathscr{K}_{B} \longrightarrow J \mathscr{K}_{B / N} \quad \text { and } \quad(-)^{N}: G \mathscr{K}_{B} \longrightarrow J \mathscr{K}_{B^{N}}
$$

obtained by passing to orbits over $N$ and to $N$-fixed points. When $B$ is a point, these last two functors are left and right adjoint to $\epsilon^{*}$, but in general change of base must enter in order to obtain such adjunctions. The following observation follows directly by inspection of the definitions. 
Proposition 2.4.1. Let $j: B^{N} \longrightarrow B$ be the inclusion and $p: B \longrightarrow B / N$ be the quotient map. Then the following factorization diagrams commute.

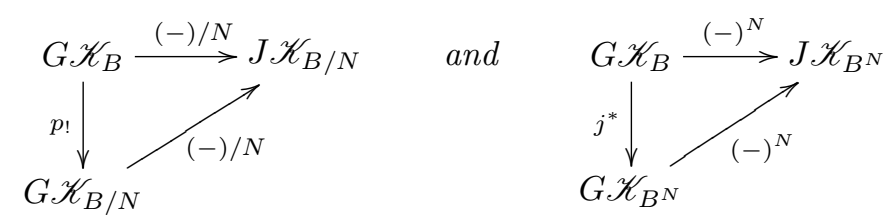

It follows that $\left((-) / N, p^{*} \epsilon^{*}\right)$ and $\left(j ! \epsilon^{*},(-)^{N}\right)$ are adjoint pairs.

We have the following analogue of Proposition 2.3.11, but to state its last part we first recall the following standard definition [79, 4.2.2] and fix a convention to be used throughout the book.

Definition 2.4.2. A $G$-space $P$ is principal if the action of $G$ is free and the translation map $\tau: \operatorname{Orb}(P) \longrightarrow G$ is continuous, where $\operatorname{Orb}(P)=\{(x, g x)\} \subset P \times P$ and $\tau(x, g x)=g$. A principal $G$-bundle is a principal $G$-space $P$ and a projection $p: P \longrightarrow B$ such that $B$ is homeomorphic under $P$ to $P / G$. Henceforward, we adopt the convention that all given free actions are understood to be principal. By Remark 3.1.8 below, this holds automatically if $G$ is compact or, more generally, the action of $G$ is proper.

Proposition 2.4.3. Let $f: A \longrightarrow B$ be a map of $G$-spaces. Then the following diagrams commute up to natural isomorphism.

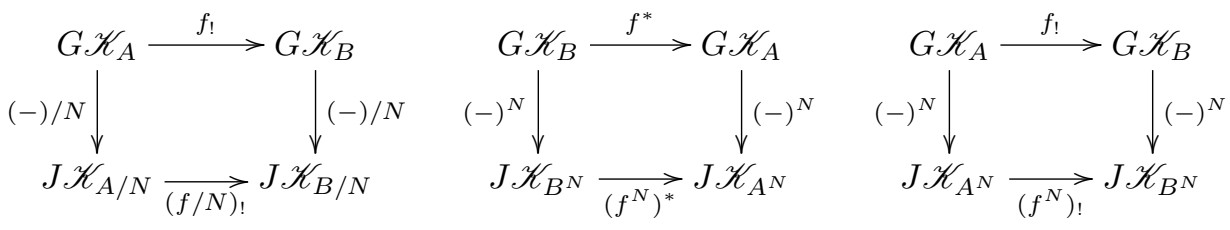

If $B$ is an $N$-free $G$-space, then the following diagram also commutes.

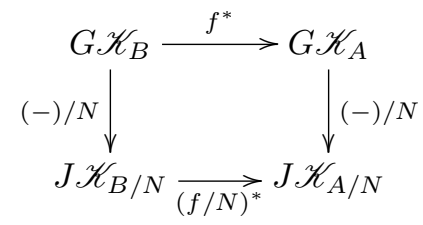

Proof. For ex- $G$-spaces $X$ over $A$ and $Y$ over $B$, the first three isomorphisms are given by the homeomorphisms

and

$$
\begin{gathered}
\left(X \cup_{A} B\right) / N \cong X / N \cup_{A / N} B / N, \\
\left(Y \times_{B} A\right)^{N} \cong Y^{N} \times_{B^{N}} A^{N},
\end{gathered}
$$

$$
\left(X \cup_{A} B\right)^{N} \cong X^{N} \cup_{A^{N}} B^{N} .
$$

As a quibble, the third requires $A \longrightarrow X$ to be a closed inclusion, but this will hold for the sections of compactly generated ex- $G$-spaces over $A$ by Lemma 1.6.2(i). For the fourth diagram, the evident canonical map $\left(f^{*} Y\right) / N \longrightarrow Y / N \times_{B / N} A / N$ is a homeomorphism. Indeed, the inverse $Y / N \times_{B / N} A / N \longrightarrow\left(f^{*} Y\right) / N$ sends $(\bar{y}, \bar{a})$ to $[n y, a]$ where $n \in N$ is the unique element such that $n p(y)=f(a)$. Here $n$ is 
unique since $B$ is $N$-free, and the continuity of the specified inverse is ensured by the tacitly assumed continuity of the translation map.

Specializing to $N$-free $G$-spaces, we obtain a factorization result that is analogous to those in Proposition 2.4.1, but is less obvious. It is a precursor of the Adams isomorphism, which we will derive in $\$ 19.7$, and will have other uses.

Proposition 2.4.4. Let $E$ be an $N$-free $G$-space, let $B=E / N$, and let $p: E \longrightarrow B$ be the quotient map. Then the diagram

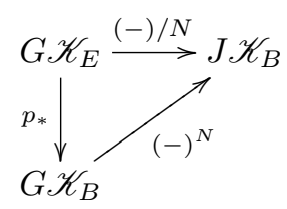

commutes up to natural isomorphism. Therefore the left adjoint $(-) / N$ of the functor $p^{*} \varepsilon^{*}$ is also its right adjoint.

Proof. Let $X$ be an ex- $G$-space over $E$ with projection $q$. Comparing the pullbacks that are used to define the functors $p_{*}$ and $\operatorname{Map}_{B}$ in Definitions 2.1.1 and 1.3.7, we find that $p_{*} X$ fits into a pullback diagram

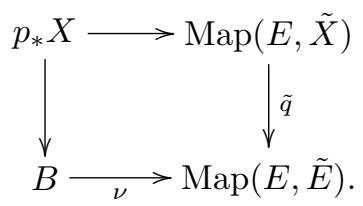

Here $\nu(b), b=N e$, corresponds as in Definition 1.3.6 to the inclusion of the closed subset $N e$ in $E$. Passing to $N$-fixed points, we see that it suffices to prove that the following commutative diagram is a pullback.

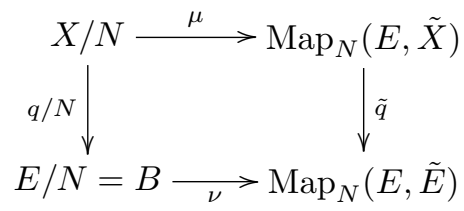

Here $\mu$ is induced from the adjoint of the map $X \times E \longrightarrow \tilde{X}$ that sends $(x, e)$ to $n x$ if $e=n q(x)$ and sends $(x, e)$ to $\omega$ otherwise. With this description, $\mu$ is well-defined since $E$ is $N$-free. It suffices to give a continuous inverse to the induced map

$$
\phi: X / N \longrightarrow \operatorname{Map}_{N}(E, \tilde{X}) \times_{\operatorname{Map}_{N}(E, \tilde{E})} E / N .
$$

If $(f, N e)$ is a point in the pullback, then $f$ corresponds to a map $N e \longrightarrow X$, and $\phi^{-1}(f, N e)=N f(e)$ in $X / N$. For continuity, note that $\phi^{-1}$ is obtained from the evaluation map $\operatorname{Map}(E, \tilde{X}) \times E \longrightarrow \tilde{X}$ by passage to subquotient spaces.

REMARK 2.4.5. This leads to a useful alternative description of the functor $\iota !: H \mathscr{K}_{A} \longrightarrow G \mathscr{K}_{\iota !} A$, where $A$ is an $H$-space and $\iota ! A=G \times_{H} A$, as a composite of right adjoints. We have the projection $\pi: G \times A \longrightarrow A$ of $(G \times H)$-spaces, where the $G \times H$ actions on the source and target are given by

$$
(g, h)\left(g^{\prime}, a\right)=\left(g g^{\prime} h^{-1}, h a\right) \quad \text { and } \quad(g, h) a=h a .
$$


Consider ex- $H$-spaces $X$ over $A$ as $(G \times H)$-spaces with $G$ acting trivially and let $\epsilon: G \times H \longrightarrow H$ be the projection. By inspection of definitions, $\iota ! X=\left(\pi^{*} \varepsilon^{*} X\right) / H$. Since $G \times A$ is an $H$-free $(G \times H)$-space, we conclude from the previous result that $\iota ! X \cong\left(p_{*} \pi^{*} \varepsilon^{*} X\right)^{H}$, where $p: G \times A \longrightarrow G \times_{H} A=\iota ! A$ is the quotient map.

\subsection{The closed symmetric monoidal category of retracts}

Let $\mathscr{B}$ be a topologically bicomplete full subcategory of a topologically bicomplete category $\mathscr{C}$. We are thinking of $\mathscr{U} \subset \mathscr{K}$ or $G \mathscr{U} \subset G \mathscr{K}$. We have the category of retracts $\mathscr{C}_{\mathscr{B}}$. The objects of $\mathscr{C}_{\mathscr{B}}$ are the retractions $B \stackrel{s}{\longrightarrow} X \stackrel{p}{\longrightarrow} B$ with $B \in \mathscr{B}$ and $X \in \mathscr{C}$, abbreviated $(X, p, s)$ or just $X$. The morphisms of $\mathscr{C}_{\mathscr{B}}$ are the evident commutative diagrams. When $\mathscr{B}=\mathscr{C}$, this is just a diagram category for the evident two object domain category.

The importance of the category $\mathscr{C}_{\mathscr{B}}$ is apparent from its role in Definition 2.1.1: focus on this category is natural when we consider base change functors. In our examples, $\mathscr{B}$ and $\mathscr{C}$ are enriched and topologically bicomplete over the appropriate category of spaces, $\mathscr{U}$ for $\mathscr{B}$ and $\mathscr{K}$ for $\mathscr{C}$. For a space $K \in \mathscr{K}$, the tensors $-\times K$ and cotensors $\operatorname{Map}(K,-)$ applied to retractions give retractions, and we have the adjunction homeomorphisms

$$
\mathscr{C}_{\mathscr{B}}(X \times K, Y) \cong \mathscr{K}\left(K, \mathscr{C}_{\mathscr{B}}(X, Y)\right) \cong \mathscr{C}_{\mathscr{B}}(X, \operatorname{Map}(K, Y)) .
$$

Now restrict attention to $G \mathscr{K}_{G \mathscr{U}}$.

Lemma 2.5.2. The category G $\mathscr{K}_{G \mathscr{U}}$ is topologically bicomplete.

Proof. Since we have tensors and cotensors, by (2.5.1), it suffices to show that $G \mathscr{K}_{G \mathscr{U}}$ is bicomplete. As a diagram category, $G \mathscr{K}_{G \mathscr{K}}$ is certainly bicomplete. Since limits in $G \mathscr{U}$ are constructed in $G \mathscr{K}$, it follows directly that $G \mathscr{K}_{G \mathscr{U}}$ is complete. Colimits in $G \mathscr{U}$ are obtained by applying the weak Hausdorffication functor $w$ to colimits in $G \mathscr{K}$. Using Proposition 1.5.4, it follows by direct verification of the universal property that colimits in $G \mathscr{K}$ on total spaces paired with colimits in $G \mathscr{U}$ on base spaces specify colimits in $G \mathscr{K}_{G \mathscr{U}}$.

The category $G \mathscr{K}_{G \mathscr{U}}$ is closed symmetric monoidal under an external smash product functor, denoted $X \bar{\wedge} Y$, and an external function ex-space functor, denoted $\bar{F}(Y, Z)$. If $X, Y$, and $Z$ are ex-spaces over $A, B$, and $A \times B$, respectively, then $X \bar{\wedge} Y$ is an ex-space over $A \times B$ and $\bar{F}(Y, Z)$ is an ex-space over $A$. We have

$$
G \mathscr{K}_{A \times B}(X \bar{\wedge} Y, Z) \cong G \mathscr{K}_{A}(X, \bar{F}(Y, Z)),
$$

which gives the required adjunction in $G \mathscr{K}_{G \mathscr{U}}$. It specializes to parts of (1.4.2) when $A$ or $B$ is a point. The ex-space $X \bar{\wedge} Y$ is the evident fiberwise smash product, with $(X \wedge Y)_{(a, b)}=X_{a} \wedge Y_{b}$. The fiber $\bar{F}(Y, Z)_{a}$ is $F_{B}\left(Y, Z_{a}\right)$, where $Z_{a}$ is the ex-space over $B$ whose fiber $Z_{a, b}$ over $b$ is the inverse image of $(a, b)$ under the projection $Z \longrightarrow A \times B$. Rather than describe the topology of the ex-space $\bar{F}(Y, Z)$ directly, we will shortly give alternative descriptions of $X \bar{\wedge} Y$ and $\bar{F}(Y, Z)$ in terms of internal smash products and internal function ex-spaces. The commutativity isomorphism of the external smash product is evident, but it requires comment because it will

play an important and subtle role later on. 
Remark 2.5.4. Consider the following diagram, where $X$ and $Y$ are ex-spaces over $A$ and $B$ and $t: A \times B \longrightarrow B \times A$ is the interchange map.

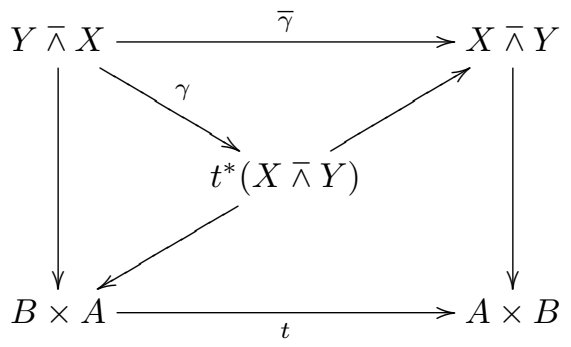

The evident interchange map $\bar{\gamma}$ is the commutativity isomorphism of the external smash product. The lower right square is a pullback, and $\gamma$ is given by the universal property. It is a map of ex-spaces over $B \times A$ and should be regarded as a symmetry isomorphism. When we introduce symmetric bicategories of exspaces and ex-spectra in $\S 17.1, \gamma$ will lead to the relevant symmetry. When we discuss products in parametrized homology and cohomology theories in $\S 20.7$, the commutativity isomorphism $\bar{\gamma}$ will play a key role.

Let $\pi_{A}$ and $\pi_{B}$ be the projections of $A \times B$ on $A$ and $B$ and observe that $\pi_{A}^{*} X \cong X \times B$ and $\pi_{B}^{*} Y \cong A \times Y$. If one likes, the following results can be taken as either a definition of the external operations together with a characterization of the internal operations, or vice versa.

LEMMA 2.5.5. The external smash product and function ex-space functors are determined by the internal functors via natural isomorphisms

$$
X \bar{\wedge} Y \cong \pi_{A}^{*} X \wedge_{A \times B} \pi_{B}^{*} Y \quad \text { and } \quad \bar{F}(Y, Z) \cong \pi_{A *} F_{A \times B}\left(\pi_{B}^{*} Y, Z\right),
$$

where $X, Y$, and $Z$ are ex-spaces over $A, B$, and $A \times B$, respectively.

With these isomorphisms taken as definitions, the adjunction (2.5.3) follows from the adjunctions $\left(\pi_{A}^{*}, \pi_{A *}\right),\left(\pi_{B}^{*}, \pi_{B *}\right)$, and $\left(\wedge_{A \times B}, F_{A \times B}\right)$.

LEMMA 2.5.6. The internal smash product and function ex-space functors are determined by the external functors via natural isomorphisms

$$
X \wedge_{B} Y \cong \Delta^{*}(X \bar{\wedge} Y) \quad \text { and } \quad F_{B}(X, Y) \cong \bar{F}\left(X, \Delta_{*} Y\right),
$$

where $X$ and $Y$ are ex-spaces over $B$ and $\Delta: B \longrightarrow B \times B$ is the diagonal map.

With these isomorphisms taken as definitions, the adjunction $\left(\wedge_{B}, F_{B}\right)$ follows from the adjunctions $\left(\Delta^{*}, \Delta_{*}\right)$ and (2.5.3). Since $\Delta^{*}$ is symmetric monoidal and the composite of either projection $\pi_{i}: B \times B \longrightarrow B$ with $\Delta$ is the identity map of $B$, we see that, if we have constructed both internal and external smash products, then they must be related by natural isomorphisms as in Lemmas 2.5.5 and 2.5.6.

REMARK 2.5.7. The referee of a preliminary version of this work suggested that we give a consistency check on fibers. The fiber $\left(\Delta_{*} Y\right)_{(b, c)}$ is a point if $b \neq c$ and is $Y_{b}$ if $b=c$. Therefore the fiber over $b$ of the restriction $\left(\Delta_{*} Y\right)_{b}$ of $\Delta_{*} Y$ to $\{b\} \times B$ is $Y_{b} \cup(B-\{b\})$, suitably topologized, and

$$
\bar{F}\left(X, \Delta_{*} Y\right)_{b}=F_{B}\left(X,\left(\Delta_{*} Y\right)_{b}\right)_{b} \cong F\left(X_{b}, Y_{b}\right)=F_{B}(X, Y)_{b} .
$$


REMARK 2.5.8. The description of the internal smash product in terms of the external smash product sheds light on the basic compatibility isomorphisms (2.2.3) and (2.2.6). For maps $f: A \longrightarrow B$ and $g: A^{\prime} \longrightarrow B^{\prime}$ and for ex-spaces $X$ over $B$ and $Y$ over $B^{\prime}$, it is easily checked that

$$
f^{*} Y \bar{\wedge} g^{*} Z \cong(f \times g)^{*}(Y \bar{\wedge} Z) .
$$

Similarly, for ex-spaces $W$ over $A$ and $X$ over $A^{\prime}$,

$$
f_{!} W \bar{\wedge} g ! X \cong(f \times g) !(W \bar{\wedge} X) .
$$

Now take $A=A^{\prime}, B=B^{\prime}$ and $f=g$. For ex-spaces $Y$ and $Z$ over $B$,

$$
f^{*}\left(Y \wedge_{B} Z\right) \cong f^{*} \Delta_{B}^{*}(Y \bar{\wedge} Z) \cong\left(\Delta_{B} \circ f\right)^{*}(Y \bar{\wedge} Z) .
$$

On the other hand, using (2.5.9),

$$
f^{*} Y \wedge_{A} f^{*} Z \cong \Delta_{A}^{*}(f \times f)^{*}(Y \bar{\wedge} Z) \cong\left((f \times f) \circ \Delta_{A}\right)^{*}(Y \bar{\wedge} Z) .
$$

The right sides are isomorphic since $\Delta_{B} \circ f=(f \times f) \circ \Delta_{A}$. Similarly,

$$
f_{!}\left(f^{*} Y \wedge_{A} X\right) \cong f_{!} \Delta_{A}^{*}(f \times \mathrm{id})^{*}(Y \bar{\wedge} X) \cong f_{!}\left((f \times \mathrm{id}) \circ \Delta_{A}\right)^{*}(Y \bar{\wedge} X),
$$

while

$$
Y \wedge_{B} f_{!} X \cong \Delta_{B}^{*}(\mathrm{id} \times f) !(Y \bar{\wedge} X)
$$

The naturality $\Delta_{B} \circ f=(f \times f) \circ \Delta_{A}$ can be rewritten as the pullback diagram

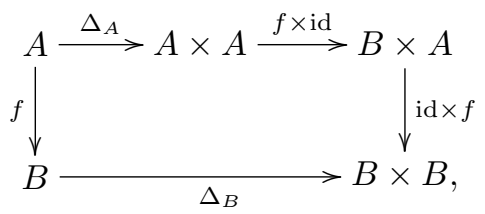

and the right sides are isomorphic by Proposition 2.2.11.

It is illuminating conceptually to go further and consider group actions from an external point of view. For groups $H$ and $G$, an $H$-space $A$, and a $G$-space $B$, we have an evident external smash product

$$
\bar{\wedge}: H \mathscr{K}_{A} \times G \mathscr{K}_{B} \rightarrow(H \times G) \mathcal{K}_{A \times B}
$$

For an ex- $H$-space $X$ over $A$ and an ex- $G$-space $Y$ over $B, X \wedge Y$ is just the internal smash product over the $(H \times G)$-space $A \times B$ of $\pi_{H}^{*} \pi_{A}^{*} X$ and $\pi_{G}^{*} \pi_{B}^{*} Y$, where the $\pi^{\prime} s$ are the projections from $H \times G$ and $A \times B$ to their coordinates. It is easily seen that this definition leads to another $(\bar{\wedge}, \bar{F})$ adjunction.

When $H=G$, the diagonal $\Delta: G \longrightarrow G \times G$ is a closed inclusion since $G$ is compactly generated. We can pull back along $\Delta$, and then our earlier external smash product $X \bar{\wedge} Y$ over the $G$-space $\Delta^{*}(A \times B)$ is given in terms of (2.5.11) as the pullback $\Delta^{*}(X \bar{\wedge} Y)$. Note that, by Proposition 2.3.10, $\Delta^{*}$ here can be viewed as a base change functor. 


\section{CHAPTER 3}

\section{Proper actions, equivariant bundles and fibrations}

\section{Introduction}

Proper actions by locally compact groups are well-behaved actions whose isotropy groups are compact. All actions by compact Lie groups are proper, and an action of a discrete group is proper if and only if it is properly discontinuous. We discuss proper actions of locally compact groups in $\S 3.1$.

In $\S 3.2$, we describe a class of equivariant bundles that will play an important role in Parts IV and V. Starting from a principal bundle $P \longrightarrow B=P / \Pi$ (with appropriate equivariance) and an associated bundle $E=P \times_{\Pi} F \longrightarrow B$, we show how to insert ex-spaces over $\mathrm{F}$ fiberwise into the functor $P \times_{\Pi}(-)$ to obtain exspaces over $E$ in $\S 3.3$, and we show that this "bundle construction" gives a closed symmetric monoidal functor that commutes with base change functors. This sets the stage for the definition and use of a spectrum level analogue, starting in §15.4.

In a different direction, we generalize Waner's equivariant versions [168] of Milnor's results about spaces of the homotopy types of CW complexes in $\S 3.4$, and we generalize classical theorems of Dold and Stasheff about Hurewicz fibrations in §3.5. We also recall an important but little known result of Steinberger and West that relates Serre and Hurewicz fibrations. We define equivariant quasifibrations in $\S 3.6$.

\subsection{Proper actions of locally compact groups}

For appropriate generality and technical convenience, we let $G$ be a locally compact topological group whose underlying topological space is compactly generated. Local compactness means that the identity element, hence any point, has a compact neighborhood. We see from Proposition 1.6.1 that $G$ is Hausdorff and, since all compact subsets are closed, it follows that each neighborhood of any point contains a compact neighborhood.

REMARK 3.1.1. We comment on the assumptions we make for $G$. If $G$ is any topological group whose underlying space is in $\mathscr{K}$, then an action of $G$ on $X$ in $\mathscr{K}$ may not come from an action in $\mathscr{T}$ op. The point is that the product $G \times X$ in $\mathscr{K}$ is defined by applying the $k$-ification functor to the product $G \times_{c} X$ in $\mathscr{T} o p$, and not every action $G \times X \longrightarrow X$ need be continuous when viewed as a function $G \times_{c} X \longrightarrow X$. However, when $G$ is locally compact, $G \times_{c} X$ is already in $\mathscr{K}$ by Proposition 1.6.1, and $k$-ification is not needed. There is then no ambiguity about what we mean by a $G$-space, and we need not worry about refining the topology on products with $G$.

Another reason for restricting to locally compact groups is that many useful properties of proper actions only hold in that case. In the literature, such results 
are usually derived for actions on Hausdorff spaces, but we shall see that weak Hausdorff generally suffices.

We begin with some standard equivariant terminology.

Definition 3.1.2. Let $X$ be a $G$-space and let $H \subset G$.

(i) An $H$-tube $U$ in $X$ is an open $G$-invariant subset of $X$ together with a $G$-map $\pi: U \longrightarrow G / H$. If $x \in U$ and $H=G_{x}$, then $U$ is a tube around $x$. A tube is contractible if $\pi$ is a $G$-homotopy equivalence.

(ii) An $H$-slice $S$ in $X$ is an $H$-invariant subset such that the canonical $G$-map $G \times{ }_{H} S \longrightarrow G S \subset X$ is an embedding onto an open subset. Then $G S$ is an $H$-tube with $S=\pi^{-1}(e H)$. Conversely, if $(U, \pi)$ is an $H$-tube in $X$, then $S=\pi^{-1}(e H)$ is an $H$-slice and $U=G S$. On isotropy subgroups, we then have $G_{y}=H_{y} \subset H$ for all $y \in S$, but equality need not hold. If $x \in S$ and $H=G_{x}$, then $S$ is a slice through $x$.

(iii) We say that $X$ has enough slices if every point $x \in X$ is contained in an $H$-slice for some compact subgroup $H$. This implies that every point $x$ has compact isotropy group, but in general it does not imply that there must be a slice through every point $x$.

(iv) A $G$-numerable cover of $X$ is a cover $\left\{U_{j}\right\}$ by tubes such that there exists a locally finite partition of unity by $G$-maps $\lambda_{j}: X \longrightarrow[0,1]$ with support $U_{j}$.

The following is the equivariant generalization of $[48,6.7]$.

Proposition 3.1.3. Any G-CW complex admits a $G$-numerable cover by contractible tubes.

Proof. The proof given by Dold [48] in the nonequivariant case goes through with only a minor change in the initial construction, which we sketch. From there, the technical details are unchanged. Let $X^{n}$ be the $n$-th skeletal filtration of a $G$ CW complex $X$. Let $\dot{X}^{n}$ denote the subspace obtained by deleting the central orbits $(G / H \times 0$ for a cell of orbit type $G / H)$ of all $n$-cells in $X^{n}$ and let $r_{n}: \dot{X}^{n} \longrightarrow X^{n-1}$ denote the obvious retract. Starting from the interior $e_{n}=G / H \times\left(D^{n}-S^{n-1}\right)$ of a fixed $n$-cell, define $V_{n}^{m}$ inductively for $m \geq n$ by setting $V_{n}^{n}=e_{n}$ and $V_{n}^{m+1}=$ $r_{m+1}^{-1}\left(V_{n}^{m}\right)$. Then the union $V_{n}^{\infty}=\bigcup_{m \geq n} V_{n}^{m}$ is a contractible tube, where the projection to $G / H$ is induced by the projection of $e_{n}$ to $G / H$.

We now give the definition of a proper group action in $\mathscr{K}$. We shall see that the definition could equivalently be made in $\mathscr{U}$. For further details, but in $\mathscr{T} o p$, see for example $[24,47]$. Recall that a continuous map is proper if it is a closed map with compact fibers.

Definition 3.1.4. A $G$-space $X$ in $G \mathscr{K}$ is proper (or $G$-proper) if the map

$$
\theta: G \times X \longrightarrow X \times X
$$

specified by $\theta(g, x)=(x, g x)$ is proper.

We warn the reader that this definition is not quite the standard one. We are working in the category $\mathscr{K}$, and the product $X \times X$ on the right hand side is the $k$-space obtained by $k$-ifying the standard product topology on $X \times_{c} X$. In $\mathscr{T}$ op there are various other notions of a proper group action; see [12] for a careful discussion. They all agree for actions of locally compact groups on completely regular spaces. If $X$ is proper, then the isotropy groups $G_{x}$ are compact since they 
are the fibers $\theta^{-1}(x, x)$. Moreover, since points are closed subsets of $G$, the diagonal $\Delta_{X}=\theta(\{e\} \times X)$ must be a closed subset of $X \times X$ and thus $X$ must be weak Hausdorff. This means that proper $G$-spaces must be in $\mathscr{U}$. Since $G$ is locally compact, we have the following useful characterizations.

Proposition 3.1.5. For a $G$-space $X$ in $G \mathscr{K}$ the following are equivalent.

(i) The action of $G$ on $X$ is proper.

(ii) The isotropy groups $G_{x}$ are compact and for any point $(x, y) \in X \times X$ and any neighborhood $U$ of $\theta^{-1}(x, y)$ in $G \times X$, there is a neighborhood $V$ of $(x, y)$ in $X \times X$ such that $\theta^{-1}(V) \subset U$.

(iii) The isotropy groups $G_{x}$ are compact and for any $(x, y) \in X \times X$ and any neighborhood $U$ of $\{g \mid g x=y\}$ in $G$, there is a neighborhood $V$ of $(x, y)$ such that

$$
\{g \in G \mid g a=b \text { for some }(a, b) \in V\} \subset U .
$$

(iv) The space $X$ is weak Hausdorff and every point $(x, y) \in X \times X$ has a neighborhood $V$ such that

$$
\{g \in G \mid g a=b \text { for some }(a, b) \in V\}
$$

has compact closure in $G$.

Proof. This holds by essentially the same proof as $[12,1.6(\mathrm{~b})]$. One must only keep in mind that we are now working in $\mathscr{K}$ rather than in $\mathscr{T}$ op and adjust the argument accordingly.

Corollary 3.1.6. If $G$ is discrete, then a $G$-space $X$ is proper if and only if any point $(x, y) \in X \times X$ has a neighborhood $V$ such that

$$
\{g \in G \mid g a=b \text { for some }(a, b) \in V\}
$$

is finite.

Corollary 3.1.7. If $G$ is compact, then any $G$-space in $G \mathscr{U}$ is proper. More generally, if $\Gamma=G \times \Pi$, where $G$ is compact and $\Pi$ is locally compact, then any $\Pi$-free $\Gamma$-space in $\Gamma \mathscr{U}$ is proper.

Remark 3.1.8. Recall Definition 2.4.2 and let $G$ act freely on $X$. Since the fibers of $\theta$ are points, the action is proper if and only if $\theta$ is a closed map. Since $\theta$ is injective, it is then a homeomorphism onto its image. However, its image is the domain $\operatorname{Orb}(X)$ of the translation map $\tau$, and $\tau$ is the composite $\pi \circ \theta^{-1}$, where $\pi: G \times X \longrightarrow G$ is the projection. Therefore $\tau: \operatorname{Orb}(X) \longrightarrow G$ is necessarily continuous, as required by our standing convention on free actions, when $G$ acts freely and properly on $X$. This always holds when $X$ is in $G \mathscr{U}$ and $G$ is compact.

REMARK 3.1.9. There is an alternative description of the set displayed in Proposition 3.1.5 that may clarify the characterization. Define

$$
\phi: G \times X \times X \longrightarrow X \times X
$$

by $\phi(g, x, y)=(g x, y)$. For $V \subset X \times X$, let $\phi_{V}$ be the restriction of $\phi$ to $G \times V$ and let $\pi: G \times V \longrightarrow G$ be the projection, which is an open map since $G \times V$ has the product topology. Then the displayed set is $\pi \phi_{V}^{-1}\left(\Delta_{X}\right)$. If $X \times X=X \times_{c} X$, then the condition in Proposition 3.1.5 is equivalent to the more familiar one that any two points $x$ and $y$ in $X$ have neighborhoods $V_{x}$ and $V_{y}$ such that

$$
\left\{g \in G \mid g V_{x} \cap V_{y} \neq \emptyset\right\}
$$


has compact closure in $G$.

Proposition 3.1.10. Proper actions satisfy the following closure properties.

(i) The restriction of a proper action to a closed subgroup is proper.

(ii) An invariant subspace of a proper $G$-space is also proper.

(iii) Products of proper $G$-spaces are proper.

(iv) If $X$ is a proper Hausdorff $G$-space in $G \mathscr{K}$ and $C$ is a compact Hausdorff $G$-space, then the $G$-space $\operatorname{Map}(C, X)$ is proper.

(v) An $H$-space $S$ is $H$-proper if and only if $G \times_{H} S$ is $G$-proper.

Proof. The first three are standard and elementary; see for example [47, I.5.10]. The fifth is $[12,2.3]$. We prove (iv). We must show that the map

$$
\theta: G \times \operatorname{Map}(C, X) \longrightarrow \operatorname{Map}(C, X) \times \operatorname{Map}(C, X)
$$

is proper, which amounts to showing that it is closed and that the isotropy groups $G_{f}$ are compact for $f \in \operatorname{Map}(C, X)$. For the latter, let $\left\{g_{i}\right\}$ be a net in $G_{f}$ and fix $c \in C$. Note that $f\left(g_{i} c\right)=g_{i} f(c)$. Since $C$ is compact, we can assume by passing to a subnet that $\left\{g_{i} c\right\}$ converges to some $\bar{c} \in C$. Let $V$ be a neighborhood of $(f(c), f(\bar{c}))$ such that

$$
B=\{g \in G \mid g a=b \text { for some }(a, b) \in V\}
$$

has compact closure. Since $C$ is compact, $C \times C \times \operatorname{Map}(C, X)$ has the usual product topology. Since the map

$$
C \times C \times \operatorname{Map}(C, X) \longrightarrow X \times X
$$

that sends $(c, d, f)$ to $(f(c), f(d))$ is continuous and the net $\left\{c, g_{i} c, f\right\}$ converges to $(c, \bar{c}, f)$, the net $\left\{\left(f(c), f\left(g_{i} c\right)\right)\right\}=\left\{\left(f(c), g_{i} f(c)\right)\right\}$ must converge to $(f(c), f(\bar{c}))$. It follows that a subnet of $\left\{g_{i}\right\}$ lies in $B$ and therefore has a converging sub-subnet.

To show that $\theta$ is closed, let $A$ be a closed subset of $G \times \operatorname{Map}(C, X)$ and let $\left\{\left(f_{i}, g_{i} f_{i}\right)\right\}$ be a net in $\theta(A)$ that converges to $(f, F)$. We must show that $(f, F)$ is in $\theta(A)$. For $c \in C$, the net $\left\{g_{i}^{-1} c\right\}$ has a subnet that converges to some $\bar{c}$, by the compactness of $C$, so we may as well assume that the original net converges to $\bar{c}$. Let $V$ be a neighborhood of $(f(\bar{c}), F(c))$ such that

$$
B^{\prime}=\{g \in G \mid g a=b \text { for some }(a, b) \in V\}
$$

has compact closure. By continuity and the compactness of $C$, there is a compact neighborhood $K_{1} \times K_{2}$ of $(\bar{c}, c)$ that $(f, F)$ maps into $V$. Since $\left\{\left(f_{i}, g_{i} f_{i}\right)\right\}$ converges to $(f, F)$, there is an $h$ such that $\left(f_{i}, g_{i} f_{i}\right)\left(K_{1} \times K_{2}\right) \subset V$ for $i \geq h$. It follows that there is a $k \geq h$ such that $\left(f_{i}\left(g_{i}^{-1} c\right), g_{i} f_{i}\left(g_{i}^{-1} c\right)\right) \in V$ for all $i \geq k$. Then the subnet $\left\{g_{i}\right\}_{i \geq k}$ is contained in $B^{\prime}$ and therefore has a sub-subnet that converges to some $g \in G$. We have now seen that our original net $\left\{\left(g_{i}, f_{i}\right)\right\}$ in $A$ has a subnet $\left\{\left(g_{i_{j}}, f_{i_{j}}\right)\right\}$ that converges to $(g, f)$, and $(g, f) \in A$ since $A$ is closed. By the continuity of $\theta,\left\{\theta\left(g_{i}, f_{i}\right)\right\}$ must converge to $(f, F)=\theta(g, f) \in \theta(A)$. In this last statement, we are using the uniqueness of limits, which we ensure by requiring $X$ and $C$ to be Hausdorff.

The following theorem of Palais [136], as generalized by Biller [12], is fundamental. Those sources work in $\mathscr{T} o p$, but the arguments work just as well in $\mathscr{U}$.

Theorem 3.1.11 (Palais). Let $X$ be a $G$-space in $G \mathscr{U}$.

(i) If $X$ has enough slices, then it is proper. 
(ii) Conversely, if $X$ is completely regular and proper, then it has enough slices.

(iii) If $G$ is a Lie group and $X$ is completely regular and proper, then there is a slice through each point of $X$.

Proof. Part (i) is given by $[12,2.4]$. Part (iii) is given by [136, 2.3.3]. Part (ii) is deduced from part (iii) in $[12,2.5]$.

\subsection{Proper actions and equivariant bundles}

We introduce here the equivariant bundles to which we will apply our basic foundational results in Part IV. The applications there will focus on compact Lie groups, but we need greater generality in Part V. As we explain, Theorem 3.1.11 allows us to generalize some basic results about such bundles from actions of compact Lie groups to proper actions of locally compact groups.

Let $\Pi$ be a normal subgroup of a locally compact group $\Gamma$ such that $\Gamma / \Pi=G$ and let $q: \Gamma \longrightarrow G$ be the quotient homomorphism. By a principal $(\Pi ; \Gamma)$-bundle we mean the quotient map $p: P \longrightarrow P / \Pi$ where $P$ is a $\Pi$-free $\Gamma$-space such that $\Gamma$ acts properly on $P$. It follows that the induced $G$-action on $B=P / \Pi$ is proper. If $F$ is a $\Gamma$-space, then we have the associated $G$-map $E=P \times_{\Pi} F \longrightarrow P \times_{\Pi} * \cong P / \Pi$, which we say is a $\Gamma$-bundle with structure group $\Pi$ and fiber $F$.

For compact Lie groups, bundles of this form are studied in [92], which generalizes the study of the classical case $\Gamma=G \times \Pi$ given in [91]. In view of Corollary 3.1.7, the classical case works equally well when $G$ but not necessarily $\Pi$ is compact. A summary and further references are given in [118, Chapter VII]. We recall an observation about bundles of this sort.

Lemma 3.2.1. For $b \in B$, the action of $\Gamma$ on $F$ induces an action of the isotropy group $G_{b}$ on the fiber $E_{b}$ through a homomorphism $\rho_{b}: G_{b} \longrightarrow \Gamma$ such that $q \circ \rho_{b}$ is the inclusion $G_{b} \longrightarrow G$ and $E_{b}$ is $G_{b}$-homeomorphic to $\rho_{b}^{*} F$.

Proof. Choose $z \in P$ such that $\pi(z)=b$. The isotropy group $\Gamma_{z}$ intersects $\Pi$ in the trivial group, and $q$ maps $\Gamma_{z}$ isomorphically onto $G_{b}$. Let $\rho_{b}$ be the composite of $q^{-1}: G_{b} \longrightarrow \Gamma_{z}$ and the inclusion $\Gamma_{z} \longrightarrow \Gamma$. Since the subspace $\{z\} \times F$ of $P \times F$ is $\Gamma_{z}$-invariant and maps homeomorphically onto $E_{b}$ on passage to orbits over $\Pi$, the conclusion follows. Note that changing the choice of $z$ changes $\rho_{b}$ by conjugation by an element of $\Pi$ and changes the identification of $E_{b}$ with $F$ correspondingly.

Bundles should be locally trivial. When $P$ is completely regular, local triviality is a consequence of Theorem 3.1.11(iii), just as in the case when $\Gamma$ is a compact Lie group [92, Lemma 3], and this justifies our bundle-theoretic terminology. Note that if $P$ is completely regular, then so is $B=P / \Pi$.

Lemma 3.2.2. A completely regular principal $(\Pi ; \Gamma)$-bundle $P$ is locally trivial. That is, for each $b \in B$, there is a slice $S_{b}$ through $b$ and a homeomorphism

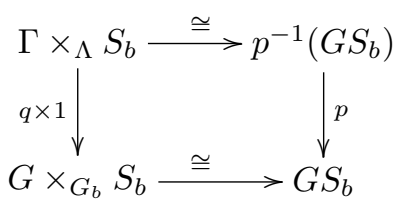

where $\Lambda \subset \Gamma$ only intersects $\Pi$ in the identity element and is mapped isomorphically to $G_{b}$ by $q$. The $\Lambda$-action on $S_{b}$ is given by pulling back the $G_{b}$-action along $q$. 


\subsection{The bundle construction}

In $\S 15.4$ we will introduce a bundle construction on parametrized spectra that will play a central role in Parts IV and V. We consider the underlying space level bundle construction here. We retain the notations of the previous section, so that $P$ is a proper $\Pi$-free $\Gamma$-space, $E=P \times_{\Pi} F$ for a $\Gamma$-space $F$, and $B=P / \Pi$.

Applying the functor $P \times_{\Pi}(-)$ to retracts gives the functor

$$
P_{F}: \Gamma \mathscr{K}_{F} \longrightarrow G \mathscr{K}_{E} \text {. }
$$

Thus, for an ex- $\Gamma$-space $K$ over $F$, the ex- $G$-space $P \times_{\Pi} K$ over $P \times_{\Pi} F$ has section and projection induced by the section and projection of $K$. Observe that if $F$ is a smooth manifold and $S^{\tau}$ is the sphere bundle obtained by fiberwise one-point compactification of the tangent bundle of $F$, then $P_{F} S^{\tau}$ is the $G$-bundle of spherical tangents along the fiber associated to $p: E \longrightarrow B$.

The bundle construction has good formal properties. For example, we have the following observation.

Lemma 3.3.1. The functor $P_{F}: \Gamma \mathscr{K}_{F} \longrightarrow G \mathscr{K}_{E}$ is both a left and a right adjoint.

Proof. Let $\pi: P \times F \longrightarrow F$ be the projection. Clearly $P_{F}$ is the composite of $\pi^{*}: \Gamma \mathscr{K}_{F} \longrightarrow \Gamma \mathscr{K}_{P \times F}$ and $(-) / \Pi: \Gamma \mathscr{K}_{P \times F} \longrightarrow G \mathscr{K}_{E}$. Certainly $\pi^{*}$ is both a left and a right adjoint and $(-) / \Pi$ is a left adjoint. By Proposition 2.4.4, $(-) / \Pi$ coincides with the right adjoint $(-)^{\Pi} \circ p_{*}$, where $p$ is the quotient map $P \times F \longrightarrow$ $P \times_{\Pi} F=E$.

For $b \in B$, we have the fiber $G_{b}$-space $E_{b}$. The following observation, which is immediate by inspection from Lemma 3.2.1, shows what happens if we apply the bundle construction and then restrict it to obtain an ex- $G_{b}$-space over $E_{b}$.

Lemma 3.3.2. Fix $b \in B$. Let $\iota: G_{b} \longrightarrow G$ and $\rho_{b}: G_{b} \longrightarrow \Gamma$ be the inclusion and the homomorphism of Lemma 3.2.1. Let $b: * \longrightarrow B$ and $i_{b}: E_{b} \longrightarrow E$ denote the evident inclusions of $G_{b}$-spaces. Then the following diagram commutes.

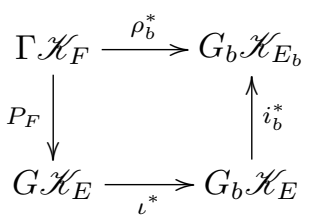

It is crucial to our applications that the functor $P_{F}$ is (strong) monoidal. A key point in the proof is the observation that we can factor the diagonal map of any $G$-space $E$ over $B$ as the composite

$$
\Delta_{E}: E \stackrel{\delta_{E}}{\longrightarrow} E \times{ }_{B} E \stackrel{\iota_{E}}{\longrightarrow} E \times E
$$

of the fiberwise diagonal $\delta_{E}$ and the inclusion $\iota_{E}$. We begin the proof by showing that the non-sectioned analogue of the functor $P_{F}$, which we also denote by $P_{F}$, preserves cartesian products.

LEMma 3.3.4. The functor

$$
P_{F}: \Gamma \mathscr{K} / F \longrightarrow G \mathscr{K} / E
$$

is cartesian. 
Proof. Clearly $P_{F}(F)=E$. Let $K$ and $L$ be spaces over $F$. Then

$$
P_{F}\left(K \times_{F} L\right) \cong\left(\pi_{F}^{*} \Delta_{F}^{*}(K \times L)\right) / \Pi,
$$

where the maps

$$
P \times F \stackrel{\pi_{F}}{\longrightarrow} F \stackrel{\Delta_{F}}{\longrightarrow} F \times F
$$

are the evident projection and diagonal. The composite $\Delta_{F} \pi_{F}$ agrees with the following composition of diagonal, inclusion, and projection maps.

$$
P \times F \stackrel{\delta_{P \times F}}{\longrightarrow}(P \times F) \times_{P}(P \times F) \stackrel{\iota_{P \times F}}{\longrightarrow} P \times F \times P \times F \stackrel{\pi}{\longrightarrow} F \times F .
$$

Recall from the last square of Proposition 2.4.3 and its proof that pullbacks commute with passage to orbits when the base is $\Pi$-free. Using this, we see that, after passage to orbits over $\Pi$, the first two maps become $\delta_{E}$ and $\iota_{E}$. Now the first and third of the following isomorphisms are clear, and the second is given by the unsectioned version of the last square of Proposition 2.4.3.

$$
\begin{aligned}
P_{F}\left(K \times_{F} L\right) & \cong\left(\delta_{P \times F}^{*} \iota_{P \times F}^{*}((P \times K) \times(P \times L))\right) / \Pi \\
& \cong \delta_{E}^{*} \iota_{E}^{*}\left(P_{F} K \times P_{F} L\right) \\
& \cong P_{F} K \times_{E} P_{F} L .
\end{aligned}
$$

We now consider the behavior of the bundle construction with respect to external smash products and diagonal maps. Taking $F=*$, we obtain a functor $P_{*}$, and $\delta_{E} \cong P_{*} \Delta_{F}$ under the identification of their targets given by Lemma 3.3.4:

$$
\delta_{E}: E=P_{*} F \stackrel{\operatorname{id} \times_{\Pi} \Delta_{F}}{\longrightarrow} P_{*}(F \times F) \cong E \times_{B} E .
$$

The inclusion $\delta_{E}$ is a special case of the inclusion $\iota: E_{1} \times_{B} E_{2} \longrightarrow E_{1} \times E_{2}$ for $G$-spaces $E_{1}$ and $E_{2}$ over $B$. We apply this inclusion when the $E_{i}$ are of the form $P \times_{\Pi} F_{i}$ for $\Gamma$-spaces $F_{i}$ to relate external smash products to the functors $P_{F}$.

Proposition 3.3.6. For $K_{i} \in \Gamma \mathscr{K}_{F_{i}}$,

$$
\iota^{*}\left(P_{F_{1}} K_{1} \wedge P_{F_{2}} K_{2}\right) \cong P_{F_{1} \times F_{2}}\left(K_{1} \bar{\wedge} K_{2}\right) .
$$

For $K \in \Gamma \mathscr{K}_{F}$ and $L \in \Gamma \mathscr{K}_{F \times F}$,

$$
P_{F \times F}\left(\Delta_{!} K\right) \cong \delta_{E !} P_{F} K \quad \text { and } \quad P_{F}\left(\Delta^{*} L\right) \cong \delta_{E}^{*} P_{F \times F} L .
$$

Proof. Using that each functor $P_{F}$ is obtained from the functor $P \times_{\Pi}(-)$ by remembering extra structure and that $P \times_{\Pi}(-)$ is cartesian, we see that

$P_{F_{1} \times F_{2}}\left(K_{1} \times K_{2}\right)=P \times_{\Pi}\left(K_{1} \times K_{2}\right) \cong\left(P \times_{\Pi} K_{1}\right) \times_{B}\left(P \times_{\Pi} K_{2}\right)=P_{F_{1}} K_{1} \times_{B} P_{F_{2}} K_{2}$ as ex- $G$-spaces over $E_{1} \times{ }_{B} E_{2}$. We can identify the ex- $G$-space $P_{F_{1}} K_{1} \wedge P_{F_{2}} K_{2}$ over $E_{1} \times E_{2}$ with the pushout of the diagram

$$
E_{1} \times E_{2} \longleftarrow\left(P_{F_{1}} K_{1} \times E_{2}\right) \cup\left(E_{1} \times P_{F_{2}} K_{2}\right) \longrightarrow P_{F_{1}} K_{1} \times P_{F_{2}} K_{2} .
$$

It follows from the pullback diagram

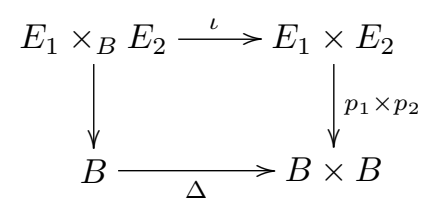


that we can identify the ex-G-space $\iota^{*}\left(P_{F_{1}} K_{1} \bar{\wedge} P_{F_{2}} K_{2}\right)$ over $E_{1} \times_{B} E_{2}$ with the pushout of the diagram

$$
E_{1} \times_{B} E_{2} \longleftarrow\left(P_{F_{1}} K_{1} \times_{B} E_{2}\right) \cup\left(E_{1} \times_{B} P_{F_{2}} K_{2}\right) \longrightarrow P_{F_{1}} K_{1} \times_{B} P_{F_{2}} K_{2} .
$$

Again using that $P_{*}$ is cartesian, we see that this pushout is isomorphic to the pushout of the diagram

$$
P \times_{\Pi}\left(F_{1} \times F_{2}\right) \longleftarrow P \times_{\Pi}\left(K_{1} \times F_{2}\right) \cup P \times_{\Pi}\left(F_{1} \times K_{2}\right) \longrightarrow P \times_{\Pi}\left(K_{1} \times K_{2}\right),
$$

which is $P \times_{\Pi}\left(K_{1} \bar{\wedge} K_{2}\right)=P_{F_{1} \times F_{2}}\left(K_{1} \bar{\wedge} K_{2}\right)$. This gives the first isomorphism.

For the second and third isomorphisms, we factor $P_{F}$ for any $F$ as the composite $\pi_{F}^{*}(-) / \Pi$, where $\pi_{F}: P \times F \longrightarrow F$ is the projection. This applies equally well with $F$ replaced by $F \times F$. Together with our identification of $\delta_{E}$ in (3.3.5), this gives the first and fourth of the following isomorphisms. The second follows from an evident pullback square of base spaces, and the third follows from the first square in Proposition 2.4.3.

$$
\begin{aligned}
P_{F \times F} \Delta_{!} K & \cong\left(\pi_{F \times F}^{*} \Delta_{!} K\right) / \Pi \\
& \cong\left((P \times \Delta) ! \pi_{F}^{*} K\right) / \Pi \\
& \cong(P \times \Pi \Delta) !\left(\pi_{F}^{*} K\right) / \Pi \\
& \cong \delta_{E !} P_{F} K
\end{aligned}
$$

This gives the second stated isomorphism. The proof of the third is completely parallel, except that we now use the fourth square in Proposition 2.4.3.

Putting these isomorphisms together, we obtain that $P_{F}$ is monoidal, and in fact closed symmetric monoidal in the sense of (2.2.1).

Proposition 3.3.7. The functor

$$
P_{F}: \Gamma \mathscr{K}_{F} \longrightarrow G \mathscr{S}_{E}
$$

is closed symmetric monoidal

Proof. Clearly $S_{E}^{0} \cong P_{F}\left(S_{F}^{0}\right)$ since $P \times_{F}(-)$ commutes with disjoint unions. Let $K$ and $L$ be ex- $\Gamma$-spaces over $F$. Applying $\delta_{E}^{*}$ to the first isomorphism in Proposition 3.3.6 and using the factorization of $\Delta_{E}$ from (3.3.3) on the left and the third isomorphism in Proposition 3.3.6 on the right, we obtain

$$
P_{F} K \wedge_{E} P_{F} L \cong \delta_{E}^{*} \iota_{E}^{*}\left(P_{F} K \bar{\wedge} P_{F} L\right) \cong \delta_{E}^{*}\left(P_{F \times F}(K \bar{\wedge} L)\right) \cong P_{F}\left(K \wedge_{F} L\right) .
$$

For the closed part, we must show that the natural map

$$
P_{F} F_{F}(K, L) \longrightarrow F_{E}\left(P_{F} K, P_{F} L\right)
$$

is an isomorphism. Using Lemma 3.3.2, we see that this map restricts over $E_{b}$ to the homeomorphism

$$
\rho_{b}^{*} F_{F}(K, L) \cong F_{E_{b}}\left(\rho_{b}^{*} K, \rho_{b}^{*} L\right)
$$

To see that the map is actually a homeomorphism and not just a continuous bijection, we use the factorization of $P_{F}$ as the composite of $(-) / \Pi$ and $\pi^{*}$ from Lemma 3.3.1. We know that $\pi^{*}$ is closed monoidal, and we use the fact that passage to orbits is ( $k$-ification of) a closed map and the methods of $[21, \S 7]$ to check that the map $F_{P \times F}\left(\pi^{*} K, \pi^{*} L\right) / \Pi \longrightarrow F_{E}\left(P_{F} K, P_{F} L\right)$ is a homeomorphism.

We have the following relations between $P_{F}$ and base change functors. 
Proposition 3.3.8. Let $f: F \longrightarrow F^{\prime}$ be a map of $\Gamma$-spaces, let $E=P \times_{\Pi} F$ and $E^{\prime}=P \times_{\Pi} F^{\prime}$, and let $g=P \times_{\Pi} f: E \longrightarrow E^{\prime}$. For $K \in \Gamma \mathscr{K}_{F}$ and $L \in \Gamma \mathscr{K}_{F^{\prime}}$, there are natural isomorphisms

$$
g_{!} P_{F} K \longrightarrow P_{F^{\prime}} f_{!} K, \quad P_{F} f^{*} L \longrightarrow g^{*} P_{F^{\prime}} L, \quad \text { and } \quad P_{F^{\prime}} f_{*} K \longrightarrow g_{*} P_{F} K .
$$

Proof. The first and second isomorphisms are proven in parallel, so we only give the details for the first. Write $P_{F} \cong\left(\pi_{F}^{*}(-)\right) / \Pi$ and similarly for $P_{F^{\prime}}$. That gives the first and fourth of the following isomorphisms. The second isomorphism follows from the evident pullback diagram by Proposition 2.2.11, and the third follows from Proposition 2.4.3.

$$
P_{F^{\prime}} f_{!} K \cong\left(\pi_{F^{\prime}}^{*} f_{!} K\right) / \Pi \cong\left((P \times f) ! \pi_{F}^{*} K\right) / \Pi \cong\left(P \times_{\Pi} f\right) !\left(\pi_{F}^{*} K\right) / \Pi \cong g_{!} P_{A} K .
$$

For the third isomorphism in the statement, recall that $f_{*} K$ is the pullback over $F^{\prime}$ of $\operatorname{Map}_{F^{\prime}}(F, K) \longrightarrow \operatorname{Map}_{F^{\prime}}(F, F)$ along $\iota: F^{\prime} \longrightarrow \operatorname{Map}_{F^{\prime}}(F, F)$. As a closed monoidal right adjoint, the functor $P_{F^{\prime}}$ (in the unsectioned sense) converts this pullback to the pullback that defines $g_{*} P_{F} K$.

\subsection{Spaces of the homotopy types of $G-\mathrm{CW}$ complexes}

In this section, we recall and generalize the equivariant version of Milnor's results [128] about spaces of the homotopy types of CW complexes. For compact Lie groups, Waner formulated and proved such results in [168, §4]. With a few observations, his proofs generalize to deal with proper actions by general Lie groups. We first note the following immediate consequence of Proposition 3.1.3 and Theorem 3.1.11.

THEOREM 3.4.1. For any locally compact group $G, a G-C W$ complex is proper if and only if it is constructed from cells of the form $G / K \times D^{n}$, where $K$ is compact.

We also note the following recent "triangulation theorem" of Illman [80, Theorem II]. It is this result that led us to try to generalize some of our results from compact Lie groups to general Lie groups, and it is also this result that forces us to now restrict from general locally compact groups to Lie groups. We recall that the classical definition of a Lie group [30, p. 129] includes all discrete groups (even though they need not be second countable).

THEOREM 3.4.2 (Illman). If $G$ is a Lie group that acts smoothly and properly on a smooth manifold $M$, then $M$ has a $G-C W$ structure.

Many of our applications of this result are based on the following observation.

LEMma 3.4.3. If $H$ and $K$ are closed subgroups of a topological group $G$ and $K$ is compact, then the diagonal action of $G$ on $G / H \times G / K$ is proper.

Proof. The proof given in [47, I.5.16] that $G$ acts properly on $G / K$ generalizes directly. Set $X=G / H \times G$. Let $G$ act diagonally from the left and let $K$ act on the second factor from the right. Note that these actions commute. It suffices to show that $\theta: G \times X \longrightarrow X \times X$ is proper. Indeed, consider the commutative square

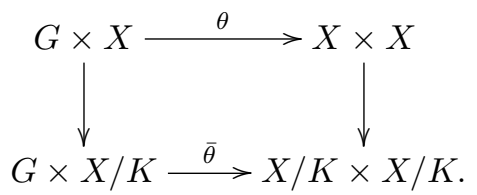


The right vertical map is proper and the left vertical map is surjective. Therefore, by [47, VI.2.13], the bottom horizontal map is proper if the top horizontal map is proper. Since $X$ is a free $G$-space, $\theta$ is proper if and only if the image $\operatorname{Im}(\theta)$ is a closed subspace of $X \times X$ and the map $\phi: \operatorname{Im}(\theta) \longrightarrow G$ specified by $\phi(x, g x)=g$ is continuous. The diagonal subspace of $G / H \times G / H$ is closed, and its preimage under the map $\zeta: X \times X \longrightarrow G / H \times G / H$ specified by

$$
\zeta((x H, y),(\bar{x} H, \bar{y}))=\left(\bar{y} y^{-1} x H, \bar{x} H\right)
$$

is precisely $\operatorname{Im}(\theta)$, which is therefore closed. The function $\phi$ is the restriction to $\operatorname{Im}(\theta)$ of the continuous map $\Phi: X \times X \longrightarrow G$ specified by

$$
\Phi((x H, y),(\bar{x} H, \bar{y}))=\bar{y} y^{-1}
$$

and is therefore continuous.

We shall also make essential use of the following corollary of Theorem 3.4.2.

Corollary 3.4.4. If $X$ is a proper $G$-CW complex, then, viewed as an $H$-space for any closed subgroup $H$ of $G, X$ has the structure of an $H$-cell complex.

Proof. Each cell $G / K \times D^{n}$ has $K$ compact. Since $G$ acts smoothly and properly on the smooth manifold $G / K$, the closed subgroup $H$ also acts smoothly and properly. We use the resulting $H$-CW structure on all of the cells to obtain an $H$-cell structure. It is homotopy equivalent to an $H$-CW complex obtained by "sliding down" cells that are attached to higher dimensional ones, but we shall not need to use that.

TheOREM 3.4.5 (Milnor, Waner). Let $G$ be a Lie group and $\left(X ; X_{i}\right)$ be an $n$-ad of closed sub-G-spaces of a proper $G$-space $X$. If $\left(X ; X_{i}\right)$ has the homotopy type of a $G$-CWn-ad and $\left(C ; C_{i}\right)$ is an $n$-ad of compact $G$-spaces, then $\left(X ; X_{i}\right)^{\left(C ; C_{i}\right)}$ has the homotopy type of a $G-C W n$-ad.

PRoOF. We only remark how the proof of Waner for the case of actions by a compact Lie group generalizes to the case of proper actions by a Lie group. Define a $G$-simplicial complex to be a $G$-CW complex such that $X / G$ with the induced cell structure is a simplicial complex. In $[168, \S 5]$, Waner proves that any $G$-CW complex is $G$-homotopy equivalent to a colimit of finite dimensional $G$-simplicial complexes and cellular inclusions and that a $G$-space dominated by a $G$-CW complex is $G$-homotopy equivalent to a $G$-CW complex. The arguments apply verbatim to any topological group $G$.

The rest of the argument requires two key lemmas. In [168, 4.2], Waner defines the notion of a $G$-equilocally convex, or $G$-ELC, $G$-space. The first lemma says that every finite dimensional $G$-simplicial complex is $G$-ELC. The essential starting point is that orbits are $G$-ELC, the proof of which uses the Lie group structure just as in $[168$, p.358] in the compact case. From there, Waner's proof $[168, \S 6]$ goes through unchanged. The second says that any completely regular, $G$-paracompact, $G$-ELC, proper $G$-space is dominated by a $G$-CW complex. When $G$ is compact Lie, this is proven in $[168, \S 7]$. However, the hypothesis on $G$ is only used to guarantee the existence of enough slices, hence the proof holds without change for proper actions of Lie groups, indeed of locally compact groups.

The rest of the proof goes as in [128, Theorem 3]. One only needs to make two small additional observations. First, if a $G$-simplicial complex $K$ has the homotopy type of a proper $G$-space $X$, then it is proper. This holds since if $f: K \longrightarrow X$ is a 
homotopy equivalence, then $G_{k} \subset G_{f(k)}$ is compact. Second, for an $n$-ad $\left(K ; K_{i}\right)$ of $G$-simplicial complexes and a compact $n$-ad $\left(C ; C_{i}\right),\left(X ; X_{i}\right)^{\left(C ; C_{i}\right)}$ is proper since it is a subspace of the proper $G$-space $X^{C}$; see (i) and (iv) of Proposition 3.1.10. Since it is also completely regular, $G$-paracompact, and $G$-ELC, it is dominated by a $G$-CW complex, and the result follows from the steps above.

\subsection{Some classical theorems about fibrations}

A basic principle of parametrized homotopy theory is that homotopical information is given on fibers. We recall two relevant classical theorems about Hurewicz fibrations and a comparison theorem relating Serre and Hurewicz fibrations. We begin with Dold's theorem [48, 6.3]. The nonequivariant proof in $[111,2.6]$ is generalized to the equivariant case in Waner $[169,1.11]$. Waner assumes throughout [169] that $G$ is a compact Lie group, but that assumption is not used in the cited proof.

TheOREM 3.5.1 (Dold). Let $G$ be any topological group and let $B$ be a $G$-space that has a $G$-numerable cover by contractible tubes. Let $X \longrightarrow B$ and $Y \longrightarrow B$ be Hurewicz fibrations. Then a map $X \longrightarrow Y$ over $B$ is a fiberwise $G$-homotopy equivalence if and only if each fiber restriction $X_{b} \longrightarrow Y_{b}$ is a $G_{b}$-homotopy equivalence.

We next recall and generalize a classical result that relates the homotopy types of fibers to the homotopy types of total spaces. Nonequivariantly, it is due to Stasheff [155] and, with a much simpler proof, Schön [143]. The generalization to the equivariant case, for compact Lie groups, is given by Waner [169, 6.1]. With Theorems 3.5.1, 3.4.5 and 3.4.2 in place, Schön's argument generalizes directly to give the following version. Since the result plays an important role in our work and the argument is so pretty, we can't resist repeating it in full.

Theorem 3.5.2 (Stasheff, Schön). Let $G$ be a Lie group and $B$ be a proper $G$-space that has the homotopy type of a $G-C W$ complex. Let $p: X \longrightarrow B$ be a Hurewicz fibration. Then $X$ has the homotopy type of a $G-C W$ complex if and only if each fiber $X_{b}$ has the homotopy type of $a G_{b}-C W$ complex.

Proof. First assume that $X$ has the homotopy type of a $G$-CW complex. For $b \in B$, let $\iota: G_{b} \longrightarrow G$ be the inclusion and consider the $G_{b^{-}}$map $\iota^{*} p: \iota^{*} X \longrightarrow \iota^{*} B$ of $G_{b}$-spaces. It is still a Hurewicz fibration, as we see by using the left adjoint $G \times{ }_{G_{b}}(-)$ of $\iota^{*}$. By Corollary 3.4.4, $\iota^{*} X$ and $\iota^{*} B$ have the homotopy types of $G_{b}$-CW complexes. Factor $\iota^{*} p$ through the inclusion into its mapping cylinder $i: \iota^{*} X \longrightarrow M \iota^{*} p$. Since $G_{b}$ is compact, it follows from Theorem 3.4.5 that the homotopy fiber $F_{b} i=\left(M \iota^{*} p ;\{b\}, \iota^{*} X\right)^{(I ; 0,1)}$ has the homotopy type of a $G_{b}$-CW complex. Since $F_{b} i$ is homotopy equivalent to $F_{b} \iota^{*} p$, by the gluing lemma, and $F_{b} \iota^{*} p$ is homotopy equivalent to the fiber $X_{b}$, this proves the forward implication.

For the converse, assume that each fiber $X_{b}$ has the homotopy type of a $G_{b^{-}}$ CW complex. Let $\gamma: \Gamma X \longrightarrow X$ be a $G$-CW approximation of $X$. The mapping path fibration of $\gamma$ gives us a factorization of $\gamma$ as the composite of a $G$-homotopy equivalence $\nu: \Gamma X \longrightarrow N \gamma$ and a Hurewicz fibration $q: N \gamma \longrightarrow X$. We may view 
$q$ as a map of fibrations over $B$.

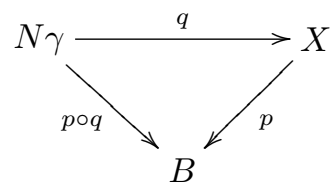

The fibers of $p \circ q$ have the homotopy types of $G_{b}-\mathrm{CW}$ complexes by the first part of the proof, since $\Gamma X$ is a $G$-CW complex, and the fibers of $p$ have the homotopy types of $G_{b}-\mathrm{CW}$ complexes by hypothesis. Comparison of the long exact sequences associated to $p \circ q$ and $p$ gives that $q$ restricts to a $G_{b}$-homotopy equivalence on each fiber. Noting that we can pull back a numerable cover by contractible tubes along a homotopy equivalence $B \longrightarrow B^{\prime}$, where $B^{\prime}$ is a $G$-CW complex, it follows from Theorem 3.5.1 that $q$ is a homotopy equivalence.

Although it no longer plays a role in our theory, the following little known result played a central role in our thinking. It shows that the dichotomy between Serre and Hurewicz fibrations diminishes greatly over CW base spaces. It is due to Steinberger and West [156], with a correction by Cauty [29].

Theorem 3.5.3 (Steinberger and West; Cauty). A Serre fibration whose base and total spaces are $C W$ complexes is a Hurewicz fibration.

We believe that this remains true equivariantly for compact Lie groups, and it certainly remains true for finite groups. Before we understood the limitations of the $q$-model structure, we planned to use this result to relate our model theoretic homotopy category of ex-spaces over a CW complex $B$ to a classical homotopy category defined in terms of Hurewicz fibrations and thereby overcome the problems illustrated in Counterexample 0.0.1. Such a comparison is still central to our theory, and it is this result that convinced us that such a comparison must hold.

\subsection{Quasifibrations}

For later reference, we recall the definition of quasifibrations. Here $G$ can be any topological group.

Definition 3.6.1. A map $p: E \longrightarrow Y$ in $\mathscr{K}$ is a quasifibration if the map of pairs $p:\left(E, E_{y}\right) \longrightarrow(Y, y)$ is a weak equivalence for all $y$ in $Y$. A map $p: E \longrightarrow Y$ in $\mathscr{K} / B$ or $\mathscr{K}_{B}$ is a quasifibration if it is a quasifibration on total spaces. A $G$-map $p: E \longrightarrow Y$ is a quasifibration if each of its fixed point maps $p^{H}: E^{H} \longrightarrow Y^{H}$ is a nonequivariant quasifibration.

The condition that $p:\left(E, E_{y}\right) \longrightarrow(Y, y)$ is a weak equivalence means that for all $e \in E_{y}$ the following two conditions hold.

(i) $p_{*}: \pi_{n}\left(E, E_{y}, e\right) \longrightarrow \pi_{n}(Y, y)$ is an isomorphism for all $n \geq 1$.

(ii) For any $x \in E, p(x)$ is in the path component of $y$ precisely when the path component of $x$ in $E$ intersects $E_{y}$. In other words, the sequence

$$
\pi_{0}\left(E_{y}, e\right) \longrightarrow \pi_{0}(E, e) \longrightarrow \pi_{0}(Y, y)
$$

of pointed sets is exact. 
WARNING 3.6.2. In contrast to the usual treatments in the literature, we do not require $p$ to be surjective and therefore $\pi_{0}(E, e) \longrightarrow \pi_{0}(Y, y)$ need not be surjective. Hurewicz and, more generally, Serre fibrations are examples of quasifibrations, and they are not always surjective, as the trivial example $\{0\} \longrightarrow\{0,1\}$ illustrates. Model categorically, one point is that the initial map $\emptyset \longrightarrow Y$ is always a Serre fibration since the empty lifting problem always has a solution.

The definition of a quasifibration is arranged so that the long exact sequence of homotopy groups associated to the triple $\left(E, E_{y}, e\right)$ is isomorphic to a long exact sequence

$$
\cdots \longrightarrow \pi_{n+1}(Y, y) \longrightarrow \pi_{n}\left(E_{y}, e\right) \longrightarrow \pi_{n}(E, e) \longrightarrow \pi_{n}(Y, y) \longrightarrow \cdots \longrightarrow \pi_{0}(Y, y)
$$

We say that a $G$-space over $B$ is quasifibrant if its projection is a quasifibration, and we have the following useful observation.

LEMMA 3.6.3. Let $i_{n}: E_{n} \longrightarrow E_{n+1}$ be a sequence of inclusions of quasifibrant $G$-spaces over $B$. Then colim $E_{n}$ is a quasifibrant $G$-space over $B$.

Proof. The essential point is that we are dealing with a fixed base space and, for $b \in B$, the fiber over $b$ of colim $E_{n}$ is homeomorphic to the colimit of the fibers over $b$ of the $E_{n}$. Since any basepoint of a fiber lies in one of the terms and since colimits of long exact sequences are exact, the conclusion follows directly from the definition of a quasifibration. 



\section{Part II}

\section{Model categories and parametrized spaces}





\section{Introduction}

In Part III, we shall develop foundations for parametrized equivariant stable homotopy theory. In making that theory rigorous, it became apparent to us that substantial foundational work was already needed on the level of ex-spaces. That work is of considerable interest for its own sake, and it involves general points about the use of model categories that should be of independent interest. Therefore, rather than rush through the space level theory as just a precursor of the spectrum level theory, we have separated it out in this more leisurely and discursive exposition.

In Chapter 4, which is entirely independent of our parametrized theory, we give general model theoretic background, philosophy, and results. In contrast to the simplicial world, we often have both a classical $h$-type and a derived $q$-type model structure in topologically enriched categories, with respective weak equivalences the homotopy equivalences and the weak homotopy equivalences. We describe what is involved in verifying the model axioms for these two types of model structures.

In Chapter 5, we describe how the parametrized world fits into this general framework. There are several different $h$-type model structures on our categories of parametrized $G$-spaces, with different homotopy equivalences based on different choices of cylinders. These mesh in unexpected ways. Understanding of this particular case leads us to a conceptual axiomatic description of how the classical $h$-type homotopy theory and the $q$-type model structure must be related in order to be able to do homotopy theory satisfactorily in a topologically enriched category.

In Chapter 6, we work nonequivariantly and develop our preferred " $q$-type" model category structure, the " $q f$-model structure", on the categories $\mathscr{K} / B$ and $\mathscr{K}_{B}$. This chapter is taken directly from the second author's thesis [152].

In Chapter 7, we give the equivariant generalization of the $q f$-model structure and begin the study of the resulting homotopy categories by discussing those adjunctions that are given by Quillen pairs. There is another new twist here in that we need to use many Quillen equivalent $q f$-type model structures. In fact, this is already needed nonequivariantly in the study of base change along bundles $f: A \longrightarrow B$.

In Chapter 8, we discuss ex-fibrations and an ex-fibrant approximation functor that better serves our purposes than model theoretic fibrant approximation in studying those adjunctions that are not given by Quillen pairs. In Chapter 9, we describe our parametrized homotopy categories in terms of classical homotopy categories of ex-fibrations and use this description to resolve the issues concerning base change functors and smash products that are discussed in the Prologue. 



\section{CHAPTER 4}

\section{Topologically bicomplete model categories}

\section{Introduction}

In $\S 4.1$, we describe a general philosophy about the role of different model structures on a given category $\mathscr{C}$. It is natural and important in many contexts, and it helps to clarify our thinking about topological categories of parametrized objects. In particular, we advertise a remarkable unpublished insight of Mike Cole. It is a pleasure to thank him for keeping us informed of his ideas. We describe how a classical " $h$-type" model structure and a suitably related Quillen " $q$-type" model structure, can be mixed together to give an " $m$-type" model structure such that the $m$-equivalences are the $q$-equivalences and the $m$-fibrations are the $h$-fibrations. This is a completely general phenomenon, not restricted to topological contexts.

In $\S \S 4.2$ and 4.3 , we describe classical structure that is present in any topologically bicomplete category $\mathscr{C}$. Here we follow up a very illuminating paper of Schwänzl and Vogt [144]. There are two classes of (Hurewicz) $h$-fibrations and two classes of $h$-cofibrations, ordinary and strong. Taking weak equivalences to be homotopy equivalences, the ordinary $h$-fibrations pair with the strong $h$-cofibrations and the strong $h$-fibrations pair with the ordinary $h$-cofibrations to give two interrelated model like structures. For each choice, all of the axioms for a proper topological model category are satisfied except for the factorization axioms, which hold in a weakened form. To prove that $\mathscr{C}$ is a model category, it suffices to prove one of the factorization axioms since the other will follow. Again, the theory can easily be adapted to other contexts than our topological one.

We signal an ambiguity of nomenclature. In the model category literature, the term "simplicial model structure" is clear and unambiguous, since there is only one model structure on simplicial sets in common use. In the topological context, we understand "topological model structures" to refer implicitly to the $h$-model structure on spaces for model structures of $h$-type and to the $q$-model structure on spaces for model structures of $q$-type. The meaning should always be clear from context.

In $\S 4.4$, we give another insight of Cole, which gains power from the work of Schwänzl and Vogt. Cole provides a simple hypothesis that implies the missing factorization axioms for an $h$-model structure of either type on a topologically bicomplete category $\mathscr{C}$. When we restrict to compactly generated spaces, the hypothesis applies to give an $h$-model structure on $\mathscr{U}$. In $\mathscr{K}$, this seems to fail, and we give a streamlined version of Strøm's original proof [160], together with his proof

that the strong $h$-cofibrations in $\mathscr{K}$ are just the closed ordinary $h$-cofibrations. This works in exactly the same way for the categories $G \mathscr{K}$ and $G \mathscr{U}$, where $G$ is any (compactly generated) topological group. 
In $\S 4.5$, we describe how to construct compactly generated $q$-type model structures, giving a slight variant of standard treatments. In particular, $G \mathscr{K}$ and $G \mathscr{U}$ have the usual $q$-model structures in which the $q$-equivalences are the weak equivalences and the $q$-fibrations are the Serre fibrations. Again, $G$ can be any topological group. However we only know that the model structure is $G$-topological when $G$ is a compact Lie group.

\subsection{Model theoretic philosophy: $h, q$, and $m$-model structures}

The point of model categories is to systematize "homotopy theory". The homotopy theory present in many categories of interest comes in two flavors. There is a "classical" homotopy theory based on homotopy equivalences, and there is a more fundamental "derived" homotopy theory based on a weaker notion of equivalence than that of homotopy equivalence. This dichotomy pervades the applications, regardless of field. It is perhaps well understood that both homotopy theories can be expressed in terms of model structures on the underlying category, but this aspect of the classical homotopy theory has usually been ignored in the model theoretical literature, a tradition that goes back to Quillen's original paper [139]. The "classical" model structure on spaces was introduced by Strøm [160], well after Quillen's paper, and the "classical" model structure on chain complexes was only introduced explicitly quite recently, by Cole [34] and Schwänzl and Vogt [144].

Perhaps for this historical reason, it may not be widely understood that these two model structures can profitably be used in tandem, with the $h$-model structure used as a tool for proving things about the $q$-model structure. This point of view is implicit in $[61,105,106]$, and a variant of this point of view will be essential to our work. In the cited papers, the terms " $q$-fibration" and " $q$-cofibration" were used for the fibrations and cofibrations in the Quillen model structures, and the term " $h$-cofibration" was used for the classical notion of a Hurewicz cofibration specified in terms of the homotopy extension property (HEP). The corresponding notion of an " $h$-fibration" defined in terms of the covering homotopy property (CHP) is fortuitously appropriate ${ }^{1}$. Just as the " $q$ " is meant to suggest Quillen, the " $h$ " is meant to suggest Hurewicz, as well as homotopy. It is logical to follow this idea further (as was not done in $[61,105,106]$ ) by writing $q$-fibrant, $q$-cofibrant, $h$-fibrant, and $h$-cofibrant for clarity. Following this still further, we should also write " $h$-equivalence" for homotopy equivalence and "q-equivalence" for (Quillen) weak equivalence. The relations among these notions are as follows in all of the relevant categories $\mathscr{C}$ :

$\begin{aligned} h \text {-equivalence } & \Longleftrightarrow q \text {-equivalence } \\ h \text {-cofibration } & \Longleftrightarrow q \text {-cofibration } \\ h \text {-cofibrant } & \Longleftrightarrow q \text {-cofibrant } \\ h \text {-fibration } & \Longleftrightarrow q \text {-fibration } \\ h \text {-fibrant } & \Longrightarrow q \text {-fibrant }\end{aligned}$

Therefore, the identity functor is the right adjoint of a Quillen adjoint pair from $\mathscr{C}$ with its $h$-model structure to $\mathscr{C}$ with its $q$-model structure. It follows that we have an adjoint pair relating the classical homotopy category, $h \mathscr{C}$ say, to the

\footnotetext{
${ }^{1}$ However, the notation conflicts with the notation often used for Dold's notion of a weak or "halb"-fibration. We shall make no use of that notion, despite its real importance in the theory of fibrations. We do not know whether or not it has a model theoretic role to play.
} 
derived homotopy category $q \mathscr{C}=$ Ho $\mathscr{C}$. This formulation packages standard information. For example, the Whitehead theorem that a weak equivalence between cell complexes is a homotopy equivalence, or its analogue that a quasi-isomorphism between projective complexes is a homotopy equivalence, is a formal consequence of this adjunction between homotopy categories.

Recently, Cole [36] discovered a profound new way of thinking about the dichotomy between the kinds of model structures that we have been discussing. He proved the following formal model theoretic result.

TheOREM 4.1.1 (Cole). Let $\left(\mathscr{W}_{h}, \mathscr{F} i b_{h}, \mathscr{C O}_{h}\right)$ and $\left(\mathscr{W}_{q}, \mathscr{F} i b_{q}, \mathscr{C} o f_{q}\right)$ be two model structures on the same category $\mathscr{C}$. Suppose that $\mathscr{W}_{h} \subset \mathscr{W}_{q}$ and $\mathscr{F} i b_{h} \subset \mathscr{F} i b_{q}$. Then there is a mixed model structure $\left(\mathscr{W}_{q}, \mathscr{F} i b_{h}, \mathscr{C} o f_{m}\right)$ on $\mathscr{C}$. The mixed cofibrations $\mathscr{C o f}_{m}$ are the maps in $\mathscr{C o f}_{h}$ that factor as the composite of a map in $\mathscr{W}_{h}$ and a map in $\mathscr{C} o f_{q}$. An object is $m$-cofibrant if and only it is $h$-cofibrant and of the $h$-homotopy type of a q-cofibrant object. If the $h$ and q-model structures are left or right proper, then so is the m-model structure.

By duality, the analogue with the inclusion $\mathscr{F} i b_{h} \subset \mathscr{F} i b_{q}$ replaced by an inclusion $\mathscr{C} o f_{h} \subset \mathscr{C} \circ f_{q}$ also holds. In the category of spaces with the $h$ and $q$-model structures discussed above, the theorem gives a mixed model structure whose $m$ cofibrant spaces are the spaces of the homotopy types of CW-complexes. This $m$-model structure combines weak equivalences with Hurewicz fibrations, and it might conceivably turn out to be as important and convenient as the Quillen model structure. It is startling that this model structure was not discovered earlier.

The pragmatic point is two-fold. On the one-hand, there are many basic results that apply to $h$-cofibrations and not just $q$-cofibrations. Use of $h$-cofibrations limits the need for $q$-cofibrant approximation and often clarifies proofs by focusing attention on what is relevant. Many examples appear in $[61,105,106]$, where properties of $h$-cofibrations serve as scaffolding in the proof that $q$-model structures are in fact model structures. We shall formalize and generalize this idea in the next chapter.

On the other hand, there are many vital results that apply only to $h$-fibrations (Hurewicz fibrations), not to $q$-fibrations (Serre fibrations). For example, a local Hurewicz fibration is a Hurewicz fibration, but that is not true for Serre fibrations. The mixed model structure provides a natural framework in which to make use of Hurewicz fibrations in conjunction with weak equivalences. While we shall make no formal use of this model structure, it has provided a helpful guide to our thinking. The philosophy here applies in algebraic as well as topological contexts, but we shall focus on the latter.

\subsection{Strong Hurewicz cofibrations and fibrations}

Fix a topologically bicomplete category $\mathscr{C}$ throughout this section and the next. With no further hypotheses on $\mathscr{C}$, we show that it satisfies most of the axioms for not one but two generally different proper topological $h$-type model structures. We alert the reader to the fact that we are here using the term " $h$-model structure" in a generic sense. When we restrict attention to parametrized spaces, we will use the term in a different specific sense derived from the $h$-model structure on underlying total spaces. The material of these sections follows and extends material in Schwänzl and Vogt [144].

We have cylinders $X \times I$ and cocylinders $\operatorname{Map}(I, X)$. When $\mathscr{C}$ is based, we focus on the based cylinders $X \wedge I_{+}$and cocylinders $F\left(I_{+}, X\right)$. In either case, these 
define equivalent notions of homotopy, which we shall sometimes call h-homotopy. We will later use these and cognate notations, but, for the moment, it is convenient to introduce the common notations $\operatorname{Cyl}(X)$ and $\operatorname{Cocyl}(X)$ for these objects. There are obvious classes of maps that one might hope would specify a model structure.

Definition 4.2.1. Let $f$ be a map in $\mathscr{C}$.

(i) $f$ is an $h$-equivalence if it is a homotopy equivalence in $\mathscr{C}$.

(ii) $f$ is a Hurewicz fibration, abbreviated $h$-fibration, if it satisfies the CHP in $\mathscr{C}$, that is, if it has the right lifting property (RLP) with respect to the maps $i_{0}: X \longrightarrow \operatorname{Cyl}(X)$ for $X \in \mathscr{C}$.

(iii) $f$ is a Hurewicz cofibration, abbreviated $h$-cofibration, if it satisfies the HEP in $\mathscr{C}$, that is, if it has the left lifting property (LLP) with respect to the maps $p_{0}: \operatorname{Cocyl}(X) \longrightarrow X$.

These sometimes do give a model structure, but then the $h$-cofibrations must be exactly the maps that satisfy the LLP with respect to the $h$-acyclic $h$-fibrations, and dually. In general, that does not hold. We shall characterize the maps in $\mathscr{C}$ that do satisfy the LLP with respect to the $h$-acyclic $h$-fibrations and, dually, the maps that satify the RLP with respect to the $h$-acyclic $h$-fibrations. For this, we need the following relative version of the above notions.

Definition 4.2.2. We define strong Hurewicz fibrations and cofibrations.

(i) A map $p: E \longrightarrow Y$ is a strong Hurewicz fibration, abbreviated $\bar{h}$-fibration, if it satisfies the relative $C H P$ with respect to all $h$-cofibrations $i: A \longrightarrow X$, in the sense that a lift exists in any diagram

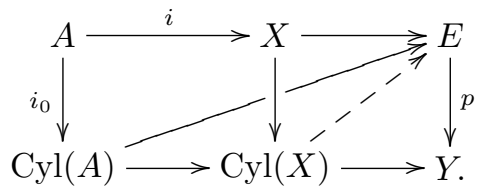

(ii) A map $i: A \rightarrow X$ is a strong Hurewicz cofibration, abbreviated $\bar{h}$-cofibration, if it satisfies the relative $H E P$ with respect to all $h$-fibrations $p: E \rightarrow Y$, in the sense that a lift exists in any diagram

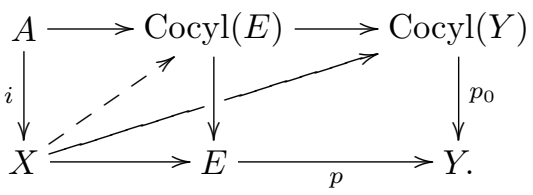

We recall the standard criteria for maps to be $h$-fibrations or $h$-cofibrations. Define the mapping cylinder $M f$ and mapping path fibration $N f$ by the usual pushout and pullback diagrams
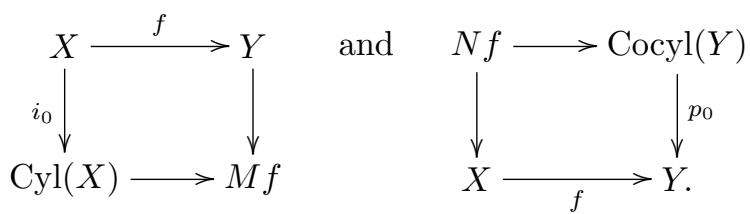

LEMMA 4.2.3. Let $f$ be a map in $\mathscr{C}$. 
(i) $f$ is an h-fibration if and only if it has the $R L P$ with respect to the map $i_{0}: N f \longrightarrow C y l(N f)$.

(ii) $f$ is an h-cofibration if and only if it has the LLP with respect to the map $p_{0}: \operatorname{Cocyl}(M f) \longrightarrow M f$.

The $\bar{h}$-fibrations and $\bar{h}$-cofibrations admit similar characterizations. These were taken as definitions in $[144,2.4]$.

Lemma 4.2.4. Consider maps $i: A \longrightarrow X$ and $p: E \longrightarrow Y$.

(i) The map $p: E \longrightarrow Y$ is an $\bar{h}$-fibration if and only if it has the $R L P$ with respect to the canonical map $M i \longrightarrow C y l(X)$ for any $h$-cofibration $i: A \rightarrow X$; this holds if and only if the canonical map Cocyl $(E) \rightarrow N p$ has the RLP with respect to all h-cofibrations.

(ii) The map $i: A \rightarrow X$ is an $\bar{h}$-cofibration if and only if it has the LLP with respect to the canonical map $\operatorname{Cocyl}(E) \longrightarrow N p$ for any $h$-fibration $p: E \rightarrow Y$; this holds if and only if the canonical map $M i \rightarrow C y l(X)$ has the LLP with respect to all h-fibrations.

Observe that the map $i_{0}: X \longrightarrow \operatorname{Cyl}(X)$ is an $\bar{h}$-cofibration and the map $p_{0}$ : $\operatorname{Cocyl}(X) \longrightarrow X$ is an $\bar{h}$-fibration. Since the cylinder objects associated to initial objects are initial objects, $\bar{h}$-fibrations are in particular $h$-fibrations. Similarly, $\bar{h}$ cofibrations are $h$-cofibrations. Observe too that every object is both $\bar{h}$-cofibrant and $\bar{h}$-fibrant, hence both $h$-cofibrant and $h$-fibrant.

We shall see in $\S 4.4$ that these distinctions are necessary in $\mathscr{K}$ but disappear in $\mathscr{U}$, where the $h$ and $\bar{h}$ notions coincide. Even there, however, the conceptual distinction sheds light on classical arguments.

The results of this section and the next are quite formal. Amusingly, the main non-formal ingredient is just the use in the following proof of the fact that $\{0,1\} \rightarrow I$ has the LLP with respect to all $h$-acyclic $h$-fibrations, which follows easily from Proposition 4.3.5(ii) below.

Lemma 4.2.5. Let $i: A \longrightarrow X$ and $p: E \longrightarrow B$ be maps in $\mathscr{C}$.

(i) If $i$ is an $h$-acyclic $h$-cofibration, then $i$ is the inclusion of a strong deformation retraction $r: X \longrightarrow A$.

(ii) If $i$ is the inclusion of a strong deformation retraction $r: X \rightarrow A$, then $i$ is a retract of $M i \rightarrow C y l(X)$.

(iii) If $p$ is an $h$-acyclic $h$-fibration, then $p$ is a strong deformation retraction.

(iv) If $p$ is a strong deformation retraction, then $p$ is a retract of $\operatorname{Cocyl}(E) \longrightarrow N p$.

Proof. The last two statements are dual to the first two. For (i), since the $h$-equivalence $i$ is an $h$-cofibration, application of the HEP shows that $i$ has a homotopy inverse $r: X \rightarrow A$ such that $r i=\operatorname{id}_{A}$. Since $\{0,1\} \longrightarrow I$ has the LLP with respect to $h$-acyclic $h$-fibrations, an adjunction argument shows that $p_{(0,1)}$ has the RLP with respect to $h$-cofibrations. Thus a lift exists in the diagram on the 
left, which means that $r$ is a strong deformation retraction with inclusion $i$.
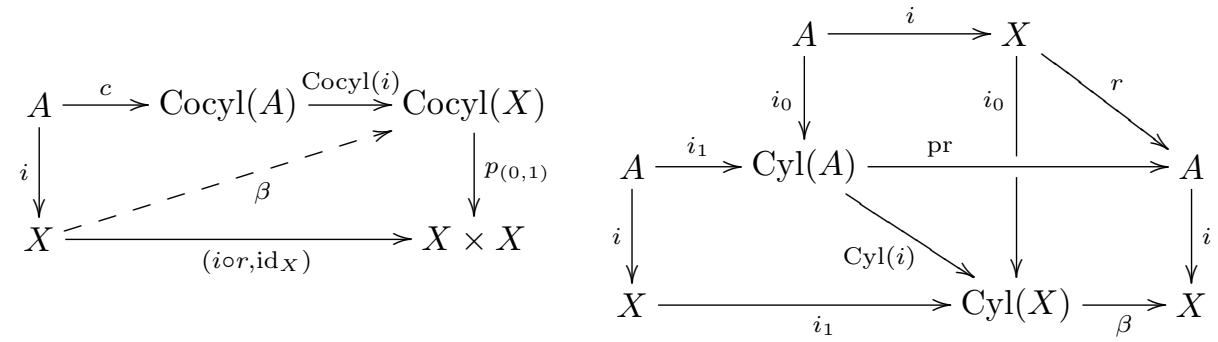

For (ii), we are given $\beta$ in the diagram on the left displaying $r$ as a strong deformation retraction with inclusion $i$. Then the diagram on the right commutes, where the composites displayed in the lower two rows are identity maps. Using the universal property of $M i$ to factor the crossing arrows $i_{0}$ and pr through $M i$, we see that $i$ is a retract of the canonical map $M i \rightarrow \operatorname{Cyl}(X)$.

\subsection{Towards classical model structures in topological categories}

We now have two candidates for a classical model structure on $\mathscr{C}$ based on the $h$-equivalences. We can either take the $h$-fibrations and the $\bar{h}$-cofibrations or the $h$-cofibrations and the $\bar{h}$-fibrations. The following result shows that all of the axioms for a proper topological model category are satisfied except that, in general, only a weakened form of the factorization axioms holds.

THEOREM 4.3.1. The following versions of the axioms for a proper topological model category hold.

(i) The classes of $h$-cofibrations, $\bar{h}$-cofibrations, $h$-fibrations and $\bar{h}$-fibrations are closed under retracts.

(ii) Let $i$ be an $h$-cofibration and $p$ be an $h$-fibration. The pair $(i, p)$ has the lifting property if $i$ is strong and $p$ is h-acyclic or if $p$ is strong and $i$ is h-acyclic.

(iii) Any map $f: X \rightarrow Y$ factors as

$$
X \stackrel{i}{\longrightarrow} M f \stackrel{r}{\longrightarrow} Y
$$

where $i$ is an $\bar{h}$-cofibration and $r$ has a section that is an $h$-acyclic $\bar{h}$-cofibration and as

$$
X \stackrel{s}{\longrightarrow} N f \stackrel{p}{\longrightarrow} Y
$$

where $p$ is an $\bar{h}$-fibration and s has a retraction that is an $h$-acyclic $\bar{h}$-fibration.

(iv) Let $i: A \rightarrow X$ be an $h$-cofibration and $p: E \rightarrow B$ be an $h$-fibration, where $i$ or $p$ is strong. Then the map

$$
\mathscr{C}^{\square}(i, p): \mathscr{C}(X, E) \rightarrow \mathscr{C}(A, E) \times_{\mathscr{C}(A, B)} \mathscr{C}(X, B)
$$

induced by $i$ and $p$ is an $h$-fibration of spaces. It is $h$-acyclic if $i$ or $p$ is acyclic and it is an $\bar{h}$-fibration if both $i$ and $p$ are strong.

(v) The h-equivalences are preserved under pushouts along $h$-cofibrations and pullbacks along $h$-fibrations.

Proof. Part (i) is clear since all classes are defined in terms of lifting properties. Part (ii) follows directly from Lemma 4.2.4 and Lemma 4.2.5. The factorizations of part (iii) are the standard ones. We consider the first. The evident 
section $j: Y \longrightarrow M f$ is an $h$-acyclic $\bar{h}$-cofibration since it is the pushout of one. Consider the lifting problem in the left diagram below, in which the middle vertical composite is $i$. Here $p$ is an $h$-acyclic $h$-fibration, and we choose a section $s$ of $p$.
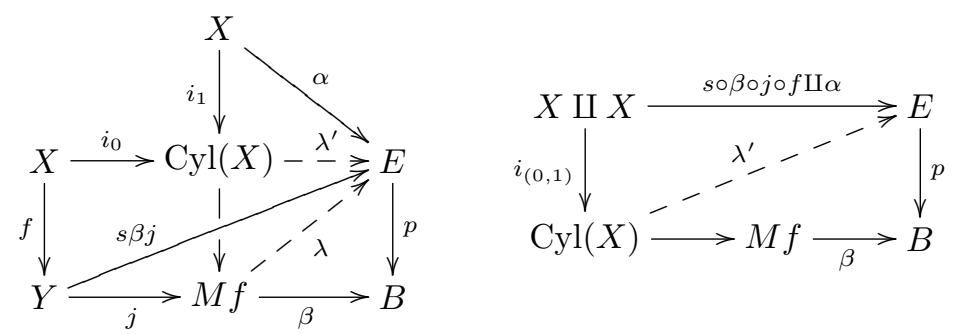

We have a lift $\lambda^{\prime}$ in the diagram on the right that makes the diagram on the left commute, and the universal property of $M f$ then gives us the lift $\lambda$. Part (iv) is a consequence of the "pairing theorem" [144], which we will state below. Finally we prove the first half of $(\mathrm{v})$. The second half follows by duality. Assume that $i$ is an $h$-cofibration and $f$ is an $h$-equivalence in the pushout diagram on the left.
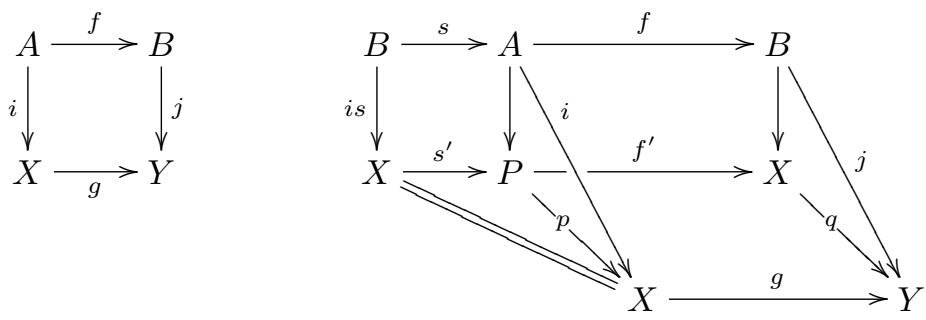

We must prove that $g$ is an $h$-equivalence. By (ii), we can factor $f$ as a composite of an $h$-acyclic $h$-cofibration and a map that has a section which is an $h$-acyclic $h$ cofibration. Since pushouts preserve $h$-acyclic $h$-cofibrations, we may assume that $f$ has a section $s: B \longrightarrow A$ that is an $h$-acyclic $h$-cofibration. We then obtain the diagram on the right. Its left back rectangle is a pushout, as is the outer back rectangle, and therefore the right back rectangle is also a pushout. This implies that the bottom square is a pushout. The map $s^{\prime}$ is an $h$-acyclic $h$-cofibration since $s$ is one, and therefore $p$ is an $h$-equivalence. The map $f^{\prime}$ is also $h$-acyclic since it has the $h$-acyclic section $s^{\prime}$. Just as we could assume that $f$ has a section that is an $h$-acyclic $h$-cofibration, we find that we may assume that $p$ has a section $t$ that is an $h$-acyclic $h$-cofibration and is a map under $A$. Chasing pushout diagrams, we find that $g$ is a retract of $f^{\prime}$ and is therefore an $h$-equivalence.

The following result is the pairing theorem of [144, 2.7 and 3.6]. We shall not repeat the proof, which consists of careful but formal adjunction arguments. Its general statement is framed so as to apply to cartesian products in the unbased situation, smash products in the based situation, and tensors in either situation.

Theorem 4.3.2 (Schwänzl and Vogt). Let $\mathscr{A}, \mathscr{B}$, and $\mathscr{C}$ be topologically bicomplete categories and let

$$
T: \mathscr{A} \times \mathscr{B} \longrightarrow \mathscr{C}, \quad U: \mathscr{A}^{o p} \times \mathscr{C} \longrightarrow \mathscr{B}, \text { and } V: \mathscr{B}^{o p} \times \mathscr{C} \longrightarrow \mathscr{A}
$$

be continuous functors that satisfy adjunctions

$$
\mathscr{C}(T(A, B), C) \cong \mathscr{B}(B, U(A, C)) \cong \mathscr{A}(A, V(B, C)) .
$$


Let $i: A \longrightarrow X$ be an h-cofibration in $\mathscr{A}, j: B \longrightarrow Y$ be an $h$-cofibration in $\mathscr{B}$, and $p: E \longrightarrow Z$ be an h-fibration in $\mathscr{C}$.

(i) Assume that $i$ or $j$ is strong. Then the map

$$
T(A, Y) \cup_{T(A, B)} T(X, B) \longrightarrow T(X, Y)
$$

induced by $i$ and $j$ is an $h$-cofibration in $\mathscr{C}$. It is $h$-acyclic if $i$ or $j$ is $h$-acyclic and it is strong if both $i$ and $j$ are strong.

(ii) Assume that $j$ or $p$ is strong. Then the map

$$
V(Y, E) \longrightarrow V(B, E) \times_{V(B, Z)} V(Y, Z)
$$

induced by $j$ and $p$ is an $h$-fibration in $\mathscr{A}$. It is h-acyclic if $j$ or $p$ is $h$-acyclic and it is strong if both $j$ and $p$ are strong.

As Schwänzl and Vogt observe, these results imply that the canonical map $M i \longrightarrow \operatorname{Cyl}(X)$ is an $h$-acyclic $\bar{h}$-cofibration for any $h$-cofibration $i: A \longrightarrow X$ and, dually, the canonical map $\operatorname{Cocyl}(X) \longrightarrow N p$ is an $h$-acyclic $\bar{h}$-fibration for any $h$-fibration $p: E \longrightarrow Y$. Together with Lemma 4.2.5 and the retract and factorization axioms of Theorem 4.3.1, this implies that all of the various classes of maps are characterized by the expected lifting properties, just as if we had actual model categories.

Proposition 4.3.3. The following characterizations hold.

(i) The h-fibrations are the maps that have the RLP with respect to the h-acyclic $\bar{h}$-cofibrations and the $h$-acyclic $\bar{h}$-cofibrations are the maps that have the LLP with respect to the h-fibrations.

(ii) The h-cofibrations are the maps that have the LLP with respect to the h-acyclic $\bar{h}$-fibrations and the $h$-acyclic $\bar{h}$-fibrations are the maps that have the $R L P$ with respect to the $h$-cofibrations.

(iii) The $\bar{h}$-fibrations are the maps that have the $R L P$ with respect to the $h$-acyclic $h$-cofibrations and the $h$-acyclic $h$-cofibrations are the maps that have the LLP with respect to the $\bar{h}$-fibrations.

(iv) The $\bar{h}$-cofibrations are the maps that have the LLP with respect to the $h$-acyclic $h$-fibrations and the $h$-acyclic $h$-fibrations are the maps that have the RLP with respect to the $\bar{h}$-cofibrations.

To show that $\mathscr{C}$ has an $h$-type model structure, it suffices to prove the factorization axioms, and it is unnecessary to prove them both.

LEMma 4.3.4. For either proposed h-model structure, if one of the factorization axioms holds, then so does the other.

Proof. For definiteness, consider the case of $h$-fibrations and $\bar{h}$-cofibrations. By Theorem 4.3.1(ii), we can factor any map $f: X \longrightarrow Y$ as the composite of an $\bar{h}$-cofibration $i: X \longrightarrow M f$ and an $h$-equivalence $r: M f \longrightarrow Y$. Suppose that we can factor $r$ as the composite of an $h$-acyclic $\bar{h}$-cofibration $j: M f \longrightarrow Z$ and an $h$-fibration $q: Z \longrightarrow Y$. Then $q$ must be $h$-acyclic, hence $f=q \circ(j \circ i)$ factors $f$ as the composite of an $\bar{h}$-cofibration and an $h$-acyclic $h$-fibration.

A homotopy $X \longrightarrow Y$ in $\mathscr{C}$ can be specified by a path $h: I \longrightarrow \mathscr{C}(X, Y)$. If $i: A \longrightarrow X$ and $p: Y \longrightarrow B$ are maps in $\mathscr{C}$, then we say that $h$ is a homotopy 
relative to $i$ or corelative to $p$ if the composite

$$
I \stackrel{h}{\longrightarrow} \mathscr{C}(X, Y) \stackrel{\mathscr{C}(i, Y)}{\longrightarrow} \mathscr{C}(A, Y) \quad \text { or } \quad I \stackrel{h}{\longrightarrow} \mathscr{C}(X, Y) \stackrel{\mathscr{C}(X, p)}{\longrightarrow} \mathscr{C}(X, B)
$$

is constant. When $i$ or $p$ is understood, we also refer to these as homotopies under $A$ or over $B$. The following result is well known and holds in any (based) topologically bicomplete category.

Proposition 4.3.5. Let $f: X \longrightarrow Y$ be an $h$-equivalence.

(i) If $i: A \longrightarrow X$ and $j: A \longrightarrow Y$ are $h$-cofibrations such that $j=f \circ i$, then $f$ is an h-equivalence under $A$.

(ii) If $p: Y \longrightarrow B$ and $q: X \longrightarrow B$ are $h$-fibrations such that $q=p \circ f$, then $f$ is an h-equivalence over $B$.

Proof. For (i), see for example [121, p.44]. The proof there, although written for spaces, goes through without change. Part (ii) follows by a dual proof.

REMARK 4.3.6. The current section, as well as the previous and the following one, applies verbatim to the $G$-topologically bicomplete $G$-categories of $\S 10.2$, where $G$ is any topological group. Of course, $\left(\mathscr{K}_{G, B}, G \mathscr{K}_{B}\right)$ is an example. The only changes occur in Theorem 4.3.1(iv), where one must take the arrow $G$-spaces $\mathscr{C}_{G}(-,-)$ rather than the non-equivariant spaces $G \mathscr{C}(-,-)$, and in Theorem 4.3.2, where the adjunction hypothesis requires a similar equivariant interpretation. See $\S 10.3$ for a discussion of $G$-topological model $G$-categories.

\subsection{Classical model structures in general and in $\mathscr{K}$ and $\mathscr{U}$}

Again, fix a topologically bicomplete category $\mathscr{C}$. Independent of the work of Schwänzl and Vogt [144], Cole [35] proved a general result concerning when $\mathscr{C}$ has an $h$-type model structure. As we now see is inevitable, the core of his argument concerns the verification of one of the factorization axioms. That requires a hypothesis.

Hypothesis 4.4.1. Let $j_{n}: Z_{n} \longrightarrow Z_{n+1}$ and $q_{n}: Z_{n} \longrightarrow Y$ be maps in $\mathscr{C}$ such that $q_{n+1} \circ j_{n}=q_{n}$ and the $j_{n}$ are $h$-acyclic $h$-cofibrations. Let $Z=\operatorname{colim} Z_{n}$ and let $q: Z \longrightarrow Y$ be obtained by passage to colimits. Then the canonical map $\operatorname{colim} N q_{n} \longrightarrow N q$ is an isomorphism in $\mathscr{C}$.

THEOREM 4.4.2 (Cole). If $\mathscr{C}$ is a topologically bicomplete category which satisfies Hypothesis 4.4.1, then the $h$-equivalences, $h$-fibrations, and $\bar{h}$-cofibrations specify a proper topological $h$-model structure on $\mathscr{C}$.

Proof. It suffices to show that a map $f: X \longrightarrow Y$ factors as the composite of an $h$-acyclic $\bar{h}$-cofibration $j: X \longrightarrow Z$ and an $h$-fibration $q: Z \longrightarrow Y$. Let $Z_{0}=X$ and $q_{0}=f$. Inductively, given $q_{n}: Z_{n} \longrightarrow Y$, construct the following diagram, in which $Z_{n+1}$ is the displayed pushout.

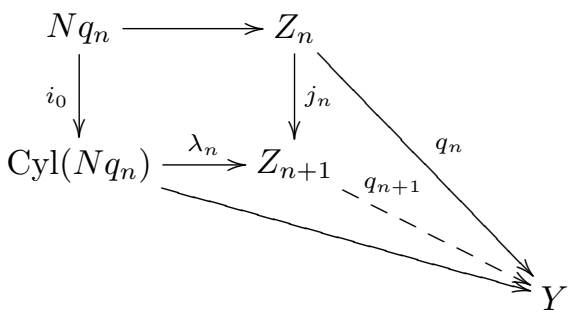


The map $\operatorname{Cyl}\left(N q_{n}\right) \longrightarrow Y$ is the adjoint of the projection $N q_{n} \longrightarrow \operatorname{Cocyl}(Y)$ given by the definition of $N q_{n}$, and $q_{n+1}$ is the induced map. The maps $j_{n}$ are $h$-acyclic $\bar{h}$-cofibrations since they are pushouts of such maps. Let $Z$ be the colimit of the $Z_{n}$ and $j$ and $q$ be the colimits of the $j_{n}$ and $q_{n}$. Certainly $f=q \circ j$ and $j$ is an $h$ acyclic $\bar{h}$-cofibration. By Hypothesis 4.4.1, $N q$ is the colimit of the $N q_{n}$. Since the cylinder functor preserves colimits, we see by Lemma 4.2 .3 that $q$ is an $h$-fibration since the $\lambda_{n}$ give a lift $\mathrm{Cyl}(N q) \longrightarrow Z$ by passage to colimits.

The dual version of Theorem 4.4.2 admits a dual proof.

THEOREM 4.4.3. If $\mathscr{C}$ is a topologically bicomplete category which satisfies the dual of Hypothesis 4.4.1, then the h-equivalences, $\bar{h}$-fibrations, and $h$-cofibrations specify a proper topological $h$-model structure on $\mathscr{C}$.

From now on, we break the symmetry by focusing on $h$-fibrations and $\bar{h}$ cofibrations. These give model structures in $\mathscr{K}$ and $\mathscr{U}$. Everything in the rest of the section works equally in $G \mathscr{K}$ and $G \mathscr{U}$. The following theorem combines several results of Strøm [158-160].

THEOREM 4.4.4 (Strøm). The following statements hold.

(i) The $h$-equivalences, $h$-fibrations, and $\bar{h}$-cofibrations give $\mathscr{K}$ a proper topological h-model structure. Moreover, a map in $\mathscr{K}$ is an $\bar{h}$-cofibration if and only if it is a closed h-cofibration.

(ii) The $h$-equivalences, $h$-fibrations, and $\bar{h}$-cofibrations give $\mathscr{U}$ a proper topological $h$-model structure. Moreover, a map in $\mathscr{U}$ is an $\bar{h}$-cofibration if and only if it is an h-cofibration.

Proof. Theorem 4.4.2 applies to prove the first statement in $(i i)$, but it does not seem to apply to prove the first statement in $(i)$. The reasons are explained in Remark 1.6.4. Taking $Z=Y^{I}$ and $p=p_{0}$ there, the comparison map $\alpha$ specializes to the map colim $N f_{n} \longrightarrow N f$ of Hypothesis 4.4.1. It may be that $\alpha$ is a homeomorphism in this special case, but we do not have a proof. It is a homeomorphism when we work in $\mathscr{U}$. The characterization of the $\bar{h}$-cofibrations in $\mathscr{U}$ follows from Lemma 1.6.2 and their characterization in $\mathscr{K}$.

For (i), we give a streamlined version of Strøm's original arguments that uses the material of the previous section to prove both statements together. We proceed in four steps. The first step is Strøm's key observation, the second and third steps give the second statement, and the fourth step proves the needed factorization axiom. Consider an inclusion $i: A \longrightarrow X$.

Step 1. By Strøm's [158, Thm. 3], if $i$ is the inclusion of a strong deformation retract and there is a map $\psi: X \longrightarrow I$ such that $\psi^{-1}(0)=A$, then $i$ has the LLP with respect to all $h$-fibrations. By Proposition 4.3.3(i), this means that $i$ is an $h$-acyclic $\bar{h}$-cofibration.

Step 2. If $i$ is an $h$-cofibration, then the canonical map $j: M i \longrightarrow X \times I$ is an $h$-acyclic $h$-cofibration and therefore, by Lemma 4.2.5, the inclusion of a strong deformation retract. If $i$ is closed, then $(X, A)$ is an NDR-pair and there exists $\phi: X \longrightarrow I$ such that $\phi^{-1}(0)=A$. Define $\psi: X \times I \longrightarrow I$ by $\psi(x, t)=t \phi(x)$. Then $\psi^{-1}(0)=M i$. Applying Step 1, we conclude that $j$ has the LLP with respect to all $h$-fibrations. By Lemma 4.2.4, this means that $i$ is an $\bar{h}$-cofibration.

Step 3. We can factor any inclusion $i$ as the composite

$$
A \stackrel{i_{0}}{\longrightarrow} E \stackrel{\pi}{\longrightarrow} X
$$


where $E$ is the subspace $X \times(0,1] \cup A \times I$ of $X \times I$ and $\pi$ is the projection. Note that $A=\psi^{-1}(0)$, where $\psi: E \longrightarrow I$ is the projection on the second coordinate. By direct verification of the CHP [160, p. 436], $\pi$ is an $h$-fibration. If $i$ is an $\bar{h}$ cofibration, then it has the LLP with respect to $\pi$, hence we can lift the identity map of $X$ to a map $\lambda: X \longrightarrow E$ such that $\lambda \circ i=i_{0}$. It follows that $i(A)$ is closed in $X$ since $i_{0}(A)$ is closed in $E$.

Step 4. Let $f: X \longrightarrow Y$ be a map. Use Theorem 4.3.1(ii) to factor $f$ as $p \circ s$, where $s: X \longrightarrow N f$ is the inclusion of a strong deformation retract and $p$ is an $\bar{h}$-fibration. Use Step 3 to factor $s$ as

$$
X \stackrel{i_{0}}{\longrightarrow} N f \times(0,1] \cup X \times I \stackrel{\pi}{\longrightarrow} N f .
$$

Here $i_{0}$ is the inclusion of a strong deformation retract and $X=\psi^{-1}(0)$, as in Step 3. By Step $1, i_{0}$ is an $h$-acyclic $\bar{h}$-cofibration. By Step $3, p \circ \pi$ is an $h$-fibration.

There are several further results of Strøm about $h$-cofibrations that deserve to be highlighted. In order, the following results are [159, Theorem 12], [160, Lemma $5]$, and [159, Corollary 5].

Proposition 4.4.5. If $p: E \longrightarrow Y$ is an h-fibration and the inclusion $X \subset Y$ is an $\bar{h}$-cofibration, then the induced map $p^{-1}(X) \longrightarrow E$ is an $\bar{h}$-cofibration.

Proposition 4.4.6. If $i: A \longrightarrow B$ and $j: B \longrightarrow X$ are maps in $\mathscr{K}$ such that $j$ and $j \circ i$ are $h$-cofibrations, then $i$ is an $h$-cofibration.

Proposition 4.4.7. If an inclusion $A \subset X$ is an $h$-cofibration, then so is the induced inclusion $\bar{A} \subset X$.

In view of the characterization of $\bar{h}$-cofibrations in Theorem 4.4.4, it is natural to ask if there is an analogous characterization of $\bar{h}$-fibrations. Only the following sufficient condition is known. It is stated without proof in [144, 4.1.1], and it gives another reason for requiring the base spaces of ex-spaces to be in $\mathscr{U}$.

Proposition 4.4.8. An h-fibration $p: E \longrightarrow Y$ with $Y \in \mathscr{U}$ is an $\bar{h}$-fibration.

Proof. Let $k: A \longrightarrow X$ be an $h$-acyclic $h$-cofibration and let $j: \bar{A} \longrightarrow X$ be the induced inclusion. By Propositions 4.4.7 and 4.4.6, $j$ and the inclusion $i: A \subset \bar{A}$ are $h$-cofibrations. By Lemma 4.2.5(i), $k$ is the inclusion of a deformation retraction $r: X \longrightarrow A$ and the deformation restricts to a homotopy from $(i \circ r) \circ j$ to the identity on $\bar{A}$. It follows that $j$ and hence also $i$ are $h$-acyclic. Since $j$ is an $h$-acyclic $\bar{h}$-cofibration, it has the LLP with respect to $p$, and we see by a little diagram chase that it suffices to verify that $i$ has the LLP with respect to $p$. Factor $p$ as the composite of $s: E \longrightarrow N p$ and $q: N p: \longrightarrow Y$, as usual. Since $q$ is an $\bar{h}$-fibration, $(i, q)$ has the lifting property, and it suffices to show that $(i, s)$ has the lifting property. Suppose given a lifting problem $f: A \longrightarrow E$ and $g: \bar{A} \longrightarrow N p$ such that $s \circ f=g \circ i$. Note that $s(e)=\left(e, c_{p(e)}\right)$ for $e \in E$, where $c_{y}$ denotes the constant path at $y$. Since $Y$ is weak Hausdorff, the constant paths give a closed subset of $Y^{I}$ and $N p=Y^{I} \times_{Y} E$ is a closed subset of $Y^{I} \times E$. Therefore $s(E)$ is closed in $N p$. We conclude that

$$
g(\bar{A}) \subset \overline{g(A)}=\overline{s(f(A)} \subset \overline{s(E)}=s(E),
$$

which means that there is a lift $\bar{A} \longrightarrow E$. 


\subsection{Compactly generated $q$-type model structures}

We give a variant of the standard procedure for constructing $q$-type model structures. The exposition prepares the way for a new variant that we will explain in $\S 5.4$ and which is crucial to our work. Although our discussion is adapted to topological examples, $\mathscr{C}$ need not be topological until otherwise specified. We first recall the small object argument in settings where compactness allows use of sequential colimits.

DeFinition 4.5.1. Let $I$ be a set of maps in $\mathscr{C}$.

(i) A relative $I$-cell complex is a map $Z_{0} \longrightarrow Z$, where $Z$ is the colimit of a sequence of maps $Z_{n} \longrightarrow Z_{n+1}$ such that $Z_{n+1}$ is the pushout $Y \cup_{X} Z_{n}$ of a coproduct $X \longrightarrow Y$ of maps in $I$ along a map $X \longrightarrow Z_{n}$.

(ii) $I$ is compact if for every domain object $X$ of a map in $I$ and every relative $I$-complex $Z_{0} \longrightarrow Z$, the map $\operatorname{colim} \mathscr{C}\left(X, Z_{n}\right) \longrightarrow \mathscr{C}(X, Z)$ is a bijection.

(iii) An $I$-cofibration is a map that satisfies the LLP with respect to any map that satisfies the RLP with respect to $I$.

Lemma 4.5.2 (Small object argument). Let $I$ be a compact set of maps in $\mathscr{C}$, where $\mathscr{C}$ is cocomplete. Then any map $f: X \longrightarrow Y$ in $\mathscr{C}$ factors functorially as a composite

$$
X \stackrel{i}{\longrightarrow} W \stackrel{p}{\longrightarrow} Y
$$

such that $p$ satisfies the $R L P$ with respect to $I$ and $i$ is a relative $I$-cell complex and therefore an $I$-cofibration.

Definition 4.5.3. A model structure on $\mathscr{C}$ is compactly generated if there are compact sets $I$ and $J$ of maps in $\mathscr{C}$ such that the following characterizations hold.

(i) The fibrations are the maps that satisfy the RLP with respect to $J$, or equivalently, with respect to retracts of relative $J$-cell complexes.

(ii) The acyclic fibrations are the maps that satisfy the RLP with respect to $I$, or equivalently, with respect to retracts of relative $I$-cell complexes.

(iii) The cofibrations are the retracts of relative $I$-cell complexes.

(iv) The acyclic cofibrations are the retracts of relative $J$-cell complexes.

The maps in $I$ are called the generating cofibrations and the maps in $J$ are called the generating acyclic cofibrations.

Remark 4.5.4. The term "compactly generated" is non-standard. Compactly generated model categories whose cofibrations are effective monomorphisms $[73$, 10.9.1] are examples of cellular model categories, which are themselves examples of cofibrantly generated model categories. In our topological context, it is immediate that all cofibrations $f: A \longrightarrow B$ that we encounter are effective monomorphisms, since the condition asserts in effect that $A$ is the intersection of the two copies of $B$ in $B \cup_{A} B$. Cellular model categories are specified in terms of a more general notion of compactness that encodes the cardinality conditions required to carry out the small object argument [73, 11.4.1] in contexts, such as Bousfield localization, where the sequential small object argument does not apply. Everything that we say about compactly generated topological model categories applies equally well to cellular topological model categories. We have restricted to the compactly generated case for simplicity and because the examples that we consider here are of this type. 
We find it convenient to separate out properties of classes of maps in a model category, starting with the weak equivalences.

Definition 4.5.5. A subcategory of $\mathscr{C}$ is a subcategory of weak equivalences if it satisfies the following closure properties.

(i) All isomorphisms in $\mathscr{C}$ are weak equivalences.

(ii) A retract of a weak equivalence is a weak equivalence.

(iii) If two out of three maps $f, g, g \circ f$ are weak equivalences, so is the third.

THEOREM 4.5.6. Let $\mathscr{C}$ be a bicomplete category with a subcategory of weak equivalences. Let $I$ and $J$ be compact sets of maps in $\mathscr{C}$. Then $\mathscr{C}$ is a compactly generated model category with generating cofibrations I and generating acyclic cofibrations $J$ if the following two conditions hold:

(i) (Acyclicity condition) Every relative $J$-cell complex is a weak equivalence.

(ii) (Compatibility condition) A map has the RLP with respect to I if and only if it is a weak equivalence and has the RLP with respect to $J$.

Proof. This is the formal part of Quillen's original proof of the $q$-model structure on topological spaces and is a variant of $[75,2.1 .19]$ or $[73,11.3 .1]$. The fibrations are defined to be the maps that satisfy the RLP with respect to $J$. The cofibrations are defined to be the $I$-cofibrations and turn out to be the retracts of relative $I$-cell complexes. The retract axioms clearly hold and, by (ii), the cofibrations are the maps that satisfy the LLP with respect to the acyclic fibrations, which gives one of the lifting axioms. The maps in $J$ satisfy the LLP with respect to the fibrations and are therefore cofibrations, which verifies something that is taken as a hypothesis in the versions in the cited sources. Applying the small object argument to $I$, we factor a map $f$ as a composite of an $I$-cofibration followed by a map that satisfies the RLP with respect to $I$; by (ii), the latter is an acyclic fibration. Applying the small object argument to $J$, we factor $f$ as a composite of a relative $J$-cell complex that is a $J$-cofibration followed by a fibration. By (i), the first map is acyclic, and it is a cofibration because it satisfies the LLP with respect to all fibrations, in particular the acyclic ones. Finally, for the second lifting axiom, if we are given a lifting problem with an acyclic cofibration $f$ and a fibration $p$, then a standard retract argument shows that $f$ is a retract of an acyclic cofibration that satisfies the LLP with respect to all fibrations.

Using the following companion to Definition 4.5.5, we codify the usual pattern for verifying the acyclicity condition.

Definition 4.5.7. A subcategory of a cocomplete category $\mathscr{C}$ is a subcategory of cofibrations if it satisfies the following closure properties.

(i) All isomorphisms in $\mathscr{C}$ are cofibrations.

(ii) All coproducts of cofibrations are cofibrations.

(iii) If $i: X \longrightarrow Y$ is a cofibration and $f: X \longrightarrow Z$ is any map, then the pushout $j: Z \longrightarrow Y \cup_{X} Z$ of $i$ along $f$ is a cofibration.

(iv) If $X$ is the colimit of a sequence of cofibrations $i_{n}: X_{n} \longrightarrow X_{n+1}$, then the induced map $i: X_{0} \longrightarrow X$ is a cofibration.

(v) A retract of a cofibration is a cofibration.

In more general contexts, (iv) should be given a transfinite generalization, but we shall not have need of that. Note that if a subcategory of cofibrations is defined in terms of a left lifting property, then all of the conditions hold automatically. 
LEMMA 4.5.8. Let $\mathscr{C}$ be a cocomplete category together with a subcategory of cofibrations, denoted $g$-cofibrations, and a subcategory of weak equivalences, satisfying the following properties.

(i) A coproduct of weak equivalences is a weak equivalence.

(ii) If $i: X \longrightarrow Y$ is an acyclic $g$-cofibration and $f: X \longrightarrow Z$ is any map, then the pushout $j: Z \longrightarrow Y \cup_{X} Z$ of $i$ along $f$ is a weak equivalence.

(iii) If $X$ is the colimit of a sequence of acyclic $g$-cofibrations $i_{n}: X_{n} \longrightarrow X_{n+1}$, then the induced map $i: X_{0} \longrightarrow X$ is a weak equivalence.

If every map in a set $J$ is an acyclic $g$-cofibration, then every relative $J$-cell complex is a weak equivalence.

We emphasize that, in practice, the $g$-cofibrations need not be the model category cofibrations and may or may not be the intrinsic $h$-cofibrations or $\bar{h}$ cofibrations. They serve as convenient scaffolding for proving the model axioms.

REMARK 4.5.9. The properties listed in Lemma 4.5.8 include some of the axioms for a "cofibration category" given by Baues [8, pp 6, 182]. However, our purpose is to describe features of categories that are more richly structured than model categories, often with several relevant subcategories of cofibrations, rather than to describe deductions from axiom systems for less richly structured categories, which is his focus. The $g$-cofibrations in Lemma 4.5.8 need not be the cofibrations of any cofibration category or model category.

The $q$-model structures on $\mathscr{K}$ and $\mathscr{U}$ are obtained by Theorem 4.5.6, taking the $q$-equivalences to be the weak equivalences, that is, the maps that induce isomorphisms on all homotopy groups, and the $q$-fibrations to be the Serre fibrations. Similarly, in $\mathscr{K}_{*}$ and $\mathscr{T}$, a based map is a $q$-equivalence or $q$-fibration if its underlying unbased map is so. We also have the equivariant generalization, which applies to any topological group $G$. We introduce the following notations, which will be used throughout.

Definition 4.5.10. Nonequivariantly, let $I$ and $J$ denote the set of inclusions $i: S^{n-1} \longrightarrow D^{n}$ (where $S^{-1}$ is empty) and the set of maps $i_{0}: D^{n} \longrightarrow D^{n} \times I$. Equivariantly, let $I$ and $J$ denote the set of all maps of the form $G / H \times i$, where $H$ is a (closed) subgroup of $G$ and $i$ runs through the maps in the nonequivariant sets $I$ and $J$. In the based categories $\mathscr{K}_{*}$ and $G \mathscr{K}_{*}$ we continue to write $I$ and $J$ for the sets obtained by adjoining disjoint base points to the specified maps.

A map $f: X \longrightarrow Y$ of $G$-spaces is said to be a weak equivalence or Serre fibration if all fixed point maps $f^{H}: X^{H} \longrightarrow Y^{H}$ are weak equivalences or Serre fibrations. Just as nonequivariantly, we also call these $q$-equivalences and $q$-fibrations. Observe that $q$-equivalences are defined in terms of the equivariant homotopy groups $\pi_{n}^{H}(X, x)=\pi_{n}\left(X^{H}, x\right)$ for $H \subset G$ and $x \in X^{H}$ and that $q$-fibrations are defined in terms of the RLP with respect to the cells in $J$.

If $X_{0} \longrightarrow X$ is a relative $I$ or $J$-cell complex, then $X / X_{0}$ is in $G \mathscr{U}$ and Lemma 1.6.5 gives all that is needed to verify the compactness hypothesis in Definition 4.5.1(ii). Taking the $g$-cofibrations to be the $h$-cofibrations, Lemma 4.5.8 applies to verify the acyclicity condition of Theorem 4.5.6. With considerable simplification, our verification of the compatibility condition for the $q f$-model structure in Chapter 6 specializes to verify it here. 
Nonequivariantly, the $q$-model structure is discussed in $[56, \S 8]$ and, with somewhat different details, in [75, 2.4]. Equivariantly, a detailed proof of the following result is given in [105, III $\S 1]$. The argument there is given for based $G$-spaces, in $G \mathscr{T}$, but it works equally well for unbased $G$-spaces, in $G \mathscr{K}$.

TheOREM 4.5.11. For any $G, G \mathscr{K}$ is a compactly generated proper model category whose q-equivalences, $q$-fibrations, and q-cofibrations are the weak equivalences, the Serre fibrations, and the retracts of relative $G$-cell complexes. The sets $I$ and $J$ are the generating q-cofibrations and the generating acyclic q-cofibrations, and all $q$-cofibrations are $\bar{h}$-cofibrations. If $G$ is a compact Lie group, then the model structure is G-topological.

The notion of a $G$-topological model category is defined in the same way as the notion of a simplicial or topological model category and is discussed formally in $\S 10.3$ below. The point of the last statement is that if $H$ and $K$ are subgroups of a compact Lie group $G$, then $G / H \times G / K$ has the structure of a $G$-CW complex. By Theorem 3.4.2, this remains true when $G$ is a Lie group and $H$ and $K$ are compact subgroups. We shall see how to use this fact model theoretically in Chapter 7.

REMARK 4.5.12. In Theorem 4.5.11, the generating acyclic cofibrations are actually $\bar{h}$-cofibrations that are inclusions of deformation retracts. A pushout of a coproduct of such maps is again such a map, the inclusion of the initial term in a sequential colimit of such maps is another such, and so is a retract of such a map. We conclude that every acyclic $q$-cofibration is an $\bar{h}$-cofibration that is an inclusion of a deformation retract. Thus, implicitly, the classical $q$-type model structures are built directly from the underlying $h$-type model structures. As soon as we work in the parametrized context, this structure will disappear. Our generating acyclic cofibrations over $B$ will be given by deformation retracts on total spaces, but the retractions will not be maps over $B$. Therefore direct arguments with weak equivalences that are wholly unnecessary in the classical case will be essential to our work, especially when we turn to the stable theory. 



\section{CHAPTER 5}

\section{Well-grounded topological model categories}

\section{Introduction}

It is essential to our theory to understand the interrelationships among the various model structures that appear naturally in the parametrized context, both in topology and in general. This understanding leads us more generally to an axiomatization of the properties that are required of a good $q$-type model structure in order that it relate well to the classical homotopy theory on a topological category. The obvious $q$-model structure on ex-spaces over $B$ does not satisfy the axioms, and in the next chapter we will introduce a new model structure, the $q f$-model structure, that does satisfy the axioms.

As we recall in $\S 5.1$, any model structure on a category $\mathscr{C}$ induces a model structure on the category of objects over, under, or over and under a given object $B$. When $\mathscr{C}$ is topologically bicomplete, so are these over and under categories. They therefore have their own intrinsic $h$-type model structures, which differ from the one inherited from $\mathscr{C}$. This leads to quite a few different model structures on the category $\mathscr{C}_{B}$ of objects over and under $B$, each with its own advantages and disadvantages. Letting $B$ vary, we also obtain a model structure on the category of retracts. We shall only be using most of these structures informally, but the plethora of model structures is eye opening.

In $\S 5.2$, we focus on spaces and compare the various classical notions of fibrations and cofibrations that are present in our over and under categories. Although elementary, this material is subtle, and it is nowhere presented accurately in the literature. In particular, we discuss $h$-type, $f$-type and $f p$-type model structures, where $f$ and $f p$ stand for "fiberwise" and "fiberwise pointed". For simplicity, we discuss this material nonequivariantly, but it applies verbatim equivariantly.

The comparisons among the $q, h, f$, and $f p$ classes of maps and model structures guide our development of parametrized homotopy theory. We think of the $f$-notions as playing a transitional role, connecting the $f p$ and $h$-notions. In the rest of the chapter, we work in a general topologically bicomplete category $\mathscr{C}$, and we sort out this structure and its relationship to a desired $q$-type model structure axiomatically.

Here we shift our point of view. We focus on three basic types of cofibrations that are in play in the general context, namely the Hurewicz cofibrations determined by the cylinders in $\mathscr{C}$, the ground cofibrations that come in practice from a given forgetful functor to underlying spaces, and the $q$-type model cofibrations. The first two are intrinsic, but we think of the $q$-type cofibrations as subject to negotiation. In $\mathscr{K}_{B}$, the Hurewicz cofibrations are the $f p$-cofibrations and the ground cofibrations are the $h$-cofibrations, which is in notational conflict with the point of view taken in the previous chapter. 
In $\S \S 5.3$ and 5.4, we ignore model theoretic considerations entirely. We describe how the two intrinsic types of cofibrations relate to each other and to colimits and tensors, and we explain how this structure relates to weak equivalences. These ideas are made precise by the notions of "well-grounded objects" and of a "ground structure" in a topologically bicomplete category and of a subcategory of "wellgrounded weak equivalences" in a category with a ground structure.

We define the notion of a "well-grounded model structure" in $§ 5.5$. We believe that this notion captures exactly the right blend of classical and model categorical homotopical structure in topological situations. It describes what is needed for a $q$ type model structure in a topologically bicomplete category to be compatible with its intrinsic $h$-type model structure and its ground structure. Crucially, the $q$-type cofibrations should be "bicofibrations", meaning that they are both Hurewicz cofibrations and ground cofibrations. We illustrate the usefulness of the axiomatization in $§ 5.6$. We show that the elementary classical theory of long exact sequences associated to cofiber sequences, homotopy colimits, and $\lim ^{1}$ exact sequences applies in any well-grounded model category.

A clear understanding of the desiderata for a good $q$-type model structure reveals that the obvious over and under $q$-model structure is essentially worthless for serious work in parametrized homotopy theory. This will lead us to introduce the new $q f$-model structure, with better behaved $q$-type cofibrations, in the next chapter. The formalization given in $§ \S 5.3-5.6$ might seem overly pedantic were it only to serve as motivation for the definition of the $q f$-model structure. However, we will encounter exactly the same structure in Part III when we construct the level and stable model structures on parametrized spectra. We hope that the formalization will help guide the reader through the rougher terrain there.

We note parenthetically that there is still another interesting model structure on the category of ex-spaces over $B$, one based on local considerations. It is due to Michelle Intermont and Mark Johnson [81]. We shall not discuss their model structure here, but we are indebted to them for illuminating discussions. It is conceivable that their model structure could be used in an alternative development of the stable theory, but that has not been worked out. Their structure suffers the defects that it is not known to be left proper and that, with their definition of weak equivalences, homotopy equivalences of base spaces need not induce equivalences of homotopy categories.

We focus mainly on the nonequivariant context in this chapter, but $G$ can be any topological group in all places where equivariance is considered.

\subsection{Over and under model structures}

Recall from $\S 1.2$ that, for any category $\mathscr{C}$ and object $B$ in $\mathscr{C}$, we let $\mathscr{C} / B$ and $\mathscr{C}_{B}$ denote the categories of objects over $B$ and of ex-objects over $B$. We also have the category $B \backslash \mathscr{C}$ of objects under $B$. If $\mathscr{C}$ is bicomplete, then so are $\mathscr{C} / B, B \backslash \mathscr{C}$ and $\mathscr{C}_{B}$. We begin with some general observations about over and under model categories before returning to topological categories.

We have forgetful functors $U: \mathscr{C} / B \longrightarrow \mathscr{C}$ and $V: \mathscr{C}_{B} \longrightarrow \mathscr{C} / B$. The first is left adjoint to the functor that sends an object $Y$ to the object $B \times Y$ over $B$ :

$$
\mathscr{C}(U X, Y) \cong \mathscr{C} / B(X, B \times Y)
$$


The second is right adjoint to the functor that sends an object $X$ over $B$ to the object $X \amalg B$ over and under $B$ :

$$
\mathscr{C}_{B}(X \amalg B, Y) \cong \mathscr{C} / B(X, V Y) .
$$

We interpolate some discussion of notation. It has been the custom since the beginnings of topology to use the same letter for a bundle and for its underlying total space. In line with this, the literature of parametrized homotopy theory systematically uses the notation $X_{+}$for the union $X \amalg B$ of a space $X$ over $B$ with a disjoint section. It seems to us that these standard abuses of notation hide structure and seriously obscure the literature of the subject. For example, they force one to the identity " $r_{!} X_{+}=X_{+}$", where $X$ is a space over $B$ with disjoint section on the left and its total space with a disjoint basepoint on the right. In line with this, we adopt the following notational convention throughout this book.

Notation 5.1.3. For a space $(X, p)$ over $B$, we use the notation $X \amalg B$ and $(X, p)_{+}$interchangeably for the space over and under $B$ obtained by adjoining a disjoint section to $(X, p)$. We shall reserve the notation $X_{+}$for the union of a space $X$ and a disjoint basepoint, so that $r_{!}(X, p)_{+}=X_{+}$.

Returning to our general discussion, we observe that, as a composite of a left and a right adjoint, the total object functor $U V: \mathscr{C}_{B} \longrightarrow \mathscr{C}$ does not enjoy good formal properties. This obvious fact plays a significant role in our work. For example, it limits the value of the model structures on $\mathscr{C}_{B}$ that are given by the following result.

Proposition 5.1.4. Let $\mathscr{C}$ be a model category. Then $\mathscr{C} / B, B \backslash \mathscr{C}$, and $\mathscr{C}_{B}$ are model categories in which the weak equivalences, cofibrations, and fibrations are the maps over $B$, under $B$, or over and under $B$ which are weak equivalences, fibrations, or cofibrations in $\mathscr{C}$. If $\mathscr{C}$ is left or right proper, then so are $\mathscr{C} / B, B \backslash \mathscr{C}$, and $\mathscr{C}_{B}$.

Proof. As observed in $[75$, p. 5$]$ and $[56,3.10]$, the statement about $\mathscr{C} / B$ is a direct verification from the definition of a model category. By the self-dual nature of the axioms, the statement about $B \backslash \mathscr{C}$ is equivalent. The statement about $\mathscr{C}_{B}$ follows since it is the category of objects under $(B$, id $)$ in $\mathscr{C} / B$. The last statement holds since pushouts and pullbacks in these over and under categories are constructed in $\mathscr{C}$.

When considering $q$-type model structures, we start with a compactly generated model category $\mathscr{C}$. Using the adjunctions (5.1.1) and (5.1.2), we then obtain the following addendum to Proposition 5.1.4.

Proposition 5.1.5. If $\mathscr{C}$ is a compactly generated model category, then $\mathscr{C} / B$ and $\mathscr{C}_{B}$ are compactly generated. The generating (acyclic) cofibrations in $\mathscr{C} / B$ are the maps $i$ such that $U i$ is a generating (acyclic) cofibration in $\mathscr{C}$. The generating (acyclic) cofibrations in $\mathscr{C}_{B}$ are the maps $i \amalg B$ where $i$ is a generating (acyclic) cofibration in $\mathscr{C} / B$.

We now return to the case when $\mathscr{C}$ is topologically bicomplete. Then it has the resulting "classical", or $h$-type, structure that was discussed in $\S 4.3$ and $\S 4.4$. If our philosophy in $\S 4.1$ applies to $\mathscr{C}$, then it also has $q$ and $m$-structures and the categories $\mathscr{C} / B$ and $\mathscr{C}_{B}$ both inherit over and under model structures that are related 
as we discussed there. However, since $\mathscr{C}$ is topologically bicomplete, so is $\mathscr{C} / B$ by Proposition 1.2.8, and $\mathscr{C}_{B}$ is based topologically bicomplete by Proposition 1.2.9. These categories therefore have classical $h$-type structures when they are regarded in their own right as topologically bicomplete categories. To fix notation and avoid confusion we give an overview of all of these structures.

We start with the $h$-classes of maps in $\mathscr{C}$ that are given in Definition 4.2 .1 and Lemma 4.2.4. As in our discussion of spaces, we work assymmetrically, ignoring the $\bar{h}$-fibrations and focusing on the candidates for $h$-type model structures given by the $h$-fibrations and $\bar{h}$-cofibrations. We agree to use the letter $h$ for the inherited classes of maps in $\mathscr{C} / B$ and $\mathscr{C}_{B}$, although that contradicts our previous use of $h$ for the classical classes of maps in an arbitrary topologically bicomplete category, such as $\mathscr{C} / B$ or $\mathscr{C}_{B}$. We shall resolve that ambiguity shortly by introducing new names for the classes of "classical" maps in those categories.

Definition 5.1.6. A map $g$ in $\mathscr{C} / B$ is an $h$-equivalence, $h$-fibration, $h$-cofibration, or $\bar{h}$-cofibration if $U g$ is such a map in $\mathscr{C}$. A map $g$ in $\mathscr{C}_{B}$ is an $h$ equivalence, $h$-fibration, $h$-cofibration, or $\bar{h}$-cofibration if $V g$ is such a map in $\mathscr{C} / B$ or, equivalently, $U V g$ is such a map in $\mathscr{C}$.

The $\bar{h}$-cofibrations are $h$-cofibrations, but not conversely in general. Since the object $*_{B}=(B$, id, id $)$ is initial and terminal in $\mathscr{C}_{B}$, an object of $\mathscr{C}_{B}$ is $h$-cofibrant (or $\bar{h}$-cofibrant) if its section is an $h$-cofibration (or $\bar{h}$-cofibration) in $\mathscr{C}$. It is $h$ fibrant if its projection is an $h$-fibration in $\mathscr{C}$.

In $\mathscr{C} / B$, we have the notion of a homotopy over $B$, defined in terms of $X \times{ }_{B} I$ or, equivalently, $\operatorname{Map}_{B}(I, X)$. The adjective "fiberwise" is generally used in the literature to describe these homotopies. See, for example, the books $[42,83]$ on fiberwise homotopy theory. To distinguish from the $h$-model structure, we agree to write $f$ rather than $h$ for the fiberwise specializations of Definition 4.2.1 and Lemma 4.2.4. To avoid any possible confusion, we formalize this, making use of Proposition 4.3.3.

Definition 5.1.7. Let $g$ be a map in $\mathscr{C} / B$.

(i) $g$ is an $f$-equivalence if it is a fiberwise homotopy equivalence.

(ii) $g$ is an $f$-fibration if it satisfies the fiberwise CHP, that is, if it has the RLP with respect to the maps $i_{0}: X \longrightarrow X \times{ }_{B} I$ for $X \in \mathscr{C} / B$.

(iii) $g$ is an $f$-cofibration if it satisfies the fiberwise HEP, that is, if it has the LLP with respect to the maps $p_{0}: \operatorname{Map}_{B}(I, X) \longrightarrow X$.

(iv) $g$ is an $\bar{f}$-cofibration if it has the LLP with respect to the $f$-acyclic $f$-fibrations.

A map $g$ in $\mathscr{C}_{B}$ is an $f$-equivalence, $f$-fibration, $f$-cofibration, or $\bar{f}$-cofibration if $V g$ is one in $\mathscr{C} / B$.

Again, $\bar{f}$-cofibrations are $f$-cofibrations, but not conversely in general. Theorem 4.4.2 often applies to show that the $f$-fibrations and $\bar{f}$-cofibrations define an $f$-model structure on $\mathscr{C} / B$ and therefore, by Proposition 5.1.4, on $\mathscr{C}_{B}$. As is always the case for an intrinsic classical model structure, every object of $\mathscr{C} / B$ is both $f$-cofibrant and $\bar{f}$-cofibrant as well as $f$-fibrant. While this is obvious from the definitions, it may seem counterintuitive. It does not follow that every object of $\mathscr{C}_{B}$ is $f$-cofibrant since the two categories have different initial objects.

In $\mathscr{C}_{B}$, we also have the notion of a homotopy over and under $B$, defined in terms of $X \wedge_{B} I_{+}$or, equivalently, $F_{B}\left(I_{+}, X\right)$. The adjective "fiberwise pointed" is 
used in $[42,83]$ to describe these homotopies. Again, for notational clarity, we agree to write $f p$ rather than $h$ for the fiberwise pointed specializations of Definition 4.2.1 and Lemma 4.2.4, and we formalize this to avoid any possible confusion.

Definition 5.1.8. Let $g$ be a map in $\mathscr{C}_{B}$.

(i) $g$ is an $f p$-equivalence if it is a fiberwise pointed homotopy equivalence.

(ii) $g$ is an $f p$-fibration if it satisfies the fiberwise pointed CHP, that is, if it has the RLP with respect to the maps $i_{0}: X \longrightarrow X \wedge_{B} I_{+}$.

(iii) $g$ is a $f p$-cofibration if it satisfies the fiberwise pointed HEP, that is, if it has the LLP with respect to the maps $p_{0}: F_{B}\left(I_{+}, X\right) \longrightarrow X$.

(iv) $g$ is an $\overline{f p}$-cofibration if it has the LLP with respect to the $f p$-acyclic $f p$ fibrations.

Again, $\overline{f p}$-cofibrations are $f p$-cofibrations, but not conversely in general, and Theorem 4.4.2 often applies to show that the $f p$-fibrations and $\overline{f p}$-cofibrations define an $f p$-model structure on $\mathscr{C}_{B}$. We summarize some general formal implications relating our classes of maps.

Proposition 5.1.9. Let $\mathscr{C}, \mathscr{C} / B$ and $\mathscr{C}_{B}$ be topologically bicomplete categories with $h, f$, and $f p$-classes of maps defined as above. Then the following implications hold for maps in $\mathscr{C}_{B}$.

\begin{tabular}{rllcll}
$f p$-equivalence & $\Longleftrightarrow$ & $f$-equivalence & $\Longleftrightarrow$ & $h$-equivalence \\
$f p$-cofibration & $\Longleftrightarrow f$-cofibration & $\Longleftrightarrow$ & $h$-cofibration \\
$\Uparrow$ & & & $\Uparrow$ \\
$f p$-cofibration & $\Longleftrightarrow$ & $\bar{f}$-cofibration & $\Longleftrightarrow$ & $\bar{h}$-cofibration \\
$f p$-fibration & $\Longleftrightarrow$ & $f$-fibration & $\Longleftrightarrow$ & $h$-fibration \\
\hline
\end{tabular}

Moreover, every object of $\mathscr{C}_{B}$ is both $\mathrm{fp}$-fibrant and fp-cofibrant.

ProOF. Trivial inspections of lifting diagrams show that an $h$-fibration is an $f$-fibration, an $f$-cofibration is an $f p$-cofibration, and an $\bar{f}$-cofibration is an $\overline{f p}$ cofibration. Use of the adjunctions (5.1.1) and (5.1.2) shows that an $f$-cofibration is an $h$-cofibration, an $\bar{f}$-cofibration is an $\bar{h}$-cofibration, and an $f p$-fibration is an $f$ fibration. The last statement holds since fiberwise pointed homotopies with domain or target $B$ are constant at the section or projection of the target or source.

REMARK 5.1.10. Assume that these classes of maps define model structures. Then the implications in Proposition 5.1.9 lead via Theorem 4.1.1 and its dual version to two new mixed model structures on $\mathscr{C}_{B}$, one with weak equivalences the $f$-equivalences and fibrations the $f p$-fibrations and one with weak equivalences the $h$-equivalences and cofibrations the $\bar{f}$-cofibrations.

The category $\mathscr{C}_{\mathscr{B}}$ of retracts introduced in $\S 2.5$ suggests an alternative model theoretic point of view. We give the basic definitions, but we shall not pursue this idea in any detail. Again, Theorem 4.4.2 often applies to verify the model category axioms. Note that the intrinsic homotopies are given by homotopies of total objects over and under homotopies of base objects.

Definition 5.1.11. Assume that $\mathscr{C}_{\mathscr{B}}$ is topologically bicomplete and let $g$ be a map in $\mathscr{C}_{\mathscr{B}}$.

(i) $g$ is an $r$-equivalence if it is a homotopy equivalence of retractions. 
(ii) $g$ is an $r$-fibration if it satisfies the retraction CHP, that is, if it has the RLP with respect to the maps $i_{0}: X \longrightarrow X \times I$ for $X \in \mathscr{C}_{\mathscr{B}}$.

(iii) $g$ is an $r$-cofibration if it satisfies the retraction HEP, that is, if it has the LLP with respect to the maps $p_{0}: \operatorname{Map}(I, X) \longrightarrow X$.

(iv) $g$ is an $\bar{r}$-cofibration if it has the LLP with respect to the $r$-acyclic $r$-fibrations.

REMARK 5.1.12. The initial and terminal object of $\mathscr{C}_{\mathscr{B}}$ are the identity retractions of the initial and terminal objects of $\mathscr{B}$ and every object is both $r$-cofibrant and $r$-fibrant. It might be of interest to characterize the retractions for which the map $*_{B} \longrightarrow(X, p, s)$ induced by $s$ is an $r$-cofibration or for which the map $(X, p, s) \longrightarrow *_{B}$ induced by $p$ is an $r$-fibration. By specialization of the lifting properties, an ex-map over $B$ that is an $r$-cofibration or $r$-fibration is an $f p$-cofibration or $f p$-fibration in $\mathscr{C}_{B}$, but we have not pursued this question further.

\subsection{The specialization to over and under categories of spaces}

Now we take $\mathscr{C}$ to be $\mathscr{K}$ or $\mathscr{U}$. We discuss the relationships among our various classes of fibrations and cofibrations in this special case, and we consider when the $f$ and $f p$ classes of maps give model structures. Everything in this section applies equally well equivariantly.

We first say a bit about based spaces, which are ex-spaces over $B=\{*\}$. Here the fact that $*$ is a terminal object greatly simplifies matters. All of the $f$-notions coincide with the corresponding $h$-notions, and our trichotomy reduces to the familiar dichotomy between free (or $h$ ) notions and based (or $f p$ ) notions. Recall that a based space is well-based, or nondegenerately based, if the inclusion of the basepoint is an $h$-cofibration. Every based space is $f p$-cofibrant, and an $f p$-cofibration between well-based spaces is an $h$-cofibration [160, Prop. 9]. Every based space is $f p$-fibrant, and an $h$-fibration of based spaces satisfies the based CHP with respect to well-based source spaces. Of course, the over and under $h$-model structure differs from the intrinsic $f p$-model structure.

None of the reverse implications in Proposition 5.1.9 holds in general. We gave details of that result since it is easy to get confused and think that more is true than we stated.

Scholium 5.2.1. On [42, p. 66], it is stated that a fiberwise pointed cofibration which is a closed inclusion is a fiberwise cofibration. That is false even when $B$ is a point, since it would imply that every point of a $T_{1}$-space is a nondegenerate basepoint. On [42, p. 69], it is stated that a fiberwise pointed map (= ex-map) is a fiberwise pointed fibration if and only if it is a fiberwise fibration. That is also false when $B$ is a point, since the unbased CHP does not imply the based CHP.

However, as for based spaces, the reverse implications in parts of Proposition 5.1.9 often do hold under appropriate additional hypotheses.

PROPOSITION 5.2.2. The following implications hold for an arbitrary topologically bicomplete category $\mathscr{C}$.

(i) A map in $\mathscr{C} / B$ between $h$-fibrant objects over $B$ is an h-equivalence if and only if it is an $f$-equivalence.

(ii) An ex-map between $f$-cofibrant ex-objects over $B$ is an $f$-equivalence if and only if it is an fp-equivalence. 
Proof. The first part follows from Proposition 4.3.5(ii) since an $f$-equivalence in $\mathscr{C} / B$ is the same as an $h$-equivalence over $B$ in $\mathscr{C}$. The second part follows similarly from Proposition 4.3.5(i) since an $f p$-equivalence in $\mathscr{C}_{B}$ is the same as an $f$-equivalence under $B$ in $\mathscr{C} / B$.

The following results hold for spaces. We are doubtful that they hold in general.

Proposition 5.2.3. The following implications hold in both $G \mathscr{K}$ and $G \mathscr{U}$.

(i) An ex-map between $\bar{f}$-cofibrant ex-spaces is an $f$-cofibration if and only if it is an $\mathrm{fp}$-cofibration.

(ii) An ex-map whose source is $\bar{f}$-cofibrant is an $f$-fibration if and only if it is an fp-fibration.

Proof. Part (ii) is $[42,16.3]$. Part (i) is stated on [160, p. 441] and the proof given there for based spaces generalizes using the following lemma.

It is easy to detect $f$-cofibrations by means of the following result, whose proof is the same as that of the standard characterization of Hurewicz cofibrations (e.g. [121, p. 43]; see also [158, Thm. 2], [159, Lem. 4] and [42, 4.3]).

Lemma 5.2.4. An inclusion $i: X \longrightarrow Y$ in $\mathscr{K} / B$ is an $f$-cofibration if and only if $(Y, X)$ is a fiberwise NDR-pair in the sense that there is a map $u: Y \longrightarrow I$ such that $X \subset u^{-1}(0)$ and a homotopy $h: Y \times{ }_{B} I \longrightarrow Y$ over $B$ such that $h_{0}=i d$, $h_{t}=i d$ on $X$ for $0 \leq t \leq 1$, and $h_{1}(y) \in X$ if $u(y)<1$. A closed inclusion $i: X \longrightarrow Y$ in $\mathscr{K} / B$ is an $\bar{f}$-cofibration if and only if the map $u$ above can be chosen so that $X=u^{-1}(0)$.

We introduce the following names here, but we defer a full discussion to §8.1.

Definition 5.2.5. An ex-space is said to be well-sectioned if it is $\bar{f}$-cofibrant. An ex-space is said to be ex-fibrant or, synonomously, to be an ex-fibration if it is both $\bar{f}$-cofibrant and $h$-fibrant. Thus an ex-fibration is a well-sectioned ex-space whose projection is an $h$-fibration.

The term ex-fibrant is more logical than ex-fibration, since we are defining a type of object rather than a type of morphism of $\mathscr{K}_{B}$, but the term ex-fibration goes better with Serre and Hurewicz fibration and is standard in the literature. We have the following implication of Propositions 5.1.9 and 5.2.2. It helps explain the usefulness of ex-fibrations.

Corollary 5.2.6. Let $g$ be an ex-map between ex-fibrations over B.

(i) $g$ is an h-equivalence if and only if $g$ is an $f$-equivalence, and this hold if and only if $g$ is an $f p$-equivalence.

(ii) $g$ is an $f$-cofibration if and only if $g$ is an fp-cofibration, and then $g$ is an $h$-cofibration.

(iii) $g$ is an $f$-fibration if and only if $g$ is an $f p$-fibration, and this holds if $g$ is an $h$-fibration.

REMARK 5.2.7. The model theoretic significance of ex-fibrations over $B$ is unclear. They are fibrant and cofibrant objects in the mixed model structure on ex-spaces over $B$ whose weak equivalences are the $h$-equivalences and whose cofibrations are the $\bar{f}$-cofibrations. However, the converse fails since there are wellsectioned $f$-fibrant ex-spaces that are $f$-equivalent to $h$-fibrant ex-spaces, hence are mixed fibrant, but are not themselves $h$-fibrant. 
The previous remark anticipated the following result on over and under model structures in the categories of spaces and ex-spaces over $B$. Note that Lemma 1.6.2 applies to $\mathscr{K} / B$ and $\mathscr{K}_{B}$ as well as to $\mathscr{K}$ to show that both $f$-cofibrations and $f p$-cofibrations are inclusions which are closed when the total spaces are in $\mathscr{U}$.

THEOREM 5.2.8. The following statements hold.

(i) The $f$-equivalences, $f$-fibrations, and $\bar{f}$-cofibrations give $\mathscr{K} / B$ a proper topological model structure. Moreover, a map in $\mathscr{K} / B$ is an $\bar{f}$-cofibration if and only if it is a closed $f$-cofibration.

(ii) The $f$-equivalences, $f$-fibrations, and $\bar{f}$-cofibrations give $\mathscr{U} / B$ a proper topological model structure. Moreover, a map in $\mathscr{U} / B$ is an $\bar{f}$-cofibration if and only if it is an $f$-cofibration.

(iii) The $f p$-equivalences, $f p$-fibrations, and $\overline{f p}$-cofibrations give $\mathscr{U}_{B}$ an $f p$-model structure.

(iv) The r-classes of maps give the category $\mathscr{U}_{\mathscr{U}}$ of retracts a proper topological $r$-model structure.

Proof. Apart from the factorization axioms, the model structures follow from the discussion in 4.3. In particular, the lifting axioms, the properness, and the topological property of all of these model structures are given by Theorem 4.3.1. In (ii), (iii), and (iv), the factorization axioms follow from Theorem 4.4.2 since the argument in Remark 1.6.4 verifies Hypothesis 4.4.1. The rest of (i) can be proven by direct mimicry of the proof of Theorem 4.4.4, using Lemma 5.2.4, and the characterization of the $\bar{f}$-cofibrations in (ii) follows.

REMARK 5.2.9. We do not know whether or not $\mathscr{K}_{B}$ is an $f p$-model category or whether the $\overline{f p}$-cofibrations in $\mathscr{K}_{B}$ are characterized as the closed $f p$-cofibrations. We also do not know whether or not $\mathscr{K}_{\mathscr{U}}$ is an $r$-model category. The problem here is related to the fact that, while the sections of ex-spaces are always inclusions, they need not be closed inclusions unless the total spaces are in $\mathscr{U}$. Steps 1 and 3 of the proof of Theorem 4.4.4 fail in $\mathscr{K}_{B}$, and we also do not see how to carry over Strøm's original proofs in $[159,160]$. Theorem 4.3 .1 still applies, giving much of the information carried by a model structure. Observe too that if $i: A \longrightarrow X$ is a map of well-sectioned ex-spaces over $B$, then $i$ is an $f p$-cofibration if and only if it is an $f$-cofibration, by Proposition 5.2.2(iii). For ex-spaces that are not well-sectioned, we have little understanding of $f p$-cofibrations, even when $B$ is a point. We also have little understanding of $\overline{f p}$-cofibrations that are not $\bar{f}$-cofibrations.

There is a certain tension between the $f p$ and $h$-notions, with the $f$-notions serving as a bridge between the two. Fiberwise pointed homotopy is the intrinsically right notion of homotopy in $\mathscr{K}_{B}$, hence the $f p$-structure is the philosopically right classical $h$-type model structure on $\mathscr{K}_{B}$, or at least on $\mathscr{U}_{B}$. It is the one that is naturally related to fiber and cofiber sequences, the theory of which works formally in any based topologically bicomplete category in exactly the same way as for based spaces, as we will recall in $\$ 5.6$. A detailed exposition in the case of ex-spaces is given in $[42,83,84]$.

However, with $h$ replaced by $f p$, we do not have the implications that we emphasized in the general philosophy of $\S 4.1$. In particular, with the over and under $q$-model structure, $q$-cofibrations need not be $f p$-cofibrations and $f p$-fibrations need not be $q$-fibrations, let alone $h$-fibrations. The $q$-model structure is still related to 
the $h$-model structure as in $\S 4.1$, but this does not serve to relate the $q$-model structure to parametrized fiber and cofiber sequences in the way familiar from the nonparametrized context. This already suggests that the $q$-model structure might not be appropriate in parametrized homotopy theory. In the following four sections, we explore conceptually what is required of a $q$-type model structure to connect it up with the intrinsic homotopy theory in a topologically bicomplete category.

\subsection{Well-grounded topologically bicomplete categories}

Let $\mathscr{C}$ be a topologically bicomplete category in either the based or the unbased sense; we use the notations of the based context. In our work here, and in other topological contexts, $\mathscr{C}$ is topologically concrete in the sense that there is a faithful and continuous forgetful functor from $\mathscr{C}$ to spaces. In practice, appropriate "ground cofibrations" can then be specified in terms of underlying spaces. These cofibrations should be thought of as helpful background structure in our category $\mathscr{C}$.

To avoid ambiguity, we use the term "Hurewicz cofibration", abbreviated notationally to cyl-cofibration, for the maps that satisfy the HEP with respect to the cylinders in $\mathscr{C}$. We also have the notion of a strong Hurewicz cofibration, which we abbreviate notationally to $\overline{c y l}$-cofibration. For example, the cyl-cofibrations in $\mathscr{K}$, $\mathscr{K} / B$, and $\mathscr{K}_{B}$ are the $h$-cofibrations, the $f$-cofibrations, and the $f p$-cofibrations, respectively, and similarly for $\overline{c y l}$-cofibrations. As we have seen, it often happens that $c y l$-cofibrations between suitably nice objects of $\mathscr{C}$, which we shall call "wellgrounded", are also ground cofibrations. We introduce language to describe this situation. The following definitions codify the behavior of the well-grounded objects with respect to the cyl-cofibrations, colimits, and tensors in $\mathscr{C}$. It is convenient to build in the appropriate equivariant generalizations of our notions, although we defer a formal discussion of $G$-topologically bicomplete $G$-categories to $\S 10.2$; see Definition 10.2.1. The examples in $\S 1.4$ give the idea.

DeFinition 5.3.1. An unbased space is well-grounded if it is compactly generated. A based space is well-grounded if it is compactly generated and well-based. The same definitions apply to $G$-spaces for a topological group $G$.

Let $\mathscr{C}$ be a topologically bicomplete category.

Definition 5.3.2. A full subcategory of $\mathscr{C}$ is said to be a subcategory of wellgrounded objects if the following properties hold.

(i) The initial object of $\mathscr{C}$ is well-grounded.

(ii) All coproducts of well-grounded objects are well-grounded.

(iii) If $i: X \longrightarrow Y$ is a cyl-cofibration and $f: X \longrightarrow Z$ is any map, where $X, Y$, and $Z$ are well-grounded, then the pushout $Y \cup_{X} Z$ is well-grounded.

(iv) The colimit of a sequence of $c y l$-cofibrations between well-grounded objects is well-grounded.

(v) A retract of a well-grounded object is well-grounded.

(vi) If $X$ is a well-grounded object and $K$ is a well-grounded space, then $X \wedge K$ $(X \times K$ in the unbased context $)$ is well-grounded.

When $\mathscr{C}$ is $G$-topologically bicomplete, we replace spaces by $G$-spaces in (vi).

Definition 5.3.3. A ground structure on $\mathscr{C}$ is a (full) subcategory of wellgrounded objects together with a subcategory of cofibrations, called the ground cofibrations and denoted $g$-cofibrations, such that every cyl-cofibration between 
well-grounded objects is a $g$-cofibration. A map that is both a $g$-cofibration and a cyl-cofibration is called a bicofibration.

Thus a cyl-cofibration between well-grounded objects is a bicofibration. The need for focusing on bicofibrations and the force of the definition come from the following fact.

WARNING 5.3.4. In practice, (iii) often fails if $i$ is a $g$-cofibration between wellgrounded objects that is not a cyl-cofibration, as we shall illustrate in $\S 6.1$. In particular, in $G \mathscr{K}_{B}$ with the canonical ground structure described below, it can already fail for an inclusion $i$ of $I$-cell complexes, where $I$ is the standard set of generators for the $q$-cofibrations.

In the next chapter, we will construct a $q$-type model structure for $G \mathscr{K}_{B}$ with a set of generating cofibrations to which the following implication of Definitions 4.5.7 and 5.3.2 applies.

LEMma 5.3.5. Let I be a set of cyl-cofibrations between well-grounded objects and let $f: X \longrightarrow Y$ be a retract of a relative $I$-cell complex $W \longrightarrow Z$. Then $f$ is a bicofibration. If $W$ is well-grounded, then so are $X, Y$, and $Z$.

Our categories of equivariant parametrized spaces have canonical ground structures. Recall that the classes of $f$ and $\bar{f}$-cofibrations in $G \mathscr{U} / B$ and $G \mathscr{U}_{B}$ coincide.

Definition 5.3.6. A space over $B$ is well-grounded if its total space is compactly generated. An ex-space over $B$ is well-grounded if it is well-sectioned and its total space is compactly generated. In both $G \mathscr{K} / B$ and $G \mathscr{K}_{B}$, define the $g$ cofibrations to be the $h$-cofibrations.

Note that the only distinction between well-sectioned and well-grounded exspaces is the condition on total spaces. The distinction is relevant when we consider relative $I$-cell complexes $X_{0} \longrightarrow X$ in $G \mathscr{K}_{B}$. If $X_{0}$ is well-sectioned, then so is $X$, whereas $X / X_{0}$ is an $I$-cell complex and is therefore well-grounded for any $X_{0}$.

Proposition 5.3.7. These definitions specify ground structures on $G \mathscr{K} / B$ and on $G \mathscr{K}_{B}$.

Proof. For $G \mathscr{K} / B$, the Hurewicz cofibrations are the $f$-cofibrations, and these are $h$-cofibrations. It is standard that $G \mathscr{U} / B$ has the closure properties specified in Definition 5.3.2. For $G \mathscr{K}_{B}$, the Hurewicz cofibrations are the $f p$ cofibrations. Between well-sectioned ex-spaces, these are $f$-cofibrations and therefore $h$-cofibrations by Proposition 5.2.3(i). Parts (i)-(v) of Definition 5.3.2 are clear since well-sectioned means $\bar{f}$-cofibrant, which is a lifting property. Finally we consider part (vi). Recall that $X \wedge_{B} K$ can be constructed as the pushout of the diagram of spaces

$$
B<(X \times *) \cup(B \times K) \longrightarrow X \times K,
$$

which also gives the pushout in the category of spaces over $B$. By the equivariant version of the NDR-pair characterization of $f$-cofibrations in Lemma 5.2.4, these spaces are $f$-cofibrant and the inclusion on the right is an $f$-cofibration. This implies that $X \wedge_{B} K$ is $f$-cofibrant. 


\subsection{Well-grounded categories of weak equivalences}

The following definition describes how the weak equivalences and the ground structure are related in practice.

DEFINITION 5.4.1. Let $\mathscr{C}$ be a topologically bicomplete category with a given ground structure. A subcategory of weak equivalences in $\mathscr{C}$ is well-grounded if the following properties hold (where acyclicity refers to the weak equivalences).

(i) A homotopy equivalence is a weak equivalence.

(ii) A coproduct of weak equivalences between well-grounded objects is a weak equivalence.

(iii) (Gluing lemma) Assume that the maps $i$ and $i^{\prime}$ are bicofibrations and the vertical arrows are weak equivalences in the following diagram.

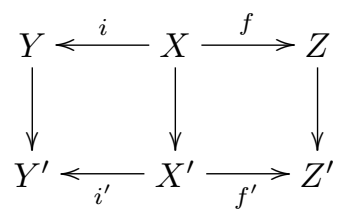

Then the induced map of pushouts is a weak equivalence. In particular, pushouts of weak equivalences along bicofibrations are weak equivalences.

(iv) (Ladder lemma) Let $X$ and $Y$ be the colimits of sequences of bicofibrations $i_{n}: X_{n} \longrightarrow X_{n+1}$ and $j_{n}: Y_{n} \longrightarrow Y_{n+1}$ such that both $X / X_{0}$ and $Y / Y_{0}$ are well-grounded. If $f: X \longrightarrow Y$ is the colimit of a sequence of compatible weak equivalences $f_{n}: X_{n} \longrightarrow Y_{n}$, then $f$ is a weak equivalence. In particular, if each $i_{n}$ is a weak equivalence, then the induced map $i: X_{0} \longrightarrow X$ is a weak equivalence.

(v) For a map $i: X \longrightarrow Y$ of well-grounded objects in $\mathscr{C}$ and a map $j: K \longrightarrow L$ of well-grounded spaces, $i \square j$ is a weak equivalence if $i$ is a weak equivalence or $j$ is a weak equivalence (that is, a $q$-equivalence of spaces).

Here, in the based context, $i \square j$ is the evident induced map

$$
(X \wedge L) \cup_{X \wedge K}(Y \wedge K) \longrightarrow Y \wedge L
$$

In practice, the conditions that we have specified are stronger than consideration of left proper $q$-type model category structures might lead one to expect. However, (v) is both stronger and weaker than one might expect.

REMARK 5.4.2. Condition (v) holds as stated for most of the examples we consider, but in Theorem 24.3.1 we shall encounter an example where $K$ and $L$ must be restricted to be $\mathrm{CW}$ complexes. Note that condition (v) does not involve cofibrations at all, and its verification depends on the particular nature of the tensor functor $\wedge$. This condition describes what actually holds, but, unfortunately, it is not well related to the question of whether or not a model structure on $\mathscr{C}$ is topological. The problem is that the model theoretic cofibrations to which we would like to apply the axiom need not have well-grounded source objects, and it is unclear whether or not (v) holds with unrestricted source objects even when $i$ and $j$ are bicofibrations. (This is already unclear to us when $\mathscr{C}=\mathscr{T}$ ). It is crucial to the usefulness of (iii) and (iv) to the study of model structures that the bicofibrations that appear in them are not required to have well-grounded source objects. 
The gluing lemma implies that acyclic bicofibrations are preserved under pushouts, as of course holds for pushouts of acyclic cofibrations in model categories. The special case mentioned in (iii) corresponds to the left proper axiom in model categories. As there, it can be used to prove the general case of the gluing lemma provided that we have suitable factorizations.

LEMMA 5.4.3. Assume the following hypotheses.

(i) Weak equivalences are preserved under pushouts along bicofibrations.

(ii) Every map factors as the composite of a bicofibration and a weak equivalence. Then the gluing lemma holds.

Proof. We use the notations of Definition 5.4.1(iii) and proceed in three cases.

If $f$ and $f^{\prime}$ are both weak equivalences, then, by (i), so are the horizontal arrows in the commutative diagram

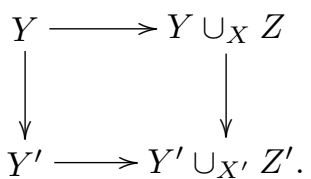

Since $Y \longrightarrow Y^{\prime}$ is a weak equivalence, the right arrow is a weak equivalence by the two out of three property of weak equivalences.

If $f$ and $f^{\prime}$ are both bicofibrations, consider the commutative diagram

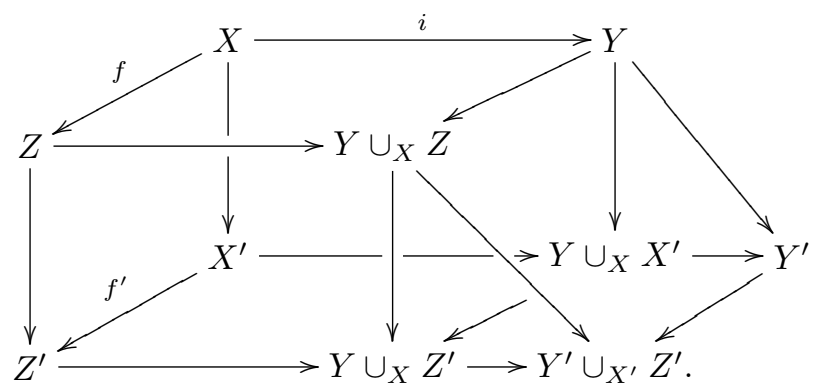

The back, front, top, and two bottom squares are pushouts, and the middle composite $X^{\prime} \longrightarrow Y^{\prime}$ is $i^{\prime}$. Since $f$ and $f^{\prime}$ are bicofibrations, so are the remaining three arrows from the back to the front. Similarly, $i$ and its pushouts are bicofibrations. Since $X \longrightarrow X^{\prime}, Y \longrightarrow Y^{\prime}$, and $Z \longrightarrow Z^{\prime}$ are weak equivalences, (i) and the two out of three property imply that $Y \longrightarrow Y \cup_{X} X^{\prime}, Y \cup_{X} X^{\prime} \longrightarrow Y^{\prime}$, $Y \cup_{X} Z \longrightarrow Y \cup_{X} Z^{\prime}$, and $Y \cup_{X} Z^{\prime} \longrightarrow Y^{\prime} \cup_{X^{\prime}} Z^{\prime}$ are weak equivalences. Composing the last two, $Y \cup_{X} Z \longrightarrow Y^{\prime} \cup_{X^{\prime}} Z^{\prime}$ is a weak equivalence.

To prove the general case, construct the following commutative diagram.

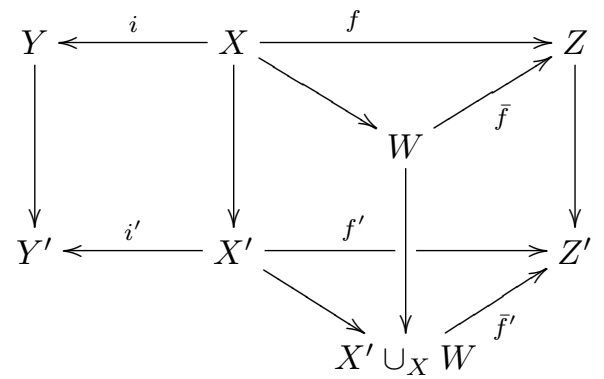


Here we first factor $f$ as the composite of a bicofibration and a weak equivalence $\bar{f}$ and then define a map $\bar{f}^{\prime}$ by the universal property of pushouts. By hypothesis (i), $W \longrightarrow X^{\prime} \cup_{X} W$ is a weak equivalence, and by the two out of three property, so is $\bar{f}^{\prime}$. By the second case,

$$
Y \cup_{X} W \longrightarrow Y^{\prime} \cup_{X^{\prime}}\left(X^{\prime} \cup_{X} W\right) \cong Y^{\prime} \cup_{X} W
$$

is a weak equivalence and by the first case, so is

$$
Y \cup_{X} Z \cong\left(Y \cup_{X} W\right) \cup_{W} Z \longrightarrow\left(Y^{\prime} \cup_{X} W\right) \cup_{\left(X^{\prime} \cup_{X} W\right)} Z^{\prime} \cong Y^{\prime} \cup_{X^{\prime}} Z^{\prime}
$$

REMARK 5.4.4. Clearly the previous result applies to any categories of weak equivalences and cofibrations that satisfy (i) and (ii). The essential point is that, in practice, we often need bicofibrations in order to verify (i).

Similarly, but more simply, the following observation reduces the verification of Definition 5.4.1(v) to special cases. Here we assume that $\mathscr{C}$ is based.

Lemma 5.4.5. Let $i: X \longrightarrow Y$ be a map in $\mathscr{C}$ and $j: K \longrightarrow L$ be a map of based spaces. Display $i \square j$ in the diagram

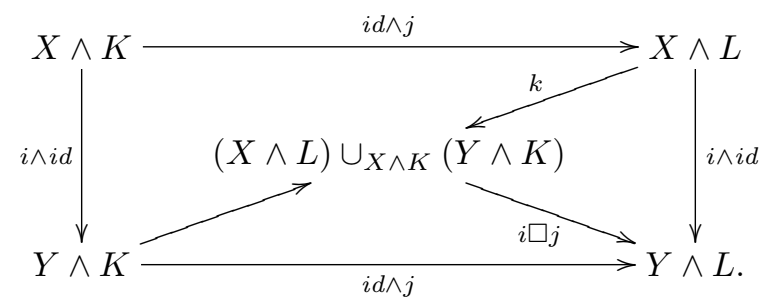

If the maps $i \wedge i d$ and the pushout $k$ of $i \wedge i d$ along $i d \wedge j$ are weak equivalences, then so is $i \square j$, and similarly with the roles of $i$ and $j$ reversed.

Together with Lemma 5.3.5, the notion of a well-grounded category of weak equivalences encodes a variant of Lemma 4.5.8 that often applies when the latter does not.

LEMMA 5.4.6. If $J$ is a set of acyclic cyl-cofibrations between well-grounded objects, then all relative $J$-cell complexes are weak equivalences.

Proof. This follows from (ii), (iii), and (iv) of Definition 5.4.1, together with the observation that if $X_{0} \longrightarrow X$ is a relative $J$-cell complex, then $X / X_{0}$ is a $J$-cell complex and is therefore well-grounded, so that (iv) applies.

There is an analogous reduction of the problem of determining when a functor preserves weak equivalences.

Lemma 5.4.7. Let $F: \mathscr{C} \longrightarrow \mathscr{D}$ be a functor between topologically bicomplete categories that come equipped with subcategories of well-grounded weak equivalences with respect to given ground structures. Let $J$ be a set of acyclic cyl-cofibrations between well-grounded objects in $\mathscr{C}$. Assume that $F$ has a continuous right adjoint and that $F$ takes maps in $J$ to weak equivalences between well-grounded objects. Then $F$ takes a retract of a relative $J$-cell complex to an acyclic map in $\mathscr{D}$.

Proof. The functor $F$ preserves cyl-cofibrations since it has a continuous right adjoint and hence $F J$ consists of acyclic cyl-cofibrations between well-grounded objects. The conclusion follows from Lemma 5.4.6, the fact that left adjoints commute with colimits, and (ii)-(iv) of Definition 5.4.1. 
The following classical example is implicit in the literature.

Proposition 5.4.8. The q-equivalences in $G \mathscr{K}$ are well-grounded with respect to the ground structure whose well-grounded objects are the compactly generated spaces and whose $g$-cofibrations are the $h$-cofibrations.

Proof. Parts (i), (ii), and, here in the unbased case, (v) of Definition 5.4.1 are clear, and (iv) follows easily from Lemma 1.6.5. The essential point is the gluing lemma of (iii). By passage to fixed point spaces, it suffices to prove this nonequivariantly. Using the gluing lemma for the proper $h$-model structure on $\mathscr{K}$, we see that $f$ and $f^{\prime}$ can be replaced by their mapping cylinders. Then the induced map of pushouts is the map of double mapping cylinders induced by the original diagram. This map is equivalent to a map of excisive triads, and in that case the result is $[117,1.3]$, whose proof is corrected in [173].

Proposition 5.4.9. The q-equivalences in $G \mathscr{K} / B$ and $G \mathscr{K}_{B}$ are well-grounded with respect to the ground structures of Proposition 5.3.7. In these cases, one need only assume that the relevant maps in the gluing and colimit lemmas are ground cofibrations (=h-cofibrations), not both ground and Cyl-cofibrations.

Proof. We verify this for $G \mathscr{K}_{B}$. Part (i) of Definition 5.4.1 holds since any $f p$ equivalence is a $q$-equivalence and part (iii) follows directly from the gluing lemma in $G \mathscr{K}$. For part (ii), the total space of $\vee_{B} X_{i}$ is the pushout in $G \mathscr{K}$ of

$$
B<\amalg B \longrightarrow \amalg X_{i} .
$$

Since the $X_{i}$ are well-grounded, the map on the right is an $h$-cofibration, hence (ii) also follows from the gluing lemma in $G \mathscr{K}$. In part (iv), the relevant quotient in $G \mathscr{K}_{B}$ is given by the pushout, $X /_{B} X_{0}$, of the diagram $*_{B} \longleftarrow X_{0} \longrightarrow X$. Since $X /{ }_{B} X_{0}$ is well-grounded, the quotient total space is in $\mathscr{U}$ and one can apply Lemma 1.6.5 just as on the space level. Finally consider (v). As in the proof of Proposition 5.3.7(vi), $X \wedge_{B} K$ can be constructed as the pushout of the following diagram of $f$-cofibrant spaces over $B$.

$$
B<(X \times *) \cup(B \times K) \longrightarrow X \times K
$$

The inclusion on the right is an $f$-cofibration. By the gluing lemma in $G \mathscr{K}$, the functor $X \wedge_{B} K$ preserves $q$-equivalences in both variables since the functor $X \times K$ evidently does so.

\subsection{Well-grounded compactly generated model structures}

Let $\mathscr{C}$ be a topologically bicomplete category or, equivariantly, a $G$-topologically bicomplete $G$-category. In the notion of a "well-grounded model structure", we formulate the properties that a compactly generated model structure on $\mathscr{C}$ should have in order to mesh well with the intrinsic Cyl-structure on $\mathscr{C}$ described in $\S 4.3$. When $\mathscr{C}$ has such a model structure, and when the classical Cyl-structure actually is a model structure, the identity functor on $\mathscr{C}$ is a Quillen left adjoint from the well-grounded model structure to the Cyl-model structure. Thus this notion gives a precise axiomatization for the implementaton of the philosophy that we advertised in $\S 4.1$. We begin with a variant of Theorem 4.5.6. Recall the definitions of a ground structure from Definitions 5.3.2 and 5.3.3, of a subcategory of wellgrounded weak equivalences from Definition 5.4.1, and of a compact set of maps from Definition 4.5.1. 
THEOREM 5.5.1. Let $\mathscr{C}$ be a topologically bicomplete category with a ground structure, a subcategory of well-grounded weak equivalences, and compact sets I and $J$ of maps that satisfy the following conditions.

(i) (Acyclicity condition) Every map in $J$ is a weak equivalence.

(ii) (Compatibility condition) A map has the RLP with respect to $I$ if and only if it is a weak equivalence and has the $R L P$ with respect to $J$.

(iii) Every map in $I$ and $J$ is a $\overline{c y l}$-cofibration between well-grounded objects.

Then $\mathscr{C}$ is a compactly generated model category with generating sets $I$ and $J$ of cofibrations and acyclic cofibrations. Every cofibration is a bicofibration and every cofibrant object is well-grounded. A pushout of a weak equivalence along a bicofibration is a weak equivalence and, in particular, the model structure is left proper. The model structure is topological or, equivariantly, G-topological if the following condition holds.

(iv) $i \square j$ is a relative $I$-cell complex if $i: X \longrightarrow Y$ is a map in $I$ and $j: K \longrightarrow L$ is a map in $J$ (that is, in the set $I$ of generating cofibrations of spaces or $G$-spaces), and $i \square j$ is a relative $J$-cell complex if $i$ is in $I$ and $j$ is in $J$ or if $i$ is in $J$ and $j$ is in $I$.

Proof. By Lemma 5.4.6, Theorem 4.5.6 applies to verify the model axioms. Condition (iii) implies the statements about cofibrations and cofibrant objects by Lemma 5.3.5, and the gluing lemma implies the statement about pushouts of weak equivalences. In the last statement, $j$ is in the set $I$ of generating cofibrations or the set $J$ of generating acyclic cofibrations in the relevant category of (based or unbased) spaces, as specified in Definition 4.5.10. By passage to coproducts, pushouts, sequential colimits, and retracts, (iv) implies that $i \square j$ is a cofibration if $i: X \longrightarrow Y$ is a cofibration in $\mathscr{C}$ and $j: K \longrightarrow L$ is a $q$-cofibration of spaces (or $G$-spaces) and is acyclic if either $i$ or $j$ is so, which means that the model structure is topological.

REMARK 5.5.2. We emphasize the fundamental difference between the acyclicity conditions stated in Theorem 4.5.6 and in Theorem 5.5.1. In the applications of the former, it is the verification of the acyclicity of $J$-cell complexes that is problemmatic, but in the latter our axiomatization has built in that verification.

REMARK 5.5.3. The conclusions of Theorem 5.5.1 still hold if in (iii) we only require $c y l$-cofibrations, rather than $\overline{c y l}$ cofibrations. The stronger assumption holds in all examples we know, and it is needed to implement our philosophy comparing $h$-type and $q$-type model structures via a Quillen adjunction. Note, however, that the theorem holds whether or not the homotopy equivalences, $c \bar{y} l$-cofibrations, and cyl-fibrations actually specify an $h$-type model structure.

Definition 5.5.4. A compactly generated model structure on $\mathscr{C}$ is said to be well-grounded if it is right proper and satisfies all of the hypotheses of the preceding theorem. It follows that $\mathscr{C}$ is proper and topological or, equivariantly, $G$-topological.

\subsection{Properties of well-grounded model categories}

Assume that $\mathscr{C}$ is a well-grounded model category throughout this section. To derive properties of its homotopy category Ho $\mathscr{C}$, we must sort out the relationship between homotopies defined in terms of cylinders and homotopies in the model 
theoretic sense, which we call "model homotopies". We adopt the following slightly non-standard definition of $[56,4.2]$.

Definition 5.6.1. A cylinder object for $X$ is an object $Y$ together with maps $i_{0}: X \longrightarrow Y, i_{1}: X \longrightarrow Y$ and $p: Y \longrightarrow X$ such that $p i_{0}=$ id $=p i_{1}$ and $p$ is a weak equivalence; $Y$ is a good cylinder object if the map $i: X \amalg X \longrightarrow Y$ given by $i_{0}$ and $i_{1}$ is a cofibration; $Y$ is a very good cylinder object if, further, $p$ is a fibration.

Of course, the cylinder objects $\mathrm{Cyl}(X)$ in $\mathscr{C}$ have maps $i_{0}, i_{1}: X \longrightarrow \mathrm{Cyl}(X)$ and $p: \operatorname{Cyl}(X) \longrightarrow X$. Since tensors with spaces preserve homotopies in the space variable, $i_{0}$ (or $i_{1}$ ) and $p$ are inverse homotopy equivalences. Definition 5.4.1(i) ensures that $p$ is therefore a weak equivalence. Thus $\mathrm{Cyl}(X)$ is a model theoretic cylinder object in $\mathscr{C}$. It need not be a good cylinder object. As pointed out in [56, p. 90], this already fails for spaces, where the inclusion $X \amalg X \longrightarrow X \times I$ need not be a $q$-cofibration unless $X$ is $q$-cofibrant. Note however that $i: X \amalg X \longrightarrow \mathrm{Cyl}(X)$ is a $\overline{C y l}$-cofibration for any $X$, by Theorem 4.3.2.

REMARK 5.6.2. The standard definition of cylinder objects in $[73,75,139]$ requires $i$ to be a cofibration. An alternative definition of "good cylinders" that includes all standard cylinders in the category of spaces is given in [144].

We record the following observations.

Lemma 5.6.3. Consider maps $f, g: X \longrightarrow Y$ in $\mathscr{C}$.

(i) If $f$ is homotopic to $g$, then $f$ is left model homotopic to $g$.

(ii) If $X$ is cofibrant, then $C y l(X)$ is a good cylinder object.

(iii) If $X$ is cofibrant and $Y$ is fibrant, then $f$ is homotopic to $g$ if and only if $f$ is left and right model homotopic to $\mathrm{g}$.

Proof. Part (i) is $[56,4.6]$, part (ii) follows from Definition 5.3.2(iii), and part (iii) follows from $[56,4.23]$.

Let $[X, Y]$ denote the set of morphisms $X \longrightarrow Y$ in $\operatorname{HoC}$ and let $\pi(X, Y)$ denote the set of homotopy classes of maps $X \longrightarrow Y$. Recall the following fact, which holds in any model category [75, I.2.10(ii)].

Lemma 5.6.4. The natural map $\pi(X, Y) \longrightarrow[X, Y]$ is a bijection if $X$ is cofibrant and $Y$ is fibrant.

We use this to obtain homotopy category conclusions from elementary classical arguments with homotopy classes of maps. In all of the following results, we transport conclusions obtained by standard arguments with morphism sets $\pi(X, Y)$ in the classical homotopy category $h \mathscr{C}$ to conclusions in the homotopy category HoC by assuming that $Y$ is fibrant and applying cofibrant approximation to the variable $X$. The essential point is to check that cofibrant approximation preserves the constructions we consider. The well-groundedness of $\mathscr{C}$ gives exactly what is needed for these verifications. We assume that $\mathscr{C}$ is based in the rest of the section.

Lemma 5.6.5 (Cofiber sequence lemma). Consider the cofiber sequence

$$
X \longrightarrow Y \longrightarrow C f \longrightarrow \Sigma X \longrightarrow \Sigma Y \longrightarrow \Sigma C f \longrightarrow \Sigma^{2} X \longrightarrow \cdots
$$

of a map $f: X \longrightarrow Y$ between well-grounded objects. For any object $Z$, the induced sequence

$$
\cdots \longrightarrow\left[\Sigma^{n+1} X, Z\right] \longrightarrow\left[\Sigma^{n} C f, Z\right] \longrightarrow\left[\Sigma^{n} Y, Z\right] \longrightarrow\left[\Sigma^{n} X, Z\right] \longrightarrow \cdots \longrightarrow[X, Z]
$$


of pointed sets (groups left of $[\Sigma X, Z]$, Abelian groups left of $\left[\Sigma^{2} X, Z\right]$ ) is exact.

Proof. As usual, giving $I$ the basepoint 1 , we define

$$
C X=X \wedge I, \quad \Sigma X=X \wedge S^{1}, \quad \text { and } \quad C f=Y \cup_{f} C X .
$$

If $X$ is cofibrant, then $X$ is well-grounded and $X \longrightarrow C X$ is a cofibration and therefore a bicofibration. If $X$ and $Y$ are cofibrant, then so is $C f$, as one sees by solving the relevant lifting problem by first using that $Y$ is cofibrant, then using that $X \longrightarrow C X$ is a cofibration, and finally using that $C f$ is a pushout. Thus, taking $Z$ to be fibrant, the conclusion follows in this case from the sequence of homotopy classes of maps

$$
\cdots \longrightarrow \pi(\Sigma X, Z) \longrightarrow \pi(C f, Z) \longrightarrow \pi(Y, Z) \longrightarrow \pi(X, Z)
$$

which is proven to be exact in the same way as on the space level. If $X$ and $Y$ are not cofibrant, let $Q f: Q X \longrightarrow Q Y$ be a cofibrant approximation to $f$. The gluing lemma applies to give that the canonical map $C Q f \longrightarrow C f$ is a weak equivalence. Therefore the conclusion follows in general from the special case of cofibrant objects.

WARNING 5.6.6. While the proof just given is very simple, it hides substantial subtleties. It is crucial that cofibrant objects $X$ be well-grounded, so that the cyl-cofibration $X \longrightarrow C X$ is a bicofibration and the gluing lemma applies.

Of course, the group structures are defined just as classically. The pinch maps

$$
S^{1} \cong I /\{0,1\} \longrightarrow I /\left\{0, \frac{1}{2}, 1\right\} \cong S^{1} \vee S^{1} \quad \text { and } \quad I \longrightarrow I /\left\{\frac{1}{2}, 1\right\} \cong I \vee S^{1}
$$

induce pinch maps

$$
\Sigma X \longrightarrow \Sigma X \vee \Sigma X \quad \text { and } \quad C f \longrightarrow C f \vee \Sigma X
$$

that give $\Sigma X$ the structure of a cogroup object in HoC and $C f$ a coaction by $\Sigma X ; \Sigma^{2} X$ is an abelian cogroup object for the same reason that higher homotopy groups are abelian. Therefore $[\Sigma X, Z]$ is a group, $[C f, Z]$ is a $[\Sigma X, Z]$-set, and $[\Sigma X, Z] \longrightarrow[C f, Z]$ is a $[\Sigma X, Z]$-map.

Lemma 5.6.7 (Wedge lemma). For well-grounded objects $X_{i}$ and any object $Z$ in $\mathscr{C},\left[\amalg X_{i}, Z\right] \cong \Pi\left[X_{i}, Z\right]$.

PROOF. Since a coproduct of weak equivalences between well-grounded objects is a weak equivalence, we see that a coproduct of cofibrant approximations of wellgrounded objects is a cofibrant approximation.

Similarly, we can develop the elementary theory of classical homotopy colimits. It would take us too far afield to go into full details of what should be standard arguments, but we give a sketch since we cannot find our preferred development in the literature.

DeFinition 5.6.8. The classical homotopy pushout, or double mapping cylinder, of a diagram

$$
X \stackrel{f}{\longleftarrow} W \stackrel{g}{\longrightarrow} Y
$$

is the ordinary pushout $M(f, g)$ of the diagram

$$
\operatorname{Cyl}(W) \stackrel{i}{<} W \amalg W \stackrel{f \amalg g}{\longrightarrow} X \amalg Y .
$$


It fits into a cofiber sequence

$$
X \amalg Y \longrightarrow M(f, g) \longrightarrow \Sigma W .
$$

LEMma 5.6.9 (Double mapping cylinder lemma). For maps $f: W \longrightarrow X$ and $g: W \longrightarrow Y$ between well-grounded objects and any object $Z$, there is a natural surjection from $[M(f, g), Z]$ to the pullback of the diagram

$$
[X, Z] \stackrel{f^{*}}{\longrightarrow}[W, Z]<\stackrel{g^{*}}{<}[Y, Z] .
$$

The kernel is the set of orbits of the right action of $[\Sigma X, Z] \times[\Sigma Y, Z]$ on $[\Sigma W, Z]$ specified by

$$
w(x, y)=(\Sigma f)^{*}(x)^{-1} w(\Sigma g)^{*}(y) .
$$

PROOF. Inspection of the cofiber sequence in Definition 5.6.8 gives the analogous conclusion in the classical homotopy category $h \mathscr{C}$. Applying cofibrant approximation and the gluing lemma, Definition 5.4.1(iii), we obtain a weak equivalence

$$
M(Q f, Q g) \longrightarrow M(f, g) .
$$

Since $M(Q f, Q g)$ is cofibrant, this is a cofibrant approximation and the stated conclusion follows directly.

DEFINITION 5.6.10. The classical homotopy coequalizer $C(f, g)$, or mapping torus, of a pair of parallel maps $f, g: X \longrightarrow Y$ is the homotopy pushout of the sum $f+g: X \amalg X \longrightarrow Y$ (which restricts to $f$ and $g$ on the two copies of $X$ ) and the codiagonal $\nabla: X \amalg X \longrightarrow X$.

Algebraic inspection from Lemma 5.6.9 gives the following calculation.

LEMma 5.6.11 (Mapping torus lemma). For maps $f, g: X \longrightarrow Y$ between wellgrounded objects and any object $Z$, there is a surjection from $[C(f, g), Z]$ to the equalizer of the maps $f^{*}$ and $g^{*}$ from $[Y, Z]$ to $[X, Z]$. Its kernel is isomorphic to the set of orbits of $[\Sigma X, Z]$ under the right action of $[\Sigma Y, Z]$ specified by

$$
x y=(\Sigma f)^{*}(y)^{-1} x(\Sigma g)^{*}(y) .
$$

Definition 5.6.12. The classical homotopy colimit or telescope Tel $X_{n}$ of maps $f_{n}: X_{n} \longrightarrow X_{n+1}$ is the homotopy coequalizer of the identity map and the coproduct of the $f_{n}$, both being self maps of the coproduct of the $X_{n}$.

We shall often use the alternative notation hocolim $X_{n}$ for Tel $X_{n}$. Algebraic inspection from Lemma 5.6.9 gives a $\mathrm{lim}^{1}$ exact sequence.

Lemma 5.6.13 (Telescope lemma). For a sequence of maps $f_{n}: X_{n} \longrightarrow X_{n+1}$ between well-grounded objects and any object $Y$, there is an exact sequence of pointed sets

$$
* \longrightarrow \lim ^{1}\left[\Sigma X_{n}, Y\right] \longrightarrow\left[\operatorname{Tel} X_{n}, Y\right] \longrightarrow \lim \left[X_{n}, Y\right] \longrightarrow * \text {. }
$$

LEMMA 5.6 .14 ( $\operatorname{Lim}^{1}$ lemma). Let $X$ be the colimit of a sequence of cylcofibrations $i_{n}: X_{n} \longrightarrow X_{n+1}$ between well-grounded objects. Then there is a natural weak equivalence Tel $X_{n} \longrightarrow X$ and a resulting lim $^{1}$ exact sequence for the calculation of $[X, Y]$. 
Proof. By a standard direct comparison, the telescope Tel $X_{n}$ is isomorphic to colim $T_{n}$, where the $T_{n}$, together with a ladder of weak equivalences $j_{n}: X_{n} \longrightarrow T_{n}$ and $r_{n}: T_{n} \longrightarrow X_{n}$, are constructed inductively by setting $T_{0}=X_{0}$ and letting $j_{n+1}$ and $r_{n+1}$ be the maps of pushouts induced by the following diagram.

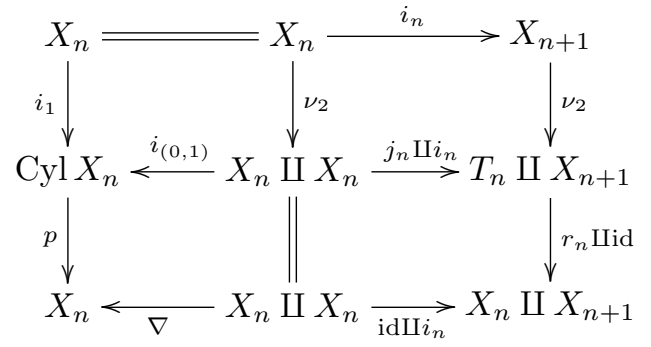

Since $j_{n+1}$ is a pushout of the bicofibration $i_{1}: X_{n} \longrightarrow \operatorname{Cyl}\left(X_{n}\right)$, the gluing and ladder lemmas, Definition 5.4.1(iii) and (iv), apply to show that the induced map Tel $X_{n} \longrightarrow \operatorname{colim} X_{n}=X$ is a weak equivalence.

REMARK 5.6.15. Let $\mathscr{C}$ be an arbitrary pointed model category with (for simplicity) a functorial cylinder construction Cyl. If $X$ is cofibrant, let $\Sigma X$ denote the quotient $\operatorname{Cyl}(X) /(X \vee X)$. Quillen [139] constructed a natural cogroup structure on $\Sigma X$ in Ho $\mathscr{C}$. For a cofibration $X \longrightarrow Y$ between cofibrant objects, he also constructed a natural coaction of $\Sigma X$ on the quotient $Y / X$. One can then define cofiber sequences in Ho $\mathscr{C}$ just as in the homotopy category of a topological model category, and one can define fiber sequences dually.

The cofiber sequences and fiber sequences each give Ho $\mathscr{C}$ a suitably weakened form of the notion of a triangulation, called a "pretriangulation" $[75,139]$, and they are suitably compatible. If HoC is closed symmetric monoidal one can take this a step further and formulate what it means for the pretriangulation to be compatible with that structure, as was done in [124] for triangulated categories. However, proving the compatibility axioms from this general point of view would at best be exceedingly laborious, if it could be done at all.

These purely model theoretic constructions of the suspension and looping functors $\Sigma$ and $\Omega$ are more closely related to the familiar topological constructions than might appear. The homotopy category of any model category is enriched and bitensored over the homotopy category of spaces (obtained from the $q$-model structure) $[55,75]$, and the suspension and loop functors are given by the (derived) tensor and cotensor with the unit circle. That is, $\Sigma X \simeq X \wedge S^{1}$ and $\Omega X \simeq F\left(S^{1}, X\right)$.

This general point of view is not one that we wish to emphasize. For topological model categories, the structure described in this section is far easier to define and work with directly, as in classical homotopy theory, and we have axiomatized what is required of a model structure in order to allow the use of such standard and elementary classical methods. In our topological context, the homotopy category HoC $\mathscr{C}$ is automatically enriched over $\operatorname{Ho} \mathscr{K}_{*}$ and $(\Sigma, \Omega)$ is a Quillen adjoint pair that descends to an adjoint pair on homotopy categories that agrees with the purely model theoretic adjoint pair just described.

The crucial point for our stable work is that a large part of this structure exists before one constructs the desired model structure. It can therefore be used as a tool for carrying out that construction. This is in fact how stable model categories were constructed in $[61,105,106]$, but there the compatibility between $q$-type and 
$h$-type structures was too evident to require much comment. The key step in our construction of the stable model structure on parametrized spectra in Chapter 12 is to show that cofiber sequences induce long exact sequences on stable homotopy groups. That will allow us to verify that the stable equivalences are suitably wellgrounded, and from there the model axioms follow as in the earlier work just cited. 


\section{CHAPTER 6}

\section{The $q f$-model structure on $\mathscr{K}_{B}$}

\section{Introduction}

In this chapter, we introduce and develop our preferred $q$-type model structure, namely the $q f$-model structure. It is a Quillen equivalent variant of the $q$-model structure that has fewer, and better structured, cofibrations. For clarity of exposition, we work nonequivariantly in this chapter, which is taken from [152].

We begin by comparing the homotopy theory of spaces and the homotopy theory of ex-spaces over $B$, starting with a comparison of the $q$-model structures that we have on both. In the category $\mathscr{K}$ of spaces, we have the familiar situation described in $\S 4.1$. The homotopy category Ho $\mathscr{K}$ that we care about is defined in terms of $q$-equivalences, the intrinsic notion of homotopy is given by the classical cylinders, and, since all spaces are $q$-fibrant, the category Ho $\mathscr{K}$ is equivalent to the classical homotopy category $h \mathscr{K}_{c}$ of $q$-cofibrant spaces (or CW complexes). Since the $q$-cofibrations are $h$-cofibrations, the $q$-model structure and the $h$-model structure on $\mathscr{K}$ mesh smoothly. Indeed, the classical and model theoretic homotopy theory have been used in tandem for so long that this meshing of structures goes without notice. In particular, although cofiber and fiber sequences are defined in terms of the $h$-model structure while the homotopy category is defined in terms of the $q$-model structure, the compatibility seems automatic.

Now consider the category $\mathscr{K}_{B}$. The homotopy category Ho $\mathscr{K}_{B}$ that we care about is defined in terms of $q$-equivalences of total spaces, but we need some justification for making that statement. A map of $q$-fibrant ex-spaces is a $q$-equivalence of total spaces if and only if all of its maps on fibers are $q$-equivalences. This reformulation captures the idea that the homotopical information in parametrized homotopy theory should be encoded on the fibers, and it is such fiberwise $q$-equivalences that we really care about. It is only for $q$-fibrant ex-spaces, or ex-spaces whose projections are at least quasifibrations, that the homotopy groups of total spaces give the "right answer". There are three notions of homotopy in sight, $h, f$, and $f p$. The last of these is the intrinsic one defined in terms of the relevant cylinders in $\mathscr{K}_{B}$, and Ho $\mathscr{K}_{B}$ is equivalent to the classical homotopy category $h \mathscr{K}_{B c f}$ of $q$-cofibrant and $q$-fibrant objects, defined with respect to $f p$-homotopy. It is still true that $q$-cofibrations are $h$-cofibrations. However, it is not true that $q$-cofibrations are $f p$ cofibrations, and it is the latter that are intrinsic to cofiber sequences. The classical and model theoretic homotopy theory no longer mesh.

Succinctly, the problem is that the $q$-model structure is not an example of a well-grounded compactly generated model category. The task that lies before us is to find a model structure which does satisfy the axioms that we set out in $\S 5.5$ and therefore can be used in tandem with the $f p$-structure to do parametrized homotopy theory. Before embarking on this, we point out the limitations of the 
$q$-model structure more explicitly in $\S 6.1$. There are two kinds of problems, those that we are focusing on in our development of the model category theory, and the more intrinsic ones that account for Counterexample 0.0.1 and which cannot be overcome model theoretically.

Ideally, to define the $q f$-model structure, we would like to take the $q f$-cofibrations to be those $q$-cofibrations that are also $f$-cofibrations. However, with that choice, we would not know how to prove the model category axioms. We get closer if we try to take as generating sets of cofibrations and acyclic cofibrations those generators in the $q$-model structure that are $f$-cofibrations, but with that choice we still would not be able to prove the compatibility condition Theorem 5.5.1(ii). However, using this generating set of cofibrations and a subtler choice of a generating set of acyclic cofibrations, we obtain a precise enough homotopical relationship to the $q$-equivalences that we can prove the cited compatibility. The construction of the $q f$-model structure is given in $\S 6.2$, but all proofs are deferred to the following three sections.

\subsection{Some of the dangers in the parametrized world}

We introduce notation for the generating (acyclic) cofibrations for the $q$-model structures on $\mathscr{K} / B$ and $\mathscr{K}_{B}$. These maps are identified in Proposition 5.1.5, starting from the sets $I$ and $J$ in $\mathscr{K}$ specified in Definition 4.5.10. We then make some comments about these maps that help explain the structure of our theory.

Definition 6.1.1. For maps $i: C \longrightarrow D$ and $d: D \longrightarrow B$ of (unbased) spaces, we have the restriction $d \circ i: C \longrightarrow B$ and may view $i$ as a map over $B$. We agree to write $i(d)$ for either the map $i$ viewed as a map over $B$ or the map $i \amalg$ id: $C \amalg B \longrightarrow$ $D \amalg B$ of ex-spaces over $B$ that is obtained by taking the coproduct with $B$ to adjoin a section. In either $\mathscr{K} / B$ or $\mathscr{K}_{B}$, define $I_{B}$ to be the set of all such maps $i(d)$ with $i \in I$, and define $J_{B}$ to be the set of all such maps $j(d)$ with $j \in J$. Observe that in $\mathscr{K}_{B}$, each map in $J_{B}$ is the inclusion of a deformation retract of spaces under, but not over, $B$.

WARNing 6.1.2. We cannot restrict the maps $d$ to be open here. That is one of the reasons we chose $\mathscr{K}_{B}$ over $\mathscr{O}_{*}(B)$ in $\S 1.3$.

WARNING 6.1.3. The maps in $I_{B}$ and $J_{B}$ are clearly not $f$-cofibrations, only $h$-cofibrations. Looking at the NDR-pair characterization of $f$-cofibrations given in Lemma 5.2.4, we see that, with our arbitrary projections $d$, there is in general no way to carry out the required deformation over $B$. Since the maps in $I_{B}$ and $J_{B}$ are maps between well-sectioned spaces, they cannot be $f p$-cofibrations in general, by Proposition 5.2.3(i).

REMARK 6.1.4. Observe that the maps $i$ in $I_{B}$ or $J_{B}$ are closed inclusions in $\mathscr{U}$, so that those maps in $I_{B}$ or $J_{B}$ which are $f$-cofibrations are necessarily $\bar{f}$-cofibrations and therefore both $\overline{f p}$-cofibrations and $\bar{h}$-cofibrations, by Proposition 5.1.9 and Theorem 5.2.8.

Warning 6.1.3 shows that the $q$-model structure is not well-grounded since its generating (acyclic) cofibrations are not $f p$-cofibrations. This may sound like a minor technicality, but that is far from the case. We record an elementary example.

Counterexample 6.1.5. Let $B=I$ and define an ex-map $i: X \longrightarrow Y$ over $I$ by letting $X=\{0\} \amalg I, Y=I \amalg I$, and $i$ be the inclusion. The second copies of $I$ 
give the sections, and the projections are given by the identity map on each copy of $I$. This is a typical generating acyclic $q$-cofibration, and it is not an $f p$-cofibration. Let $Z$ be the pushout of $i$ and $p: X \longrightarrow I$, where the latter is viewed as a map of ex-spaces over $I$. Then $Z$ is the one-point union $I \vee I$ obtained by identifying the points 0 . The section $I \longrightarrow Z$ is not an $f$-cofibration, so that $Z$ is not wellsectioned. The same is true if we replace $Y$ by $Y^{\prime}=\{1 /(n+1) \mid n \in \mathbb{N}\} \amalg I$ and obtain $Z^{\prime}$. The map $Z^{\prime} \longrightarrow C_{I} Z^{\prime}$ of $Z^{\prime}$ into its cone over $I$ is not an $h$-cofibration (and therefore not a $q$-cofibration).

Thus we cannot apply the classical gluing lemma to develop cofiber sequences, as we did in $\$ 5.6$. This and related problems prevent use of the $q$-model structure in a rigorous development of parametrized stable homotopy theory. For example, consider $q$-fibrant approximation. If we have a map $f: X \longrightarrow Y$ with $q$-fibrant approximation $R f: R X \longrightarrow R Y$, there is no reason to believe that $C_{B} R f$ is $q$ equivalent to $R C_{B} f$.

We are about to overcome model-theoretically the problems pointed out in the warnings above. Turning to the intrinsic problems that must hold in any $q$-type model structure, we explain why the base change functor $f^{*}$ and the internal smash product cannot be Quillen left adjoints.

Warning 6.1.6. If $f: A \longrightarrow B$ is a map and $d: D \longrightarrow B$ is a disk over $B$, we have no homotopical control over the pullback $A \times_{B} D \longrightarrow A$ in general.

WARNING 6.1.7. In sharp contrast to the nonparametrized case, the generating sets do not behave well with respect to internal smash products, although they do behave well with respect to external smash products. We have

$$
(D \amalg A) \bar{\wedge}(E \amalg B) \cong(D \times E) \amalg(A \times B) .
$$

If the projections of $D$ and $E$ are $d$ and $e$, then the projection of $D \times E$ is $d \times e$. However, if $A=B$, then

$$
(D \amalg B) \wedge_{B}(E \amalg B) \cong(d \times e)^{-1}(\Delta B) \amalg(A \times B) .
$$

We have no homotopical control over the space $(d \times e)^{-1}(\Delta B)$ in general.

This has the unfortunate consequence that, when we go on to parametrized spectra in Part III, we will not be able to develop a homotopically well-behaved theory of point-set level parametrized ring spectra. However, we will be able to develop a satisfactory point-set level theory of parametrized module spectra over nonparametrized ring spectra.

As a final warning of problems ahead, we observe that the finiteness (or compactness) of our generating cells is no longer present on fibers after fibrant approximation, which throws out some of our intuitions. This observation will imply that finite cell spectra are generally not dualizable in the traditional fiberwise sense. We will develop a quite different duality theory, Costenoble-Waner duality, under which they are dualizable in Chapter 18.

WARNING 6.1.8. No matter what $q$-type model structure one considers, the generating cells are not fibrant. Let $\left(D^{n}, p\right)$ be a disk over $B$ and let $(\tilde{B}, q)$ be the universal cover of $B$. Lift $p$ to a map $\tilde{p}: D^{n} \longrightarrow \tilde{B}$. If $B=K(\pi, 1)$, then $\tilde{p}$ is an equivalence and $(\tilde{B}, q)$ is a fibrant approximation to $\left(D^{n}, p\right)$. For example, if $B=S^{1}$, then $\mathbb{R}$ is a fibrant approximation of any disk over $B$, and the derived 
fiber is $\mathbb{Z}$. We can adjoin a disjoint section to form an ex-space $\left(D^{n}, p\right)_{+}$over $B$, and $(\tilde{B}, q)_{+}$is a fibrant approximation to it in the category of ex-spaces over $B$.

\subsection{The $q f$ model structure on the category $\mathscr{K} / B$}

Rather than start with a model structure on $\mathscr{K}$ to obtain model structures on $\mathscr{K} / B$ and $\mathscr{K}_{B}$, we can start with a model structure on $\mathscr{K} / B$ and then apply Proposition 5.1.4 to obtain a model structure on $\mathscr{K}_{B}$. This gives us the opportunity to restrict the classes of generating (acyclic) cofibrations present in the $q$-model structure on $\mathscr{K} / B$ to ones that are $f$-cofibrations, retaining enough of them that we do not lose homotopical information. This has the effect that the generating (acyclic) cofibrations are $f$-cofibrations between well-grounded spaces over $B$, as is required of a well-grounded model structure. Such maps have closed images, hence are $\bar{f}$-cofibrations, and therefore all of the cofibrations in the resulting model structure on $\mathscr{K} / B$ are $\bar{f}$-cofibrations.

We call the resulting model structure the " $q f$-model structure", where $f$ refers to the fiberwise cofibrations that are used and $q$ refers to the weak equivalences. The latter are the same as in the $q$-model structure, namely the weak equivalences on total spaces, or $q$-equivalences. This model structure restores us to a situation in which the philosophy advertised in $\S 4.1$ applies, with the $q$ and $h$-model structures on spaces replaced by the $q f$ and $f$-model structures on spaces over $B$. Since $f$-cofibrations in $\mathscr{K}_{B}$ are $f p$-cofibrations, by Proposition 5.1 .9 , the philosophy also applies to the $q f$ and $f p$-model structures on $\mathscr{K}_{B}$, or at least on $\mathscr{U}_{B}$ (see Theorem 5.2.8 and Remark 5.2.9).

We need some notations and recollections in order to describe the generating (acyclic) $q f$-cofibrations and the $q f$-fibrations.

Notation 6.2.1. For each $n \geq 1$, embed $\mathbb{R}^{n-1}$ in $\mathbb{R}^{n}=\mathbb{R}^{n-1} \times \mathbb{R}$ by sending $x$ to $(x, 0)$. Let $e_{n}=(0,1) \in \mathbb{R}^{n}$. For $n \geq 0$, define the following subspaces of $\mathbb{R}^{n}$.

$$
\begin{aligned}
\mathbb{R}_{+}^{n} & =\left\{(x, t) \in \mathbb{R}^{n} \mid t \geq 0\right\} & \mathbb{R}_{-}^{n} & =\left\{(x, t) \in \mathbb{R}^{n} \mid t \leq 0\right\} \\
D^{n} & =\left\{\left.(x, t) \in \mathbb{R}^{n}|| x\right|^{2}+t^{2} \leq 1\right\} & S^{n-1} & =\left\{\left.(x, t) \in \mathbb{R}^{n}|| x\right|^{2}+t^{2}=1\right\} \\
S_{+}^{n-1} & =S^{n-1} \cap \mathbb{R}_{+}^{n} & S_{-}^{n-1} & =S^{n-1} \cap \mathbb{R}_{-}^{n}
\end{aligned}
$$

Here $\mathbb{R}^{0}=\{0\}$ and $S^{-1}=\emptyset$. We think of $S^{n} \subset \mathbb{R}^{n+1}$ as having equator $S^{n-1}$, upper hemisphere $S_{+}^{n}$ with north pole $e_{n+1}$ and lower hemisphere $S_{-}^{n}$.

There is a conflict of notation here, but it should not cause confusion since we shall not be adding disjoint basepoints to any spaces in this chapter. We recall a characterization of Serre fibrations.

Proposition 6.2.2. The following conditions on a map $p: E \longrightarrow Y$ in $\mathscr{K}$ are equivalent; $p$ is called a Serre fibration, or $q$-fibration, if they are satisfied.

(i) The map p satisfies the covering homotopy property with respect to disks $D^{n}$; that is, there is a lift in the diagram

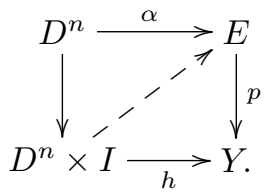


(ii) If $h$ is a homotopy relative to the boundary $S^{n-1}$ in the diagram above, then there is a lift that is a homotopy relative to the boundary.

(iii) The map $p$ has the $R L P$ with respect to the inclusion $S_{+}^{n} \longrightarrow D^{n+1}$ of the upper hemisphere into the boundary $S^{n}$ of $D^{n+1}$; that is, there is a lift in the diagram

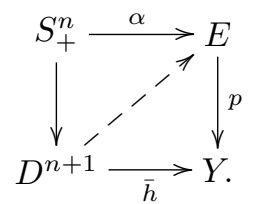

Proof. Serre fibrations $p: E \longrightarrow Y$ are usually characterized by the first condition. Since the pairs $\left(D^{n} \times I, D^{n}\right)$ and $\left(D^{n} \times I, D^{n} \cup\left(S^{n-1} \times I\right)\right)$ are homeomorphic, one easily obtains that the first condition implies the second. Similarly a homeomorphism of the pairs $\left(D^{n+1}, S_{+}^{n}\right)$ and $\left(D^{n} \times I, D^{n}\right)$ gives that the first and third conditions are equivalent. A homotopy $h: D^{n} \times I \longrightarrow Y$ relative to the boundary $S^{n-1}$ factors through the quotient map $D^{n} \times I \longrightarrow D^{n+1}$ that sends $(x, t)$ to $\left(x,(2 t-1) \sqrt{1-|x|^{2}}\right)$. Conversely, any map $\bar{h}: D^{n+1} \longrightarrow Y$ gives rise to a homotopy $h: D^{n} \times I \longrightarrow Y$ relative to the boundary $S^{n-1}$. It follows that the second condition implies the third.

Property (ii) states that Serre fibrations are the maps that satisfy the "disk lifting property" and that is the way we shall think about the $q f$-fibrations. In view of property (iii), we sometimes abuse language by calling a map $h: D^{n+1} \longrightarrow Y$ a disk homotopy. The restriction to the upper hemisphere $S_{+}^{n}$ gives the "initial disk" and the restriction to the lower hemisphere $S_{-}^{n}$ gives the "terminal disk".

Definition 6.2.3. A disk $d: D^{n} \longrightarrow B$ in $\mathscr{K} / B$ is said to be an $f$-disk if $i(d): S^{n-1} \longrightarrow D^{n}$ is an $f$-cofibration. An $f$-disk $d: D^{n+1} \longrightarrow B$ is said to be a relative $f$-disk if the lower hemisphere $S_{-}^{n}$ is also an $f$-disk, so that the restriction $i(d): S^{n-1} \longrightarrow S_{-}^{n}$ is an $f$-cofibration; the upper hemisphere $i(d): S^{n-1} \longrightarrow S_{+}^{n}$ need not be an $f$-cofibration.

Definition 6.2.4. Define $I_{B}^{f}$ to be the set of inclusions $i(d): S^{n-1} \longrightarrow D^{n}$ in $\mathscr{K} / B$, where $d: D^{n} \longrightarrow B$ is an $f$-disk. Define $J_{B}^{f}$ to be the set of inclusions $i(d): S_{+}^{n} \longrightarrow D^{n+1}$ of the upper hemisphere into a relative $f$-disk $d: D^{n+1} \longrightarrow B$; note that these initial disks are not assumed to be $f$-disks. A map in $\mathscr{K} / B$ is said to be

(i) a $q f$-fibration if it has the RLP with respect to $J_{B}^{f}$ and

(ii) a $q f$-cofibration if it has the LLP with respect to all $q$-acyclic $q f$-fibrations, that is, with respect to those $q f$-fibrations that are $q$-equivalences.

Note that $J_{B}^{f}$ consists of relative $I_{B}^{f}$-cell complexes and that a map is a $q f$-fibration if and only if it has the "relative $f$-disk lifting property."

With these definitions in place, we have the following theorem. Recall the definition of a well-grounded model category from Definition 5.5.4 and recall from Propositions 5.3.7 and 5.4.9 that we have ground structures on $\mathscr{K} / B$ and $\mathscr{K}_{B}$ with respect to which the $q$-equivalences are well-grounded. Also recall the definition of a quasifibration from Definition 3.6.1. 
TheOREM 6.2.5. The category $\mathscr{K} / B$ of spaces over $B$ is a well-grounded model category with respect to the $q$-equivalences, $q f$-fibrations and $q f$-cofibrations. The sets $I_{B}^{f}$ and $J_{B}^{f}$ are the generating $q f$-cofibrations and the generating acyclic $q f$ cofibrations. All qf-cofibrations are also $\bar{f}$-cofibrations and all $q f$-fibrations are quasifibrations.

Using Proposition 5.1.4 and Proposition 5.1.5, we obtain the $q f$-model structure on $\mathscr{K}_{B}$. We define a $q f$-fibration in $\mathscr{K}_{B}$ to be a map which is a $q f$-fibration when regarded as a map in $\mathscr{K} / B$, and similarly for $q f$-cofibrations.

TheOREM 6.2.6. The category $\mathscr{K}_{B}$ of ex-spaces over $B$ is a well-grounded model category with respect to the q-equivalences, $q f$-fibrations, and $q f$-cofibrations. The sets $I_{B}^{f}$ and $J_{B}^{f}$ of generating $q f$-cofibrations and generating acyclic $q f$-cofibrations are obtained by adjoining disjoint sections to the corresponding sets of maps in $\mathscr{K} / B$. All $q f$-cofibrations are $\bar{f}$-cofibrations and all $q f$-fibrations are quasifibrations.

Since the $q f$-model structures are well-grounded, they are in particular proper and topological. Furthermore, the $q f$-cofibrant spaces over $B$ are well-grounded and the $q f$-fibrant spaces over $B$ are quasifibrant, but of course not conversely. Since $q f$-cofibrations are $q$-cofibrations, we have an obvious comparison.

THEOREM 6.2.7. The identity functor is a left Quillen equivalence from $\mathscr{K} / B$ with the $q f$-model structure to $\mathscr{K} / B$ with the q-model structure, and similarly for the identity functor on $\mathscr{K}_{B}$.

The following result, which implements the philosophy of $\S 4.1$, is false for the $q$-model structure. It implies that an acyclic $q f$-cofibration is an $f$-equivalence and not just an $h$-equivalence, although it still need not be an $f p$-equivalence in the case of $\mathscr{K}_{B}$.

THEOREM 6.2.8. The identity functor is a left Quillen adjoint from $\mathscr{K} / B$ with the $q f$-model structure to $\mathscr{K} / B$ with the $f$-model structure. Therefore the identity functor is a left Quillen adjoint from $\mathscr{K}_{B}$ with the qf-model structure to $\mathscr{K}_{B}$ with the $f$-model structure.

The one real advantage that the $q$-model structure has over the $q f$-model structure is that naturally occurring $q$-cofibrant ex-spaces are far more common than naturally occurring $q f$-cofibrant ex-spaces. The scarcity of $q f$-cofibrations will force us to introduce a number of variants of the $q f$-model structure in the next chapter, as the following warning illustrates.

WARNing 6.2.9. Let $B$ be a cell complex. Then $S_{B}^{0}=(B \text {,id })_{+}$is $q$-cofibrant in $G \mathscr{K}_{B}$, but it is not $q f$-cofibrant with the $q f$-model structure specified in this chapter. In fact, $(K, p)_{+}$is $q$-cofibrant but not necessarily $q f$-cofibrant for any cell complex $K$.

We state and prove two technical lemmas in $\S 6.3$, prove that $\mathscr{K} / B$ is a compactly generated model category in $\S 6.4$, and prove that the $q f$-fibrations are quasifibrations and the model structure is right proper in $\S 6.5$.

\subsection{Statements and proofs of the thickening lemmas}

We need two technical "thickening lemmas". They encapsulate the idea that no information about homotopy groups is lost if we restrict from the general disks 
and cells used in the $q$-model structure to the $f$-disks and $f$-cells that we use in the $q f$-model structure.

Lemma 6.3.1. Let $\left(S^{m}, q\right)$ be a sphere over $B$. Then there is an h-equivalence $\mu:\left(S^{m}, \bar{q}\right) \longrightarrow\left(S^{m}, q\right)$ in $\mathscr{K} / B$ such that $\left(S^{m}, \bar{q}\right)$ is an $I_{B}^{f}$-cell complex with two cells in each dimension.

Lemma 6.3.2. Let $\left(D^{n}, q\right)$ be a disk over B. Then there is an h-equivalence $\nu:\left(D^{n}, \bar{q}\right) \longrightarrow\left(D^{n}, q\right)$ relative to the upper hemisphere $S_{+}^{n-1}$ such that $\left(D^{n}, \bar{q}\right)$ is a relative $f$-disk.

The rest of the section is devoted to the proofs of these lemmas. The reader may prefer to skip ahead to $\S 6.4$ to see how they are used to prove Theorem 6.2.5.

Proof of Lemma 6.3.1. To define the map $\mu:\left(S^{m}, \bar{q}\right) \longrightarrow\left(S^{m}, q\right)$, we begin by defining some auxiliary maps for each natural number $n \leq m$. They will in fact be continuous families of maps, defined for each $s \in\left[\frac{1}{2}, 1\right]$. The parameter $s$ will show that $\mu$ is an $h$-equivalence.

First we define the map

$$
\phi_{+}^{n}: D^{n} \cap \mathbb{R}_{+}^{n} \longrightarrow A_{s} \cup s \cdot S_{+}^{n-1}
$$

from the upper half of the disk $D^{n}$ to the union of the equatorial annulus

$$
A_{s}=\overline{D^{n-1}-s \cdot D^{n-1}}=\left\{(x, 0) \in \mathbb{R}^{n}: s \leq|x| \leq 1\right\}
$$

and the upper hemisphere

$$
s \cdot S_{+}^{n-1}=\left\{(x, t) \in \mathbb{R}^{n}: t \geq 0 \text { and }|(x, t)|=s\right\}
$$

to be the projection from the south pole $-e_{n}$. Similarly, we define

$$
\phi_{-}^{n}: D^{n} \cap \mathbb{R}_{-}^{n} \longrightarrow A_{s} \cup s \cdot S_{-}^{n-1}
$$

to be the projection from the north pole $e_{n}$. The map $\phi_{+}^{n}$ is drawn schematically in the following picture. Each point in the upper half of the larger disk lies on a unique ray from $-e_{n}$. The map $\phi_{+}^{n}$ sends it to the intersection of that ray with $A_{s} \cup s \cdot S^{n-1}$; two such points of intersection are marked with dots in the picture.

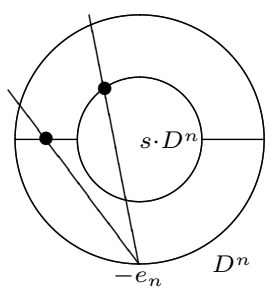

Next we use the maps $\phi_{ \pm}^{n}$ to define a continuous family of maps $f_{s}^{n}: D^{n} \longrightarrow D^{n}$ for $s \in\left[\frac{1}{2}, 1\right]$ by induction on $n$. We let $f_{s}^{0}: D^{0} \longrightarrow D^{0}$ be the unique map and we define $f_{s}^{1}: D^{1} \longrightarrow D^{1}$ by

$$
f_{s}^{1}(t)= \begin{cases}t / s & \text { if }|t| \leq s \\ 1 & \text { if } t \geq s \\ -1 & \text { if } t \leq-s\end{cases}
$$


it maps $[-s, s]$ homeomorphically to $[-1,1]$. We define $f_{s}^{n}: D^{n} \longrightarrow D^{n}$ by

$$
f_{s}^{n}(x, t)= \begin{cases}s^{-1} \cdot(x, t) & \text { if }|(x, t)| \leq s \\ s^{-1} \cdot \phi_{+}^{n}(x, t) & \text { if }|(x, t)| \geq s, t \geq 0 \text { and }\left|\phi_{+}^{n}(x, t)\right|=s \\ f_{s}^{n-1}\left(\phi_{+}^{n}(x, t)\right) & \text { if }|(x, t)| \geq s, t \geq 0 \text { and }\left|\phi_{+}^{n}(x, t)\right| \geq s \\ s^{-1} \cdot \phi_{-}^{n}(x, t) & \text { if }|(x, t)| \geq s, t \leq 0 \text { and }\left|\phi_{-}^{n}(x, t)\right|=s \\ f_{s}^{n-1}\left(\phi_{-}^{n}(x, t)\right) & \text { if }|(x, t)| \geq s, t \leq 0 \text { and }\left|\phi_{-}^{n}(x, t)\right| \geq s\end{cases}
$$

The map $f_{s}^{n}$ is drawn in the following picture. The smaller ball $s \cdot D^{n}$ is mapped homeomorphically to $D^{n}$ by radial expansion from the origin. Next comes the region in the upper half of the larger ball that is inside the cone and outside the smaller ball. Each segment of a ray from the south pole $-e_{n}$ that lies in that region is mapped to a point which is determined by where we mapped the intersection of that ray-segment with the smaller ball (which was radially from the origin to the boundary of $D^{n}$ ). Third is the region in the upper half of the larger ball that is outside the cone. Each segment of a ray from the south pole $-e_{n}$ that lies in that region is first projected to the annulus in the equatorial plane of the two balls; we then apply the previously defined map $f_{s}^{n-1}$ to map the projected points to the equator of $D^{n}$. The lower half of the ball is mapped similarly.

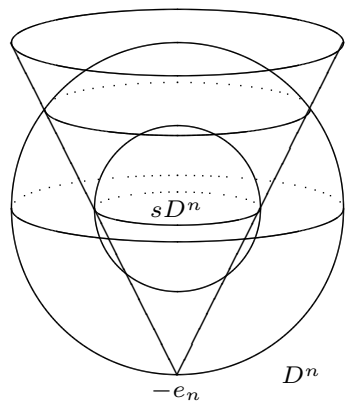

It is clear that $f_{s}^{n}$ gives a homotopy from $f_{1 / 2}^{n}$ to the identity and, given any disk $\left(D^{n}, q\right)$ in $\mathscr{K} / B$, the map $f_{s}^{n}$ induces an $h$-equivalence from the $f$-disk $\left(D^{n}, q \circ f_{1 / 2}^{n}\right)$ to the disk $\left(D^{n}, q\right)$.

Finally we define the required cell structure on the domain of the desired map $\mu:\left(S^{m}, \bar{q}\right) \longrightarrow\left(S^{m}, q\right)$. For each $n \leq m$, the boundary sphere $\left(S^{n}, q \circ f_{1 / 2}^{n+1} \mid S^{n}\right)$ is constructed from two copies of the $f$-disk $\left(D^{n}, q \circ f_{1 / 2}^{n}\right)$ by gluing them along their boundary. The inclusions $\left(D^{n}, q \circ f_{1 / 2}^{n}\right) \longrightarrow\left(S^{n}, q \circ f_{1 / 2}^{n+1} \mid S^{n}\right)$ of the two cells are given by projecting $D^{n}$ to the upper hemisphere from the south pole $-e_{n+1}$ and, similarly, by projecting $D^{n}$ to the lower hemisphere from the north pole $e_{n+1}$. The map

$$
\mu=f_{1 / 2}^{m+1} \mid S^{m}:\left(S^{m}, q \circ f_{1 / 2}^{m+1} \mid S^{m}\right) \longrightarrow\left(S^{m}, q\right) .
$$

is then the required $f$-cell sphere approximation.

Proof of Lemma 6.3.2. Define $\nu_{s}: D^{n} \longrightarrow D^{n}$ for $s \in\left[\frac{1}{2}, 1\right]$ by

$$
\nu_{s}(x, t)= \begin{cases}s^{-1} \cdot(x, t) & \text { if }|(x, t)| \leq s, \\ |(x, t)|^{-1} \cdot(x, t) & \text { if }|(x, t)| \geq s, t \geq 0 \text { and }|x| \geq s, \\ s^{-1} \cdot \phi_{-}^{n+1}(x, t) & \text { if }|(x, t)| \geq s, t \leq 0 \text { and }\left|\phi_{-}^{n+1}(x, t)\right|=s, \\ \left|\phi_{-}^{n+1}(x, t)\right|^{-1} \cdot \phi_{-}^{n+1}(x, t) & \text { if }|(x, t)| \geq s, t \leq 0 \text { and }\left|\phi_{-}^{n+1}(x, t)\right| \geq s,\end{cases}
$$


where $\phi_{-}^{n}$ is the projection as in the previous proof. Then $\nu_{s}$ maps $s \cdot D^{n}$ homeomorphically to $D^{n}$, it is radially constant on the region in the upper half space between the disks $D^{n}$ and $s \cdot D^{n}$ with respect to projection from the origin, and it is radially constant on the region in the lower half space between the two disks with respect to projection from the north pole.

\subsection{The compatibility condition for the $q f$-model structure}

This section is devoted to the proof that $\mathscr{K} / B$ is a compactly generated topological model category. Since our generating sets $I_{B}^{f}$ and $J_{B}^{f}$ certainly satisfy conditions (i) and (iii) of Theorem 5.5.1, it only remains to verify the compatibility condition (ii) and the $\square$-product condition (iv).

For the first, we must show that a map has the RLP with respect to $I_{B}^{f}$ if and only if it is a $q$-equivalence and has the RLP with respect to $J_{B}^{f}$. Thus suppose that $p: E \longrightarrow Y$ has the RLP with respect to $I_{B}^{f}$. Since all maps in $J_{B}^{f}$ are relative $I_{B^{-}}^{f}$ cell complexes, $p$ has the RLP with respect to $J_{B}^{f}$. To show that $\pi_{n}(p)$ is injective, let $\alpha: S^{n} \longrightarrow E$ represent an element in $\pi_{n}(E)$ such that $p \circ \alpha: S^{n} \longrightarrow Y$ is nullhomotopic. Then there is a nullhomotopy $\beta: C S^{n} \longrightarrow Y$ that gives rise to a lifting problem

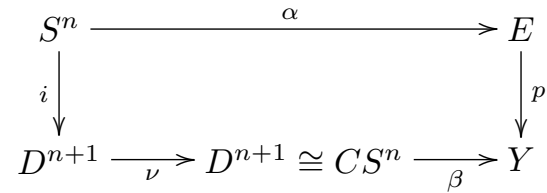

where $\nu: D^{n+1} \longrightarrow D^{n+1}$ is defined by

$$
\nu(x)= \begin{cases}2 x & \text { if }|x| \leq \frac{1}{2} \\ |x|^{-1} \cdot x & \text { if }|x| \geq \frac{1}{2}\end{cases}
$$

Then $i$ is an $f$-disk and we are entitled to a lift, which can be viewed as a nullhomotopy of $\alpha$ after we identify $D^{n+1}$ with $C S^{n}$.

To show that $\pi_{n}(p)$ is surjective, choose a representative $\alpha: S^{n} \longrightarrow Y$ of an element in $\pi_{n}(Y)$. The projection of $Y$ induces a projection $q: S^{n} \longrightarrow B$ and by Lemma 6.3.1 there is an $h$-equivalence $\mu:\left(S^{n}, \bar{q}\right) \longrightarrow\left(S^{n}, q\right)$ such that $\left(S^{n}, \bar{q}\right)$ is an $I_{B}^{f}$-complex with two cells in each dimension. We may therefore assume that the source of $\alpha$ is an $I_{B}^{f}$-cell complex. Inductively, we can then solve the lifting problems for the diagrams

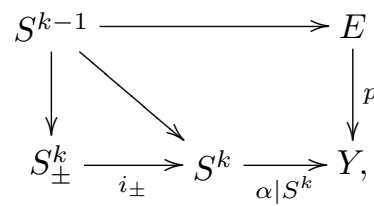

where $S^{k-1} \longrightarrow S^{k}$ is the inclusion of the equator and $i_{ \pm}: S_{ \pm}^{k} \longrightarrow S^{k}$ are the inclusions of the upper and lower hemispheres. We obtain a lift $S^{n} \longrightarrow E$.

Conversely, suppose that $p: E \longrightarrow Y$ is an acyclic $q f$-fibration. We must show that $p$ has the RLP with respect to any cell $i$ in $I_{B}^{f}$. We are therefore faced with a 
lifting problem

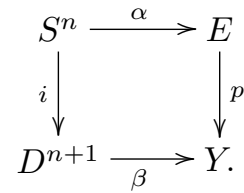

Identifying $D^{n+1}$ with $C S^{n}$ we see that $\beta$ gives a nullhomotopy of $p \circ \alpha$. Since $\pi_{n}(p)$ is injective there is a nullhomotopy $\gamma: C S^{n} \longrightarrow E$ such that $\alpha=\gamma \circ i$, but it may not cover $\beta$. Gluing $\beta$ and $p \circ \gamma$ along $p \circ \alpha$ gives $\delta: S^{n+1} \longrightarrow Y$ such that $\delta \mid S_{+}^{n+1}=\beta$ and $\delta \mid S_{-}^{n+1}=p \circ \gamma$. Surjectivity of $\pi_{n+1}(p)$ gives a map $\Delta: S^{n+1} \longrightarrow E$ and a homotopy $h: S^{n+1} \wedge I_{+} \longrightarrow Y$ from $p \circ \Delta$ to $\delta$. We now construct a diagram

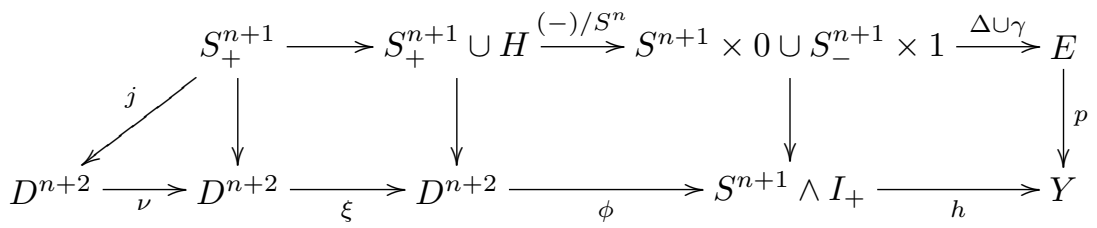

where the downward maps, except $p$, are inclusions. Part of the bottom row of the diagram is drawn schematically below. Let $H$ be the region on $S_{-}^{n+1}$ between the equator $S^{n}$ and the circle through $e_{1}$ and $-e_{n+2}$ with center on the line $\mathbb{R} \cdot\left(e_{1}-e_{n+2}\right)$. Let $\xi$ be a homeomorphism whose restriction to $S_{+}^{n+1}$ maps it homeomorphically to $S_{+}^{n+1} \cup H$. Define $\phi: D^{n+2} \longrightarrow D^{n+2} / S^{n} \cong S^{n+1} \wedge I_{+}$as the composite of the quotient map that identifies the equator $S^{n}$ of $D^{n+2}$ to a point and a homeomorphism that maps the upper hemisphere $S_{+}^{n+1}$ to $S^{n+1} \times 0$, maps $H$ to $S_{-}^{n+1} \times 1$, and is such that $(h \circ \phi \circ \xi) \mid S_{-}^{n+1}=\beta$. The map $\nu$ is defined as above.
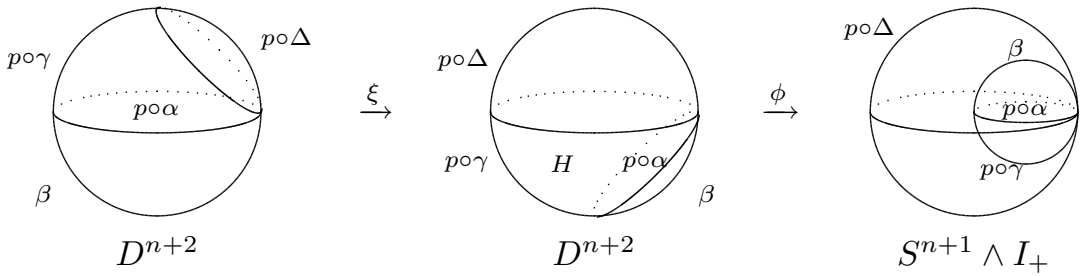

Since the restriction $S^{n} \longrightarrow S_{-}^{n+1} \cong D^{n+1}$ of $j$ agrees with the $f$-cofibration $i$ in our original lifting problem, we see that $j$ is a $J_{B}^{f}$-cell. Since $p$ is a $q f$-fibration we get a lift in the outer trapezoid. Denote its restriction to $S_{-}^{n+1} \cong D^{n+1}$ by $k: D^{n+1} \longrightarrow E$. Then $k$ solves our original lifting problem.

Turning to the $\square$-product condition, Theorem 5.5.1(iv), let $i: X \longrightarrow Y$ be a map in $\mathscr{K} / B$ and $j: K \longrightarrow L$ be a map in $\mathscr{K}$. We must show that if $i$ is in $I_{B}^{f}$ and $j$ is in $I$, then $i \square j$ is in $I_{B}^{f}$. If we were working with the $q$-model structure, this would be evident from the fact that a product of cell-boundary pairs is homeomorphic to a cell-boundary pair. In our situation, by the fiberwise NDR-pair characterization of $f$-cofibrations and a standard argument for product pairs (for example, [121, p. $43]$ ), the boundary inclusion $i \square j$ is an $f$-cofibration since $i$ is an $f$-cofibration and $j$ is an $h$-cofibration. This gives that $i \square j$ is in $I_{B}^{f}$, as required, and it follows that the $\square$-product of a $q f$-cofibration in $\mathscr{K}_{B}$ and a $q$-cofibration in $\mathscr{K}$ is a $q f$-cofibration. Elaborating on this observation, we can show that if $i$ is in $J_{B}^{f}$ and $j$ is in $I$ or if $i$ is in $I_{B}^{f}$ and $j$ is in $J$, then $i \square j$ is in $J_{B}^{f}$. However, it is worth observing that we do 
not need such precision to conclude that the $q f$-model structure is topological. It is simpler and more general to observe that $i \square j$ is a generating acyclic $q$-cofibration if $i \in I_{B}$ and $j \in J$ or $i \in J_{B}$ and $j \in I$, by inspection of cells exactly as in the unparametrized context. Therefore the acyclicity condition required for the $q$-model structure to be topological holds. The acyclicity condition required for the $q f$-model structure to be topological is a special case.

\subsection{The quasifibration and right properness properties}

We have now established the $q f$-model structures on both $\mathscr{K} / B$ and $\mathscr{K}_{B}$. We will derive the right properness of $\mathscr{K} / B$, and therefore of $\mathscr{K}_{B}$, from the fact that every $q f$-fibration is a quasifibration.

Proposition 6.5.1. If $p: E \longrightarrow Y$ is a $q f$-fibration in $\mathscr{K} / B$, then $p$ is a quasifibration. Therefore, for any choice of $e \in E$, there results a long exact sequence of homotopy groups

$$
\cdots \longrightarrow \pi_{n+1}(Y, y) \longrightarrow \pi_{n}\left(E_{y}, e\right) \longrightarrow \pi_{n}(E, e) \longrightarrow \pi_{n}(Y, y) \longrightarrow \cdots \longrightarrow \pi_{0}(Y, y),
$$

where $y=p(e)$ and $E_{y}=p^{-1}(y)$.

Proof. We must prove that $p$ induces an isomorphism

$$
\pi_{n}(p): \pi_{n}\left(E, E_{y}, e\right) \longrightarrow \pi_{n}(Y, y)
$$

for all $n \geq 1$ and verify exactness at $\pi_{0}(E, e)$. We begin with the latter. Let $e^{\prime} \in E$ and suppose that $p\left(e^{\prime}\right)$ is in the component of $y^{\prime}$. Let $\gamma: I \longrightarrow Y$ be a path in $Y$ from $p\left(e^{\prime}\right)$ to $y^{\prime}$ such that $\gamma$ is constant at $p\left(e^{\prime}\right)$ for time $t \leq \frac{1}{2}$. Let $q$ be the projection of $Y$. Then $(I, q \circ \gamma)$ is a relative $f$-disk, and we obtain a lift $\bar{\gamma}: I \longrightarrow E$ such that $\gamma=p \circ \bar{\gamma}$. But then $e^{\prime}$ is in the same component as the endpoint of $\bar{\gamma}$, which lies in $E_{y}$.

Now assume that $n \geq 1$. Recall that an element of $\pi_{n}(X, A, *)$ can be represented by a map of triples $\left(D^{n}, S^{n-1}, S_{+}^{n-1}\right) \longrightarrow(X, A, *)$. We begin by showing surjectivity. Let $\alpha:\left(D^{n}, S^{n-1}\right) \longrightarrow(Y, y)$ represent an element of $\pi_{n}(Y, y)$. We can view $D^{n}$ as a disk over $B$, and Lemma 6.3.2 gives an approximation $\nu: D^{n} \longrightarrow D^{n}$ by a relative $f$-disk. Then we can solve the lifting problem

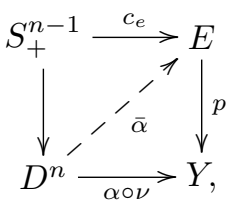

where the top map is the constant map at $e \in E$. A lift is a map of triples $\bar{\alpha}:\left(D^{n}, S^{n-1}, S_{+}^{n-1}\right) \longrightarrow\left(E, E_{y}, e\right)$ such that $p_{*}([\bar{\alpha}])=[\alpha]$.

For injectivity, let $\alpha:\left(D^{n}, S^{n-1}, S_{+}^{n-1}\right) \longrightarrow\left(E, E_{y}, e\right)$ represent an element of $\pi_{n}\left(E, E_{y}, e\right)$ such that $p_{*}([\alpha])=0$. Then there is a homotopy $h: D^{n} \times I \longrightarrow Y$ rel $S^{n-1}$ such that $h \mid D^{n} \times 0=p \circ \alpha$ and $h$ maps the rest of the boundary of $D^{n} \times I$ to $y$. Let $A=D^{n} \times\{0,1\} \cup S_{+}^{n-1} \times I \subset \partial\left(D^{n} \times I\right)$ and define $\beta: A \longrightarrow E$ by setting $\beta(x)=\alpha(x)$ if $x \in D^{n} \times 0$ and $\beta(x)=e$ otherwise. We then have a homeomorphism of pairs $\phi:\left(D^{n} \times I, A\right) \longrightarrow\left(D^{n+1}, S_{+}^{n}\right)$ and an approximation $\nu: D^{n+1} \longrightarrow D^{n+1}$ 
by an $f$-disk by Lemma 6.3 .2 . We can now solve the lifting problem

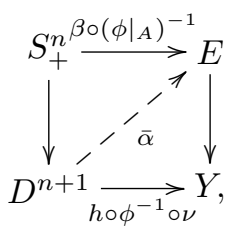

and this shows that $[\alpha]=0$ in $\pi_{n}\left(E, E_{y}, e\right)$.

Corollary 6.5.2. The $q f$-model structure on $\mathscr{K} / B$ is right proper.

Proof. Since $q f$-fibrations are preserved under pullbacks, this is a five lemma comparison of long exact sequences as in Proposition 6.5.1. 


\section{CHAPTER 7}

\section{Equivariant $q f$-type model structures}

\section{Introduction}

We return to the equivariant context in this chapter, letting $G$ be a Lie group throughout. Actually, our definitions of the $q$ and $q f$-model structures work for arbitrary topological groups $G$, but we must restrict to Lie groups to obtain structures that are $G$-topological and behave well with respect to change of groups and smash products. A discussion of details special to the non-compact Lie case is given in $\S 7.1$, which describes the role played by the family $\mathscr{G}$ of compact subgroups of a Lie group $G$, but after that the generalization from compact to non-compact Lie groups requires no extra work. However, we alert the reader that passage to stable equivariant homotopy theory raises new problems in the case of non-compact Lie groups that will not be dealt with in this book; see $\$ 11.6$.

The equivariant $q$-model structure on $G \mathscr{K}_{B}$ is just the evident over and under $q$ model structure. However, the equivariant generalization of the $q f$-model structure is subtle. In fact, the subtlety is already relevant nonequivariantly when we study base change along the projection of a bundle. The problem is that there are so few generating $q f$-cofibrations that many functors that take generating $q$-cofibrations to $q$-cofibrations do not take generating $q f$-cofibrations to $q f$-cofibrations. We show how to get around this in $\S 7.2$. For each such functor that we encounter, we find an enlargement of the obvious sets of (acyclic) generating $q f$-cofibrations on the target of the functor so that it is still a model category, but now the functor does send generating (acyclic) $q f$-cofibrations to (acyclic) $q f$-cofibrations.

The point is that there are many different useful choices of Quillen equivalent $q f$-type model structures, and they can be used in tandem. For all of our choices, the weak equivalences are the $\mathscr{G}$-equivalences and all cofibrations are both $q$-cofibrations and $f$-cofibrations. Given a finite number of adjoint pairs with composable left adjoints such that each is a Quillen adjunction with its own choice of $q f$-type model structure, we can successively expand generating sets in target categories of the left adjoints to arrange that the composite be one of Quillen left adjoints with respect to well chosen $q f$-type model structures.

In $\S 7.2$, we describe the $q f(\mathscr{C})$-model structure associated to a "generating set" $\mathscr{C}$ of $\mathscr{G}$-complexes. Each such model structure is $G$-topological. In $\S 7.3$, we show that external smash products are Quillen adjunctions when $\mathscr{C}$ is a "closed" generating set, as can always be arranged, and we show that all base change adjunctions $\left(f_{!}, f^{*}\right)$ are Quillen adjunctions. We show further that there are generating sets for which $\left(f^{*}, f_{*}\right)$ is a Quillen adjunction when $f$ is a bundle with cellular fibers. In $\S 7.4$, we show similarly that various change of group functors are given by Quillen adjunctions when the generating sets are well chosen. In $\S 7.5$, we show that HoG $\mathscr{K}_{B}$ has the properties required for application of the Brown representability theorem. 
Those adjunctions between our basic functors that are not given by Quillen adjoint pairs in any choice of $q f$-model structure are studied in Chapter 9.

We let $[X, Y]_{G, B}$ denote the set of maps $X \longrightarrow Y$ in HoG $\mathscr{K}_{B}$. The reader wishing to focus on the nonequivariant theory should skip $\S \S 7.1,7.2$, and 7.4 on a first reading; $\S \S 7.3$ and 7.5 work the same way equivariantly and nonequivariantly and, except for some use of the generalized $q f$-model structures of $\$ 7.2$, are largely independent of the other sections.

\subsection{Families and non-compact Lie groups}

There are several sources of problems in the equivariant homotopy theory of general topological groups $G$. Two key examples are that we only know that orbit types $G / K$ are $H$-CW complexes for $H \subset G$ when $G$ is a Lie group and $K$ is a compact subgroup, and we only know that a product of orbits $G / H \times G / K$ is a $G$-CW complex when $G$ is a Lie group and either $H$ or $K$ is a compact subgroup. This motivates us to restrict to Lie groups, for which these conclusions are ensured by Theorem 3.4.2 and Lemma 3.4.3.

The compactness requirements force us to restrict orbit types when we prove properties of our model structures, and the family $\mathscr{G}$ of all compact subgroups of our Lie group $G$ plays an important role. We recall the relevant definitions, which apply to any topological group $G$ and are familiar and important in a variety of contexts. They provide a context that allows us to work with non-compact Lie groups with no more technical work than is required for compact Lie groups.

A family $\mathscr{F}$ in $G$ is a set of subgroups that is closed under passage to subgroups and conjugates. An $\mathscr{F}$-space is a $G$-space all of whose isotropy groups are in $\mathscr{F}$. An $\mathscr{F}$-equivalence is a $G$-map $f$ such that $f^{H}$ is a weak equivalence for all $H \in \mathscr{F}$. If $X$ is an $\mathscr{F}$-space, then the only non-empty fixed point sets $X^{H}$ are those for groups $H \in \mathscr{F}$. In particular, an $\mathscr{F}$-equivalence between $\mathscr{F}$-spaces is the same as a $q$-equivalence. For based $G$-spaces, the definition of an $\mathscr{F}$-space must be altered to require that all isotropy groups except that of the $G$-fixed base point must be in $\mathscr{F}$. The notion of an $\mathscr{F}$-equivalence remains unchanged.

A map in $G \mathscr{K} / B$ or $G \mathscr{K}_{B}$ is an $\mathscr{F}$-equivalence if its map of total $G$-spaces is an $\mathscr{F}$-equivalence. If $B$ is an $\mathscr{F}$-space, then so is any $G$-space $X$ over $B$ and any fiber $X_{b}$. The only orbits that can then appear in our parametrized theory are of the form $G / H$ with $H \in \mathscr{F}$ and the only non-empty fixed point sets $X^{H}$ are those for groups $H \in \mathscr{F}$. In particular, $H$ must be subconjugate to some $G_{b}$. An $\mathscr{F}$-equivalence of $G$-spaces over an $\mathscr{F}$-space $B$ is the same as a $q$-equivalence.

It is well-known that equivariant $q$-type model structures generalize naturally to families. One takes the weak equivalences to be the $\mathscr{F}$-equivalences, and one restricts the orbits $G / H$ that appear as factors in the generating (acyclic) cofibrations to be those such that $H \in \mathscr{F}$. The resulting cell complexes are called $\mathscr{F}$-cell complexes. Restricting tensors from $G$-spaces to $\mathscr{F}$-spaces, we obtain a restriction of the notion of a $G$-topological model category to an $\mathscr{F}$-topological model category that applies here; see Remark 10.3.5.

Proper $G$-spaces are particularly well-behaved $\mathscr{G}$-spaces, and $\mathscr{G}$-cell complexes are proper $G$-spaces. Restricting base $G$-spaces to be proper, or more generally to be $\mathscr{G}$-spaces, has the effect of restricting all relevant orbit types $G / H$ to ones where $H$ is compact. However, this is too restrictive for some purposes. For example, we are interested in developing nonparametrized equivariant homotopy theory for 
non-compact Lie groups $G$. Here $B=*$ is a $G$-space which, in the unbased sense, is not a $\mathscr{G}$-space.

We therefore do not make the blanket assumption that $B$ is a $\mathscr{G}$-space. We give the $q$-model structure in complete generality, in Theorem 7.2.3, but after that we restrict to $\mathscr{G}$-model structures throughout. That is, our weak equivalences will be the $\mathscr{G}$-equivalences. This ensures that, after cofibrant approximation, our total $G$-spaces are $\mathscr{G}$-spaces. This convention enables us to arrange that all of our model categories are $G$-topological. Everything in this chapter applies more generally to the study of parametrized $\mathscr{F}$-homotopy theory for any family $\mathscr{F}$; see Remark 7.2.14.

The reader may prefer to think in terms of either the case when $B=*$ or the case when $B$ is proper. Indeed, in order to resolve the problems intrinsic to the parametrized context that are described in the Prologue, which we do in Chapter 9 , it seems essential that we restrict to proper actions on base spaces. The reason is that Stasheff's Theorem 3.5.2 relating the equivariant homotopy types of fibers and total spaces plays a fundamental role in the solution. Alternatively, the reader may prefer to focus just on compact Lie groups, reading $q$-equivalence instead of $\mathscr{G}$-equivalence and $G$-space instead of $\mathscr{G}$-space throughout.

\subsection{The equivariant $q$ and $q f$-model structures}

Recall from Definition 4.5.10 that the sets $I$ and $J$ of generating cofibrations and generating acyclic cofibrations of $G$-spaces are defined as the sets of all maps of the form $G / H \times i$, where $i$ is in the corresponding set $I$ or $J$ of maps of spaces.

Definition 7.2.1. Starting from the sets $I$ and $J$ of maps of $G$-spaces, define sets $I_{B}$ and $J_{B}$ of maps of ex- $G$-spaces over $B$ in exactly the same way that their nonequivariant counterparts were defined in terms of the sets $I$ and $J$ of maps of spaces in Definition 6.1.1. Note that if $B$ is a $\mathscr{G}$-space, then only orbits $G / H$ with $H$ compact appear in the sets $I_{B}$ and $J_{B}$.

Taking $Y=B$ in the usual composite adjunction

$$
G \mathscr{K}(G / H \times T, Y) \cong H \mathscr{K}(T, Y) \cong \mathscr{K}\left(T, Y^{H}\right)
$$

for non-equivariant spaces $T$ and $G$-spaces $Y$, we can translate back and forth between equivariant homotopy groups and cells for $G$-spaces over $B$ on the one hand and nonequivariant homotopy groups and cells for spaces over $B^{H}$ on the other. Maps in each of the equivariant sets specified in Definition 7.2.1 correspond by adjunction to maps in the nonequivariant set with the same name. Systematically using this translation, it is easy to use Theorem 4.5.6 to generalize the $q$-model structures on $\mathscr{K} / B$ and $\mathscr{K}_{B}$ to corresponding model structures on $G \mathscr{K} / B$ and $G \mathscr{K}_{B}$. We obtain the following theorem.

TheOREm 7.2 .3 ( $q$-model structure). The categories $G \mathscr{K} / B$ and $G \mathscr{K}_{B}$ are compactly generated proper $\mathscr{G}$-topological model categories whose q-equivalences, $q$ fibrations, and $q$-cofibrations are the maps whose underlying maps of total $G$-spaces are q-equivalences, q-fibrations, and q-cofibrations. The sets $I_{B}$ and $J_{B}$ are the generating $q$-cofibrations and generating acyclic q-cofibrations, and all $q$-cofibrations are $\bar{h}$-cofibrations. If $B$ is a $\mathscr{G}$-space, then the model structure is $G$-topological.

To show that the $q$-model structures are $\mathscr{G}$-topological, and $G$-topological if $B$ is a $\mathscr{G}$-space, we must inspect the maps $i \square j$ in $G \mathscr{K} / B$, where $i$ is a generating 
$q$-cofibration in $G \mathscr{K} / B$ and $j$ is a generating cofibration in $G \mathscr{K}$. They can be written in the form

$$
i \square j: G / H \times G / K \times \partial\left(D^{m} \times D^{n}\right) \longrightarrow G / H \times G / K \times D^{m} \times D^{n},
$$

where $i \square j$ is the product of the identity map of $G / H \times G / K$ with the inclusion of the boundary of $D^{m} \times D^{n}$. By Lemma 3.4.3, $G / H \times G / K$ is a proper $G$-space if $H$ or $K$ is compact. Since we are assuming that $G$ is a Lie group, we can then triangulate $G / H \times G / K$ as a $\mathscr{G}$-CW complex and use the triangulation to write $i \square j$ as a relative $I_{B}$-cell complex. The case when either $i$ or $j$ is acyclic works in the same way. As explained in Warning 6.1.7, there is no problem with projection maps in this external context. Moreover, if $i$ and $j$ are $f$-cofibrations, then so is $i \square j$, as we see from the fiberwise NDR pair characterization.

One might be tempted to generalize the $q f$-model structure to the equivariant context in exactly the same way as we just did for the $q$-model structure. This certainly works to give a model structure. However, there is no reason to think that it is either $G$ or $\mathscr{G}$-topological. The problem is that we need $i \square j$ above to be a $q f$-cofibration when $i$ is a generating $q f$-cofibration, and triangulations into $f$-cells are hard to come by. Therefore the $G$-CW structure on $G / H \times G / K$ will rarely produce a relative $I_{B}^{f}$-cell complex. This means that we must be careful when selecting the generating (acyclic) $q f$-cofibrations if we want the resulting model structure to be $G$-topological. We will build the solution into our definition of $q f$-type model structures, but we need a few preliminaries.

We shall make repeated use of the adjunction

$$
G \mathscr{K}(C \times T, Y) \cong \mathscr{K}\left(T, \operatorname{Map}_{G}(C, Y)\right)
$$

for non-equivariant spaces $T$ and $G$-spaces $C$ and $Y$. This is a generalization of (7.2.2). Taking $Y=B$, we note in particular that it gives a correspondence between maps $f: T \longrightarrow T^{\prime}$ over $\operatorname{Map}_{G}(C, B)$ and $G$-maps id $\times f: C \times T \longrightarrow C \times T^{\prime}$ over $B$.

Lemma 7.2.5. For a $\mathscr{G}$-cell complex $C$, the functor $\operatorname{Map}_{G}(C,-): G \mathscr{K} \longrightarrow \mathscr{K}$ preserves q-equivalences.

Proof. The functor $\operatorname{Map}(C,-)$ is a Quillen right adjoint since the $q$-model structure on $G \mathscr{K}$ is $\mathscr{G}$-topological. The $G$-fixed point functor is also a Quillen right adjoint, for example by Proposition 7.4.3 below. The composite $\operatorname{Map}_{G}(C,-)$ therefore preserves $q$-equivalences between $q$-fibrant $G$-spaces. However, every $G$ space is $q$-fibrant.

Observe that Lemma 3.4.3 gives that the collection of $\mathscr{G}$-cell complexes is closed under products with arbitrary orbits $G / H$ of $G$.

Definition 7.2.6. Let $\mathscr{O}_{G}$ denote the set of all orbits $G / H$ of $G$. Any set $\mathscr{C}$ of $\mathscr{G}$-cell complexes in $G \mathscr{K}$ that contains all orbits $G / K$ with $K \in \mathscr{G}$ and is closed under products with arbitrary orbits in $\mathscr{O}_{G}$ is called a generating set. It is a closed generating set if it is closed under finite products. The closure of a generating set $\mathscr{C}$ is the generating set consisting of the finite products of the $\mathscr{G}$-cell complexes in $\mathscr{C}$. We define sets of generating $q f(\mathscr{C})$-cofibrations and acyclic $q f(\mathscr{C})$-cofibrations in $G \mathscr{K} / B$ associated to any generating set $\mathscr{C}$ as follows.

(i) Let $I_{B}^{f}(\mathscr{C})$ consist of the maps

$$
(\mathrm{id} \times i)(d): C \times S^{n-1} \longrightarrow C \times D^{n}
$$


such that $C \in \mathscr{C}, d: C \times D^{n} \longrightarrow B$ is a $G$-map, $i$ is the boundary inclusion, and the corresponding map $\tilde{\imath}$ over $\operatorname{Map}_{G}(C, B)$ is a generating $q f$-cofibration in $\mathscr{K} / \operatorname{Map}_{G}(C, B)$; that is, $\tilde{\imath}$ must be an $f$-cofibration.

(ii) Similarly let $J_{B}^{f}(\mathscr{C})$ consist of the maps

$$
(\mathrm{id} \times i)(d): C \times S_{+}^{n} \longrightarrow C \times D^{n+1}
$$

such that $C \in \mathscr{C}, d: C \times D^{n+1} \longrightarrow B$ is a $G$-map, $i$ is the inclusion, and the corresponding map $\tilde{\imath}$ over $\operatorname{Map}_{G}(C, B)$ is a generating acyclic $q f$-cofibration in $\mathscr{K} / \operatorname{Map}_{G}(C, B)$.

Adjoining disjoint sections to these maps, we obtain the corresponding sets $I_{B}^{f}(\mathscr{C})$ and $J_{B}^{f}(\mathscr{C})$ in $G \mathscr{K}_{B}$.

Fix a generating set $\mathscr{C}$. We define a $q f$-type model structure based on $\mathscr{C}$, called the $q f(\mathscr{C})$-model structure. Its weak equivalences are the $\mathscr{G}$-equivalences, which are the same as the $q$-equivalences when $B$ is a $\mathscr{G}$-space. We define the $q f(\mathscr{C})$-fibrations.

Definition 7.2.7. A map $f$ in $G \mathscr{K} / B$ is a $q f(\mathscr{C})$-fibration if $\operatorname{Map}_{G}(C, f)$ is a $q f$-fibration in $\mathscr{K} / \operatorname{Map}_{G}(C, B)$ for all $C \in \mathscr{C}$. A map in $G \mathscr{K}_{B}$ is a $q f(\mathscr{C})$ fibration if the underlying map in $G \mathscr{K} / B$ is one. In either category, a map $f$ is a $\mathscr{G}$-quasifibration if $f^{H}$ is a quasifibration for $H \in \mathscr{G}$.

TheOREM 7.2.8 ( $q f$-model structure). For any generating set $\mathscr{C}$, the categories $G \mathscr{K} / B$ and $G \mathscr{K}_{B}$ are well-grounded (hence $G$-topological) model categories. The weak equivalences and fibrations are the $\mathscr{G}$-equivalences and the $q f(\mathscr{C})$-fibrations. The sets $I_{B}^{f}(\mathscr{C})$ and $J_{B}^{f}(\mathscr{C})$ are the generating $q f(\mathscr{C})$-cofibrations and the generating acyclic $q f(\mathscr{C})$-cofibrations. All $q f(\mathscr{C})$-cofibrations are both $q$-cofibrations and $\bar{f}$ cofibrations, and all $q f(\mathscr{C})$-fibrations are $\mathscr{G}$-quasifibrations.

Proof. Recall from Proposition 5.4.9 that the $q$-equivalences in $G \mathscr{K} / B$ and $G \mathscr{K}_{B}$ are well-grounded with respect to the ground structure given in Definition 5.3.6 and Proposition 5.3.7. It follows that the $\mathscr{G}$-equivalences are also wellgrounded. It suffices to verify conditions (i)-(iv) of Theorem 5.5.1. The acyclicity condition (i) is inherited from the $q$-model structure.

Consider the compatibility condition (ii). By the adjunction (7.2.4), a map $f$ has the RLP with respect to $I_{B}^{f}(\mathscr{C})$ if and only if $\operatorname{Map}_{G}(C, f)$ has the RLP with respect to $I_{\operatorname{Map}_{G}(C, B)}^{f}$ for all $C \in \mathscr{C}$. By the compatibility condition for the nonequivariant $q f$-model structure, that holds if and only if $\operatorname{Map}_{G}(C, f)$ is a $q$-equivalence and has the LLP with respect to $J_{\operatorname{Map}_{G}(C, B)}^{f}$ for all $C \in \mathscr{C}$. By Lemma 7.2.5, $\operatorname{Map}_{G}(C, f)$ is a $q$-equivalence if $f$ is one. Conversely, if $\operatorname{Map}_{G}(C, f)$ is a $q$-equivalence for all $C \in \mathscr{C}$, then the case $C=G / K$ shows that $f^{K}$ is a $q$ equivalence for every compact $K$ and thus $f$ is a $\mathscr{G}$-equivalence. By the adjunction again, we see that $f$ has the RLP with respect to $I_{B}^{f}(\mathscr{C})$ if and only if $f$ is a $\mathscr{G}$-equivalence which has the RLP with respect to $J_{B}^{f}(\mathscr{C})$.

The fiberwise NDR characterization of $\bar{f}$-cofibrations given in Lemma 5.2.4 shows that $I_{B}^{f}(\mathscr{C})$ and $J_{B}^{f}(\mathscr{C})$ consist of $\bar{f}$-cofibrations, as stipulated in (iii). More precisely, if $(u, h), u: D^{n} \longrightarrow I$ and $h: D^{n} \times I \longrightarrow D^{n}$, represents $\left(D^{n}, S^{n-1}\right)$ as a fiberwise NDR-pair over $\operatorname{Map}_{G}(C, B)$, then the map $v=u \circ \pi: C \times D^{n} \longrightarrow D^{n} \longrightarrow I$ and the homotopy given by the maps id $\times h_{t}$ over $B$ corresponding to the $h_{t}$ represent $\left(C \times D^{n}, C \times S^{n-1}\right)$ as a fiberwise NDR pair over $B$. 
Since $\operatorname{Map}_{G}(G / K, f) \cong f^{K}$ is a nonequivariant $q f$-fibration for any $q f(\mathscr{C})$ fibration $f$, every $q f(\mathscr{C})$-fibration is a $\mathscr{G}$-quasifibration by Proposition 6.5.1. That the model structure is right proper follows as in Corollary 6.5.2.

Finally, we must verify the $\square$-product condition (iv). The relevant maps $i \square j$,

$$
i: C \times S^{m-1} \longrightarrow C \times D^{m} \text { and } j: G / H \times S^{n-1} \longrightarrow G / H \times D^{n},
$$

are of the form

$$
C \times G / H \times k: C \times G / H \times \partial\left(D^{m} \times D^{n}\right) \longrightarrow C \times G / H \times D^{m} \times D^{n},
$$

where $k$ is the boundary inclusion. Now $C \times G / H \in \mathscr{C}$ by the closure property of the generating set, so we don't need to triangulate. The projection of the target factors through the projection of the target $C \times D^{m}$ of $i$. To see that the corresponding map $k$ over $\operatorname{Map}_{G}(C \times G / H, B)$ is an $\bar{f}$-cofibration, let $(u, h)$ represent $\left(D^{m}, S^{m-1}\right)$ as a fiberwise NDR-pair over $\operatorname{Map}_{G}(C, B)$ and let $(v, j)$ represent $\left(D^{n}, S^{n-1}\right)$ as an NDR-pair; we can think of the latter as a fiberwise NDR-pair over $*=\operatorname{Map}_{G}(G / H, *)$. Then the usual product pair representation (for example, $\left[121\right.$, p. 43]) exhibits $k$ as a fiberwise NDR over $\operatorname{Map}_{G}(C, B) \times \operatorname{Map}_{G}(G / H, *)$ and thus, by the factorization of the projection of $i \square j$, also over $\operatorname{Map}_{G}(C \times G / H, B \times$ $*)$.

THEOREM 7.2.9. If $\mathscr{C} \subset \mathscr{C}^{\prime}$ is an inclusion of generating sets, then the identity functor is a left Quillen equivalence from $G \mathscr{K} / B$ with the $q f(\mathscr{C})$-model structure to $G \mathscr{K} / B$ with the $q f\left(\mathscr{C}^{\prime}\right)$-model structure. The identity functor is also a left Quillen equivalence from $G \mathscr{K} / B$ with the $q f(\mathscr{C})$-model structure to $G \mathscr{K} / B$ with the q-model structure. Both statements also hold for the identity functor on $G \mathscr{K}_{B}$.

Proof. The first statement is obvious. For the second, if $\operatorname{id}_{C} \times i$ is a generating $q f(\mathscr{C})$-cofibration, then $C$ is a $\mathscr{G}$-cell complex and we can use the triangulation to write $\operatorname{id}_{C} \times i$ as a relative $I_{B}$-cell complex.

THEOREM 7.2.10. For any $\mathscr{C}$, the identity functor is a left Quillen adjoint from $G \mathscr{K} / B$ with the $q f(\mathscr{C})$-model structure to $G \mathscr{K} / B$ with the $f$-model structure. Therefore, the identity functor is a left Quillen adjoint from $G \mathscr{K}_{B}$ with the $q f(\mathscr{C})$ model structure to $G \mathscr{K}_{B}$ with the $f$-model structure.

REMARK 7.2.11. The smallest generating set $\mathscr{C}$ is the set of all (non-empty) finite products of orbits $G / H$ of $G$ such that at least one of the factors has $H$ compact. Clearly it is a closed generating set. Henceforward, by the $q f$-model structure, we mean the $q f(\mathscr{C})$-model structure associated to this choice of $\mathscr{C}$. In the nonequivariant case, this is the $q f$-model structure of the previous chapter.

REMARK 7.2.12. In the nonparametrized setting, the $\mathscr{G}$-model structure associated to the $q$-model structure and the $q f(\mathscr{C})$-model structures on $G \mathscr{K}=G \mathscr{K} / *$ coincide, and similarly for $G \mathscr{K}_{*}$. This holds since the $f$-cofibrations and $h$-cofibrations over a point coincide and since the $C \in \mathscr{C}$ for any choice of $\mathscr{C}$ are $\mathscr{G}$-cell complexes. Of course, the $q f(\mathscr{C})$-model structures have more generating (acyclic) cofibrations.

REMARK 7.2.13. It might be useful to combine the various $q f(\mathscr{C})$-model structures by taking the union of the $q f(\mathscr{C})$-cofibrations over some suitable collection of generating sets $\mathscr{C}$ and so obtain a "closure" of the $q f$-model structure whose cofibrations are as close as possible to being the intersection of the $q$-cofibrations with the $\bar{f}$-cofibrations. We do not know whether or not that can be done. 
REMARK 7.2.14. As noted in the introduction, we can generalize the $q$ and $q f(\mathscr{C})$-model structures to the context of families $\mathscr{F}$. We generalize the $q$-model structure to the $\mathscr{F}$-model structure by taking the $\mathscr{F}$-equivalences and $\mathscr{F}$-fibrations and by restricting the sets $I_{B}$ and $J_{B}$ to be constructed from orbits $G / H$ with $H \in \mathscr{F}$. The resulting model structure will then be $(\mathscr{F} \cap \mathscr{G})$-topological and $\mathscr{F}$ topological if the base space $B$ is a $\mathscr{G}_{\text {-space. }}$

To generalize the $q f(\mathscr{C})$ model structure, we take the weak equivalences to be the $\mathscr{F} \cap \mathscr{G}$-equivalences and we require the generating set $\mathscr{C}$ to consist of $\mathscr{F} \cap \mathscr{G}$ cell complexes, to contain the orbits $G / K$ for $K \in \mathscr{F} \cap \mathscr{G}$, and to be closed under products with orbits $G / K$ where $K \in \mathscr{F}$. With that modification, everything else above goes through unchanged.

\subsection{External smash product and base change adjunctions}

The following results relate the $q$ and $q f(\mathscr{C})$-model structures to smash products and base change functors and show that various of our adjunctions are given by Quillen adjoint pairs and therefore induce adjunctions on passage to homotopy categories. For uniformity, since we are not assuming that our base $G$-space is proper, we must understand the $q$-model structure to mean the associated $\mathscr{G}$-model structure, although many of the results do apply to the full $q$-model structure. Those results that refer to $q$-equivalences by name work equally well for $\mathscr{G}$-equivalences.

Most of the results in this section and the next apply both to the $q$-model structure and to the $q f(\mathscr{C})$-model structure for any generating set $\mathscr{C}$. We agree to omit the $q$ or $q f(\mathscr{C})$ from the notations in those cases. In the remaining cases, we will have to restrict to well chosen generating sets $\mathscr{C}$. Here the idea is to expand the collection of generating cofibrations in the target category of a left adjoint $F$ to make it large enough that $F$ takes generating (acyclic) cofibrations to (acyclic) cofibrations and is therefore a Quillen left adjoint.

With these conventions, our first result is clear from the fact that our model structures are $G$-topological.

Proposition 7.3.1. For a based $G-C W$ complex $K$, the functor $(-) \wedge_{B} K$ preserves cofibrations and acyclic cofibrations, hence the functor $F_{B}(K,-)$ preserves fibrations and acyclic fibrations. Thus $\left((-) \wedge_{B} K, F_{B}(K,-)\right)$ is a Quillen adjoint pair of endofunctors of $G \mathscr{K}_{B}$.

For the rest of our results, recall from Lemma 5.4.7 that a left adjoint that takes generating acyclic cofibrations to acyclic cofibrations preserves acyclic cofibrations. The following two results apply to the $q f(\mathscr{C})$-model structure for any closed generating set $\mathscr{C}$.

Proposition 7.3.2. If $i: X \longrightarrow Y$ and $j: W \longrightarrow Z$ are cofibrations over base $G$-spaces $A$ and $B$, then

$$
i \square j:(Y \bar{\wedge} W) \cup_{X \bar{\wedge} W}(X \bar{\wedge} Z) \longrightarrow Y \bar{\wedge} Z
$$

is a cofibration over $A \times B$ which is acyclic if either $i$ or $j$ is acyclic.

Proof. It suffices to inspect $i \square j$ for generating (acyclic) cofibrations as was done for the case $A=*$ in the proof of Theorem 7.2.8. For generating cofibrations, the argument there generalizes without change to this setting. The assumption that $\mathscr{C}$ is closed avoids the need for triangulations here. For the acyclicity, it suffices to work in the $q$-model structure, for which the conclusion is both more general 
and easier to prove. There it is easily checked using triangulations of products of $\mathscr{G}$-cell complexes, that if $i$ is a generating cofibration and $j$ is a generating acyclic cofibration, then $i \square j$ is an acyclic cofibration.

Of course, by Warning 6.1.7, the analogue for internal smash products fails. Taking $W=*_{B}$ and changing notations, we obtain the following special case.

Corollary 7.3.3. Let $Y$ be a cofibrant ex-space over B. Then the functor $(-) \bar{\wedge}$ from ex-spaces over $A$ to ex-spaces over $A \times B$ preserves cofibrations and acyclic cofibrations, hence the functor $\bar{F}(Y,-)$ from ex-spaces over $A \times B$ to exspaces over $A$ preserves fibrations and acyclic fibrations. Thus $((-) \bar{\wedge} Y, \bar{F}(Y,-))$ is a Quillen adjoint pair of functors between $G \mathscr{K}_{A}$ and $G \mathscr{K}_{A \times B}$.

The next two results apply to the $q f(\mathscr{C})$-model structures for any $\mathscr{C}$, provided that we use the same generating set $\mathscr{C}$ for both $G \mathscr{K}_{A}$ and $G \mathscr{K}_{B}$.

Proposition 7.3.4. Let $f: A \longrightarrow B$ be a $G$-map. Then the functor $f_{!}$preserves cofibrations and acyclic cofibrations, hence $\left(f_{!}, f^{*}\right)$ is a Quillen adjoint pair. The functor $f$ ! also preserves q-equivalences between well-sectioned ex-spaces. If $f$ is a $q$-fibration, then the functor $f^{*}$ preserves all q-equivalences.

Proof. If $(D, p)$ is a space over $A$, then $f_{!}((D, p) \amalg A)=(D, f \circ p) \amalg B$. Therefore $f$ ! takes generating (acyclic) $q$-cofibrations over $A$ to such maps over $B$.

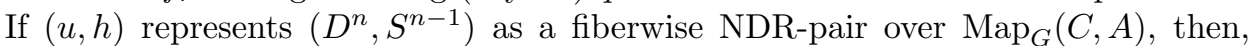
after composing the projection maps with $\operatorname{Map}_{G}(C, A) \longrightarrow \operatorname{Map}_{G}(C, B)$, it also represents $\left(D^{n}, S^{n-1}\right)$ as a fiberwise NDR-pair over $\operatorname{Map}_{G}(C, B)$. It follows that $f_{\text {! }}$ also preserves the generating (acyclic) $q f$-cofibrations. Recall that the wellsectioned ex-spaces are those that are $\bar{f}$-cofibrant and that $f$-cofibrations are $h$ cofibrations. Since $f_{!} X$ is defined by a pushout in $G \mathscr{K}$, the gluing lemma in $G \mathscr{K}$ implies that $f$ ! preserves $q$-equivalences between well-sectioned ex-spaces.

If $f$ is a $q$-fibration and $k: Y \longrightarrow Z$ is a $q$-equivalence of ex-spaces over $B$, consider the diagram

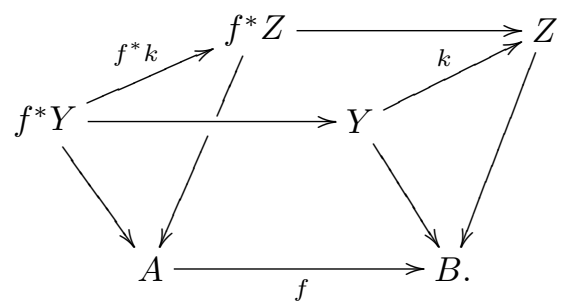

The relation $\left(A \times_{B} Z\right) \times_{Z} Y \cong A \times_{B} Y$ shows that the top square is a pullback, and the pullback $f^{*} Z \longrightarrow Z$ of $f$ is a $q$-fibration. Since the $q$-model structure on the category of $G$-spaces is right proper, it follows that $f^{*} k$ is a $q$-equivalence.

Proposition 7.3.5. If $f: A \longrightarrow B$ is a q-equivalence, then $\left(f_{!}, f^{*}\right)$ is a Quillen equivalence.

Proof. The conclusion holds if and only if the induced adjunction on homotopy categories is an adjoint equivalence $[75,1.3 .3]$, so it suffices to verify the usual defining condition for a Quillen adjunction in either model structure. The condition for the other model structure follows formally. We choose the $q$-model structure. Let $X$ be a $q$-cofibrant ex-space over $A$ and $Y$ be a $q$-fibrant ex-space over $B$, so 
that $A \longrightarrow X$ is a $q$-cofibration and $Y \longrightarrow B$ is a $q$-fibration of $G$-spaces. Since the model structure on the category of $G$-spaces is left and right proper, inspection of the defining diagrams in Definition 2.1.1 shows that the canonical maps $X \longrightarrow f_{!} X$ and $f^{*} Y \longrightarrow Y$ of total spaces are $q$-equivalences. For an ex-map $k: f_{!} X \longrightarrow Y$ with adjoint $\tilde{k}: X \longrightarrow f^{*} Y$, the commutative diagram

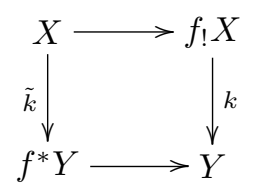

of total spaces then implies that $k$ is a $q$-equivalence if and only if $\tilde{k}$ is a $q$ equivalence.

In view of Counterexample 0.0.1, we can at best expect only a partial and restricted analogue of Proposition 7.3.4 for $\left(f^{*}, f_{*}\right)$. We first give a result for the $q$-model structure and then show how to obtain the analogue for the $q f(\mathscr{C})$-model structures using well chosen generating sets $\mathscr{C}$.

Proposition 7.3.6. Let $f: A \longrightarrow B$ be a $G$-bundle such that $B$ is a $\mathscr{G}$-space and each fiber $A_{b}$ is a $G_{b}$-cell complex. Then $\left(f^{*}, f_{*}\right)$ is a Quillen adjoint pair with respect to the q-model structures. Moreover, if the total space of an ex-G-space $Y$ over $B$ is a $\mathscr{G}$-cell complex, then so is the total space of $f^{*} Y$.

Proof. Since $f$ is a $q$-fibration, $f^{*}$ preserves $q$-equivalences. It therefore suffices to show that $f^{*}$ takes generating cofibrations in $I_{B}$ to relative $I_{A}$-cell complexes. Observe first that if $\phi: G / H \longrightarrow B$ is a $G$-map with $\phi(e H)=b$, then $H \subset G_{b}$ and the pullback $G$-bundle $\phi^{*} f: f^{*}(G / H, \phi) \longrightarrow G / H$ of $f$ along $\phi$ is $G$-homeomorphic to $G \times_{H} A_{b} \longrightarrow G / H$. We can triangulate orbits in a $G_{b}$-cell decomposition of $A_{b}$ as $H$-CW complexes, by Theorem 3.4.2, and so give $A_{b}$ the structure of an $H$-cell complex. Then $G \times_{H} A_{b}$ has an induced structure of a $\mathscr{G}$-cell complex and thus so does $f^{*}(G / H, \phi)$.

For a space $d: E \longrightarrow B$ over $B$ with associated ex-space $E \amalg B$ over $B$, we have $f^{*}(E \amalg B)=f^{*} E \amalg A$. Let $E=G / H \times D^{n}$ and let $i: G / H \longrightarrow G / H \times D^{n}$ be the inclusion $i(g H)=(g H, 0)$. The composite $d \circ i$ is a map $\phi$ as above. Since the identity map on $G / H \times D^{n}$ is homotopic to the composite $i \circ \pi: G / H \times D^{n} \longrightarrow$ $G / H \times D^{n}$, where $\pi$ is the projection, the pullback $G$-bundle $d^{*} f: f^{*}(E, d) \longrightarrow E$ is equivalent to the pullback bundle $(\phi \circ \pi)^{*} f: f^{*}(E, \phi \circ \pi) \longrightarrow E$. But the latter is the product of $\phi^{*} f: f^{*}(G / H, \phi) \longrightarrow G / H$ and the identity map of $D^{n}$ as we see from the following composite of pullbacks

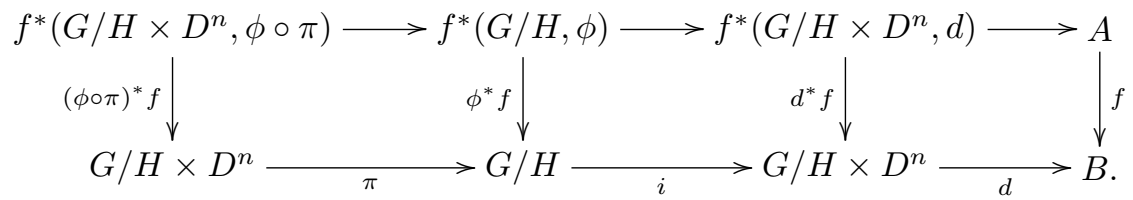

The $\mathscr{G}$-cell structure on $f^{*}(G / H, \phi)$ gives a canonical decomposition of the inclusion $f^{*}(G / H, \phi) \times S^{n-1} \longrightarrow f^{*}(G / H, \phi) \times D^{n}$ as a relative $\mathscr{G}_{\text {-cell complex. The last }}$ statement follows by applying this analysis inductively to the cells of $Y$.

The previous result fails for the $q f$-model structure. In fact, it already fails nonequivariantly for the unique map $r: A \longrightarrow *$, where $A$ is a cell complex. The 
proof breaks down when we try to use a cell decomposition of $A$ (the fiber over $*$ ) to decompose cells $A \times S^{n-1} \longrightarrow A \times D^{n}$ over $A$ as relative $I_{A}^{f}$-cell complexes. Similarly, the equivariant proof above breaks down when we try to use the $G$-cell structure of $f^{*}(G / H, \phi)$ to obtain a relative $I_{A}^{f}$-cell complex. Note, however, that there is no problem when the fibers are homogeneous spaces $G / H$; the nonequivariant analogue is just the trivial case when $f$ is a homeomorphism, but principal bundles and projections $G / H \times B \longrightarrow B$ give interesting equivariant examples. For the general equivariant case, we choose a closed generating set $\mathscr{C}(f)$ that depends on the $G$-bundle $f$ and a given closed generating set $\mathscr{C}$. Using the $q f(\mathscr{C}(f))$-model structures on $G \mathscr{K}_{A}$ and $G \mathscr{K}_{B}$, we then recover the Quillen adjunction.

Construction 7.3.7. Let $f: A \longrightarrow B$ be a $G$-bundle such that $B$ is a $\mathscr{G}$-space and each fiber $A_{b}$ is a $G_{b}$-cell complex and let $\mathscr{C}$ be a closed generating set. We construct the set $\mathscr{C}(f)$ inductively. Let $\mathscr{C}(f)_{0}=\mathscr{C}$ and suppose that we have constructed a set $\mathscr{C}(f)_{n}$ of $\mathscr{G}$-cell complexes in $G \mathscr{K}$ that is closed under both finite products and products with arbitrary orbits $G / H$ of $G$. Let

$$
\mathscr{A}_{n}=\left\{f^{*}(C, \phi) \mid C \in \mathscr{C}(f)_{n} \text { and } \phi \in G \mathscr{K}(C, B)\right\} .
$$

Then let $\mathscr{C}(f)_{n+1}$ consist of all finite products of spaces in $\mathscr{C}(f)_{n} \cup \mathscr{A}_{n}$. Note that $\mathscr{C}(f)_{n+1}$ contains $\mathscr{C}(f)_{n}$ and that the $f^{*}(C, \phi)$ are $\mathscr{G}$-cell complexes by the last statement of Proposition 7.3.6. Finally, let $\mathscr{C}(f)=\bigcup \mathscr{C}(f)_{n}$. Clearly $\mathscr{C}(f) \supset \mathscr{C}$ is a closed generating set that contains $f^{*}(C, \phi)$ for all $C \in \mathscr{C}(f)$ and all $G$-maps $\phi: C \longrightarrow B$.

Proposition 7.3.8. Let $f: A \longrightarrow B$ be a $G$-bundle such that $B$ is a $\mathscr{G}$-space and all fibers $A_{b}$ are $G_{b}$-cell complexes. Then $\left(f^{*}, f_{*}\right)$ is a Quillen adjoint pair with respect to the $q f(\mathscr{C}(f))$-model structures on $G \mathscr{K}_{A}$ and the $q f(\mathscr{C})$-model structure on $G \mathscr{K}_{B}$.

Proof. Reexamining the proof of Proposition 7.3.6, but starting with a map $d: E=C \times D^{n} \longrightarrow B$ where $C \in \mathscr{C}(f)$, we see that

$$
f^{*} E \cong f^{*}(C, \phi) \times D^{n}
$$

where $\phi=d \circ i$. Since $f^{*}(C, \phi)$ is a $\mathscr{G}$-cell complex in $\mathscr{C}(f)$, it remains only to show that $f^{*}(C, \phi) \times S^{n-1} \longrightarrow f^{*}(C, \phi) \times D^{n}$ is an $f$-cofibration. Let $(u, h)$ represent $\left(D^{n}, S^{n-1}\right)$ as a fiberwise NDR-pair over $\operatorname{Map}_{G}(C, B)$. Applying $f^{*}$ to the corresponding maps $h_{t}: C \times D^{n} \longrightarrow C \times D^{n}$ over $B$, we obtain maps $f^{*} h_{t}: f^{*} E \longrightarrow f^{*} E$ over $A$. Under the displayed isomorphism, these maps give a homotopy $f^{*} h: D^{n} \times I \longrightarrow D^{n}$ that, together with $u$, represents $\left(D^{n}, S^{n-1}\right)$ as a fiberwise NDR-pair over $\operatorname{Map}_{G}\left(f^{*}(C, \phi), A\right)$.

The following important corollary should be compared with Warning 6.2.9.

Corollary 7.3.9. If $B$ is a $G$-cell complex, then $\left(r^{*}, r_{*}\right)$ is a Quillen adjoint pair with respect to the $q f(\mathscr{C}(r))$-model structure on $G \mathscr{K}_{B}$ and the q-model structure on $G \mathscr{K}_{*}$.

\subsection{Change of group adjunctions}

We consider change of groups in the $q$ and the $q f$-model structures, starting with the former. The context of the following results is given in $\S 2.3$ and $\S 2.4$. 
Proposition 7.4.1. Let $\theta: G \longrightarrow G^{\prime}$ be a homomorphism of Lie groups. The restriction of action functor

$$
\theta^{*}: G^{\prime} \mathscr{K}_{B} \longrightarrow G \mathscr{K}_{\theta^{*} B}
$$

preserves q-equivalences and $q$-fibrations. If $B$ is a $\mathscr{G}^{\prime}$-space, then it also preserves q-cofibrations.

Proof. Since $\left(\theta^{*} A\right)^{H}=A^{\theta(H)}$ for any subgroup $H$ of $G$ and a map $f$ : $X \longrightarrow Y$ of $G$-spaces is a $q$-equivalence or $q$-fibration if and only if each $f^{H}$ is a $q$-equivalence or $q$-fibration, it is clear that $\theta^{*}$ preserves $q$-equivalences and $q$ fibrations. To study $q$-cofibrations, recall that $\theta$ factors as the composite of a quotient homomorphism, an isomorphism, and an inclusion. If $\theta$ is an inclusion and $H^{\prime}$ is a compact subgroup of $G^{\prime}$, then we can triangulate $G^{\prime} / H^{\prime}$ as a $G$-CW complex by Theorem 3.4.2. If $\theta$ is a quotient homomorphism with kernel $N$ and $H^{\prime}$ is a subgroup of $G^{\prime}$, then $H^{\prime}=H / N$ for a subgroup $H$ of $G$ and $\theta^{*}\left(G^{\prime} / H^{\prime}\right)=G / H$ so that no triangulations are required. Thus in both of these cases, $\theta^{*}$ takes generating $q$-cofibrations to $q$-cofibrations. Since $\theta^{*}$ is also a left adjoint in both cases, it preserves $q$-cofibrations in general.

Remark 7.4.2. We did not require $\theta^{*} B$ to be a $\mathscr{G}$-space in Proposition 7.4.1. However, if the kernel of $\theta$ is compact and $B$ is a $\mathscr{G}^{\prime}$-space, then $\theta^{*} B$ is a $\mathscr{G}$-space. Indeed, $\theta$ is then a proper map and $G_{b}=\theta^{-1}\left(G_{b}^{\prime}\right)$ is compact since $G_{b}^{\prime}$ is compact. The restriction to compact kernels is the price we must pay in order to stay in the context of compact isotropy groups. We might instead consider $G^{\prime}$-spaces $B$ such that the isotropy groups of both $B$ as a $G^{\prime}$-space and $\theta^{*} B$ as a $G$-space are compact, but the assumption on $\theta^{*} B$ would be unnatural. Note however that one of the main reasons for restricting to compact isotropy groups is to obtain $G$-CW structures. If $X$ is a $G^{\prime}$-CW complex where $G^{\prime}=G / N$ is a quotient group of $G$, then $\theta^{*} X$ is a $G$-CW complex with the same cells since the relevant orbits $G^{\prime} / H^{\prime}$ can be identified with $G / H$, where $H^{\prime}=H / N$.

For the $q f$-model structures, and to study adjunctions, it is convenient to consider quotient homomorphisms and inclusions separately. For the former, we consider the adjunctions of Proposition 2.4.1.

Proposition 7.4.3. Let $\epsilon: G \longrightarrow J$ be a quotient homomorphism of $G$ by a normal subgroup $N$. For a $G$-space $B$, consider the functors

$$
(-) / N: G \mathscr{K}_{B} \longrightarrow J \mathscr{K}_{B / N} \text { and }(-)^{N}: G \mathscr{K}_{B} \longrightarrow J \mathscr{K}_{B^{N}} \text {. }
$$

Let $j: B^{N} \longrightarrow B$ be the inclusion and $p: B \longrightarrow B / N$ be the quotient map.

(i) $\left((-) / N, p^{*} \epsilon^{*}\right)$ and $\left(j ! \epsilon^{*},(-)^{N}\right)$ are Quillen adjoint pairs with respect to the q-model structures on both $G \mathscr{K}_{B}$ and $J \mathscr{K}_{B / N}$.

Let $\mathscr{C}_{G}$ and $\mathscr{C}_{J}$ be generating sets of $G$-cell complexes and $J$-cell complexes. Consider $G \mathscr{K}_{B}$ with the $q f\left(\mathscr{C}_{G}\right)$-model structure and $J \mathscr{K}_{B / N}$ and $J \mathscr{K}_{B^{N}}$ with the $q f\left(\mathscr{C}_{J}\right)$-model structure.

(ii) $\left((-) / N, p^{*} \epsilon^{*}\right)$ is a Quillen adjunction if $C / N \in \mathscr{C}_{J}$ for $C \in \mathscr{C}_{G}$.

(iii) $\left(j_{!} \epsilon^{*},(-)^{N}\right)$ is a Quillen adjunction if $\varepsilon^{*} C \in \mathscr{C}_{G}$ for $C \in \mathscr{C}_{J}$.

Proof. Since $\left(j_{!}, j^{*}\right)$ and $\left(p_{!}, p^{*}\right)$ are Quillen adjoint pairs in both the $q$ and the $q f$ contexts, it suffices to consider the case when $N$ acts trivially on $B$, so that $j$ and $p$ are identity maps. Then $\varepsilon^{*}$ is right adjoint to $(-) / N$ and left adjoint 
to $(-)^{N}$. The properties of $\varepsilon^{*}$ in the previous result give the conclusion for the $q$-model structures. The functors $\varepsilon^{*}$ and $(-)^{N}$ preserve $q$-equivalences. Since

$$
\operatorname{Map}_{G}\left(C, \varepsilon^{*} f^{\prime}\right) \cong \operatorname{Map}_{G}\left(C / N, f^{\prime}\right) \text { and } \operatorname{Map}_{J}\left(C^{\prime}, f^{N}\right) \cong \operatorname{Map}_{G}\left(\epsilon^{*} C^{\prime}, f\right)
$$

for a $J$-map $f^{\prime}$ and a $G$-map $f$, the conditions on generating sets in (i) and (ii) ensure that $\varepsilon^{*}$ and $(-)^{N}$ preserve the relevant $q f$-fibrations.

REMARK 7.4.4. In (i), we can take $\mathscr{C}_{J}$ to consist of all finite products of quotients $C / N$ with $C \in \mathscr{C}_{G}$ and orbits $J / H$ to arrange that $\mathscr{C}_{J}$ be closed and contain these $C / N$. In (ii), we can take $\mathscr{C}_{G}$ to consist of all products of pullbacks $\varepsilon^{*} C$ for $C \in \mathscr{C}_{J}$ with finite products of orbits $G / H$. This set will be closed if $\mathscr{C}_{J}$ is closed since $\varepsilon^{*}$ preserves products.

Using Proposition 7.4.3 in conjunction with the additional change of group relations of Propositions 2.4.3 and 2.4.4, we obtain the following compendium of equivalences in homotopy categories.

Proposition 7.4.5. Let $A$ and $B$ be $G$-spaces. Let $j: B^{N} \longrightarrow B$ be the inclusion and $p: B \longrightarrow B / N$ be the quotient map, and let $f: A \longrightarrow B$ be a $G$-map. Then, for ex-G-spaces $X$ over $A$ and $Y$ over $B$,

$$
\begin{array}{ll}
\left(p_{!} Y\right) / N \simeq Y / N, & \left(f_{!} X\right) / N \simeq(f / N)_{!}(X / N), \\
\left(j^{*} Y\right)^{N} \simeq Y^{N}, & \left(f^{*} Y\right)^{N} \simeq\left(f^{N}\right)^{*}\left(Y^{N}\right), \\
\left(p_{*} Y\right)^{N} \simeq Y / N, & \left(f_{!} X\right)^{N} \simeq\left(f^{N}\right) !\left(X^{N}\right) \\
& \left(f^{*} Y\right) / N \simeq(f / N)^{*}(Y / N) .
\end{array}
$$

Here $B$ is assumed to be $N$-free for the last equivalence in the first column, and both $A$ and $B$ are assumed to be $N$-free for the last equivalence in the second column.

Proof. The equivalences displayed in the first line come from isomorphisms between Quillen left adjoints and are therefore clear. Similarly the equivalences in the second line come from isomorphisms between Quillen right adjoints. The first equivalence in the third line (in which we have changed notations from Proposition 2.4.4) comes from an isomorphism between a Quillen right adjoint on the left hand side, by Proposition 7.3.6, and a Quillen left adjoint on the right hand side and therefore also descends directly to an equivalence on homotopy categories. For the second equivalence on the third line, note that $(-)^{N}$ preserves all $q$-equivalences and also preserves well-grounded ex-spaces and that $\left(f^{N}\right)$ ! preserves $q$-equivalences between well-grounded ex-spaces. Letting $Q$ and $R$ denote cofibrant and fibrant replacement functors, as usual, it follows that the maps

$$
\left(R\left(f_{!} X\right)\right)^{N} \longleftarrow\left(f_{!} X\right)^{N} \cong\left(f^{N}\right) !\left(X^{N}\right) \longleftarrow\left(f^{N}\right) !\left(Q\left(X^{N}\right)\right)
$$

are $q$-equivalences on ex-spaces $X$ that are $q f$-fibrant and $q f$-cofibrant. As noted in the proof of Proposition 2.4.3, the point set level isomorphism $\left(f_{!} X\right)^{N} \cong\left(f^{N}\right)_{!}\left(X^{N}\right)$ is only valid for an ex-space $X$ whose section is a closed inclusion. However, if $X$ is $q f$-cofibrant, then it is compactly generated and this holds by Lemma 1.6.2(i). Thus the equivalence holds in general in the homotopy category. For the last equivalence, take $A$ and $B$ to be $N$-free and let $q: A \longrightarrow A / N$ be the quotient map. Then $(-) / N \cong\left(q_{*}(-)\right)^{N}$ on ex- $G$-spaces over $A$ and $(-) / N \cong\left(p_{*}(-)\right)^{N}$ on ex- $G$-spaces over $B$. Since $p$ and $q$ are $J$-bundles satisfying the hypothesis of Proposition 7.3.8 
(compare $\S 3.2$ ), these isomorphisms exhibit both orbit functors $(-) / N$ as composites of Quillen right adjoints. Therefore the point-set level isomorphism

$$
\left(f^{*} Y\right) / N \cong(f / N)^{*}(Y / N)
$$

(which only required $B$ to be $N$-free) is a comparison between Quillen right adjoints and so descends to the claimed equivalence of derived functors.

The result Proposition 2.4.4 describing passage to orbits as a right adjoint also descends to homotopy categories.

Proposition 7.4.6. Let $E$ be an $N$-free $G$-space, let $B=E / N$, and let $p: E \longrightarrow B$ be the quotient map. For $X \in G \mathscr{K}_{E}, X / N \cong\left(p_{*} X\right)^{N}$, and this isomorphism descends to an isomorphism in HoJ $\mathscr{K}_{B}$. Therefore the left adjoint $(-) / N$ of the functor $p^{*} \varepsilon^{*}$ is also its right adjoint on homotopy categories.

Proof. The point set level isomorphism is given in Proposition 2.4.4. Since it gives an isomorphism between a Quillen left adjoint on the left hand side and a composite of Quillen right adjoints on the right hand side, it descends directly to homotopy categories.

The context for the next result is given in Definition 2.3.7 and Proposition 2.3.9.

Proposition 7.4.7. Let $\iota: H \longrightarrow G$ be the inclusion of a subgroup and let $A$ be an $H$-space. The adjoint equivalence $\left(\iota !, \nu^{*} \iota^{*}\right)$ relating $H \mathscr{K}_{A}$ and $G \mathscr{K}_{\iota_{1} A}$ is a Quillen equivalence in the q-model structures and also in the $q f\left(\mathscr{C}_{H}\right)$ and $q f\left(\mathscr{C}_{G}\right)$ model structures for any generating sets $\mathscr{C}_{H}$ and $\mathscr{C}_{G}$ of $H$-cell complexes and $G$-cell complexes such that $\iota_{!} C=G \times{ }_{H} C \in \mathscr{C}_{G}$ for $C \in \mathscr{C}_{H}$. If $A$ is proper and completely regular, then the functor $\iota$ ! is also a Quillen right adjoint with respect to the $q$ and qf-model structures.

Proof. Recall that $\nu: A \longrightarrow \iota^{*} \iota ! A=G \times_{H} A$ is the natural inclusion of $H$ spaces and that $\left(\nu_{!}, \nu^{*}\right)$ is a Quillen adjunction in both the $q$ and $q f$ contexts. The functor $\iota^{*}$ preserves $q$-equivalences and $q$-fibrations. It takes $q f\left(\mathscr{C}_{G}\right)$-fibrations to $q f\left(\mathscr{C}_{H}\right)$-fibrations when $\iota_{!} C \in \mathscr{C}_{G}$ for $C \in \mathscr{C}_{H}$ since

$$
\operatorname{Map}_{H}\left(C, \iota^{*} f\right) \cong \operatorname{Map}_{G}(\iota ! C, f) \text {. }
$$

To show that $\left(\iota !, \nu^{*} \iota^{*}\right)$ is a Quillen equivalence, we may as well check the defining condition in the $q$-model structure. Let $X$ be a $q$-cofibrant ex- $H$-space over $A$ and $Y$ be a $q$-fibrant ex- $G$-space over $\iota ! A$. Consider a $G$-map $f: \iota ! X \longrightarrow Y$. We must show that $f$ is a q-equivalence if and only if its adjoint $H$-map $\tilde{f}: X \longrightarrow \nu^{*} \iota^{*} Y$ is a $q$-equivalence. Since $\iota$ ! preserves acyclic $q$-cofibrations, we can extend $f$ to $f^{\prime}: \iota ! R X \longrightarrow Y$, where $R X$ is a $q$-fibrant approximation. Since $f^{\prime}$ is a $q$-equivalence if and only if $f$ is one, and similarly for their adjoints, we may assume without loss of generality that $X$ is $q$-fibrant. Recall from Proposition 2.3.9 that $\iota$ ! and $\nu^{*} \iota^{*}$ are inverse equivalences of categories and observe that $\nu^{*} \iota^{*} Y$ can be viewed as the restriction, $\left.Y\right|_{A}$, of $Y$ along the inclusion of $H$-spaces $\nu: A \longrightarrow G \times_{H} A$. From that point of view, $\tilde{f}: X \longrightarrow \nu^{*} \iota^{*} Y$ is just the map $X \longrightarrow Y \mid A$ of ex- $H$-spaces over $A$ obtained by restriction of $\iota^{*} f$ along $\nu$.

Now $f$ is a $q$-equivalence if and only if $f$ restricts to a $q$-equivalence $f_{[g, a]}$ on each fiber, meaning that this restriction is a weak equivalence after passage to fixed points under all subgroups of the isotropy group of $[g, a]$. For $a \in A$, the isotropy subgroup $H_{a} \subset H$ of $a$ coincides with the isotropy subgroup $G_{[e, a]} \subset G$ of 
$[e, a] \in G \times_{H} A$. For $g \in G$, the isotropy subgroup of $[g, a]$ is $g H_{a} g^{-1}$. Since the action by $g \in G$ induces a homeomorphism between the fibers over $[e, a]$ and over $[g, a]$, we see that $f$ is a $q$-equivalence if and only if each of the restrictions $f_{[e, a]}$ is a $q$-equivalence. But that holds if and only if $\tilde{f}$ is a $q$-equivalence.

For the last statement, recall the description of $\iota$ in Remark 2.4.5 as the composite $\left(p_{*} \pi^{*} \varepsilon^{*}(-)\right)^{H}$, where $\varepsilon: G \times H \longrightarrow H$ and $\pi: G \times A \longrightarrow A$ are the projections and $p: G \times A \longrightarrow G \times_{H} A$ is the quotient map. Since $G \times A$ is completely regular, $p$ is a bundle with fiber $G / H_{a}$ over $[g, a]$, and $H_{a}$ is compact since $A$ is proper. Therefore, by Propositions 7.3.6 and 7.3.8, $p_{*}$ is a Quillen right adjoint with respect to the $q$ and $q f$-model structures. In view of Proposition 7.4.3, this displays $\iota$ ! as a composite of Quillen right adjoints.

REMARK 7.4.8. We can take $\mathscr{C}_{G}$ to consist of all finite products of the $\iota_{!} C$ with $C \in \mathscr{C}_{H}$ and orbits $G / K$ to arrange that $\mathscr{C}_{G}$ be closed and contain these $\iota ! C$.

We shall prove that $\left(\iota_{!}, \nu^{*} \iota^{*}\right)$ descends to a closed symmetric monoidal equivalence of homotopy categories in Proposition 9.4.9 below. The first statement of Proposition 7.4.7 implies that the description of $\iota^{*}$ in terms of base change that is given in Proposition 2.3.10 descends to homotopy categories.

Corollary 7.4.9. The functor $\iota^{*}: H o G \mathscr{K}_{B} \longrightarrow H o H \mathscr{K}_{\iota^{*} B}$ is the composite

$$
H o G \mathscr{K}_{B} \stackrel{\mu^{*}}{\longrightarrow} H o G \mathscr{K}_{\iota ! \iota^{*} B} \simeq \mathrm{HoH} \mathscr{K}_{\iota^{*} B}
$$

\subsection{Fiber adjunctions and Brown representability}

For a point $b$ in $B$, we combine the special case $\tilde{b}: G / G_{b} \longrightarrow B$ of Proposition 7.3.4 with the special case $\iota: G_{b} \longrightarrow G$ and $A=*$, hence $\nu: * \longrightarrow G / G_{b}$, of Proposition 7.4.7 to obtain the following result concerning passage to fibers. Recall from Example 2.3.12 that the fiber functor $(-)_{b}: G \mathscr{K}_{B} \longrightarrow G_{b} \mathscr{K}_{*}$ is given by $\nu^{*} \iota^{*} \tilde{b}^{*}=b^{*} \iota^{*}$. By conjugation, its left adjoint $(-)^{b}$ therefore agrees with $\tilde{b}_{!} \iota !$

Proposition 7.5.1. For $b \in B$, the pair of functors $\left((-)^{b},(-)_{b}\right)$ relating $G_{b} \mathscr{K}_{*}$ and $G \mathscr{K}_{B}$ is a Quillen adjoint pair.

We use certain objects $X^{b}$ to verify the formal hypotheses of Brown's representability theorem [26] for the category $\mathrm{Ho} G \mathscr{K}_{B}$, or rather for a suitable subcategory. We need a few preliminaries and some discussion of the nature and limitations of the Brown representability theorem.

Definition 7.5.2. Define weak colimits in any category $\mathscr{H}$ by requiring the existence but not the uniqueness property of colimits. When $\mathscr{H}$ has (specified) weak sequential colimits, denoted hocolim $Y_{n}$ or Tel $Y_{n}$, we say that an object $X$ of $\mathscr{H}$ is compact if

$$
\operatorname{colim} \mathscr{H}\left(X, Y_{n}\right) \cong \mathscr{H}\left(X, \operatorname{hocolim} Y_{n}\right)
$$

for any sequence of maps $Y_{n} \longrightarrow Y_{n+1}$ in $\mathscr{H}$.

Of course, homotopy colimits often provide weak colimits, but the two notions are conceptually distinct. If $\mathscr{H}$ has coproducts and weak pushouts, then it has weak coequalizers and weak sequential colimits, as in $§ 5.6$. Definitions 5.6.8, 5.6.10, and 5.6.12 together with Lemmas 5.6.9, 5.6.11, and 5.6.13 give the following result. 
Lemma 7.5.3. Applied to diagrams of cofibrant objects, the classical homotopy pushouts, homotopy coequalizers, and telescopes in a well-grounded model category give weak pushouts, weak coequalizers, and weak sequential colimits in its homotopy category.

WARNING 7.5.4. When verifying weak colimit properties by use of homotopy classes of maps, as in $\S 5.6$, one is studying maps out of homotopy colimits, and it suffices to restrict attention to cofibrant objects. However, when trying to verify compactness similarly, one must study maps into homotopy colimits, and one must therefore apply fibrant approximation to homotopy colimits in order to pass to homotopy classes of maps. This destroys the point-set level control that classical homotopy colimits provide. Said another way, topological intuition relates to the hom sets $\pi(X,-)$ of the classical homotopy category and not the hom sets $[X,-]$ of the relevant homotopy category. For this reason, compactness is a quite subtle notion in the homotopy categories of topological model categories in which not all objects are fibrant.

We also need generating sets of objects and, in unstable homotopy theory, it is useful to distinguish these from detecting sets of objects. Variant forms of the following definition appear in $[76,134]$ and elsewhere. In topological or triangulated categories, it is natural to insist that these classes be closed under suspension, and we shall do so implicitly.

Definition 7.5.5. A set $\mathscr{D}$ of objects in a pointed category $\mathscr{H}$ is a detecting set if $\mathscr{H}(D, X)=*$ for all objects $D \in \mathscr{D}$ implies that $X \cong *$. A set $\mathscr{D}$ is a generating set if a map $f: X \longrightarrow Y$ such that $f_{*}: \mathscr{H}(D, X) \longrightarrow \mathscr{H}(D, Y)$ is a bijection for all $D \in \mathscr{D}$ is an isomorphism.

REMARK 7.5.6. Clearly any generating set is a detecting set. The converse holds in triangulated categories, as we shall see in Lemma 13.1.6. The term "detecting set" is new, but we have followed the literature in defining generating sets, although they might more reasonably be called detecting sets. The reason for the name "generating set" is given by Theorem 13.1.14 below.

The point of the distinction is that Brown's theorem requires the use of a generating set and not just a detecting set of objects, and for that reason it requires a connectedness hypothesis in unstable situations. The following abstract representability theorem was proven by Brown [26, 2.9], although he stated it differently.

Theorem 7.5.7 (Brown). Let $\mathscr{H}$ be a category with coproducts and weak pushouts and therefore weak sequential colimits. Assume that $\mathscr{H}$ has a generating set of compact objects. Let $k: \mathscr{H} \longrightarrow$ Sets be a contravariant functor that takes coproducts to products and weak pushouts to weak pullbacks. Then there is an object $Y \in \mathscr{H}$ and a natural isomorphism $k(X) \cong \mathscr{H}(X, Y)$ for $X \in \mathscr{H}$.

The conditions on $k$ are called the wedge and Mayer-Vietoris axioms. Of course, the representing object $Y$ is unique up to isomorphism. The hypotheses are satisfied in appropriate subcategories of unstable homotopy categories.

Definition 7.5.8. A $G$-space $K$ is said to be $G$-connected if each of its fixed point sets $K^{H}$ is non-empty and path connected. Let HoG $\mathscr{K}_{*}^{c}$ be the full subcategory of $G$-connected $G$-spaces in $\operatorname{Ho} G \mathscr{K}_{*}$. Say that an ex- $G$-space $X$ over $B$ is $G$-connected if, after fibrant approximation, each of its fibers is $G_{b}$-connected. Let $\mathrm{Ho} G \mathscr{K}_{B}^{c}$ be the full subcategory of $G$-connected ex- $G$-spaces in $\mathrm{Ho} G \mathscr{K}_{B}$. 
Model categorically, one has the following general result of Hovey [75, 7.3.1]. We need some language to state it.

Notation 7.5.9. By a standard abuse of language, define the cofiber $Z$ of a cofibration $f: X \longrightarrow Y$ in a pointed model category $\mathscr{C}$ to be the quotient $Y / X$, that is, the pushout of $f$ along the trivial map $X \longrightarrow *$. Observe that if $\mathscr{C}$ is cofibrantly generated, then the cell complexes in $\mathscr{C}$ consisting of a single cell, the 1-cell complexes, are precisely the cofibers of the generating cofibrations.

THEOREM 7.5.10. Let $\mathscr{C}$ be a pointed cofibrantly generated model category. Then the set of all iterated suspensions $\Sigma^{n} Z, n \geq 0$, of 1 -cell complexes $Z$ in $\mathscr{C}$ is a set of detecting objects for $H o \mathscr{C}$.

This specializes to give the first statement in the following familiar example.

LEMmA 7.5.11. In $H o G \mathscr{K}_{*}$, the set of spheres $S_{H}^{n}=G / H_{+} \wedge S^{n}, n \geq 0$, is a detecting set. On the subcategory HoG $\mathscr{K}_{*}^{c}$ of $G$-connected $G$-spaces, the set of such spheres with $n>0$ is a generating set.

We emphasize that, already nonequivariantly, we do not know of a convenient generating set in Ho $\mathscr{K}_{*}$. Naive variants of the sets of spheres given in Lemma 7.5.11 are incorrect, as consideration of based versus unbased homotopy classes of maps makes clear. The notion of a weak equivalence of spaces, as a condition on homotopy groups for all choices of basepoint and thus all components, is more subtle than appears at first sight. Lemma 7.5.11 leads to the appropriate parametrized analogue, but here we can restrict to smaller detecting and generating sets than Theorem 7.5.10 would suggest.

Definition 7.5.12. For $n \geq 0, b \in B$, and $H \subset G_{b}$, let $S_{H}^{n, b}$ be the ex- $G$ space $\left(G_{b} / H_{+} \wedge S^{n}\right)^{b}$ over $B$. Explicitly, $S_{H}^{n, b}=\tilde{b}_{!} S_{H}^{n}=\left(G / H_{+} \wedge S^{n}\right) \vee_{b} B$, where the wedge is taken with respect to the standard basepoint of $G / H_{+} \wedge S^{n}$ and the basepoint $b \in B$. The inclusion of $B$ gives the section, and the projection maps $G / H_{+} \wedge S^{n}$ to the point $b$ and maps $B$ by the identity map. Equivalently, taking $d$ to be the constant map at $b, S_{H}^{n, b}$ is the quotient ex-G-space associated to the generating cofibration $i(d), i: G / H \times S^{n-1} \longrightarrow G / H \times D^{n}$. Therefore, $S_{H}^{n, b}$ is cofibrant in both the $q$ and the $q f$-model structures. Let $\mathscr{D}_{B}$ be the set of all such ex-G-spaces $S_{H}^{n, b}$, and let $\mathscr{D}_{B}^{c}$ be the subset of those $S_{H}^{n, b}$ such that $n>0$.

LEMma 7.5.13. $\mathscr{D}_{B}$ is a detecting set in $H o G \mathscr{K}_{B}$ and $\mathscr{D}_{B}^{c}$ is a generating set in $\mathrm{HoG} \mathscr{K}_{B}^{c}$.

Proof. We need only observe that, when $n>0$, the fiber over $a \in B$ of a fibrant approximation $R S_{H}^{n, b}$ to $S_{H}^{n, b}$ is $G_{a}$-connected, so that $S_{H}^{n, b}$ is $G$-connected and $\mathscr{D}_{B}^{c}$ is contained in $\operatorname{HoG} \mathscr{K}_{B}^{c}$.

LEMMA 7.5.14. Each $X$ in $\mathscr{D}_{B}$ is a compact object.

Proof. Let $X=S_{H}^{n, b}$. Then

$$
[X, Y]_{G, B} \cong\left[G_{b} / H_{+} \wedge S^{n}, Y_{b}\right]_{G_{b}} \cong \pi_{n}\left(Y_{b}^{H}\right) \equiv \pi_{n}^{H}\left(Y_{b}\right)
$$

for any $q f$-fibrant object $Y$. We must insist on the fibrancy since the homotopy groups of fibers must be understood in the derived sense. We must show that

$$
\operatorname{colim}\left[X, Y_{q}\right]_{G, B} \cong\left[X, \operatorname{hocolim} Y_{q}\right]_{G, B}
$$


for any sequence of maps $f_{q}: Y_{q} \longrightarrow Y_{q+1}$ in HoG $\mathscr{S}_{B}$. Since we are working in the homotopy category, we may assume that the $Y_{q}$ are $q f$-fibrant and $q f$-cofibrant. We choose representative maps $f_{q}$ for the given homotopy classes and, since we are studying maps into homotopy colimits, we take our representative for the homotopy colimit to be a fibrant approximation $R$ Tel $Y_{q}$ of the classical telescope of these $f_{q}$. Now our desired isomorphism becomes

$$
\operatorname{colim} \pi_{n}^{H}\left(\left(Y_{q}\right)_{b}\right) \cong \pi_{n}^{H}\left(\left(R \operatorname{Tel} Y_{q}\right)_{b}\right) .
$$

In $\mathrm{Ho} H \mathscr{K}_{*}$, where every object is $q$-fibrant, homotopy groups of telescopes are given as colimits, so that

$$
\operatorname{colim} \pi_{n}^{H}\left(\left(Y_{q}\right)_{b}\right) \cong \pi_{n}^{H}\left(\operatorname{Tel}\left(Y_{q}\right)_{b}\right) .
$$

By direct inspection of the telescope construction, we have

$$
\operatorname{Tel}\left(Y_{q}\right)_{b} \cong\left(\operatorname{Tel} Y_{q}\right)_{b}
$$

The desired isomorphism is immediate from the following result.

LEMMA 7.5.15. For a sequence of maps $f_{n}: Y_{n} \longrightarrow Y_{n+1}$ between $q f$-fibrant and $q f$-cofibrant objects of $G \mathscr{K}_{B}$ and a point $b \in B$, a qf-fibrant approximation $\mathrm{TelY}_{q} \longrightarrow R \mathrm{Rel} Y_{q}$ induces a weak $G_{b}$-equivalence $\left(\mathrm{Tel} Y_{q}\right)_{b} \longrightarrow\left(\operatorname{RTel} Y_{q}\right)_{b}$ for each $b \in B$.

PROOF. Inductively, starting with $Z_{1}=Y_{1}$ and $\pi_{1}=\mathrm{id}$, we construct acyclic $q f$-fibrations $\pi_{q}: Z_{q} \longrightarrow Y_{q}$ together with $q f$-cofibrations $g_{q}: Z_{q} \longrightarrow Z_{q+1}$ such that $\pi_{q+1} \circ g_{q}=f_{q} \circ \pi_{q}$ by applying the appropriate factorization axiom to $f_{q} \circ \pi_{q}$. Then the $Z_{q}$ are also $q f$-fibrant and $q f$-cofibrant. Therefore the $\pi_{q}$ are $f p$-equivalences, so induce homotopy equivalences of fibers. Comparing constructions as in the proof of Lemma 5.6.14, we see that the telescope of the maps $f_{q}$ is weakly equivalent to the telescope and therefore to the colimit of the sequence of maps $g_{q}$. Because the latter colimit is quasifibrant, by Lemma 3.6.3, the homotopy groups of its fibers are isomorphic to the homotopy groups of the fibers of its $q f$-fibrant approximation. The conclusion follows.

WARNING 7.5.16. We do not know whether or not a general 1-cell complex is compact. At first sight, that might seem obvious from the compactness of discs, but, as pointed out in Warning 7.5.4, the need for $q f$-fibrant approximation of targets before passage to homotopy classes of maps obstructs such an argument.

THEOREM 7.5.17 (Brown). A contravariant set-valued functor on the category $H o G \mathscr{K}_{B}^{c}$ is representable if and only if it satisfies the wedge and Mayer-Vietoris axioms. 



\section{CHAPTER 8}

\section{Ex-fibrations and ex-quasifibrations}

To complete the space level foundations of parametrized homotopy theory, we are faced with two problems that were discussed in the Prologue. In our preferred $q f$-model structure, the base change adjunction $\left(f_{!}, f^{*}\right)$ is a Quillen pair for any map $f$ and is a Quillen equivalence if $f$ is an equivalence. As shown by Counterexample 0.0.1, this implies that the base change adjunction $\left(f^{*}, f_{*}\right)$ cannot be a Quillen adjoint pair, and some such defect must hold for any model structure. Therefore, we cannot turn to model theory to construct the functor $f_{*}$ on the level of homotopy categories. The same counterexample illustrates that passage to derived functors is not functorial in general, so that a relation between composites of functors that holds on the point-set level need not imply a corresponding relation on homotopy categories.

In any attempt to solve those two problems, one runs into a third one that concerns a basic foundational problem in ex-space theory. Model theoretical considerations lead to the use of Serre fibrations as projections, or to the even weaker class of $q f$-fibrations. However, only Hurewicz fibrations are considered in most of the literature. There is good reason for that. Fiberwise smash products, suspensions, cofibers, function spaces, and other fundamental constructions in ex-space theory do not preserve Serre fibrations.

The solutions to all three problems are obtained by the use of ex-fibrations. Recall that these are the well-sectioned $h$-fibrant ex-spaces. We study their properties in $\S 8.1$. They seem to give the definitively right kind of "fibrant ex-space" from the point of view of classical homotopy theory, and they behave much better under the cited constructions than do Serre fibrations, as we show in $\S 8.2$. Many variants of this notion appear in the literature. Precisely this variant, with this name, appears in Monica Clapp's paper [31], and we are indepted to her work for an understanding of the centrality of the notion. Perversely, as we noted in Remark 5.2.7, it is unclear how it fits into the model categorical framework.

We construct an elementary ex-fibrant approximation functor in $\S 8.3$. It plays a key role in bridging the gap between the model theoretic and classical worlds. In a different context, the classification of sectioned fibrations, the first author introduced this construction in $[111, \S 5]$. We record some of its properties in $\S 8.4$.

We define quasifibrant ex-spaces and ex-quasifibrations and show that they inherit some of the good properties of ex-fibrations in $\S 8.5$. They will play a key role in the stable theory.

Everything in this chapter works just as well equivariantly as nonequivariantly for any topological group $G$ of equivariance. 


\subsection{Ex-fibrations}

Under various names, the following notions were in common use in the 1970's. We shall see shortly that these definitions agree with those given in Definition 5.2.5.

Definition 8.1.1. Let $(X, p, s)$ be an ex-space over $B$.

(i) $(X, p, s)$ is well-sectioned if $s$ is a closed inclusion and there is a retraction

$$
\rho: X \times I \longrightarrow X \cup_{B}(B \times I)=M s
$$

over $B$.

(ii) $(X, p, s)$ is well-fibered if there is a coretraction, or path-lifting function,

$$
\iota: N p=X \times{ }_{B} B^{I} \longrightarrow X^{I}
$$

under $B^{I}$, where $B^{I}$ maps to $N p$ via $\alpha \longrightarrow(s \alpha(0), \alpha)$.

(iii) $(X, p, s)$ is an ex-fibration if it is both well-sectioned and well-fibered.

The requirement in (i) that the retraction $\rho$ be a map over $B$ ensures that it restricts on fibers to a retraction that exhibits the nondegeneracy of the basepoint $s(b)$ in $X_{b}$ for each $b \in B$. In view of Theorem 5.2.8(i), we have the following characterization of well-sectioned ex-spaces, in agreement with Definition 5.2.5.

Lemma 8.1.2. An ex-space $X$ is well-sectioned if and only if $X$ is $\bar{f}$-cofibrant.

We use the term "well-sectioned" since it goes well with "well-based". The category of well-sectioned ex-spaces is the appropriate parametrized generalization of the category of well-based spaces, and restricting to well-sectioned ex-spaces is analogous to restricting to well-based spaces.

Note that the section of $X$ provides a canonical way of lifting a path in $B$ that starts at $b$ to a path in $X$ that starts at $s(b)$. The requirement in Definition 8.1.1(ii) that the path-lifting function $\iota$ be a map under $B^{I}$ says that $\iota(s \alpha(0), \alpha)(t)=s(\alpha(t))$ for all $\alpha \in B^{I}$ and $t \in I$. That is, $\iota$ is required to restrict to the canonical lifts provided by the section, so that paths in $X$ that start in $s(B)$ remain in $s(B)$. In contrast with Lemma 8.1.2, the well-fibered condition does not by itself fit naturally into the model theoretic context of Chapter 5 . However, we have the following characterization of ex-fibrations, which again is in agreement with the original definition we gave in Definition 5.2.5.

Lemma 8.1.3. If $X$ is well-fibered, then $X$ is h-fibrant. If $X$ is well-sectioned, then $X$ is an ex-fibration if and only if $X$ is h-fibrant.

Proof. The first statement is clear since the coretraction $\iota$ is a path-lifting function. This gives the forward implication of the second statement, and the converse is a special case of the following result of Eggar [57, 3.2].

Lemma 8.1.4. Let $i: X \longrightarrow Y$ be an $\bar{f}$-cofibration of ex-spaces over $B$, where $Y$ is $h$-fibrant. Then any map $\iota: X \times_{B} B^{I} \longrightarrow Y^{I}$ such that the composite

$$
X \times_{B} B^{I} \stackrel{\iota}{\longrightarrow} Y^{I} \longrightarrow Y \times_{B} B^{I}
$$

is the inclusion can be extended to a coretraction $Y \times_{B} B^{I} \longrightarrow Y^{I}$. 
Proof. The inclusion $X \times_{B} B^{I} \longrightarrow Y \times_{B} B^{I}$ is an $\bar{h}$-cofibration by Proposition 4.4.5. Therefore there is a lift $\nu$ in the diagram

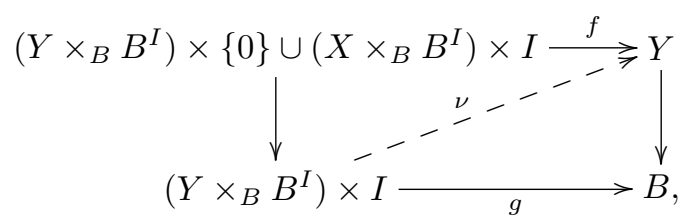

where $f(y, \omega, 0)=y, f(x, \omega, t)=\iota(x, \omega)(t)$, and $g(y, \omega, t)=\omega(t)$. The adjoint $Y \times{ }_{B} B^{I} \longrightarrow Y^{I}$ of $\nu$ is the required extension to a coretraction.

REMARK 8.1.5. We comment on the terminology and history.

(1) We are following $[42,83]$ and others in saying that an $\bar{f}$-cofibrant ex-space is well-sectioned; the term "fiberwise well-pointed" is also used. For a based space, the terms "nondegenerately based" and "well-based" or "well-pointed" are used interchangeably to mean that the inclusion of the basepoint is an $h$-cofibration. In contrast, for an ex-space, the term "fiberwise nondegenerately pointed" is used in $[42,83]$ to indicate a somewhat weaker condition than well-sectioned.

(2) The term "well-fibered" is new but goes naturally with well-sectioned. The concept itself is old. We believe that it is due to Eggar [57, 3.3], who calls a coretraction under $B^{I}$ a special lifting function.

(3) Becker and Gottlieb [9] may have been the first to use the term "exfibration", but for a slightly different notion with sensible CW restrictions. As noted in the introduction, precisely our notion is used by Clapp [31]. Earlier, in [111, §5] and [113], the first author called ex-fibrations " $\mathscr{T}$-fibrations", and he studied their classification and their fiberwise localizations and completions. The equivariant generalization appears in Waner [170]. A more recent treatment of the classification of ex-fibrations has been given by Booth [20].

\subsection{Preservation properties of ex-fibrations}

We have a series of results that show that ex-fibrations behave well with respect to standard constructions. In some of them, one must use the equivariant version of Lemma 5.2.4 to verify that the given construction preserves well-sectioned objects. In all of them, if we only assume that the input ex-spaces are well-sectioned, then we can conclude that the output ex-spaces are well-sectioned. It is the fact that the given constructions preserve well-fibered objects that is crucial. Few if any of these results hold with Serre rather than Hurewicz fibrations as projections.

Proposition 8.2.1. Ex-fibrations satisfy the following properties.

(i) A wedge over $B$ of ex-fibrations is an ex-fibration.

(ii) If $X, Y$ and $Z$ are ex-fibrations and $i$ is an $\bar{f}$-cofibration in the following pushout diagram of ex-spaces over $B$, then $Y \cup_{X} Z$ is an ex-fibration.

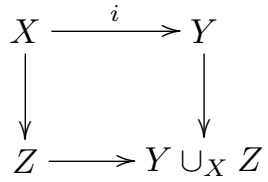

(iii) The colimit of a sequence of $\bar{f}$-cofibrations $X_{i} \longrightarrow X_{i+1}$ between ex-fibrations is an ex-fibration. 
If the input ex-spaces are only assumed to be well-sectioned, then the output exspaces are well-sectioned.

Proof. The last statement is clear. Using it, we see that the colimits in (i), (ii), and (iii) are well-sectioned, hence it suffices to prove that they are $h$-fibrant. This is done by constructing path lifting functions for the colimits from path lifting functions for their inputs. In (i), we start with path lifting functions under $B^{I}$ for the wedge summands and see that they glue together to define a path lifting function under $B^{I}$ for the wedge. Part (ii) is due to Clapp [31, 1.3], and we omit full details. She starts with a path lifting function for $X$ and uses Lemma 8.1.4 to extend it to a path lifting function for $Y$. She also starts with a path lifting function for $Z$. She then uses a representation $(h, u)$ of $(Y, X)$ as a fiberwise NDR pair to build a path lifting function for the pushout from the given path lifting function for $Z$ and a suitably deformed version of the path lifting function for $Y$. In (iii), Lemma 8.1.4 shows that we can extend a path lifting function for $X_{i}$ to a path lifting function for $X_{i+1}$. Inductively, this allows the construction of compatible path lifting functions for the $X_{i}$ that glue together to give a path lifting function for their colimit.

Although of little use to us, since the $f$-homotopy category is not the right one for our purposes, many of our adjunctions give Quillen adjoint pairs with respect to the $f$-model structure. For example, the following result, which should be compared with Proposition 7.3.4, implies that $\left(f_{!}, f^{*}\right)$ is a Quillen adjoint pair in the $f$-model structures and that it is a Quillen equivalence if $f$ is an $h$-equivalence.

Proposition 8.2.2. Let $f: A \longrightarrow B$ be a map, let $X$ be a well-sectioned exspace over $A$, and let $Y$ be a well-sectioned ex-space over $B$. Then $f_{!} X$ and $f^{*} Y$ are well-sectioned. If $Y$ is an ex-fibration, then so is $f^{*} Y$, and the functor $f^{*}$ preserves $f$-equivalences. If $f$ is an h-equivalence, then $\left(f_{!}, f^{*}\right)$ induces an equivalence of $f$-homotopy categories.

Proof. It is easy to check that representations of $(X, A)$ and $(Y, B)$ as fiberwise NDR-pairs induce representations of $\left(f_{!} X, B\right)$ and $\left(f^{*} Y, A\right)$ as fiberwise NDRpairs. As a pullback, the functor $f^{*}$ preserves both $f$-fibrant and $h$-fibrant exspaces, and $f^{*}$ preserve $f$-equivalences since it preserves $f$-homotopies. For the last statement, if $f$ is a homotopy equivalence with homotopy inverse $g$, then standard arguments with the CHP imply that $f^{*}$ induces an equivalence of $f$-homotopy categories with inverse $g^{*}$; see, for example, $[111,2.5]$. It follows that $g^{*}$ is equivalent to $f_{!}$and that $\left(f_{!}, f^{*}\right)$ is a Quillen equivalence.

The following result appears in [57] and $[111,3.6]$. It also leads to a Quillen adjoint pair with respect to the $f$-model structure; compare Corollary 7.3.3.

Proposition 8.2.3. Let $X$ and $Y$ be well-sectioned ex-spaces over $A$ and $B$. Then $X \wedge Y$ is a well-sectioned ex-space over $A \times B$. If $X$ and $Y$ are ex-fibrations, then $X \bar{\wedge} Y$ is an ex-fibration.

Proof. Representations of $(X, A)$ and $(Y, B)$ as fiberwise NDR-pairs determine a representation of $(X \bar{\wedge} Y, A \times B)$ as a fiberwise NDR-pair, by standard formulas [121, p. 43]. Similarly, path lifting functions for $X$ and $Y$ can be used to write down a path lifting function for $X \bar{\wedge} Y$.

Corollary 8.2.4. If $X$ and $Y$ are ex-fibrations over $B$, then so is $X \wedge_{B} Y$. 
Corollary 8.2.5. If $X$ is an ex-fibration over $B$ and $K$ is a well-based space, then $X \wedge_{B} K$ is an ex-fibration over $B$.

Proposition 8.2.6. Let $X$ and $Y$ be well-sectioned and let $f: X \longrightarrow Y$ be an ex-map that is an h-equivalence. Then $f \wedge_{B} i d: X \wedge_{B} Z \longrightarrow Y \wedge_{B} Z$ is an h-equivalence for any ex-fibration $Z$. In particular, $f \wedge_{B} i d: X \wedge_{B} K \longrightarrow Y \wedge_{B} K$ is an $h$-equivalence for any well-based space $K$.

Proof. As observed by Clapp [31, 2.7], this follows from the gluing lemma by comparing the defining pushouts.

As in ordinary topology, function objects work less well, but we do have the following analogue of Corollary 8.2.5.

Proposition 8.2.7. If $X$ is an ex-fibration over $B$ and $K$ is a compact wellbased space, then $F_{B}(K, X)$ is an ex-fibration over $B$.

Proof. Let $(h, u)$ represent $(X, B)$ as a fiberwise NDR-pair. Then $(j, v)$ represents $\left(F_{B}(K, X), B\right)$ as a fiberwise NDR-pair, where

$$
v(f)=\sup _{k \in K} u(f(k)) \quad \text { and } \quad j_{t}(f)(k)=h_{t}(f(k))
$$

for $f \in F_{B}(K, X)$. Note for this that $F_{B}(K, B)=B$ and that, by Proposition 1.3.16, $F_{B}(K, X)$ is $h$-fibrant.

\subsection{The ex-fibrant approximation functor}

We describe an elementary ex-fibrant replacement functor $P$. It is just the composite of a whiskering functor $W$ with a version of the mapping path fibration functor $N$. The functor $P$ replaces ex-spaces by naturally $h$-equivalent ex-fibrations. From the point of view of model theory, $P$ can be thought of as a kind of $q$-fibrant replacement functor that gives Hurewicz fibrations rather than just Serre fibrations as projections. The nonequivariant version of $P$ appears in $[111,5.3,5.6]$, and the equivariant version appears in $[170, \S 3]$. With motivation from the theory of transports in fibrations, those sources work with Moore paths of varying length. Surprisingly, that choice turns out to be essential for the construction to work.

We therefore begin by recalling that the space of Moore paths in $B$ is given by

$$
\Lambda B=\left\{(\lambda, l) \in B^{[0, \infty]} \times[0, \infty) \mid \lambda(r)=\lambda(l) \text { for } r \geq l\right\}
$$

with the subspace topology. We write $\lambda$ for $(\lambda, l)$ and $l_{\lambda}$ for $l$, which is the length of $\lambda$. Let $e: \Lambda B \longrightarrow B$ be the endpoint projection $e(\lambda)=\lambda\left(l_{\lambda}\right)$. The composite of Moore paths $\mu$ and $\lambda$ such that $\lambda\left(l_{\lambda}\right)=\mu(0)$ is defined by $l_{\mu \lambda}=l_{\mu}+l_{\lambda}$ and

$$
(\mu \lambda)(r)= \begin{cases}\lambda(r) & \text { if } r \leq l_{\lambda}, \\ \mu\left(r-l_{\lambda}\right) & \text { if } r \geq l_{\lambda} .\end{cases}
$$

Embed $B$ and $B^{I}$ in $\Lambda B$ as the paths of length 0 and 1. For a Moore path $\lambda$ in $B$ and real numbers $u$ and $v$ such that $0 \leq u \leq v$, let $\left.\lambda\right|_{u} ^{v}$ denote the Moore path $r \mapsto \lambda(u+r)$ of length $v-u$.

Definition 8.3.1. Consider an ex-space $X=(X, p, s)$ over $B$.

(i) Define the whiskering functor $W$ by letting

$$
W X=\left(X \cup_{B}(B \times I), q, t\right),
$$


where the pushout is defined with respect to $i_{0}: B \longrightarrow B \times I$. The projection $q$ is given by the projection $p$ of $X$ and the projection $B \times I \longrightarrow B$, and the section $t$ is given by $t(b)=(b, 1)$.

(ii) Define the Moore mapping path fibration functor $L$ by letting

$$
L X=\left(X \times{ }_{B} \Lambda B, q, t\right),
$$

where the pullback is defined with respect to the map $\Lambda B \longrightarrow B$ given by evaluation at 0 . The projection $q$ is given by $q(x, \lambda)=e(\lambda)$ and the section $t$ is given by $t(b)=(s(b), b)$, where $b$ is viewed as a path of length 0 .

Thus $W X$ is obtained by growing a whisker on each point in the section of $X$, and the endpoints of the whiskers are used to give $W X$ a section. Similarly, $L X$ is obtained by attaching to $x \in X$ all Moore paths in $B$ starting at $p(x)$. The endpoints of the paths give the projection. In the language of $\S 4.3, W X$ is the standard mapping cylinder construction of the section of $X$, thought of as a map in $G \mathscr{K} / B$. The section $t$ of $W X$ is just the $f$-cofibration in the standard factorization $\rho \circ t$ of $s$ through its mapping cylinder. In particular, $W X$ is well-sectioned. Similarly, $L X$ is a modification of the mapping path fibration $N p$ in $G \mathscr{K}$. The projection $p$ of $X$ factors through the projection $q$ of $L X$, which is an $h$-fibration; a path lifting function $\xi: L X \times_{B} B^{I} \longrightarrow(L X)^{I}$ is given by $\xi((x, \lambda), \gamma)(t)=\left(x,\left.\gamma\right|_{0} ^{t} \lambda\right)$. Thus $L X$ is $h$-fibrant, but it need not be well-fibered.

We can display all of this conveniently in the following diagram. The third square on the top is a pushout and the second square on the bottom is a pullback. That defines the maps $\phi$ and $\pi$, and the maps $\rho$ and $\iota$ are induced by the universal properties from the identity map of $X$.

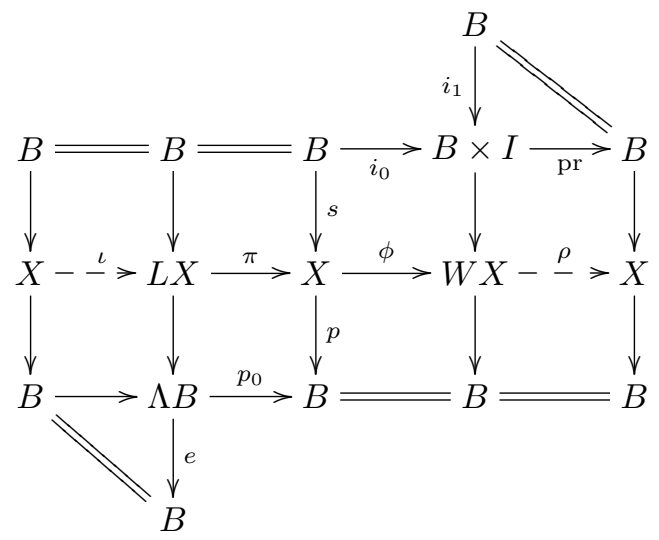

Thus $\rho$ projects whiskers on fibers to the original basepoints and $\iota$ is the inclusion $x \mapsto(x, p(x))$, where $p(x)$ is the path of length zero. Note that $\phi$ is not a map under $B$ and $\pi$ is not a map over $B$. They give an inverse $f$-equivalence to $\rho$ and an inverse $h$-equivalence to $\iota$.

Proposition 8.3.2. The map $\rho: W X \longrightarrow X$ is a natural $f$-equivalence of ex-spaces and $W X$ is well-sectioned. The map $\iota: X \longrightarrow L X$ is a natural $h$ equivalence of ex-spaces and $L X$ is $h$-fibrant. Therefore $W$ takes $f$-equivalences to $f p$-equivalences and $L$ takes $h$-equivalences to $f$-equivalences.

The last statement follows from Proposition 5.2.2. We think of $\rho$ and $\iota$ as giving a well-sectioned approximation and an $h$-fibrant approximation in the category of 
ex-spaces. We will combine them to obtain the promised ex-fibrant approximation, but we first insert a technical lemma.

LEMMA 8.3.3. If $X$ is an ex-space with a closed section, then $W L X$ is an ex-fibration. If $X$ is well-fibered, then $W X$ is an ex-fibration.

Proof. A path lifting function $\xi: N W L X=W L X \times_{B} B^{I} \longrightarrow(W L X)^{I}$ for $W L X$ is obtained by letting

$$
\xi(z, \gamma)(t)= \begin{cases}\left(x,\left.\gamma\right|_{0} ^{t} \lambda\right) \in L X & \text { if } z=(x, \lambda) \in L X \\ (\gamma(t), u-t) \in B \times I & \text { if } z=(b, u) \text { and } t \leq u \\ \left(s(\gamma(u)),\left.\gamma\right|_{u} ^{t}\right) \in L X & \text { if } z=(b, u) \text { and } t \geq u\end{cases}
$$

It is easy to verify that, as a map of sets, $\xi$ gives a well-defined section of the canonical retraction $\pi:(W L X)^{I} \longrightarrow W L X \times_{B} B^{I}$. Continuity is a bit more delicate, but if the section of $X$ is closed, then one verifies that

$$
\Phi=\{(z, \gamma) \mid z \text { is the equivalence class of }(s(b), b) \sim(b, 0)\}
$$

is a closed subset of WLX and hence $N \Phi$ is a closed subset of $N W L X$. To see the implication, note that $(-) \times B^{I}$ preserves closed inclusions and $Z \times{ }_{B} B^{I} \subset Z \times B^{I}$ is a closed inclusion because $B$ is in $\mathscr{U}$ (see Remark 1.6.4). Continuity follows since we are then piecing together continuous functions on closed subsets.

If $X$ is well-fibered and $\xi: X \times_{B} B^{I} \longrightarrow X^{I}$ is a path-lifting function under $B^{I}$, we can define a path lifting function $\bar{\xi}: W X \times_{B} B^{I} \longrightarrow(W X)^{I}$ for $W X$ by

$$
\bar{\xi}(x, \gamma)= \begin{cases}\xi(x, \gamma) & \text { if } x \in X \\ (\gamma, u) & \text { if } x=(b, u)\end{cases}
$$

To check that $\bar{\xi}$ is continuous, we use the fact that the functor $N(-)=B^{I} \times_{B}(-)$ commutes with pushouts to write $N W X$ as a pushout. We then see that $\bar{\xi}$ is the map obtained by passage to pushouts from a pair of continuous maps.

Recall that the sections of ex-spaces in $G \mathscr{U}_{B}$ are closed, by Lemma 1.6.2. Since we shall only need to apply the constructions of this section to ex-spaces in $G \mathscr{U}_{B}$, the closed section hypothesis need not concern us.

Definition 8.3.4. Define the ex-fibrant approximation functor $P$ by the natural zig-zag of $h$-equivalences $\phi=(\rho, W \iota)$ displayed in the diagram

$$
X<^{\rho} W X \stackrel{W \iota}{\longrightarrow} W L X=P X .
$$

By Proposition 8.3.2, $P$ takes $h$-equivalences between arbitrary ex-spaces to $f p$ equivalences. If $X$ has a closed section, then $P X$ is an ex-fibration. If $X$ is an ex-fibration, then it has a closed section, and the above display is a natural zig-zag of $f p$-equivalences between ex-fibrations.

\subsection{Preservation properties of ex-fibrant approximation}

One advantage of ex-fibrant approximation over $q$ or $q f$-fibrant approximation is that there are explicit commutation natural transformations relating it to many constructions of interest. The following result is an elementary illustrative example. 
LEMma 8.4.1. Let $\mathscr{D}$ be a small category, $X: \mathscr{D} \longrightarrow G \mathscr{K}_{B}$ be a functor, and

$$
\omega: \operatorname{colim} W X_{d} \longrightarrow W \operatorname{colim} X_{d} \quad \text { and } \quad \nu: \operatorname{colim} L X_{d} \longrightarrow L \operatorname{colim} X_{d}
$$

be the evident natural maps. Then $\omega$ is a map over colim $X_{d}$ and $\nu$ is a map under colim $X_{d}$, so that the following diagrams commute. All maps in these diagrams are $h$-equivalences.
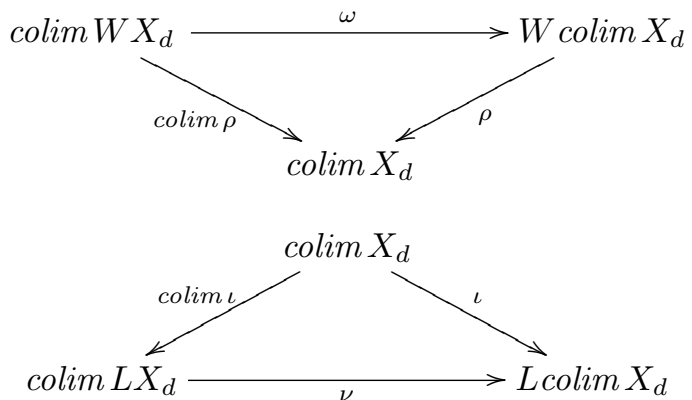

Let $\mu=W \nu \circ \omega:$ colim $P X_{d} \longrightarrow P \operatorname{colim} X_{d}$. Then the following diagram of $h$ equivalences commutes.

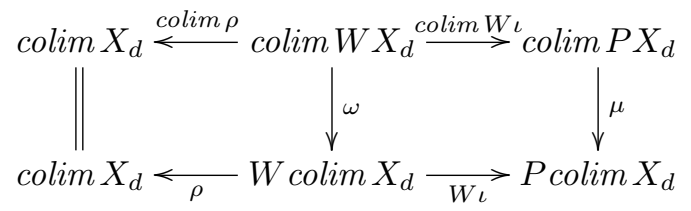

The analogous statements for limits also hold.

Proof. This is clear from the construction of limits and colimits in Proposition 1.2.9. The relevant $h$-equivalences of total spaces are natural and piece together to pass to limits and colimits.

WARNING 8.4.2. We would like an analogue of the previous result for tensors. In particular, we would like a natural map $(L X) \wedge K \longrightarrow L(X \wedge K)$ under $X \wedge K$ for ex-spaces $X$ over $B$ and based spaces $K$. Inspection of definitions makes clear that there is no such map. The obvious map that one might write down, as in the erroneous $[111,5.6]$, is not well-defined. In Part III, this complicates the extension of $P$ to a functor on spectra over $B$.

Lemma 8.4.3. Let $f: A \longrightarrow B$ be a map.

(i) Let $X$ be an ex-space over $A$. Then there are natural maps

$$
\omega: f_{!} W X \longrightarrow W f_{!} X \quad \text { and } \quad \nu: f_{!} L X \longrightarrow L f_{!} X
$$

of ex-spaces over $B$ such that $\omega$ is a map over $f_{!} X$ and $\nu$ is a map under $f_{!} X$. Let $\mu=W \nu \circ \omega: f_{!} P X \longrightarrow P f_{!} X$. Then the following diagram commutes.

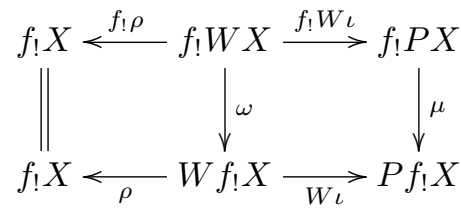


(ii) Let $Y$ be an ex-space over B. Then there are natural maps

$$
\omega: W f^{*} Y \longrightarrow f^{*} W Y \text { and } \nu: L f^{*} Y \longrightarrow f^{*} L Y
$$

of ex-spaces over $A$, the first an isomorphism, such that $\omega$ is a map over $f^{*} Y$ and $\nu$ is a map under $f^{*} Y$. Let $\mu=\omega \circ W \nu: P f^{*} Y \longrightarrow f^{*} P Y$. Then the following diagram commutes.

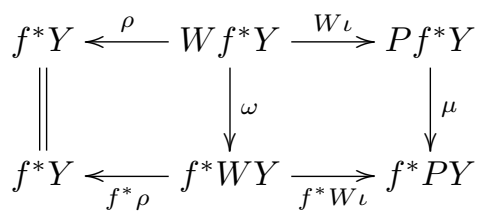

If $Y$ is an ex-fibration, then $\mu$ is an fp-equivalence.

(iii) Let $X$ be an ex-space over A. Then there are natural maps

$$
\omega: W f_{*} X \longrightarrow f_{*} W X \quad \text { and } \quad \nu: L f_{*} X \longrightarrow f_{*} L X
$$

of ex-spaces over $B$ such that $\omega$ is a map over $f_{*} X$ and $\nu$ is a map under $f_{*} X$. Let $\mu=\omega \circ W \nu: P f_{*} X \longrightarrow f_{*} P X$. Then the following diagram commutes.

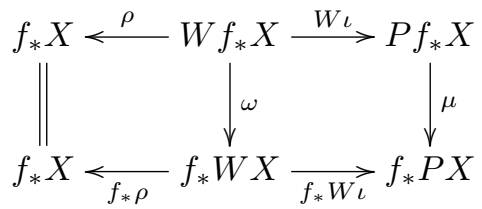

Proof. Again, the proof is by inspection of definitions. Since $f_{!}$does not preserve ex-fibrations, we do not have an analogue for $f$ ! of the last statement about $f^{*}$ in (ii).

WARNING 8.4.4. We offer another example of the technical dangers lurking in this subject. The maps $\mu$ in the previous proposition are not $h$-equivalences in general, the problem in (ii), say, being that $f^{*}$ does not preserve $h$-equivalences in general. If $\mu: P f^{*} Y \longrightarrow f^{*} P Y$ were always an $h$-equivalence, then one could prove by the methods in $\S 9.3$ below that the relations (2.2.12) descend to homotopy categories for all pullbacks of the form displayed in Proposition 2.2.11. In view of Counterexample 0.0.1, that conclusion is false. This is a pitfall we fell into, and it invalidated much work in an earlier draft.

\subsection{Quasifibrant ex-spaces and ex-quasifibrations}

By analogy with the fact that an ex-fibration is a well-sectioned $h$-fibrant exspace, we adopt the following terminology.

Definition 8.5.1. An ex-space $X$ is quasifibrant if its projection $p$ is a quasifibration. An ex-quasifibration is a well-sectioned quasifibrant ex-space.

If $X$ is quasifibrant, there is a long exact sequence of homotopy groups

$\cdots \longrightarrow \pi_{q+1}^{H}(B, b) \longrightarrow \pi_{q}^{H}\left(X_{b}, x\right) \longrightarrow \pi_{q}^{H}(X, x) \longrightarrow \pi_{q}^{H}(B, b) \longrightarrow \cdots \longrightarrow \pi_{0}^{H}(B, b)$

for any $b \in B, x \in X_{b}$ and $H \subset G_{b}$. Using this and the long exact sequences of the pairs $\left(X, X_{b}\right)$, five lemma comparisons give the following observations. 
Lemma 8.5.2. Let $f: X \longrightarrow Y$ be a q-equivalence of ex-spaces over B. Then each map of fibers $f: X_{b} \longrightarrow Y_{b}$ is a q-equivalence if and only if each map of pairs $f:\left(X, X_{b}\right) \longrightarrow\left(Y, Y_{b}\right)$ is a q-equivalence. If $X$ and $Y$ are quasifibrant, then these maps of pairs are q-equivalences. Conversely, if these maps of pairs are $q$ equivalences and either $X$ or $Y$ is quasifibrant, then so is the other.

Working in $G \mathscr{U}_{B}$, we obtain the following result. The same pattern of proof gives many other results of the same nature that we leave to the reader.

Proposition 8.5.3. The following statements hold.

(i) A wedge over $B$ of ex-quasifibrations is an ex-quasifibration.

(ii) If $f: X \longrightarrow Y$ is a map such that $X$ is an ex-quasifibration and $Y$ is quasifibrant, then the cofiber $C_{B} f$ is quasifibrant.

(iii) If $X$ is an ex-quasifibration and $K$ is a well-based space, then $X \wedge_{B} K$ is an ex-quasifibration.

Proof. This follows from Lemma 8.5.2, the natural zig-zag

$$
X<W X \longrightarrow P X
$$

of $h$-equivalences, the corresponding preservation properties for ex-fibrations, and the properties of $q$-equivalences between well-grounded ex-spaces; see Definition 5.4.1 and Proposition 5.4.9. It is also relevant that in each case passage to fibers gives the nonparametrized analogue of the construction under consideration. Since this result plays a vital role in our work, we give more complete details of (ii) and (iii); (i) works the same way.

The cofiber $C_{B} f$ is the pushout of the diagram

$$
C_{B} X \longleftarrow X \stackrel{f}{\longrightarrow} Y .
$$

If $X$ is well-sectioned, then the left arrow is an $h$-cofibration and $W X$ and $P X$ are well-sectioned. Replacing $f$ by $W f$ and $P f$ we obtain three such cofiber diagrams. Together with our original zig-zag this gives a $3 \times 3$-diagram. Applying the gluing lemma, Definition 5.4.1(iii), we obtain a zig-zag of $q$-equivalences

$$
C_{B} f \longleftarrow C_{B} W f \longrightarrow C_{B} P f .
$$

Similarly, on fibers we obtain zig-zags of $q$-equivalences

$$
C f_{b} \longleftarrow C(W f)_{b} \longrightarrow C W(L f)_{b} .
$$

There results a zig-zag of $q$-equivalences of pairs

$$
\left(C_{B} f, C f_{b}\right) \longleftarrow\left(C_{B} W f, C W f_{b}\right) \longrightarrow\left(C_{B} P f, C W(L f)_{b}\right) .
$$

Since $C_{B} P f$ is ex-fibrant and in particular quasifibrant, $C_{B} f$ is quasifibrant.

Similarly, by Definition 5.4.1(v), we have natural zig-zags of $q$-equivalences

$$
X \wedge_{B} K \prec W X \wedge_{B} K \longrightarrow P X \wedge_{B} K
$$

and

$$
X_{b} \wedge K \longleftarrow W X_{b} \wedge K \longrightarrow W(L X)_{b} \wedge K .
$$

We therefore have a zig-zag of $q$-equivalences of pairs

$$
\left(X \wedge_{B} K, X_{b} \wedge K\right) \longleftarrow\left(W X \wedge_{B} K, W X_{b} \wedge K\right) \longrightarrow\left(P X \wedge_{B} K, W(L X)_{b} \wedge K\right) .
$$

Since $P X \wedge_{B} K$ is ex-fibrant and hence quasifibrant, $X \wedge_{B} K$ is quasifibrant. 


\section{CHAPTER 9}

\section{The equivalence between $\operatorname{Ho} G \mathscr{K}_{B}$ and $h G \mathscr{W}_{B}$}

\section{Introduction}

We developed the point-set level properties of the category $G \mathscr{K}_{B}$ of ex- $G$-spaces over $B$ in Chapters 1 and 2, and we developed those homotopical properties that are accessible to model theoretic techniques in Chapters $4-7$. In this chapter, we use ex-fibrations to prove that certain structure on the point-set level that seems inaccessible from the point of view of model category theory nevertheless descends to homotopy categories. In particular, we prove that Ho $G \mathscr{K}_{B}$ is closed symmetric monoidal and that the right derived functor $f^{*}$ of the Quillen adjunction $\left(f_{!}, f^{*}\right)$ in the $q f$-model structure is closed symmetric monoidal. We use Brown representability to show that $f^{*}$ has a partially defined right adjoint. Here on the ex-space level, the classical limitation of Brown representability to connected spaces forces us to restrict the construction of $f_{*}$ to $G$-connected ex-fibrations, which have $G_{b}$-connected fibers.

In $\S 9.1$ we use the ex-fibrant approximation functor and the good properties of the $q f$-model structure to prove that our model theoretic homotopy category of ex$G$-spaces over $B$ is equivalent to the classical homotopy category of ex- $G$-fibrations over $B$. In $\S 9.2$, we discuss how to compare derived functors as constructed on either side of that equivalence in certain general cases. Replacing the model-theoretic method of constructing derived functors by a more classical method given in terms of ex-fibrant approximation, we construct the functors $f_{*}$ and $F_{B}$ on homotopy categories in $\S 9.3$. By a combination of methods, we prove that Ho $G \mathscr{K}_{B}$ is a symmetric monoidal category and that the base change functor $f^{*}$ descends to a closed symmetric monoidal functor on homotopy categories in $\S 9.4$. We also obtain such descent results for change of group adjunctions and for passage to fibers in that section. These results are central to the theory, and there seem to be no shortcuts to their proofs.

Everything is understood to be equivariant in this chapter, and we abbreviate ex- $G$-fibration and ex- $G$-space to ex-fibration and ex-space throughout. We shall retreat just a bit from all-embracing generality. We assume that $G$ is a Lie group and that all given base $G$-spaces $B$ are proper and are of the homotopy types of $G$-CW complexes. The reader may prefer to assume that $G$ is compact, but there is no gain in simplicity. In view of the properties of the base change adjunction $\left(f_{!}, f^{*}\right)$ given in Proposition 7.3.4, there would be no real loss of generality if we restricted further to base spaces that are actual $G$-CW complexes, but that would be inconveniently restrictive. 


\subsection{The equivalence of $\mathrm{Ho} G \mathscr{K}_{B}$ and $h G \mathscr{W}_{B}$}

Recall that $X \wedge_{B} I_{+}$is a cylinder object in the sense of the $q f$-model structure. When we restrict to $q f$-fibrant and $q f$-cofibrant objects, homotopies in the $q f$ model sense are the same as $f p$-homotopies, by Lemma 5.6.3. The morphism set $[X, Y]_{G, B}$ in Ho $G \mathscr{K}_{B}$ is naturally isomorphic to $[R Q X, R Q Y]_{G, B}$, and this is the set of $f p$-homotopy classes of maps $R Q X \longrightarrow R Q Y$. Here $R$ and $Q$ denote the functorial $q f$-fibrant and $q f$-cofibrant approximation functors obtained from the small object argument. The total space of $R Q X$ has the homotopy type of a $G$ CW complex since $B$ does. This leads us to introduce the following categories.

Definition 9.1.1. Define $G \mathscr{V}_{B}$ to be the full subcategory of $G \mathscr{K}_{B}$ whose objects are well-grounded and $q f$-fibrant with total spaces of the homotopy types of $G$-CW complexes. Define $G \mathscr{W}_{B}$ to be the full subcategory of $G \mathscr{V}_{B}$ whose objects are the ex-fibrations over $B$. Let $h G \mathscr{W}_{B}$ denote the category obtained from $G \mathscr{W}_{B}$ by passage to $f p$-homotopy classes of maps.

Note that the definition of $G \mathscr{W}_{B}$ makes no reference to model category theory. Recall that well-grounded means well-sectioned and compactly generated. When $B=*, G \mathscr{W}_{*}$ is just the category of well-based compactly generated $G$-spaces of the homotopy types of $G$-CW complexes, and it is standard that its classical homotopy category is equivalent to the homotopy category of based $G$-spaces with respect to the $q$-model structure. We shall prove a parametrized generalization.

We think of $G \mathscr{V}_{B}$ as a convenient half way house between $G \mathscr{K}_{B}$ and $G \mathscr{W}_{B}$. It turns out to be close enough to the category of $q f$-cofibrant and $q f$-fibrant objects in $G \mathscr{K}_{B}$ to serve as such for our purposes, while already having some of the properties of $G \mathscr{W}_{B}$. The following crucial theorem fails for the $q$-model structure. It is essential for this result that we only require the objects of $\mathscr{V}_{B}$ to be well-sectioned, rather than requiring them to be $q f$-cofibrant. This will force an assymmetry when we deal with left and right derived functors in Proposition 9.2.2 below.

THEOREM 9.1.2. The $q f$-cofibrant and $q$-fibrant approximation functor $R Q$ and the ex-fibrant approximation functor $P$, together with the forgetful functors $I$ and $J$, induce the following equivalences of homotopy categories.

$$
\text { Ho } G \mathscr{K}_{B} \underset{I}{\stackrel{R Q}{\rightleftarrows}} H o G \mathscr{V}_{B} \underset{J}{\stackrel{P}{\rightleftarrows}} h G \mathscr{W}_{B}
$$

Proof. For $X$ in $G \mathscr{K}_{B}$, we have a natural zig-zag of $q$-equivalences in $G \mathscr{K}_{B}$

$$
X \longleftarrow Q X \longrightarrow R Q X \text {. }
$$

Therefore $X$ and $I R Q X$ are naturally $q$-equivalent in $G \mathscr{K}_{B}$. If $X$ is in $G \mathscr{V}_{B}$, then it is $q f$-fibrant and therefore so is $Q X$. Then the above zig-zag is in $G \mathscr{V}_{B}$ and thus $X$ and $R Q I X$ are naturally $q$-equivalent in $G \mathscr{V}_{B}$.

Since $q$-equivalences in $G \mathscr{V}_{B}$ are $h$-equivalences, and $P$ takes $h$-equivalences to $f p$-equivalences, it is clear that $P$ induces a functor on homotopy categories. Conversely, since $f p$-equivalences are in particular $q$-equivalences, the forgetful functor $J$ induces a functor in the other direction. For $X$ in $G \mathscr{V}_{B}$ we have the natural zig-zag of $h$-equivalences

$$
X<^{\rho} W X \stackrel{W \iota}{\longrightarrow} P X
$$


of Definition 8.3.4. However $W X$ may not be in $G \mathscr{V}_{B}$ since it may not be $q f$-fibrant. Applying $q f$-fibrant approximation, we get a natural zig-zag of $q$-equivalences in $G \mathscr{V}_{B}$ connecting $X$ and $P X$. It follows that $X$ and $J P X$ are naturally $q$-equivalent in $G \mathscr{V}_{B}$. Starting with $X$ in $G \mathscr{W}_{B}$, the above display is a zig-zag of $f p$-equivalences in $G \mathscr{W}_{B}$, by Proposition 8.3.2. It follows that $X$ and $J P X$ are naturally $f p$ equivalent in $G \mathscr{W}_{B}$.

\subsection{Derived functors on homotopy categories}

Model category theory tells us how Quillen functors $V: G \mathscr{K}_{A} \longrightarrow G \mathscr{K}_{B}$ induce derived functors on the homotopy categories on the left hand side of the equivalence displayed in Theorem 9.1.2. We now seek an equivalent way of passing to derived functors on the right hand side. We begin with an informal discussion. We focus on functors of one variable, but functors of several variables work the same way.

Following the custom in algebraic topology, we have been abusing notation by using the same notation for point-set level functors and for derived homotopy category level functors. We will continue to do so. However, the more accurate notations of algebraic geometry, $L V$ and $R V$ for left and right derived functors, might

clarify the discussion. As we have already seen in Counterexample 0.0.1, passage to derived functors is not functorial in general, so that a relation between composites of functors that holds on the point-set level need not imply a corresponding relation on passage to homotopy categories.

Recall that, model theoretically, if $V$ is a Quillen right adjoint, then the right derived functor of $V$ is obtained by first applying fibrant approximation $R$ and then applying $V$ on homotopy categories, which makes sense since $V$ preserves weak equivalences between fibrant objects. The left derived functor of a Quillen left adjoint $V$ is defined dually, via $V Q$. Problems arise when one tries to compose left and right derived functors, which is what we must do to prove some of our compatibility relations.

The equivalence of categories proven in Theorem 9.1.2 gives us a way of putting the relevant left and right adjoints on the same footing, giving a "straight" passage to derived functors that is neither "left" nor "right". We do not attempt a formal categorical analysis. We need mild good behavior for this to work.

Definition 9.2.1. A functor $V: G \mathscr{K}_{A} \longrightarrow G \mathscr{K}_{B}$ is good if it is continuous, takes well-grounded ex-spaces to well-grounded ex-spaces, and takes ex-spaces whose total spaces are of the homotopy types of $G$-CW complexes to ex-spaces with that property. Since $V$ is continuous, it preserves $f p$-homotopies.

Proposition 9.2.2. Let $V: G \mathscr{K}_{A} \longrightarrow G \mathscr{K}_{B}$ be a good functor that is a left or a right Quillen adjoint. If $V$ is a Quillen left adjoint, assume further that it preserves $q$-equivalences between well-grounded ex-spaces. Then, under the equivalence of categories in Theorem 9.1.2, the derived functor HoG $\mathscr{K}_{A} \longrightarrow$ HoG $\mathscr{K}_{B}$ induced by $V Q$ or $V R$ is equivalent to the functor $P V J: h G \mathscr{W}_{A} \longrightarrow h G \mathscr{W}_{B}$ obtained by passage to homotopy classes of maps.

Proof. If $V$ is a Quillen right adjoint, then it preserves $q$-equivalences between $q f$-fibrant objects. If $V$ is a Quillen left adjoint, then we are assuming that it preserves $q$-equivalences between well-grounded objects. Since $G \mathscr{V}_{A}$ consists of well-sectioned $q f$-fibrant objects, it follows in both cases that $V: G \mathscr{V}_{A} \longrightarrow G \mathscr{V}_{B}$ passes straight to homotopy categories to give $V: \mathrm{Ho} \mathscr{V}_{A} \longrightarrow \mathrm{Ho} G \mathscr{V}_{B}$. Since $V$ 
preserves $G$-CW homotopy types on total spaces, $V$ takes $q$-equivalences to $h$ equivalences. Therefore $P V$ takes $q$-equivalences to $f p$-equivalences and induces a functor $\mathrm{Ho} \mathscr{V}_{A} \longrightarrow h G \mathscr{W}_{B}$. To show that $P V J$ and either $V Q$ or $V R$ agree under the equivalence of categories, it suffices to verify that the following diagram commutes.

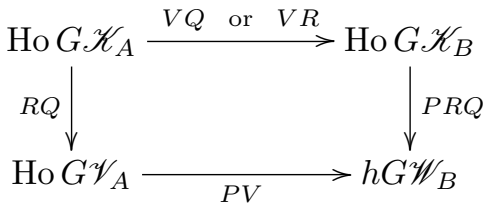

We have a natural acyclic $q f$-fibration $Q X \longrightarrow X$ and a natural acyclic $q f$ cofibration $X \longrightarrow R X$. If $V$ is a Quillen left adjoint, then we have a zig-zag of natural $q$-equivalences

$$
R Q V Q \longrightarrow R V Q \longleftarrow V Q \longrightarrow V R Q
$$

because $V$ preserves acyclic $q f$-cofibrations. If $V$ is a Quillen right adjoint, then we have a zig-zag of natural $q$-equivalences

$$
R Q V R \longleftarrow R Q V R Q \longrightarrow R V R Q \longleftarrow V R Q
$$

because $V$ preserves $q$-equivalences between $q f$-fibrant objects. In both cases, all objects have total spaces of the homotopy types of $G$-CW complexes, so in fact we have zig-zags of $h$-equivalences. Therefore, applying $P$ gives us zig-zags of $f p$ equivalences in $G \mathscr{W}_{B}$, by Proposition 8.3.2.

REMARK 9.2.3. When $V$ preserves ex-fibrations, $P V$ is naturally $f p$-equivalent to $V$ on ex-fibrations, by Proposition 8.3.2. The derived functor of $V$ can then be obtained directly by applying $V$ and passing to equivalence classes of maps under fp-homotopy.

\subsection{The functors $f_{*}$ and $F_{B}$ on homotopy categories}

We use the equivalence between $\mathrm{Ho}_{G} \mathscr{K}_{B}$ and $h G \mathscr{W}_{B}$ to prove that, for any map $f: A \longrightarrow B$ between spaces of the homotopy types of $G$-CW complexes, the adjunction $\left(f^{*}, f_{*}\right)$ descends to homotopy categories. We begin by verifying that $f^{*}$ satisfies the hypotheses of Proposition 9.2.2.

Proposition 9.3.1. Let $f: A \longrightarrow B$ be a map of base spaces. Then the base change functor $f^{*}$ restricts to a functor $f^{*}: G \mathscr{W}_{B} \longrightarrow G \mathscr{W}_{A}$.

Proof. Consider $Y$ in $G \mathscr{W}_{B}$. Since the total space of $Y$ is of the homotopy type of a $G$-CW complex, the fibers $Y_{b}$ are of the homotopy types of $G_{b}-\mathrm{CW}$ complexes by Theorem 3.5.2. The fiber $\left(f^{*} Y\right)_{a}$ is a copy of $Y_{f(a)}$, and $G_{a}$ acts through the evident inclusion $G_{a} \subset G_{f(a)}$. Therefore $\left(f^{*} Y\right)_{a}$ is of the homotopy type of a $G_{a}$-CW complex. The total space of $f^{*} Y$ is therefore of the homotopy type of a $G$-CW complex, again by Theorem 3.5.2. Moreover, $f^{*} Y$ is an ex-fibration by Proposition 8.2.2. Thus $f^{*}$ restricts to a functor $f^{*}: G \mathscr{W}_{B} \longrightarrow G \mathscr{W}_{A}$.

Recalling the discussion of $\S 7.5$, we write $G \mathscr{W}_{B}^{c}$ for the full subcategory of $G \mathscr{W}_{B}$ whose objects are the $G$-connected ex-fibrations. 
Theorem 9.3.2. For any map $f: A \longrightarrow B$ of base spaces, the right derived functor $f^{*}: H o G \mathscr{K}_{B}^{c} \longrightarrow H_{o} G \mathscr{K}_{A}^{c}$ has a right adjoint $f_{*}$, so that

$$
\left[f^{*} Y, X\right]_{G, A} \cong\left[Y, f_{*} X\right]_{G, B}
$$

for $X$ in $G \mathscr{K}_{A}^{c}$ and $Y$ in $G \mathscr{K}_{B}^{c}$.

Proof. Clearly $f^{*} Y$ is $G$-connected if $Y$ is $G$-connected. In view of the equivalence of categories in Theorem 9.1.2 and the fact that $f^{*}$ descends directly to a functor $f^{*}: h G \mathscr{W}_{B}^{c} \longrightarrow h G \mathscr{W}_{B}^{c}$ on homotopy categories, by Propositions 9.2.2 and 9.3.1, it suffices to construct a right adjoint $f_{*}: h G_{A}^{c} \longrightarrow h \mathscr{W}_{A}^{c}$. We do that using the Brown representability theorem. By Theorem 7.5.17, Ho $G \mathscr{K}_{B}^{c}$ satisfies the formal hypotheses for Brown representability, and therefore so does $h G^{2} \mathscr{W}_{B}^{c}$. In fact $G \mathscr{W}_{B}^{c}$ has the required wedges over $B$, double mapping cylinders and telescopes since these constructions preserve ex-fibrations by Proposition 8.2.1 and Corollary 8.2.5 and since they clearly preserve $G$-CW homotopy types on the total space level and stay within $G \mathscr{U}_{B}$. The objects in the detecting set $\mathscr{D}_{B}$ of Definition 7.5 .12 are not in $G \mathscr{W}_{B}$, but we can apply the ex-fibrant approximation functor $P$ to them to obtain a detecting set of objects in $h G \mathscr{W}_{B}$. They still satisfy the requisite compactness by Lemma 7.5.14 and our equivalence of categories. Therefore a contravariant set-valued functor on $h G \mathscr{W}_{B}^{c}$ is representable if and only if it satisfies the wedge and Mayer-Vietoris axoms.

For a fixed ex-fibrant space $X$ over $A$, consider the functor $\pi\left(f^{*} Y, X\right)_{G, A}$ on objects $Y$ of $G \mathscr{W}_{B}^{c}$, where $\pi$ denotes $f p$-homotopy classes of maps. Since the functor $\pi(W, X)_{G, A}$ on objects $W$ of $G \mathscr{W}_{A}^{c}$ is represented and is computed using homotopy classes of maps, it clearly satisfies the wedge and Mayer-Vietoris axioms. Moreover, the functor $f^{*}$ preserves wedges, double mapping cylinders, and telescopes. Indeed, $f^{*}: G \mathscr{K}_{B}^{c} \longrightarrow G \mathscr{K}_{A}^{c}$ is a continuous left adjoint and therefore preserves colimits and tensors, and this implies that $f^{*}: G \mathscr{W}_{B}^{c} \longrightarrow G \mathscr{W}_{A}^{c}$ preserves the relevant homotopy colimits. Moreover, $f^{*}$ preserves $f p$-homotopies and so induces a functor on homotopy categories that still preserves these homotopy colimits. Therefore the functor $\pi\left(f^{*} Y, X\right)_{G, A}$ of $Y$ also satisfies the wedge and Mayer-Vietoris axioms. We conclude that there is an object $f_{*} X \in G \mathscr{W}_{B}^{c}$ that represents this functor. It follows formally that $f_{*}$ is a functor of $X$ and that the required adjunction holds.

REMARK 9.3.3. When thinking in terms of $\mathrm{HoG} \mathscr{K}_{B}$ rather than $h G \mathscr{W}_{B}$, it is vital to remember that $f^{*}$ here refers to the right derived functor on homotopy categories. While $f^{*}$ preserves some cofibrant objects, it still cannot be computed in terms of such point-set level objects. For example, working nonequivariantly, if $f$ is an inclusion, then, on the point-set level, $f^{*} S^{n, b} \cong S^{n, a}$ if $f(a)=b$ while $f^{*} S^{n, b} \cong *_{A}$ if $b \notin \operatorname{Im}(f)$. Our adjunction gives

$$
\left[f^{*} S^{n, b}, X\right]_{A}=\left[S^{n, b}, f_{*} X\right]_{B}=\pi_{n}\left(\left(f_{*} X\right)_{b}\right) .
$$

Clearly, this cannot have the erroneously predicted value $\pi_{n}\left(X_{a}\right)$ if $f(a)=b$ and 0 otherwise. In particular, if $A$ and $B$ are connected and $A$ is not all of $B$, this would imply that $\pi_{n}\left(X_{a}\right)=0$ for all $a$ and any $X$, which is nonsense.

We agree to write $\simeq$ for natural equivalences on homotopy categories.

REMARK 9.3.4. For composable maps $f$ and $g, g_{*} \circ f_{*} \simeq(g \circ f)_{*}$ on homotopy categories since $f^{*} \circ g^{*} \simeq(g \circ f)^{*}$ on homotopy categories. The latter equivalence 
is clear since $f^{*}$ and $g^{*}$ are derived from Quillen right adjoints. More sophisticated commutation laws are proven in the next section.

Applying Theorem 9.3.2 to diagonal maps and composing with the homotopy category level adjunction between the external smash product and function ex-space functors, we obtain the following basic result; compare Lemma 2.5.6. Implicitly, in everything that follows in this section and the next, we restrict to $G$-connected ex-spaces whenever we apply base change functors $f_{*}$, such as $\Delta_{*}$. Conclusions that do not involve these functors apply in general.

Theorem 9.3.5. Define $\wedge_{B}$ and $F_{B}$ on $H o G \mathscr{K}_{B}$ as the composite (derived) functors

$$
X \wedge_{B} Y=\Delta^{*}(X \bar{\wedge} Y) \quad \text { and } \quad F_{B}(X, Y)=\bar{F}\left(X, \Delta_{*} Y\right) .
$$

Then

$$
\left[X \wedge_{B} Y, Z\right]_{G, B} \cong\left[X, F_{B}(Y, Z)\right]_{G, B}
$$

for $X, Y$, and $Z$ in $H o G \mathscr{K}_{B}^{c}$.

Proof. The displayed adjunction is the composite of adjunctions for the (derived) external smash and function ex-space functors and for the (derived) adjoint pair $\left(\Delta^{*}, \Delta_{*}\right)$.

REMARK 9.3.6. The referee (of the preliminary version) pointed out that the ex-space analogue of $[22,7.2]$ shows that we can work directly with the pointset topology to show that the $\left(\wedge_{B}, F_{B}\right)$ adjunction on the original category $G \mathscr{K}_{B}$ is continuous and so descends to (classical) $f p$-homotopy categories to give the adjunction

$$
h G \mathscr{K}_{B}\left(X \wedge_{B} Y, Z\right) \cong h G \mathscr{K}_{B}\left(X, F_{B}(Y, Z)\right) .
$$

Similar point-set topological arguments work to show that, for a map $f: A \longrightarrow B$, we have an adjunction

$$
h G \mathscr{K}_{A}\left(f^{*} X, Y\right) \cong h G \mathscr{K}_{B}\left(X, f_{*} Y\right) .
$$

These adjunctions do not imply our Theorems 9.3.2 and 9.3.5. By definition, our category $h G \mathscr{W}_{B}$ is a full subcategory of $h G \mathscr{K}_{B}$, but it is not an equivalent full subcategory. The objects of $G \mathscr{W}_{B}$ are very restricted, and general function exspaces $F_{B}(Y, Z)$ are not $f p$-homotopy equivalent to such objects. The force of our theorems is that, after restricting to our subcategories $h G \mathscr{W}_{B}^{c}$, we still have right adjoints in these categories. It is this fact that we need to obtain right adjoints in our preferred homotopy categories Ho $G \mathscr{K}_{B}^{c}$.

\subsection{Compatibility relations for smash products and base change}

We first prove that Ho $G \mathscr{K}_{B}$ satisfies the associativity, commutativity and unity conditions required of a symmetric monoidal category. We then show that all of the isomorphisms of functors in Proposition 2.2.2 and some of the isomorphisms of functors in Proposition 2.2.11 still hold after passage to homotopy categories. Finally, we relate change of groups and passage to fibers to the symmetric monoidal structure on homotopy categories. In some of our arguments, it is natural to work in Ho $G \mathscr{K}_{B}$. In others, it is natural to work in the equivalent category $h G \mathscr{W}_{B}$. 
Proposition 9.4.1. For maps $f: A \longrightarrow B$ and $g: A^{\prime} \longrightarrow B^{\prime}$ of base spaces and for ex-spaces $X$ over $B$ and $Y$ over $B^{\prime}$,

$$
\left(f^{*} Y \bar{\wedge} g^{*} Z\right) \simeq(f \times g)^{*}(Y \bar{\wedge} Z)
$$

in HoG $\mathscr{K}_{A}$. For ex-spaces $W$ over $A$ and $X$ over $A^{\prime}$,

$$
\left(f_{!} W \bar{\wedge} g_{!} X\right) \simeq(f \times g)_{!}(W \bar{\wedge} X)
$$

in $\mathrm{Ho}_{\mathrm{G}} \mathscr{K}_{B}$.

Proof. For (9.4.2), we work with ex-fibrations, starting in $h G \mathscr{W}_{B \times B^{\prime}}$. By Propositions 8.2.2 and 8.2.3, the functors we are dealing with preserve ex-fibrations and therefore descend straight to homotopy categories. The conclusion is thus immediate from its point-set level analogue. For (9.4.3), we work with model theoretic homotopy categories, starting in Ho $G \mathscr{K}_{A \times A^{\prime}}$. Since $(f \times g) ! \simeq(f \times$ id $) ! \circ($ id $\times g)$ !, we can proceed in two steps and so assume that $g=$ id. By Corollary 7.3.3 and Proposition 7.3.4, we are then composing Quillen left adjoints. Starting with $q f$ cofibrant objects, we do not need to apply $q f$-cofibrant approximation, and the conclusion follows directly from its point-set level analogue.

We use this to complete the proof that $\mathrm{Ho}_{G} \mathscr{K}_{B}$ is symmetric monoidal.

TheOREM 9.4.4. The category Ho G $\mathscr{K}_{B}$ is symmetric monoidal under the functor $\wedge_{B}$, and $H o G \mathscr{K}_{B}^{c}$ is closed symmetric monoidal under $F_{B}$.

Proof. In view of Theorem 9.3.5, we need only prove the associativity, commutativity, and unity of $\wedge_{B}$ up to coherent natural isomorphism. The external smash product has evident associativity, commutativity, and unity isomorphisms, and these descend directly to homotopy categories, either by using that the external smash produce of $q f$-cofibrant ex-spaces over $A$ and $B$ is $q f$-cofibrant over $A \times B$ or by using that the external smash product of $K \in G \mathscr{W}_{A}$ and $L \in G \mathscr{W}_{B}$ is in $G \mathscr{W}_{A \times B}$. To see that these isomorphisms are inherited after internalization along $\Delta^{*}$, we use (9.4.2). For the associativity of $\wedge_{B}$, we have

$$
\begin{aligned}
& \Delta^{*}\left(\Delta^{*}(X \bar{\wedge} Y) \wedge Z\right) \simeq \Delta^{*}(\Delta \times \mathrm{id})^{*}((X \bar{\wedge} Y) \bar{\wedge} Z) \simeq((\Delta \times \mathrm{id}) \Delta)^{*}((X \bar{\wedge} Y) \wedge Z) \\
& \quad \simeq((\operatorname{id} \times \Delta) \Delta)^{*}(X \bar{\wedge}(Y \wedge Z)) \simeq \Delta^{*}(\operatorname{id} \times \Delta)^{*}(X \bar{\wedge}(Y \bar{\wedge} Z)) \simeq \Delta^{*}\left(X \wedge \Delta^{*}(Y \wedge Z)\right) .
\end{aligned}
$$

The commutativity of $\wedge_{B}$ is similar but simpler. For the unit, we observe that $S_{B}^{0} \simeq r^{*} S^{0}, r: B \longrightarrow *$. Therefore, since $(\mathrm{id} \times r) \Delta=\mathrm{id}$,

$$
X \wedge_{B} S_{B}^{0} \simeq \Delta^{*}\left(X \bar{\wedge} r^{*} S^{0}\right) \simeq \Delta^{*}(\operatorname{id} \times r)^{*}\left(X \bar{\wedge} S^{0}\right) \simeq((\operatorname{id} \times r) \Delta)^{*}(X)=X .
$$

We turn next to the derived versions of the base change compatibilities of Propositions 2.2.2 and 2.2.11. Observe that the functor $f_{!}$is good since the section of a well-sectioned ex-space is an $h$-cofibration and since $G$-CW homotopy types are preserved under pushouts one leg of which is an $h$-cofibration. Moreover, $f$ ! preserves $q$-equivalences between well-sectioned ex-spaces by Proposition 7.3.4. Therefore Proposition 9.2.2 applies to $f_{!}$. In the remaining results, when we refer to closed structures and to right adjoints to base change functors $f^{*}$, restriction to $G$-connected ex-spaces is assumed implicitly.

Theorem 9.4.5. For a G-map $f: A \longrightarrow B, f^{*}: H o G \mathscr{K}_{B} \longrightarrow$ HoG $\mathscr{K}_{A}$ is a closed symmetric monoidal functor. 
Proof. The isomorphism $f^{*} S_{B}^{0} \cong S_{A}^{0}$ implies the equivalence $f^{*} S_{B}^{0} \simeq S_{A}^{0}$ since $S_{B}^{0}$ is $q f$-fibrant. Alternatively, since $S_{B}^{0}$ is in $G \mathscr{W}_{B}, f^{*} S_{B}^{0} \cong S_{A}^{0}$ is in $G \mathscr{W}_{A}$, and the isomorphism implies that $f^{*} S_{B}^{0} \simeq S_{A}^{0}$ in $h G^{2} \mathscr{W}_{A}$. We must prove that the isomorphisms (2.2.3) through (2.2.7) descend to equivalences on homotopy categories. Categorical arguments in $[62, \S \S 2,3]$ show that it suffices to show that the two isomorphisms (2.2.3) and (2.2.6) descend to equivalences on homotopy categories. These two isomorphisms do not involve the right adjoints $f_{*}$ or $\Delta_{*}$ and are therefore more tractable than the other three (which require $G$-connected ex-spaces). First consider (2.2.3):

$$
f^{*}\left(Y \wedge_{B} Z\right) \cong f^{*} Y \wedge_{A} f^{*} Z
$$

If $Y$ and $Z$ are in $G \mathscr{W}_{B}$, then the two sides of this isomorphism are both in $G \mathscr{W}_{A}$, by Proposition 8.2.2 and Proposition 8.2.3. Therefore the point-set level isomorphism descends directly to the desired homotopy category level equivalence. Next, consider (2.2.6):

$$
f_{!}\left(f^{*} Y \wedge_{A} X\right) \cong Y \wedge_{B} f_{!} X
$$

Assume that $X$ is in $G \mathscr{W}_{A}$ and $Y$ is in $G \mathscr{W}_{B}$. The functor $f_{!}$does not preserve exfibrations so, to pass to derived categories, we must replace it by $P f$ ! on both sides. By Proposition 8.2.6, the functor $Y \wedge_{B}(-)$ preserves $h$-equivalences between wellsectioned ex-spaces. Since $P$ sends $h$-equivalences to $f p$-equivalences, we therefore have $f p$-equivalences, natural up to $f p$-homotopy,

$$
P f_{!}\left(f^{*} Y \wedge_{A} X\right) \cong P\left(Y \wedge_{B} f_{!} X\right) \stackrel{P\left(\mathrm{id} \wedge_{B} \phi\right)}{\longrightarrow} P\left(Y \wedge_{B} P f_{!} X\right){ }^{\phi} Y \wedge_{B} P f_{!} X,
$$

where $\phi=(\rho, W \iota)$ is the zigzag of $h$-equivalences of Definition 8.3.4. This implies the desired equivalence in the homotopy category.

The reader is invited to try to prove directly that the projection formula holds in the homotopy category. Even the simple case of $f: * \longrightarrow B$, the inclusion of a point, should demonstrate the usefulness of Proposition 9.2.2.

TheOREM 9.4.6. Suppose given a pullback diagram of $G$-spaces

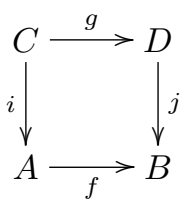

in which $f$ (or $j$ ) is a q-fibration. Then there are natural equivalences of functors on homotopy categories

$$
j^{*} f_{!} \simeq g_{!} i^{*}, \quad f^{*} j_{*} \simeq i_{*} g^{*}, \quad f^{*} j_{!} \simeq i_{!} g^{*}, \quad j^{*} f_{*} \simeq g_{*} i^{*} .
$$

Proof. As in Proposition 2.2.11, the second and fourth equivalences (which again require $G$-connected ex-spaces) are conjugate to the first and third. However, since the situation is no longer symmetric, we must prove both the first and third equivalences, assuming $f$ is a $q$-fibration.

First consider the desired equivalence $f^{*} j_{!} \simeq i_{!} g^{*}$. We work with ex-fibrations, starting with $X \in h G \mathscr{W}_{D}$. We must replace $j_{\text {! }}$ and $i_{\text {! }}$ by $P j_{\text {! }}$ and $P i_{\text {! }}$ before passing to homotopy categories. By Proposition 7.3.4, $f^{*}$ preserves $q$-equivalences since $f$ is a $q$-fibration. Moreover, our $q$-equivalences are $h$-equivalences since we are dealing with total spaces of the homotopy types of $G$-CW complexes. By the diagram in 
Lemma 8.4.3(ii), we see that $\mu: P f^{*} \longrightarrow f^{*} P$ is a natural $h$-equivalence here. This would be false for arbitrary maps $f$, as observed in Warning 8.4.4. Since $\mu$ is an $h$-equivalence between ex-fibrations, it is an $f p$-equivalence. Therefore

$$
f^{*} P j_{!} X \simeq P f^{*} j_{!} X \cong P i_{!} g^{*} X
$$

Now consider the desired equivalence $j^{*} f_{!} X \simeq g_{!} i^{*} X$ in Ho $G \mathscr{K}_{D}$. Our assumption that $f$ is a $q$-fibration gives us no direct help with this. However, we may factor $j$ as the composite of a homotopy equivalence and an $h$-fibration. Expanding our pullback diagram as a composite of pullbacks, we see that it suffices to prove our commutation relation when $j$ is an $h$-fibration and when $j$ is a homotopy equivalence. The first case is immediate by symmetry from the first part. Thus assume that $j$ is a homotopy equivalence. Then, since $f$ is a $q$-fibration, $i$ is also a homotopy equivalence. By Proposition 7.3.4, $\left(i_{!}, i^{*}\right)$ and $\left(j_{!}, j^{*}\right)$ are adjoint equivalences of homotopy categories. Therefore

$$
j^{*} f_{!} \simeq j^{*} f_{!} i_{!} i^{*} \simeq j^{*} j_{!} g ! i^{*} \simeq g_{!} i^{*} .
$$

REMARK 9.4.8. As illustrated in this proof, it is a useful general procedure to factor a map $f: A \longrightarrow B$ as the composite of a homotopy equivalence and a Hurewicz fibration. If $f$ is an equivalence, then $f$ ! on derived categories is an equivalence with inverse $f^{*}$, hence $f$ ! is equivalent to $g^{*}$ for a homotopy inverse $g$ of $f$. Therefore good properties of $(-)^{*}$ translate to good properties of $(-)$ !. When $f$ is a fibration, we have derived commutation relations coming from Theorem 9.4.6 that can help in the study of $f_{!}$. Note, however, that this factorization procedure cannot be used to get around the fibration hypothesis in Theorem 9.4.6. It was our attempt to achieve this that led to the discovery of Counterexample 0.0.1.

Finally, we turn to a promised compatibility relationship between products and change of groups. We observed in Proposition 7.4.7 that the point-set level closed symmetric monoidal equivalence of Proposition 2.3.9 is given by a Quillen equivalence. The following addendem shows that the resulting equivalence on homotopy categories is again closed symmetric monoidal.

Proposition 9.4.9. Let $\iota: H \longrightarrow G$ be the inclusion of a subgroup and $A$ be an $H$-space. The Quillen equivalence $\left(\iota_{!}, \nu^{*} \iota^{*}\right)$ descends to a closed symmetric monoidal equivalence between $H o H \mathscr{K}_{A}$ and $H o G \mathscr{K}_{L ! A}$.

Proof. Let $\Delta: A \longrightarrow A \times A$ be the diagonal map. The isomorphisms

$$
\iota^{*} \Delta^{*}(X \bar{\wedge} Y) \cong \Delta^{*} \iota^{*}(X \bar{\wedge} Y) \cong \Delta^{*}\left(\iota^{*} X \bar{\wedge} \iota^{*} Y\right)
$$

descend to equivalences on homotopy categories, the first since it is between Quillen right adjoints, the second since $\iota^{*}$ preserves all $q$-equivalences. It follows that $\nu^{*} \iota^{*}$ is a symmetric monoidal functor on homotopy categories. Since it is also an equivalence, it follows formally that it is closed symmetric monoidal.

Combined with Theorem 9.4.5 applied to the inclusion $\tilde{b}: G / G_{b} \longrightarrow B$, this last observation gives us the following conclusion.

TheOREM 9.4.10. The derived fiber functor $(-)_{b}: H_{o} G \mathscr{K}_{B} \longrightarrow H_{o} G_{b} \mathscr{K}_{b}$ is closed symmetric monoidal, and it has a left adjoint $(-)^{b}$ and a right adjoint ${ }^{b}(-)$.

We emphasize that this innocent looking result packages highly non-trivial and important information. It gives in particular that, for ex-spaces $X$ and $Y$, the 
(derived) fiber $F_{B}(X, Y)_{b}$ of the (derived) function space $F_{B}(X, Y)$ is equivalent in Ho $G_{b} \mathscr{K}_{b}$ to the (derived) function space $F\left(X_{b}, Y_{b}\right)$ of the (derived) fibers $X_{b}$ and $Y_{b}$. On the point set level, that is what motivated the definition of the internal function ex-space. That it still holds on the level of homotopy categories is a reassuring consistency result. The annoying implicit restriction to $G$-connected ex-spaces will disappear when we pass to a stable situation in Part III. 


\section{Part III}

\section{Parametrized equivariant stable homotopy theory}





\section{Introduction}

We develop rigorous foundations for parametrized equivariant stable homotopy theory. The idea is to start with a fixed base $G$-space $B$ and to build a good category, here denoted $G \mathscr{S}_{B}$, of $G$-spectra over $B$. We assume once and for all that our base spaces $B$ must be compactly generated and must have the homotopy types of $G$-CW complexes. By "good" we mean that $G \mathscr{S}_{B}$ is a closed symmetric monoidal topological model category whose associated homotopy category has properties analogous to those of the ordinary equivariant stable homotopy category. Informally, the homotopy theory of $G \mathscr{S}_{B}$ is specified by the homotopy theory that is seen on the fibers of $G$-spectra over $B$. This builds a natural home in which one can do stable homotopy theory while still keeping track of such unstable data as the fundamental groupoid of the base space.

There are many foundational problems that make the development of the parametrized theory a less than obvious generalization of the nonparametrized theory. Problems on the space level were dealt with in Parts I and II, and we deal with the analogous spectrum level problems here. We give some categorical preliminaries on enriched equivariant categories in Chapter 10. We define and develop the basic properties of our preferred category of parametrized $G$-spectra in Chapter 11, working on the point-set level. We study its model structures in Chapter 12, and we study adjunctions and compatibility relations in Chapter 13. All of the problems that we faced on the space level are still there, but their solutions are more difficult. In Chapter 14, we go on to study further such compatibilities that more fundamentally involve equivariance.

The theory of highly structured spectra is highly cumulative. We build on the theory of equivariant orthogonal spectra of Mandell and May [105]. In turn, that theory builds on the theory of nonequivariant orthogonal spectra. A self-contained treatment of nonequivariant diagram spectra, including orthogonal spectra, is given by Mandell, May, Schwede, Shipley in [106]. The treatments of [105] and [106], like this one, are topological as opposed to simplicial. That seems to be essential when dealing with infinite groups of equivariance. It also allows use of orthogonal spectra rather than symmetric spectra. These are much more natural equivariantly and, even nonequivariantly, they have the major convenience that their weak equivalences are exactly the maps that induce isomorphisms of homotopy groups.

The theory of equivariant parametrized spectra can be thought of as the pushout over the theory of spectra of the theories of equivariant spectra and of nonequivariant parametrized spectra. In fact, given [105], the equivariance adds few serious difficulties to the passage from spectra to parametrized spectra, although it does add many interesting new features. The reader primarily interested in classical homotopy theory should ignore all details of equivariance in reading Chapters 11-13. 
However, there is no nonequivariant precursor of the present treatment of parametrized spectra in the literature. There are preliminary forms of such a theory $[9,10,31,32,42]$, but these either do not go beyond suspension spectra or are based on obsolescent technology. None go far enough for our purposes, although the early first approximation of Monica Clapp [31], written up in more detail with Dieter Puppe [32], deserves much credit. Clapp's emphasis on ex-fibrations, together with some key technical results about them, have been very helpful.

There are several possible alternative approaches. Rather than building on the theory of orthogonal $G$-spectra of $[105,106]$, one can build on the theory of $G$-spectra, $S$-modules, and $S_{G}$-modules of [98], [61], and [105], respectively. Po Hu [78] began work on such a treatment, using parametrized $G$-spectra. However, she followed the first author's unpublished notes [122] by taking the $q$-model structure on ex-G-spaces as her starting point, and the stable model structure cannot be made rigorous from there. It seems to us that resolving the foundational issues concerning smash products, function spectra, base change functors, compatibility relations, and so forth considered here would be more difficult in that framework than in the one we have chosen. In $\S 24.4$, we at least give a rigorous definition of the parametrized stable homotopy category from that point of view.

Alternatively, for finite groups $G$, one can build on the theory of symmetric spectra of Hovey, Smith, and Shipley [77] and its equivariant generalization due to Mandell [104]. Such an approach would avoid the point-set topological technicalities of the present approach and would presumably lead to rather different looking problems with fibrations and cofibrations. The problems with the stable homotopy category level adjunctions that involve base change functors, smash products, and function spectra are intrinsic and would remain. Our solutions to these problems do not seem to carry over to the simplicial context in an obvious way, and an alternative simplicial treatment could prove to be quite illuminating.

In view of the understanding of unstable equivariant homotopy theory for proper actions of non-compact Lie groups that was obtained in Part II, it might seem that there should be no real difficulty in obtaining a good stable theory along the same lines as the theory for compact Lie groups. However, equivariant stable homotopy theory for non-compact Lie groups is in rudimentary form. We leave its study to future work, explaining in $\$ 11.6$ where some of the problems lie. Except in that section, $G$ is asssumed to be a compact Lie group from Chapter 11 onwards.

A few other notes on terminology may be helpful. We shall not use the term "ex-spectrum over $B$ " since, stably, there is no meaningful unsectioned theory. Instead, we shall use the term "spectrum over $B$ ". This is especially convenient when considering base change. We write out "orthogonal $G$-spectrum over $B$ " until $§ 11.4$. However, since we consider no other kinds of $G$-spectra and work equivariantly throughout, we later abbreviate this to "spectrum over $B$ " when there is no danger of confusion. That is, we work equivariantly throughout, but we only draw attention to this fact when it plays a significant mathematical role.

In Part II, the main objects of study were ex-spaces over $B$, and we used letters such as $X$ and $Y$ for them, using $K$ and $L$ for spaces. In this part, the main objects of study are spectra over $B$, and we change conventions by now using letters such as $X$ and $Y$ for them, using letters such as $K$ and $L$ for ex-spaces, and using letters such as $T$ for based spaces. 


\section{CHAPTER 10}

\section{Enriched categories and $G$-categories}

\section{Introduction}

To give context for the structure enjoyed by the categories of parametrized orthogonal $G$-spectra that we shall define, we first describe the kind of equivariant parametrized enrichments that we shall encounter. In fact, our categories have several layers of enrichment, and it is helpful to have a consistent language, somewhat non-standard from a categorical point of view, to keep track of them. In $\S \S 10.1$ and 10.2, we give some preliminaries on enriched categories, working nonequivariantly in $\S 10.1$ and adding considerations of equivariance in $\S 10.2$. We discuss the role of the several enrichments in sight in our $G$-topological model $G$-categories in $\$ 10.3$. In this chapter, $G$ can be any topological group.

\subsection{Parametrized enriched categories}

As discussed in $\S 1.2$, all of our categories $\mathscr{C}$ are topological, meaning that they are enriched over the category $\mathscr{K}_{*}$ of based spaces (= $k$-spaces). In contrast with general enriched category theory and our further enrichments, the topological enrichment is given just by a topology on the underlying set of morphisms, and we denote the space of morphisms $X \longrightarrow Y$ by $\mathscr{C}(X, Y)$. We say that a topological category $\mathscr{C}$ is topologically bicomplete if it is bicomplete and bitensored over $\mathscr{K}_{*}$. In fact, we shall have enrichments and bitensorings over the category $\mathscr{K}_{B}$ of exspaces over $B$ that imply the topological enrichment and bitensoring by restriction to ex-spaces $B \times T$ for $T \in \mathscr{K}_{*}$.

Recall from $\S 1.3$ that $\mathscr{K}_{B}$ is topologically bicomplete, with tensors and cotensors denoted by $K \wedge_{B} T$ and $F_{B}(T, K)$ for $T \in \mathscr{K}_{*}$ and $K \in \mathscr{K}_{B}$. It is also closed symmetric monoidal under its fiberwise smash product and function space functors, which are also denoted by $\wedge_{B}$ and $F_{B}$; its unit object is $S_{B}^{0}=B \times S^{0}$. It is therefore enriched and bitensored over itself. The two enrichments are related by natural based homeomorphisms

$$
\mathscr{K}_{B}(K, L) \cong \mathscr{K}_{B}\left(S_{B}^{0}, F_{B}(K, L)\right) .
$$

This is the case $T=S^{0}$ of the more general based homeomorphism

$$
\mathscr{K}_{*}\left(T, \mathscr{K}_{B}(K, L)\right) \cong \mathscr{K}_{B}\left(S_{B}^{0} \wedge_{B} T, F_{B}(K, L)\right)
$$

for $T \in \mathscr{K}_{*}$ and $K, L \in \mathscr{K}_{B}$. The Yoneda lemma, (10.1.1), and the bitensoring adjunctions imply that the two bitensorings are related by the equivalent natural isomorphisms of ex-spaces

$$
K \wedge_{B} T \cong K \wedge_{B}\left(S_{B}^{0} \wedge_{B} T\right) \quad \text { and } \quad F_{B}(T, K) \cong F_{B}\left(S_{B}^{0} \wedge_{B} T, K\right)
$$

These in turn imply the equivalent generalizations

(10.1.4) $K \wedge_{B}\left(L \wedge_{B} T\right) \cong\left(K \wedge_{B} L\right) \wedge_{B} T$ and $F_{B}\left(T, F_{B}(K, L)\right) \cong F_{B}\left(K \wedge_{B} T, L\right)$. 
Formally, rather than defining the enrichments and bitensorings over $\mathscr{K}_{*}$ independently, we can take (10.1.2) and (10.1.3) as definitions of these structures in terms of the enrichment and bitensoring over $\mathscr{K}_{B}$. Then (10.1.4) and the bitensoring adjunction homeomorphisms

$$
\mathscr{K}_{B}\left(K \wedge_{B} T, L\right) \cong \mathscr{K}_{*}\left(T, \mathscr{K}_{B}(K, L)\right) \cong \mathscr{K}_{B}\left(K, F_{B}(T, L)\right)
$$

follow directly.

REMARK 10.1.6. We make frequent use of the functor $r^{*}(-)=S_{B}^{0} \wedge_{B}(-)$ from based spaces to ex-spaces over $B$, and we henceforward abbreviate notation by setting

$$
T_{B}=B \times T=S_{B}^{0} \wedge_{B} T
$$

for a based space $T$, and similarly for maps. Observe that $K \wedge_{B} T$ and $K \wedge_{B} T_{B}$ are two names for the same ex-space over $B$. When working on a formal conceptual level, it is often best to think in terms of tensors over $\mathscr{K}_{*}$ and use the first name. However, on a pragmatic level, to avoid confusion, it is perhaps best to view based spaces as embedded in ex-spaces via $r^{*}$ and to use the second notation, working only with tensors over $\mathscr{K}_{B}$.

We generalize and formalize several aspects of the discussion above.

DeFinition 10.1.7. A topological category $\mathscr{C}$ is topological over $B$ if it is enriched and bitensored over $\mathscr{K}_{B}$. It is topologically bicomplete over $B$ if it is also bicomplete. We write $P_{B}(X, Y)$ for the hom ex-space over $B$, and we write $X \wedge_{B} K$ and $F_{B}(K, X)$ for the tensor and cotensor in $\mathscr{C}$, where $X, Y \in \mathscr{C}$ and $K \in \mathscr{K}_{B}$. Explicitly, we require bitensoring adjunction homeomorphisms of based spaces

$$
\mathscr{C}\left(X \wedge_{B} K, Y\right) \cong \mathscr{K}_{B}\left(K, P_{B}(X, Y)\right) \cong \mathscr{C}\left(X, F_{B}(K, Y)\right) .
$$

By Yoneda lemma arguments, these imply unit and transitivity isomorphisms in $\mathscr{C}$, namely

$$
X \cong X \wedge_{B} S_{B}^{0} \text { and } X \wedge_{B}\left(K \wedge_{B} L\right) \cong\left(X \wedge_{B} K\right) \wedge_{B} L
$$

and also bitensoring adjunction isomorphisms of ex-spaces

$$
P_{B}\left(X \wedge_{B} K, Y\right) \cong F_{B}\left(K, P_{B}(X, Y)\right) \cong P_{B}\left(X, F_{B}(K, Y)\right) .
$$

Conversely, there is a natural homeomorphism

$$
\mathscr{C}(X, Y) \cong \mathscr{K}_{B}\left(S_{B}^{0}, P_{B}(X, Y)\right),
$$

and the isomorphisms (10.1.8) follow from (10.1.10) by applying $\mathscr{K}_{B}\left(S_{B}^{0},-\right)$.

If we do not require $\mathscr{C}$ to be topological to begin with, we can take (10.1.11) as the definition of the space $\mathscr{C}(X, Y)$ and so recover the topological enrichment. With the notation of Remark 10.1.6, we obtain tensors and cotensors with based spaces $T$ by setting

$$
X \wedge_{B} T=X \wedge_{B} T_{B} \quad \text { and } \quad F_{B}(T, X)=F_{B}\left(T_{B}, X\right) .
$$

The adjunction homeomorphisms

$$
\mathscr{C}\left(X \wedge_{B} T, Y\right) \cong \mathscr{K}_{*}(T, \mathscr{C}(X, Y)) \cong \mathscr{C}\left(X, F_{B}(T, Y)\right)
$$

are obtained by replacing $K$ by $T_{B}$ in (10.1.8) and using (10.1.2) and (10.1.11).

In the cases of interest, $\mathscr{C}$ is closed symmetric monoidal, and then the hom ex-spaces $P_{B}(X, Y)$ can be understood in terms of the internal hom in $\mathscr{C}$ by the following definition and result. 
Definition 10.1.14. Let $\mathscr{C}$ be a topological category over $B$ with a closed symmetric monoidal structure given by a product $\wedge_{B}$ and function object functor $F_{B}$, with unit object $S_{B}$. We say that $\mathscr{C}$ is a topological closed symmetric monoidal category over $B$ if the tensors and products are related by a natural isomorphism

$$
X \wedge_{B} K \cong X \wedge_{B}\left(S_{B} \wedge_{B} K\right)
$$

in $\mathscr{C}$ for $K \in \mathscr{K}_{B}$ and $X \in \mathscr{C}$.

Proposition 10.1.15. Let $\mathscr{C}$ be a topological closed symmetric monoidal category over $B$. Then, for $K \in \mathscr{K}_{B}$ and $X, Y, Z \in \mathscr{C}$, there are natural isomorphisms

$$
\begin{gathered}
F_{B}(K, Y) \cong F_{B}\left(S_{B} \wedge_{B} K, Y\right), \\
P_{B}(X, Y) \cong P_{B}\left(S_{B}, F_{B}(X, Y)\right), \\
P_{B}\left(X \wedge_{B} Y, Z\right) \cong P_{B}\left(X, F_{B}(Y, Z)\right)
\end{gathered}
$$

in $\mathscr{C}$ and a natural homeomorphism of based spaces

$$
\mathscr{K}_{B}\left(K, P_{B}(X, Y)\right) \cong \mathscr{C}\left(S_{B} \wedge_{B} K, F_{B}(X, Y)\right) .
$$

\subsection{Equivariant parametrized enriched categories}

Turning to the equivariant generalization, we give details of the context of topological $G$-categories, continuous $G$-functors, and natural $G$-maps that we first alluded to in $\S 1.4$. The discussion elaborates on that given in [105, II $\S 1]$. Generically, we use notations of the form $\mathscr{C}_{G}$ and $G \mathscr{C}$ to denote a category $\mathscr{C}_{G}$ enriched over the category $G \mathscr{K}_{*}$ of based $G$-spaces and its associated " $G$-fixed category" $G \mathscr{C}$ with the same objects and the $G$-maps between them; $G \mathscr{C}$ is enriched over $\mathscr{K}_{*}$. We shall write $\left(\mathscr{C}_{G}, G \mathscr{C}\right)$ for such a pair, and we shall refer to the pair as a "G-category".

In the terminology of enriched category theory, $G \mathscr{C}$ is the underlying topological category of $\mathscr{C}_{G}$. The hom objects of $\mathscr{C}_{G}$ are $G$-spaces $\mathscr{C}_{G}(X, Y) ; G$-functors and natural $G$-maps just mean functors and natural transformations enriched over $G \mathscr{K}_{*}$. Consistently with enriched category theory, the space $G \mathscr{C}(X, Y)=\mathscr{C}_{G}(X, Y)^{G}$ can be identified with the space of $G$-maps $S^{0} \longrightarrow \mathscr{C}_{G}(X, Y)$. We call the points of $\mathscr{C}_{G}(X, Y)$ "arrows" to distinguish them from the points of $G \mathscr{C}(X, Y)$, which we call "G-maps", or often just "maps", with the equivariance understood.

We cannot expect $\mathscr{C}_{G}$ to have limits and colimits, but $G \mathscr{C}$ is usually bicomplete. In many of our examples, both $\mathscr{C}_{G}$ and $G \mathscr{C}$ are closed symmetric monoidal under functors $\wedge_{B}$ and $F_{B}$. For example, we have the closed symmetric monoidal $G$ category $\left(\mathscr{K}_{G, B}, G \mathscr{K}_{B}\right)$ of ex-G-spaces over a $G$-space $B$ described in $\S 1.4$.

Definition 10.2.1. A $G$-category $\left(\mathscr{C}_{G}, G \mathscr{C}\right)$ is $G$-topological over $B$ if $\mathscr{C}_{G}$ is enriched over $G \mathscr{K}_{B}$ and bitensored over $\mathscr{K}_{G, B}$. It follows that $G \mathscr{C}$ is enriched over $\mathscr{K}_{B}$ and bitensored over $G \mathscr{K}_{B}$. We say that $\left(\mathscr{C}_{G}, G \mathscr{C}\right)$ is $G$-topologically bicomplete over $B$ if, in addition, $G \mathscr{C}$ is bicomplete. We write $P_{B}(X, Y)$ for the hom ex- $G$ space over $B$, and we write $X \wedge_{B} K$ and $F_{B}(K, X)$ for the tensor and cotensor in $\mathscr{C}_{G}$, where $X, Y \in \mathscr{C}_{G}$ and $K \in \mathscr{K}_{G, B}$. Explicitly, we require bitensoring adjunction homeomorphisms of based $G$-spaces

$$
\mathscr{C}_{G}\left(X \wedge_{B} K, Y\right) \cong \mathscr{K}_{G, B}\left(K, P_{B}(X, Y)\right) \cong \mathscr{C}_{G}\left(X, F_{B}(K, Y)\right)
$$

There result coherent unit and transitivity isomorphisms in $G \mathscr{C}$

$$
X \cong X \wedge_{B} S_{B}^{0} \text { and } X \wedge_{B}\left(K \wedge_{B} L\right) \cong\left(X \wedge_{B} K\right) \wedge_{B} L
$$


and also bitensoring adjunction isomorphisms of ex- $G$-spaces

$$
P_{B}\left(X \wedge_{B} K, Y\right) \cong F_{B}\left(K, P_{B}(X, Y)\right) \cong P_{B}\left(X, F_{B}(K, Y)\right) .
$$

Conversely, there is a natural homeomorphism of based $G$-spaces

$$
\mathscr{C}_{G}(X, Y) \cong \mathscr{K}_{G, B}\left(S_{B}^{0}, P_{B}(X, Y)\right),
$$

and the isomorphisms (10.2.2) follow from (10.2.4) by applying $\mathscr{K}_{G, B}\left(S_{B}^{0},-\right)$. Passage to $G$-fixed points from $(10.2 .2)$ gives the bitensoring adjunction homeomorphisms of based spaces

$$
G \mathscr{C}\left(X \wedge_{B} K, Y\right) \cong G \mathscr{K}_{B}\left(K, P_{B}(X, Y)\right) \cong G \mathscr{C}\left(X, F_{B}(K, Y)\right) .
$$

We warn the reader that we shall not always adhere strictly to the notational pattern of Definition 10.2.1 for our several layers of enrichment. In particular, in the domain categories for our equivariant diagram spaces and diagram spectra, only $\mathscr{C}_{G}$ is of interest, not $G \mathscr{C}$, and our notations will reflect that. On the other hand, when studying model categories, it is always the bicomplete category $G \mathscr{C}$ that is of fundamental interest.

If $\left(\mathscr{C}_{G}, G \mathscr{C}\right)$ is $G$-topological over $B$, then it is automatically $G$-topological (over $*$ ). This enrichment is recovered by taking (10.1.11), read equivariantly, as the definition of the based $G$-space $\mathscr{C}_{G}(X, Y)$. Just as in the nonequivariant case, for based $G$-spaces $T$ and objects $X$ of $\mathscr{C}_{G}$, the tensors and cotensors in $\mathscr{C}_{G}$ and $G \mathscr{C}$ are given on objects by

$$
X \wedge_{B} T=X \wedge_{B} T_{B} \quad \text { and } \quad F_{B}(T, X)=F_{B}\left(T_{B}, X\right),
$$

using the notation of Remark 10.1.6 equivariantly. The required $G$-homeomorphisms

$$
\mathscr{C}_{G}\left(X \wedge_{B} T, Y\right) \cong \mathscr{K}_{G, *}\left(T, \mathscr{C}_{G}(X, Y)\right) \cong \mathscr{C}_{G}\left(X, F_{B}(T, Y)\right)
$$

follow directly.

We have equivariant analogues of Definition 10.1.14 and Proposition 10.1.15.

Definition 10.2.9. Let $\left(\mathscr{C}_{G}, G \mathscr{C}\right)$ be a $G$-topological $G$-category over $B$ with a closed symmetric monoidal structure given by a product $G$-functor $\wedge_{B}$ and a function object $G$-functor $F_{B}$, with unit object $S_{B}$. We say that $\left(\mathscr{C}_{G}, G \mathscr{C}\right)$ is a $G$-topological closed symmetric monoidal $G$-category over $B$ if the tensors and products are related by a natural isomorphism

$$
X \wedge_{B} K \cong X \wedge_{B}\left(S_{B} \wedge_{B} K\right)
$$

in $G \mathscr{C}$ for $K \in G \mathscr{K}_{B}$ and $X \in G \mathscr{C}$.

Proposition 10.2.10. Let $\left(\mathscr{C}_{G}, G \mathscr{C}\right)$ be a $G$-topological closed symmetric monoidal $G$-category over $B$. Then, for $K \in \mathscr{K}_{B}$ and $X, Y, Z \in \mathscr{C}$, there are natural isomorphisms

$$
\begin{gathered}
F_{B}(K, Y) \cong F_{B}\left(S_{B} \wedge_{B} K, Y\right), \\
P_{B}(X, Y) \cong P_{B}\left(S_{B}, F_{B}(X, Y)\right), \\
P_{B}\left(X \wedge_{B} Y, Z\right) \cong P_{B}\left(X, F_{B}(Y, Z)\right)
\end{gathered}
$$

in $G \mathscr{C}$ and there is a natural homeomorphism of based $G$-spaces

$$
\mathscr{K}_{G, B}\left(K, P_{B}(X, Y)\right) \cong \mathscr{C}_{G}\left(S_{B} \wedge_{B} K, F_{B}(X, Y)\right) .
$$




\section{3. $G$-topological model $G$-categories}

We explain what it means for a $G$-topological $G$-category $\left(\mathscr{C}_{G}, G \mathscr{C}\right)$ over $B$ to have a $G$-topological model structure. This structure implies in particular that the homotopy category $\operatorname{HoG} \mathscr{C}$ is bitensored over the homotopy category HoG $\mathscr{K}$. We need some notation. Throughout this section, we consider maps

$$
i: W \longrightarrow X, j: V \longrightarrow Z \text {, and } p: E \longrightarrow Y
$$

in $G \mathscr{C}$ and a map $k: K \longrightarrow L$ in either $G \mathscr{K}_{B}$ or $G \mathscr{K}_{*}$; in the latter case we apply the functor $(-)_{B}=B \times(-)$ to $k$ and so regard it as a map in $G \mathscr{K}_{B}$, as suggested in Remark 10.1.6. We shall define the notion of a $G$-topological model category in terms of the induced map

$$
\mathscr{C}_{G}^{\square}(i, p): \mathscr{C}_{G}(X, E) \longrightarrow \mathscr{C}_{G}(W, E) \times_{\mathscr{C}_{G}(W, Y)} \mathscr{C}_{G}(X, Y)
$$

of based $G$-spaces. Passing to $G$-fixed points, this gives rise to a map

$$
G \mathscr{C}^{\square}(i, p): G \mathscr{C}(X, E) \longrightarrow G \mathscr{C}(W, E) \times_{G \mathscr{C}(W, Y)} G \mathscr{C}(X, Y)
$$

of based spaces, and we have the following motivating observation.

LEMMA 10.3.3. The pair $(i, p)$ has the lifting property if and only if the function $G \mathscr{C}^{\square}(i, p)$ is surjective.

Definition 10.3.4. Let $\left(\mathscr{C}_{G}, G \mathscr{C}\right)$ be a $G$-topological $G$-category over $B$ such that $G \mathscr{C}$ is a model category. We say that the model structure is $G$-topological if $\mathscr{C}_{G}^{\square}(i, p)$ is a fibration in $G \mathscr{K}_{*}$ when $i$ is a cofibration and $p$ is a fibration and is acyclic when, further, either $i$ or $p$ is acyclic.

REMARK 10.3.5. The definition must refer consistently to either $h$-type or $q$ type model structures; the resulting notions are quite different. We usually have in mind a $q$-type model structure, in which case the weak equivalences and fibrations are often characterized by conditions on the $H$-fixed point maps $f^{H}$ of a map $f$. If $\mathscr{F}$ is a family of subgroups of $G$, such as the family $\mathscr{G}$ of compact subgroups, then we can restrict attention to those $H$ that are in $\mathscr{F}$. The resulting $\mathscr{F}$-equivalences and $\mathscr{F}$-fibrations usually specify another model structure on $G \mathscr{C}$. In particular, we have the $\mathscr{F}$-model structure on $G \mathscr{K}_{*}$. For the $q f$-type model structures of $\S 7.2$, we must start with a generating set $\mathscr{C}$ that contains the orbits $G / H$ with $H \in \mathscr{F} \cap \mathscr{G}$ and consists of $\mathscr{F} \cap \mathscr{G}$-cell complexes. We say that an $\mathscr{F}$-model structure on $G \mathscr{C}$ is $\mathscr{F}$-topological if the condition of the previous definition holds when we use the $\mathscr{F}$ notions of fibration, cofibration and weak equivalence throughout. The observations of this section generalize to $\mathscr{F}$-topological model categories for any family $\mathscr{F}$.

In addition to the map of $G$-spaces displayed in (10.3.1), we have a map

$$
P_{B}^{\square}(i, p): P_{B}(X, E) \longrightarrow P_{B}(W, E) \times_{P_{B}(W, Y)} P_{B}(X, Y)
$$

of ex- $G$-spaces over $B$.

WARNING 10.3.7. We can define what it means for $\left(\mathscr{C}_{G}, G \mathscr{C}\right)$ to be a $G$ topological model $G$-category over $B$, using the map $P_{B}^{\square}(i, p)$ of ex- $G$-spaces rather than the map $\mathscr{C}_{G}^{\square}(i, p)$ of $G$-spaces. However,we know of no $q$-type examples (even nonequivariant ones) where this condition is satisfied. For example, $\left(\mathscr{K}_{G, B}, G \mathscr{K}_{B}\right)$ is $G$-topological, by Theorems 7.2.3 and 7.2.8, but, as Warning 6.1.7 makes clear by adjunction, we cannot expect it to be $G$-topological over $B$. 
Just as in the classical theory of simplicial or topological model categories, there are various equivalent reformulations of what it means for $G \mathscr{C}$ to be $G$-topological. To explain them, observe that the tensors and cotensors with ex- $G$-spaces over $B$ give rise to induced maps

$$
i \square_{B} k:\left(X \wedge_{B} K\right) \cup_{W \wedge_{B} K}\left(W \wedge_{B} L\right) \longrightarrow X \wedge_{B} L
$$

and

$$
F_{B}^{\square}(k, p): F_{B}(L, E) \longrightarrow F_{B}(K, E) \times_{F_{B}(K, Y)} F_{B}(L, Y)
$$

of ex-G-spaces over $B$. If $\left(\mathscr{C}_{G}, G \mathscr{C}\right)$ is closed symmetric monoidal, then we also have the induced maps

$$
i \square_{B} j:\left(X \wedge_{B} V\right) \cup_{W \wedge_{B} V}\left(W \wedge_{B} Z\right) \longrightarrow X \wedge_{B} Z
$$

and

$$
F_{B}^{\square}(j, p): F_{B}(Z, E) \longrightarrow F_{B}(V, E) \times_{F_{B}(V, Y)} F_{B}(Z, Y)
$$

in $G \mathscr{C}$. We have various adjunction isomorphisms relating these various $\square$-product maps and $\square$-function object maps.

Proposition 10.3.12. If $k$ is a map of ex-G-spaces over $B$, then there are adjunction isomorphisms

$$
P_{B}^{\square}\left(i \square_{B} k, p\right) \cong F_{B}^{\square}\left(k, P_{B}^{\square}(i, p)\right) \cong P_{B}^{\square}\left(i, F_{B}^{\square}(k, p)\right)
$$

of maps of ex-G-spaces over $B$ and

$$
\mathscr{C}_{G}^{\square}\left(i \square_{B} k, p\right) \cong \mathscr{K}_{G, B}^{\square}\left(k, P_{B}^{\square}(i, p)\right) \cong \mathscr{C}_{G}^{\square}\left(i, F_{B}^{\square}(k, p)\right)
$$

of maps of based $G$-spaces. If $k$ is a map of based $G$-spaces, then the last pair of isomorphisms can be rewritten as

$$
\mathscr{C}_{G}^{\square}\left(i \square_{B} k, p\right) \cong \mathscr{K}_{G, *}^{\square}\left(k, \mathscr{C}_{G}^{\square}(i, p)\right) \cong \mathscr{C}_{G}^{\square}\left(i, F_{B}^{\square}(k, p)\right) .
$$

When $\left(\mathscr{C}_{G}, G \mathscr{C}\right)$ is closed symmetric monoidal there are adjunction isomorphisms

$$
P_{B}^{\square}\left(i \square_{B} k, p\right) \cong P_{B}^{\square}\left(i, F_{B}^{\square}(k, p)\right)
$$

of maps of ex-G-spaces over $B$ and

$$
\mathscr{C}_{G}^{\square}\left(i \square_{B} k, p\right) \cong \mathscr{C}_{G}^{\square}\left(i, F_{B}^{\square}(k, p)\right)
$$

of maps of based G-spaces.

Together with Lemma 10.3.3, this implies the promised alternative equivalent conditions that describe when a model category is $G$-topological.

Proposition 10.3.18. Let $\left(\mathscr{C}_{G}, G \mathscr{C}\right)$ be a $G$-topological $G$-category over $B$ such that $G \mathscr{C}$ has a model structure. Then the following conditions are equivalent.

(i) The map $i \square_{B} k$ of (10.3.8) is a cofibration in $G \mathscr{C}$ if $i$ is a cofibration in $G \mathscr{C}$ and $k$ is a cofibration in $G \mathscr{K}_{*}$. It is acyclic if either $i$ or $k$ is acyclic.

(ii) The map $F_{B}^{\square}(k, p)$ of (10.3.9) is a fibration in $G \mathscr{C}$ if $p$ is a fibration in $G \mathscr{C}$ and $k$ is a cofibration in $G \mathscr{K}_{*}$. It is acyclic if either $p$ or $k$ is acyclic.

(iii) The map $\mathscr{C}_{G}^{\square}(i, p)$ of (10.3.1) is a fibration in $G \mathscr{K}_{*}$ if $i$ is a cofibration in $G \mathscr{C}$ and $p$ is a fibration in $G \mathscr{C}$. It is acyclic if either $i$ or $p$ is acyclic. 
Proof. The third condition is our definition of the model structure being $G$-topological. We prove that the first condition is equivalent to the third. A similar argument shows that the second condition is also equivalent to the third. The map $\mathscr{C}_{G}^{\square}(i, p)$ is a fibration if and only if $\left(k, \mathscr{C}_{G}^{\square}(i, p)\right)$ has the lifting property with respect to all acyclic cofibrations $k$ in $G \mathscr{K}_{*}$. By Lemma 10.3.3 and the first adjunction isomorphism in (10.3.15), that holds if and only if $\left(i \square_{B} k, p\right)$ has the lifting property, that is, if and only if $i \square_{B} k$ is an acyclic cofibration. If either $i$ or $p$ is acyclic, then we take $k$ to be a cofibration in $G \mathscr{K}_{*}$ and argue similarly. 



\section{CHAPTER 11}

\section{The category of orthogonal $G$-spectra over $B$}

\section{Introduction}

Intuitively, an orthogonal spectrum $X$ over $B$ consists of ex-spaces $X(V)$ over $B$ and ex-maps $\sigma: X(V) \wedge_{B} S^{W} \longrightarrow X(V \oplus W)$ for suitable inner product spaces $V$ and $W$. The orthogonal group $O(V)$ must act on $X(V)$, and $\sigma$ must be $(O(V) \times O(W))$ equivariant. The orthogonal group actions enable the definition of a good external smash product. Moreover, they will later allow us to define stable weak equivalences in terms of homotopy groups, as would not be possible if we only had actions by symmetric groups.

Similarly, use of general inner product spaces allows us to build in actions by a compact Lie group $G$ without difficulty. For non-compact Lie groups, we should ignore inner products and use linear isomorphisms, replacing the compact orthogonal group $O(V)$ by the general linear group $G L(V)$. However, as we explain in $\S 11.6$, there are more serious problems in generalizing to non-compact Lie groups; except in that section, we require $G$ to be a compact Lie group.

Working equivariantly, we first ignore the structure maps $\sigma$ and describe the diagram category of ex- $G$-spaces in $\S 11.1$. We build in the structure maps in $\S 11.2$, where we define the category of orthogonal $G$-spectra over $B$. In $\S 11.3$, we show that this category too can be described as a diagram category of ex- $G$-spaces, of course over a different domain category. The formal properties of the category of ex- $G$-spaces over $B$ carry over to the category of orthogonal $G$-spectra over $B$, but there are some new twists. For example, our category of $G$-spectra over $B$ is enriched not just over based $G$-spaces, but more generally over ex- $G$-spaces over $B$. We discussed the relevant formalities in the previous chapter. This enhanced enrichment is essential to the definition of function $G$-spectra over $B$.

We show in $\S 11.4$ that the base change functors and their properties also carry over to these categories of parametrized $G$-spectra, and we discuss change of group functors and restriction to fibers in $\S 11.5$.

\subsection{The category of $\mathscr{I}_{G}$-spaces over $B$}

We recall the $G$-category $\left(\mathscr{I}_{G}, G \mathscr{I}\right)$ from [105, II.2.1]. The objects and arrows of $\mathscr{I}_{G}$ are finite dimensional $G$-inner product spaces and linear isometric isomorphisms. The maps of $G \mathscr{I}$ are $G$-linear isometries. More precisely, as dictated by the general theory of $[105,106]$, we take $\mathscr{I}_{G}(V, W)$ as based with basepoint disjoint from the space of linear isometric isomorphisms $V \longrightarrow W$. The objects $V$ run over a collection $\mathscr{V}$ of $G$-representations that is closed under direct sums and contains the trivial representation $\mathbb{R}$. We generally focus on the collection $\mathscr{V}(U)$ of all representations that embed up to isomorphism in a given " $G$-universe" $U$, where a 
$G$-universe is a sum of countably many copies of representations in a set of representations that includes the trivial representation. We usually think in terms of a "complete $G$-universe", one that contains all representations of $G$, but the choice is irrelevant until otherwise stated.

Based $G$-spaces are ex- $G$-spaces over $*$, and $\mathscr{I}_{G}$-spaces are defined in $[105$, II.2.3] as $G$-functors $\mathscr{I}_{G} \longrightarrow \mathscr{T}_{G}$, where $\mathscr{T}_{G}$ is the $G$-category of compactly generated based $G$-spaces. One can just as well drop the weak Hausdorff condition, which plays no necessary mathematical role in $[105,106]$, and allow general $k$ spaces. With the notations of Part II, we can thus change the target $G$-category to $\mathscr{K}_{G, *}$. Then we generalize the definition to the parametrized context simply by changing the target $G$-category to the category $\mathscr{K}_{G, B}$ of ex- $G$-spaces over a $G$ -

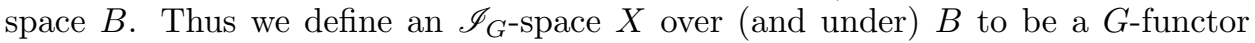
$X: \mathscr{I}_{G} \longrightarrow \mathscr{K}_{G, B}$. Using nonequivariant arrows and equivariant maps, we obtain the $G$-category $\left(\mathscr{I}_{G} \mathscr{K}_{B}, G \mathscr{I} \mathscr{K}_{B}\right)$ of $\mathscr{I}_{G \text {-spaces. }}$

To unravel definitions, for each representation $V \in \mathscr{V}$ we are given an ex- $G$ space $X(V)$ over $B$, for each arrow (linear isometric isomorphism) $f: V \longrightarrow W$ we are given an arrow (non-equivariant map)

$$
X(f): X(V) \longrightarrow X(W)
$$

of ex- $G$-spaces over $B$, and the continuous function

$$
X: \mathscr{I}_{G}(V, W) \longrightarrow \mathscr{K}_{G, B}(X(V), X(W))
$$

is a based $G$-map. An arrow $\alpha: X \longrightarrow Y$ is just a natural transformation, and a $G$-map is a $G$-natural transformation, for which each $\alpha_{V}: X(V) \longrightarrow Y(V)$ is a $G$-map. For both arrows and $G$-maps, the naturality diagrams

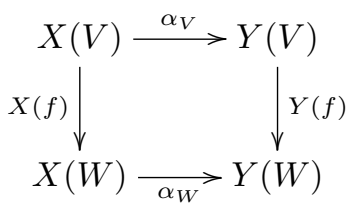

must commute for all arrows $f: V \longrightarrow W$. The group $G$ acts on the space $\mathscr{I}_{G} \mathscr{K}_{B}(X, Y)$ of arrows by levelwise conjugation. The $G$-fixed category is denoted

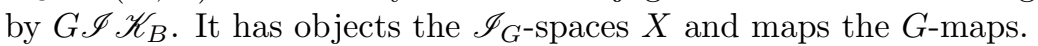

To study the parametrized enrichment of the $G$-category of orthogonal $G$ spectra over $B$, it is convenient to extend the domain category $\mathscr{I}_{G}$, which is enriched over $\mathscr{K}_{G, *}$, to a new domain category $\mathscr{I}_{G, B}$ that is enriched over $\mathscr{K}_{G, B}$. Departing from the notational pattern of Definition 10.2.1 and using Remark 10.1.6, we define the hom ex- $G$-spaces over $B$ of $\mathscr{I}_{G, B}$ by

$$
\mathscr{I}_{G, B}(V, W)=\mathscr{I}_{G}(V, W)_{B} \equiv B \times \mathscr{I}_{G}(V, W) .
$$

If $X: \mathscr{I}_{G} \longrightarrow \mathscr{K}_{G, B}$ is an $\mathscr{I}_{G}$-space, then the given based $G$-maps

$$
X: \mathscr{I}_{G}(V, W) \longrightarrow \mathscr{K}_{G, B}(X(V), X(W))
$$

correspond by adjunction (see (10.2.7) and (10.2.8)) to ex-G-maps

$$
X(V) \wedge_{B} \mathscr{I}_{G, B}(V, W) \longrightarrow X(W) .
$$

In turn, these correspond by the internal hom adjunction to ex- $G$-maps

$$
X: \mathscr{I}_{G, B}(V, W) \longrightarrow F_{B}(X(V), X(W)) .
$$


These give an equivalent version of the original $G$-functor $X$, but now in terms of categories enriched over the category $G \mathscr{K}_{B}$.

Lemma 11.1.2. The G-category $\left(\mathscr{I}_{G} \mathscr{K}_{B}, G \mathscr{I} \mathscr{K}_{B}\right)$ of $\mathscr{I}_{G}$-spaces is equivalent to the $G$-category of $\mathscr{I}_{G, B}$-spaces, where an $\mathscr{I}_{G, B}$-space is a $G$-functor

$$
X: \mathscr{I}_{G, B} \longrightarrow \mathscr{K}_{G, B}
$$

enriched over $G \mathscr{K}_{B}$.

Proposition 11.1.3. The G-category $\left(\mathscr{I}_{G} \mathscr{K}_{B}, G \mathscr{I} \mathscr{K}_{B}\right)$ is G-topological over $B$ and thus also $G$-topological. Therefore the category $G \mathscr{I}_{\mathscr{K}_{B}}$ is topologically bicomplete over $B$.

Proof. We define tensor and cotensor $\mathscr{I}_{G}$-spaces over $B$

$$
X \wedge_{B} K \quad \text { and } \quad F_{B}(K, X)
$$

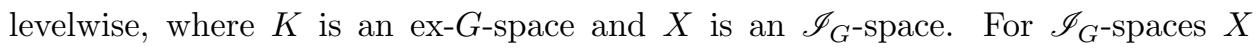
and $Y$, we must define a parametrized morphism ex-G-space $P_{B}(X, Y)$ over $B$. Parallelling a standard formal description of the $G$-space $\mathscr{I}_{G} \mathscr{K}_{B}(X, Y)$, we define $P_{B}(X, Y)$ to be the end

$$
P_{B}(X, Y)=\int_{\mathscr{I}_{G, B}} F_{B}(X(V), Y(V)) .
$$

Explicitly, it is the equalizer displayed in the following diagram of ex- $G$-spaces.

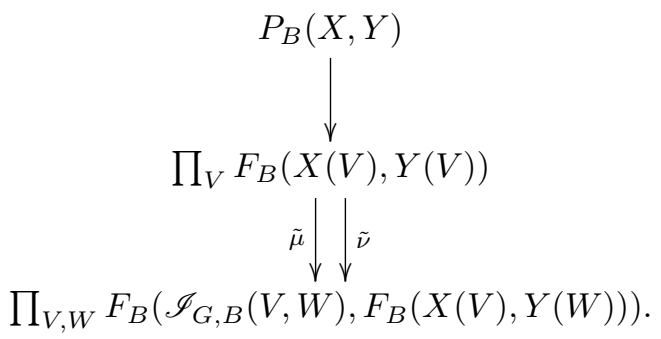

The products run over the objects and pairs of objects of a skeleton $s k \mathscr{I}_{G}$ of $\mathscr{I}_{G}$. The $(V, W)$ th coordinate of $\tilde{\mu}$ is given by the composite of the projection to $F_{B}(X(W), Y(W))$ and the $G$-map

$$
F_{B}(X(W), Y(W)) \longrightarrow F_{B}\left(\mathscr{I}_{G, B}(V, W), F_{B}(X(V), Y(W))\right)
$$

adjoint to the composite ex- $G$-map

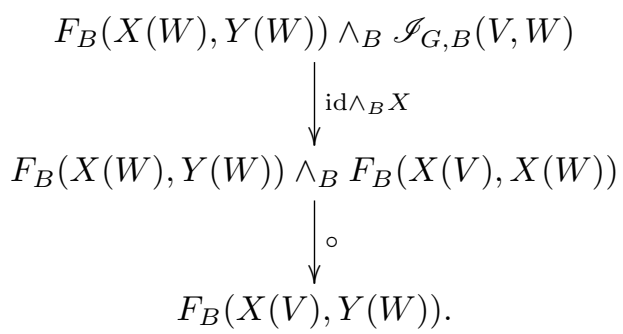

The $(V, W)$ th coordinate of $\tilde{\nu}$ is the composite of the projection to $F_{B}(X(V), Y(V))$ and the $G$-map

$$
\tilde{\nu}_{V, W}: F_{B}(X(V), Y(V)) \longrightarrow F_{B}\left(\mathscr{I}_{G, B}(V, W), F_{B}(X(V), Y(W))\right.
$$


adjoint to the composite ex- $G$-map

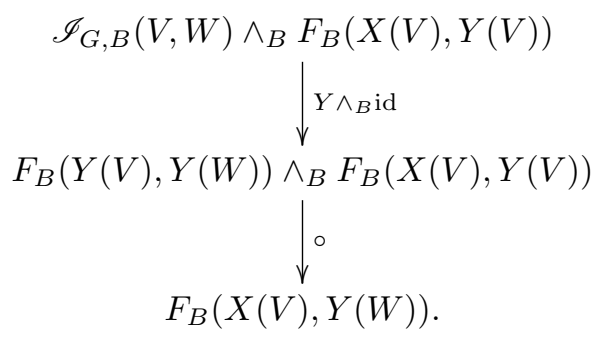

Passage to ends from the isomorphisms of ex- $G$-spaces

$$
F_{B}\left(X(V) \wedge_{B} K, Y(V)\right) \cong F_{B}\left(K, F_{B}(X(V), Y(V))\right) \cong F_{B}\left(X(V), F_{B}(K, Y(V))\right)
$$

gives natural isomorphisms of ex- $G$-spaces

$$
P_{B}\left(X \wedge_{B} K, Y\right) \cong F_{B}\left(K, P_{B}(X, Y)\right) \cong P_{B}\left(X, F_{B}(K, Y)\right) .
$$

With these constructions, we see that $\left(\mathscr{I}_{G} \mathscr{K}_{B}, G \mathscr{I}_{\mathscr{K}_{B}}\right)$ is $G$-topological over $B$; compare Definition 10.2.1 and the discussion following it. The last statement follows since $G \mathscr{I} \mathscr{K}_{B}$ is complete and cocomplete, with limits and colimits constructed levelwise from the limits and colimits in $G \mathscr{K}_{B}$.

We have several kinds of smash products and function objects in this context. For $\mathscr{I}_{G}$-spaces $X$ and $Y$ over $B$, define the "external" smash product $X \bar{\wedge}_{B} Y$ by

$$
X \bar{\wedge}_{B} Y=\wedge_{B} \circ(X \times Y): \mathscr{I}_{G} \times \mathscr{I}_{G} \longrightarrow \mathscr{K}_{G, B}
$$

Thus $\left(X{\wedge_{B}} Y\right)(V, W)=X(V) \wedge_{B} Y(W)$. Here we have used the word "external" to refer to the use of pairs of representations, as is usual in the theory of diagram spectra. It is standard category theory $[43,106]$ to use left Kan extension to internalize this external smash product over $B$; relevant general definitions are recalled

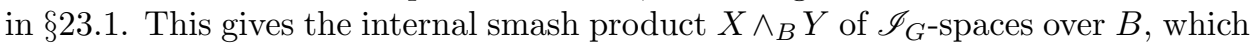
is again an $\mathscr{I}_{G}$-space over $B$. For an $\mathscr{I}_{G}$-space $Y$ over $B$ and an $\left(\mathscr{I}_{G} \times \mathscr{I}_{G}\right)$-space $Z$ over $B$, define the external function $\mathscr{I}_{G}$-space over $B$, denoted $\bar{F}_{B}(Y, Z)$, by

$$
\bar{F}_{B}(Y, Z)(V)=P_{B}(Y, Z\langle V\rangle),
$$

where $Z\langle V\rangle(W)=Z(V, W)$. It is mainly to allow this definition that weneed the morphism ex- $G$-spaces $P_{B}(-,-)$. It is also formal to obtain an internal function

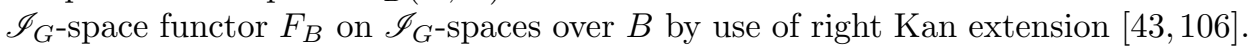
Using these internal smash product and function $\mathscr{I}_{G}$-space functors, we obtain the following result. Recall Definition 10.2.9 and Proposition 10.2.10.

Theorem 11.1.6. $\left(\mathscr{I}_{G} \mathscr{K}_{B}, G \mathscr{I} \mathscr{K}_{B}\right)$ is a $G$-topological closed symmetric monoidal $G$-category over $B$.

REMARK 11.1.7. In the theory of ex-spaces, we also have the "external smash product" of ex-spaces over different base spaces defined in $§ 2.5$. Using the two different notions of "external" together, we obtain the definition of the "external external smash product" of an $\mathscr{I}_{G}$-space $X$ over $A$ and an $\mathscr{I}_{G}$-space $Y$ over $B$; it is an $\left(\mathscr{I}_{G} \times \mathscr{I}_{G}\right)$-space over $A \times B$. We write $X \bar{\wedge} Y$ for the left Kan extension internalization of this smash product. Thus $X \wedge Y$ is an $\mathscr{I}_{G^{-}}$space over $A \times B$. Similarly, using the external function ex-space construction $\bar{F}$ of $\S 2.5$, for an $\mathscr{I}_{G^{-}}$ space $Y$ over $B$ and an $\mathscr{I}_{G}$-space $Z$ over $A \times B$, we obtain the "internalized external 
function $\mathscr{I}_{G}$-space" $\bar{F}(Y, Z)$ over $A$. Notationally, use of $\bar{\wedge}$ and $\bar{F}$ without an ensuing subscript always denotes these internalized external operations with respect to varying base spaces. We shall return to these functors in Proposition 11.4.10.

Similarly, but more simply, we have the "external tensor" $K \wedge Y$ of an ex- $G$ space $K$ over $A$ and an $\mathscr{I}_{G}$-space $Y$ over $B$, which again is an $\mathscr{I}_{G}$-space over $A \times B$. When $A=*$, this just constructs the tensor of a based $G$-space with an $\mathscr{I}_{G}$-space over $B$. When $B=*$, this constructs an $\mathscr{I}_{G}$-space over $A$ from an ex- $G$-space over $A$ and a nonparametrized $\mathscr{I}_{G}$-space. Since these external tensors can be view as special cases of external smash products, via variants of Definition 10.2.9 and (11.2.6) below, we shall not treat them formally and shall not repeat the definitions on the $G$-spectrum level. However, we shall find several uses for them.

\subsection{The category of orthogonal $G$-spectra over $B$}

For a representation $V$ of $G$ and an $\mathscr{I}_{G}$-space $X$, we define

$$
\Sigma_{B}^{V} X=X \wedge_{B} S_{B}^{V} \quad \text { and } \quad \Omega_{B}^{V} X=F_{B}\left(S_{B}^{V}, X\right),
$$

where $S^{V}$ is the one-point compactification of $V$.

Definition 11.2.2. Define the $G$-sphere $S_{B}$, written $S_{G, B}$ when necessary for clarity, to be the $\mathscr{I}_{G}$-space over $B$ that sends $V$ to $S_{B}^{V}$.

Clearly $S_{B}^{V} \wedge_{B} S_{B}^{W} \cong S_{B}^{V \oplus W}$, and the functor $S_{B}$ is strong symmetric monoidal, where the monoidal structure on $\mathscr{I}_{G}$ is given by direct sums. It follows that $S_{B}$ is a commutative monoid in the symmetric monoidal category $G \mathscr{I} \mathscr{K}_{B}$, and we can define $S_{B}$-modules $X$ in terms of (right) actions $X \wedge_{B} S_{B} \longrightarrow X$. These $S_{B}$-modules are our orthogonal $G$-spectra over $B$, but it is more convenient to give the definition using the equivalent reformulation in terms of the external smash product.

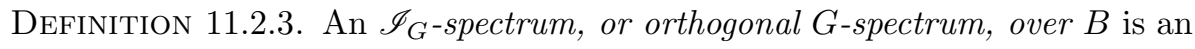

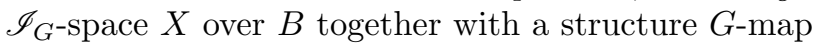

$$
\sigma: X \bar{\wedge}_{B} S_{B} \longrightarrow X \circ \oplus
$$

such that the evident unit and associativity diagrams commute. Thus we have compatible equivariant structure maps

$$
\sigma: \Sigma_{B}^{W} X(V)=X(V) \wedge_{B} S_{B}^{W} \longrightarrow X(V \oplus W) .
$$

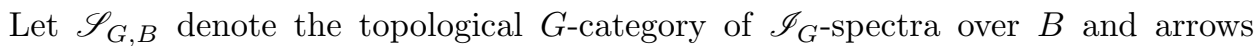
$f: X \longrightarrow Y$ that commute with the structure maps, with $G$ acting by conjugation on arrows. Let $G \mathscr{S}_{B}$ denote the topological category of $\mathscr{I}_{G}$-spectra over $B$ and $G$-maps (equivariant arrows) between them.

Definition 11.2.4. Define the suspension orthogonal $G$-spectrum functor and the 0 th ex- $G$-space functor

$$
\Sigma_{B}^{\infty}: \mathscr{K}_{G, B} \longrightarrow \mathscr{S}_{G, B} \text { and } \Omega_{B}^{\infty}: \mathscr{S}_{G, B} \longrightarrow \mathscr{K}_{G, B}
$$

by $\left(\Sigma_{B}^{\infty} K\right)(V)=\Sigma_{B}^{V} K$, with the evident isomorphisms as structure maps, and $\Omega_{B}^{\infty} X=X(0)$. Then $\Sigma_{B}^{\infty}$ and $\Omega_{B}^{\infty}$ give left and right adjoints between $\mathscr{K}_{G, B}$ and $\mathscr{S}_{G, B}$ and, on passage to $G$-fixed points, between $G \mathscr{K}_{B}$ and $G \mathscr{S}_{B}$.

The category $G \mathscr{S}_{B}$ is our candidate for a good category of parametrized $G$ spectra over $B$. It inherits all of the properties of the category $G \mathscr{I} \mathscr{K}_{B}$ of $\mathscr{I}_{G}$-spaces that were discussed in the previous section and, in the case $B=*$, it is exactly 
the category $G \mathscr{S}$ of orthogonal $G$-spectra that is studied in [105]. We summarize its formal properties in the following omnibus theorem. In the language of $\S 10.2$, much of it can be summarized by the assertion that the $G$-category $\left(\mathscr{S}_{G, B}, G \mathscr{S}_{B}\right)$ is a $G$-topological closed symmetric monoidal $G$-category over $B$, but we prefer to be more explicit than that.

TheOREM 11.2.5. The G-category $\mathscr{S}_{G, B}$ is enriched over G $\mathscr{K}_{B}$ and is tensored and cotensored over $\mathscr{K}_{G, B}$. The category $G \mathscr{S}_{B}$ is enriched over $\mathscr{K}_{B}$ and is tensored and cotensored over $G \mathscr{K}_{B}$. The $G$-category $\mathscr{S}_{G, B}$ and the category $G \mathscr{S}_{B}$ admit smash product and function spectrum functors $\wedge_{B}$ and $F_{B}$ under which they are closed symmetric monoidal with unit object $S_{B}$. Let $X$ and $Y$ be orthogonal $G$-spectra over $B$ and $K$ be an ex-G-space over $B$. The morphism ex-G-spaces $P_{B}(X, Y)$ can be specified by

$$
P_{B}(X, Y)=\Omega_{B}^{\infty} F_{B}(X, Y)
$$

and there are natural isomorphisms

$$
\Sigma_{B}^{\infty} K \cong S_{B} \wedge_{B} K \text { and } \Omega_{B}^{\infty} X \cong P_{B}\left(S_{B}, X\right) .
$$

The tensors and cotensors are related to smash products and function $G$-spectra by natural isomorphisms

$$
X \wedge_{B} K \cong X \wedge_{B} \Sigma_{B}^{\infty} K \quad \text { and } \quad F_{B}(K, X) \cong F_{B}\left(\Sigma_{B}^{\infty} K, X\right)
$$

of orthogonal $G$-spectra. There are natural isomorphisms

$$
P_{B}\left(\Sigma_{B}^{\infty} K, X\right) \cong F_{B}\left(K, \Omega_{B}^{\infty} X\right)
$$

and

$$
P_{B}\left(X \wedge_{B} K, Y\right) \cong F_{B}\left(K, P_{B}(X, Y)\right) \cong P_{B}\left(X, F_{B}(K, Y)\right)
$$

of ex-G-spaces,

$$
\mathscr{S}_{G, B}\left(X \wedge_{B} K, Y\right) \cong \mathscr{K}_{G, B}\left(K, P_{B}(X, Y)\right) \cong \mathscr{S}_{G, B}\left(X, F_{B}(K, Y)\right)
$$

of based $G$-spaces, and

$$
G \mathscr{S}_{B}\left(X \wedge_{B} K, Y\right) \cong G \mathscr{K}_{B}\left(K, P_{B}(X, Y)\right) \cong G \mathscr{S}_{B}\left(X, F_{B}(K, Y)\right)
$$

of based spaces. Moreover, G $\mathscr{S}_{B}$ is G-topologically bicomplete over $B$.

Proof. For the enrichment, the $G$-space $\mathscr{S}_{G, B}(X, Y)$ is the evident sub $G$ space of $\mathscr{I}_{G} \mathscr{K}_{B}(X, Y)$, and the space $G \mathscr{S}_{B}(X, Y)$ is the evident sub space of $G \mathscr{I} \mathscr{K}_{B}(X, Y)$. The tensors and cotensors in $\mathscr{S}_{G, B}$ are constructed in $\mathscr{I}_{G} \mathscr{K}_{B}$ and given induced structure maps. The limits and colimits in $G \mathscr{S}_{B}$ are constructed in the same way. As in [105, II $\S 3]$, we think of orthogonal $G$-spectra over $B$ as $S_{B}$-modules, and we construct the smash product and function spectra functors by passage to coequalizers and equalizers from the smash product and function $\mathscr{I}_{G}$-space functors, exactly as in the definition of tensor products and hom functors in algebra. We have defined $P_{B}(X, Y)$ in the statement, but we shall give a more intrinsic alternative description later. The first isomorphism of (11.2.6) is given by unit and associativity relations

$$
X \wedge_{B} K \cong\left(X \wedge_{B} S_{B}\right) \wedge_{B} K \cong X \wedge_{B} \Sigma_{B}^{\infty} K
$$


The second follows from the Yoneda lemma since

$$
\begin{aligned}
G \mathscr{S}_{B}\left(X, F_{B}(K, Y)\right) & \cong G \mathscr{S}_{B}\left(X \wedge_{B} K, Y\right) \\
& \cong G \mathscr{S}_{B}\left(X \wedge_{B} \Sigma_{B}^{\infty} K, Y\right) \\
& \cong G \mathscr{S}_{B}\left(X, F_{B}\left(\Sigma_{B}^{\infty} K, Y\right)\right) .
\end{aligned}
$$

Now (11.2.7) and (11.2.8) follow from already established adjunctions. For part of the latter, we apply $\Omega_{B}^{\infty}$ to the composite isomorphism

$$
\begin{aligned}
F_{B}\left(X \wedge_{B} K, Y\right) & \cong F_{B}\left(X \wedge_{B} \Sigma_{B}^{\infty} K, Y\right) \\
& \cong F_{B}\left(X, F_{B}\left(\Sigma_{B}^{\infty} K, Y\right)\right) \\
& \cong F_{B}\left(X, F_{B}(K, Y)\right) .
\end{aligned}
$$

Comparisons of definitions, seen more easily from (11.3.2) below, give

$$
\mathscr{S}_{G, B}(X, Y)=\mathscr{K}_{G, B}\left(S_{B}^{0}, P_{B}(X, Y)\right)
$$

and

$$
G \mathscr{S}_{B}(X, Y) \cong G \mathscr{K}_{B}\left(S_{B}^{0}, P_{B}(X, Y)\right) .
$$

Therefore the isomorphisms (11.2.9) and (11.2.10) follow from (11.2.8).

As noted in $\S 10.1$, we obtain the following corollary by replacing $K$ with $T_{B}$ for a based $G$-space $T$ in the tensors and cotensors of the theorem. Of course, these tensors and cotensors with $G$-spaces could just as well be defined directly. It will be important in our discussion of model category structures to keep separately in mind the tensors and cotensors over ex- $G$-spaces over $B$ and over based $G$-spaces.

Corollary 11.2.13. The $G$-category $\mathscr{S}_{G, B}$ is enriched over $G \mathscr{K}_{*}$ and is tensored and cotensored over $\mathscr{K}_{G, *}$. The category $G \mathscr{S}_{B}$ is enriched over $\mathscr{K}_{G, *}$ and is tensored and cotensored over $G \mathscr{K}_{*}$. Thus, for orthogonal $G$-spectra $X$ and $Y$ and based $G$-spaces $T$,

$$
\mathscr{S}_{G, B}\left(X \wedge_{B} T, Y\right) \cong \mathscr{K}_{G, *}\left(T, \mathscr{S}_{G, B}(X, Y)\right) \cong \mathscr{S}_{G, B}\left(X, F_{B}(T, Y)\right)
$$

and

$$
G \mathscr{S}_{B}\left(X \wedge_{B} T, Y\right) \cong G \mathscr{K}_{*}\left(T, \mathscr{S}_{G, B}(X, Y)\right) \cong G \mathscr{S}_{B}\left(X, F_{B}(T, Y)\right) \text {. }
$$

We have the parallel definition of $G$-prespectra over $B$.

Definition 11.2.16. A $G$-prespectrum $X$ over $B$ consists of ex- $G$-spaces $X(V)$ over $B$ for $V \in \mathscr{V}$ together with structure $G$-maps $\sigma: \Sigma_{B}^{W} X(V) \longrightarrow X(V \oplus W)$ such that $\sigma$ is the identity if $W=0$ and the following diagrams commute.

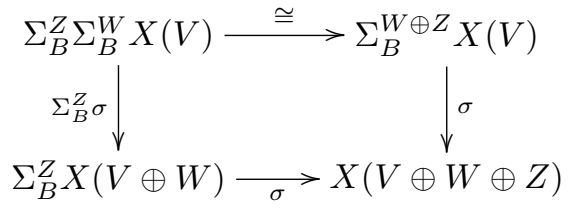

Let $\mathscr{P}_{G, B}$ denote the $G$-category of $G$-prespectra and nonequivariant arrows, and let $G \mathscr{P}_{B}$ denote its $G$-fixed category of $G$-prespectra and $G$-maps. There result forgetful functors

$$
\mathbb{U}: \mathscr{S}_{G, B} \longrightarrow \mathscr{P}_{G, B} \quad \text { and } \quad \mathbb{U}: G \mathscr{S}_{B} \longrightarrow G \mathscr{P}_{B}
$$


The categories $\mathscr{P}_{G, B}$ and $G \mathscr{P}_{B}$ enjoy the same properties that were specified for $\mathscr{S}_{G, B}$ and $G \mathscr{S}_{B}$ in Theorem 11.2.5 and Corollary 11.2.13, except for the statements about smash product and function spectra. Here, since we do not have the internal hom functor $F_{B}$, we must give an alternative direct description of $P_{B}(X, Y)$, as in (11.3.2) below.

\subsection{Orthogonal $G$-spectra as diagram ex- $G$-spaces}

Arguing as in [106, §2] and [105, II $\S 4]$, we construct a new domain category $\mathscr{J}_{G, B}$ which has the same object set $\mathscr{V}$ as $\mathscr{I}_{G}$ and, like $\mathscr{I}_{G, B}$, is enriched over $G \mathscr{K}_{B}$. It builds in spheres in such a way that the category of $\mathscr{I}_{G}$-spectra over $B$ is equivalent to the category of $\mathscr{J}_{G, B}$-spaces over $B$. Here, just as for $\mathscr{I}_{G, B}$ in Lemma 11.1.2, we understand a $\mathscr{J}_{G, B}$-space to be an enriched $G$-functor $X: \mathscr{J}_{G, B} \longrightarrow \mathscr{K}_{G, B}$. Thus it is specified by ex- $G$-spaces $X(V)$ and ex- $G$-maps

$$
X: \mathscr{J}_{G, B}(V, W) \longrightarrow F_{B}(X(V), X(W)) .
$$

To construct $\mathscr{J}_{G, B}$, recall from [105, II $\left.\S 4\right]$ that we have a topological $G$-category $\mathscr{J}_{G}$ with object set $\mathscr{V}$ such that the category of $\mathscr{I}_{G}$-spectra is equivalent to the category of $\mathscr{J}_{G}$-spaces. We define

$$
\mathscr{J}_{G, B}(V, W)=\mathscr{J}_{G}(V, W)_{B},
$$

just as we defined $\mathscr{I}_{G, B}$ in (11.1.1), and the desired equivalence of categories follows. Several constructions of $\mathscr{J}_{G}$ are given in $[105,106]$, and we shall shortly give an alternative direct description of $\mathscr{J}_{G, B}$ that is based on one of them. The intuition is that an extension of an $\mathscr{I}_{G, B}$-space to a $\mathscr{J}_{G, B}$-space builds in an action by $S_{B}$.

The alternative description of $G \mathscr{S}_{B}$ as the category of enriched $G$-functors $\mathscr{J}_{G, B} \longrightarrow \mathscr{K}_{G, B}$ and enriched $G$-natural transformations leads to a more conceptual proof of Theorem 11.2.5: it is a specialization of general results about diagram categories of enriched functors. In analogy with (11.1.4) we could have defined $P_{B}(X, Y)$ to be the end

$$
P_{B}(X, Y)=\int_{\mathscr{J}_{G, B}} F_{B}(X(V), Y(V))
$$

and derived the isomorphism (11.2.8) just as we derived (11.1.5) in the previous section. By the Yoneda lemma, the two definitions of $P_{B}(X, Y)$ agree. With this description of $P_{B}$, some of the adjunctions in Theorem 11.2.5 become more transparent.

This leads us to the promised alternative description of $\mathscr{J}_{G, B}$. It is given in terms of $\mathscr{I}_{G, B}$ rather than $\mathscr{J}_{G}$ and follows the formal definition of [106, 2.1]. We have the represented functors $V^{*}: \mathscr{I}_{G} \longrightarrow \mathscr{K}_{G, B}$ specified by $V^{*}(W)=\mathscr{I}_{G, B}(V, W)$. If $X$ is an $\mathscr{I}_{G}$-space, such as $V^{*}$, then the smash product $X \wedge_{B} S_{B}$ in the category of $\mathscr{I}_{G}$-spaces should be thought of as the "free" orthogonal $G$-spectrum over $B$ generated by $X$. Let

$$
\mathscr{J}_{G, B}(V, W)=P_{B}\left(W^{*} \wedge_{B} S_{B}, V^{*} \wedge_{B} S_{B}\right),
$$

with the evident composition. Then we can mimic the arguments of $[106, \S \S 2,23]$ to check that the category of $\mathscr{J}_{G, B}$-spaces is equivalent to the category of $\mathscr{I}_{G \text {-spectra }}$ over $B$. An enriched Yoneda lemma argument $[87,2.4]$ shows that this description of $\mathscr{J}_{G, B}$ coincides up to isomorphism with our original one. 
Although we will not have occasion to quote it formally, we record the following consequence of the identification of $\mathscr{I}_{G}$-spectra over $B$ with $\mathscr{J}_{G, B}$-spaces.

Lemma 11.3.4. For any enriched $G$-functor $T: \mathscr{K}_{G, B} \longrightarrow \mathscr{K}_{G, B}$ and orthogonal $G$-spectrum $X$ over $B$, the composite functor $T \circ X$ is an orthogonal $G$-spectrum over $B$. Similarly, an enriched natural transformation $\xi: T \longrightarrow T^{\prime}$ induces a natural $G$ $\operatorname{map} \xi: T \circ X \longrightarrow T^{\prime} \circ X$.

Proof. The enriched functor $T$ is given by maps

$$
T: F_{B}(K, L) \longrightarrow F_{B}(T(K), T(L)) .
$$

Composing levelwise with $X$ gives maps

$$
\mathscr{J}_{G, B}(V, W) \longrightarrow F_{B}(T(X(V)), T(X(W)))
$$

that specify $T \circ X$. It is a direct categorical implication of the fact that $T$ is an enriched functor that there are natural maps of ex- $G$-spaces

$$
T(K) \wedge_{B} L \longrightarrow T\left(K \wedge_{B} L\right) \text { and } T F_{B}(K, L) \longrightarrow F_{B}(K, T(L))
$$

for ex- $G$-spaces $K$ and $L$. This explains more concretely why the structure maps of $X$ induce structure maps for $T \circ X$. Similarly, since $\xi$ is enriched, it is given by maps from the unit ex- $G$-space $S_{B}^{0}$ to $F_{B}\left(T(K), T^{\prime}(K)\right)$ such that the appropriate diagrams commute. We specialize to $K=X(V)$ to obtain $\xi: T \circ X \longrightarrow T^{\prime} \circ X$.

The following functors relating ex- $G$-spaces to orthogonal $G$-spectra over $B$ play a central role in our theory. In particular, they give "negative dimensional" spheres $\Sigma_{V}^{\infty} S_{B}^{0}=S_{B}^{-V}$.

Definition 11.3.5. Let $V^{*}=V_{B}^{*}$ denote the represented $\mathscr{J}_{G, B}$-space specified by $V^{*}(W)=\mathscr{J}_{G, B}(V, W)$. Define the shift desuspension functor

$$
F_{V}: \mathscr{K}_{G, B} \longrightarrow \mathscr{S}_{G, B}
$$

by letting $F_{V} K=V^{*} \wedge_{B} K$ for an ex-G-space $K$. Let $\operatorname{Ev}_{V}: \mathscr{S}_{G, B} \longrightarrow \mathscr{K}_{G, B}$ be the functor given by evaluation at $V$. The alternative notations

$$
\Sigma_{V}^{\infty} K=F_{V} K \text { and } \Omega_{V}^{\infty} K=\mathrm{Ev}_{V}
$$

are often used. In particular, $F_{0}=\Sigma_{0}^{\infty}=\Sigma_{B}^{\infty}$ and $\mathrm{Ev}_{0}=\Omega_{0}^{\infty}=\Omega_{B}^{\infty}$.

LEMma 11.3.6. The functors $F_{V}$ and $E v_{V}$ are left and right adjoint, and there is a natural isomorphism

$$
F_{V} K \wedge_{B} F_{W} L \cong F_{V \oplus W}\left(K \wedge_{B} L\right) .
$$

Proof. The first statement is clear, and the verification of the second statement is formal, as in $[106, \S 1]$.

\subsection{The base change functors $f^{*}, f_{!}$, and $f_{*}$}

From now on, we drop the adjective "orthogonal" (or prefix $\mathscr{I}_{G}$ ), and we generally take the equivariance for granted, referring to orthogonal $G$-spectra over $B$ just as spectra over $B$. We return $G$ to the notations when considering change of groups, or for emphasis, but otherwise $G$-actions are tacitly assumed throughout.

We first show that the results on base change functors proven for ex-spaces in $\S 2.2$ extend to parametrized spectra. We then show that the results in $\S 2.5$ relating external and internal smash product and function ex-spaces also extend to parametrized spectra. Let $A$ and $B$ be base $G$-spaces. 
Theorem 11.4.1. Let $f: A \longrightarrow B$ be a $G$-map. Let $X$ be in $\mathscr{S}_{G, A}$ and let $Y$ and $Z$ be in $\mathscr{S}_{G, B}$. There are $G$-functors

$$
f_{!}: \mathscr{S}_{G, A} \longrightarrow \mathscr{S}_{G, B}, \quad f^{*}: \mathscr{S}_{G, B} \longrightarrow \mathscr{S}_{G, A}, \quad f_{*}: \mathscr{S}_{G, A} \longrightarrow \mathscr{S}_{G, B}
$$

and $G$-adjunctions

$$
\mathscr{S}_{G, B}\left(f_{!} X, Y\right) \cong \mathscr{S}_{G, A}\left(X, f^{*} Y\right) \quad \text { and } \quad \mathscr{S}_{G, A}\left(f^{*} Y, X\right) \cong \mathscr{S}_{G, B}\left(Y, f_{*} X\right) .
$$

On passage to $G$-fixed points levelwise, there result functors

$$
f_{!}: G \mathscr{S}_{A} \longrightarrow G \mathscr{S}_{B}, \quad f^{*}: G \mathscr{S}_{B} \longrightarrow G \mathscr{S}_{A}, \quad f_{*}: G \mathscr{S}_{A} \longrightarrow G \mathscr{S}_{B}
$$

and adjunctions

$$
G \mathscr{S}_{B}\left(f_{!} X, Y\right) \cong G \mathscr{S}_{A}\left(X, f^{*} Y\right) \quad \text { and } \quad G \mathscr{S}_{A}\left(f^{*} Y, X\right) \cong G \mathscr{S}_{B}\left(Y, f_{*} X\right) .
$$

The functor $f^{*}$ is closed symmetric monoidal. Therefore, by definition and implication, $f^{*} S_{B} \cong S_{A}$ and there are natural isomorphisms

$$
\begin{gathered}
f^{*}\left(Y \wedge_{B} Z\right) \cong f^{*} Y \wedge_{A} f^{*} Z, \\
F_{B}\left(Y, f_{*} X\right) \cong f_{*} F_{A}\left(f^{*} Y, X\right), \\
f^{*} F_{B}(Y, Z) \cong F_{A}\left(f^{*} Y, f^{*} Z\right), \\
f_{!}\left(f^{*} Y \wedge_{A} X\right) \cong Y \wedge_{B} f_{!} X, \\
F_{B}\left(f_{!} X, Y\right) \cong f_{*} F_{A}\left(X, f^{*} Y\right) .
\end{gathered}
$$

Proof. We define the functors $f^{*}, f_{!}$, and $f_{*}$ levelwise. This certainly gives well-defined functors on $\mathscr{I}_{G}$-spaces that satisfy the appropriate adjunctions there. We shall show shortly that these functors preserve $\mathscr{I}_{G}$-spectra. For a based $G$ space $T, f^{*}\left(T_{B}\right) \cong T_{A}$, and this implies $f^{*} S_{B} \cong S_{A}$. If we replace $\mathscr{I}_{G \text {-spectra }}$ by $\mathscr{I}_{G}$-spaces and replace the internal smash product and function object functors $(\wedge$ and $F)$ by their external precursors $(\bar{\wedge}$ and $\bar{F})$, then everything is immediate by levelwise application of the corresponding results for ex-spaces. Still working with $\mathscr{I}_{G}$-spaces, we first show how to internalize the isomorphisms (11.4.2) and (11.4.5) by use of the universal property of left Kan extension. Indeed, noting that $\left(f_{*} X\right) \circ \oplus \cong f_{*}(X \circ \oplus)$, and similarly for $f^{*}$ and $f_{!}$, we have

$$
\begin{aligned}
\mathscr{I}_{G} \mathscr{K}_{A}\left(f^{*}\left(Y \wedge_{B} Z\right), X\right) & \cong \mathscr{I}_{G} \mathscr{K}_{B}\left(Y \wedge_{B} Z, f_{*} X\right) \\
& \cong\left(\mathscr{I}_{G} \times \mathscr{I}_{G}\right) \mathscr{K}_{B}\left(Y \bar{\wedge}_{B} Z, f_{*} X \circ \oplus\right) \\
& \cong\left(\mathscr{I}_{G} \times \mathscr{I}_{G}\right) \mathscr{K}_{A}\left(f^{*}\left(Y \bar{\wedge}_{B} Z\right), X \circ \oplus\right) \\
& \cong\left(\mathscr{I}_{G} \times \mathscr{I}_{G}\right) \mathscr{K}_{A}\left(f^{*} Y \bar{\wedge}_{A} f^{*} Z, X \circ \oplus\right) \\
& \cong \mathscr{I}_{G} \mathscr{K}_{A}\left(f^{*} Y \wedge_{A} f^{*} Z, X\right)
\end{aligned}
$$

and

$$
\begin{aligned}
\mathscr{I}_{G} \mathscr{K}_{B}\left(f_{!} X \wedge_{B} Y, Z\right) & \cong\left(\mathscr{I}_{G} \times \mathscr{I}_{G}\right) \mathscr{K}_{B}\left(f ! X \bar{\wedge}_{B} Y, Z \circ \oplus\right) \\
& \cong\left(\mathscr{I}_{G} \times \mathscr{I}_{G}\right) \mathscr{K}_{B}\left(f_{!}\left(X \bar{\wedge}_{A} f^{*} Y\right), Z \circ \oplus\right) \\
& \cong\left(\mathscr{I}_{G} \times \mathscr{I}_{G}\right) \mathscr{K}_{A}\left(X \bar{\wedge}_{A} f^{*} Y, f^{*} Z \circ \oplus\right) \\
& \cong \mathscr{I}_{G} \mathscr{K}_{A}\left(X \wedge_{A} f^{*} Y, f^{*} Z\right) \\
& \cong \mathscr{I}_{G} \mathscr{K}_{A}\left(f_{!}\left(X \wedge_{A} f^{*} Y\right), Z\right) .
\end{aligned}
$$


As explained in $[62, \S \S 2-3]$, the remaining isomorphisms on the $\mathscr{I}_{G}$-space level follow formally.

We must show that our functors on $\mathscr{I}_{G}$-spaces preserve $\mathscr{I}_{G}$-spectra. The given structure map $\sigma: Y \bar{\wedge}_{B} S_{B} \longrightarrow Y \circ \oplus$ gives rise via the external version of (11.4.2) to the required structure map

$$
f^{*} Y \bar{\wedge}_{A} S_{A} \cong f^{*}\left(Y \bar{\wedge}_{B} S_{B}\right) \longrightarrow f^{*} Y \circ \oplus .
$$

Similarly, the given structure map $\sigma: X \wedge S_{A} \longrightarrow X \circ \oplus$ gives rise to the required structure map

$$
f_{!} X \bar{\wedge}_{B} S_{B} \cong f_{!}\left(X \bar{\wedge}_{A} S_{A}\right) \longrightarrow f_{!} X \circ \oplus .
$$

As in $[62,(3.6)]$, there is a canonical natural map, not usually an isomorphism,

$$
\pi: f_{*} X \bar{\wedge}_{B} Y \longrightarrow f_{*}\left(X \bar{\wedge}_{A} f^{*} Y\right)
$$

Taking $Y=S_{B}$, we see that $\sigma$ also induces the required structure map

$$
f_{*} X \bar{\wedge}_{B} S_{B} \longrightarrow f_{*}\left(X \bar{\wedge}_{A} S_{A}\right) \longrightarrow f_{*} X \circ \oplus .
$$

Now the spectrum level adjunctions follow directly from their $\mathscr{I}_{G}$-space analogues. The spectrum level isomorphisms (11.4.2) and (11.4.5) follow from their $\mathscr{I}_{G}$-space analogues by comparisons of coequalizer diagrams, and the remaining isomorphisms again follow formally.

Remark 11.4.7. Since the base change functors are defined levelwise, they commute with the evaluation functors $\mathrm{Ev}_{V}$. These commutation relations for the right adjoints $f_{*}$ and $f^{*}$ imply conjugate commutation isomorphisms

$$
f^{*} F_{V} \cong F_{V} f^{*} \quad \text { and } \quad f_{!} F_{V} \cong F_{V} f_{!}
$$

of left adjoints. In particular,

$$
f^{*} \Sigma_{B}^{\infty} \cong \Sigma_{A}^{\infty} f^{*} \quad \text { and } \quad f_{!} \Sigma_{A}^{\infty} \cong \Sigma_{B}^{\infty} f_{!} .
$$

Via (11.2.6), these isomorphisms and the isomorphisms of the theorem imply isomorphisms relating base change functors to tensors and cotensors. For example (11.4.5) implies isomorphisms

$$
f_{!}\left(f^{*} Y \wedge_{A} K\right) \cong Y \wedge_{B} f_{!} K \quad \text { and } \quad f_{!}\left(f^{*} L \wedge_{A} X\right) \cong L \wedge_{B} f_{!} X .
$$

Here $K$ and $L$ are ex-spaces over $A$ and $B$ and $X$ and $Y$ are spectra over $A$ and $B$.

The following result is immediate from its precursor Proposition 2.2.11 for exspaces.

Proposition 11.4.8. Suppose given a pullback diagram of $G$-spaces

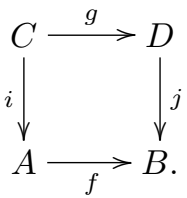

Then there are natural isomorphisms of functors

$$
j^{*} f_{!} \cong g_{!} i^{*}, \quad f^{*} j_{*} \cong i_{*} g^{*}, \quad f^{*} j_{!} \cong i_{!} g^{*}, \quad j^{*} f_{*} \cong g_{*} i^{*} .
$$

Returning to Remark 11.1.7, we have the following important results on external smash product and function spectra and their internalization by means of base change along diagonal maps. 
Proposition 11.4.10. Let $X$ be a spectrum over $A, Y$ be a spectrum over $B$, and $Z$ be a spectrum over $A \times B$. There is an external smash product functor that assigns a spectrum $X \bar{\wedge} Y$ over $A \times B$ to $X$ and $Y$ and an external function spectrum functor that assigns a spectrum $\bar{F}(Y, Z)$ over $A$ to $Y$ and $Z$, and there is a natural isomorphism

$$
G \mathscr{S}_{A \times B}(X \bar{\wedge} Y, Z) \cong G \mathscr{S}_{A}(X, \bar{F}(Y, Z)) .
$$

The internal smash products are determined from the external ones via

$$
X \wedge_{B} Y \cong \Delta^{*}(X \bar{\wedge} Y) \quad \text { and } \quad F_{B}(X, Y) \cong \bar{F}\left(X, \Delta_{*} Y\right),
$$

where $X$ and $Y$ are spectra over $B$ and $\Delta: B \longrightarrow B \times B$ is the diagonal map.

Proof. It is not hard to start from Remark 11.1.7 and construct these functors directly. We instead follow Lemma 2.5.5 and observe that the spectrum level external functors can and, up to isomorphism, must be defined in terms of the internal functors as

$$
X \wedge Y \cong \pi_{A}^{*} X \wedge_{A \times B} \pi_{B}^{*} Y \quad \text { and } \quad \bar{F}(Y, Z) \cong \pi_{A *} F_{A \times B}\left(\pi_{B}^{*} Y, Z\right),
$$

where $\pi_{A}: A \times B \longrightarrow A$ and $\pi_{B}: A \times B \longrightarrow B$ are the projections. The displayed adjunction is immediate from the adjunctions $\left(\pi_{A}^{*}, \pi_{A *}\right),\left(\pi_{B}^{*}, \pi_{B *}\right)$, and $\left(\wedge_{A \times B}, F_{A \times B}\right)$. The second statement follows formally, as in Lemma 2.5.6.

Proposition 11.4.11. For ex-spaces $K$ over $A$ and $L$ over $B$, there is a natural isomorphism

$$
\Sigma_{A \times B}^{\infty}(K \bar{\wedge} L) \cong \Sigma_{A}^{\infty} K \bar{\wedge} \Sigma_{B}^{\infty} L .
$$

Proof. This is most easily seen using adjunction and the Yoneda lemma. Using external function objects, we see that $\bar{F}\left(\Sigma_{B}^{\infty} L, Z\right) \cong \bar{F}(L, Z)$ for $Z \in G \mathscr{S}_{A \times B}$. This has zeroth ex-space $\bar{F}(L, Z(0))$ over $A$.

\subsection{Change of groups and restriction to fibers}

We give the analogues for parametrized spectra of the results concerning change of groups and restriction to fibers that were given for parametrized ex-spaces in $\S 2.3$. We shall say more about change of groups in Chapter 14. Fix an inclusion $\iota: H \longrightarrow G$ of a (closed) subgroup $H$ of $G$ and let $A$ be an $H$-space and $B$ be a $G$-space. We index $H$-spectra over $A$ on the collection $\iota^{*} \mathscr{V}$ of $H$-representations $\iota^{*} V$ with $V \in \mathscr{V}$. As we discuss in $\S \S 14.2$ and 14.3 , when $\mathscr{V}$ is the collection of all representations of $G$, we can change indexing to the collection of all representations of $H$ since our assumption that $G$ is compact ensures that every representation of $H$ is a direct summand of a representation $\iota^{*} V$. We have an evident forgetful functor

$$
\iota^{*}: G \mathscr{S}_{B} \longrightarrow H \mathscr{S}_{\iota^{*} B}
$$

On the space level, we write $\iota$ ambiguously for both the based and unbased induction functors $G_{+} \wedge_{H}(-)$ and $G \times_{H}(-)$, and similarly for coinduction $\iota_{*}$. Context should make clear which is intended. Applying the unbased versions to retracts, we defined induction and coinduction functors $\iota_{!}$and $\iota_{*}$ on ex-spaces in Definition 2.3.7. These functors extend to the spectrum level. Since we are now considering change of groups, we write $S_{G, B}$ instead of $S_{B}$ for the $G$-sphere spectrum over $B$.

Proposition 11.5.2. Levelwise application of $\iota$ ! and $\iota_{*}$ gives functors

$$
\iota !: H \mathscr{S}_{A} \longrightarrow G \mathscr{S}_{\iota_{1} A} \quad \text { and } \quad \iota_{*}: H \mathscr{S}_{A} \longrightarrow G \mathscr{S}_{\iota_{*} A} \text {. }
$$


Proof. We must show that the structure $H$-maps $\sigma: X \bar{\wedge} S_{H, A} \longrightarrow X \circ \oplus$ of an

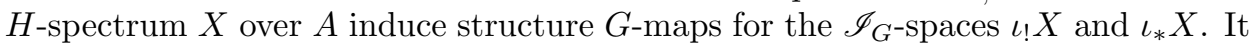
is clear that $\iota_{!}(X \circ \oplus) \cong \iota_{!} X \circ \oplus$ and $\iota_{*}(X \circ \oplus) \cong \iota_{*} X \circ \oplus$. Using (2.3.4), we see that $S_{G, \iota ! A} \cong \iota_{!} S_{H, A}$. Since the functor $\iota$ ! on the ex-space level is symmetric monoidal by Proposition 2.3.9, its levelwise $\mathscr{I}_{G}$-space analogue commutes up to isomorphism with the external smash product $\bar{\wedge}$. Thus $\sigma$ induces a structure $G$-map

$$
\iota ! X \bar{\wedge}_{\iota ! A} S_{G, \iota ! A} \cong \iota_{!}\left(X \bar{\wedge}_{A} S_{H, A}\right) \longrightarrow \iota !(X \circ \oplus) \cong \iota ! X \circ \oplus .
$$

For $\iota_{*}$, let $\mu: \iota^{*} \iota_{*} \longrightarrow$ Id be the counit of the space level adjunction $\left(\iota_{*}, \iota^{*}\right)$ (see (2.3.2)). For an $H$-space $A, \mu$ is the $H$-map $\operatorname{Map}_{H}(G, A) \longrightarrow A$ given by evaluation at the identity element of $G$. Applied to an ex-space $K$ over $A$, thought of as a retract, $\mu$ gives a map $\iota^{*} \iota_{*} K \longrightarrow K$ of total spaces over and under the map $\mu: \iota^{*} \iota_{*} A \longrightarrow A$ of base spaces in the category of retracts of $\S 2.5$. We can apply this to $X$ levelwise. We also have the projection pr : $\mu^{*} S_{H, A} \longrightarrow S_{H, A}$ over $\mu$. Together, these maps give

$$
\iota^{*}\left(\iota_{*} X \bar{\wedge}_{\iota_{*} A} S_{G, \iota_{*} A}\right) \cong \iota^{*} \iota_{*} X \bar{\wedge}_{\iota^{*} \iota_{*} A} \mu^{*} S_{H, A} \stackrel{\mu \bar{\wedge} \mathrm{pr}}{\longrightarrow} X \bar{\wedge}_{A} S_{H, A} .
$$

For the isomorphism, we have used the facts that $\iota^{*}$ is strong monoidal and that $\iota^{*} S_{G, \iota_{*} A} \cong S_{H, \iota^{*} \iota_{*} A} \cong \mu^{*} S_{H, A}$. The adjoint of the composite of this map with the structure map $\sigma: X \bar{\wedge}_{A} S_{H, A} \longrightarrow X \circ \oplus$ gives the required structure map $\iota_{*} X \bar{\wedge}_{\iota_{*} A} S_{G, \iota_{*} A} \longrightarrow \iota_{*} X \circ \oplus$.

As on the ex-space level, the categories $H \mathscr{S}_{A}$ and $G \mathscr{S}_{\iota_{1} A}=G \mathscr{S}_{G \times H} A$ can be used interchangeably. The following result is immediate from Proposition 2.3.9.

Proposition 11.5.3. Let $\nu: A \longrightarrow \iota^{*} \iota ! A$ be the natural inclusion of $H$-spaces. Then $\iota_{!}: H \mathscr{S}_{A} \longrightarrow G \mathscr{S}_{\iota ! A}$ is a closed symmetric monoidal equivalence of categories with inverse the composite $\nu^{*} \circ \iota^{*}: G \mathscr{S}_{\iota ! A} \longrightarrow H \mathscr{S}_{\iota^{*} \iota !} \longrightarrow \longrightarrow H \mathscr{S}_{A}$.

In particular, if $A=*$ then $\nu$ maps $*$ to the identity coset $e H \in G / H$ and we see that $H \mathscr{S}$ and $G \mathscr{S}_{G / H}$ can be used interchangeably. Arguing as in Proposition 2.3.1, we could more easily prove this directly.

COROLlary 11.5.4. The category HS is equivalent as a closed symmetric monoidal category to G $\mathscr{S}_{G / H}$. Under this equivalence,

$$
\iota^{*} \cong r^{*}, \quad \iota ! \cong r_{!}, \quad \text { and } \quad \iota_{*} \cong r_{*},
$$

where $r: G / H \longrightarrow *$.

Looking at the fiber $X_{b}(V)=X(V)_{b}$ over $b$ of a $G$-spectrum $X$ over $B$, we see a $G_{b}$-spectrum $X_{b}$ of the sort that has been studied in [105], where $G_{b}$ is the isotropy group of $b$. Our homotopical analysis of parametrized $G$-spectra will be based on the idea of applying the results of [105] fiberwise. By the previous result, we can think of this fiber as a $G$-spectrum over $G / G_{b}$. The following spectrum level analogues of Example 2.3.12 and Example 2.3.13 analyze the relationships among passage to fibers, base change, and change of groups.

EXAmple 11.5.5. For $b \in B$, we write $b: * \longrightarrow B$ for the $G_{b}$-map that sends * to $b$ and $\tilde{b}: G / G_{b} \longrightarrow B$ for the induced inclusion of orbits. Under the equivalence $G \mathscr{S}_{G / G_{b}} \cong G_{b} \mathscr{S}, \tilde{b}^{*}$ may be interpreted as the fiber functor $G \mathscr{S}_{B} \longrightarrow G_{b} \mathscr{S}$ that sends $Y$ to $Y_{b}$. Its left and right adjoints $\tilde{b}_{!}$and $\tilde{b}_{*}$ may be interpreted as the functors that send a $G_{b}$-spectrum $X$ to the $G$-spectra $X^{b}$ and ${ }^{b} X$ over $B$ obtained by levelwise 
application of the corresponding ex-space level adjoints of Example 2.3.12. With these notations, the isomorphisms of Theorem 11.4.1 specialize to the following natural isomorphisms, where $Y$ and $Z$ are in $G \mathscr{S}_{B}$ and $X$ is in $G_{b} \mathscr{S}$.

$$
\begin{aligned}
&\left(Y \wedge_{B} Z\right)_{b} \cong Y_{b} \wedge Z_{b}, \\
& F_{B}\left(Y,{ }^{b} X\right) \cong{ }^{b} F\left(Y_{b}, X\right), \\
& F_{B}(Y, Z)_{b} \cong F\left(Y_{b}, Z_{b}\right), \\
&\left(Y_{b} \wedge X\right)^{b} \cong Y \wedge_{B} X^{b}, \\
& F_{B}\left(X^{b}, Y\right) \cong{ }^{b} F\left(X, Y_{b}\right) .
\end{aligned}
$$

ExAmple 11.5.6. Let $f: A \longrightarrow B$ be a $G$-map and let $i_{b}: A_{b} \longrightarrow B$ be the inclusion of the fiber over $b$, which is a $G_{b}$-map. As in Example 2.3.13, we have the compatible pullback squares
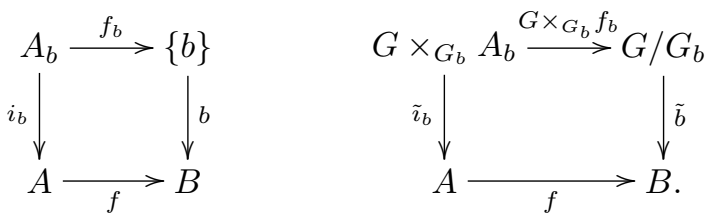

Applying Proposition 11.4.8 to the right-hand square and interpreting the conclusion in terms of fibers, we obtain canonical isomorphisms of $G_{b}$-spectra

$$
\left(f_{!} X\right)_{b} \cong f_{b !} i_{b}^{*} X \quad \text { and } \quad\left(f_{*} X\right)_{b} \cong f_{b *} i_{b}^{*} X,
$$

where $X$ is a $G$-spectrum over $A$, regarded on the right-hand sides as a $G_{b}$-spectrum over $A$ by pullback along $\iota: G_{b} \longrightarrow G$.

\subsection{Some problems concerning non-compact Lie groups}

In equivariant stable homotopy theory, the key idea is that the one-point compactification of a representation $V$ of dimension $n$ is a $G$-sphere and that smashing with that sphere should be a self-equivalence of the equivariant stable homotopy category. That is, the idea is to invert $G$-spheres in just the way that we invert spheres when constructing the nonequivariant stable homotopy category. For compact Lie groups of equivariance, the philosophy and its implementation and applications are well understood. When we invert representation spheres, we invert other homotopy spheres as well, and the relevant Picard group is analyzed in [63].

For non-compact Lie groups, the philosophy is less clear and its technical implementation is problematic. The focus on finite dimensional representations is intrinsic to the philosophy just expressed, but fails to come to grips with basic features of the representation theory of non-compact Lie groups. A theory based on finite dimensional representations may still have its uses, but there are real difficulties in obtaining even that much. In particular, a focus on spheres associated to linear representations, rather than on less highly structured homotopy spheres, may be misplaced.

A non-compact semi-simple Lie group will generally have no non-trivial finite dimensional unitary or orthogonal representations, hence our theory of "orthogonal" $G$-spectra is clearly too restrictive. This, at least, is easily remedied. The use of linear isometries in the definition of orthogonal spectra is a choice dictated more 
by the history than by the mathematics. In the alternative approach to equivariant stable homotopy theory based on Lewis-May spectra and EKMM [61,98, 105], use of orthogonal complements is certainly convenient and perhaps essential. However, the diagram orthogonal spectra of $[105,106]$ could just as well have been developed in terms of diagram "general linear spectra". In the few places where complements are used, they can by avoided. For consistency with the previous literature, we have chosen to give our exposition in the compact case using the word "orthogonal" and the language from the cited references, but for general Lie groups of equivariance, we should eliminate all considerations of isometries.

More precisely, for the complete case, we redefine $\mathscr{I}$ by taking $\mathscr{V}$ to be the collection of all finite dimensional representations $V$ of $G$. More generally, we can index on any subcollection that contains the trivial representation and is closed under finite direct sums. Since we are only interested in a skeleton of $\mathscr{I}$, we may as well restrict to orthogonal representations in $\mathscr{V}$ when $G$ is compact. We replace linear isometries by linear isomorphims when defining the $G$-spaces $\mathscr{I}(V, W)$. Thus we replace orthogonal groups by general linear groups. Otherwise, the formal definitional framework developed in this chapter (or, in the nonparametrized case, $[105, \mathrm{II}])$ goes through verbatim for general topological groups $G$.

However, we emphasize the formality. When considering change of groups, for example, the significance changes drastically. As noted at the start of the previous section, for an inclusion $\iota: H \longrightarrow G$ of a (closed) subgroup $H$ of $G$, we index $H$-spectra on the collection $\iota^{*} \mathscr{V}$ of $H$-representations $\iota^{*} V$ with $V \in \mathscr{V}$. We also pointed out the relevance of the compact case of the following result.

Proposition 11.6.1. If $G$ is either a compact Lie group or a matrix group and $W$ is a representation of a subgroup $H$, then there is a representation $V$ of $G$ and an embedding of $W$ as a subrepresentation of $\iota^{*} V$.

This is clear in the compact case and is given by [136, 3.1] for matrix groups. However, the following striking counterexample, which we learned from Victor Ginzburg, shows just how badly this basic result fails in general.

Counterexample 11.6.2 (Ginzburg). Let $\mathbb{H}$ be the Heisenberg group of $3 \times 3$ matrices

$$
\left(\begin{array}{lll}
1 & a & c \\
0 & 1 & b \\
0 & 0 & 1
\end{array}\right)
$$

where $a, b$, and $c$ are real numbers. Embed $\mathbb{R}$ in $\mathbb{H}$ as the subgroup of matrices with $a=b=0$. Embed $\mathbb{Z}$ in $\mathbb{R}$ as usual. Then $\mathbb{R}$ is a central subgroup of $\mathbb{H}$. Define $G=\mathbb{H} / \mathbb{Z}$. Then $T=\mathbb{R} / \mathbb{Z}$ is a circle subgroup of $G$. Moreover, $T$ is the center of $G$ and coincides with the commutator subgroup $[G, G]$. Let $V$ be any finite dimensional (complex linear) representation of $G$. Since $T$ is compact, the action of $T$ on $V$ is semisimple, and since $T$ is central, any weight space of $T$ is a $G$-submodule. Therefore $V$ is a direct sum of $G$-submodules $V_{i}$ such that $T$ acts on each $V_{i}$ by scalar matrices. Since $T=[G, G]$, this scalar action of $T$ on $V_{i}$ is trivial: the determinant of $g$ is 1 for any $g \in[G, G]$. Therefore no nontrivial 1-dimensional character of $T$ can embed in $V$. Reinterpreting in terms of real representations, as we may, we conclude that, for $\iota: T \longrightarrow G, \iota^{*} \mathscr{V}$ is the trivial $T$-universe.

For a compact Lie group $G$ and an inclusion $\iota: H \subset G, \iota^{*} X$ is a dualizable $H$ spectrum if $X$ is a dualizable $G$-spectrum, and an $H$-spectrum indexed on the trivial 
$H$-universe is dualizable if and only if it is a retract of a finite $H$-CW spectrum built up from trivial orbits. We conclude that duality theory (in the nonparametrized context) cannot work as one would wish in the context of the previous example.

Looking ahead, some of the theory of the following three chapters also works formally in the context of non-compact Lie groups. However, there is an obvious mismatch between our emphasis on the one-point compactifications of representations, which have at least two fixed points even if the isotropy groups away from zero are compact, and the desire to focus on proper actions. Even ignoring 0 and $\infty$, it is not clear that anything like Illman's Theorem 3.4.2 applies to give homotopical control on spheres of representations, however they might be interpreted, or on orbits. A serious attempt to come to grips with these and other issues is beyond the scope of this book. 


\section{CHAPTER 12}

\section{Model structures for parametrized $G$-spectra}

\section{Introduction}

We define and study two model structures on the category $G \mathscr{S}_{B}$ of (orthogonal) $G$-spectra over $B$. We emphasize that, except for the theory of smash products, everything in this chapter applies equally well to the category $G \mathscr{P}_{B}$ of $G$-prespectra over $B$. That fact will become important in the next chapter.

We start in $\S 12.1$ by defining a "level model structure" on $G \mathscr{S}_{B}$, based on the $q f$-model structure on $G \mathscr{K}_{B}$. In $\S 12.2$, we record analogues for this model structure of the results on external smash product and base change functors that were given for $G \mathscr{K}_{B}$ in $\$ 7.2$. The level model structure serves as a stepping stone to the stable model structure, which we define in $§ 12.3$. It has the same cofibrations as the level model structure, and we therefore call these "s-cofibrations". An essential point in our approach is a fiberwise definition of the homotopy groups of a parametrized $G$-spectrum that throws much of our work onto the theory of nonparametrized orthogonal $G$-spectra developed by Mandell and the first author in [105]. We define homotopy groups using the level $q f$-fibrant replacement functor provided by the level model structure, and we define stable equivalences to be the $\pi_{*}$-isomorphisms. It is essential to think in terms of fibers and not total spaces since the total spaces of a parametrized spectrum do not assemble into a spectrum. We show in $\S 12.4$ that the $\pi_{*}$-isomorphisms give a well-grounded subcategory of weak equivalences, and we complete the proofs of the model axioms in $\S 12.5$. We return to the context of $\S 12.2$ in $\S 12.6$, where we prove that various Quillen adjoint pairs in the level model structures are also Quillen adjoint pairs in the stable model structures.

The basic conclusion is that $G \mathscr{S}_{B}$ is a well-grounded model category in the sense of $\$ 5.5$ under the stable model structure. Although not very noticeable on the surface, essential use is made of the $q f$-model structure on $G \mathscr{K}_{B}$ throughout this chapter. As we observe in Remark 12.1.8, the $q$-model structure on $G \mathscr{K}_{B}$ does give rise to a level model structure on $G \mathscr{S}_{B}$. However this model structure is not wellgrounded and does not provide the necessary tools to work out the technical details of $\S 12.4$. The results there are crucial to prove that the relative cell complexes over $B$ defined in terms of the appropriate generating acyclic $s$-cofibrations are acyclic. ${ }^{1}$ It was our fruitless attempt to obtain a stable model structure starting from the level $q$-model structure that led us to the construction of the $q f$-model structure on $G \mathscr{K}_{B}$ and to the notion of a well-grounded model category.

When there are no issues of equivariance, we generally abbreviate $G$-spectrum over $B$, ex- $G$-space, and $G$-space to spectrum over $B$, ex-space, and space; $G$ is a compact Lie group throughout.

\footnotetext{
${ }^{1}$ In $[78,3.4]$, such acyclicity of relative cell complexes is assumed without proof.
} 


\subsection{The level model structure on $G \mathscr{S}_{B}$}

After changing the base space from $*$ to $B$, the level model structure works in much the same way as in the nonparametrized case of [105].

Definition 12.1.1. Let $f: X \longrightarrow Y$ be a map of spectra over $B$. With one exception, for any type of ex-space and any type of map of ex-spaces, we say that $X$ or $f$ is a level type of spectrum over $B$ or a level type of map of spectra over $B$ if each $X(V)$ or $f(V): X(V) \longrightarrow Y(V)$ is that type of ex-space or that type of map. Thus, for example, we have level $h$, level $f$ and level $f p$-fibrations, cofibrations and equivalences from $\S 5.1$ together with the corresponding fibrant and cofibrant objects. We have level $q$-equivalences and level $q$ and $q f$-fibrations from $\S 7.1$ and we have level ex-fibrations and level ex-quasifibrations from $\S 8.1$ and $\S 8.5$. The exceptions concern cofibrations and cofibrant objects. We shall never be interested in "level $q$-cofibrations" or "level $q f$-cofibrations", nor in "level $q$-cofibrant" or "level $q f$-cofibrant" objects, since these do not correspond to cofibrations and cofibrant objects in the model structures that we consider. Instead we have the following definitions.

(i) $f$ is an $s$-cofibration if it satisfies the LLP with respect to the level acyclic $q f$-fibrations.

(ii) $f$ is a level acyclic s-cofibration if it is both a level $q$-equivalence and an $s$-cofibration.

To reiterate, in the phrase "level acyclic $q f$-fibration", the adjective "level" applies to "acyclic $q f$-fibration", but in the phrase "level acyclic $s$-cofibration" it applies only to "acyclic"; the cofibrations are not defined levelwise.

Definition 12.1.2. A spectrum $X$ over $B$ is well-sectioned if it is level wellsectioned, so that each ex-space $X(V)$ is $\bar{f}$-cofibrant. It is well-grounded if it is level well-grounded, so that each $X(V)$ is well-sectioned and compactly generated.

The discussion of $\S 4.3$ applies to the category $G \mathscr{S}_{B}$ of $G$-spectra over $B$ with homotopies defined in terms of the cylinders $X \wedge_{B} I_{+}$. In particular, we have the notion of a Hurewicz cofibration in $G \mathscr{S}_{B}$, abbreviated cyl-cofibration, defined in terms of these cylinders, and we also have the notion of strong Hurewicz cofibration, abbreviated $\overline{c y l}$-cofibration.

LEMma 12.1.3. A cyl-cofibration of spectra over $B$ is a level $f p$-cofibration and a cyl-fibration of spectra over $B$ is a level $f p$-fibration. A cyl-cofibration between well-sectioned spectra over $B$ is a level $f$-cofibration and therefore both a level $h$ cofibration and a level $f p$-cofibration.

ProOF. By the mapping cylinder retraction characterization of Hurewicz cofibrations, a $c y l$-cofibration of spectra over $B$ is a level $f p$-cofibration. The statement about fibrations follows similarly from the path lifting function characterization of Hurewicz fibrations. An $f p$-cofibration between well-sectioned ex-spaces is an $f$ cofibration by Proposition 5.2.3, and all $f$-cofibrations are $h$-cofibrations.

Recall the notions of a ground structure and of a well-grounded subcategory of weak equivalences from Definitions 5.3.2, 5.3.3, and 5.4.1.

Proposition 12.1.4. The well-grounded spectra over $B$ give $G \mathscr{S}_{B}$ a ground structure whose ground cofibrations, or g-cofibrations, are the level h-cofibrations. 
The level q-equivalences specify a well-grounded subcategory of weak equivalences with respect to this ground structure. In the gluing and colimit lemmas, one need only assume that the relevant maps are level $h$-cofibrations, not necessarily also cyl-cofibrations.

Proof. That we have a ground structure follows levelwise from the ground structure on ex-spaces in Proposition 5.3.7. That the level q-equivalences are wellgrounded follows levelwise from Proposition 5.4.9.

We construct the level model structure on $G \mathscr{S}_{B}$ from the $q f$-model structure on $G \mathscr{K}_{B}$ specified in Remark 7.2 .11 , but all results apply verbatim starting from the $q f(\mathscr{C})$-model structure for any closed generating set $\mathscr{C}$ (as defined in Definition 7.2.6). We shall need the extra generality for the reasons discussed in Chapter 7. Recall that $I_{B}^{f}$ and $J_{B}^{f}$ denote the sets of generating $q f$-cofibrations and generating acyclic $q f$-cofibrations in $G \mathscr{K}_{B}$. We use the shift desuspension functors $F_{V}$ of Definition 11.3.5 to obtain corresponding sets on the spectrum level. We need the following observations.

Lemma 12.1.5. The functor $F_{V}$ enjoys the following properties.

(i) If $K$ is a well-grounded ex-space over $B$, then $F_{V} K$ is well-grounded. If $K$ is an ex-fibration, then $F_{V} K$ is a level ex-fibration.

(ii) If $i: K \longrightarrow L$ is an h-equivalence between well-grounded ex-spaces over $B$, then $F_{V} i$ is a level h-equivalence.

(iii) If $i: K \longrightarrow L$ is an $f p$-cofibration, then $F_{V} i$ is a cyl-cofibration and therefore a level fp-cofibration. If, further, $K$ and $L$ are well-sectioned, then $F_{V} i$ is a level $f$-cofibration and therefore a level $h$-cofibration.

(iv) If $i: K \longrightarrow L$ is an $\overline{f p}$-cofibration, then $F_{V} i$ is a $\overline{c y l}$-cofibration.

(v) If $i: K \longrightarrow L$ is an $\bar{f}$-cofibration between well-grounded ex-spaces over $B$, then $F_{V} i$ is a $\overline{c y l}$-cofibration which is a level $\bar{f}$-cofibration and therefore both a level $\overline{f p}$-cofibration and a level $\bar{h}$-cofibration.

Proof. By Definition 11.3.5, $\left(F_{V} K\right)(W)=\mathscr{J}_{G}(V, W)_{B} \wedge_{B} K$, and the $G$ space $\mathscr{J}_{G}(V, W)$ is well-based. Now (i) holds by Corollary 8.2 .5 and (ii) holds by Proposition 8.2.6. Since $F_{V}$ is left adjoint to the evaluation functor $\mathrm{Ev}_{V}$ and since $c y l$-fibrations are level $f p$-fibrations, (iv) and the first statement of (iii) follow from the definitions by adjunction. The second statement of (iii) follows from Proposition 5.2.3. The first half of (v) follows from (iv) since $\bar{f}$-cofibrations are $\overline{f p}$-cofibrations, and the second half follows from (iii) since $F_{V} i$ is a level $f$ cofibration between well-grounded spectra and therefore a level $\bar{f}$-cofibration by Theorem 5.2.8(ii).

Definition 12.1.6. Define $F I_{B}^{f}$ to be the set of maps $F_{V} i$ with $V$ in a skeleton $s k \mathscr{I}_{G}$ of $\mathscr{I}_{G}$ and $i$ in $I_{B}^{f}$. Define $F J_{B}^{f}$ to be the set of maps $F_{V} j$ with $V$ in $s k \mathscr{I}_{G}$ and $j$ in $J_{B}^{f}$.

Recall the notion of a well-grounded model structure from Definition 5.5.4. Among other properties, such model structures are compactly generated, proper, and $G$-topological.

TheOREM 12.1.7. The category $G \mathscr{S}_{B}$ is a well-grounded model category with respect to the level q-equivalences, the level $q f$-fibrations and the $s$-cofibrations. The 
sets $F I_{B}^{f}$ and $F J_{B}^{f}$ give the generating s-cofibrations and the generating level acyclic s-cofibrations. All s-cofibrations are both Cyl-cofibrations and level $\bar{f}$-cofibrations, hence level $\overline{f p}$ and level $\bar{h}$-cofibrations, and all s-cofibrant spectra over $B$ are wellgrounded.

Proof. By Lemma 12.1.5, the maps in $F I_{B}^{f}$ and $F J_{B}^{f}$ are $\overline{c y l}$-cofibrations between well-grounded objects and $\bar{f}$-cofibrations, hence the $s$-cofibrations are bicofibrations by Lemma 5.3.5. Moreover, the maps in $F J_{B}^{f}$ are level acyclic. Therefore, to prove the model axioms, we need only verify the compatibility condition (ii) in Theorem 5.5.1. Adjunction arguments show that a map is a level $q f$-fibration if and only if it has the RLP with respect to $F J_{B}^{f}$ and that it is a level acyclic $q$-fibration if and only if it has the RLP with respect to $F I_{B}^{f}$. This implies that the classes of $s$-cofibrations and of $F I_{B}^{f}$-cofibrations (in the sense of Definition 4.5.1(iii)) coincide. Therefore, if a map has the RLP with respect to $F I_{B}^{f}$, then it is a level acyclic $q f$-fibration. The required compatibility condition now follows from its analogue for $G \mathscr{K}_{B}$. Condition (iv) in Theorem 5.5.1 holds by its ex-space level analogue and the fact that $\left(F_{V} K\right) \wedge_{B} T \cong F_{V}\left(K \wedge_{B} T\right)$ for an ex-space $K$ over $B$ and a based space $T$. Right properness follows directly from the space level analogue.

Remark 12.1.8. Just as in Definition 12.1.6, we can also define sets $F I_{B}$ and $F J_{B}$ based on the generating sets $I_{B}$ and $J_{B}$ for the $q$-model structure on $G \mathscr{K}_{B}$. We can then use Theorem 4.5.6 to prove the analogue of Theorem 12.1.7 stating that $G \mathscr{S}_{B}$ is a compactly generated model category under the level $q$-model structure. Since the compatibility condition holds by the same proof as for the level $q f$-model structure, we need only verify the acyclicity condition to show this.

For a generating acyclic $q$-cofibration $j \in J_{B}$, we have $F_{V} j=V^{*} \wedge_{B} j$, where $V^{*}(W)=\mathscr{J}_{G, B}(V, W)$. This map is a level $h$-equivalence by Lemma 12.1.5(ii). Although $j$ is an $h$-cofibration, it is not immediate that $F_{V} j$ is a level $h$-cofibration. (This holds for $j \in J_{B}^{f}$ by Lemma 12.1.5(iii), since $j$ is then an $f p$-cofibration). Indeed, for general ex-spaces $K$ and $h$-cofibrations $f, K \wedge_{B} f$ need not be an $h$ cofibration. However, since $\mathscr{J}_{G, B}(V, W)=\mathscr{J}_{G}(V, W)_{B}$, we see directly that $F_{V} j$ is indeed a level $h$-cofibration. By inspection of the definition of wedges over $B$ in terms of pushouts, the gluing lemma in $\mathscr{K}$ then applies to show that wedges over $B$ of maps in $F J_{B}$ are level acyclic $h$-cofibrations. Since pushouts and colimits in $\mathscr{S}_{B}$ are constructed levelwise on total spaces, it follows that relative $F J_{B}$ complexes are acyclic $h$-cofibrations since the $q$-model structure on $\mathscr{K}$ is well-grounded.

REMARK 12.1.9. As in the nonparametrized case [105], "positive" model structures would be needed to obtain a comparison with the as yet undeveloped alternative approach to parametrized stable homotopy theory based on [61,98]. Such model structures can be defined as in [105, p. 44], starting from the subsets $\left(F I_{B}^{f}\right)^{+}$ and $\left(F J_{B}^{f}\right)^{+}$that are obtained by restricting to those $V$ such that $V^{G} \neq 0$. One then defines the positive level versions of all of the types of maps specified in Definition 12.1 .1 by restricting to those levels $V$ such that $V^{G} \neq 0$. The positive level analogue of Theorem 12.1.7 holds, where the positive $s$-cofibrations are the $s$-cofibrations that are isomorphisms at all levels $V$ such that $V^{G}=0$; compare [105, III.2.10]. However, we shall make no use of the positive model structure in this paper, and we will make little further reference to it.

The same proof as in [105, I.2.10, II.4.10, III.2.12] gives the following result. 
THEOREM 12.1.10. The forgetful functor $\mathbb{U}$ from spectra over $B$ to prespectra over $B$ has a left adjoint $\mathbb{P}$ such that $(\mathbb{P}, \mathbb{U})$ is a Quillen equivalence.

\subsection{Some Quillen adjoint pairs relating level model structures}

This section gives the analogues for the level model structure of some of the ex-space level results in $\S \S 7.2-7.4$. These results are also analogues of results in [105, III.§2], which in turn have non-equivariant precursors in $[106, \S 6]$. They admit essentially the same proofs as in Chapter 7 or in the cited references. The level $q f$ model structure is understood throughout. More precisely, where a $q f(\mathscr{C})$-model structure was used in Chapter 7 , we must use the corresponding level $q f(\mathscr{C})$-model structure here. Since we want our model structures to be $G$-topological, we only use generating sets $\mathscr{C}$ that are closed under finite products.

Our first observation is immediate from the fact that equivalences and fibrations are defined levelwise, the next follows directly from its ex-space analogue Proposition 7.3.1, and the third and fourth are proven in the same way as their ex-space analogues 7.3.2 and Corollary 7.3.3. All apply to the level $q f(\mathscr{C})$-model structures for any choice of $\mathscr{C}$.

Proposition 12.2.1. The pair of adjoint functors $\left(F_{V}, E v_{V}\right)$ between $G \mathscr{K}_{B}$ and $G \mathscr{S}_{B}$ is a Quillen adjoint pair.

Proposition 12.2.2. For a based $G-C W$ complex $T,\left((-) \wedge_{B} T, F_{B}(T,-)\right)$ is a Quillen adjoint pair of endofunctors of $G \mathscr{S}_{B}$.

Proposition 12.2.3. If $i: X \longrightarrow Y$ and $j: W \longrightarrow Z$ are $s$-cofibrations of spectra over base spaces $A$ and $B$, then

$$
i \square j:(Y \bar{\wedge} W) \cup_{X \bar{\wedge} W}(X \bar{\wedge} Z) \longrightarrow Y \bar{\wedge} Z
$$

is an s-cofibration over $A \times B$ which is level acyclic if either $i$ or $j$ is acyclic.

As in $\S 7.2$, we cannot expect this result to hold for internal smash products over $B$. The case $A=*$, which relates spectra to spectra over $B$, is particularly important. As we explain in $\S 14.1$, it leads to a fully satisfactory theory of parametrized module spectra over nonparametrized ring spectra.

COROLlary 12.2.4. If $Y$ is s-cofibrant over $B$, then the functor $(-) \wedge Y$ from $G \mathscr{S}_{A}$ to $G \mathscr{S}_{A \times B}$ is a Quillen left adjoint with Quillen right adjoint $\bar{F}(Y,-)$.

Again the next result is a direct consequence of its ex-space analogue Proposition 7.3.4 and applies with any choice of $\mathscr{C}$.

Proposition 12.2.5. Let $f: A \longrightarrow B$ be a $G$-map. Then $\left(f_{!}, f^{*}\right)$ is a Quillen adjoint pair. The functor $f_{!}$preserves level q-equivalences between well-sectioned $G$-spectra over $B$. If $f$ is a $q f$-fibration, then $f^{*}$ preserves all level q-equivalences.

Proposition 12.2.6. If $f: A \longrightarrow B$ is a q-equivalence, then $\left(f_{!}, f^{*}\right)$ is a Quillen equivalence.

Proof. We mimic the proof of Proposition 7.3.5, but with $X$ and $Y$ taken to be an $s$-cofibrant $G$-spectrum over $A$ and a level $q f$-fibrant $G$-spectrum over $B$. It is clear that $f^{*} Y \longrightarrow Y$ is a level $q$-equivalence since $A \longrightarrow B$ is a $q$-equivalence. Since $X$ is $s$-cofibrant, $*_{A} \longrightarrow X$ is a level $h$-cofibration. Note that it is essential for this statement that we start from the $q f$ and not the $q$-model structure on ex-spaces. 
Since pushouts along level $h$-cofibrations preserve level $q$-equivalences, $X \longrightarrow f_{!} X$ is a level $q$-equivalence. The conclusion follows as in Proposition 7.3.5.

Proposition 12.2.7. Let $f: A \longrightarrow B$ be a $G$-bundle whose fibers $A_{b}$ are $G_{b^{-}}$ $C W$ complexes. Then the functor $f^{*}$ preserves level $q$-equivalences and s-cofibrations. Therefore $\left(f^{*}, f_{*}\right)$ is a Quillen adjoint pair.

Proof. Here we must use a generating set $\mathscr{C}(f)$ as specified in Proposition 7.3.8. The proof that $f^{*}$ preserves $s$-cofibrations reduces to showing that the maps $f^{*} F_{V} i \cong F_{V} f^{*} i$ are $s$-cofibrations for generating $s$-cofibrations $i$. Since $F_{V}$ is a Quillen left adjoint it takes $q f$-cofibrations to $s$-cofibrations, so we are reduced to the ex-space level, where $f^{*} i$ is shown to be a $q f$-cofibration in Proposition 7.3.8.

Corollary 12.2.8. If $B$ is a $G$-cell complex, then $\left(r^{*}, r_{*}\right)$ is a Quillen adjoint pair.

Now consider the change of groups functors of $\S 11.5$. The following result shows that the equivalence of Proposition 11.5.3 descends to homotopy categories. It is proven by levelwise application of its ex-space analogue Proposition 7.4.7, together with change of universe considerations that are deferred until $\S 14.2$ and $\S 14.3$.

Proposition 12.2.9. Let $\iota: H \longrightarrow G$ be the inclusion of a subgroup. The pair of functors $\left(\iota !, \nu^{*} \iota^{*}\right)$ relating $H \mathscr{S}_{A}$ and $G \mathscr{S}_{\iota !} A$ give a Quillen equivalence. If $A$ is completely regular, then $\iota$ ! is also a Quillen right adjoint.

For a point $b$ in $B$, we combine the special case $\tilde{b}: G / G_{b} \longrightarrow B$ of Proposition 12.2.5 with Proposition 12.2.9, where $\iota: G_{b} \longrightarrow G$ and $\nu: * \longrightarrow G / G_{b}$, to obtain the following analogue of Proposition 7.5.1. Recall from Example 11.5.5 that the fiber functor $(-)_{b}: G \mathscr{S}_{B} \longrightarrow G_{b} \mathscr{S}$ is given by $\nu^{*} \iota^{*} \tilde{b}^{*}=b^{*} \iota^{*}$. Its left adjoint $(-)^{b}$ therefore agrees with $\tilde{b}_{!} \iota !$.

Proposition 12.2.10. For $b \in B$, the pair of functors $\left((-)^{b},(-)_{b}\right)$ relating $G_{b} \mathscr{S}_{*}$ and $G \mathscr{S}_{B}$ is a Quillen adjoint pair.

\subsection{The stable model structure on $G \mathscr{S}_{B}$}

The essential point in the construction of the stable model structure is to define the appropriate (stable) homotopy groups. The weak equivalences will then be the maps of parametrized spectra that induce isomorphisms on all homotopy groups. We refer to them as the $\pi_{*}$-isomorphisms or $s$-equivalences, using these terms interchangeably. There are several motivating observations for our definitions. We return the group $G$ to the notations for much of this section.

For the first, let $*_{B}$ denote the terminal $G$-spectrum over $B$, so that each $*_{B}(V)$ is the terminal ex- $G$-space $*_{B}$. Then a $G$-spectrum $X$ over $B$ is level $q f$-fibrant if and only if each projection $X(V) \longrightarrow *_{B}(V)=*_{B}$ is a $q f$-fibration of ex-G-spaces. It is equivalent that each fixed point map $X(V)^{H} \longrightarrow B^{H}$ be a non-equivariant $q f$-fibration, and, by Proposition 6.5.1, we have resulting long exact sequences of homotopy groups

$$
\cdots \longrightarrow \pi_{q+1}^{H}(B) \longrightarrow \pi_{q}^{H}\left(X_{b}(V)\right) \longrightarrow \pi_{q}^{H}(X(V)) \longrightarrow \pi_{q}^{H}(B) \longrightarrow \cdots
$$

for each $b \in B^{H}$. Here, for a $G$-space $T, \pi_{q}^{H}(T)$ denotes $\pi_{q}\left(T^{H}\right)$. 
Second, as we have already discussed in $\S 11.4$, the fibers $X_{b}$ of a $G$-spectrum $X$ are $G_{b}$-spectra, and our guiding principle is to use these nonparametrized spectra to encode the homotopical information about our parametrized spectra. Proposition 12.2.10 allows us to encode levelwise information in the level homotopy groups of fibers, and it is plausible that we can similarly encode the full structure of our parametrized $G$-spectrum $X$ in the spectrum level homotopy groups of the fiber $G_{b}$-spectra $X_{b}$. However, we can only expect to do so when $X$ is level $q f$-fibrant and we have the long exact sequences (12.3.1).

Recall that the homotopy groups $\pi_{q}^{H}(Y)$ of a nonparametrized $G$-spectrum $Y$ are defined in [105, III.3.2] as the colimits of the groups $\pi_{q}^{H}\left(\Omega^{V} Y(V)\right)$, where the maps of the colimit system are induced in the evident way by the adjoint structure maps $\tilde{\sigma}: Y(V) \longrightarrow \Omega^{W-V} Y(W)$ of $Y$. The functor $\Omega^{V}$ on based $G$ spaces preserves $q$-fibrations and the functor $\Omega_{B}^{V}=F_{B}\left(S^{V},-\right)$ on $G$-spectra over $B$ preserves level $q f$-fibrations. Formally, these hold since $S^{V}$ is a $q$-cofibrant $G$-space and the relevant model structures are $G$-topological. This leads to two families of long exact sequences relating the homotopy groups $\pi_{q}^{H}\left(\Omega^{V} X_{b}(W)\right)$ of fibers to the homotopy groups of the base space $B$ and of the total spaces $X(W)$. First, if $X$ is a level $q$-fibrant $G$-spectrum over $B$, then, using basepoints determined by a point $b \in B^{H}$ for any $H \subset G_{b}$, the $q$-fibrations $\Omega^{V} X(W) \longrightarrow \Omega^{V} B$ of based $G$-spaces with fibers $\Omega^{V} X_{b}(W)$ induce long exact sequences

$$
\cdots \longrightarrow \pi_{q+1}^{H}\left(\Omega^{V} B\right) \longrightarrow \pi_{q}^{H}\left(\Omega^{V} X_{b}(W)\right) \longrightarrow \pi_{q}^{H}\left(\Omega^{V} X(W)\right) \longrightarrow \pi_{q}^{H}\left(\Omega^{V} B\right) \longrightarrow \cdots
$$

Second, if $X$ is level $q f$-fibrant, then the $q f$-fibrations $\left(\Omega_{B}^{V} X\right)(W) \longrightarrow *_{B}$ of ex-Gspaces over $B$ with fibers $\Omega^{V} X_{b}(W)$ induce long exact sequences

$$
\cdots \longrightarrow \pi_{q+1}^{H}(B) \longrightarrow \pi_{q}^{H}\left(\Omega^{V} X_{b}(W)\right) \longrightarrow \pi_{q}^{H}\left(\left(\Omega_{B}^{V} X\right)(W)\right) \longrightarrow \pi_{q}^{H}(B) \longrightarrow \cdots .
$$

The first allows us to relate the homotopy groups of the $X_{b}$ to the homotopy groups of the ordinary loops $\Omega^{V} X(W)$ on total spaces. The second allows us to relate the homotopy groups of the $X_{b}$ to the homotopy groups of the parametrized loop exspaces $\left(\Omega_{B}^{V} X\right)(W)$. It is the second that is most relevant to our work.

Definition 12.3.4. The homotopy groups of a level $q f$-fibrant $G$-spectrum over $B$, or of a level $q f$-fibrant $G$-prespectrum $X$, are all of the homotopy groups $\pi_{q}^{H}\left(X_{b}\right)$ of all of the fibers $X_{b}$, where $H \subset G_{b}$. The homotopy groups of a general $G$-spectrum, or $G$-prespectrum, $X$ over $B$ are the homotopy groups $\pi_{q}^{H}\left((R X)_{b}\right)$ of a level $q f$-fibrant approximation $R X$ to $X$. We still denote these homotopy groups by $\pi_{q}^{H}\left(X_{b}\right)$. In either category, a map $f: X \longrightarrow Y$ is said to be a $\pi_{*}$-isomorphism or, synonymously, an $s$-equivalence, if, after level $q f$-fibrant approximation, it induces an isomorphism on all homotopy groups.

There are also homotopy groups specified in terms of maps out of sphere spectra over $B$, but we choose to ignore them in setting up our model theoretic foundations. Our choice captures the intuitive idea that spectra over $B$ should be parametrized spectra: the fiber spectra should carry all of the homotopy theoretical information. With this choice, a good deal of the work needed to set up the stable model structure reduces to work that has already been done in [105]. The following observation is a starting point that illustrates the pattern of proof.

Lemma 12.3.5. A level q-equivalence of $G$-spectra over $B$ is a $\pi_{*}$-isomorphism. 
Proof. A level $q f$-fibrant approximation to the given level $q$-equivalence is a level acyclic $q f$-fibration, and it induces a level $q$-equivalence on fibers over points of $B$ by Proposition 12.2.10. This allows us to apply [105, III.3.3], which gives the same conclusion for nonparametrized $G$-spectra, one fiber at a time.

To exploit our definition of homotopy groups, we need the following accompanying definition and proposition.

Definition 12.3.6. An $\Omega$-G-prespectrum over $B$ is a level $q f$-fibrant prespectrum $X$ over $B$ whose adjoint structure maps $\tilde{\sigma}: X(V) \longrightarrow \Omega_{B}^{W-V} X(W)$ are $q$ equivalences of ex- $G$-spaces over $B$, that is, q-equivalences of total $G$-spaces. An (orthogonal) $\Omega$-G-spectrum over $B$ is a level $q f$-fibrant $G$-spectrum over $B$ whose adjoint structure maps are $q$-equivalences; equivalently, its underlying prespectrum must be an $\Omega-G$-prespectrum over $B$.

Since we are omitting the adjective "orthogonal" from "orthogonal $G$-spectrum over $B$ ", we must use the term " $\Omega-G$-prespectrum over $B$ " on the prespectrum level to avoid confusion; the usual term " $\Omega$ - $G$-spectrum" was used in [105]. We state the following two results for $\Omega$ - $G$-spectra, but they hold equally well for $\Omega$ - $G$-prespectra.

Proposition 12.3.7. A level fibrant $G$-spectrum $X$ over $B$ is an $\Omega$-G-spectrum over $B$ if and only if each fiber $X_{b}$ is an $\Omega-G_{b}$-spectrum.

Proof. By the five lemma, this is immediate from a comparison of the long exact sequences in (12.3.1) and (12.3.3).

This result leads to the following partial converse to Lemma 12.3.5.

THEOREM 12.3.8. A $\pi_{*}$-isomorphism between $\Omega$-G-spectra over $B$ is a level q-equivalence.

Proof. The analogue for nonparametrized $\Omega$ - $G$-spectra is [105, III.3.4]. In view of Proposition 12.3.7, we can apply that result on fibers and then use that $\Omega$ - $G$-spectra over $B$ are required to be level $q f$-fibrant to deduce the claimed level $q$-equivalence on total spaces from (12.3.1).

Technically, the real force of our definition of homotopy groups is that this result describing the $\pi_{*}$-isomorphisms between $\Omega$ - $G$-spectra over $B$ is an immediate consequence of the work in [105]. Given this relationship between $\Omega$ - $G$-spectra and homotopy groups, many of the arguments of [105] apply fiberwise to allow the development of the stable model structure. However, as discussed in the next section, careful use of level fibrant approximation is required. We shall use the terms "stable model structure" and "s-model structure" interchangeably. The $s$ cofibrations are the same as those of the level $q f$-model structure and the $s$-fibrant $G$-spectra over $B$ turn out to be the $\Omega$ - $G$-spectra over $B$.

Now that we have seen how the equivariance appears in the definition of homotopy groups and the deduction of results about $G$-spectra over $B$ from results about $G_{b}$-spectra, we revert to our custom of generally deleting $G$ from the notations.

Definition 12.3.9. A map of spectra or prespectra over $B$ is

(i) an acyclic $s$-cofibration if it is a $\pi_{*}$-isomorphism and an $s$-cofibration,

(ii) an $s$-fibration if it satisfies the RLP with respect to the acyclic $s$-cofibrations,

(iii) an acyclic $s$-fibration if it is a $\pi_{*}$-isomorphism and an $s$-fibration. 
We shall prove the following basic theorem in the next two sections.

THEOREM 12.3.10. The categories $G \mathscr{S}_{B}$ and $G \mathscr{P}_{B}$ are well-grounded model categories with respect to the $\pi_{*}$-isomorphisms (=s-equivalences), s-fibrations and $s$-cofibrations. The $s$-fibrant objects are the $\Omega$-spectra over $B$.

REMARK 12.3.11. Recall Remark 12.1.9. We can define positive $\Omega$-prespectra and positive analogues of our $s$-classes of maps, starting with the positive level $q f$ model structure. As in [105, III $\S 5]$, the positive analogue of the previous theorem also holds, with the same proof. The identity functor is the left adjoint of a Quillen equivalence from $G \mathscr{S}_{B}$ or $G \mathscr{P}_{B}$ with its positive stable model structure to $G \mathscr{S}_{B}$ or $G \mathscr{P}_{B}$ with its stable model structure.

The proof of the following result is virtually the same as the proof of its nonparametrized precursor [105, III.4.16 and III.5.7] and will not be repeated.

THEOREM 12.3.12. The adjoint pair $(\mathbb{P}, \mathbb{U})$ relating the categories $G \mathscr{P}_{B}$ and $G \mathscr{S}_{B}$ of prespectra and spectra over $B$ is a Quillen equivalence with respect to either the stable model structures or the positive stable model structures.

As in $[105$, III.§6], Theorem 12.3.10 leads to the following definition and theorem, whose proof is the same as the proof of [105, III.6.1].

Definition 12.3.13. Let $[X, Y]^{\ell}$ denote the morphism sets in the homotopy category associated to the level $q f$-model structure on $G \mathscr{P}_{B}$ or $G \mathscr{S}_{B}$. A map $f: X \longrightarrow Y$ is a stable equivalence if $f^{*}:[Y, E]^{\ell} \longrightarrow[X, E]^{\ell}$ is an isomorphism for all $\Omega$-spectra $E$ over $B$. Define the positive analogues similarly. Let $[X, Y]$ denote the morphism sets in the stable homotopy category Ho $G \mathscr{S}_{B}$ of spectra over $B$.

THEOREM 12.3.14. The following are equivalent for a map $f: X \longrightarrow Y$ of spectra or prespectra over $B$.

(i) $f$ is a stable equivalence.

(ii) $f$ is a positive stable equivalence.

(iii) $f$ is a $\pi_{*}$-isomorphism.

Moreover $[X, E]=[X, E]^{\ell}$ if $E$ is an $\Omega$-spectrum.

Lemma 12.6.1 below should make it clear why the last statement is true.

\subsection{Cofiber sequences and $\pi_{*}$-isomorphisms}

In the main, the proof of Theorem 12.3.10 is obtained by applying the results in [105] fiberwise. Since total spaces are no longer assumed to be weak Hausdorff, we have to be a little careful: we are quoting results proven for $\mathscr{T}$ and using them for $\mathscr{K}_{*}$. However, we can just as well interpret [105] in terms of $\mathscr{K}_{*}$. The total spaces $X(V)$ of an $s$-cofibrant spectrum over $B$ are weak Hausdorff, hence $s$-cofibrant approximation places us in a situation where total spaces are in $\mathscr{U}$ and therefore fibers are in $\mathscr{T}$.

There is a more substantial technical problem to overcome in adapting the proofs of $[105,106]$ to the present setting. In the situations encountered in those references, all objects were level $q$-fibrant, and that simplified matters considerably. Here, level $q f$-fibrant approximation entered into our definition of homotopy groups, and for that reason the results of this section are considerably more subtle than their 
counterparts in the cited sources. Their proofs depend on the full strength of our topological model structures in general and the $q f$-model structure in particular.

We begin by noting that any level ex-quasifibrant approximation, not necessarily a $q f$-fibrant approximation, can be used to calculate the homotopy groups of parametrized spectra.

LEMMA 12.4.1. A zig-zag of level q-equivalences connecting a spectrum $X$ over $B$ to a level ex-quasifibrant spectrum $Y$ over $B$ induces an isomorphism between the homotopy groups of $X$ and of $Y$, and the latter can be computed directly in terms of the fibers of $Y$.

Proof. This follows from Lemma 12.3 .5 by applying a level $q f$-fibrant approximation functor to the zig-zag.

Theorem 12.4.2. Let $f: X \longrightarrow Y$ be a map between $G$-spectra over $B$. For any $H \subset G$ and $b \in B^{H}$, there is a natural long exact sequence

$$
\cdots \longrightarrow \pi_{q+1}^{H}\left(Y_{b}\right) \longrightarrow \pi_{q}^{H}\left(\left(F_{B} f\right)_{b}\right) \longrightarrow \pi_{q}^{H}\left(X_{b}\right) \longrightarrow \pi_{q}^{H}\left(Y_{b}\right) \longrightarrow \cdots
$$

and, if $X$ is well-sectioned, there is also a natural long exact sequence

$$
\cdots \longrightarrow \pi_{q}^{H}\left(X_{b}\right) \longrightarrow \pi_{q}^{H}\left(Y_{b}\right) \longrightarrow \pi_{q}^{H}\left(\left(C_{B} f\right)_{b}\right) \longrightarrow \pi_{q-1}^{H}\left(X_{b}\right) \longrightarrow \cdots .
$$

Proof. For the first long exact sequence, let $R$ be a level $q f$-fibrant approximation functor and consider $R f$. We claim that the induced map $F_{B} f \longrightarrow F_{B} R f$ is a level $q$-equivalence and that $F_{B} R f$ is level $q f$-fibrant. This means that $F_{B} R f$ is a level $q f$-fibrant approximation to $F_{B} f$, so that the homotopy groups of the fibers $\left(F_{B} R f\right)_{b} \cong F\left((R f)_{b}\right)$ are the homotopy groups of $F_{B} f$. When restricted to fibers over $b$, the parametrized fiber sequence $R X \longrightarrow R Y \longrightarrow F_{B} R f$ of spectra over $B$ gives the nonparametrized fiber sequence $(R X)_{b} \longrightarrow(R Y)_{b} \longrightarrow F\left((R f)_{b}\right)$, and the long exact sequence follows from [105, III.3.5]. To prove the claim, observe that since $F_{B}(I, Y) \longrightarrow Y$ is a Hurewicz fibration, it has a path-lifting function which levelwise shows that $F_{B}(I, Y) \longrightarrow Y$ is a level $f p$-fibration and therefore a level $q f$-fibration (since all $q f$-cofibrations are $f p$-cofibrations in $G \mathscr{K}_{B}$ ). The dual gluing lemma (see Definition 5.4.1(iii)) then gives that the induced map $F_{B} f \longrightarrow F_{B} R f$ is a level $q$-equivalence. Since $F_{B}(I,-)$ preserves level $q f$-fibrant objects and since pullbacks of level $q f$-fibrant objects along a level $q f$-fibration are level $q f$-fibrant, $F_{B} R f$ is level $q f$-fibrant.

Since the maps $X \longrightarrow C_{B} X$ and $R X \longrightarrow C_{B} R X$ are $c y l$-cofibrations between well-sectioned spectra and therefore level $h$-cofibrations by Lemma 12.1.3, the gluing lemma gives that $C_{B} f \longrightarrow C_{B} R f$ is a level $q$-equivalence. Since $R X$ and $R Y$ are level well-sectioned and level $q f$-fibrant, they are level ex-quasifibrations. It follows from Proposition 8.5.3 that $C_{B} R f$ is a level ex-quasifibration. We cannot conclude that $C_{B} R f$ is level $q f$-fibrant, but by Lemma 12.4 .1 we can nevertheless use $C_{B} R f$ to calculate the homotopy groups of $C_{B} f$. On fibers over $b$, the cofiber sequence of $R f$ is just the cofiber sequence of $(R f)_{b}$, and the long exact sequence follows from [105, III.3.5].

Recall Proposition 12.1.4, which specifies the ground structure in $G \mathscr{S}_{B}$ and shows that the level $q$-equivalences give a well-grounded subcategory of weak equivalences; the $g$-cofibrations are just the level $h$-cofibrations. The following result shows that the same is true for the $\pi_{*}$-isomorphisms. However, in contrast to Proposition 12.1.4, it is crucial to assume that the relevant maps in the gluing 
and colimit lemmas are both $c y l$-cofibrations and $g$-cofibrations, as prescribed in Definition 5.4.1.

THEOREM 12.4.3. The $\pi_{*}$-isomorphisms in $G_{\mathscr{S}_{B}}$ give a well-grounded subcategory of weak equivalences. In detail, the following statements hold.

(i) A homotopy equivalence is a $\pi_{*}$-isomorphism.

(ii) The homotopy groups of a wedge of well-grounded spectra over $B$ are the direct sums of the homotopy groups of the wedge summands.

(iii) The $\pi_{*}$-isomorphisms are preserved under pushouts along maps that are both cyl and $g$-cofibrations.

(iv) Let $X$ be the colimit of a sequence $i_{n}: X_{n} \longrightarrow X_{n+1}$ of maps that are both cyl and $g$-cofibrations and assume that $X /{ }_{B} X_{0}$ is well-grounded. Then the homotopy groups of $X$ are the colimits of the homotopy groups of the $X_{n}$.

(v) For a map $i: X \longrightarrow Y$ of well-grounded spectra over $B$ and a map $j: K \longrightarrow L$ of well-based spaces, $i \square j$ is a $\pi_{*}$-isomorphism if either $i$ is a $\pi_{*}$-isomorphism or $j$ is a q-equivalence.

PROOF. The conclusion that the $\pi_{*}$-isomorphisms give a well-grounded subcategory of weak equivalences, as prescribed in Definition 5.4.1, follows directly from the listed properties, using Lemma 5.4.3 to derive the gluing lemma. Since level $q$ equivalences are $\pi_{*}$-isomorphisms, $s$-cofibrant approximation in the level $q f$-model structure gives the factorization hypothesis Lemma 5.4.3(ii).

A homotopy equivalence of spectra is a level $f p$-equivalence and hence a level $q$-equivalence, so (i) follows from Lemma 12.3.5. For finite wedges, (ii) is immediate from the evident split cofiber sequences and Theorem 12.4.2. For arbitrary wedges of well-grounded spectra over $B, \vee_{B} X_{i} \longrightarrow \vee_{B} R X_{i}$ is a level $q$-equivalence since the level $q$-equivalences are well-grounded and $\vee_{B} R X_{i}$ is level quasifibrant by Proposition 8.5.3. By Lemma 12.4.1 we can use $\vee_{B} R X_{i}$ to calculate the homotopy groups of $\vee_{B} X_{i}$. Over a point $b$ in $B, \vee_{B} R X_{i}$ is just $\vee\left(R X_{i}\right)_{b}$ and the result follows from the nonparametrized analogue [105, III.3.5].

Now consider (iii). Let $i: X \longrightarrow Y$ be both a cyl-cofibration and a $g$-cofibration and let $f: X \longrightarrow Z$ be a $\pi_{*}$-isomorphism. Since $i$ and its $s$-cofibrant approximation $Q i$ are both $c y l$ and $g$-cofibrations and since the level $q$-equivalences give a well-grounded subcategory of weak equivalences, the gluing lemma shows that we may approximate our given pushout diagram by one in which all objects are wellsectioned. Let $j: Z \longrightarrow Y \cup_{X} Z$ be the pushout of $i$ along $f$. Since $i$ and $j$ are $c y l$-cofibrations and $j$ is the pushout of $i$, their cofibers are homotopy equivalent. Comparing the long exact sequences of homotopy groups associated to the cofiber sequences of $i$ and $j$ gives that the pushout $Y \longrightarrow Y \cup_{X} Z$ of $f$ along $i$ is a $\pi_{*^{-}}$ isomorphism.

For (iv), we may use $s$-cofibrant approximation in the level model structure to replace our given tower by one in which all objects are well-sectioned. We note as in the proof of Lemma 5.6.14 that the natural map Tel $X_{n} \longrightarrow \operatorname{colim} X_{n}$ is a level $q$-equivalence and therefore a $\pi_{*}$-isomorphism. Relating the telescope to a classical homotopy coequalizer as in the cited proof, we reduce the calculation of the homotopy groups of the telescope to an algebraic inspection based on (ii). Alternatively, one can commute double colimits to reduce the verification to its space level analogue.

For (v), it suffices to show that the tensor $X \wedge_{B} T$ preserves $\pi_{*}$-isomorphisms in either variable, by Lemma 5.4.5. That follows from Proposition 12.4.4 below. 
Proposition 12.4.4. Let $X$ and $Y$ be well-grounded spectra over $B$ and $T$ and $W$ be well-based spaces.

(i) If $g: T \longrightarrow W$ is a q-equivalence, then

$$
i d \wedge_{B} g: X \wedge_{B} T \longrightarrow X \wedge_{B} W
$$

is a level q-equivalence and therefore a $\pi_{*}$-isomorphism.

(ii) If $f: X \longrightarrow Y$ is a $\pi_{*}$-isomorphism, then

$$
f \wedge_{B} i d: X \wedge_{B} T \longrightarrow Y \wedge_{B} T
$$

is a $\pi_{*}$-isomorphism; if $T$ is a finite based $C W$ complex, then

$$
F_{B}(i d, f): F_{B}(T, X) \longrightarrow F_{B}(T, Y)
$$

is a $\pi_{*}$-isomorphism.

(iii) For a representation $V$ in $\mathscr{V}, f: X \longrightarrow Y$ is a $\pi_{*}$-isomorphism if and only if $\Sigma_{B}^{V} f$ is a $\pi_{*}$-isomorphism.

Proof. Part (i) holds since the level $q$-equivalences are well-grounded. Therefore, for the first part of (ii), we may assume by $q$-cofibrant approximation in the space variable that $T$ is a based CW complex. Using Proposition 8.5.3, it also implies that $-\wedge_{B} T$ preserves approximations of well-grounded spectra over $B$ by level ex-quasifibrations. Now the first part of (ii) follows fiberwise from its nonparametrized analogue [105, III.3.11] and (iii) follows fiberwise from its nonparametrized analogue [105, III.3.6]. Since $F_{B}(-, X)$ takes cofiber sequences of based spaces to fiber sequences of spectra over $B$, the second part of (iii) follows from the first exact sequence in Theorem 12.4.2, as in the proof of [105, III.3.9].

This leads to the following result, which shows that we are in a stable situation.

Proposition 12.4.5. For all well-grounded spectra $X$ over $B$ and all representations $V$ in $\mathscr{I}_{G}$, the unit $\eta: X \longrightarrow \Omega_{B}^{V} \Sigma_{B}^{V} X$ and counit $\varepsilon: \Sigma_{B}^{V} \Omega_{B}^{V} X \longrightarrow X$ of the $\left(\Sigma_{B}^{V}, \Omega_{B}^{V}\right)$ adjunction are $\pi_{*}$-isomorphisms. Therefore, if $f: X \longrightarrow Y$ is a map between well-grounded spectra over $B$, then the natural maps $\eta: F_{B} f \longrightarrow \Omega_{B} C_{B} f$ and $\epsilon: \Sigma_{B} F_{B} f \longrightarrow C_{B} f$ are $\pi_{*}$-isomorphism.

Proof. For $\eta$, after approximation of $X$ by an ex-quasifibration, the conclusion follows fiberwise from its nonparametrized analogue [105, III.3.6]. Using the two out of three property and the triangle equality for the adjunction, it follows that $\Omega_{B}^{V} \varepsilon$ is a $\pi_{*}$-isomorphism, hence so is $\varepsilon$. For the last statement, the maps $\eta$ and $\varepsilon$ are the parametrized analogues of the maps defined for ordinary loops and suspensions in [121, p. 61], and they fit into diagrams relating fiber and cofiber sequences like those displayed there. Now the last statement follows from the five lemma and the exact sequences in Theorem 12.4.2.

\subsection{Proofs of the model axioms}

We need some $G$-spectrum level recollections from [105] and their analogues for $G$-spectra over $B$ to describe the generating acyclic $s$-cofibrations. Let $\left(\mathscr{S}_{G}, G \mathscr{S}\right)$ denote the $G$-category of $G$-spectra. To keep track of enrichments, we again return $G$ to the notations for the moment. 
We have a shift desuspension functor $F_{V}$ from based $G$-spaces to $G$-spectra given by $F_{V} T=V^{*} \wedge T$, where $V^{*}(W)=\mathscr{J}_{G}(V, W)$ [105, III.4.6]. It is left adjoint to evaluation at $V$. For $G$-spectra $X$, the adjoint structure $G$-map

$$
\tilde{\sigma}: X(V) \longrightarrow \Omega^{W} X(V \oplus W)
$$

may be viewed by adjunction as a $G$-map

$$
\tilde{\sigma}: \mathscr{S}_{G}\left(F_{V} S^{0}, X\right) \longrightarrow \mathscr{S}_{G}\left(F_{V \oplus W} S^{W}, X\right) .
$$

Passing to $G$-fixed points and taking $X=F_{V} S^{0}$, the image of the identity map gives a map of $G$-spectra

$$
\lambda^{V, W}: F_{V \oplus W} S^{W} \longrightarrow F_{V} S^{0} .
$$

(The notation $\lambda_{V, W}$ was used in [105], but we need room for a subscript). A Yoneda lemma argument then shows that the map of $G$-spaces

$$
\mathscr{S}_{G}\left(\lambda^{V, W}, \mathrm{id}\right): \mathscr{S}_{G}\left(F_{V} S^{0}, X\right) \longrightarrow \mathscr{S}_{G}\left(F_{V \oplus W} S^{W}, X\right)
$$

can be identified with $\tilde{\sigma}: X(V) \longrightarrow \Omega^{W} X(V \oplus W)$.

We need the analogue for $G$-spectra over $B$. Recall from Definition 11.3.5 that, for an ex- $G$-space $K$ over $B,\left(F_{V} K\right)(W)=V^{*}(W) \wedge_{B} K$, where

$$
V^{*}(W)=\mathscr{J}_{G, B}(V, W)=\mathscr{J}_{G}(V, W)_{B}=\left(F_{V} S^{0}\right)(W) \wedge_{B} S_{B}^{0} .
$$

It follows that we can identify $F_{V} K$ with the evident external tensor $F_{V} S^{0} \wedge_{B} K$ of the $G$-spectrum $F_{V} S^{0}$ and the ex- $G$-space $K$ over $B$; compare Remark 11.1.7. We have used the notation $\wedge_{B}$ for this generalized tensor, but viewing it as a special case of the external smash product of spectra over $*$ and over $B$ would suggest the alternative notation $\bar{\wedge}$.

Definition 12.5.1. For ex- $G$-spaces $K$ over $B$, we define a natural map

$$
\lambda_{B}^{V, W}: F_{V \oplus W} \Sigma_{B}^{W} K \longrightarrow F_{V} K
$$

by identifying the source and target with external tensor products and setting

$$
\lambda_{B}^{V, W}=\lambda^{V, W} \wedge_{B} \text { id }:\left(F_{V \oplus W} S^{W}\right) \wedge_{B} K \longrightarrow\left(F_{V} S^{0}\right) \wedge_{B} K .
$$

We can describe the adjoint structure maps of $G$-spectra over $B$ in terms of these maps $\lambda_{B}^{V, W}$.

LEMMA 12.5.2. Under the adjunctions

$$
P_{B}\left(F_{V} S_{B}^{0}, X\right) \cong F_{B}\left(S_{B}^{0}, X(V)\right) \cong X(V)
$$

and

$$
P_{B}\left(F_{V \oplus W} S_{B}^{W}, X\right) \cong F_{B}\left(S_{B}^{0}, \Omega_{B}^{W} X(V \oplus W)\right) \cong \Omega_{B}^{W} X(V \oplus W),
$$

the map

$$
P_{B}\left(\lambda_{B}^{V, W}, i d\right): P_{B}\left(F_{V} S_{B}^{0}, X\right) \longrightarrow P_{B}\left(F_{V \oplus W} S_{B}^{W}, X\right)
$$

corresponds to

$$
\tilde{\sigma}: X(V) \longrightarrow \Omega_{B}^{W} X(V \oplus W) .
$$

Proof. When $X=F_{V} S_{B}^{0}$, the conclusion holds by comparison with the case of $G$-spectra. The general case follows from the Yoneda lemma of enriched category theory. See, for example, [23, 6.3.5]. 
We could have started off by defining $\lambda_{B}^{V, W}$ in a conceptual manner analogous to our definition of $\lambda^{V, W}$, but we want the explicit description of $\lambda_{B}^{V, W}$ in terms of $\lambda^{V, W}$ in order to deduce homotopical properties in the parametrized context from homotopical properties in the nonparametrized context. For that and other purposes, we need the following observation. We return to our convention of deleting $G$ from the notations, on the understanding that everything is equivariant.

Lemma 12.5.3. If $\phi: X \longrightarrow Y$ is an s-equivalence of level well-based nonparametrized spectra and $K$ is a well-grounded ex-space with total space of the homotopy type of a $G-C W$ complex, then $\phi \wedge_{B} i d: X \wedge_{B} K \longrightarrow Y \wedge_{B} K$ is an s-equivalence.

Proof. We use the ex-fibrant approximation functor $P$ of Definition 8.3.4. We have a natural zig-zag of $h$-equivalences between $K$ and $P K$. By Proposition 8.2.6, it induces a zig-zag of level $h$-equivalences between $X \wedge_{B} K$ and $X \wedge_{B} P K$ and, by Corollary 8.2.5, $X \wedge_{B} P K$ is a level ex-fibration. Therefore, by Lemma 12.4.1, it suffices to consider the case when $K$ is an ex-fibration. Since $\left(X \wedge_{B} K\right)_{b}=X \wedge K_{b}$ and $K_{b}$ is of the homotopy type of a $G_{b}$-CW complex, by Theorem 3.5.2, each $\left(\phi \wedge_{B} \text { id }\right)_{b}$ is an $s$-equivalence by [105, III.3.11].

The following result is crucial.

Proposition 12.5.4. Let $K$ be a well-grounded ex-space with total space of the homotopy type of a $C W$ complex. Then

$$
\lambda_{B}^{V, W}: F_{V \oplus W} \Sigma_{B}^{W} K \longrightarrow F_{V} K
$$

and

$$
\lambda^{V, W} \bar{\wedge} i d: F_{V \oplus W} S^{W} \bar{\wedge} F_{Z} K \longrightarrow F_{V} S^{0} \bar{\wedge} F_{Z} K
$$

are $\pi_{*}$-isomorphisms of spectra over $B$.

Proof. Since $\lambda_{B}^{V, W}=\lambda^{V, W} \wedge_{B}$ id, Lemma 12.5.3 and the corresponding nonparametrized statement [105, III.4.5] imply the first statement. For the second statement, observe that for spectra $X$ we have the associativity relation

$$
X \bar{\wedge} F_{Z} K \cong X \bar{\wedge}\left(F_{Z} S^{0} \wedge_{B} K\right) \cong\left(X \wedge F_{Z} S^{0}\right) \wedge_{B} K .
$$

Taking $X=F_{V} T$ for a based space $T$ and using Lemma 11.3.6, we see that

$$
F_{V} T \wedge F_{Z} K \cong F_{V \oplus Z}\left(T \wedge_{B} K\right) .
$$

Using equivalences of this form and checking definitions, we conclude that the map $\lambda^{V, W} \bar{\wedge}$ id of the statement can be identified with the map

$$
\lambda^{V \oplus Z, W} \wedge_{B} \text { id }:\left(F_{V \oplus Z \oplus W} S^{W}\right) \wedge_{B} K \longrightarrow\left(F_{V \oplus Z} S^{0}\right) \wedge_{B} K .
$$

Thus the second $\pi_{*}$-isomorphism is a special case of the first.

From here, the proof of Theorem 12.3.10 closely parallels arguments in [105, III.§4], but simplified a little by Theorem 5.5.1. The generating set of $s$-cofibrations is again $F I_{B}^{f}$. The generating set $F K_{B}^{f}$ of acyclic s-cofibrations is given by a variant of the definition in the nonparametrized case [105, III.4.6].

Definition 12.5.5. Recall the factorization of $\lambda^{V, W}$ through the mapping cylinder (in the category of spectra) as

$$
\lambda^{V, W}: F_{V \oplus W} S^{W} \stackrel{k^{V, W}}{\longrightarrow} M \lambda^{V, W} \stackrel{r^{V, W}}{\longrightarrow} F_{V} S^{0} .
$$


Here $k^{V, W}$ is an $s$-cofibration and $r^{V, W}$ is a deformation retraction. For $i: C \longrightarrow D$ in $I_{B}^{f}$, the map

$$
i \square k^{V, W}: C \wedge_{B} M \lambda^{V, W} \cup_{C \wedge_{B} F_{V \oplus W} S^{W}} D \wedge_{B} F_{V \oplus W} S^{W} \longrightarrow D \wedge_{B} M \lambda^{V, W}
$$

is an $s$-cofibration in $G \mathscr{S}_{B}$ by Proposition 12.2.3, and it is therefore also a $c y l$ cofibration by Theorem 12.1.7. It is a $\pi_{*}$-isomorphism by Proposition 12.5 .4 and inspection of definitions. The $s$-cofibrations in $F J_{B}^{f}$ are level acyclic and are therefore also $\pi_{*}$-isomorphisms. Restricting to $V$ and $W$ in sk $\mathscr{I}_{G}$, define the generating set $F K_{B}^{f}$ of acyclic s-cofibrations to be the union of $F J_{B}^{f}$ and the set of all maps of the form $i \square k^{V, W}$ with $i \in I_{B}^{f}$.

A fortiori, the following result identifies the $s$-fibrations, but it must be proven a priori as a first step towards the verification of the model axioms.

Proposition 12.5.6. A map $f: X \longrightarrow Y$ satisfies the $R L P$ with respect to $F K_{B}^{f}$ if and only if $f$ is a level $q f$-fibration and the diagrams

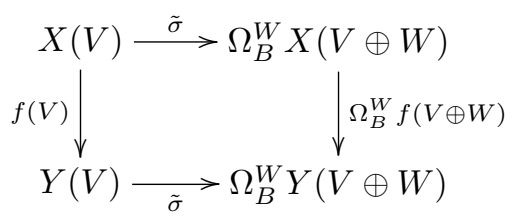

are homotopy pullbacks for all $V$ and $W$.

Proof. As in [105, III.4.7], the homotopy pullback property must be interpreted as requiring a $q$-equivalence from $X(V)$ into the pullback in the displayed diagram. Recall that $F J_{B}^{f}$ is contained in $F K_{B}^{f}$ and that a map has the RLP with respect to $F J_{B}^{f}$ if and only if it is a level $q f$-fibration. This gives part of both implications. It remains to show that a level $q f$-fibration $f$ has the RLP with respect to $i \square k^{V, W}$ for all $i \in I_{B}^{f}$ if and only if the displayed diagram is a homotopy pullback. This is a formal but not altogether trivial exericise from the fact that the level $q f$-model structure is $G$-topological in the sense characterized in Proposition 10.3.18. Notice that the map $i \square k^{V, W}$ is isomorphic to the map $i \square k_{B}^{V, W}$, where $k_{B}^{V, W}=k^{V, W} \wedge_{B} S_{B}^{0}$. With notation as in (10.3.6), $f$ has the RLP with respect to $i \square k_{B}^{V, W}$ for all $i \in I_{B}^{f}$ if and only if the pair $\left(i, P_{B}^{\square}\left(k_{B}^{V, W}, f\right)\right)$ has the lifting property for all $i \in I_{B}^{f}$, which holds if and only if the map $P_{B}^{\square}\left(k_{B}^{V, W}, f\right)$ of ex-spaces over $B$ is an acyclic $q f$-fibration. This map is a $q f$-fibration since, for $j \in J_{B}^{f}$, the map $j \square k^{V, W} \cong j \square k_{B}^{V, W}$ is a level acyclic $s$-cofibration of spectra over $B$ by Proposition 12.2.3. Since $f$ is a level $q f$-fibration, $\left(j \square k_{B}^{V, W}, f\right)$ has the lifting property, hence, by adjunction, so does $\left(j, P_{B}^{\square}\left(k_{B}^{V, W}, f\right)\right)$. Finally, $P_{B}^{\square}\left(k_{B}^{V, W}, f\right)$ is homotopy equivalent to $P_{B}^{\square}\left(\lambda_{B}^{V, W}, f\right)$ so one is a $q$-equivalence if and only if the other is. Under the isomorphisms in Lemma 12.5.2, the map $P_{B}^{\square}\left(\lambda_{B}^{V, W}, f\right)$ coincides with the map from $X(V)$ into the pullback in the displayed diagram and is thus a $q$-equivalence if and only if that diagram is a homotopy pullback.

Observe that $*_{B}$ is an $\Omega$-spectrum with trivial homotopy groups.

COROLlaRY 12.5.8. The terminal map $F \longrightarrow *_{B}$ satisfies the $R L P$ with respect to $F K_{B}$ if and only if $F$ is an $\Omega$-spectrum over $B$. 
Corollary 12.5.9. If $f: X \longrightarrow Y$ is a $\pi_{*}$-isomorphism that satisfies the RLP with respect to $F K_{B}$, then $f$ is a level acyclic $q f$-fibration.

Proof. Since $f$ is a level $q f$-fibration by Proposition 12.5.6, the dual of the gluing lemma applied to the diagram

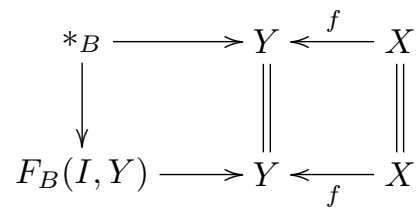

gives that the induced map $F \longrightarrow F_{B} f$ of pullbacks is a level $q$-equivalence. Since $f$ has the RLP with respect to $F K_{B}$, so does its pullback $F \longrightarrow *_{B}$. By the previous corollary, $F$ is thus an $\Omega$-spectrum over $B$. In particular, it is level $q f$ fibrant. We conclude that $F$ is a level $q f$-fibrant approximation for $F_{B} f$. Since $f$ is a $\pi_{*}$-isomorphism, Theorem 12.4.2 gives that $F$ is acyclic. By Theorem 12.3.8, this implies that $F \longrightarrow *_{B}$ is a level $q$-equivalence. Thus the fibers $F(V)_{b}$ all have trivial homotopy groups. We conclude (with a bit of extra argument as in $[106,9.8]$ to handle $\left.\pi_{0}\right)$ that each map of fibers $f(V)_{b}$ induces an isomorphism on homotopy groups. Therefore, since each $f(V)$ is a $q f$-fibration, each $f(V)$ induces an isomorphism on homotopy groups.

The proof of the model axioms for the stable model structure is now immediate.

Proof of Theorem 12.3.10. The $\pi_{*}$-isomorphisms give a well-grounded subcategory of weak equivalences, by Theorem 12.4.3. Conditions (i), (iii), and (iv) in Theorem 5.5.1 are clear from our specification of the generating acyclic $s$ cofibrations and the result for the level $q f$-model structure. For condition (ii), a $\pi_{*}$-isomorphism that satisfies the RLP with respect to $F K_{B}$ has the RLP with respect to $F I_{B}$ by Corollary 12.5.9. Conversely, a map that has the RLP with respect to $F I_{B}$ is a level acyclic $q f$-fibration and therefore has the RLP with respect to $F K_{B}$ by Proposition 12.5.6. It is a $\pi_{*}$-isomorphism since it is level acyclic. Since all $s$-fibrations are level $q f$-fibrations, right properness follows from the slightly stronger observation in the following result.

Proposition 12.5.10. The $\pi_{*}$-isomorphisms in $G \mathscr{S}_{B}$ are preserved under pullbacks along level $q f$-fibrations.

Proof. Let $g$ be the pullback of a level $q f$-fibration $f$ along a $\pi_{*}$-isomorphism. Then $g$ is a level $q f$-fibration and the fibers of $g(V)$ are isomorphic to the fibers of $f(V)$. Therefore the homotopy fibers $F_{B} g$ are level $q$-equivalent to the homotopy fibers $F_{B} f$. The result follows by comparison of the first long exact sequence in Theorem 12.4.2 for $f$ and $g$.

\subsection{Some Quillen adjoint pairs relating stable model structures}

We prove here that all of the adjoint pairs that were shown to be Quillen adjoints with respect to the level model structure in $\S 12.2$ are still Quillen adjoints with respect to the stable model structure. In view of the role played by level $q f$-fibrant approximation in our definition of homotopy groups, it is helpful to first understand the relationship between $s$-fibrant approximation and level $q f$ fibrant approximation. Now that the model structures have been established, we 
henceforward use the term $s$-equivalence rather than the synonymous term $\pi_{*}$ isomorphism.

LEMmA 12.6.1. Let $\nu: X \longrightarrow R X$ and $\nu_{\ell}: X \longrightarrow R_{\ell} X$ be an s-fibrant approximation of $X$ and a level $q f$-fibrant approximation of $X$. Then there is an s-equivalence $\xi: R_{\ell} X \longrightarrow R X$ under $X$.

ProOF. Since $\nu_{\ell}$ is a level acyclic $s$-cofibration, it is an acyclic $s$-cofibration by Lemma 12.3.5. Since $R X$ is $s$-fibrant, the RLP gives a map $\xi$ under $X$, and it is an $s$-equivalence since $\nu$ and $\nu_{\ell}$ are $s$-equivalences.

We have the following relationship between the homotopy categories of exspaces over $B$ and of spectra over $B$.

Proposition 12.6.2. The pair $\left(\Sigma_{B}^{\infty}, \Omega_{B}^{\infty}\right)$ is a Quillen adjunction relating $G \mathscr{S}_{B}$ and $G \mathscr{K}_{B}$. More generally, $\left(\Sigma_{V}^{\infty}, \Omega_{V}^{\infty}\right)=\left(F_{V}, E v_{V}\right)$ is a Quillen adjunction for any representation $V \in \mathscr{V}$.

Proof. The maps $\Sigma_{V}^{\infty} i$, where $i \in I_{B}^{f}$ is a generating cofibration for the $q f$ model structure on $G \mathscr{K}_{B}$, are among the generating cofibrations of the $s$-model structure on $G \mathscr{S}_{B}$, and it follows that $\Sigma_{V}^{\infty}$ preserves cofibrations. Since $\Sigma_{V}^{\infty}$ takes acyclic $q f$-cofibrations to level acyclic $q f$-cofibrations, and these are acyclic by Lemma $12.3 .5, \Sigma_{V}^{\infty}$ also preserves acyclic cofibrations.

Now consider an adjoint pair $(F, V)$ between categories of parametrized spectra that is a Quillen adjunction with respect to the level model structures. Since the cofibrations are the same in the level model structure and in the stable model structure, the left adjoint $F$ certainly preserves cofibrations. Thus, to show that $(F, V)$ is also a Quillen adjunction with respect to the stable model structures, we need only show that $F$ carries acyclic $s$-cofibrations to $s$-equivalences. When $F$ preserves all $s$-equivalences, this is obvious; otherwise, by Lemma 5.4.7, it suffices to verify this for the generating acyclic $s$-cofibrations. The cited result applies in general to subcategories of well-grounded weak equivalences, and in our context it applies to both the level $q$-equivalences and the $s$-equivalences. Recall that a Quillen left adjoint in any model structure preserves weak equivalences between cofibrant objects, by Ken Brown's lemma [75, 1.1.12]. The following parenthetical observation applies to give a stronger conclusion for the Quillen left adjoints that we shall encounter. It will play a crucial role in exploiting the equivalence of homotopy categories that we will establish in the next chapter. Note that the $s$ cofibrant spectra are the cofibrant objects in both the level and the stable model structures, and they are well-grounded.

Proposition 12.6.3. Let $F$ be a Quillen left adjoint between categories of parametrized spectra with their stable model structures and suppose that $F$ preserves level q-equivalences between well-grounded spectra. Then $F$ preserves s-equivalences between well-grounded spectra.

Proof. If $g: X \longrightarrow Y$ is an $s$-equivalence, where $X$ and $Y$ are well-grounded, factor $g$ in the level model structure as

$$
X \stackrel{g^{\prime}}{\longrightarrow} W \stackrel{g^{\prime \prime}}{\longrightarrow} Y,
$$

where $g^{\prime}$ is an $s$-cofibration and $g^{\prime \prime}$ is a level acyclic $q f$-fibration. Then $W$ is wellgrounded and $F g^{\prime \prime}$ is a level $q$-equivalence by assumption. Since $F$ is a Quillen left 
adjoint in the $s$-model structures, $F g^{\prime}$ is an $s$-equivalence. Since level $q$-equivalences are $s$-equivalences it follows that $F g=F g^{\prime \prime} \circ F g^{\prime}$ is an $s$-equivalence.

The following sequence of results consists of analogues for the stable model structures of results proven for the level model structures in $\$ 12.2$. Recall that we actually have well-grounded stable model structures $s(\mathscr{C})$ for any closed generating set $\mathscr{C}$. As in $\$ 12.2$, wherever a $q f(\mathscr{C})$-model structure was used in Chapter 7 for some particularly well chosen $\mathscr{C}$, we must use the corresponding $s(\mathscr{C})$-model structure here.

Proposition 12.6.4. Let $T$ be a based $G-C W$ complex. Then $\left(-\wedge_{B} T, F_{B}(T,-)\right)$ is a Quillen adjunction on $G \mathscr{S}_{B}$. When $T=S^{V}$, it is a Quillen equivalence.

Proof. This is immediate from the fact that the stable model structure is $G$-topological, together with Propositions 12.4.4 and 12.4.5.

Proposition 12.6.5. If $i: X \longrightarrow Y$ and $j: W \longrightarrow Z$ are $s$-cofibrations of spectra over base spaces $A$ and $B$, then

$$
i \square j:(Y \bar{\wedge} W) \cup_{X \overline{ }}(X \bar{\wedge} Z) \longrightarrow Y \bar{\wedge} Z
$$

is an s-cofibration over $A \times B$ which is s-acyclic if either $i$ or $j$ is s-acyclic.

Proof. The statement about $s$-cofibrations is part of the analogue, Proposition 12.2.3, for the level model structure. As usual, it suffices to show that $i \square j$ is an $s$-equivalence if $i \in F I_{B}^{f}$ and $j \in F K_{B}^{f}$, where $F K_{B}^{f}$ is the set of generating acyclic $s$-cofibrations specified in Definition 12.5.5. Arguing as in Lemma 5.4.5 and using properness, this will hold if smashing the source and the target of $i$ with $j$ give $s$-equivalences. The reduction so far would work just as well for internal smash products. The required last step reduces via inspection of Definition 12.5.5 to an application of Proposition 12.5.4, with base space taken to be $A \times B$. The reason that this last step works for external smash products but fails for internal smash products is made clear in Warning 6.1.7.

CoROLlary 12.6.6. If $Y$ is an s-cofibrant spectrum over $B$, then the functor $(-) \bar{\wedge} Y$ from $G \mathscr{S}_{A}$ to $G \mathscr{S}_{A \times B}$ is a Quillen left adjoint with Quillen right adjoint $\bar{F}(Y,-)$.

Proposition 12.6.7. Let $f: A \longrightarrow B$ be a G-map. Then $\left(f_{!}, f^{*}\right)$ is a Quillen adjoint pair. If $f$ is a q-equivalence, then $\left(f_{!}, f^{*}\right)$ is a Quillen equivalence.

Proof. We must show that $f$ ! takes acyclic $s$-cofibrations to $s$-equivalences. Since $f_{\text {! }}$ preserves well-grounded objects and level $q$-equivalences between wellgrounded objects by Proposition 12.2.5, it suffices by Lemma 5.4 .7 to prove that $f_{!} k$ is an $s$-equivalence for each map $k$ in $F K_{A}^{f}$. This follows from the corresponding Quillen adjunction with respect to the level model structure if $k \in F J_{A}^{f}$, so assume that $k$ is of the form $i \square k, W \cong i \square k_{A}^{V, W}$. We claim that $f_{!} k$ is a map in $F K_{B}^{f}$ and is therefore an $s$-equivalence. Observe that $k_{A}^{V, W} \cong f^{*} k_{B}^{V, W}$. Using (11.4.5) and the fact that $f_{!}$preserves pushouts, we see from the definition of the $\square$-product that $f_{!}\left(i \square f^{*} k_{B}^{V, W}\right) \cong\left(f_{!} i\right) \square k_{B}^{V, W}$. Since $i$ is obtained from a map over $A$ by adjoining a disjoint section, $f_{!} i$ is obtained from a map over $B$ by adjoining a disjoint section and is thus in $I_{B}^{f}$.

Now assume that $f$ is a $q$-equivalence. By $[75,1.3 .16],\left(f_{!}, f^{*}\right)$ is a Quillen equivalence if and only if $f^{*}$ reflects $s$-equivalences between $s$-fibrant objects and 
the composite $X \longrightarrow f^{*} f_{!} X \longrightarrow f^{*} R f_{!} X$ given by the unit of the adjunction and $s$-fibrant approximation is an $s$-equivalence for all $s$-cofibrant $X$. Since the $s$ fibrant objects are the $\Omega$-spectra over $B$ and the $s$-equivalences between $\Omega$-spectra over $B$ are the level $q$-equivalences, the reflection property follows directly from the corresponding Quillen equivalence with respect to the level model structure. That result also gives that the composite $X \longrightarrow f^{*} f_{!} X \longrightarrow f^{*} R_{\ell} f_{!} X$ is a level $q$ equivalence and hence an $s$-equivalence. Applying Lemma 12.6.1 with $X$ replaced by $f_{!} X$ and observing that $f^{*}$ preserves $s$-equivalences between level $q f$-fibrant $G$-spectra over $B$ since $\left(f^{*} Y\right)_{a} \cong Y_{f(a)}$, a little diagram chase shows that the composite $X \longrightarrow f^{*} f_{!} X \longrightarrow f^{*} R f_{!} X$ is an $s$-equivalence.

Observe that Proposition 12.6.3 applies to $f_{!}$.

Proposition 12.6.8. Let $f: A \longrightarrow B$ be a $G$-bundle whose fibers $A_{b}$ are $G_{b}$ $C W$ complexes. Then $\left(f^{*}, f_{*}\right)$ is a Quillen adjoint pair.

Proof. We must show that $f^{*}$ preserves acyclic $s$-cofibrations. Again it suffices by Lemma 5.4.7 to prove that $f^{*} k$ is an $s$-equivalence between well-grounded spectra for each map $k \in F K_{B}^{f}$. That $f^{*} k$ is a map between well-grounded spectra follows from the fact that if $K \amalg B$ is a space over $B$ with a disjoint section, then $f^{*} F_{V}(K \amalg B)=F_{V} f^{*} K \amalg A$ is well-grounded. To see that $f^{*} k$ is an $s$-equivalence, it is enough, as in the proof of Proposition 12.6.7, to consider $k=i \square k_{B}^{V, W}$ with $i \in I_{B}^{f}$. We have that $f^{*} k_{B}^{V, W}=k_{A}^{V, W}$ and, since $f^{*}$ preserves pushouts, smash products, and factorizations through mapping cylinders, we see as in the cited proof that $f^{*} k \cong f^{*} i \square k_{A}^{V, W}$, which is an acyclic $s$-cofibration.

Corollary 12.6.9. If $B$ is a $G$-cell complex, then $\left(r^{*}, r_{*}\right)$ is a Quillen adjoint pair.

Proposition 12.6.10. Let $\iota: H \longrightarrow G$ be the inclusion of a subgroup. The pair of functors $\left(\iota !, \nu^{*} \iota^{*}\right)$ relating $H \mathscr{S}_{A}$ and $G \mathscr{S}_{\iota !}$ a gives a Quillen equivalence. If $A$ is completely regular, then $\iota$ is also a Quillen right adjoint.

Proof. By Proposition 14.3.1 below, $\left(\iota !, \nu^{*} \iota^{*}\right)$ is a Quillen adjoint pair. The proof that it is a Quillen equivalence is the same as the proof of the ex-space level analogue in Proposition 7.4.7. The last statement is less obvious. As in the proof of the corresponding statement in Proposition 7.4.7, it follows from the spectrum level analogue of Remark 2.4.5, which in turn requires the spectrum level analogue of Proposition 2.4.4, and the analogue in the stable model structure of Proposition 7.4.3. The required analogues are proven in $\$ 14.4$ below.

We shall see that $\left(\iota !, \nu^{*} \iota^{*}\right)$ descends to a closed symmetric monoidal equivalence of homotopy categories in Proposition 13.7.9 below.

Corollary 12.6.11. The functor $\iota^{*}: H o G \mathscr{S}_{B} \longrightarrow H o H \mathscr{S}_{\iota^{*} B}$ is the composite

$$
H o G \mathscr{S}_{B} \stackrel{\mu^{*}}{\longrightarrow} H o G \mathscr{K}_{\iota ! \iota^{*} B} \simeq \mathrm{HoH} \mathscr{K}_{\iota^{*} B}
$$

Using Example 11.5.5 as in Proposition 12.2.10, the following result is now a special case of Propositions 12.6.10 and 12.6.7.

Proposition 12.6.12. For $b \in B$, the pair of functors $\left((-)^{b},(-)_{b}\right)$ relating $G_{b} \mathscr{S}$ and $G \mathscr{S}_{B}$ is a Quillen adjoint pair. 



\section{CHAPTER 13}

\section{Adjunctions and compatibility relations}

\section{Introduction}

The utility of the stable homotopy category Ho $G \mathscr{S}_{B}$ depends on the fact that the usual functors and adjunctions descend to it and still satisfy appropriate commutation relations. We consider such matters in this chapter. Many of our basic adjunctions are Quillen adjunctions in the stable model structure. We recorded those in $\S 12.6$. The crucial adjunction missing from $\S 12.6$ is $\left(f^{*}, f_{*}\right)$ for a general map $f$ of base spaces. This cannot be a Quillen adjoint pair by the argument in Counterexample 0.0.1. We used Brown representability to construct the right adjoint $f_{*}$ between homotopy categories of ex-spaces in Theorem 9.3.2. Analogously, in $\S 13.1$ we use Brown representability to construct $f_{*}$ between homotopy categories of parametrized spectra, and we use base change along diagonal maps to internalize smash products and function spectra. There is an interesting twist here. It is not easy to verify the Mayer-Vietoris axiom directly. Rather, we use the triangulated category variant of the Brown representability theorem, whose hypotheses turn out to be easier to check.

In $§ 13.7$, we complete the proof that our stable homotopy categories are symmetric monoidal and prove some basic compatibility relations among smash products and base change functors. These results involve commutation of Quillen left and right adjoints, and we would not know how to prove them using only model theoretic fibrant and cofibrant replacement functors. Rather, their proofs depend on an equivalence between our model theoretic stable homotopy category of parametrized $G$-prespectra and a classical homotopy category of what we call "excellent" parametrized $G$-prespectra. We used an analogous, but more elementary, equivalence of categories in Chapter 9. It is essential to use parametrized $G$-prespectra rather than parametrized $G$-spectra to make the comparison since the relevant constructions do not all preserve functoriality on linear isometries; that is, they do not preserve $\mathscr{I}_{G}$-spaces. Results proven using the comparison are then translated to parametrized $G$-spectra along the Quillen equivalence between parametrized $G$-prespectra and parametrized $G$-spectra.

These equivalences of categories allow us to use a prespectrum level analogue $T$ of the ex-fibrant approximation functor $P$ to study derived functors. We define excellent parametrized $G$-prespectra in $\S 13.2$. We lift the ex-fibrant approximation functor $P$ from ex- $G$-spaces to parametrized $G$-spectra in $\S 13.3$. There are several further twists here. First, the functor $P$ on ex- $G$-spaces does not behave well with respect to tensors, so extending it to a functor on parametrized $G$-prespectra is subtle. Second, with the extension, the zig-zag of $h$-equivalences connecting $P$ to the identity functor is no longer given by honest maps of parametrized $G$-prespectra, only weak maps. Third, the functor $P$ does not take parametrized $G$-prespectra to 
excellent ones. To remedy this, we introduce two auxiliary functors $K$ and $E$ in $\S 13.4$. The composite $T=K E P$ does land in excellent parametrized $G$-prespectra, and $K$ converts weak maps to honest maps. In $\S \S 13.5$ and 13.6 we use $T$ to prove the promised equivalence of homotopy categories and show how to study derived funtors in this context.

There are few issues of equivariance in this chapter, and we generally continue to omit the (compact Lie) group $G$ from the notations. We adopt the convention of calling isomorphisms in homotopy categories equivalences and we denote them by $\simeq$ rather than $\cong$.

\subsection{Brown representability and the functors $f_{*}$ and $F_{B}$}

We need some preliminaries about the two versions of Brown representability that are applicable in stable situations. Recall Example 11.5.5.

Definition 13.1.1. For $n \in \mathbb{Z}$ and $H \subset G$, we have an $s$-cofibrant sphere $G$-spectrum $S_{H}^{n}$ such that $\pi_{n}^{H}(X)=\left[S_{H}^{n}, X\right]_{G}$ for all $G$-spectra $X$. Explicitly,

$$
S_{H}^{n}= \begin{cases}\Sigma^{\infty}\left(G / H_{+} \wedge S^{n}\right) & \text { if } n \geq 0, \\ F_{-n}\left(G / H_{+} \wedge S^{0}\right) & \text { if } n<0\end{cases}
$$

(as in [105, II.4.7]), where $F_{-n}$ is the shift desuspension by $\mathbb{R}^{n}$. We may allow the ambient group to vary. Replacing $G$ by $G_{b}$ for $b \in B$ and letting $H \subset G_{b}$, define $S_{H}^{n, b}$ to be the $G$-spectrum $\tilde{b}_{!} S_{H}^{n}=\left(S_{H}^{n}\right)^{b}$ over $B$. Note that $S_{H}^{n, b}$ is $s$-cofibrant, by Proposition 12.2.10. By adjunction, for $G$-spectra $X$ over $B, \pi_{n}^{H}\left(X_{b}\right)$ is isomorphic to $\left[S_{H}^{n, b}, X\right]_{G, B}$. Let $\mathscr{D}_{B}$ be the set of all such $G$-spectra $S_{H}^{n, b}$ over $B$.

From here, the following three results work in much the same way as their exspace analogues in $\S 7.5$. The category $\mathrm{Ho} G \mathscr{S}_{B}$ has coproducts and weak pushouts, hence weak sequential colimits. Again, care must be taken with compactness, as defined in Definition 7.5.2, since maps into homotopy colimits must be interpreted in the derived sense.

Lemma 13.1.2. Each $X \in \mathscr{D}_{B}$ is compact.

Proof. The sphere $H$-spectra $S_{H}^{n}$ are compact in $H \mathscr{S}$, where all objects are $s$-fibrant, so that the analogue in that category is clear. We mimic the ex-space level proofs of Lemmas 7.5.14 and 7.5.15. Remember that $s$-equivalences are defined in terms of homotopy groups of fibers after level fibrant approximation. As in the proof of Lemma 7.5.14, for a sequence of maps $f_{q}: Y_{q} \longrightarrow Y_{q+1}$ between $s$-fibrant and $s$-cofibrant $G$-spectra over $B$, we take $R \operatorname{Tel} Y_{q}$ as our model for the derived homotopy colimit of the $Y_{q}$, where $R$ denotes $s$-fibrant approximation. As a fibrant approximation, the map Tel $Y_{q} \longrightarrow R \operatorname{Tel} Y_{q}$ is an $s$-equivalence. To show that the homotopy groups of $R \mathrm{Tel} Y_{q}$ are given as colimits of the homotopy groups of the $Y_{q}$, we must show that the homotopy groups of the source can be computed directly, without level fibrant approximation. Arguing as in the proof of Lemma 7.5.15, we approximate our given sequence of maps $f_{q}: Y_{q} \longrightarrow Y_{q+1}$ by a sequence of $s$ cofibrations $g_{q}: Z_{q} \longrightarrow Z_{q+1}$ of $s$-fibrant and $s$-cofibrant $G$-spectra over $B$. Here the $Z_{q}$ are again $s$-fibrant and $s$-cofibrant, and the approximating maps $Z_{q} \longrightarrow Y_{q}$ are homotopy equivalences and therefore level homotopy equivalences on fibers. We use Theorem 12.4.3(iv) to compute the homotopy groups of colim $Z_{q}$ as the colimit of homotopy groups of the $Z_{q}$, and the conclusion follows. 
Proposition 13.1.3. A map $\xi: Y \longrightarrow Z$ in $G \mathscr{S}_{B}$ is an s-equivalence if and only if the induced map $\xi_{*}:[X, Y]_{G, B} \longrightarrow[X, Z]_{G, B}$ is a bijection for all $X \in \mathscr{D}_{B}$.

Proof. This is a tautology since as $X$ ranges through the $S_{H}^{n, b},[X, Y]_{G, B}$ ranges through the homotopy groups $\pi_{n}^{H}\left(Y_{b}\right)$ that define the $s$-equivalences.

THEOREM 13.1.4 (Brown). A contravariant set-valued functor on the category $H o G \mathscr{S}_{B}$ is representable if and only if it satisfies the wedge and Mayer-Vietoris axioms.

Since we have the Quillen adjoint pair $\left(f_{!}, f^{*}\right)$, we have the right derived functor $f^{*}:$ Ho $G \mathscr{S}_{B} \longrightarrow$ Ho $G \mathscr{S}_{A}$. As in the proof of the analogous result on the level of ex-spaces, Theorem 9.3.2, we can obtain the desired right adjoint $f_{*}$ to $f^{*}$ by use of Brown's theorem provided that we can show that $f^{*}$ preserves the relevant homotopy colimits. However, since $f^{*}: G \mathscr{S}_{B} \longrightarrow G \mathscr{S}_{A}$ does not preserve $s$-cofibrant objects, this is not obvious. We will later give results that would allow us to carry out the proof in a manner analogous to the proof of Theorem 9.3.2, but it is instructive to switch gears and give a more direct proof. It is based on the use of triangulated categories, which are reviewed briefly in $\S 16.6$, and it would not have applied on the ex-space level.

Lemma 13.1.5. The category Ho G $\mathscr{S}_{B}$ is triangulated.

Proof. The treatment of triangulated categories in [124] gives a general pattern of proof for showing that homotopy categories associated to appropriate model categories, generally called stable model categories, are triangulated. It applies here. The distinguished triangles are those equivalent in $\mathrm{Ho}_{G} \mathscr{S}_{B}$ to cofiber sequences of well-grounded spectra or, equivalently by Proposition 12.4.5, those equivalent to the negatives of fiber sequences. It is relevant to note that, by the proof of Theorem 12.4.2, every cofiber sequence is equivalent in Ho $G \mathscr{S}_{B}$ to a cofiber sequence of level ex-quasifibrations.

In triangulated categories, there is an alternative version of Brown's representability theorem due to Neeman [132]. It requires a "generating set of compact objects". We defined detecting sets and generating sets in Definition 7.5.5, but here the distinction disappears; see, for example, $[134,6.2 .9]$ or $[76,1.4 .5]$. Contrary to the usual practice in the literature, we insist that detecting sets and generating sets in triangulated categories be closed under the suspension equivalence $\Sigma$.

Lemma 13.1.6. Any detecting set in a triangulated category is a generating set.

Together with Theorem 7.5.10, this has the following consequence.

THEOREM 13.1.7. The set of iterated suspensions of cell complexes with a single cell in any cofibrantly generated stable model category $\mathscr{C}$ is a set of generating objects for $H o \mathscr{C}$.

Of course, our generating set $\mathscr{D}_{B}$ is smaller than the one given by this general result. Turning to compactness, we assume that any given triangulated category $\mathscr{A}$ has all small coproducts.

Definition 13.1.8. An object $X$ in a triangulated category $\mathscr{A}$ is said to be compact if the natural map $\oplus\left[X, Y_{i}\right] \cong\left[X, \amalg Y_{i}\right]$ is an isomorphism for any set of objects $Y_{i} ; \mathscr{A}$ is said to be compactly generated if it has a generating set $\mathscr{D}$ of compact objects. 
The following obvious lemma describes a general way to prove compactness.

Lemma 13.1.9. Let $T: \mathscr{A} \longrightarrow \mathscr{B}$ be a left adjoint between triangulated categories $\mathscr{A}$ and $\mathscr{B}$ such that the right adjoint $U: \mathscr{B} \longrightarrow \mathscr{A}$ preserves coproducts. If $A$ is compact in $\mathscr{A}$, then $T A$ is compact in $\mathscr{B}$.

REMARK 13.1.10. We are following Neeman [132-134] in using the term "compact"; the term "small" is used for the same notion in [76]. Neither term is fully satisfactory. Observe that this is a different notion from the sequential one that we specified in Definition 7.5.2. It is clear from the construction of sequential homotopy colimits (see Definition 16.6.5) that a compact object in the sense of Definition 13.1 .8 is compact in the sense of Definition 7.5.2. We doubt that the converse holds in general triangulated categories, but we have not looked for a counterexample. The notion in Definition 13.1.8 is the more fundamental one; the notion in Definition 7.5.2 serves only to specify exactly what is relevant to the classical Brown representability theorem.

Warnings 7.5.4 and 7.5.16 apply equally well to the present notion of compactness, but the required compactness of objects of $\mathscr{D}_{B}$ reduces to the compactness of spheres, as in the proofs of Lemmas 7.5.14 and 13.1.2.

LEMMA 13.1.11. $\mathscr{D}_{B}$ is a generating set of compact objects in $\mathrm{HoG} \mathscr{S}_{B}$.

Proof. Take $U$ in Lemma 13.1 .9 to be the fiber functor $(-)_{b}$ : Ho $G \mathscr{S}_{B} \longrightarrow$ Ho $G_{b} \mathscr{S}$ for some $b \in B$. Arguing as in the proof of Lemma 13.1.2, it preserves coproducts since the homotopy groups of coproducts are the direct sums of their homotopy groups. Clearly the objects of $\mathscr{D}_{B}$ are of the form $T A$ for some compact $A$ in some $\mathrm{Ho}_{b} \mathscr{S}$, as in Lemma 13.1.2.

Theorems 13.1.12, 13.1.14, and 13.1 .17 below are proven in $[132,2.13 .1,3.2$, 4.1]. Related results that compare compactness to dualizability appear in [76, 2.1.3] and will become relevant in $\S 16.7$; see also $[62, \S 8]$.

THEOREM 13.1.12. Let $\mathscr{A}$ be a compactly generated triangulated category. A functor $k: \mathscr{A}^{o p} \longrightarrow \mathscr{A} b$ that converts coproducts to products and takes distinguished triangles to exact sequences is representable.

DEFinition 13.1.13. Let $\mathscr{B}$ be a full subcategory of a triangulated category $\mathscr{A}$. Then $\mathscr{B}$ is a thick subcategory if the third term of a distinguished triangle with two terms in $\mathscr{B}$ is in $\mathscr{B}$ and if a direct summand of an object in $\mathscr{B}$ is in $\mathscr{B}$. A thick subcategory $\mathscr{B}$ is localizing if it is also closed under coproducts.

THEOREM 13.1.14. Let $\mathscr{A}$ be a triangulated category with a generating set $\mathscr{D}$ of compact objects. Then the thick subcategory of $\mathscr{A}$ generated by $\mathscr{D}$ is the full subcategory $\mathscr{B}$ of compact objects in $\mathscr{A}$, and the localizing subcategory of $\mathscr{A}$ generated by $\mathscr{D}$ is $\mathscr{A}$ itself.

One can be more precise about how this works, giving a kind of cellular theory in triangulated categories, as in [76, 2.3.1].

THEOREM 13.1.15. Let $\mathscr{A}$ be a triangulated category with a generating set $\mathscr{D}$ of compact objects. Then every object $X$ is isomorphic to a homotopy colimit of a sequence of maps $f_{i}: X_{i} \longrightarrow X_{i+1}$ such that $X_{0}=*$ and the cofiber of $f_{i}$ is a coproduct of generating objects. 
Remark 13.1.16. This result applies to our generating set $\mathscr{D}_{B}$ in Ho $\mathscr{S}_{B}$. Here the conclusion seems quite surprising since the cell complexes generated by just those generating $s$-cofibrations whose cofiber 1-cell complexes are in $\mathscr{D}_{B}$ are very restricted. In fact, one finds by inspection of pushouts and colimits that such a cell spectrum $X$ is isomorphic to the wedge over $B$ of the $\left(X_{b}\right)^{b}=b_{!} X_{b}$. That is, one sees only fibers, glued together trivially. This illustrates the fundamental difference between generating sets of cofibrations and cellular objects in a stable model category and generating sets of objects and cellular objects (as in Theorem 13.1.15) in its derived triangulated homotopy category. Concretely, the difference is explained by the need for fibrant approximation in the comparison of $[-,-]$ and $\pi(-,-)$.

Theorem 13.1.12 specializes to give an adjoint functor theorem for exact functors between triangulated categories (as defined in Definition 16.6.6).

THEOREM 13.1.17. Let $\mathscr{A}$ be a compactly generated triangulated category and let $\mathscr{B}$ be any triangulated category. An exact functor $F: \mathscr{A} \longrightarrow \mathscr{B}$ that preserves coproducts has a right adjoint $G$.

Theorem 13.1.18. For any $G$-map $f: A \longrightarrow B$, there is a right adjoint $f_{*}$ to the functor $f^{*}: H o G \mathscr{S}_{B} \longrightarrow H o G \mathscr{S}_{A}$, so that

$$
\left[f^{*} Y, X\right]_{G, A} \cong\left[Y, f_{*} X\right]_{G, B}
$$

for $X$ in $G \mathscr{S}_{A}$ and $Y$ in $G \mathscr{S}_{B}$.

Proof. The left adjoint $f_{!}$commutes with $\Sigma$ and preserves cofiber sequences, and this remains true after passage to derived homotopy categories. Therefore the derived functor $f_{!}$is exact. Since $f^{*}$ is Quillen right adjoint to $f_{!}$, the derived functor $f^{*}$ is right adjoint to $f_{!}$and is therefore also exact; see, for example, [131, 3.9]. If $X$ is in $\mathscr{D}_{A}$, then $f_{!} X$ is compact in Ho $G \mathscr{S}_{B}$, as we see from commutation relations between relevant Quillen left adjoints given in Remark 11.4.7. It follows formally that $f^{*}$ preserves coproducts, by $[132,5.1]$ or $[62,7.4]$.

REMARK 13.1.19. For composable maps $f$ and $g$, there is a natural equivalence $g_{*} \circ f_{*} \simeq(g \circ f)_{*}$ on homotopy categories since $f^{*} \circ g^{*} \simeq(g \circ f)^{*}$.

Exactly as for ex-spaces in Theorem 9.3.5, we apply change of base along the diagonal map $\Delta: B \longrightarrow B \times B$ to obtain internal smash product and function spectra functors in $\mathrm{Ho} G \mathscr{S}_{B}$.

Theorem 13.1.20. Define $\wedge_{B}$ and $F_{B}$ on $H o G \mathscr{S}_{B}$ to be the composite (derived) functors

$$
X \wedge_{B} Y=\Delta^{*}(X \bar{\wedge} Y) \text { and } F_{B}(X, Y)=\bar{F}\left(X, \Delta_{*} Y\right)
$$

Then

$$
\left[X \wedge_{B} Y, Z\right]_{G, B} \cong\left[X, F_{B}(Y, Z)\right]_{G, B}
$$

for $X, Y$ and $Z$ in $H_{o} G \mathscr{S}_{B}$.

Proof. The displayed adjunction is the composite of the adjunction for the external smash product and function spectra functors given by Corollary 12.6.6 and the adjunction $\left(\Delta^{*}, \Delta_{*}\right)$. 


\subsection{The category $G \mathscr{E}_{B}$ of excellent prespectra over $B$}

We must still prove that Ho $G \mathscr{S}_{B}$ is a closed symmetric monoidal category under the derived internal smash product, that the derived functor $f^{*}$ is closed symmetric monoidal, and that various compatibility relations that hold on the point-set level descend to homotopy categories. In particular, since our right adjoints $f_{*}, \Delta_{*}$, and therefore $F_{B}$ come from Brown's representability theorem, it is not at all obvious how to prove that they are well-behaved homotopically. In Chapter 9, we solved the corresponding ex-space level problems by proving that $\mathrm{Ho}_{\mathrm{K}} G \mathscr{K}_{B}$ is equivalent to the more classical and elementary homotopy category $h G \mathscr{W}_{B}$. Here $G \mathscr{W}_{B}$ is the category of ex-fibrations over $B$ whose total spaces are compactly generated and of the homotopy types of $G$-CW complexes, and $h G \mathscr{W}_{B}$ is obtained from $G \mathscr{W}_{B}$ simply by passage to homotopy classes of maps. This equivalence allowed us to exploit the ex-fibrant approximation functor $P$ of $\S 8.3$ to resolve the cited problems.

We shall resolve our spectrum level problems similarly, and the following definitions give the appropriate analogues of $G \mathscr{W}_{B}$ and $h G^{2} \mathscr{W}_{B}$. However, to keep closer to the ex-space level, it is essential to work with parametrized prespectra rather than parametrized spectra. It is safe to do so in view of the Quillen equivalence $(\mathbb{P}, \mathbb{U})$ of Theorem 12.3 .12 relating $G \mathscr{P}_{B}$ and $G \mathscr{S}_{B}$.

Definition 13.2.1. Let $X$ be a $G$-prespectrum over $B$.

(i) $X$ is well-structured if each level $X(V)$ is in $G \mathscr{W}_{B}$.

(ii) $X$ is $\Sigma$-cofibrant if it is well-grounded and each structure map

$$
\sigma: \Sigma_{B}^{W} X(V) \longrightarrow X(V \oplus W)
$$

is an $f p$-cofibration and therefore an $f$-cofibration.

We can now give the definition of excellent $G$-prespectra over $B$ and of the associated classical homotopy category. Working with classical nonequivariant and nonparametrized coordinatized prespectra $\left\{E_{n}\right\}$, it has been known since the 1960's that the following definition gives the simplest quick and dirty rigorous construction of the stable homotopy category.

Definition 13.2.2. The category $G \mathscr{E}_{B}$ of excellent $G$-prespectra over $B$ is the full subcategory of $G \mathscr{P}_{B}$ whose objects are the well-structured $\Sigma$-cofibrant $\Omega$ - $G$ prespectra over $B$. Let $h G \mathscr{E}_{B}$ denote the classical homotopy category obtained from $G \mathscr{E}_{B}$ by passage to homotopy classes of maps.

We comment on the conditions we require of excellent prespectra over $B$. We require that they be well-structured so that we can exploit levelwise our equivalence of homotopy categories on the ex-space level. We require that they be $\Sigma$-cofibrant since that provides "homotopical glue" that is necessary for the transition from the known equivalence on the ex-space level to the desired equivalence on the prespectrum level. We shall make this idea precise shortly, in Proposition 13.2.5. We require that they be $\Omega$-prespectra over $B$ since it is clearly sensible to restrict attention to $s$-fibrant objects in $G \mathscr{S}_{B}$ if we hope to compare homotopy categories. Recall that $X$ is an $\Omega$-prespectrum if it is a level $q f$-fibrant prespectrum over $B$ whose adjoint structure maps

$$
\tilde{\sigma}: X(V) \longrightarrow \Omega_{B}^{W-V} X(W)
$$


are $q$-equivalences. Since excellent prespectra over $B$ are required to be level exfibrations, they are automatically level $q f$-fibrant. The condition on the adjoint structure maps is stronger than it appears on the surface.

Lemma 13.2.3. For excellent $G$-prespectra $X$ over $B$, the adjoint structure maps

$$
\tilde{\sigma}: X(V) \longrightarrow \Omega_{B}^{W} X(V \oplus W)
$$

are fp-equivalences.

Proof. The $\tilde{\sigma}$ are $q$-equivalences between $G$-CW homotopy types and are therefore $h$-equivalences. Since they are maps between ex-fibrations, they are $f p$ equivalences by Proposition 5.2.2.

This implies, for example, that homotopy-preserving functors $G \mathscr{E}_{B} \longrightarrow G \mathscr{P}_{B}$ that may not preserve level $q$-equivalences nevertheless do preserve the equivalence property required of the adjoint structure maps.

REMARK 13.2.4. Our definition of excellent parametrized prespectra is close to that used by Clapp and Puppe [31,32], who in turn were influenced by definitions in [112]. Curiously, while Clapp [31] focuses on ex-fibrations, Clapp and Puppe [32] never mention fibration conditions. These papers are nonequivariant, but the second is written in terms of what the authors call "coordinate-free spectra" over $B$. These are the same as our nonequivariant prespectra over $B$, except that their adjoint structure maps $\tilde{\sigma}$ are required to be closed inclusions, which holds automatically for $\Sigma$-cofibrant prespectra. Clapp and Puppe [32] use the term "cofibrant" for our notion of $\Sigma$-cofibrant.

A crucial result of Clapp and Puppe makes the idea of homotopical glue precise. It is stated nonequivariantly in $[32,6.1]$, but it works just as well equivariantly. Translated to our language, it reads as follows.

Proposition 13.2.5 (Clapp-Puppe). If $f: X \longrightarrow Y$ is a level $f p$-equivalence between $\Sigma$-cofibrant prespectra over $B$, then $f$ is a homotopy equivalence of prespectra over $B$. Therefore, if $f: X \longrightarrow Y$ is a level h-equivalence between wellstructured $\Sigma$-cofibrant prespectra over $B$, then $f$ is a homotopy equivalence of prespectra over $B$.

SkETCH PROOF. The proof is analogous to the proof that a ladder of homotopy equivalences connecting sequences of cofibrations induces a homotopy equivalence on passage to colimits. The point is that, for $\Sigma$-cofibrant parametrized prespectra $Y$, we can carry out inductive arguments just as if $Y$ were such a colimit. Using standard cofibration arguments, carried over to the parametrized case, we can extend an $f p$-homotopy inverse of $\Sigma_{B}^{W_{i}} X\left(V_{i}\right) \longrightarrow \Sigma_{B}^{W_{i}} Y\left(V_{i}\right)$ to an $f p$-homotopy inverse of $X\left(V_{i+1}\right) \longrightarrow Y\left(V_{i+1}\right)$ and proceed inductively. The last statement follows by Corollary 5.2.6(i), which shows that a level $h$-equivalence between well-structured prespectra over $B$ is a level $f p$-equivalence.

REMARK 13.2.6. The category $G \mathscr{E}_{B}$ inherits many of the preservation properties for ex-fibrations that are catalogued in $\S 8.2$ and are inherited by $G \mathscr{W}_{B}$. The condition of being well-structured is defined levelwise, so that any construction that takes $G \mathscr{W}_{B}$ to itself will preserve well-structured $G$-prespectra over $B$. By the retract characterization of $f p$-cofibrations, any functorial construction that commutes 
with $\Sigma_{B}$ will preserve $\Sigma$-cofibrant prespectra over $B$. Any homotopy-preserving functorial construction that commutes with $\Omega_{B}$ will preserve $\Omega$ - $G$-prespectra over $B$. For example, base change functors $f^{*}$ preserve excellent $G$-prespectra. Functors that take excellent $G$-prespectra to well-structured $G$-prespectra can be composed with the approximation functor $T=K E$ defined in $\$ 13.4$ below to rectify them to functors that preserve excellent $G$-prespectra.

\subsection{The level ex-fibrant approximation functor $P$ on prespectra}

We seek an approximation functor to play the role on the parametrized prespectrum level that the functor $P$ played on the ex-space level functor. We shall introduce three approximation functors, $P, E$ and $K$, that successively build in the properties of being well-structured, being an $\Omega$-prespectrum, and being $\Sigma$-cofibrant, each preserving the properties already obtained. We define $P$ in this section and $E$ and $K$ in the next.

Lifting the ex-space level functor $P$ of $\S 8.3$ to the prespectrum level requires care. Recall that $P$ is the composite of the whiskering functor $W$ and the Moore mapping path space functor $L$, together with the natural zig-zag of $h$-equivalences

$$
K<\stackrel{\rho}{<} W \stackrel{W \iota}{\longrightarrow} W L K=P K
$$

of Definition 8.3.4 for ex-spaces $K$ over $B$. The functors $W$ and $L$ do not commute with tensors with based spaces, hence cannot be enriched over $G \mathscr{K}_{B}$, by Lemma 11.3.4. There is therefore no canonical way of inducing structure maps after applying $P$ levelwise to a prespectrum, as one might at first hope. We shall resolve this by constructing by hand certain non-canonical but natural maps

$$
\alpha_{V}: W K \wedge_{B} S^{V} \longrightarrow W\left(K \wedge_{B} S^{V}\right)
$$

and

$$
\beta_{V}: L K \wedge_{B} S^{V} \longrightarrow L\left(K \wedge_{B} S^{V}\right)
$$

such that $\alpha_{0}=\mathrm{id}, \beta_{0}=\mathrm{id}$ and the following associativity diagram commutes, where $\left(F, f_{V}\right)$ stands for either $\left(W, \alpha_{V}\right)$ or $\left(L, \beta_{V}\right)$.

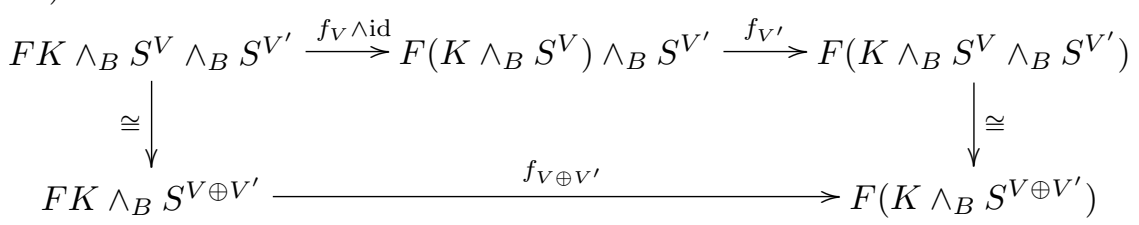

The definitions of these maps and the proofs that these diagrams commute depend on chosen decompositions of $V$ and $V^{\prime}$ as direct sums of indecomposable representations, and we cannot choose compatible decompositions for all representations $V$ and $V^{\prime}$ at once. For this reason, and for other reasons that will become apparent later, we must switch gears and work with sequentially indexed prespectra.

Thus, to be precise about the constructions in this section and the next, we restrict our original collection $\mathscr{V}$ of indexing representations to a countable cofinal sequence $\mathscr{W}$ of expanding representations in our given universe $U$. More precisely, $\mathscr{W}$ consists of representations $V_{i}$ for $i \geq 0$ such that $V_{0}=0$ and $V_{i} \subset V_{i+1}$. We set $W_{i}=V_{i+1}-V_{i}$. Such a sequence can be chosen in any universe. We could just as well start with representations $W_{i}$ and define $V_{i}$ inductively by $V_{i+1}=V_{i} \oplus W_{i}$. There is 
no need to use orthogonal complements. We shall write in terms of complements, but on the understanding that that is just a notational convenience.

REMARK 13.3.5. There is a small quibble here since we originally defined our categories of parametrized prespectra only on collections of representations that are closed under finite direct sums, which $\mathscr{W}$ clearly is not. However, if we let $\mathscr{W}^{\prime}$ consist of all finite sums of the $W_{i}$, then we recover such a collection. As in $\S 11.3$ (or $[106, \S 2]$ ), we can interpret $G \mathscr{P}_{B}^{\mathscr{W}^{\prime}}$ as a diagram category indexed on a certain small category, say $\mathscr{D}_{G}^{\mathscr{W}^{\prime}}$, with object set $\mathscr{W}^{\prime}$, and we can interpret $G \mathscr{P}_{B}^{\mathscr{W}}$ as a diagram category indexed on the full subcategory $\mathscr{D}_{G}^{\mathscr{W}}$ of $\mathscr{D}_{G}^{\mathscr{W}^{\prime}}$ whose object set is $\mathscr{W}$. This gives a restriction functor $\mathbb{U}: G \mathscr{P}_{B}^{\mathscr{W}^{\prime}} \longrightarrow G \mathscr{P}_{B}^{\mathscr{W}}$ that is right adjoint to a prolongation functor $\mathbb{P}[106, \S 3]$, and $(\mathbb{P}, \mathbb{U})$ induces an adjoint equivalence of homotopy categories. We shall study such "change of universe" adjunctions in $\S 14.2$. They allow us to lift all results we prove about the categories of parametrized prespectra indexed on cofinal sequences to our usual ones indexed on collections of representations closed under direct sums.

Definition 13.3.6. Let $X$ be a prespectrum over $B$ indexed on the countable cofinal sequence $\mathscr{W}=\left\{V_{i}\right\}$, where $V_{0}=0$ and $V_{i+1}=V_{i} \oplus W_{i}$. Let $X$ have structure maps $\sigma_{i}: \Sigma_{B}^{W_{i}} X\left(V_{i}\right) \longrightarrow X\left(V_{i+1}\right)$. Then the maps

$$
W \sigma_{i} \circ \alpha: W X\left(V_{i}\right) \wedge_{B} S^{W_{i}} \longrightarrow W X\left(V_{i+1}\right)
$$

and

$$
L \sigma_{i} \circ \beta: L X\left(V_{i}\right) \wedge_{B} S^{W_{i}} \longrightarrow L X\left(V_{i+1}\right)
$$

specify structure maps for prespectra $W X$ and $L X$ over $B$. Therefore $P X=W L X$ is a prespectrum over $B$.

Unfortunately, as will be clear from the following construction, the maps in the zig-zag (13.3.1) do not lift to the prespectrum level. They only induce weak maps of prespectra, that is, levelwise maps that only commute with the structure maps up to (canonical) $f p$-homotopy. Fortunately, the last approximation functor $K$, which arranges $\Sigma$-cofibrancy and will be discussed in the next section, turns weak maps into honest ones.

Construction 13.3.7. We define $\alpha_{V}$ and $\beta_{V}$. Fix a decomposition of $V$ into a direct sum of irreducible representations and let $\mathscr{P}_{V}$ be the set of the projections from $V$ to the irreducible subrepresentations in this fixed decomposition. Define three equivariant maps from $V$ to the real numbers by setting

$$
\|v\|_{V}=\max _{\pi \in \mathscr{P}_{V}}|\pi v|, \quad \mu_{V}(v)=\prod_{\pi \in \mathscr{P}_{V}}(1-|\pi v|), \quad \nu_{V}(v)=\prod_{\pi \in \mathscr{P}_{V}} \max (1,|\pi v|) .
$$

Applying the same definitions to another representation $V^{\prime}$ and to $V \oplus V^{\prime}$ with its induced decomposition as a sum of irreducible representations, we see that the following equations hold.

$$
\begin{gathered}
\left\|v \oplus v^{\prime}\right\|_{V \oplus V^{\prime}}=\max \left\{\|v\|_{V},\left\|v^{\prime}\right\|_{V^{\prime}}\right\} \\
\mu_{V \oplus V^{\prime}}\left(v \oplus v^{\prime}\right)=\mu_{V}(v) \mu_{V^{\prime}}\left(v^{\prime}\right) \\
\nu_{V \oplus V^{\prime}}\left(v \oplus v^{\prime}\right)=\nu_{V}(v) \nu_{V^{\prime}}\left(v^{\prime}\right) .
\end{gathered}
$$

Define a natural map

$$
h_{V}: W K \wedge_{B} S^{V} \wedge_{B}[1, \infty)_{+} \longrightarrow W\left(K \wedge_{B} S^{V}\right),
$$


by setting

$$
\begin{gathered}
h_{V}(x \wedge v \wedge t)= \begin{cases}x \wedge \mu\left(t^{-1} v\right)^{-1} \cdot v & \text { if }\|v\| \leq t, \\
\left(p(x), 1-\nu\left(t^{-1} v\right)^{-1}\right) & \text { if }\|v\| \geq t,\end{cases} \\
h_{V}((b, s) \wedge v \wedge t)= \begin{cases}(b, s) & \text { if }\|v\| \leq t, \\
\left(b, 1-(1-s) \nu\left(t^{-1} v\right)^{-1}\right) & \text { if }\|v\| \geq t .\end{cases}
\end{gathered}
$$

At time $t=1$ this specifies $\alpha_{V}$ and it is easy to verify that the associativity diagram (13.3.4) commutes. Further, the map $\rho \circ h_{V}$ extends to $t=\infty$ to give an $f p$-homotopy from $\rho \circ \alpha_{V}$ to $\rho \wedge_{B}$ id. It follows that $\rho$ induces levelwise a weak map of prespectra $W X \longrightarrow X$.

Similarly define

$$
k_{V}: L K \wedge_{B} S^{V} \wedge_{B}[1, \infty)_{+} \longrightarrow L\left(K \wedge_{B} S^{V}\right),
$$

by setting

$$
k_{V}((x, \lambda) \wedge v \wedge t)= \begin{cases}(x \wedge v, \lambda) & \text { if }\|v\| \leq t \\ \left(x \wedge v, \nu\left(t^{-1} v\right) \lambda\right. & \text { if }\|v\| \geq t\end{cases}
$$

Here, if $1 \leq a<\infty$, and $\lambda \in \Lambda B$, then $a \lambda$ denotes the Moore path of length $l_{\lambda} / a$ given by $a \lambda(t)=\lambda(a t)$. At time $t=1$ this specifies $\beta_{V}$, and it is again easy to check the required associativity. The map $k_{V} \circ(\iota \wedge$ id) extends to an $f p$-homotopy from $\beta_{V} \circ\left(\iota \wedge_{B}\right.$ id $)$ to $\iota$, hence $\iota$ induces levelwise a weak map of prespectra $X \longrightarrow L X$, to which we can apply $W$ to obtain a weak map $W X \longrightarrow W L X=P X$.

In view of Definition 8.3.4, naturality arguments from Definition 13.3.6 and Construction 13.3.7 prove the following theorem.

Theorem 13.3.8. There are functors $L, W$, and $P=W L$ on $G \mathscr{P}_{B}$ that are given levelwise by the functors $L, W$, and $P$ on $G \mathscr{K}_{B}$. There are natural weak maps $\rho: W X \longrightarrow X$ and $\iota: X \longrightarrow L X$ that are given levelwise by the ex-space maps $\rho$ and $\iota$. Therefore, there is a natural zig-zag of weak maps $\phi=(\rho, W \iota)$ as displayed in the diagram

$$
X \stackrel{\rho}{\longleftarrow} W X \stackrel{W \iota}{\longrightarrow} W L X=P X .
$$

These maps are level h-equivalences, and $P$ converts level $h$-equivalences to level fp-equivalences. If each $X(V)$ is compactly generated and of the homotopy type of a $G-C W$ complex, then $P X$ is well-structured. If $X$ is well-structured, then the weak maps in the above display are level $f p$-equivalences between well-structured $G$ prespectra over B. If, further, the adjoint structure maps of $X$ are $h$-equivalences or q-equivalences, then so are the adjoint structure maps of $L X, W X$, and $P X$.

Proof. The only point that may need elaboration is the last clause. For a weak map $f: X \longrightarrow Y$, we have a homotopy commutative diagram

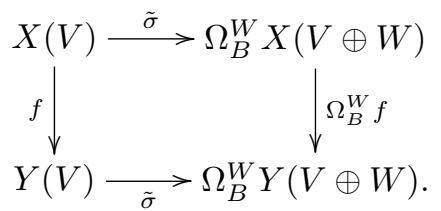

The functor $\Omega_{B}^{W}$ preserves $f p$-equivalences. Therefore, if $f$ is an $f p$-equivalence, then the $\tilde{\sigma}$ for $X$ are $h$-equivalences or $q$-equivalences if and only if the $\tilde{\sigma}$ for $Y$ are so. We apply this to $f=\rho$ and $f=W \iota$. 


\subsection{The auxiliary approximation functors $K$ and $E$}

We begin with the parametrized $\Omega$-prespectrum approximation functor $E$. This is a folklore construction when $B$ is a point. In the parametrized context, the construction makes essential use of Stasheff's theorem, Theorem 3.5.2.

Proposition 13.4.1. There is a functor $E: G \mathscr{P}_{B} \longrightarrow G \mathscr{P}_{B}$ and a natural map $\alpha: X \longrightarrow E X$ with the following properties.

(i) The functor $E$ preserves level fp-equivalences and well-grounded prespectra.

(ii) If $X$ is well-structured, then $E X$ is a well-structured $\Omega$-prespectrum and the map $\alpha: X \longrightarrow E X$ is an s-equivalence.

Proof. Define $E X$ by letting $E X\left(V_{i}\right)$ be the telescope over $j \geq i$ of the exspaces $\Omega_{B}^{V_{j}-V_{i}} X\left(V_{j}\right)$ with respect to the adjoint structure maps

$$
\Omega_{B}^{V_{j}-V_{i}} \tilde{\sigma}: \Omega_{B}^{V_{j}-V_{i}} X\left(V_{j}\right) \longrightarrow \Omega_{B}^{V_{j}-V_{i}} \Omega_{B}^{W_{j}} X\left(V_{j+1}\right) \cong \Omega_{B}^{V_{j+1}-V_{i}} X\left(V_{j+1}\right) .
$$

Since the functor $\Omega_{B}^{W_{i}}$ commutes with telescopes, $\Omega_{B}^{W_{i}} E X\left(V_{i+1}\right)$ is isomorphic to the telescope over $j \geq i+1$ of the ex-spaces $\Omega_{B}^{V_{j}-V_{i+1}} X\left(V_{j}\right)$. The adjoint structure map $E X\left(V_{i}\right) \longrightarrow \Omega_{B}^{W_{i}} E X\left(V_{i+1}\right)$ is induced by the maps $\Omega_{B}^{V_{j}-V_{i}} \tilde{\sigma}_{j}$ for $j \geq i$. The map $\alpha: X \longrightarrow E X$ is given by the inclusion of the bases of the telescopes. If $f: X \longrightarrow Y$ is a level $f p$-equivalence, then $E f: E X \longrightarrow E Y$ is a level $f p$-equivalence since a standard inductive argument (applicable in any topologically bicomplete category) shows that the telescope of a ladder of $f p$-equivalences is an $f p$-equivalence.

If $X$ is well-grounded or level ex-fibrant, then so is $E X$ since the construction clearly stays in the category of compactly generated spaces and since it preserves the conditions of being well-sectioned or level ex-fibrant by results in $\S 8.2$. To show that $E$ preserves well-structured prespectra, it remains to show that if $X$ has total spaces of the homotopy types of $G$-CW complexes, then so does $E X$. By Stasheff's theorem (Theorem 3.5.2), the fibers $X(V)_{b}=X_{b}(V)$ have the homotopy types of $G_{b}$-CW complexes. We have the analogous construction $E$ in the category of $G_{b}$-prespectra and, by Milnor's theorem (Theorem 3.4.5) and standard facts about telescopes, the $\left(E\left(X_{b}\right)\right)(V)$ have the homotopy types of $G_{b}$-CW complexes. It is clear from the definition of $E$ that $\left(E\left(X_{b}\right)\right)(V)=((E X)(V))_{b}$. That is, the $G_{b}$-prespectrum $E\left(X_{b}\right)$ is the fiber $(E X)_{b}$ of the $G$-prespectrum $E X$ over $B$. By Stasheff's theorem again, it follows that the $(E X)(V)$ have the homotopy types of $G$-CW complexes.

To check that the adjoint structure maps are q-equivalences when $X$ is wellstructured, it suffices to check that they induce $q$-equivalences on the fibers over $b$ for all $b \in B$. That holds by inspection of the homotopy groups of the colimits that define $(E X)_{b} \cong E\left(X_{b}\right)$. Similarly, we see that $\alpha$ is a $\pi_{*}$-equivalence when $X$ is well-structured by fiberwise comparison of the colimits of homotopy groups of fibers that define the homotopy groups of $X$ and $E X$.

To approximate parametrized prespectra by level $f p$-equivalent $\Sigma$-cofibrant prespectra, we use the elementary cylinder construction $K$ that was first defined in [108] and has been used in various papers since. We recall the construction and its main properties from $[98,6.8]$, which carries over verbatim to the parametrized context. A more sophisticated but less convenient treatment is given in [61].

Proposition 13.4.2. There is a functor $K: G \mathscr{P}_{B} \longrightarrow G \mathscr{P}_{B}$ and a natural level $f p$-equivalence $\pi: K X \longrightarrow X$. Therefore $K$ preserves level $f p$-equivalences. 
If $X$ is well-grounded, then $K X$ is $\Sigma$-cofibrant. If $X$ is well-structured, then $K X$ is well-structured. If $X$ is a well-structured $\Omega$-prespectrum, then so is $K X$ and thus $K X$ is excellent. There is a natural weak map $\iota: X \longrightarrow K X$ that is a right inverse of $\pi$, and $K$ takes weak maps $f$ to honest maps $K f$ such that $\iota \circ f=K f \circ \iota$.

Proof. Define $K X$, a level inclusion $\iota: X \longrightarrow K X$, and a level $f p$-deformation retraction $\pi: K X \longrightarrow X$ right inverse to $\iota$ as follows. Let $K X(0)=X(0)$ and $\iota(0)=\pi(0)=\mathrm{id}$. Inductively, suppose given $K X\left(V_{i}\right)$, an inclusion $\iota\left(V_{i}\right): X\left(V_{i}\right) \longrightarrow$ $K X\left(V_{i}\right)$ and an inverse $f p$-deformation retraction $\pi\left(V_{i}\right): K X\left(V_{i}\right) \longrightarrow X\left(V_{i}\right)$. Let $K X\left(V_{i+1}\right)$ be the double mapping cylinder in $G \mathscr{K}_{B}$ of the pair of maps

$$
\Sigma_{B}^{W_{i}} K X\left(V_{i}\right) \stackrel{\Sigma_{B}^{W_{i}} \iota\left(V_{i}\right)}{\longleftarrow} \Sigma_{B}^{W_{i}} X\left(V_{i}\right) \stackrel{\sigma}{\longrightarrow} X\left(V_{i+1}\right)
$$

in $G \mathscr{K}_{B}$. Let $\sigma: \Sigma_{B}^{W_{i}} K X\left(V_{i}\right) \longrightarrow K X\left(V_{i+1}\right)$ be the inclusion of the left base of the double mapping cylinder, which is an $f p$-cofibration, and let $\iota\left(V_{i+1}\right): X\left(V_{i+1}\right) \longrightarrow$ $K X\left(V_{i+1}\right)$ be the inclusion of the right base. Let $\pi\left(V_{i+1}\right): K X\left(V_{i+1}\right) \longrightarrow X\left(V_{i+1}\right)$ be the map obtained by first using the $f p$-equivalence $\Sigma_{B}^{W_{i}} \pi\left(V_{i}\right)$ on the left base to map to the mapping cylinder of $\sigma$ and then using the evident deformation retraction to the right base. There is an equivalent description as a finite telescope. Certainly $\pi$ is a map of prespectra over $B$ and a level $f p$-deformation retraction with level inverse the weak map $\iota$. The functoriality of the construction is clear.

If $X$ is well-grounded, then $K X$ is clearly also well-grounded and thus $K X$ is $\Sigma$-cofibrant. If $X$ is well-structured, then so is $K X$ by Propositions 8.2.1 and 8.2.3. If, further, the adjoint structure maps of $X$ are $q$-equivalences, then they are $f p$ equivalences since $X$ is well-structured. Since $K$ preserves $f p$-homotopies, it follows that $K X$ is also an $\Omega$-prespectrum. Alternatively, since $\Omega_{B}^{V}$ is a Quillen right adjoint in the $q f$-model structure, it preserves $q$-equivalences between $q f$-fibrant ex-spaces. In particular, the maps $\Omega_{B}^{W} \pi\left(V_{i}\right)$ are $q$-equivalences.

If $f: X \longrightarrow Y$ is a weak map with $f p$-homotopies

$$
h_{i}: \Sigma_{B}^{W_{i}} X\left(V_{i}\right) \wedge_{B} I_{+} \longrightarrow Y\left(V_{i+1}\right)
$$

from $\sigma_{Y} \circ \Sigma^{W_{i}} f\left(V_{i}\right)$ to $f\left(V_{i+1}\right) \circ \sigma_{X}$, define $K f$ inductively by setting $K f(0)=$ $f(0)$ and letting $K f\left(V_{i+1}\right)$ be $\Sigma_{B}^{W_{j}} K f\left(V_{i}\right)$ on the left end of the mapping cylinder, $f\left(V_{i+1}\right)$ on the right end and as follows on the cylinder itself:

$$
K f\left(V_{i+1}\right)[x, t]= \begin{cases}{\left[\Sigma_{B}^{W_{i}} f\left(V_{i}\right)(x), 2 t\right]} & \text { if } 0 \leq t \leq \frac{1}{2} \\ h_{i}(x, 2 t-1) & \text { if } \frac{1}{2} \leq t \leq 1 .\end{cases}
$$

Then $K f$ is a map of prespectra over $B$ and $\iota \circ f=K f \circ \iota$.

The composite approximation functor $T=K E P$ has various good preservation properties. The ex-space level properties of $P$ recorded in $\S 8.4$ are inherited on the prespectrum level, and we have the following sample result for $E$ and $K$.

Lemma 13.4.3. For a $G$-map $f: A \longrightarrow B$, a prespectrum $Y$ over $B$ and $a$ prespectrum $X$ over $A$, there are natural isomorphisms

$$
f^{*} E Y \cong E f^{*} Y, \quad f^{*} K Y \cong K f^{*} Y \text { and } K f_{!} X \cong f_{!} K X .
$$

Proof. The relevant telescopes commute with $f^{*}$ since it is a symmetric monoidal left adjoint and with $f_{\text {! }}$ since it is a left adjoint and the projection formula (2.2.6) holds. 


\subsection{The equivalence between $\operatorname{Ho} G \mathscr{P}_{B}$ and $h G \mathscr{E}_{B}$}

We can now extend the results of $\S 9.1$ to parametrized prespectra. As in the previous section, our parametrized prespectra are indexed on a countable cofinal sequence of expanding representations in our given universe. We begin by collating the results of the previous two sections.

TheOREM 13.5.1. Let $X$ be a well-grounded $G$-prespectrum over $B$ whose total spaces are of the homotopy types of $G-C W$ complexes and define $T X=K E P X$.

(i) $T X$ is an excellent $G$-prespectrum.

(ii) $T$ takes level q-equivalences between $G$-prespectra over $B$ that satisfy the hypotheses on $X$ to homotopy equivalences of $G$-prespectra.

(iii) There is a zig-zag of $s$-equivalences between $X$ and $T X$.

(iv) If $X$ is an excellent $G$-prespectrum over $B$, then the zig-zag consists of level fp-equivalences, and it gives rise to a zig-zag of homotopy equivalences of $G$-prespectra over $B$ connecting $X$ and $T X$.

Proof. We have that $P X$ is well-structured by Theorem 13.3.8, EPX is a well-structured $\Omega$-prespectrum by Proposition 13.4.1, and $T X$ is excellent by Proposition 13.4.2. In (ii), a level $q$-equivalence is a level $h$-equivalence. By the results just quoted, $P$ takes level $h$-equivalences to level $f p$-equivalences, which are preserved by $E$, and $K$ takes level $f p$-equivalences to homotopy equivalences. Since $K$ converts weak maps to genuine maps, we have the following diagram of maps of $G$-presepectra over $B$.

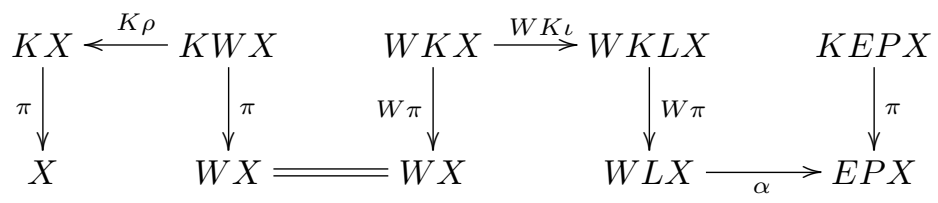

The vertical maps $\pi$, hence also the vertical maps $W \pi$, are level $f p$-equivalences. The map $\rho$ is a level $f$-equivalence. The map $\iota$ is a level $h$-equivalence, hence so is $W K \iota$. The map $\alpha$ is an $s$-equivalence because $P X$ is well-structured. Since level $q$-equivalences are also $s$-equivalences, the diagram displays a zig-zag of $s$-equivalences between $X$ and $T X$.

For the last statement, observe that all prespectra in the diagram are wellstructured $\Omega$-prespectra over $B$. Moreover, $\alpha$ is a level $q$-equivalence by Theorem 12.3.8. It is therefore a level $h$-equivalence since our total spaces have the homotopy types of $G$-CW complexes. Since all prespectra in our diagram are wellstructured, our level $h$-equivalences are level $f p$-equivalences, by Proposition 8.3.2. Applying $K$ where needed, we can expand the diagram to a zig-zag of level $f p$ equivalences between $\Sigma$-cofibrant prespectra. By Proposition 13.2.5, this gives a zig-zag of homotopy equivalences connecting $X$ and $T X$.

We introduce a category that is intermediate between $G \mathscr{P}_{B}$ and $G \mathscr{E}_{B}$.

Definition 13.5.3. Define $G \mathscr{Q}_{B}$ to be the full subcategory of $G \mathscr{P}_{B}$ consisting of the well-grounded $\Omega$-prespectra over $B$ whose total spaces are of the homotopy types of $G$-CW complexes. Define $\operatorname{Ho} G \mathscr{Q}_{B}$ to be the homotopy category obtained by inverting the $s$-equivalences in $G \mathscr{Q}_{B}$; by the proof of the next theorem, there are no set-theoretic problems in defining $\operatorname{Ho} G \mathscr{Q}_{B}$. Define $T=K E P: G \mathscr{Q}_{B} \longrightarrow G \mathscr{E}_{B}$. 
Since the $\Omega$-prespectra over $B$ are the $s$-fibrant prespectra over $B$ and since $s$-cofibrant spectra are well-grounded and have total spaces of the homotopy types of $G$-CW complexes, all $G$-prespectra over $B$ that are $s$-cofibrant and $s$-fibrant are in $G \mathscr{Q}_{B}$. We prove that $\operatorname{Ho} G \mathscr{P}_{B}$ is equivalent to $h G \mathscr{E}_{B}$ by proving that these categories are both equivalent to $\mathrm{Ho} G \mathscr{Q}_{B}$.

THEOREM 13.5.4. The canonical s-cofibrant and s-fibrant approximation functor $R Q$ and the composite approximation functor $T=K E P$, together with the forgetful functors, induce the following equivalences of homotopy categories.

$$
H o G \mathscr{P}_{B} \stackrel{R Q}{\gtrless} \underset{I}{\gtrless} H o G \mathscr{Q}_{B} \stackrel{T}{\gtrless} h \mathscr{E}_{J}
$$

Proof. For $X$ in $G \mathscr{P}_{B}$, we have a natural zig-zag of $s$-equivalences in $G \mathscr{P}_{B}$

$$
X<Q X \longrightarrow R Q X \text {. }
$$

Therefore $X$ and $I R Q X$ are naturally $s$-equivalent in $G \mathscr{P}_{B}$. If $X$ is in $G \mathscr{Q}_{B}$, then it is $s$-fibrant and therefore so is $Q X$. Then the above zig-zag is in $G \mathscr{Q}_{B}$, hence $X$ and $R Q I X$ are naturally $s$-equivalent in $G \mathscr{Q}_{B}$.

By Theorem 12.3.8, s-equivalences in $G \mathscr{Q}_{B}$ are level $q$-equivalences, and $T$ takes level $q$-equivalences to homotopy equivalences by Theorem 13.5.1. Conversely, since homotopy equivalences are $s$-equivalences, the forgetful functor $J$ induces a functor in the other direction.

For $X$ in $G \mathscr{Q}_{B}$ we have the natural zig-zag of $s$-equivalences displayed in (13.5.2). Applying $s$-fibrant approximation, we get a natural zig-zag of $s$-equivalences in $G \mathscr{Q}_{B}$ so $X$ and $J T X$ are naturally $s$-equivalent in $G \mathscr{Q}_{B}$. Starting with $X$ in $G \mathscr{E}_{B}$, the last statement of Theorem 13.5.1 shows that $X$ and $T J X$ are naturally homotopy equivalent in $G \mathscr{E}_{B}$.

\subsection{Derived functors on homotopy categories}

With $P$ replaced by $T$, the discussion of derived functors in $\S 9.2$ carries over from the level of ex-spaces to the level of parametrized prespectra indexed on cofinal sequences. In $\S 13.7$ and $\S 14.2$ we will discuss how to pass from there to conclusions on the level of parametrized spectra indexed on our usual collections of representations closed under direct sums. We must show that if $V$ is a Quillen left or right adjoint, then its model theoretic left or right derived functor agrees under our equivalences of categories with the functor obtained simply by passing to homotopy classes of maps from the composite $T V$. As on the ex-space level, we need some mild good behavior for this to work.

Definition 13.6.1. A functor $V: G \mathscr{P}_{A} \longrightarrow G \mathscr{P}_{B}$ is good if it is continuous, preserves well-grounded parametrized prespectra, and takes prespectra over $A$ whose levelwise total spaces are of the homotopy types of $G$-CW complexes to prespectra over $B$ with that property. Since $V$ is continuous, it preserves homotopies. There are evident variants for functors $V$ with source or target $G \mathscr{K}_{*}: V$ must be continuous, preserve well-grounded objects, and preserve $G$-CW homotopy type conditions on objects.

Note that a good functor $V$ need not take $\Omega$ - $G$-prespectra to $\Omega$ - $G$-prespectra and recall that a Quillen right adjoint must preserve fibrant objects and thus, in our context, must preserve $\Omega-G$-prespectra. 
Proposition 13.6.2. Let $V: G \mathscr{P}_{A} \longrightarrow G \mathscr{P}_{B}$ be a good functor that is a part of a Quillen adjoint pair. If $V$ is a Quillen left adjoint, assume further that it preserves level q-equivalences between well-grounded objects. Then the derived functor $H_{o} G \mathscr{P}_{A} \longrightarrow H_{o} G \mathscr{P}_{B}$, induced by $V Q$ or $V R$, is equivalent to the functor $T V J: h G \mathscr{E}_{A} \longrightarrow h G \mathscr{E}_{B}$ under the equivalence of categories in Theorem 13.5.4

Proof. If $V$ is a Quillen right adjoint, then it preserves $s$-equivalences between $s$-fibrant objects. If $V$ is a Quillen left adjoint, then it preserves $s$-equivalences between well-grounded objects by Proposition 12.6.3. Therefore, since $G \mathscr{Q}_{A}$ consists of well-sectioned $s$-fibrant objects, the functor $V: G \mathscr{Q}_{A} \longrightarrow G \mathscr{P}_{B}$ passes straight to homotopy categories to give $V: \operatorname{Ho} G \mathscr{Q}_{A} \longrightarrow \operatorname{HoG} \mathscr{P}_{B}$ in both cases.

If $V$ is a Quillen right adjoint, then it takes an $s$-equivalence $f$ in $G \mathscr{Q}_{A}$ to an $s$-equivalence since the objects of $G \mathscr{Q}_{A}$ are $s$-fibrant. Then $V f$ is a level $q$ equivalence by Theorem 12.3 .8 and, since $V$ is good, it is a level $h$-equivalence. On the other hand, if $V$ is a Quillen left adjoint, then Theorem 12.3 .8 gives that $f$ is a level $q$-equivalence and, by assumption, $V f$ is then a level $q$-equivalence. Since $V$ is good, $V f$ is actually a level $h$-equivalence. In both cases it follows that $V$ takes $s$-equivalences to level $h$-equivalences and therefore $T V$ passes to a functor Ho $G \mathscr{Q}_{A} \longrightarrow h G \mathscr{E}_{B}$.

To show that $T V J$ and either $V Q$ or $V R$ agree under the equivalence of categories, it suffices to verify that the following diagram commutes.

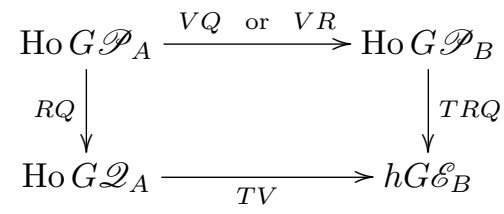

We have functorial $s$-cofibrant and $s$-fibrant approximation functors $Q$ and $R$, with natural acyclic $s$-fibrations $Q X \longrightarrow X$ and acyclic $s$-cofibrations $X \longrightarrow R X$. Clearly $Q$ and $R$ preserve $s$-equivalences. If $V$ is a Quillen left adjoint, then we have a zig-zag of natural $s$-equivalences

$$
R Q V Q \longrightarrow R V Q \longleftarrow V Q \longrightarrow V R Q
$$

because $V$ preserves acyclic $s$-cofibrations. If $V$ is a Quillen right adjoint, then we have a zig-zag of natural $s$-equivalences

$$
R Q V R \prec R Q V R Q \longrightarrow R V R Q \prec V R Q
$$

because $V$ preserves $s$-equivalences between $s$-fibrant objects. In both cases, all objects have total spaces of the homotopy types of $G$-CW complexes, hence we have zig-zags of level $h$-equivalences. Applying $T$, we obtain a zig-zag of homotopy equivalences in $G \mathscr{E}_{B}$ by Theorem 13.5.1.

REMARK 13.6.3. If $V$ preserves excellent parametrized prespectra, then $T V$ is naturally homotopy equivalent to $V$ on excellent parametrized prespectra. The derived functor of $V$ can then be obtained directly by applying $V$ and passing to homotopy classes of maps.

\subsection{Compatibility relations for smash products and base change}

This section is parallel to $\S 9.3$. The main change is just that we must replace the functor $P$ used there with the functor $T=K E P$ that we have here. This 
gives us results for the categories $G \mathscr{P}_{B}^{\mathscr{W}}$ of parametrized prespectra indexed on a collection $\mathscr{W}$ consisting of a cofinal sequence in some universe $U$. In order to obtain statements about $G \mathscr{S}_{B}^{\mathscr{V}}$, where $\mathscr{V}=\mathscr{V}(U)$, we have two pairs of Quillen equivalences, both of which can be viewed as consisting of a prolongation functor left adjoint to a forgetful functor that creates the weak equivalences; see [105, 1.2].

$$
G \mathscr{P}_{B}^{\mathscr{W}} \underset{j^{*}}{\stackrel{j_{*}}{\rightleftarrows}} G \mathscr{P}_{B}^{\mathscr{V}} \underset{\mathbb{U}}{\stackrel{\mathbb{P}}{\rightleftarrows}} G \mathscr{S}_{B}^{\mathscr{V}}
$$

We postpone until $\S 14.2$ consideration of the pair $\left(j_{*}, j^{*}\right)$ and the extension from $G \mathscr{P}_{B}^{\mathscr{W}}$ to $G \mathscr{P}_{B}^{\mathscr{V}}$ and deal with the extension from $G \mathscr{P}_{B}^{\mathcal{V}}$ to $G \mathscr{S}_{B}^{\mathscr{V}}$ in this section.

One general remark is in order, though. The forgetful functors $j^{*}$ and $\mathbb{U}$ create weak equivalences and therefore pass directly to homotopy categories. If they commute on the point set level with a functor $V$ which is defined and part of a Quillen adjoint pair (on each of our three categories), then they will also commute with its derived functor on the level of homotopy categories. It follows formally that the derived prolongation functors $\mathbb{P}$ and $j_{*}$ then also commute with the derived functor $V$ and its adjoints. This holds in particular for the base change functor $V=f^{*}$. Extending commutation results for such functors from $G \mathscr{P}_{B}^{\mathscr{W}}$ to $G \mathscr{S}_{B}^{\mathscr{V}}$ is therefore easy. However, some of the functors $V$ that we need to consider only exist on some of the categories in the above display, and such functors require special care. These include the change of universe functors that we discuss in $\S 14.2$, which don't exist on the level of $G \mathscr{P}_{B}^{\mathscr{W}}$, and the smash product $\wedge_{B}$, which we have only specified on the spectrum level and which we now discuss on the prespectrum level.

REMARK 13.7.1. Because the domain category for the diagram category of (equivariant and parametrized) prespectra is only monoidal, not symmetric monoidal, we cannot use left Kan extension to internalize "external" smash products of prespectra; see [106, 4.1]. Here "external" is understood in the sense of indexing on pairs of representations. Therefore, on the equivariant parametrized prespectrum level, when we write $X \bar{\wedge} Y$ for prespectra $X$ over $A$ and $Y$ over $B$, we should understand the external external smash product, in the sense of Remark 11.1.7. When passing from prespectrum level arguments to spectrum level conclusions using $(\mathbb{P}, \mathbb{U})$, we are implicitly using composites of the general form $\mathbb{P} V \mathbb{U}$, and similarly for functors of several variables involving smash products. We can carry out the several variable arguments externally on the prespectrum level, only internalizing with left Kan extension after passage to spectra, where we have good homotopical control by Corollary 12.6.6.

Alternatively, we can make use of classical "handicrafted smash products" of prespectra, which are defined by use of arbitrary choices of sequences of representations. As discussed on the nonequivariant nonparametrized level in [106, §11], handicrafted smash products of prespectra agree under the adjoint equivalence $(\mathbb{P}, \mathbb{U})$ with the internalized smash products. Provided that we use external parametrized handicrafted smash products over varying base spaces, only internalizing along diagonal maps at the end, the discussion there adapts readily to give the same conclusion for homotopy categories of equivariant parametrized prespectra and spectra. The advantage of handicrafted smash products is that their definition involves only direct use of ex-space level constructions that enjoy good preservation properties with respect to ex-fibrations. This often allows direct transposition of ex-space level arguments in $h G \mathscr{W}_{B}$ to parametrized prespectrum level arguments in $h G \mathscr{E}_{B}$. 
We state the following results in terms of parametrized spectra, and we indicate which parts of the proofs require the use of $h G \mathscr{E}_{B}$ and which parts work directly in the stable homotopy category $\operatorname{HoG} \mathscr{S}_{B}$.

Proposition 13.7.2. Let $f: A \longrightarrow B$ and $g: A^{\prime} \longrightarrow B^{\prime}$ be $G$-maps. If $W$ and $X$ are spectra over $A$ and $A^{\prime}$, then

$$
f_{!} W \wedge g_{!} X \simeq(f \times g) !(W \bar{\wedge} X)
$$

in $H o G \mathscr{S}_{B \times B^{\prime}}$. If $Y$ and $Z$ are spectra over $B$ and $B^{\prime}$, then

$$
f^{*} Y \bar{\wedge} g^{*} Z \simeq(f \times g)^{*}(Y \bar{\wedge} Z)
$$

in $H o G \mathscr{S}_{A \times A^{\prime}}$.

Proof. Working directly in $\mathrm{Ho}_{G} \mathscr{S}_{B \times B^{\prime}}$, the first equivalence reduces to its point-set level analogue by consideration of Quillen left adjoints, as in the corresponding proof of Proposition 9.4.1. We work in $h G \mathscr{E}_{A \times A^{\prime}}$ to prove the second equivalence. Here $f^{*}$ and $\bar{\wedge}$ (understood in the external or handicrafted sense) are both good, and both preserve excellent prespectra. Indeed, they preserve wellstructured prespectra by levelwise application of Propositions 8.2.2 and 8.2.3, they preserve $\Sigma$-cofibrant prespectra since $f^{*}$ and $\bar{\wedge}$ on ex-spaces preserve $f p$-cofibrations because they are left adjoints that commute with $f p$-homotopies, and they preserve $\Omega$-prespectra by Lemma 13.2.3 since they preserve $f p$-homotopies. Therefore, using excellent prespectra, we can pass straight to homotopy categories, without use of $T$, as in the corresponding proof of Proposition 9.4.1.

TheOREM 13.7.3. The category Ho $\operatorname{HS}_{B}$ is closed symmetric monoidal under the functors $\wedge_{B}$ and $F_{B}$.

Proof. Working either in $h G \mathscr{E}_{B}$ or in $\mathrm{Ho}_{G} \mathscr{S}_{B}$, one easily proves the external analogues of the required associativity, commutativity, and unity isomorphisms, noting for the argument in $h G \mathscr{E}_{B}$ that the previous proof shows that there is no need to use the functor $T$. The internal isomorphisms for $\wedge_{B}$ follow by pullback along diagonal maps, using the second equivalence in the previous result in the same way as in the proof of Theorem 9.4.4.

We have a commutation relation between change of base and suspension spectrum functors that is analogous to the relation between change of base and smash products recorded in Proposition 13.7.2.

Proposition 13.7.4. For a $G$-map $f: A \longrightarrow B$, there are natural equivalences

$$
\Sigma_{B}^{\infty} f_{!} \simeq f_{!} \Sigma_{A}^{\infty} \text { and } \Sigma_{A}^{\infty} f^{*} \simeq f^{*} \Sigma_{B}^{\infty}
$$

of (derived) functors. The same conclusion holds more generally for the shift desuspension functors $F_{V}=\Sigma_{V}^{\infty}$.

Proof. Working in Ho $G \mathscr{S}_{B}$, the first equivalence is clear since it is a comparison of Quillen left adjoints that commute on the point-set level. For the second equivalence, we start in $h G \mathscr{W}_{B}$ and end in $h G \mathscr{E}_{A}$. For $K \in G \mathscr{W}_{B}$, the point set level suspension prespectrum $\Sigma_{B}^{\infty} K$ is both $\Sigma$-cofibrant and well-structured, by Corollary 8.2 .5 , but of course it is not an $\Omega$-prespectrum over $B$. Since $\Sigma_{B}^{\infty}$ is good and takes well-grounded $q$-equivalences to well-grounded level $q$-equivalences, $T \Sigma_{B}^{\infty}$ is equivalent to the model theoretic left derived functor of the Quillen left adjoint $\Sigma_{B}^{\infty}$. Here we may omit $P$ from the composite functor $T$ and, since $f^{*}$ commutes with both $K$ and $E$, the conclusion follows on passage to homotopy categories. 
Applying this to $\Delta: B \longrightarrow B \times B$ and using Proposition 11.4.11, we obtain the following consequence.

Proposition 13.7.5. For ex-spaces $K$ and $L$ over $B$,

$$
\Sigma_{B}^{\infty}\left(K \wedge_{B} L\right) \simeq \Sigma_{B}^{\infty} K \wedge_{B} \Sigma_{B}^{\infty} L
$$

in $\mathrm{Ho} G \mathscr{S}_{B}$.

For $f: A \longrightarrow B$, evident properties of the functor $f_{!}$on ex-spaces imply that

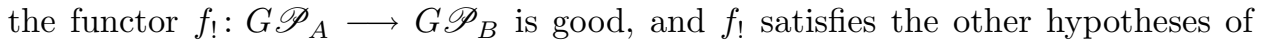
Proposition 13.6.2 by Proposition 12.2.5. We use this to prove the following basic result.

TheOrem 13.7.6. For a $G$-map $f: A \longrightarrow B$ between base spaces, the derived functor $f^{*}: H_{o} G \mathscr{S}_{B} \longrightarrow$ HoG $\mathscr{S}_{A}$ is closed symmetric monoidal.

Proof. Since $S_{B}$ is not $s$-fibrant, the isomorphism $f^{*} S_{B} \cong S_{A}$ in $G \mathscr{S}_{B}$ does not immediately imply the required equivalence $f^{*} S_{B} \simeq S_{A}$ in HoG $\mathscr{S}_{A}$, where $f^{*} S_{B}$ means $f^{*} R S_{B}$. However, Proposition 13.7.4 s pecializes to give this equivalence. For the rest, we must show that the isomorphisms (11.4.2) through (11.4.6) descend to equivalences on homotopy categories. By category theory in [62], it suffices to consider (11.4.2) and (11.4.5), and the proofs are similar to those in Theorem 9.4.5. Since $\bar{\wedge}$ and $\Delta^{*}$ both preserve excellent prespectra, so do the internalized smash products $\wedge_{A}$ and $\wedge_{B}$. For excellent prespectra $Y$ and $Z$ over $B$, it follows that both sides of

$$
f^{*}\left(Y \wedge_{B} Z\right) \cong f^{*} Y \wedge_{A} f^{*} Z
$$

are excellent prespectra over $A$, hence the point-set level isomorphism descends directly to the desired equivalence on the homotopy category level. Next consider

$$
f_{!}\left(f^{*} Y \wedge_{A} X\right) \cong Y \wedge_{B} f_{!} X
$$

where $X$ is an excellent prespectrum over $A$. Here we must replace $f_{\text {! }}$ by $T f_{\text {! }}$ on both sides. By Theorem 13.5.1 we have a natural zig-zag $\phi$ of level $h$-equivalences connecting $T$ to the identity functor which, when applied to excellent parametrized prespecra gives rise to a zig-zag $\psi$ of actual homotopy equivalences. We obtain the following zig-zag.

$$
T f_{!}\left(f^{*} Y \wedge_{A} X\right) \cong T\left(Y \wedge_{B} f_{!} X\right) \stackrel{T\left(\mathrm{id} \wedge_{B} \phi\right)}{\longrightarrow} T\left(Y \wedge_{B} T f_{!} X\right) \stackrel{\psi}{\longrightarrow} Y \wedge_{B} T f_{!} X .
$$

Using handicrafted products with their termwise construction in terms of smash products of ex-spaces, it follows from Proposition 8.2.6 that id $\wedge_{B}-$ preserves level $h$-equivalences between well-sectioned spectra. Thus id $\wedge_{B} \phi$ is a zig-zag of level $h$-equivalences and $T\left(\mathrm{id} \wedge_{B} \phi\right)$ is a zig-zag of actual homotopy equivalences.

THEOREM 13.7.7. Suppose given a pullback diagram of $G$-spaces

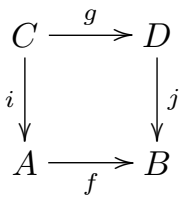


in which $f$ (or $j$ ) is a q-fibration. Then there are natural equivalences of (derived) functors on stable homotopy categories

$$
j^{*} f_{!} \simeq g_{!} i^{*}, \quad f^{*} j_{*} \simeq i_{*} g^{*}, \quad f^{*} j_{!} \simeq i_{!} g^{*}, \quad j^{*} f_{*} \simeq g_{*} i^{*} .
$$

Proof. Working in $h G \mathscr{E}_{B}$, the proof is similar to that of Theorem 9.4.6 but with $P$ replaced by $T$. Again it suffices to consider the first equivalence, and, as explained there, since $f$ is a $q$-fibration there is a level $f p$-equivalence $\mu: P f^{*} \longrightarrow$ $f^{*} P$. Since $f^{*}$ commutes with both $K$ and $E$, we obtain a level $f p$-equivalence $\mu: T f^{*} \longrightarrow f^{*} T$ between $\Sigma$-cofibrant prespectra over $A$ so it is in fact a homotopy equivalence by Proposition 13.2.5. Then $f^{*} T j_{!} X \simeq T f^{*} j_{!} X \cong T i_{!} g^{*} X$.

The following observation holds by the same proof as the analogous ex-space level result Proposition 9.4.9.

Proposition 13.7.9. Let $\iota: H \longrightarrow G$ be the inclusion of a subgroup and $A$ be an $H$-space. The closed symmetric monoidal Quillen equivalence $\left(\iota_{1}, \nu^{*} \iota^{*}\right)$ descends to a closed symmetric monoidal equivalence between $H o H \mathscr{S}_{A}$ and $H o G \mathscr{S}_{L ! A}$.

Combined with Theorem 13.7.6, applied to the inclusion $\tilde{b}: G / G_{b} \longrightarrow B$, and Proposition 13.7.4, this last observation gives us the following stable analogue of Theorem 9.4.10.

TheOREM 13.7.10. The derived fiber functor $(-)_{b}: H_{o} G \mathscr{K}_{B} \longrightarrow H_{o} G_{b} \mathscr{K}_{*}$ is closed symmetric monoidal and it has both a left adjoint $(-)^{b}$ and a right adjoint ${ }^{b}(-)$. Moreover, the derived fiber functor commutes with the derived suspension spectrum functor, $\left(\Sigma_{B}^{\infty} K\right)_{b} \simeq \Sigma^{\infty}\left(K_{b}\right)$ as $G_{b}$-spectra.

For emphasis, we repeat a remark that we made after the analogous ex-space level result. This innocent looking result packages highly non-trivial and important information. In particular, it gives that $F_{B}(X, Y)_{b} \simeq F\left(X_{b}, Y_{b}\right)$ in Ho $G_{b} \mathscr{S}$ for $X, Y \in \operatorname{Ho} G \mathscr{S}_{B}$, where the fiber and function object functors are understood in the derived sense. This reassuring consistency result is central to our applications in Part IV, where parametrized duality is studied fiberwise. 



\section{CHAPTER 14}

\section{Module categories, change of universe, and change of groups}

\section{Introduction}

We first give a discussion of module categories of parametrized spectra over nonparametrized ring spectra. One motivation is to set up the homotopical foundations for studying the special properties of generalized homology and cohomology theories on parametrized spectra that are represented by such nonparametrized ring spectra. The good behavior of the external smash product $G \mathscr{S} \times G \mathscr{S}_{B} \longrightarrow G \mathscr{S}_{B}$ makes it easy to do this. While the mathematics here is evident, it deserves emphasis since the ideas are likely to have future applications.

In the rest of the chapter, we focus on problems that are special to the equivariant context. We give the parametrized generalization of some of the work in [105] concerning change of universe, change of groups, and fixed point and orbit spectra. As usual, an essential point is to determine which of the standard adjunctions are given by Quillen adjoint pairs and to prove that other adjunctions and compatibilities that are evident on the point set level also descend to homotopy categories.

We discuss change of universe in $\S 14.2$. Here the use of prespectra indexed on cofinal sequences in the previous chapter introduces some minor difficulties that were not studied in the nonparametrized theory of $[105, \mathrm{~V} \S 1]$ and are already relevant nonequivariantly. We study subgroups and fixed point spectra in $\S 14.3$. We study quotient groups and orbit spectra in $§ 14.4$. Aside from some analogues for parametrized spectra of earlier results for parametrized spaces, these sections are precisely parallel to [105, V§§2 and 3]. We have not written down the parametrized analogue of [105, V§4], which gives the theory of geometric fixed point spectra, since it would be tedious to repeat the constructions given there. It will be apparent to the interested reader that, mutatis mutandis, the definitions and results in $[105, \mathrm{~V} \S 4]$ generalize directly to the parametrized context.

\subsection{Parametrized module $G$-spectra}

We can define a parametrized (strict) ring $G$-spectrum $R$ over $B$ to be a monoid in the symmetric monoidal category $G \mathscr{S}_{B}$, and we can then define parametrized $R$-modules and $R$-algebras in the usual way, as has become standard in stable homotopy theory $[61,77,105,106]$. However, even though the smash product $\wedge_{B}$ in $G \mathscr{S}_{B}$ gives a point-set level symmetric monoidal structure, we cannot expect to obtain Quillen model structures on the categories of such $R$-modules or $R$-algebras, as was done for orthogonal $G$-spectra in $[105, \operatorname{III} \S \S 7,8]$. To do that, we would need better homotopical behavior than we can prove here. We have only set up adequate foundations for the classical style theory of up to homotopy parametrized module 
spectra over up to homotopy parametrized ring spectra. From that point of view, our homotopical foundations are entirely satisfactory. The source of the problem is Warning 6.1.7, which implies that $X \wedge_{B}(-)$ in $G \mathscr{S}_{B}$ cannot be a Quillen functor.

However, in applications, it is natural to start with a nonparametrized orthogonal ring $G$-spectrum $R$. We are then interested in understanding the $R$-homology and $R$-cohomology theories of $G$-spectra over $B$ and their relationships with the $R$-homology and $R$-cohomology of the fibers. For this study, just as in the nonparametrized work of $[61,77,105,106]$, one is interested in the theory of $R$-modules. The external smash product $\bar{\wedge}: G \mathscr{S} \times G \mathscr{S}_{B} \longrightarrow G \mathscr{S}_{B}$ has enough of the good properties of the nonparametrized smash product $G \mathscr{S} \times G \mathscr{S} \longrightarrow G \mathscr{S}$ to give us homotopical control over parametrized module spectra over nonparametrized ring spectra. We devote this section to developing the relevant theory, which is parallel to $[105, \mathrm{III} \S 7]$. Let $R$ be a ring spectrum in $G \mathscr{S}$ which is well-grounded when viewed as a spectrum, meaning that each $R(V)$ is well-based and compactly generated.

Definition 14.1.1. A (left) $R$-module over $B$ is a $G$-spectrum $M$ over $B$ together with a left action $R \bar{\wedge} M \longrightarrow M$ satisfying the usual associativity and unit conditions. The category $G R \mathscr{M}_{B}$ of left $R$-modules over $B$ consists of the $G$-spectra $M$ over $B$ and the maps of $G$-spectra over $B$ that preserve the action by $R$.

Since $(R \bar{\wedge} X)_{b}=R \wedge X_{b}$, a parametrized $R$-module over $B$ is precisely that: each $X_{b}$ is an $R$-module $G_{b}$-spectrum. More formally, we have the $G$-category $\left(R \mathscr{M}_{G, B}, G R \mathscr{M}_{B}\right)$, as discussed in $\S 1.4$ and $\S 10.2$, and the following result is clear.

Proposition 14.1.2. The G-category $\left(R \mathscr{M}_{G, B}, G R \mathscr{M}_{B}\right)$ is $G$-topologically bicomplete in the sense of Definition 10.2.1. All of the required limits, colimits, tensors, and cotensors are constructed in the underlying $G$-category $\left(\mathscr{S}_{G, B}, G \mathscr{S}_{B}\right)$ and then given induced $R$-module structures in the evident way. A cyl-cofibration of $R$-modules is a cyl-cofibration of underlying $G$-spectra over $B$.

The last statement holds by the retract of mapping cylinders characterization of cyl-cofibrations. This immediately implies that $G R \mathscr{M}_{B}$ inherits a ground structure from $G \mathscr{S}_{B}$, in the sense of Definition 5.3.2. Recall that the well-grounded $G$-spectra over $B$ are those that are level well-grounded (well-sectioned and compactly generated) and that the $g$-cofibrations of $G$-spectra over $B$ are the level $h$-cofibrations; see Definition 12.1.2 and Proposition 12.1.4.

Definition 14.1.3. An $R$-module over $B$ is well-grounded if its underlying $G$ spectrum over $B$ is well-grounded. A map of $R$-modules over $B$ is a $g$-cofibration, level $q$-equivalence, or $s$-equivalence if its underlying map of $G$-spectra over $B$ is such a map.

Also recall the notion of a subcategory of well-grounded weak equivalences from Definition 5.4.1. Since colimits and tensors for $R$-modules are defined in terms of the underlying $G$-spectra over $B$, the following theorem is immediate from its counterpart for $G$-spectra over $B$, which is given by Proposition 12.1 .4 and Theorem 12.4.3.

THEOREM 14.1.4. Definition 14.1.3 specifies a ground structure on $G R \mathscr{M}_{B}$ such that the level q-equivalences and the $s$-equivalences both give subcategories of well-grounded weak equivalences. 
Finally, recall the definition of a well-grounded model structure from Definition 5.5.4. Such model structures are compactly generated, and we must define the generators of $G R \mathscr{M}_{B}$. The free $R$-module functor $\mathbb{F}_{R}=R \bar{\wedge}-: G \mathscr{S}_{B} \longrightarrow G R \mathscr{M}_{B}$ is left adjoint to the forgetful functor $\mathbb{U}: G R \mathscr{M}_{B} \longrightarrow G \mathscr{S}_{B}$. Adjunction arguments from the definitions show that $\mathbb{F}_{R}$ preserves cyl-cofibrations and $\overline{c y l}$-cofibrations.

Definition 14.1.5. Define $\mathbb{F}_{R} F I_{B}^{f}, \mathbb{F}_{R} F J_{B}^{f}$ and $\mathbb{F}_{R} F K_{B}^{f}$ by applying the free $R$-module functor to the maps in the sets specified in Definition 12.1.6 and Definition 12.5.5. A map of $R$-modules over $B$ is

(i) a level $q f$-fibration or an $s$-fibration if it is one in $G \mathscr{S}_{B}$,

(ii) an $s$-cofibration if it satisfies the LLP with respect to the level acyclic $q f$ fibrations,

THEOREM 14.1.6. The category $G R \mathscr{M}_{B}$ is a well-grounded model category with respect to the level q-equivalences, the level $q f$-fibrations, and the $s$-cofibrations. The sets $\mathbb{F}_{R} F I_{B}^{f}$ and $\mathbb{F}_{R} F J_{B}^{f}$ give the generating s-cofibrations and generating level acyclic s-cofibrations. All s-cofibrations of R-modules over $B$ are s-cofibrations of $G$-spectra over $B$.

We omit the proof since it is virtually the same as the proof of the following theorem, which gives the starting point for serious work on the homology and cohomology theory of parametrized $G$-spectra.

THEOREM 14.1.7. The category GR $\mathscr{M}_{B}$ is a well-grounded model category with respect to the s-equivalences, the s-fibrations, and the s-cofibrations; $\mathbb{F}_{R} F K_{B}^{f}$ gives the generating acyclic s-cofibrations.

Proof. The compatibility condition in Theorem 5.5.1 is automatic by adjunction from the parametrized spectrum level, and we have already observed that the free $R$-module functor $\mathbb{F}_{R}$ preserves $\overline{c y l}$-cofibrations. It also preserves the relevant $\square$-products, and $\mathbb{F}_{R} F_{V} K=\left(R \wedge F_{V} S^{0}\right) \bar{\wedge} K$ is well-grounded if $K$ is a well-grounded ex-space. Only the acyclicity condition remains. If $R$ is $s$-cofibrant as a ring spectrum, then $R$ is also $s$-cofibrant as a spectrum, by [105, III.7.6(iv) and (v)]. In that case, the functor $R \bar{\wedge}(-)=\mathbb{U F}_{R}$ is a Quillen left adjoint by Corollary 12.6.6 and therefore preserves level acyclic $s$-cofibrations. It follows that the maps in $\mathbb{F}_{R} K_{B}^{f}$ are $s$-equivalences. The case of a general well-grounded $R$ reduces to the cofibrant case by use of the next result; compare Proposition 14.1.9 below.

Proposition 14.1.8. The following statements hold.

(i) For an s-cofibrant spectrum $X$ over $B$, the functor $-\bar{\wedge} X: G \mathscr{S} \longrightarrow G \mathscr{S}_{B}$ preserves s-equivalences between well-grounded spectra in $G \mathscr{S}$.

(ii) If $Y$ is well-grounded in $G \mathscr{S}, j: A \longrightarrow X$ is an acyclic s-cofibration in $G \mathscr{S}_{B}$, and $A$ is well-grounded, then $Y \bar{\wedge}: Y \bar{\wedge} A \longrightarrow Y \bar{\wedge} X$ is an s-equivalence.

Proof. Let $\phi: Y \longrightarrow Z$ be an $s$-equivalence between well-grounded spectra. By parts (ii)-(iv) of Definition 5.4.1, it suffices to show that $\phi \bar{\wedge} F_{V} K$ is an $s$ equivalence if $K$ is the source or target of a map in $I_{B}^{f}$. This map is isomorphic to the map $\left(\phi \wedge F_{V} S^{0}\right) \wedge_{B} K$, where $F_{V} S^{0}$ is the shift desuspension in $G \mathscr{S}$, not $G \mathscr{S}_{B}$. Here $\phi \wedge F_{V} S^{0}$ is an $s$-equivalence by the nonparametrized analogue [105, III.7.3], and the conclusion follows from Lemma 12.5.3. (The comment on the notations $\bar{\wedge}$ and $\wedge_{B}$ above Definition 12.5.1 is relevant: the former is an external smash product and the latter is a tensor). 
For (ii), we apply an argument from [106, 12.5]. We let $Z=X / B A$, which is $s$-cofibrant, and we let $Q Y \longrightarrow Y$ be an $s$-cofibrant approximation. Since $j$ is an $s$-cofibration, it is a cyl-cofibration and $C j$ is homotopy equivalent to $Z$. Since $A$ is well-grounded, we can apply the long exact sequence of homotopy groups of Theorem 12.4.2 to conclude that $Z$ is $s$-acyclic. The map $Z \longrightarrow *_{B}$ is then an $s$-equivalence between $s$-cofibrant spectra over $B$. Since $Q Y \bar{\wedge}-$ is a Quillen left adjoint, by Proposition 12.2.3, $Q Y \bar{\wedge} Z \longrightarrow Q Y \bar{\wedge} *_{B} \cong *_{B}$ is an $s$-equivalence. Since $Q Y \bar{\wedge} \longrightarrow Y \bar{\wedge} Z$ is an $s$-equivalence by part (i), $Y \bar{\wedge} Z$ is $s$-acyclic. Since the functor $Y \bar{\wedge}$ - preserves cofiber sequences, another application of Theorem 12.4.2 shows that $Y \bar{\wedge} j$ is an $s$-equivalence.

Proposition 14.1.9. If $\phi: Q \longrightarrow R$ is an s-equivalence of well-grounded ring spectra, then the functors

$$
\phi_{*}=R \wedge_{Q}(-): G Q \mathscr{M}_{B} \longrightarrow G R \mathscr{M}_{B} \text { and } \phi^{*}: G R \mathscr{M}_{B} \longrightarrow G Q \mathscr{M}_{B}
$$

given by extension of scalars and restriction of action define a Quillen equivalence $\left(\phi_{*}, \phi^{*}\right)$ between the categories of $Q$-modules and of $R$-modules over $B$.

Proof. Since $s$-fibrations and $s$-equivalences are created in the underlying category of spectra over $B$, it is clear that they are preserved by $\phi^{*}$, so that we have a Quillen pair. If $M$ is an $s$-cofibrant $Q$-module, then, by the previous result, the unit map $\phi \wedge$ id: $M \cong Q \wedge_{Q} M \longrightarrow \phi^{*}\left(R \wedge_{Q} M\right)$ of the adjunction is an $s$ equivalence of spectra over $B$. Therefore, if $N$ is an $s$-fibrant $R$-module, then a map $M \longrightarrow \phi^{*} N$ of $Q$-modules is an $s$-equivalence if and only if its adjoint map $R \wedge_{Q} M \longrightarrow N$ of $R$-modules is an $s$-equivalence.

Implicitly, we have been studying modules over the sphere spectrum $S$ in earlier chapters, and we can redo all of our model theoretic work with $S$ replaced by $R$. The results of $\S 12.6$ and $\S 13.1$ carry over directly. For $f: A \longrightarrow B$, base change preserves $R$-modules, $\left(f_{!}, f^{*}\right)$ gives a Quillen adjoint pair relating the categories of $R$-modules over $A$ and over $B$, and we obtain a Quillen equivalence if $f$ is a $q$ equivalence. If $f$ is a bundle with cellular fibres, we obtain a Quillen pair $\left(f^{*}, f_{*}\right)$, and we can apply the triangulated category version of Brown representability to construct a right adjoint $f_{*}$ in general.

However, we do not know how to generalize the rest of Chapter 13 to the module context since we have not worked out a theory of excellent $R$-modules with an accompanying excellent $R$-module approximation functor. In view of the retreat to prespectra with their primitive handicrafted smash products in that theory, it seems unlikely to us that any such construction can be expected.

We also have the notion of a right $R$-module over a nonparametrized ring spectrum $R$. If $M$ and $N$ are right and left $R$-modules over $A$ and $B$ and $L$ is a left $R$-module over $A \times B$, then we define spectra $M \bar{\wedge}_{R} N$ over $A \times B$ and $\bar{F}_{R}(N, L)$ over $A$ by the usual coequalizer

$$
M \bar{\wedge} R \bar{\wedge} \Longrightarrow M \bar{\wedge} N \longrightarrow M \bar{\wedge}_{R} N
$$

and equalizer

$$
\bar{F}_{R}(N, L) \longrightarrow \bar{F}(N, L) \Longrightarrow \bar{F}(R \bar{\wedge} N, L) .
$$

If $R$ is commmutative, then $M \wedge_{R} N$ and $F_{R}(N, L)$ are naturally $R$-modules.

We have good homotopical control over these external constructions, as in Propositions 12.2.3 and 12.6.5. For example, if we take $A=*$, then we have good 
homotopical control over the smash product spectrum $M \wedge_{R} N$ over $B$ and the non-parametrized function spectrum $F_{R}(N, L)$, where $M$ is a non-parametrized right $R$-module and $N$ and $L$ are left $R$-modules over $B$. However, if we take $A=B$ and internalize $M \bar{\wedge}_{R} N$ along the diagonal $\Delta: B \longrightarrow B \times B$ by setting $M \wedge_{R} N=\Delta^{*} M \bar{\wedge}_{R} N$ and $F_{R}(M, N)=\bar{F}_{R}\left(M, \Delta_{*} N\right)$, we lose homotopical control.

Similarly, when $R$ is commutative, $R \mathscr{M}_{B}$ has the structure of a closed symmetric monoidal category, and that allows us to define (commutative) $R$-algebras over $B$ to be (commutative) monoids in $R \mathscr{M}_{B}$. However, because of the lack of homotopical control, in the absence of the theory of Chapter 13, we cannot give the categories of $R$-algebras and of commutative $R$-algebras over $B$ model structures.

REMARK 14.1.10. There are interesting examples of rings and modules that allow varying base spaces and are defined in terms of the external smash product. We shall work out one rather surprising way of realizing this idea in Chapter 23, but it seems likely that there are others. For example, one might consider $G$ spectra $R_{n}$ over $B^{n}$ with products $R_{m} \bar{\wedge} R_{n} \longrightarrow R_{m+n}$, or one might consider "globally defined" parametrized ring spectra $R$ consisting of spectra $R_{B}$ over $B$ for all $B$ together with appropriate products $R_{A} \bar{\wedge} R_{B} \longrightarrow R_{A \times B}$. The $R_{B}$ would in particular be module spectra over the nonparametrized ring spectrum $R_{*}$. As in the nonparametrized theory, one must use the positive stable model structures to study such ring objects model theoretically when $R_{*}$ is commutative. The essential point is that the external smash product is sufficiently well-behaved homotopically that there is no obstacle to passage from point-set level constructions to homotopy category level conclusions.

\subsection{Change of universe}

Recall that $G$-spectra over $B$ are defined in terms of a chosen collection $\mathscr{V}$ of representations of $G$. As usual in equivariant stable homotopy theory, we must introduce functors that allow us to change the collection $\mathscr{V}$. Such functors are usually referred to as "change of universe" functors, since $\mathscr{V}$ is often given as the collection $\mathscr{V}(U)$ of all representations that embed up to isomorphism in a given $G$-universe $U$. However, it is often convenient to restrict $\mathscr{V}$ to be a cofinal subcollection of $\mathscr{V}(U)$ that is closed under direct sums, and when we dealt with excellent prespectra it became essential to restrict $\mathscr{V}$ further to a countable cofinal sequence of expanding representations in $U$. In both cases, it is usual to insist that the trivial representation $\mathbb{R}$ be included in $\mathscr{V}$. Observe that such change of universe functors are already essential nonequivariantly. In order to deal with the change functors in all of the above cases at once, we adopt a slightly different approach from the one that was used in $[105, \mathrm{~V} . \S 1]$. We then explain how it specializes to the more explicit approach given there.

Let $G \mathscr{S}_{B}^{\mathscr{V}}$ denote the category of $G$-spectra over $B$ indexed on $\mathscr{V}$. If $\mathscr{V}$ is not closed under direct sums, then we are thinking of $G \mathscr{S}_{B}^{\mathscr{V}}$ as the restriction of the diagram category corresponding to $G \mathscr{S}_{B}^{\mathscr{V}^{\prime}}$, where $\mathscr{V}^{\prime}$ is the closure of $\mathscr{V}$ under sums, as discussed in Remark 13.3.5.

Let $i: \mathscr{V} \subset \mathscr{V}^{\prime}$ be the inclusion of one collection of representations in another. Thinking of parametrized spectra as diagram ex-spaces, we see that the evident forgetful functor

$$
i^{*}: G \mathscr{S}^{\mathscr{V}^{\prime}} \longrightarrow G \mathscr{S}^{\mathscr{V}}
$$


has a left adjoint $i_{*}$ given by the prolongation, or expansion of universe, functor

$$
\left(i_{*} X\right)\left(V^{\prime}\right)=\mathscr{J}_{G}^{\mathscr{V}^{\prime}}\left(-, V^{\prime}\right) \otimes_{\mathscr{J}_{G}^{\mathscr{V}}} X .
$$

Such prolongation functors are discussed in detail in [106, I§3] and [105, I§2]. By [105, I.2.4], the unit Id $\longrightarrow i^{*} i_{*}$ of the adjunction is a natural isomorphism.

We have more concrete descriptions of the functor $i_{*}$ when $\mathscr{V}$ consists of a cofinal sequence of representations in some universe $U$. Recall that $\mathscr{J}_{G}^{\mathscr{V}}(V, V)$ is the orthogonal group $O(V)$ with a disjoint base point.

LEMMA 14.2.1. If $\mathscr{V}=\left\{V_{i}\right\} \subset \mathscr{V}^{\prime}$ is a countable expanding sequence in some $G$-universe $U$, then

$$
\left(i_{*} X\right)\left(V^{\prime}\right) \cong \mathscr{J}_{G}^{\mathscr{V}^{\prime}}\left(V_{i}, V^{\prime}\right) \wedge_{O\left(V_{i}\right)} X\left(V_{i}\right)
$$

where $i$ is the largest natural number such that there is a linear isometry $V_{i} \longrightarrow V^{\prime}$.

Proof. The forgetful functor $i^{*}$ is restriction along a functor $\iota: \mathscr{J}_{G}^{\mathscr{V}} \longrightarrow \mathscr{J}_{G}^{\mathscr{V}^{\prime}}$ and $\left(i_{*} X\right)\left(V^{\prime}\right)$ is constructed as the coequalizer of the pair of parallel maps

$$
\bigvee_{j, k} \mathscr{J}_{G}^{\mathscr{V}^{\prime}}\left(V_{j}, V^{\prime}\right) \wedge_{B} \mathscr{J}_{G}^{\mathscr{V}}\left(V_{k}, V_{j}\right) \wedge_{B} X\left(V_{k}\right) \Longrightarrow \bigvee_{j} \mathscr{J}_{G}^{\mathscr{V}^{\prime}}\left(V_{j}, V^{\prime}\right) \wedge_{B} X\left(V_{j}\right)
$$

given by composition in $\mathscr{J}_{G}^{\mathscr{V}^{\prime}}$ and by the evaluation maps associated to the diagram $X$. A cofinality argument that is easily made precise by use of the explicit description of the category $\mathscr{J}_{G}^{\mathscr{V}^{\prime}}$ given in $[105$, II. $\S 4]$ shows that the above coequalizer agrees with the coequalizer of the subdiagram

$$
\mathscr{J}_{G}^{\mathscr{V}^{\prime}}\left(V_{i}, V^{\prime}\right) \wedge_{B} \mathscr{J}_{G}^{\mathscr{V}}\left(V_{i}, V_{i}\right) \wedge_{B} X\left(V_{i}\right) \Longrightarrow \mathscr{J}_{G}^{\mathscr{V}^{\prime}}\left(V_{i}, V^{\prime}\right) \wedge_{B} X\left(V_{i}\right)
$$

This coequalizer is the space that we have denoted by $\mathscr{J}_{G}^{\mathscr{V}^{\prime}}\left(V_{i}, V^{\prime}\right) \wedge_{O\left(V_{i}\right)} X\left(V_{i}\right)$.

REMARK 14.2.2. The argument above works in the same way for prespectra. It gives the conclusion that, for parametrized prespectra $X$ in $G \mathscr{P}_{B}^{\mathscr{V}}$,

$$
\left(i_{*} X\right)(V) \cong \Sigma_{B}^{V-V_{i}} X\left(V_{i}\right)
$$

REMARK 14.2.3. Assume that $\mathscr{V}$ and $\mathscr{V}^{\prime}$ are closed under finite sums and contain the trivial representation. We can then define the change of universe functors

$$
I_{\mathscr{V}^{\prime}}^{\mathscr{V}}=i_{*} i^{* *}: G \mathscr{S}_{B}^{\mathscr{V}^{\prime}} \longrightarrow G \mathscr{S}_{B}^{\mathscr{V}}
$$

where $i:\left\{\mathbb{R}^{n}\right\} \subset \mathscr{V}$ and $i^{\prime}:\left\{\mathbb{R}^{n}\right\} \subset \mathscr{V}^{\prime}$. Explicitly

$$
\left(I_{\mathscr{V}}^{\mathscr{V}} X\right)(V) \cong \mathscr{J}_{G}^{\mathscr{V}}\left(\mathbb{R}^{n}, V\right) \wedge_{O(n)} X\left(\mathbb{R}^{n}\right) .
$$

This is the definition given in [105, V.1.2]. These change of universe functors $I_{\mathscr{V}}^{\mathscr{V}}$ are exceptionally well behaved on the point set level, and they agree with those that we are using when $\mathscr{V} \subset \mathscr{V}^{\prime}$. They are symmetric monoidal equivalences of categories. For collections $\mathscr{V}, \mathscr{V}^{\prime}$ and $\mathscr{V}^{\prime \prime}$, they satisfy

$$
I_{\mathscr{V}^{\prime}}^{\mathscr{V}} \circ \Sigma_{B}^{\mathscr{V}^{\prime}} \cong \Sigma_{B}^{\mathscr{V}}, \quad I_{\mathscr{V}^{\prime}}^{\mathscr{V}} \circ I_{\mathscr{V}^{\prime \prime}}^{\mathscr{V}^{\prime}} \cong I_{\mathscr{V}^{\prime \prime}}^{\mathscr{V}}, \quad I_{\mathscr{V}}^{\mathscr{V}} \cong \mathrm{Id} .
$$

Moreover, $I_{\mathscr{V}}^{\mathscr{V}}$ is continuous and commutes with smash products with ex-spaces. In particular, it is homotopy preserving and therefore induces equivalences of the classical homotopy categories. Unfortunately, however, the functors $I_{\mathscr{V}}^{\mathscr{V}}$ are as poorly behaved on the homotopy level as they are well behaved on the point set level. They do not preserve either level $q$-equivalences or $s$-equivalences in general, and the point set level relations above do not descend to the model theoretic homotopy 
categories that we are interested in. Furthermore, these functors $I_{\mathscr{V}}^{\mathscr{V}}$, do not exist if $\mathscr{V}$ is a cofinal expanding sequence. We shall therefore not make much use of them.

Returning to our original context, let $i: \mathscr{V} \subset \mathscr{V}^{\prime}$. The adjoint pair $\left(i_{*}, i^{*}\right)$ has good homotopical properties.

TheOREM 14.2.4. Let $i: \mathscr{V} \subset \mathscr{V}^{\prime}$. Then $i^{*}$ preserves level q-equivalences, level $q$ f-fibrations, s-fibrations, and s-acyclic s-fibrations. Therefore $\left(i_{*}, i^{*}\right)$ is a Quillen adjoint pair in the level $q f$-model structure and in the s-model structure. Moreover, $i_{*}$ on homotopy categories is symmetric monoidal. If $\mathscr{V}$ is cofinal in $\mathscr{V}^{\prime}$, then $i^{*}$ creates the weak equivalences and $\left(i_{*}, i^{*}\right)$ is a Quillen equivalence.

Proof. It is clear from its levelwise definition that $i^{*}$ preserves level $q$-equivalences and level $q f$-fibrations. It follows that its left adjoint $i_{*}$ preserves $s$ cofibrations and level acyclic $s$-cofibrations. This in turn implies that $i^{*}$ preserves $s$-acyclic $s$-fibrations, since those are the maps that satisfy the RLP with respect to the $s$-cofibrations. The levelwise description of $s$-fibrations in Proposition 12.5.6 implies that $i^{*}$ preserves $s$-fibrations. The last statement follows from the definition of homotopy groups and the fact that the unit id $\longrightarrow i^{*} i_{*}$ is an isomorphism. The functor $i_{*}$ commutes with $\bar{\wedge}$ on the point set level, by [105, I.2.14], and this commutation relation descends directly to homotopy categories. Applying Proposition 14.2.8 below to the diagonal map of $B$, it follows that the derived functor $i_{*}$ is symmetric monoidal.

Many results now carry over verbatim from the case of $G$-spectra. For example, $i^{*} \Sigma_{, B \mathscr{V}^{\prime}}^{\infty}$ is isomorphic to $\Sigma_{B, \mathscr{V}}^{\infty}$ on the poinst set level, and this isomorphism descends to homotopy categories. We shall concentrate in the rest of the section on properties special to the parametrized setting. We have constructed the change of universe functors on both the spectrum and prespectrum level and they are compatible with the restriction functors $\mathbb{U}$. However, in order to make use of excellent parametrized prespectra, we must restrict to parametrized prespectra indexed on cofinal sequences $j: \mathscr{W} \subset \mathscr{V}$ and $j^{\prime}: \mathscr{W}^{\prime} \subset \mathscr{V}^{\prime}$ of indexing representations in the given universes $U \subset U^{\prime}$. But then there need not be an induced inclusion $i: \mathscr{W} \subset \mathscr{W}^{\prime}$. We therefore also define change of universe functors for prespectra indexed on cofinal sequences.

Definition 14.2.5. Let $i: \mathscr{V} \subset \mathscr{V}^{\prime}$ and choose cofinal sequences $\mathscr{W}=\left\{V_{i}\right\}$ and $\mathscr{W}^{\prime}=\left\{V_{i}^{\prime}\right\}$ in $\mathscr{V}$ and $\mathscr{V}^{\prime}$ such that $V_{i+1}=V_{i} \oplus W_{i}$ and $V_{i}^{\prime}=V_{i} \oplus Z_{i}$, where $Z_{i+1}=Z_{i} \oplus W_{i}^{\prime}$ and thus $V_{i+1}^{\prime}=V_{i}^{\prime} \oplus W_{i} \oplus W_{i}^{\prime}$. Define a pair of adjoint functors

$$
G \mathscr{P}_{B}^{\mathscr{W}} \underset{\bar{\imath}^{*}}{\stackrel{\bar{\imath}_{*}}{\rightleftarrows}} G \mathscr{P}_{B}^{\mathscr{W}^{\prime}}
$$

by setting

$$
\left(\bar{\imath}_{*} X\right)\left(V_{i}^{\prime}\right)=\Sigma_{B}^{Z_{i}} X\left(V_{i}\right) \text { and }\left(\bar{\imath}^{*} Y\right)\left(V_{i}\right)=\Omega_{B}^{Z_{i}} Y\left(V_{i}^{\prime}\right)
$$

The structure maps are induced from the given structure maps in the evident way.

Proposition 14.2.6. The pair $\left(\bar{\imath}_{*}, \bar{\imath}^{*}\right)$ is a Quillen adjoint pair with respect to both the level qf-model structure and the stable model structure. The following 
diagram commutes when the vertical arrows point in the same direction.

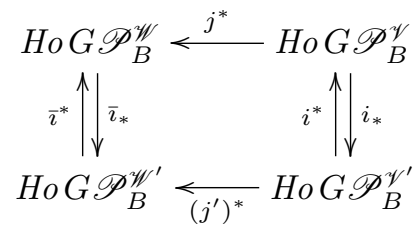

Proof. This is clearly a Quillen adjunction in the level $q f$-model structure, and to see that it is a Quillen adjunction in the stable model structure it therefore suffices to observe that the homotopy pullback condition of Proposition 12.5.6 is preserved by the right adjoint $\bar{\imath}^{*}$. This holds since the homotopy pullbacks (12.5.7) associated to the pairs $\left(V_{i}, W_{i}\right)$ and an $s$-fibration $f: X \longrightarrow Y$ are still homotopy pullbacks after we apply $\Omega_{B}^{Z_{i}}$ to them. The resulting diagrams display the required homotopy pullback diagrams (12.5.7) for the map $\bar{\imath}^{*} f$.

For the diagram with downwards pointing vertical arrows, we have

$$
\left(\bar{\imath}_{*} j^{*} X\right)\left(V_{i}^{\prime}\right)=\Sigma_{B}^{Z_{i}} X\left(V_{i}\right) \cong \Sigma_{B}^{V_{i}^{\prime}-V_{i}} X\left(V_{i}\right)=\left(\left(j^{\prime}\right)^{*} i_{*} X\right)\left(V_{i}^{\prime}\right) .
$$

This point set level isomorphism descends to homotopy categories since the functors $j^{*}$ and $\left(j^{\prime}\right)^{*}$ preserve all $s$-equivalences. For the diagram with upwards pointing arrows, the adjoint structure maps of $X \in G \mathscr{P}_{B}^{\mathscr{V}^{\prime}}$ induce maps

$$
\left(j^{*} i^{*} X\right)\left(V_{i}\right)=X\left(V_{i}\right) \longrightarrow \Omega_{B}^{Z_{i}} X\left(V_{i}^{\prime}\right)=\left(\bar{\imath}^{*}\left(j^{\prime}\right)^{*} X\right)\left(V_{i}\right) .
$$

When $X$ is $s$-fibrant, its adjoint structure maps are level $q$-equivalences, and we thus obtain an equivalence $j^{*} i^{*} \simeq \bar{\imath}^{*}\left(j^{\prime}\right)^{*}$ on homotopy categories.

On the point-set level, we have the following commutation relations between change of universe functors and change of base functors.

Lemma 14.2.7. Let $i: \mathscr{V} \subset \mathscr{V}^{\prime}$ and let $f: A \longrightarrow B$ be a $G$-map. Then $i^{*}$ commutes up to natural isomorphism with the change of base functors $f_{!}, f^{*}$, and $f_{*}$, and $i_{*}$ commutes up to natural isomorphism with $f_{!}$and $f_{*}$.

Proof. The first statement is clear from the levelwise constructions of the base change functors, and the second statement follows by conjugation since $i_{*}, f_{!}$, and $f^{*}$ are left adjoints of $i^{*}, f^{*}$, and $f_{*}$.

The missing relation, $i_{*} f^{*} \cong f^{*} i_{*}$, would hold with the alternative point-set level definitions of Remark 14.2.3, where $i^{*}$ and $i_{*}$ are inverse equivalences. However, these are point-set level relationships that need not descend to model theoretic homotopy categories. With our preferred definition of $i_{*}$ in terms of prolongation, the following result shows that $i_{*} f^{*} \simeq f^{*} i_{*}$ on homotopy categories even though we need not have an isomorphism on the point-set level.

Proposition 14.2.8. Let $i: \mathscr{V} \subset \mathscr{V}^{\prime}$ and let $f: A \longrightarrow B$ be a $G$-map. Then there are natural equivalences of derived functors

$$
i^{*} f^{*} \simeq f^{*} i^{*}, \quad i_{*} f_{!} \simeq f_{!} i_{*}, \quad i_{*} f^{*} \simeq f^{*} i_{*}, \quad i^{*} f_{*} \simeq f_{*} i^{*}, \quad i^{*} f_{!} \simeq f_{!} i^{*}
$$

in the relevant homotopy categories. 
Proof. The first two equivalences are clear since we are commuting Quillen right adjoints and their corresponding Quillen left adjoints. The fourth will follow by adjunction from the third. If $f$ is a homotopy equivalence, then $f^{*} \simeq\left(f_{!}\right)^{-1}$ and in this case the third follows from the second and the fifth from the first. Factoring $f$ as the composite of an $h$-fibration and a homotopy equivalence, we see that the third will hold in general if it holds when $f$ is an $h$-fibration. Similarly, factoring $f$ as the composite of an $h$-cofibration and a homotopy equivalence, we see that the fifth will hold in general if it holds when $f$ is an $h$-cofibration.

Further, for the third equivalence, it suffices to show that $\bar{\imath}_{*} f^{*} \simeq f^{*} \bar{\imath}_{*}$ since Proposition 14.2.6 then gives that

$$
i_{*} f^{*} \simeq i_{*} j_{*} j^{*} f^{*} \simeq\left(j^{\prime}\right)_{*} \bar{\imath}_{*} f^{*} j^{*} \simeq\left(j^{\prime}\right)_{*} f^{*} \bar{\imath}^{*} j^{*} \simeq f^{*}\left(j^{\prime}\right)_{*}\left(j^{\prime}\right)^{*} i^{*} \simeq f^{*} i^{*} .
$$

Similarly, for the fifth equivalence, it suffices to show that $\bar{\imath}^{*} f_{!} \simeq f_{!} \imath^{*}$, for then

$$
i^{*} f_{!} \simeq i^{*}\left(j^{\prime}\right)_{*}\left(j^{\prime}\right)^{*} f_{!} \simeq j_{*} \bar{\imath}^{*} f_{!}\left(j^{\prime}\right)^{*} \simeq j_{*} f_{!} \imath^{*}\left(j^{\prime}\right)^{*} \simeq f_{!}\left(j^{\prime}\right)_{*}\left(j^{\prime}\right)^{*} i^{*} \simeq f_{!} i^{*} .
$$

We have reduced the proof of the third equivalence to the situation when $f$ is an $h$-fibration and $i_{*}$ is replaced by $\bar{\imath}_{*}$. The functor $f^{*}$ preserves excellent prespectra over $B$, but we must apply $T$ to $\bar{\imath}_{*}$ before passing to homotopy categories. As in the proof of Theorem 13.7.7, since $f$ is assumed to be an $h$-fibration we have a natural homotopy equivalence $\mu: T f^{*} \longrightarrow f^{*} T$ in our categories indexed on $\mathscr{W}$ or on $\mathscr{W}^{\prime}$. Therefore

$$
T \bar{\imath}_{*} f^{*} \cong T f^{*} \bar{\imath}_{*} \simeq f^{*} T \bar{\imath}_{*} .
$$

Similarly, we have reduced the proof of the fifth equivalence to the situation when $f$ is an $h$-cofibration and $i^{*}$ is replaced by $i^{*}$. Then $f$ ! preserves level $h$ equivalences, and so does $\bar{\imath}^{*}$ since it preserves level $q$-equivalences and preserves objects whose total spaces are of the homotopy types of $G$-CW complexes. Since $T$ takes zig-zags of level $h$-equivalences to homotopy equivalences,

$$
T f_{!} T \bar{\imath}^{*} \stackrel{\simeq}{\longrightarrow} T f_{!} \bar{\imath}^{*} \cong T \bar{\imath}^{*} f_{!} \stackrel{\simeq}{\longrightarrow} T \bar{\imath}^{*} T f_{!}
$$

displays a zig-zag of homotopy equivalences showing that $f_{!} \bar{\imath}^{*} \simeq \bar{\imath}^{*} f_{!}$.

\subsection{Restriction to subgroups}

Let $\theta: G^{\prime} \longrightarrow G$ be a homomorphism and let $\theta^{* \mathscr{V}}$ be the collection of $G^{\prime}$ representations $\theta^{*} V$ for $V \in \mathscr{V}$, where $\mathscr{V}$ is our chosen collection of indexing $G$ representations. We have implicitly used the following result in our earlier results on change of groups.

Proposition 14.3.1. The functor $\theta^{*}: G \mathscr{S}_{B} \longrightarrow G^{\prime} \mathscr{S}_{\theta^{*} B}^{\theta^{*} \mathcal{V}}$ preserves level $q$ equivalences, level $q f$-fibrations, s-fibrations, and s-equivalences provided that the model structures are defined with respect to generating sets $\mathscr{C}_{G}$ and $\mathscr{C}_{G^{\prime}}$ of $G$-cell complexes and $G^{\prime}$-cell complexes such that $\theta_{!} C=G \times_{G^{\prime}} C \in \mathscr{C}_{G}$ for $C \in \mathscr{C}_{G^{\prime}}$.

Proof. Since $\left(\theta^{*} A\right)^{H}=A^{\theta^{*}(H)}$ for a $G$-space $A$ and a subgroup $H$ of $G^{\prime}$, this is clear from the definitions of homotopy groups and from the characterizations of fibrations given in Definition 7.2.7 and Proposition 12.5.6. Note in particular that $\theta^{*}$ preserves the level $q f$-fibrant approximations that are used in the definition of the stable homotopy groups. 
For the remainder of this section fix a subgroup $H$ of $G$ and consider the inclusion $\iota: H \subset G$. For an $H$-space $A$, we simplify notation by letting $H \mathscr{S}_{A}^{\mathscr{V}}$ denote the category of $H$-spectra over $A$ indexed on $\iota^{*} \mathscr{V}$. Clearly, we then have the restriction of action functor

$$
\iota^{*}: G \mathscr{S}_{B}^{\mathscr{V}} \longrightarrow H \mathscr{S}_{\iota^{*} B}^{\mathscr{V}} \text {. }
$$

For $i: \mathscr{V} \subset \mathscr{V}^{\prime}$, we have $\iota^{*} i^{*}=i^{*} \iota^{*}$ since with either composite we are just restricting from the representations in $\mathscr{V}^{\prime}$ to the representations in $\mathscr{V}$ and viewing all $G$-spaces in sight as $H$-spaces.

When $\mathscr{V}=\mathscr{V}(U)$ for a $G$-universe $U$, there is a quibble here (as was discussed in $[105, \mathrm{~V} .10])$. We are using $\iota^{*} \mathscr{V}$ as the corresponding indexing collection for $H$. However, if $V$ is an irreducible representation of $G, \iota^{*} V$ is generally not an irreducible representation of $H$ and we should expand $\iota^{*} \mathscr{V}$ to include all representations that embed up to isomorphism in $\iota^{*} U$ to fit the definitions into our usual framework. However, there is a change of universe functor associated to the inclusion $i: \iota^{*} \mathscr{V}(U) \subset \mathscr{V}\left(\iota^{*} U\right)$ that fixes this. The functor $i^{*}$ preserves all $s$-equivalences and descends to an equivalence on homotopy categories. We can and should use these rectifications when restricting to $H$-spectra over $\iota^{*} B$ for a fixed chosen $H$.

Remark 14.3.2. Consider passage to fibers and recall Proposition 12.6.12.

(i) Applied to inclusions of orbits, Proposition 14.2.8 implies that the functors $i^{*}$ for $i: \mathscr{V} \subset \mathscr{V}^{\prime}$ are compatible with passage to fibers, in the sense that

$$
\left(i^{*} X\right)_{b} \cong i^{*}\left(X_{b}\right) \text { for } b \in B,
$$

where $i^{*}$ on the right is the change of universe functor on $G_{b}$-spectra.

(ii) When $\mathscr{V}=\mathscr{V}(U)$, we can view the fiber functor

$$
(-)_{b}: G \mathscr{S}_{B} \longrightarrow G_{b} \mathscr{S}
$$

as landing in spectra indexed on $\mathscr{V}\left(\iota^{*} U\right), \iota: G_{b} \longrightarrow G$, by composing with $i_{*}$ for $i: \iota^{*} \mathscr{V}(U) \subset \mathscr{V}\left(\iota^{*} U\right)$. However, these change of universe functors must be used with caution since they are not compatible as $b$ and therefore $G_{b}$ vary.

Recall from Propositions 12.6.10 and 13.7.9 that the equivalence of categories $\left(\iota !, \nu^{*} \iota^{*}\right)$ between $H \mathscr{S}_{A}$ and $G \mathscr{S}_{\iota ! A}$ induces a closed symmetric monoidal equivalence of categories between $\operatorname{HoH} \mathscr{S}_{A}$ and $\operatorname{HoG} \mathscr{S}_{\iota_{1} A}$. By Corollary 12.6.11, we can interpret the restriction functor $\iota^{*}: \operatorname{HoG} \mathscr{S}_{B} \longrightarrow \mathrm{HoH} \mathscr{S}_{\iota^{*} B}$ as the composite of base change $\mu^{*}$ along $\mu: \iota ! \iota^{*} B \longrightarrow B$ and this equivalence applied to $A=\iota^{*} B$. The following spectrum level analogue of Proposition 2.3.11 gives compatibility relations between change of base functors and these results on change of groups.

Proposition 14.3.3. Let $f: A \longrightarrow \iota^{*} B$ be a map of $H$-spaces and $\tilde{f}: \iota ! A \longrightarrow B$ be its adjoint map of $G$-spaces. Then the following diagrams commute up to natural isomorphism, where $\mu: \iota ! \iota^{*} B \longrightarrow B$ and $\nu: A \longrightarrow \iota^{*} \iota ! A$ are the counit and unit of the adjunction $\left(\iota !, \iota^{*}\right)$.
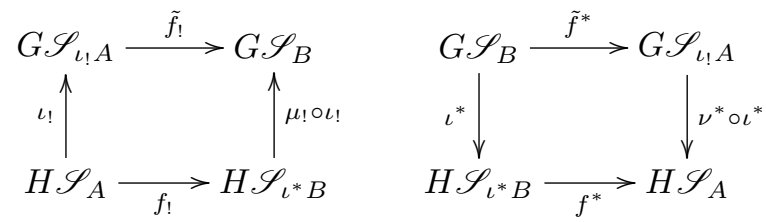
These diagrams descend to natural equivalences of composites of derived functors on homotopy categories.

Proof. The point set level diagrams commute by Proposition 2.3.11, applied levelwise. The left diagram is one of Quillen left adjoints and the right diagram is one of Quillen right adjoints, by Propositions 12.6.7 and 12.6.10 and Corollary 12.6.11.

We now define a parametrized fixed point functor associated to the inclusion $\iota: H \longrightarrow G$. Its target is a category of nonequivariant parametrized spectra. In the next section we will consider a fixed point functor that takes values in a category of parametrized $W H$-spectra, where $W H=N H / H$ is the Weyl group.

Write $G \mathscr{S}_{B}^{\text {triv }}$ for $G$-spectra over $B$ indexed on trivial representations. These are "naive" parametrized $G$-spectra. As usual, to define fixed point spectra, we must change to the trivial universe before taking fixed points levelwise. Thus let $\mathscr{V}^{G}=\left\{V^{G} \mid V \in \mathscr{V}\right\}$. It is contained in $\mathscr{V}$ if $\mathscr{V}=\mathscr{V}(U)$ for some universe $U$.

Definition 14.3.4. The $G$-fixed point functor $(-)^{G}: G \mathscr{S}_{B} \longrightarrow \mathscr{S}_{B^{G}}$ is the composite of $i^{*}, i: \mathscr{V}^{G} \subset \mathscr{V}$, and levelwise passage to fixed points. For a subgroup $H$ of $G$ the $H$-fixed point functor $(-)^{H}: G \mathscr{S}_{B} \longrightarrow \mathscr{S}_{B^{H}}$ is the composite of $\iota^{*}$, $\iota: H \subset G$, and $(-)^{H}$.

Since the homotopy groups of a level $q f$-fibrant $G$-spectrum $X$ over $B$ are the homotopy groups $\pi_{q}^{H}\left(X_{b}\right)$, we see from the nonparametrized analogue [105, V.3.2] that these are then the homotopy groups of $X^{H}$. Recall in particular that the $s$-fibrant $G$-spectra over $B$ are the $\Omega$ - $G$-spectra over $B$, which are level $q f$-fibrant. Therefore, for all subgroups $H$ of $G$, the homotopy groups of a parametrized $G$ spectrum $X$ are the nonequivariant homotopy groups of the nonequivariant spectra $X^{H}$, provided that $(-)^{H}$ is understood to mean the derived fixed point functor.

On the point-set level, the functor $(-)^{G}$ is a right adjoint. Thinking of the homomorphism $\varepsilon: G \longrightarrow e$ to the trivial group, let $\varepsilon^{*}: \mathscr{S}_{A} \longrightarrow G \mathscr{S}_{\varepsilon^{*} A}^{\text {triv }}$ be the functor that sends spectra over a space $A$ to $G$-trivial $G$-spectra over $A$ regarded as a $G$-trivial $G$-space. The following result is immediate by passage to fibers from its nonparametrized special case [105, V.3.4]. Let $\mathscr{A} \ell \ell$ denote the collection of all representations of $G$.

Proposition 14.3.5. Let $A$ be a space. Let $Y$ be a naive $G$-spectrum over $\varepsilon^{*} A$ and $X$ be a spectrum over $A$. There is a natural isomorphism

$$
G \mathscr{S}_{\varepsilon^{*} A}^{\text {triv }}\left(\varepsilon^{*} X, Y\right) \cong \mathscr{S}_{A}\left(X, Y^{G}\right) .
$$

For (genuine) $G$-spectra $Y$ over $\varepsilon^{*} A$, there is a natural isomorphism

$$
G \mathscr{S}_{\varepsilon^{*} A}\left(i_{*} \varepsilon^{*} X, Y\right) \cong \mathscr{S}_{A}\left(X,\left(i^{*} Y\right)^{G}\right),
$$

where $i:$ triv $\subset \mathscr{A l \ell}$. Both of these adjunctions are given by Quillen adjoint pairs relating the respective level and stable model structures.

Returning to $G$-spaces $B$ and comparing Definition 11.3.5 with the proof of [105, V.3.5-3.6], we obtain the following curious results.

Proposition 14.3.6. For a representation $V$ and an ex-G-space $K$, we have that $\left(F_{V} K\right)^{G}=*_{B^{G}}$ unless $G$ acts trivially on $V$, when $\left(F_{V} K\right)^{G} \cong F_{V}\left(K^{G}\right)$ as a nonequivariant spectrum over $B^{G}$. The functor $(-)^{G}$ preserves s-cofibrations, but it does not preserve acyclic s-cofibrations. 
Corollary 14.3.7. For ex-G-spaces $K$,

$$
\left(\Sigma_{B}^{\infty} K\right)^{G} \cong \Sigma_{B}^{\infty}\left(K^{G}\right)
$$

This isomorphism of spectra over $B^{G}$ does not descend to the homotopy category Ho $G \mathscr{S}_{B^{G}}$. The reader is warned to consult [105, V§3] for the meaning of these results. There is also an analogue of the comparison between $G$-fixed points and smash products in [105, V.3.8], but only when $B=B^{G}$ and only with good behavior with respect to cofibrant objects when external smash products are used. We shall not state the result formally.

\subsection{Normal subgroups and quotient groups}

We now turn to quotient homomorphisms and associated orbit and fixed point functors. The material of this section generalizes a number of results from $\S 2.4$, $\S 7.3$, and $\S 9.5$ to the level of parametrized spectra.

Just as we have been using $\iota$ generically for inclusions of subgroups, we shall use $\varepsilon$ generically for quotient homomorphisms. In particular, for an inclusion $\iota: H \subset G$, we let $W H=N H / H$, where $N H$ is the normalizer of $H$ in $G$, and we have the quotient homomorphism $\varepsilon: N H \longrightarrow W H$. We can study this situation by first restricting from $G$ to $N H$, thus changing the ambient group. Therefore, there is no loss of generality if we focus attention on a normal subgroup $N$ of $G$ with quotient group $J=G / N$, as we do throughout this section.

DEFINITION 14.4.1. Let $G \mathscr{S}_{B}^{N \text {-triv }}$ be the category of $G$-spectra over $B$ indexed on the $N$-trivial representations of $G$. Regard representations of $J$ as $N$-trivial representations of $G$ by pullback along $\varepsilon: G \longrightarrow J$. For a $J$-space $A$, define

$$
\varepsilon^{*}: J \mathscr{S}_{A} \longrightarrow G \mathscr{S}_{\varepsilon^{*} A}^{N \text {-triv }}
$$

levelwise by regarding ex- $J$-spaces over $A$ as $N$-trivial $G$-spaces over $\varepsilon^{*} A$. For a $G$-space $B$, define

$$
(-) / N: G \mathscr{S}_{B}^{N \text {-triv }} \longrightarrow J \mathscr{S}_{B / N} \quad \text { and } \quad(-)^{N}: G \mathscr{S}_{B}^{N \text {-triv }} \longrightarrow J \mathscr{S}_{B^{N}}
$$

by levelwise passage to orbits over $N$ and to $N$-fixed points.

LEMmA 14.4.2. The $N$-fixed point functor $(-)^{N}$ preserves level $q$-equivalences, level qf-fibrations, s-fibrations, and s-equivalences, provided that the model structures are defined with respect to generating sets $\mathscr{C}_{G}$ and $\mathscr{C}_{J}$ of $G$-cell complexes and $J$-cell complexes such that $C / N \in \mathscr{C}_{J}$ for $C \in \mathscr{C}_{G}$.

Proof. This is a special case of Proposition 14.3.1; it also follows directly from the ex-space level analogue in Proposition 7.4.3, the characterization of $s$-fibrations in Proposition 12.5.6, and inspection of the definition of the $s$-equivalences.

Proposition 14.4.3. Let $j: B^{N} \longrightarrow B$ be the inclusion and $p: B \longrightarrow B / N$ be the quotient map. Then the following factorization diagrams commute.
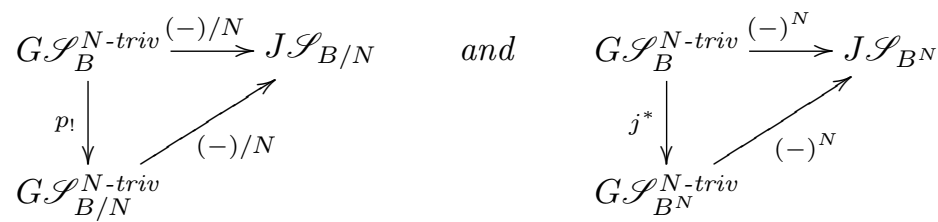
These diagrams descend to give natural equivalences on homotopy categories

$$
\left(p_{!} X\right) / N \simeq X / N \quad \text { and } \quad\left(j^{*} X\right)^{N} \simeq X^{N}
$$

for $X$ in $H o G \mathscr{S}_{B}^{N-t r i v}$. The following adjunction isomorphisms follow.

(i) For $Y \in G \mathscr{S}_{B}^{N-\text { triv }}$ and $X \in J \mathscr{S}_{B / N}$,

$$
J \mathscr{S}_{B / N}(Y / N, X) \cong G \mathscr{S}_{B}^{N-\text { triv }}\left(Y, p^{*} \varepsilon^{*} X\right) .
$$

(ii) For $Y \in G \mathscr{S}_{B}^{N-t r i v}$ and $X \in J \mathscr{S}_{B^{N}}$,

$$
G \mathscr{S}_{B}^{N-t r i v}\left(j ! \varepsilon^{*} X, Y\right) \cong J \mathscr{S}_{B^{N}}\left(X, Y^{N}\right) .
$$

(iii) For (genuine) $G$-spectra $Y \in G \mathscr{S}_{B}$ and $X \in J \mathscr{S}_{B^{N}}$,

$$
G \mathscr{S}_{B}\left(i_{*} j ! \varepsilon^{*} X, Y\right) \cong J \mathscr{S}_{B^{N}}\left(X,\left(i^{*} Y\right)^{N}\right),
$$

where $i:$ triv $\subset \mathscr{A l l}$.

All of these adjunctions are Quillen adjoint pairs with respect to both the level and the stable model structures and so descend to homotopy categories.

Proof. The factorizations follow from the ex-space level analogue, Proposition 2.4.1. The statement about Quillen adjunctions holds since $(-)^{N}, \epsilon^{*}$ and $i^{*}$ preserve level $q$-equivalences, level fibrations, $s$-equivalences, and level $s$-fibrations by Lemma 14.4.2, Proposition 14.3.1, and Theorem 14.2.4.

The following result records the behavior of the orbit and fixed point functors with respect to base change.

Proposition 14.4.4. Let $f: A \longrightarrow B$ be a map of $G$-spaces. Then the following diagrams commute up to natural isomorphism.

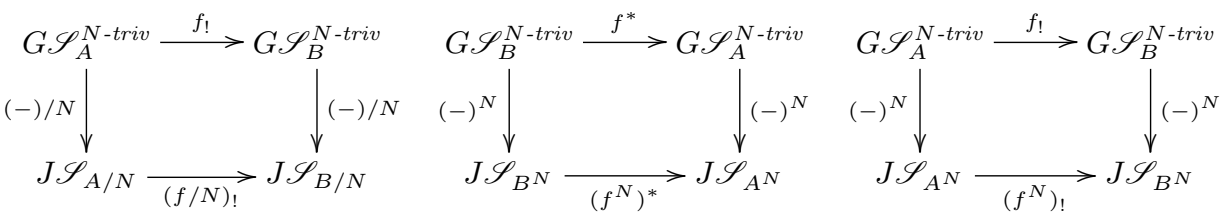

They descend to give the following natural equivalences on homotopy categories:

$$
\left(f_{!} X\right) / N \simeq(f / N)_{!}(X / N), \quad\left(f^{*} X\right)^{N} \simeq\left(f^{N}\right)^{*}\left(Y^{N}\right), \quad\left(f_{!} X\right)^{N} \simeq\left(f^{N}\right)_{!}(X / N),
$$

where $X \in H o G \mathscr{S}_{A}^{N \text {-triv }}$ and $Y \in H o G \mathscr{S}_{B}^{N-\text { triv }}$. If $B$ is an $N$-free $G$-space, then the following diagram also commutes up to natural isomorphism.

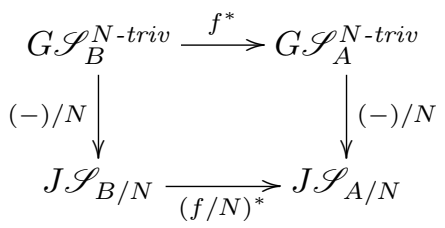

If $A$ and $B$ are both $N$-free, it descends to give a natural equivalence

$$
\left(f^{*} Y\right) / N \simeq(f / N)^{*}(Y / N)
$$

on homotopy categories. 
Proof. The point-set level commutative diagrams are obtained levelwise from the analogous diagrams for ex-spaces given in Proposition 2.4.3. The proof that these isomorphisms descend to equivalences on homotopy categories is the same as the proof of the analogous result for ex-spaces given in Proposition 7.4.5.

The spectrum level analogue of Propositions 2.4.4 and 7.4.6 also holds.

Proposition 14.4.5. Let $E$ be an $N$-free $G$-space, let $B=E / N$, and let $p: E \longrightarrow B$ be the quotient map. Then the diagram

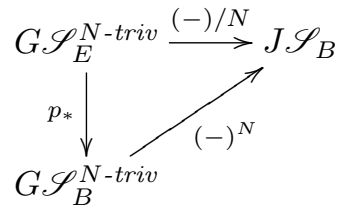

commutes up to a natural isomorphism. It descends to give a natural equivalence

$$
X / N \simeq\left(p_{*} X\right)^{N}
$$

in $\operatorname{HoJ}_{S_{B}}$ for $X \in G \mathscr{S}_{E}^{N-t r i v}$. Therefore the left adjoint $(-) / N$ of the functor $p^{*} \varepsilon^{*}$ is also its right adjoint.

Proof. The point-set level commutative diagram is obtained levelwise from the analogous diagram for ex-spaces given in Proposition 2.4.4, and this isomorphism descends to homotopy categories by the same proof as that of Proposition 7.4.6. 


\section{Part IV}

\section{Parametrized duality theory}





\section{Introduction}

We put the foundations of Part III to work in this part. Unless otherwise stated, we work in derived homotopy categories, and all functors should be understood in the derived sense. For example, we have the derived fiber functor

$$
(-)_{b}: \operatorname{Ho} \mathscr{S}_{B} \longrightarrow \mathrm{Ho}_{b} \mathscr{S} \text {. }
$$

Since passage to fibers is a Quillen right adjoint, this means that we replace $G$ spectra $X$ over $B$ by $s$-fibrant approximations before taking point-set level fibers. For emphasis, and to make the notation $X_{b}$ clear and unambiguous, we may sometimes assume explicitly that $X$ is $s$-fibrant, but this hypothesis is always implicit. A map $f$ in Ho $G \mathscr{S}_{B}$ is an equivalence if and only if $f_{b}$ is an equivalence for all $b \in B$, and that allows us to transfer information back and forth between the parametrized and nonparametrized homotopy categories with impunity. Here we use the word "equivalence" to mean an isomorphism in $\mathrm{Ho} \mathscr{S}_{B}$, and we use the notation $\simeq$ for this relation. We reserve the symbol $\cong$ to mean an isomorphism that already holds on the point set level.

We have proven that the basic structure enjoyed by the category $G \mathscr{S}_{B}$ of parametrized spectra descends coherently to the homotopy category Ho $G \mathscr{S}_{B}$. In particular, Ho $G \mathscr{S}_{B}$ is a closed symmetric monoidal category, and the derived fiber functor is closed symmetric monoidal. In any symmetric monoidal category, we have standard categorical notions of dualizable and invertible objects. Early work in parametrized stable homotopy theory of Clapp, Puppe, Becker, and Gottlieb $[9,10,31,32]$ was aimed at the understanding of fiberwise duality, viewed primarily as a tool for the study of transfer maps in the nonparametrized setting. We rework and generalize results of this sort in Chapter 15. A particularly interesting new construction explains precisely how to define bundles of spectra rigorously.

Classically, categorical duality in the symmetric monoidal category of spectra gives the definitively right context for the study of Spanier-Whitehead duality and duality between homology and cohomology theories. Categorical duality in symmetric monoidal categories of parametrized spectra is related fiberwise to this classical duality theory. However, this fiberwise duality theory is not the right context for the study of parametrized Spanier-Whitehead duality and duality between parametrized homology and cohomology theories.

The right duality theory was discovered by Costenoble and Waner [41], and we therefore call it Costenoble-Waner duality. To place it in context, we proceed from the general to the particular. We develop categorical duality theory in closed symmetric bicategories in Chapter 16. This chapter gives category theory that has applications to many other subjects, and it can be read independently of anything else in this book. 
We construct a closed symmetric bicategory of parametrized spectra with varying base spaces in Chapter 17, and we relate duality to base change using "base change spectra" in that bicategory. In Chapter 18, we focus on the category of $G$-spectra over a fixed $B$ and explain Costenoble-Waner duality. We reiterate how different this is from fiberwise duality. Nonequivariantly, the sphere spectrum over $B$, which is invertible and thus fiberwise dualizable, can only be Costenoble-Waner dualizable when $B$ is dualizable, that is, when $B$ is equivalent to a finite $\mathrm{CW}$ complex. Parametrized finite cell spectra are generally not fiberwise dualizable, but we show that they are Costenoble-Waner dualizable. We also give a self-contained proof of a parametrized version of Atiyah duality for smooth $G$-manifolds. This

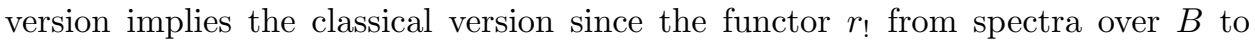
spectra preserves dualizability.

In Chapter 19, we show how to insert parametrized Costenoble-Waner duality fiberwise into bundles of equivariant spectra. The result gives what we think of as a fiberwise version of Costenoble-Waner duality. The proof of the result is of considerable conceptual interest. We generalize the symmetric bicategory of parametrized spectra to a symmetric bicategory of parametrized spectra over spaces over a given base space $B$. With appropriate equivariance, the bundle construction obtained from a fixed principal bundle $P \longrightarrow B$ maps the bicategory of parametrized spectra to this bicategory of parametrized spectra over spaces over $B$. The construction preserves dual pairs, and fiberwise Costenoble-Waner duality is obtained by starting from dual pairs in the bicategory of spectra, applying the bundle construction to obtain dual pairs of parametrized spectra over spaces over $B$, and then applying base change to obtain dual pairs of spectra over $B$. Hiding here is the use of a version of tricategories, but we shall not make that point of view explicit.

We emphasize that fiberwise Costenoble-Waner duality, like the earlier results in this chapter, is of considerable interest nonequivariantly. However, it can also be thought of as a fiberwise generalization of the Wirthmüller isomorphism in nonparametrized equivariant stable homotopy theory. In fact, parametrized Atiyah duality specializes directly to give a generalization of the Wirthmüller isomorphism from orbits to arbitrary $G$-manifolds, and the fiberwise version specializes directly to give the Adams isomorphism in equivariant stable homotopy theory. 


\section{CHAPTER 15}

\section{Fiberwise duality and transfer maps}

\section{Introduction}

In $\S 15.1$, we prove the fiberwise duality theorem, which says that a $G$-spectrum $X$ over $B$ is dualizable or invertible if and only if each fiber $X_{b}$ is dualizable or invertible. This allows us to recognize dualizable or invertible $G$-spectra over $B$ when we see them, and it gives a powerful tool for using parametrized stable homotopy theory for studying classical stable homotopy theory.

We recall the definition and properties of trace maps in symmetric monoidal categories in $\$ 15.2$, and in $\S 15.3$ we explain how the fiberwise duality theorem leads to a simple conceptual definition of transfer maps for Hurewicz fibrations. We regard a Hurewicz fibration $p: E \longrightarrow B$ with stably dualizable fibers as a space over $B$. We adjoin a copy of $B$ to obtain a section, and we suspend to obtain a $G$-spectrum over $B$. It is dualizable since its fibers are dualizable, hence it has a transfer map defined by categorical nonsense. Pushing down to $G$-spectra by base change along the map $r: B \longrightarrow *$, we obtain the transfer map of $G$-spectra $\Sigma^{\infty} B_{+} \longrightarrow \Sigma^{\infty} E_{+}$.

This definition of transfer maps is a direct generalization of various earlier ones $[9,10,28,31,170]$, most of which restrict to finite dimensional base spaces and are nonequivariant. The properties of the transfer now follow immediately from the general properties of trace maps in symmetric monoidal categories. An essential point is that the homotopy category of $G$-spectra over $B$ is closed symmetric monoidal with a "compatible triangulation", in the sense specified in [124] and recalled later; see $\S \S 16.6,16.7$, and 17.5. This point implies that our traces and transfers satisfy additivity relations as well as the more elementary standard properties.

Some of the classical constructions of the transfer work only for bundles, but have various properties that are inaccessible to the more general construction and are important in calculations. These transfers also admit a perhaps more satisfying construction. Rather than relying on duality on the level of parametrized spectra, they are obtained by inserting duality maps for fibers fiberwise into bundles. In the literature, the construction again usually requires finite dimensional base spaces and is nonequivariant. We give a general conceptual version of this alternative construction in $\S 15.6$.

As a first preliminary, in $\S 15.4$ we show how to insert parametrized spectra fiberwise into the standard construction of bundles associated to principal bundles. The construction on the ex-space level was studied in $\S 3.3$, and we show that the properties proven there remain true on the spectrum level. The construction is likely to have many further applications, and it is basic to twisted homology and cohomology theories. It is of considerable interest nonequivariantly, but we develop 
it the context of equivariant bundles with structure group $\Pi$ and ambient group $G$ related by an extension $1 \longrightarrow \Pi \longrightarrow \Gamma \longrightarrow G \longrightarrow 1$.

As a second preliminary, in $\S 15.5$ we develop the theory of $\Pi$-free parametrized $\Gamma$-spectra. This is a direct generalization of the nonparametrized theory and is important in many contexts. In particular, it will play a role in our proof of the Adams isomorphism in $§ 19.7$.

The application to transfer maps in $\S 15.6$ can be described as follows. It is a special case of a more general construction that uses the full strength of the bundle construction functor $\mathbb{P}_{F}$ of $\S 15.4$. For the special case, we take $F=*$ and use $\mathbb{P}=\mathbb{P}_{*}$. To avoid confusion with the general case, we rename the fiber $M$ and assume that it is dualizable. We then have a transfer map $\tau: S_{\Gamma} \longrightarrow \Sigma_{\Gamma}^{\infty} M_{+}$of (nonparametrized) $\Gamma$-spectra. We insert this into the functor $\mathbb{P}$ to obtain a map

$$
\mathbb{P} \tau: \mathbb{P} S_{\Gamma} \longrightarrow \mathbb{P} \Sigma_{\Gamma}^{\infty} M_{+}
$$

of $G$-spectra over $B$. Again pushing down to a map of $G$-spectra along $r: B \longrightarrow *$, we obtain the transfer $G$-map $\Sigma_{G}^{\infty} B_{+} \longrightarrow \Sigma_{G}^{\infty} E_{+}$.

This description hides a subtlety. The construction of $\mathbb{P}$ involves composition with a change of universe functor $i^{*}$. Although $i^{*}$ is not in general symmetric monoidal, it restricts to a symmetric monoidal equivalence between categories of parametrized $\Pi$-free $\Gamma$-spectra. This allows us to prove that $\mathbb{P}$ is symmetric monoidal in general. Since transfer maps are natural with respect to symmetric monoidal functors, this fact makes it transparent that the fiberwise transfer map of a bundle agrees with its transfer map as a Hurewicz fibration.

We assume throughout that all given groups $G$ are compact Lie groups and all given base $G$-spaces $B$ are $G$-CW complexes. This ensures that $\left(r^{*}, r_{*}\right)$ is a Quillen adjunction, $r: B \longrightarrow *$, but only a few details of proofs would be changed if we allowed $B$ just to have the homotopy type of a $G$-CW complex.

\subsection{The fiberwise duality theorem}

We characterize the dualizable and invertible $G$-spectra over $B$. A recent exposition of the general theory of duality in closed symmetric monoidal categories appears in [123], to which we refer the reader for discussion of the relevant categorical definitions and arguments. It is based on the more thorough and topological treatment of [98], which is carried further in [63]. The following theorem is a substantial generalization of various early results of the same nature about ex-fibrations. Results of this form were proven, for example, by Becker and Gottlieb [9, §4], Clapp $[31,3.5]$, and Waner $[170,4.6]$.

Theorem 15.1.1 (The fiberwise duality theorem). Let $X$ be an (s-fibrant) $G$ spectrum over $B$. Then $X$ is dualizable (respectively, invertible) if and only if $X_{b}$ is dualizable (respectively, invertible) as a $G_{b}$-spectrum for each $b \in B$.

Proof. By definition, $X$ is dualizable if and only if the natural map

$$
\nu: D_{B} X \wedge_{B} X \longrightarrow F_{B}(X, X)
$$

in Ho $G \mathscr{S}_{B}$ is an equivalence, where $D_{B} X=F_{B}\left(X, S_{B}\right)$. Passing to (derived) fibers $\left(D_{B} X\right)_{b} \simeq D X_{b}$, this holds if and only if the resulting map

$$
D X_{b} \wedge X_{b} \simeq\left(D_{B} X \wedge_{B} X\right)_{b} \stackrel{\nu_{b}}{\longrightarrow} F_{B}(X, X)_{b} \simeq F\left(X_{b}, X_{b}\right)
$$


in $\mathrm{Ho}_{G_{b}} \mathscr{S}$ is an equivalence for all $b \in B$. By the categorical coherence observation Remark 2.2.10, the latter map is the corresponding natural map $\nu$ in $\mathrm{Ho}_{b} \mathscr{S}$. Again by definition, that map is an equivalence if and only if $X_{b}$ is dualizable.

Similarly, $X$ is invertible if and only if the evaluation map

$$
\text { ev: } D_{B} X \wedge_{B} X \longrightarrow S_{B}
$$

in $\mathrm{Ho}_{G} \mathscr{S}_{B}$ is an equivalence. Passing to (derived) fibers, this holds if and only if the resulting map

$$
D X_{b} \wedge X_{b} \simeq\left(D_{B} X \wedge_{B} X\right)_{b} \stackrel{\mathrm{ev}_{b}}{\longrightarrow}\left(S_{B}\right)_{b} \simeq S
$$

in $\mathrm{Ho}_{b} \mathscr{S}$ is an equivalence for all $b \in B$. Again by Remark 2.2.10, the latter map is the evaluation map for $X_{b}$ in $\mathrm{Ho}_{b} \mathscr{S}$, and that map is an equivalence if and only if $X_{b}$ is invertible.

Therefore, to recognize parametrized dualizable and invertible $G$-spectra, it suffices to recognize nonparametrized dualizable and invertible $G$-spectra. As we now recall from [63], these are well understood.

Recall that a $G$-space $X$ is dominated by a $G$-space $Y$ if $X$ is a retract up to homotopy of $Y$, so that the identity map of $X$ is homotopic to a composite $X \longrightarrow Y \longrightarrow X$. If $Y$ has the homotopy type of a $G$-CW complex, then so does $X$. We say that $X$ is finitely dominated if it is dominated by a finite $G$-CW complex. This does not imply that $X$ has the homotopy type of a finite $G$-CW complex, even when $X$ and all of its fixed point spaces $X^{H}$ are simply connected and therefore, since they are finitely dominated, homotopy equivalent to finite $\mathrm{CW}$ complexes.

For example, a $G$-space $X$ is a $G$-ENR (Euclidean neighborhood retract) if it can be embedded as a retract of an open subset of some representation $V$. Such open subsets are triangulable as $G$-CW complexes, so $X$ has the homotopy type of a $G$-CW complex. A compact $G$-ENR is a retract of a finite $G$-CW complex and is thus finitely dominated, but it need not have the homotopy type of a finite $G$-CW complex. Non-smooth topological $G$-manifolds give examples of such non-finite compact $G$-ENRs.

The following result is $[63,2.1]$.

THEOREM 15.1.2. Up to equivalence, the dualizable $G$-spectra are the $G$-spectra of the form $\Sigma^{-V} \Sigma^{\infty} X$ where $X$ is a finitely dominated based $G-C W$ complex and $V$ is a representation of $G$.

DeFinition 15.1.3. A generalized homotopy representation $X$ is a finitely dominated based $G$-CW complex such that, for each subgroup $H$ of $G, X^{H}$ is equivalent to a sphere $S^{n(H)}$. A stable homotopy representation is a $G$-spectrum of the form $\Sigma^{-V} \Sigma^{\infty} X$, where $X$ is a generalized homotopy representation and $V$ is a representation of $G$.

The following result is $[63,0.5]$.

THEOREM 15.1.4. Up to equivalence, the invertible G-spectra are the stable homotopy representations.

Combining results, we obtain the following conclusion about ex- $G$-fibrations.

THEOREM 15.1.5. Let $E$ be an ex-G-fibration over $B$. If each fiber $E_{b}$ is a finitely dominated $G_{b}$-space, then $\Sigma_{B}^{\infty} E$ is a dualizable $G$-spectrum over B. If each 
$E_{b}$ is a generalized homotopy representation of $G_{b}$, then $\Sigma_{B}^{\infty} E$ is an invertible $G$ spectrum over $B$.

ProOF. Since the derived suspension spectrum functor commutes with passage to derived fibers, by Theorem 13.7.10, the derived fiber $\left(\Sigma_{B}^{\infty} E\right)_{b}$ is equivalent to $\Sigma^{\infty} E_{b}$. The conclusion follows from Theorems 15.1.1, 15.1.2, and 15.1.4.

In particular, sphere $G$-bundles and, more generally, spherical $G$-fibrations over $B$, have invertible suspension $G$-spectra over $B$.

\subsection{Duality and trace maps in symmetric monoidal categories}

Since the stable homotopy category $\mathrm{Ho}_{G} \mathscr{S}_{B}$ is closed symmetric monoidal, we have the following generalized trace maps at our disposal. We state the definition and recall its properties in full generality in this section, and we specialize to show how it gives a simple conceptual definition of the transfer maps associated to equivariant Hurewicz fibrations in the next.

DEFinition 15.2.1. Let $\mathscr{C}$ be any closed symmetric monoidal category with unit object $S$. For a dualizable object $X$ of $\mathscr{C}$ with a "coaction" map $\Delta_{X}: X \longrightarrow$ $X \wedge C_{X}$ for some object $C_{X} \in \mathscr{C}$, define the trace $\tau(f)$ of a self map $f$ of $X$ by the diagram

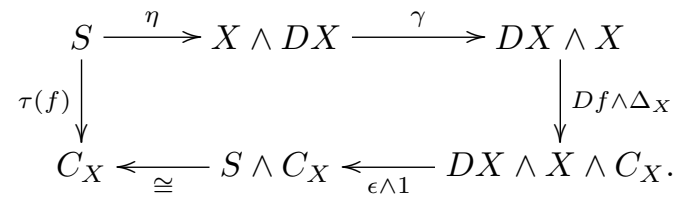

REMARK 15.2.2. Such a categorical description of generalized trace maps was first given by Dold and Puppe [51], where they showed that it gives the right framework for trace maps in algebra, the transfer maps of Becker and Gottlieb $[9,10]$, and the fixed point theory of Dold [50]. These early constructions of transfer maps had finiteness conditions that were first eliminated by Clapp [31,32]. Indeed, she gave an early construction of a parametrized stable homotopy category and proved a precursor of our fiberwise duality theorem. The equivariant analogue of the attractive space level treatment of Spanier-Whitehead duality given by Dold and Puppe was worked out in [98], and a recent categorical exposition of duality has been given in [123].

Two cases are of particular interest. The first is when $C_{X}=S$ and $\Delta_{X}$ is the unit isomorphism. Then $\tau(f)$ is called the Lefschetz constant of $f$ and is denoted by $\chi(f)$; in the special case when $f=$ id it is called the Euler characteristic of $X$ and denoted by $\chi(X)$. The second is when $C_{X}=X$. We then think of $\Delta_{X}$ as a diagonal map, and $\tau_{X}=\tau(\mathrm{id})$ is called the transfer map of $X$.

REMARK 15.2.3. If $C_{X}$ comes with a "counit" map $\xi: C_{X} \longrightarrow S$ such that the composite

$$
X \stackrel{\Delta}{\longrightarrow} X \wedge C_{X} \stackrel{\text { id } \wedge \xi}{\longrightarrow} X
$$

is the identity, then $\chi(f)=\xi \circ \tau(f)$ by a little diagram chase. The reason for the terminology "coaction" and "counit" for the maps $\Delta_{X}$ and $\xi$ is that in many situations $C_{X}$ is a comonoid and $\Delta_{X}$ is a coaction of $C_{X}$ on $X$. 
The following basic properties of the trace are proven in [98, III§7] and in [124], where more detailed statements are given. Define a map

$$
(f, \alpha):\left(X, \Delta_{X}\right) \longrightarrow\left(Y, \Delta_{Y}\right)
$$

to be a pair of maps $f: X \longrightarrow Y$ and $\alpha: C_{X} \longrightarrow C_{Y}$ such that the following diagram commutes.

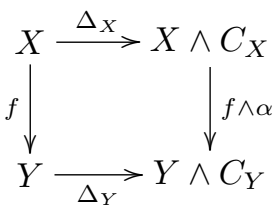

Proposition 15.2.4. The trace satisfies the following properties, where $X$ and $Y$ are dualizable and $\Delta_{X}$ and $\Delta_{Y}$ are given.

(i) (Naturality) If $\mathscr{C}$ and $\mathscr{D}$ are closed symmetric monoidal categories and $F: \mathscr{C} \longrightarrow \mathscr{D}$ is a lax symmetric monoidal functor such that $F S_{\mathscr{C}} \cong S_{\mathscr{D}}$ and $F(X) \wedge F(D X) \cong F(X \wedge D X)$, then

$$
\tau(F f)=F \tau(f),
$$

where $C_{F X}=F C_{X}$ and $\Delta_{F X}=F \Delta_{X}$.

(ii) (Unit property) If $f$ is a self map of the unit object, then $\chi(f)=f$.

(iii) (Fixed point property) If $(f, \alpha)$ is a self map of $\left(X, \Delta_{X}\right)$, then

$$
\alpha \circ \tau(f)=\tau(f) \text {. }
$$

(iv) (Invariance under retracts) If $X \stackrel{i}{\longrightarrow} Y \stackrel{r}{\longrightarrow} X$ is a retract, $f$ is a self map of $X$, and $(i, \alpha)$ is a map $\left(X, \Delta_{X}\right) \longrightarrow\left(Y, \Delta_{Y}\right)$, then

$$
\alpha \circ \tau(f)=\tau(i f r) .
$$

(v) (Commutation with $\wedge$ ) If $f$ and $g$ are self maps of $X$ and $Y$, then

$$
\tau(f \wedge g)=\tau(f) \wedge \tau(g),
$$

where $\Delta_{X \wedge Y}=(i d \wedge \gamma \wedge i d) \circ\left(\Delta_{X} \wedge \Delta_{Y}\right)$ with $\gamma$ the transposition.

(vi) (Commutation with $\vee$ ) If $\mathscr{C}$ is additive and $h: X \vee Y \longrightarrow X \vee Y$ induces $f: X \longrightarrow X$ and $g: Y \longrightarrow Y$ by inclusion and retraction, then

$$
\tau(h)=\tau(f)+\tau(g),
$$

where $C_{X}=C_{Y}=C_{X \vee Y}$ and $\Delta_{X \vee Y}=\Delta_{X} \vee \Delta_{Y}$.

(vii) (Anticommutation with suspension) If $\mathscr{C}$ is triangulated, then

$$
\tau(\Sigma f)=-\tau(f)
$$

for all self maps $f$, where $\Delta_{\Sigma X}=\Sigma \Delta_{X}$.

In the triangulated context, there is another and very much deeper property.

THEOREM 15.2.5 (Additivity). Let $\mathscr{C}$ be a closed symmetric monoidal category with a "compatible triangulation". Let $X$ and $Y$ be dualizable and let $\Delta_{X}$ and $\Delta_{Y}$ be given, where $C=C_{X}=C_{Y}$. Let $(f, i d)$ be a map $\left(X, \Delta_{X}\right) \longrightarrow\left(Y, \Delta_{Y}\right)$ and extend $f$ to a distinguished triangle

$$
X \stackrel{f}{\longrightarrow} Y \stackrel{g}{\longrightarrow} Z \stackrel{h}{\longrightarrow} \Sigma X .
$$


Assume given maps $\phi$ and $\psi$ that make the left square commute in the first of the following two diagrams.
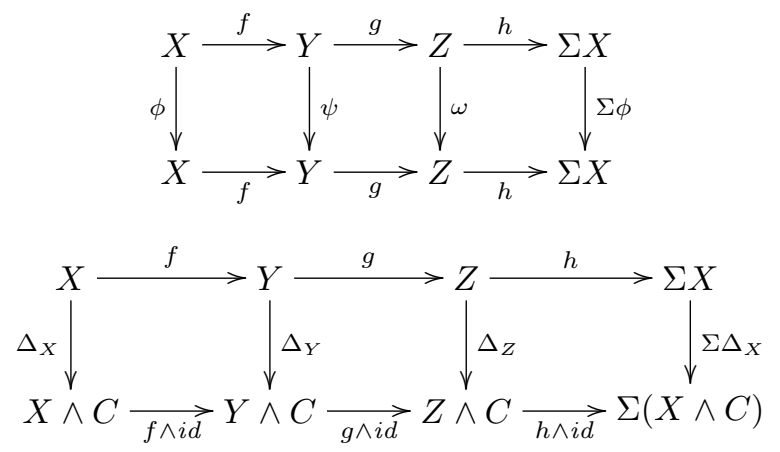

Then there are maps $\omega$ and $\Delta_{Z}$ such that the diagrams commute and

$$
\tau(\psi)=\tau(\omega)+\tau(\phi) .
$$

The most important case starts with only the distinguished triangle $(f, g, h)$ and concludes with the fundamental additivity relation

$$
\chi(Y)=\chi(X)+\chi(Z) .
$$

The additivity of traces was studied in [98, III $\S 7$ in the equivariant stable homotopy category, but the proof there is incorrect. A thorough investigation of precisely what is needed to prove the additivity of traces is given in [124], where the axioms for a "compatible triangulation" are formulated. These axioms hold in all situations previously encountered in algebraic topology and algebraic geometry. Although the verification is a little more subtle, they also hold here. Since it would be digressive at this point, we defer discussion of triangulated categories and the proof of the following theorem to $\S \S 16.6,16.7$, and 17.5.

TheOREM 15.2.6. The category HoG $\mathscr{S}_{B}$ is a closed symmetric monoidal category with a compatible triangulation.

\subsection{Transfer maps of Hurewicz fibrations}

With these foundations in place, we can now generalize the classical construction of transfer maps. The results above specialize to give more information about them than is to be found in the literature. If $X$ is a dualizable $G$-spectrum over $B$ with a diagonal map $\Delta_{X}: X \longrightarrow X \wedge_{B} X$, then we have the transfer map $\tau_{X}: S_{B} \longrightarrow X$. We shall apply this to suspension $G$-spectra associated to $G$ fibrations $p: E \longrightarrow B$, but we do not assume that $p$ has a section. Recall that $(E, p)_{+}$denotes $(E, p)$ with a disjoint section, and observe that $(E, p)_{+}$is an ex- $G-$ fibration if $p$ is a Hurewicz $G$-fibration.

Recall the desription of the base change functors associated to $r: B \longrightarrow *$ from Example 2.1.8. As we have said before, the spectrum level versions of these functors are central to the deduction of results in classical stable homotopy theory from results in parametrized stable homotopy theory. Many such deductions start from the following observation.

Lemma 15.3.1. For a $G$-map $p: E \longrightarrow B$, thought of as a $G$-space over $B$,

$$
r_{!} \Sigma_{B}^{\infty}(E, p)_{+} \simeq \Sigma^{\infty} E_{+},
$$


where $r: B \longrightarrow *$. In particular, $r ! S_{B} \simeq \Sigma^{\infty} B_{+}$.

Proof. We have $r ! \Sigma_{B}^{\infty} \simeq \Sigma^{\infty} r_{!}$. This is a commutation relation between Quillen left adjoints, and the corresponding commutation relation for right adjoints holds since

$$
r^{*} \Omega^{\infty} X=B \times X_{0} \cong \Omega_{B}^{\infty} r^{*} X
$$

for a $G$-spectrum $X$. It therefore suffices to show that $r_{!}(E, p)_{+}$is equivalent to $E_{+}$, where $r$ ! denotes the functor on derived categories. By Proposition 7.3.4, $r$ ! preserves $q$-equivalences between well-sectioned ex-spaces and it follows that

$$
r_{!} Q(E, p)_{+} \simeq r_{!}(E, p)_{+} \cong E_{+},
$$

where the first equivalence is induced by $q f$-cofibrant approximation of $(E, p)_{+}$.

To be precise about diagonal maps on the parametrized level, we consider base change along $\Delta: B \longrightarrow B \times B$. We have the obvious commutative diagram

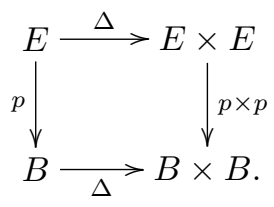

We consider $E$ as a space over $B \times B$ via this composite. The diagonal map of $E$ then specifies a natural map

$$
\Delta_{!}\left((E, p)_{+}\right)=(E, \Delta \circ p)_{+} \longrightarrow(E \times E, p \times p)_{+} \cong(E, p)_{+} \bar{\wedge}(E, p)_{+}
$$

of ex-spaces over $B \times B$. This is a comparison map between Quillen left adjoints and therefore descends to a natural map in $\mathrm{Ho} G \mathscr{K}_{B \times B}$. Its adjoint is a natural map $(E, p)_{+} \longrightarrow(E, p)_{+} \wedge_{B}(E, p)_{+}$in Ho $G \mathscr{K}_{B}$. Apply the (derived) suspension functor $\Sigma_{B}^{\infty}$ to this map and note that the target is equivalent to $\Sigma_{B}^{\infty}(E, p)_{+} \wedge_{B} \Sigma_{B}^{\infty}(E, p)_{+}$, by Proposition 13.7.5. This gives the required natural diagonal map

$$
\Delta_{(E, p)_{+}}: \Sigma_{B}^{\infty}(E, p)_{+} \longrightarrow \Sigma_{B}^{\infty}(E, p)_{+} \wedge_{B} \Sigma_{B}^{\infty}(E, p)_{+}
$$

in $\operatorname{Ho} G \mathscr{S}_{B}$.

Definition 15.3.3 (The transfer map). Let $p: E \longrightarrow B$ be a Hurewicz $G$ fibration over $B$ such that each fiber $E_{b}$ is homotopy equivalent to a retract of a finite $G_{b}$-CW-complex. Then $\Sigma_{B}^{\infty}(E, p)_{+}$is a dualizable $G$-spectrum over $B$ by Theorem 15.1.5 and we obtain the transfer map

$$
\tau_{(E, p)_{+}}: S_{B} \longrightarrow \Sigma_{B}^{\infty}(E, p)_{+}
$$

in Ho $G \mathscr{S}_{B}$. Define the transfer map of $E$ to be the map

$$
\tau_{E}=r_{!} \tau_{(E, p)_{+}}: \Sigma^{\infty} B_{+} \cong r ! S_{B} \longrightarrow r_{!} \Sigma_{B}^{\infty}(E, p)_{+} \cong \Sigma^{\infty} E_{+}
$$

in $\mathrm{Ho}_{\mathrm{G}} \mathscr{S}$.

With this definition, all of the standard properties of transfer maps are direct consequences of the general categorical results Proposition 15.2.4 and Theorem 15.2.5 and the properties of $r$. 


\subsection{The bundle construction on parametrized spectra}

The construction of the transfer in the previous section works "globally", starting on the parametrized spectrum level. We now give a fiberwise construction of "stable bundles" that leads to an alternative fiberwise perspective. However, it is natural to work in greater generality than is needed for the construction of the transfer. The extra generality will be needed in Chapter 19 and later. The relevant bundle theoretic background was recalled in $\S 3.2$ and the basic properties of the point-set level bundle construction were developed in $\S 3.3$.

Let $\Pi$ be a normal subgroup of a compact Lie group $\Gamma$ such that $\Gamma / \Pi=G$ and let $q: \Gamma \longrightarrow G$ be the quotient homomorphism. Let $p: E \longrightarrow B$ be a $(\Pi ; \Gamma)$-bundle with fiber a $\Gamma$-space $F$ and with associated principal $(\Pi ; \Gamma)$-bundle $\pi: P \longrightarrow B$. Then $P$ is a $\Pi$-free $\Gamma$-space, $\pi$ is the quotient map to the orbit $G$-space $B=P / \Pi$, and $p$ is the associated $G$-bundle $E \cong P \times_{\Pi} F \longrightarrow B$. To simplify the homotopical analysis, we assume for the rest of this section that $F$ and $P$ are $\Gamma$-CW complexes such that $P$ is $\Pi$-free. We let $E=P \times_{\Pi} F$ and $B=P \times_{\Pi} *$. Note that $B$ is a $G$-CW complex. We are thinking of the cases when $F$ is a point or when $F$ is a smooth $\Gamma$-manifold.

Recall the bundle construction of $\S 3.3$ :

$$
P_{F}=P \times_{\Pi}(-): \Gamma \mathscr{K}_{F} \longrightarrow G \mathscr{K}_{E} .
$$

We can extend the functor $P_{F}$ from ex-spaces to ex-spectra. Change of universe must enter since $\Gamma$-spectra are indexed on representations of $\Gamma$ and $G$-spectra are indexed on representations of $G$. We view representations of $G$ as $\Pi$-trivial representations of $\Gamma$. This gives $i: q^{*} \mathscr{V}_{G} \longrightarrow \mathscr{V}_{\Gamma}$. It is important to keep track of which universe we are working in, and we introduce the following notations.

Notation 15.4.1. Define $\mathbb{P}_{F}=P_{F} \circ i^{*}: \Gamma \mathscr{S}_{F} \longrightarrow G \mathscr{S}_{B}$, where

$$
i^{*}: \Gamma \mathscr{S}_{F}=\Gamma \mathscr{S}_{F}^{\mathscr{N}_{\Gamma}} \longrightarrow \Gamma \mathscr{S}_{F}^{q^{*} \mathscr{V}_{G}}=\Gamma \mathscr{S}_{F}^{\Pi-\text { triv }}
$$

is the change of universe functor and

$$
P_{F}: \Gamma \mathscr{S}_{F}^{\text {ח-triv }} \longrightarrow G \mathscr{S}_{E}
$$

is the levelwise bundle construction of $\S 3.3$. We use the same notations for the corresponding functors on prespectra.

Working in the universe $\mathscr{V}_{G}$, with $\Pi$ acting trivially on our representations $V$, we have

$$
P_{F} K \wedge_{E} S^{V} \cong P_{F}\left(K \wedge_{F} S^{V}\right)
$$

Therefore, for a $\Gamma$-spectrum $X$ over $F$, the ex- $G$-spaces $P_{F} X(V)$ over $E$ inherit structure maps from $X$, so that $P_{F} X$ is a well-defined $G$-spectrum over $E$ and the functor $P_{F}$ makes sense.

Except where explicitly indicated otherwise, we index both $G$-spectra and $\Gamma$ spectra on $\mathscr{V}_{G}$ for the rest of this section, working with $P_{F}$. We return to $\mathbb{P}_{F}$ in the following sections. In fact, the functors $P_{F}$ and $\mathbb{P}_{F}$ are of independent interest. The latter is essential in this chapter and in Chapter 19. However, only the former will be relevant for the study of twisted homology, and, when indexing on $\Pi$-trivial representations, there is no need to restrict $\Pi$ and $\Gamma$ to be compact Lie groups. The case $\Gamma=\Pi \times G$, with $G$ but not necessarily $\Pi$ a compact Lie group is of particular interest.

The functor $P_{F}$ is exceptionally well-behaved, as the following result shows. 
Proposition 15.4.2. The functor $P_{F}: \Gamma \mathscr{S}_{F}^{\Pi-\text { triv }} \longrightarrow G \mathscr{S}_{E}$ is both a left and a right Quillen adjoint with respect to the level and stable model structures. Moreover, the functor $P_{F}: \Gamma \mathscr{P}_{F}^{\Pi-\text { triv }} \longrightarrow G \mathscr{P}_{E}$ takes excellent $\Gamma$-prespectra over $F$ to excellent $G$-prespectra over $E=P \times_{\Pi} F$.

Proof. The two adjunctions are defined as in Lemma 3.3.1. Thus $P_{F}$ is the composite of $\pi^{*}: \Gamma \mathscr{S}_{F} \longrightarrow \Gamma \mathscr{S}_{P \times F}$, where $\pi: P \times F \longrightarrow F$ is the projection, and $(-) / \Pi: \Gamma \mathscr{S}_{P \times F} \longrightarrow G \mathscr{S}_{E}$. By Propositions 12.2.5, 12.2.7, 12.6.7, and 12.6.8, $\pi^{*}$ is both a left and a right Quillen adjoint, provided we use appropriate generating sets in our definitions of the model structures. By Proposition 14.4.3, the functor $(-) / \Pi$ is a Quillen left adjoint. By Proposition 14.4.5, it coincides with the right adjoint $(-)^{\Pi} \circ p_{*}^{\prime}$, where $p^{\prime}$ is the quotient map $P \times F \longrightarrow P \times_{\Pi} F=E$. Using Lemma 3.2.1, we see that $p: E \longrightarrow B$ is a $G$-bundle with $\mathrm{CW}$ fibers. Therefore $p_{*}$ is a Quillen right adjoint by Propositions 12.2 .7 and 12.6.8, and $(-)^{\Pi}$ is a Quillen right adjoint by Proposition 14.4.3. The last statement is easily checked from Definition 13.2.2 and Lemma 13.2.3.

We need an observation about the behavior of $P_{F}$ on fibers.

LEMmA 15.4.3. Fix $b \in B$. Let $\iota: G_{b} \longrightarrow G$ and $\rho_{b}: G_{b} \longrightarrow \Gamma$ be the inclusion and the homomorphism of Lemma 3.2.1. Let $b: * \longrightarrow B$ and $j_{b}: E_{b} \longrightarrow E$ denote the evident inclusions of $G_{b}$ spaces. The following diagrams commute, and these commutation relations descend to homotopy categories.

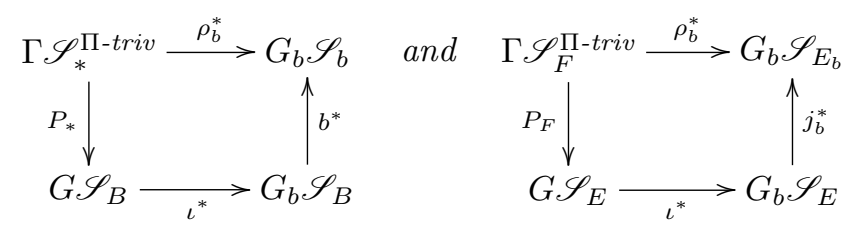

Proof. On the level of ex-spaces, this is Lemma 3.3.2. The diagrams extend levelwise to parametrized spectra, and passage to homotopy categories is clear from the previous result.

Write $\Sigma_{G, E}^{\infty}$ for the suspension $G$-spectrum over $E$, where the relevant universe is understood to be complete, and write $\Sigma_{\Pi \text {-triv, } F}^{\infty}$ for the suspension $\Gamma$-spectrum over $F$ indexed on the $\Pi$-trivial $\Gamma$-universe $q^{*} \mathscr{V}_{G}$, where $i: q^{*} \mathscr{V}_{G} \subset \mathscr{V}_{\Gamma}$.

Proposition 15.4.4. There are natural isomorphisms of functors

$$
\begin{gathered}
i^{*} \Sigma_{\Gamma, F}^{\infty} \cong \Sigma_{\Pi-\text { triv }, F}^{\infty}: \Gamma \mathscr{K}_{F} \longrightarrow \Gamma \mathscr{S}_{F}^{\Pi-t r i v}, \\
P_{F} \Sigma_{\Pi-\text { triv }, F}^{\infty} \cong \Sigma_{G, E}^{\infty} P_{F}: \Gamma \mathscr{S}_{F}^{\Pi-t r i v} P_{F} \longrightarrow G \mathscr{S}_{E},
\end{gathered}
$$

and therefore

$$
\mathbb{P}_{F} \Sigma_{\Gamma, F}^{\infty} \cong \Sigma_{G, E}^{\infty} P_{F}: \Gamma \mathscr{K}_{F} \longrightarrow G \mathscr{S}_{E}
$$

and these isomorphisms descend to homotopy categories. In particular,

$$
\mathbb{P}_{F} S_{\Gamma, F} \simeq S_{G, E}
$$

Proof. The statement about $i^{*}$ is clear from $\S 14.2$. For the statement about $P_{F}$, let $K$ be an ex- $\Gamma$-space over $F$ and observe that we have isomorphisms

$$
\left(P_{F} \Sigma_{\Pi \text {-triv }, F}^{\infty} K\right)(V)=P_{F}\left(K \wedge_{F} S_{F}^{V}\right) \cong\left(P_{F} K\right) \wedge_{E} S_{E}^{V}=\left(\Sigma_{G, E}^{\infty} P_{F} K\right)(V)
$$


since $\Pi$ acts trivially on $V$. This gives a natural isomorphism of $G$-spectra over $E$, and it descends to homotopy categories since it is a comparison of composites of Quillen left adjoints.

The following result relates external smash products and diagonal maps to the functor $P_{F}$. In $\S 15.6$, we shall use the theory of $\Pi$-free $\Gamma$-spectra developed in $\S 15.5$ to prove the analogue for $\mathbb{P}_{F}$. Recall the factorization $\Delta_{E}=\iota_{E} \circ \delta_{E}$ of (3.3.3).

Proposition 15.4.5. For $X_{i} \in \Gamma \mathscr{S}_{F_{i}}^{\Pi \text {-triv }}$,

$$
P_{F_{1} \times F_{2}}\left(X_{1} \bar{\wedge} X_{2}\right) \simeq \iota^{*}\left(P_{F_{1}} X_{1} \bar{\wedge} P_{F_{2}} X_{2}\right)
$$

where $\iota$ is the inclusion $E_{1} \times_{B} E_{2} \longrightarrow E_{1} \times E_{2}, E_{i}=P \times_{\Pi} F_{i}$. For $X \in \Gamma \mathscr{S}_{F}^{\Pi \text {-triv }}$ and $Y \in \Gamma \mathscr{S}_{F \times F}^{\Pi-t r i v}$,

$$
P_{F \times F}\left(\Delta_{F !} X\right) \simeq \delta_{E !} P_{F} X \quad \text { and } \quad P_{F} \Delta_{F}^{*} Y \simeq \delta_{E}^{*} P_{F \times F} Y .
$$

Therefore the functor $P_{F}$ is monoidal.

Proof. On the point-set level, these isomorphisms follow levelwise from Proposition 3.3.6. By Proposition 15.4.2 and the proof of Proposition 13.7.2, the functors $P_{F}, \bar{\wedge}$, and $\iota^{*}$ all preserve excellent prespectra, and it follows that our first equivalence passes directly to homotopy categories. The second equivalence only involves Quillen left adjoints and the third only involves Quillen right adjoints. The last statement follows as in Proposition 3.3.7.

We have the following relations between $P_{F}$ and base change functors. They can be used in conjunction with the general relations between change of universe and base change functors that were proven in Proposition 14.2.8 to obtain the analogues for $\mathbb{P}_{F}$.

Proposition 15.4.6. Let $f: F \longrightarrow F^{\prime}$ be a map of $\Gamma$-spaces, let $E=P \times_{\Pi} F$ and $E^{\prime}=P \times_{\Pi} F^{\prime}$, and let $g=P \times_{\Pi} f: E \longrightarrow E^{\prime}$. For $X \in \Gamma \mathscr{S}_{F}^{\Pi-t r i v}$ and $Y \in \Gamma \mathscr{S}_{F^{\prime}}^{\Pi-t r i v}$, there are natural isomorphisms

$$
g_{!} P_{F} X \longrightarrow P_{F^{\prime}} f_{!} X, \quad P_{F} f^{*} Y \longrightarrow g^{*} P_{F^{\prime}} Y \quad \text { and } \quad P_{F^{\prime}} f_{*} X \longrightarrow g_{*} P_{F} X .
$$

The first two isomorphisms descend to derived homotopy categories, and the third does so provided that $f$ is a bundle with $C W$ fibres.

Proof. The corresponding result on the ex-space level is given in Proposition 3.3.8. Applied levelwise, those point-set level isomorphisms carry over directly to parametrized prespectra and spectra. We must show that they descend to equivalences in homotopy categories. The first commutation relation is between composites of left Quillen adjoints, the second is between composites of right Quillen adjoints, and, with the proviso on $f$, so is the third.

\section{5. $\Pi$-free parametrized $\Gamma$-spectra}

We retain the notations of the previous section in this section and the next. In the next section, we show that the bundle construction on parametrized spectra leads to a fiberwise generalization of the restriction to bundles of the trace and transfer maps for fibrations that we described in $\$ 15.2$. The definition depends on a result that is proven by use of the theory of $\Pi$-free $\Gamma$-spectra that we present here.

We first recall what it means to say that a $\Gamma$-spectrum $X$ (indexed on any universe) is $\Pi$-free. Let $\mathscr{F}(\Pi ; \Gamma)$ be the family of subgroups $\Lambda$ of $\Gamma$ such that 
$\Lambda \cap \Pi=e$. A $\Gamma$-CW complex $T$ is $\Pi$-free if and only if the only orbit types $\Gamma / \Lambda$ that appear in its construction have $\Lambda \in \mathscr{F}(\Pi ; \Gamma)$. We then say that $T$ is an $\mathscr{F}(\Pi ; \Gamma)$-CW complex. We can make the same definitions for $\Gamma$-CW spectra, and in general we say that a $\Gamma$-spectrum is $\Pi$-free if it is isomorphic in $Н$ Ho $\mathscr{S}$ to an $\mathscr{F}(\Pi ; \Gamma)-C W$ spectrum. There is a more conceptual homotopical reformulation that is the one relevant to the parametrized point of view and that does not depend on the theory of $\Gamma$-CW spectra.

Let $E(\Pi ; \Gamma)$ be the universal $\Pi$-free $\Gamma$-space, so that $E(\Pi ; \Gamma)^{\Lambda}$ is contractible if $\Lambda \cap \Pi=e$ and is empty otherwise. We may take $E(\Pi ; \Gamma)$ to be an $\mathscr{F}(\Pi ; \Gamma)-\mathrm{CW}$ complex. Let $B(\Pi ; \Gamma)=E(\Pi ; \Gamma) / \Pi$ and observe that $B$ is a $G$-CW complex and therefore also a $\Gamma$-CW complex. We note parenthetically that the quotient map $p: E(\Pi ; \Gamma) \longrightarrow B(\Pi ; \Gamma)$ is the universal principal $(\Pi ; \Gamma)$-bundle. That is, pullback along $p$ gives a bijection

$$
[X, B(\Pi ; \Gamma)]_{G} \longrightarrow \mathscr{B}(\Pi ; \Gamma)(X),
$$

where $\mathscr{B}(\Pi ; \Gamma)(X)$ denotes the set of equivalence classes of principal $(\Pi ; \Gamma)$-bundles over the $G$-space $X$; see [92] or [118, VII $\S 2]$.

Definition 15.5.1. Let $r: E(\Pi ; \Gamma) \longrightarrow *$ be the projection and let $\sigma$ be the counit of the (derived) adjunction $\left(r_{!}, r^{*}\right)$. A $\Gamma$-spectrum $X$ is said to be $\Pi$-free if $\sigma: r_{!} r^{*} X \longrightarrow X$ is an equivalence.

The definition should seem reasonable since $r ! r^{*} T \cong E(\Pi ; \Gamma)_{+} \wedge T$ for a $\Gamma$ space $T$. It is equivalent to the original definition in terms of an equivalence in Ho $G \mathscr{S}$ to an $\mathscr{F}(\Pi ; \Gamma)$-CW spectrum; see [98, II.2.12] or [105, VI§4]. This definition generalizes readily to the parametrized context.

Definition 15.5.2. Let $\pi: E(\Pi ; \Gamma) \times F \longrightarrow F$ be the projection and let $\sigma$ be the counit of the (derived) adjunction $\left(\pi_{!}, \pi^{*}\right)$. An ex- $\Gamma$-space or $\Gamma$-spectrum $X$ over a $\Gamma$-space $F$ is said to be $\Pi$-free if $\sigma: \pi ! \pi^{*} X \longrightarrow X$ is an equivalence.

Since the fiber $\left(\pi_{!} \pi^{*} X\right)_{f}$ is $E(\Pi ; \Gamma)_{+} \wedge X_{f}$, the definition should seem reasonable. Since equivalences are detected fiberwise, we have the following results.

Lemma 15.5.3. A $\Gamma$-spectrum $X$ over $F$ is $\Pi$-free if and only if each of its fibers $X_{f}$ is a $\left(\Pi \cap \Gamma_{f}\right)$-free $\Gamma_{f}$-spectrum.

Proof. The fiber of $E(\Pi ; \Gamma) \times F \longrightarrow F$ over $f \in F$ is the $\Gamma$-space $E(\Pi ; \Gamma)$ with the action restricted along $\iota: \Gamma_{f} \longrightarrow \Gamma$. It is a model of the universal $\left(\Pi \cap \Gamma_{f}\right)$-free $\Gamma_{f}$-space $E\left(\Pi \cap \Gamma_{f}, \Gamma_{f}\right)$. Applying $(-)_{f}$ to the counit $\pi_{!} \pi^{*} X \longrightarrow X$ and using Theorem 13.7.7 we obtain the counit $r ! r^{*} X_{f} \longrightarrow X_{f}$ where $r: \iota^{*} E(\Pi ; \Gamma) \longrightarrow *$.

Lemma 15.5.4. If $P$ is a $\Pi$-free $\Gamma$-space and $X$ is any ex- $\Gamma$-space or $\Gamma$-spectrum over $F$, then $P \times X$ is a $\Pi$-free ex- $\Gamma$-space or $\Gamma$-spectrum over $P \times F$.

A useful slogan asserts that " $\Pi$-free $\Gamma$-spectra live in the $\Pi$-trivial universe". To explain it, consider the inclusion $i: q^{*} \mathscr{V}_{G} \longrightarrow \mathscr{V}_{\Gamma}$ of the complete $G$-universe $\mathscr{V}_{G}$ as the universe of $\Pi$-trivial representations in the complete $\Gamma$-universe $\mathscr{V} \Gamma$. Then the slogan is given meaning by the following result. In the nonparametrized case $F=*$, it is proven in [98, II $\S 2]$ and is discussed further in [105, VI $\S 4]$. Since the parametrized case presents no complications and the proof is quite easy, we only give a sketch. 
Proposition 15.5.5. The change of universe adjunction $\left(i_{*}, i^{*}\right)$ descends to a symmetric monoidal equivalence between the homotopy categories of $\Pi$-free $\Gamma$ spectra over $F$ indexed on $\Pi$-trivial representations of $\Gamma$ on the one hand and indexed on all representations of $\Gamma$ on the other. For $\Pi$-free $\Gamma$-spectra $X$ over $F$ indexed on $\mathscr{V}_{\Gamma}$, there is a natural equivalence $i_{*}\left(E(\Pi ; \Gamma)_{+} \wedge i^{*} X\right) \simeq X$.

Sketch Proof. If $\Lambda \cap \Pi=e$, then the quotient map $q: \Gamma \longrightarrow G$ maps $\Lambda$ isomorphically onto a subgroup of $G$. Any representation $V$ of $\Lambda$ is therefore of the form $q^{*} W$ for a representation $W$ of $q(\Lambda)$. It follows that the restrictions to $\Lambda$ of the universes $\mathscr{V}_{\Gamma}$ and $q^{*} \mathscr{V}_{G}$ have the same representations. Therefore, on $\Pi$-free $\Gamma$-spectra over $F$, the unit and counit of the adjunction $\left(i_{*}, i^{*}\right)$ are $\mathscr{F}(\Pi ; \Gamma)$ equivalences, in the sense that they are $\Lambda$-equivalences for any $\Lambda$ in $\mathscr{F}(\Pi ; \Gamma)$. Smashing the unit and counit with $E(\Pi ; \Gamma)_{+}$, which has trivial fixed point sets for subgroups not in $\mathscr{F}(\Pi ; \Gamma)$, we obtain natural equivalences, and it follows from Definition 15.5.1 that the unit and counit are themselves equivalences when applied to $\Pi$-free $\Gamma$-spectra. Alternatively, restricting to $s$-fibrant $\Gamma$-spectra over $F$, the conclusion follows fiberwise from its nonparametrized precursor. Since $i_{*}$ is symmetric monoidal, by Theorem 14.2.4, so is the equivalence. The last statement holds since

$$
i_{*}\left(E(\Pi ; \Gamma)_{+} \wedge i^{*} X\right) \simeq E(\Pi ; \Gamma)_{+} \wedge i_{*} i^{*} X \simeq X
$$

\subsection{The fiberwise transfer for $(\Pi ; \Gamma)$-bundles}

Returning to the context of $\S 15.4$, consider a fixed given principal ( $\Pi ; \Gamma)$-bundle $P$, where $\Pi$ is a normal subgroup of $\Gamma$ with quotient group $G$ and quotient map $q: \Gamma \longrightarrow G$. We also consider a $\Gamma$-space $F$ and the associated $(\Pi ; \Gamma)$-bundle

$$
p: E=P \times_{\Pi} F \longrightarrow P \times_{\Pi} *=B .
$$

We have the inclusion $i: q^{*} \mathscr{V}_{G} \longrightarrow \mathscr{V}_{\Gamma}$ of the complete $G$-universe $\mathscr{V}_{G}$ as the universe of $\Pi$-trivial representations in the complete $\Gamma$-universe $\mathscr{V}_{\Gamma}$.

The change of universe functor $i^{*}: \Gamma \mathscr{S}_{F} \longrightarrow \Gamma \mathscr{S}_{F}^{\Pi \text {-triv }}$ is not symmetric monoidal, and it does not preserve dualizable objects. For example, with $F=*$ and $\Pi=e$, the orbit spectrum $i^{*} \Sigma^{\infty} \Gamma / \Lambda$ is not dualizable if $\Lambda$ is a non-trivial subgroup of $\Gamma$. The bundle theoretic study of transfer maps is based on the following result.

Proposition 15.6.1. For $X_{i} \in \Gamma \mathscr{S}_{F_{i}}$,

$$
\mathbb{P}_{F_{1} \times F_{2}}\left(X_{1} \bar{\wedge} X_{2}\right) \simeq \iota^{*}\left(\mathbb{P}_{F_{1}} X_{1} \wedge \mathbb{P}_{F_{2}} X_{2}\right)
$$

where $\iota$ is the inclusion $E_{1} \times_{B} E_{2} \longrightarrow E_{1} \times E_{2}, E_{i}=P \times_{\Pi} F_{i}$. For $X \in \Gamma \mathscr{S}_{F}$ and $Y \in \Gamma \mathscr{S}_{F \times F}$,

$$
\mathbb{P}_{F \times F}\left(\Delta_{F !} X\right) \simeq \delta_{E !} \mathbb{P}_{F} X \quad \text { and } \quad \mathbb{P}_{F} \Delta_{F}^{*} Y \simeq \delta_{E}^{*} \mathbb{P}_{F \times F} Y .
$$

Therefore the functor $\mathbb{P}_{F}=P_{F} i^{*}: H o \Gamma \mathscr{S}_{F} \longrightarrow$ HoG $\mathscr{S}_{E}$ is symmetric monoidal.

Proof. We proved the analogue for the functor $P_{F}$ in Proposition 15.4.5, and $\mathbb{P}_{F}=P_{F} i^{*}$. Since $i^{*}$ commutes with all base change functors, by Proposition 14.2.8, the second and third statements follow immediately from the result for $P_{F}$. However, since $i^{*}$ is not monoidal, we cannot commute it directly with $\bar{\wedge}$ to obtain the first statement. We get around this by using the factorization of $P_{F_{1} \times F_{2}}$ as the composite $\pi^{*}(-) / \Pi$, where $\pi: P \times F_{1} \times F_{2} \longrightarrow F_{1} \times F_{2}$ is the projection. We have 
the following chain of equivalences, the second of which is given by Lemma 15.5.4 and Proposition 15.5.5.

$$
\begin{aligned}
\pi^{*} i^{*}\left(X_{1} \bar{\wedge} X_{2}\right) & \simeq i^{*} \pi^{*}\left(\pi_{1}^{*} X_{1} \wedge_{F_{1} \times F_{2}} \pi_{2}^{*} X_{2}\right) \\
& \simeq i^{*} \pi^{*} \pi_{1}^{*} X_{1} \wedge_{P \times F_{1} \times F_{2}} i^{*} \pi^{*} \pi_{2}^{*} X_{2} \\
& \simeq \pi^{*} \pi_{1}^{*} i^{*} X_{1} \wedge_{P \times F_{1} \times F_{2}} \pi^{*} \pi_{2}^{*} i^{*} X_{2} \\
& \simeq \pi^{*}\left(i^{*} X_{1} \wedge i^{*} X_{2}\right)
\end{aligned}
$$

Passing to orbits over $\Pi$, we obtain the first of the following equivalences, and the second follows from the result for $P_{F}$

$$
\mathbb{P}_{F_{1} \times F_{2}}\left(X_{1} \bar{\wedge} X_{2}\right) \simeq P_{F_{1} \times F_{2}}\left(i^{*} X_{1} \bar{\wedge} i^{*} X_{2}\right) \simeq \iota^{*}\left(\mathbb{P} X_{1} \bar{\wedge} \mathbb{P} X_{2}\right) .
$$

Now Proposition 15.2.4(i) shows that $\mathbb{P}_{F}$ commutes with trace maps.

TheOrem 15.6.2. Let $X \in H o \Gamma \mathscr{S}_{F}$ be dualizable. Then $\mathbb{P}_{F} X \in H o G \mathscr{S}_{E}$ is dualizable. Suppose given a coaction map $\Delta_{X}: X \rightarrow X \wedge_{F} C_{X}$ and a self map $\phi: X \longrightarrow X$. Then

$$
\tau\left(\mathbb{P}_{F} \phi\right) \simeq \mathbb{P}_{F} \tau(\phi): S_{E} \longrightarrow \mathbb{P}_{F} C_{X},
$$

where $\mathbb{P}_{F} X$ is given the coaction map

$$
\mathbb{P}_{F}\left(\Delta_{X}\right): \mathbb{P}_{F} X \longrightarrow \mathbb{P}_{F}\left(X \wedge_{F} C_{X}\right) \simeq \mathbb{P}_{F} X \wedge_{E} \mathbb{P}_{F} C_{X}
$$

These trace maps are maps of $G$-spectra over $E$, rather than over $B$. We can apply $r_{!}, r: E \longrightarrow *$, to obtain trace maps of nonparametrized spectra. This kind of trace map can be viewed as a fiberwise generalization of the kind of nonparametrized trace map that is defined bundle theoretically in the literature. To connect up with the latter, we specialize and change our point of view so as to arrive at bundle theoretic trace maps over $B$. Specializing further to transfer maps, we obtain the promised comparison with the transfer maps of Definition 15.3.3.

With these goals in mind, we now focus on the case $F=*$, so that $E$ above becomes $B$, with $p$ the identity map, and our trace maps are parametrized over $B$. We study our original fixed given $(\Pi ; \Gamma)$-bundle $p: E \longrightarrow B$ in a different fashion. We rename its fiber $M$ to avoid confusion with respect to the role that space is playing. In the theory above, $F$ was a base space for paramentrized spectra and there was no need for $F$ to be dualizable. We now consider the case when $M$ is stably dualizable, so that $\Sigma^{\infty} M_{+}$is dualizable, and we write $\tau_{M}$ for the transfer map $S \longrightarrow \Sigma^{\infty} M_{+}$in $\Gamma \mathscr{S}$, as defined in and after Definition 15.2.1. We apply Theorem 15.6.2 with $F=*$ and $X=\Sigma^{\infty} M_{+}$to obtain the following special case. Here we use the diagonal map induced by the diagonal map of $M$. We have

$$
P_{*}: \Gamma \mathscr{K}_{*} \longrightarrow G \mathscr{K}_{B} \text { and } \mathbb{P}_{*}: \Gamma \mathscr{S} \longrightarrow G \mathscr{S}_{B}
$$

and we observe that, by Proposition 15.4.4,

$$
\mathbb{P}_{*} \Sigma^{\infty} M_{+} \simeq \Sigma^{\infty} P_{*} M_{+}=\Sigma_{B}^{\infty}(E, p)_{+} .
$$

TheOREM 15.6.3. Let $M$ be a compact $\Gamma$-ENR and let $p: E \longrightarrow B$ be a $(\Pi ; \Gamma)$ bundle with fiber $M$ and associated principal $(\Pi ; \Gamma)$-bundle $P$. Let $\phi$ be a self-map of $\Sigma^{\infty} M_{+}$. Then

$$
\tau\left(\mathbb{P}_{*} \phi\right) \simeq \mathbb{P}_{*}(\tau(\phi)): S_{B} \rightarrow \Sigma_{B}^{\infty}(E, p)_{+} .
$$

Therefore, taking $\phi=i d$ and applying $r, r: B \longrightarrow *$,

$$
\tau_{E} \simeq r_{!} \mathbb{P}_{*} \tau_{M}: \Sigma^{\infty} B_{+} \longrightarrow \Sigma^{\infty} E_{+} .
$$


This result gives a clear and precise comparison between the specialization to bundles of the globally defined transfer map for Hurewicz fibrations and the fiberwise transfer map for bundles. Effectively, we have inserted the transfer map for $M_{+}$fiberwise into $P \times_{\Pi}(-)$ to obtain an alternative description of the transfer map for the dualizable $G$-spectrum $\Sigma^{\infty}(E, p)_{+}$over $B$.

There is a useful reinterpretation of the description of transfer maps given by Theorem 15.6.3. Consider $\pi: P \longrightarrow *$. By Proposition 14.4.4, instead of applying $r_{!}, r: B \longrightarrow *$, to orbit spectra under the action of $\Pi$, we could first apply $\pi_{!}$and then pass to orbits. For a $\Gamma$-spectrum $X$, we have a natural isomorphism

$$
\pi ! \pi^{*} i^{*} X \cong P_{+} \wedge i^{*} X
$$

and a natural equivalence

$$
i_{*}\left(P_{+} \wedge i^{*} X\right) \simeq P_{+} \wedge X
$$

Corollary 15.6.4. let $M$ be a compact $\Gamma$-ENR and let $p: E \longrightarrow B$ be a $(\Pi ; \Gamma)$ bundle with fiber $M$ and associated principal $(\Pi ; \Gamma)$-bundle $P$. Then the transfer $\tau_{E}: \Sigma^{\infty} B_{+} \longrightarrow \Sigma^{\infty} E_{+}$is obtained by passage to orbits over $\Pi$ from the map

$$
\tilde{\tau}=i d \wedge i^{*} \tau_{M}: P_{+} \wedge i^{*} S \longrightarrow P_{+} \wedge i^{*} \Sigma^{\infty} M_{+},
$$

and $i_{*} \tilde{\tau}$ can be identified with

$$
i d \wedge \tau_{M}: P_{+} \wedge S \longrightarrow P_{+} \wedge \Sigma^{\infty} M_{+} .
$$

REMARK 15.6.5. The corollary gives exactly the transfer map as defined by Lewis and May [98, IV.3.1]. Working in the nonparametrized context, they tried in vain to obtain a spectrum level transfer map for Hurewicz fibrations over general base spaces. The comparison here also sheds light on the relationship between the two constructions of Becker and Gottlieb $[9,10]$, both of which require finite dimensional base spaces. The first is bundle theoretic and is easily seen to be equivalent to the construction in this section by using Atiyah duality to interpret $\tau_{M}$ for a $\Gamma$-manifold $M$. Precisely, by [98, IV.2.3], if $M$ is embedded in $V$ with normal bundle $\nu$ and $\tau$ is the tangent bundle of $M$, then the transfer map $\tau_{M}$ is homotopic to the map obtained by applying the functor $\Sigma^{-V} \Sigma^{\infty}$ to the composite of the Pontryagin-Thom map $S^{V} \longrightarrow T \nu$ and the map $T \nu \longrightarrow T(\nu \oplus \tau) \cong M_{+} \wedge S^{V}$ induced by the inclusion $\nu \longrightarrow \nu \oplus \tau$. The second, which is generalized to the equivariant setting by Waner [170], is fibration theoretic and is easily seen to be equivalent to the construction of $\$ 15.2$. Another approach to the comparison is to show that suitable Hurewicz fibrations are equivalent to bundles, as is done by Casson and Gottlieb in [28].

REMARK 15.6.6. Since our definition coincides with that of [98, IV.3.1], the properties of the transfer catalogued in [98, IV $\S 3-7]$ apply verbatim. Many of these properties generalize directly to the parametrized trace and transfer maps of Theorem 15.6.2. The definition of [98, IV.3.1] actually works more generally, with $P$, or rather $i^{*} \Sigma^{\infty} P_{+}$, replaced by a general $\Pi$-free $\Gamma$-spectrum indexed on $\mathscr{V}_{G}$. The constructions here admit similar generalizations. One way to achieve this with minimal work is to use the case $P=E(\Pi ; \Gamma)$ of the construction already on hand. Thus, for a $\Pi$-free $\Gamma$-spectrum $\bar{P}$ over $F$ indexed on $\mathscr{V}_{G}$, we can define

$$
\mathbb{P}_{F} X=E(\Pi ; \Gamma)_{F}\left(\bar{P} \wedge_{F} i^{*} X\right)
$$

and develop parametrized trace and transfer maps from there. We leave the further development of the theory to the interested reader. 


\section{CHAPTER 16}

\section{Closed symmetric bicategories}

\section{Introduction}

In Chapter 18, we describe the parametrized analogue of Spanier-Whitehead duality. When we turn to parametrized homology and cohomology theories, it will give duality there exactly as Spanier-Whitehead duality does in classical homology and cohomology. That theory is due to Costenoble and Waner [41]. However, in their work, which is based on the foundations given by earlier drafts of this book, ${ }^{1}$ everything is done directly in terms of the constructions that we have already presented. A new conceptual framework gives the theory greater clarity and force, as our applications in Chapter 18 will show. We describe that framework here.

In fact, Costenoble-Waner duality is an application of a specialization to parametrized spectra of a general categorical duality theory in closed symmetric bicategories. After recalling basic language about bicategories in $\$ 16.1$, we describe symmetric bicategories and closed symmetric bicategories informally in $\S 16.2$ and $\S 16.3$. Details of the relevant categorical coherence theory will appear elsewhere, but the reader should have no difficulty accepting them on faith. Such structures arise in other branches of mathematics, as we illustrate with the closed symmetric bicategory of bimodules. That example should make the idea clear. Analogues for differential graded bimodules and bimodules over highly structured ring spectra also promise to be of interest. As we explain in $\S 16.4$, formal duality theory in such a bicategory works in much the same way as the duality theory in the special case of closed symmetric monoidal categories that we used in the previous chapter. In the brief $\S 16.5$, we give a simple result with powerful implications. It shows how one can sometimes compose dualities to create new ones.

The most interesting examples of closed symmetric bicategories, in both algebra and topology, are suitably triangulated. To make use of this, one must formulate appropriate compatibility relations between the triangulation and the bicategory structure. After a quick review of triangulated categories in $\$ 16.6$, we give a start by explaining the most basic axioms for such a structure in $\S 16.7$, following [124]. We discuss the behavior of duality in triangulated symmetric bicategories in $\S 16.8$, largely following [76].

We emphasize that this chapter is just a beginning and not a full development. We believe that this theory will have many future applications, and it raises quite a few new questions in higher category theory. We shall mention in passing a few concepts relevant to giving a full categorical depiction of the structures at hand, but we shall focus on the bare essentials.

\footnotetext{
${ }^{1}$ Costenoble and Waner plan to revise [41] for publication based on the current version.
} 


\subsection{Recollections about bicategories}

We outline a description of bicategories that is slanted towards our new concepts and introduces an absolute minimum of relevant categorical terminology.

A bicategory has three layers of structure: objects (or 0-cells), morphisms between objects (or 1-cells) and morphisms between morphisms (or 2-cells). The standard example is categories, functors, and natural transformations, but a more relevant example is rings, bimodules, and homomorphisms between bimodules, as discussed in Example 16.2.2 below. A particularly relevant point of view is that bicategories naturally extend the notion of a monoidal category. Recall that a monoidal category $\mathscr{M}$ is a category together with a product functor $\mathscr{M} \times \mathscr{M} \longrightarrow \mathscr{M}$ and a unit functor $* \longrightarrow \mathscr{M}$, where $*$ is the trivial category, that are associative and left and right unital up to coherent natural isomorphism. One can think of $\mathscr{M}$ as a bicategory with a single 0 -cell such that the 1-cells and 2-cells are the objects and morphisms of $\mathscr{M}$. Bicategories can be thought of as monoidal categories with "many objects", and that point of view leads to the correct formal definition.

Precisely, a bicategory $\mathscr{C}$ has a class of objects, or 0 -cells, and for each pair of 0 -cells $A, B$ it has a category $\mathscr{C}(A, B)$. Each $\mathscr{C}(A, B)$ has a class of objects, or 1-cells, and for each pair of 1-cells $X, Y$ there is a set $\mathscr{C}(X, Y)$ of morphisms, or 2-cells, $X \longrightarrow Y$. We write composition of 2-cells $\alpha: X \longrightarrow Y$ and $\beta: Y \longrightarrow Z$ simply by juxtaposition, $\beta \alpha$; note that $X, Y$, and $Z$ must all be 1-cells $A \longrightarrow B$ for the same $A$ and $B$. This is called vertical composition. Finally, $\mathscr{C}$ also has a horizontal composition functor

$$
\odot: \mathscr{C}(B, C) \times \mathscr{C}(A, B) \longrightarrow \mathscr{C}(A, C)
$$

for each triple of 0-cells and a unit functor $U_{A}: * \longrightarrow \mathscr{C}(A, A)$ for each 0-cell, that are associative and left and right unital up to coherent natural isomorphism.

The bulk of the definition is the specification of "coherence" but, exactly as in the case of monoidal categories, that is given just by a unit triangle and the standard associativity pentagon for a quadruple of composable 1-cells [99, XII§6]. A bicategory is a 2-category if the associativity and unit 2-cells are identity maps.

We shall have many maps between bicategories, but we shall rarely be very categorically explicit. Still, it will be helpful to have the relevant language on hand. A lax functor (alias morphism) $F: \mathscr{C} \longrightarrow \mathscr{D}$ between bicategories is the many object generalization of a lax monoidal functor between monoidal categories. It consists of a function $F$ on 0 -cells and, for each pair $A, B$ of 0 -cells of $\mathscr{C}$, a functor $F=F_{A, B}: \mathscr{C}(A, B) \longrightarrow \mathscr{D}(F A, F B)$, together with natural 2-cells

$$
F Y \odot F X \longrightarrow F(Y \odot X) \text { and } U_{F A} \longrightarrow F U_{A}
$$

that satisfy suitable coherence conditions. Dually, an oplax functor $F: \mathscr{C} \longrightarrow \mathscr{D}$ has the direction of its unit and composition 2-cells reversed:

$$
F(Y \odot X) \longrightarrow F Y \odot F X \text { and } F U_{A} \longrightarrow U_{F A} .
$$

In either case, $F$ is called a pseudo-functor (alias homomorphism) if these naturality 2-cells are isomorphisms and a strict functor (alias strict homomorphism) if these 2-cells are identity maps.

For a property $P$ of functors, a lax functor $F$ is said to be locally $P$ if each $F_{A, B}$ has property $P$. Local equivalences are of particular interest. There is also a notion of internal equivalence between 0-cells $A$ and $B$ of $\mathscr{C}$, namely a pair of 1cells $X: A \longrightarrow B$ and $Y: B \longrightarrow A$ together with isomorphism 2-cells $U_{A} \cong Y \odot X$ 
and $U_{B} \cong X \odot Y$. A biequivalence of bicategories is a local equivalence $F$ that is essentially surjective on 0 -cells, in the sense that every 0 -cell of the target is internally isomorphic to a 0 -cell coming from the source. In our examples, $F$ will be a bijection on 0-cells.

A lax natural transformation (alias transformation) $\sigma$ between lax functors $F, G: \mathscr{C} \longrightarrow \mathscr{D}$ consists of 1-cells $\sigma_{A}: F A \longrightarrow G A$ and, for a 1-cell $X: A \longrightarrow B$ of $\mathscr{C}, 2$-cells $\sigma_{X}: G X \odot \sigma_{A} \longrightarrow \sigma_{B} \odot F X$, natural in $X$, such that appropriate coherence diagrams relating these 2-cells to the unit and associativity isomorphism 2-cells commute. We replace lax by strong or strict if these 2-cells are isomorphisms or identity maps. Dually, we have oplax natural transformations between oplax functors.

Finally, a modification (no known alias) between transformations $\sigma, \sigma^{\prime}$ from $F$ to $G$ consists of 2-cells $\Gamma_{A}: \sigma_{A} \longrightarrow \sigma_{A}^{\prime}$ making the appropriate diagram commute; there are no variants at this level.

\subsection{The definition of symmetric bicategories}

The most interesting monoidal categories are the symmetric monoidal ones. We could view a symmetric monoidal category as a "symmetric bicategory with a single object", if only somebody had previously defined the notion of a symmetric bicategory. It appears that nobody has. This is perhaps not surprising, for two reasons. First, it seems counterintuitive to talk about commutativity in view of the directionality of 1-cells when one has many 0-cells, and in fact it will be essential to make a distinction between symmetry and commutativity (as presaged in Remark 2.5.4). Second, it turns out that the sensible many object generalization, when restricted to one object bicategories, actually gives a more general structure than a symmetric monoidal category, as we shall see. In any case, we proceed to fill the gap.

The opposite bicategory $\mathscr{C}^{\text {op }}$ of $\mathscr{C}$ has the same 0-cells as $\mathscr{C}$ does but has $\mathscr{C}^{\mathrm{op}}(B, A)=\mathscr{C}(A, B)$. This reverses the source and target of 1-cells $X$; if we write $X^{\text {op }}$ for a 1-cell $A \longrightarrow B$ of $\mathscr{C}$ regarded as a 1-cell $B \longrightarrow A$ of $\mathscr{C}^{\text {op }}$, then the 2-cells $X^{\mathrm{op}} \longrightarrow Y^{\mathrm{op}}$ in $\mathscr{C}^{\mathrm{op}}(B, A)$ are the same as the 2-cells $X \longrightarrow Y$ in $\mathscr{C}(A, B)$. Intuitively, an involution $t$ on a bicategory is a biequivalence between $\mathscr{C}$ and $\mathscr{C}^{o p}$ that is a bijection on 0-cells, but we prefer to be more explicit.

Definition 16.2.1. An involution on a bicategory $\mathscr{C}$ consists of the following data.

(i) A bijection $t$ on the 0 -cells of $\mathscr{C}$ such that $t t A=A$.

(ii) Equivalences of categories $t: \mathscr{C}(A, B) \longrightarrow \mathscr{C}(t B, t A)=\mathscr{C}^{\mathrm{op}}(t A, t B)$, with the equivalences given by isomorphism 2-cells $\xi$ : id $\cong t t$.

(iii) Natural isomorphism 2-cells $\iota=\iota_{A}: U_{t A} \longrightarrow t U_{A}$ for 0-cells $A$ and

$$
\gamma=\gamma_{X, Y}: t Y \odot{ }^{\mathrm{op}} t X \equiv t X \odot t Y \longrightarrow t(Y \odot X)
$$

for 1-cells $X: A \longrightarrow B$ and $Y: B \longrightarrow C$; the left and right unit 2-cells $\lambda$ and $\rho$ must be related by the equivalent equalities of 2 -cells

$$
t\left(\lambda_{X}\right) \gamma_{X, U_{X}}(\mathrm{id} \odot \iota)=\rho_{t X} \quad \text { and } \quad t\left(\rho_{X}\right) \gamma_{U_{X}, X}(\iota \odot \mathrm{id})=\lambda_{t X},
$$


the appropriate hexagonal coherence diagram relating $\gamma$ to the associativity 2cell $\alpha$ must commute, and the following diagram relating $\xi$ to $\gamma$ must commute:

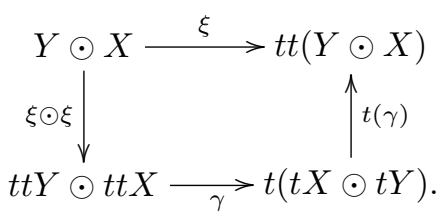

A symmetric bicategory $\mathscr{C}$ is a bicategory equipped with an involution. We say that $\mathscr{C}$ is a symmetric 2 -category if its associativity and unit 2-cells are identity maps. A 0 -cell $A$ of $\mathscr{C}$ is said to be commutative if $t A=A$.

The functors $t$ in (ii) are often isomorphisms of categories, with $\xi=$ id and $t(\gamma) \gamma=$ id. It would not be illuminating to write down the hexagon. Actually, the hexagon in the usual definition of a pseudo-functor [93], applied to our involution $t$, and the hexagon adapted from the usual definition of a symmetric monoidal category $[99$, p. 184] do not look exactly the same, but they are equivalent by elementary diagram chases. Observe that if we have a single 0-cell $A$, then $t: \mathscr{C}(A, A) \longrightarrow \mathscr{C}(A, A)$ need not be the identity functor, as it would be if $\mathscr{C}$ were symmetric monoidal.

We require of a lax functor $F: \mathscr{C} \longrightarrow \mathscr{D}$ between symmetric bicategories that $F t=t F$ on 0 -cells, that the functors $t F$ and $F t$ from $\mathscr{C}(A, B)$ to $\mathscr{D}(t F B, t F A)$ be naturally isomorphic, and that the 2-cells giving the isomorphism commute appropriately with the 2 -cells $\xi$ and $\gamma$ in $\mathscr{C}$ and $\mathscr{D}$. The details of this definition and of the symmetric versions of other standard bicategorical concepts are straightforward.

We have not fully adopted the philosophy of higher category theory, which would require us to weaken all identities in our definition to isomorphisms. Rather, we have adopted a pragmatic compromise dictated in part by our desire to keep close to the classical theory of symmetric monoidal categories and in larger part by the nature of the examples that we know. We could weaken our involutory conditions, perhaps relaxing the identity and equivalence conditions of (i) and (ii) and certainly dropping the displayed diagram, at the expense of adding further coherence diagrams. Doing so, we reach the as yet unexplored notion of a braided bicategory, the many object version of a braided monoidal category. Lest the reader think that our definition is esoteric, we give an elementary paradigmatic example.

EXAMPLE 16.2.2. Let $R$ be a commutative ring, perhaps $\mathbb{Z}$. We define the closed symmetric bicategory $\mathscr{B}_{R}$ of bimodules over $R$-algebras. The 0 -cells are the $R$-algebras (rings if $R=\mathbb{Z}$ ). For a 0 -cell $A, t A$ is the opposite $R$-algebra, namely the same $R$-module but with the opposite multiplication. It is usual to write $a^{\text {op }}$ for an element of $A$ regarded as an element of $A^{\mathrm{op}}$ and to write $a^{\mathrm{op}} b^{\mathrm{op}}=b a$ for the product, but it would be more logical to write $a{ }^{\circ \mathrm{op}} b=b \cdot a$ to indicate that it is only the product and not the underlying $R$-module that is changed. The commutative 0 -cells are the commutative $R$-algebras. The 1-cells $X: A \longrightarrow B$ are the $(B, A)$ bimodules, and $t X: t B \longrightarrow t A$ is the $R$-module $X$ regarded as a $(t A, t B)$-bimodule under the standard identification of left actions by $A$ with right actions by $t A$, $a x=x a^{\mathrm{op}}$ or, in more logical notation, $a \cdot x=x \cdot{ }^{\mathrm{op}} a$. The 2-cells $\alpha: X \longrightarrow Y$ are the morphisms of $(B, A)$-bimodules, and $t(\alpha)$ is the same morphism of $R$-modules regarded as a morphism of $(t A, t B)$-bimodules. Finally, we define $\odot$ by

$$
\odot=\otimes_{B}: \mathscr{B}_{R}(B, C) \times \mathscr{B}_{R}(A, B) \longrightarrow \mathscr{B}_{R}(A, C) .
$$


(Our left-right conventions are dictated by the order of composition here.) The unit $U_{A}$ is just $A$ regarded as an $(A, A)$-bimodule under left and right multiplication, and the associativity and unity isomorphisms are the usual ones. Obviously $t U_{A}=U_{t A}$. The symmetry isomorphism

$$
\gamma: t X \otimes_{t B} t Y \longrightarrow t\left(Y \otimes_{B} X\right)
$$

is the usual bimodule symmetry isomorphism, $\gamma(x \otimes y)=y \otimes x$. Observe that $\mathscr{B}_{R}(A, R)$ and $\mathscr{B}_{R}(R, A)$ are the categories of right and left $A$-modules, respectively. If we restrict attention to the full sub bicategory with a single commutative 0-cell $A=t A$, we obtain the category of $(A, A)$-bimodules, on which $t$ is not the identity functor. The symmetric monoidal category of $A$-modules sits inside this as the subcategory of central $(A, A)$-modules $X$, namely those for which $a x=x a$ for all $a$ and $x$.

EXAMPLE 16.2.3. The previous example works equally well if we take $R$ to be a commutative differential graded $k$-algebra for a commutative ring $k$. Here we take the 0 -cells to be the differential graded $R$-algebras $A$ and the 1-cells $A \longrightarrow B$ to be the differential graded $(B, A)$-bimodules. Of course, we use the graded symmetry $\gamma$, with the usual sign. We may pass to derived categories of bimodules, keeping the 0 -cells and 1-cells the same, to obtain another example. Similarly, topologically, we can take $R$ to be a commutative ring spectrum in one of the modern categories of spectra, such as the category of orthogonal spectra. Here we take the 0-cells to be the $R$-algebras $A$ and the 1 -cells to be the $(B, A)$-bimodules. Again, we can pass to derived homotopy categories of bimodules. In fact, formalizing the construction of Example 16.2.2, we obtain a symmetric bicategory of algebras and bimodules over any commutative monoid $R$ in any cocomplete symmetric monoidal category $\mathscr{M}$. A similar remark applies to the additional structure on $\mathscr{B}_{R}$ discussed in Examples 16.2.6, 16.3.7, and 16.3.9 below.

The definition of symmetric bicategories would be of little use without a coherence theorem, and the expected result reads as follows.

TheOREM 16.2.4. Any symmetric bicategory is biequivalent to a symmetric 2-category.

The one object case is closely related to the well-known equivalence between symmetric monoidal categories and permutative categories (see, for example, [110, 4.2]). The non-symmetric analogue is a well-known result relating bicategories and 2-categories [93], and its proof adapts readily. The bicategories $\mathscr{B}_{R}$ and our topological examples have much further structure, and the relevant coherence problems, in particular that of determining which diagrams relating the coherence constraints must commute, have generally not yet been addressed. However, several constituents of the structure in our examples have been studied separately.

EXAMPLE 16.2.5. A sensible categorical way to incorporate maps of $R$-algebras along with the other structure that we have described is to observe that $\mathscr{B}_{R}$ is part of a "pseudo (or semi-strict) double category" [69, App]. A pseudo double category is a more general structure than that of a bicategory which has vertical as well as horizontal 1-cells. We can extend $\mathscr{B}_{R}$ to such a structure by letting the maps of $R$ algebras give the vertical 1-cells and allowing correspondingly more general 2-cells. However, in both this algebraic example and our topological examples, we shall encode base change functors induced by maps in terms of horizontal composition, 
and it is usually the base change functors, rather than the maps that induce them, that are of primary interest. However, we will encounter several places in the theory where not working in the more general categorical framework will leave us without categorical language to express the phenomena we encounter.

We also have the following fundamental piece of structure, whose relationship with the closed symmetric bicategory structure has yet to be fully formalized. In fact, while our notion of a "symmetric bicategory" is new, there is an earlier and more obvious notion of a "symmmetric monoidal bicategory", and $\mathscr{B}_{R}$ has both such structures.

EXAMPLE 16.2.6. The bicategory $\mathscr{B}_{R}$ is a symmetric monoidal bicategory under the tensor product

$$
\otimes=\otimes_{R}: \mathscr{B}_{R} \times \mathscr{B}_{R} \longrightarrow \mathscr{B}_{R}
$$

(where $\otimes$ is applied to $R$-algebras, to $R$-bimodules, and to morphisms thereof). The unit $* \longrightarrow \mathscr{B}_{R}$ is given by $R$ (regarded as a 0-cell, a 1-cell $(R, R)$-bimodule, and a 2 -cell identity map). The unity, associativity, and commutativity constraints are evident.

We shall encounter precisely analogous structure in our bicategory of parametrized spectra over varying base spaces. The external smash product there is the analogue of $\otimes_{R}$, and the external commutativity isomorphism induced from that of Remark 2.5.4 is the analogue of the commutativity constraint.

Under the name Gray category, monoidal bicategories and symmetric monoidal bicategories with appropriate strictness conditions have been studied extensively. See, for example, the papers $[44,70,157]$ of Gray, Day, and Street.

REMARK 16.2.7. Just as monoidal categories are one object bicategories, so monoidal bicategories are one object tricategories [68]. In fact, we actually have something like a tricategory in sight, with 0-cells commutative rings, 1-cells algebras, 2-cells bimodules, and 3-cells maps of bimodules. However, it does not have the strictly hierarchical structure of a tricategory since we do not view algebras as morphisms of rings. In line with Example 16.2.5, we can codify the structure on hand, incorporating the maps of commutative rings and the maps of algebras, in a previously unexplored categorical structure that has both vertical and horizontal cells. We will encounter the same formal structure in parametrized homotopy theory and, again, the lack of the relevant categorical theory will leave us without language to express some of the phenomena we encounter. With Michael Shulman, we intend to give an exposition elsewhere.

\subsection{The definition of closed symmetric bicategories}

While the literature of bicategories is extensive, closed bicategories have been less studied and less exploited. This is unfortunate, since such structures appear quite commonly in mathematics. We shall indicate the basic facts, but, here again, a thorough study of categorical coherence is needed. Coherence in closed monoidal and closed symmetric monoidal categories has been studied by Eilenberg and Kelly [58] and Kelly and Mac Lane [88].

Definition 16.3.1. A bicategory $\mathscr{C}$ is right and left closed (or $\odot$-closed), if there are right and left internal hom (or $\odot$-hom) functors

$$
\triangleright: \mathscr{C}(A, B)^{\text {op }} \times \mathscr{C}(A, C) \longrightarrow \mathscr{C}(B, C)
$$


and

$$
\triangleleft: \mathscr{C}(A, C) \times \mathscr{C}(B, C)^{\text {op }} \longrightarrow \mathscr{C}(A, B)
$$

for all triples of 0-cells $A, B, C$ and "internal adjunction" natural isomorphisms

$$
\mathscr{C}(X, Z \triangleleft Y) \cong \mathscr{C}(Y \odot X, Z) \cong \mathscr{C}(Y, X \triangleright Z)
$$

for 1-cells $X: A \longrightarrow B, Y: B \longrightarrow C$, and $Z: A \longrightarrow C$.

The adjoints of identity 2-cells then give unit and counit 2-cells

$$
\begin{aligned}
& \varepsilon:(X \triangleright Z) \odot X \longrightarrow Z \text { and } \eta: Y \longrightarrow X \triangleright(Y \odot X) \\
& \varepsilon: Y \odot(Z \triangleleft Y) \longrightarrow Z \text { and } \eta: X \longrightarrow(Y \odot X) \triangleleft Y .
\end{aligned}
$$

Of course, $\mathscr{C}$ might be right closed without being left closed, and vice versa.

DEFINITION 16.3.5. A symmetric bicategory is closed if it is left closed, in which case it is also right closed with

$$
X \triangleright Z \cong t(t Z \triangleleft t X): B \longrightarrow C
$$

for $X: A \longrightarrow B$ and $Z: A \longrightarrow C$.

REMARK 16.3.6. The notations $\triangleright$ and $\triangleleft$ for the internal $\odot$-hom functors seem to be nonstandard, but are convenient and have been used previously for this purpose, for example in [89]. (There does not seem to be a standard notation). One can think of $X \triangleright Z: B \longrightarrow C$ as the 1-cell of "maps pointing right from $X$ to $Z$ " and $Z \triangleleft Y: A \longrightarrow B$ as the 1-cell of "maps pointing left from $Y$ to $Z$ ". The triangles represent arrowheads pointing in the appropriate direction. Mnemonically, $X \triangleright Z$ is a 1-cell from the target of $X$ to the target of $Z$ (with $X$ and $Z$ having the same source) and $Z \triangleleft Y$ is a 1-cell from the source of $Z$ to the source of $Y$ (with $Y$ and $Z$ having the same target). We write sources on the left and targets on the right.

ExAMPLE 16.3.7. The symmetric bicategory $\mathscr{B}_{R}$ of bimodules over $R$-algebras is closed. For a $(B, A)$-bimodule $X,(C, B)$-bimodule $Y$, and $(C, A)$-bimodule $Z$, the left and right internal $\odot$-homs are given by

$$
Z \triangleleft Y=\operatorname{Hom}_{C}(Y, Z) \quad \text { and } \quad X \triangleright Z=\operatorname{Hom}_{A}(X, Z) .
$$

Here $Z \triangleleft Y$ is a $(B, A)$-bimodule and $X \triangleright Z$ is a $(C, B)$-bimodule. The required internal adjunctions

$$
\mathscr{B}_{R}\left(Y, \operatorname{Hom}_{A}(X, Z)\right) \cong \mathscr{B}_{R}\left(Y \otimes_{B} X, Z\right) \cong \mathscr{B}_{R}\left(X, \operatorname{Hom}_{C}(Y, Z)\right)
$$

relating the morphism categories $\mathscr{B}_{R}(B, C), \mathscr{B}_{R}(A, C)$ and $\mathscr{B}_{R}(A, B)$ are evident.

EXAMPLE 16.3.8. The closed symmetric bicategory structure on $\mathscr{B}_{R}$ encodes base change along maps of $R$-algebras. For a map $f: A^{\prime} \longrightarrow A$ of $R$-algebras, thought of as 0-cells, we have "base change bimodules", namely the 1-cells

$$
A_{f}: A^{\prime} \longrightarrow A \text { and }{ }_{f} A=t A_{f}: A \longrightarrow A^{\prime} \text {, }
$$

where $A_{f}$ and ${ }_{f} A$ denote $A$ regarded as an $\left(A, A^{\prime}\right)$-bimodule or as an $\left(A^{\prime}, A\right)$ bimodule. The action of $A^{\prime}$ is given by pullback along $f$, the action of $A$ is the evident one, and the equality ${ }_{f} A=t A_{f}$ is evident. For any $R$-algebra $B$, pullback of right actions along $f$ gives a functor $f^{*}: \mathscr{B}_{R}(A, B) \longrightarrow \mathscr{B}_{R}\left(A^{\prime}, B\right)$, which has a left adjoint $f_{\text {! }}$ and a right adjoint $f_{*}$ given by extension and coextension of scalars. By inspection, we have

$$
f^{*} M \cong M \odot A_{f}, \quad f_{!} M^{\prime} \cong M^{\prime} \odot{ }_{f} A, \text { and } f_{*} M^{\prime} \cong A_{f} \triangleright M^{\prime} .
$$


Similarly, for a map $g$ : $B^{\prime} \longrightarrow B$ of $R$-algebras and any $R$-algebra $A$, the pullback of action functor $g^{*}: \mathscr{B}_{R}(A, B) \longrightarrow \mathscr{B}_{R}\left(A, B^{\prime}\right)$ has a left adjoint $g$ ! and a right adjoint $g_{*}$, and these satisfy

$$
g^{*} N \cong{ }_{g} B \odot N, \quad g_{!} N^{\prime} \cong B_{g} \odot N^{\prime}, \text { and } g_{*} N^{\prime} \cong{ }_{g} B \triangleleft N^{\prime} .
$$

We also have a closed structure for the symmetric monoidal bicategory structure on $\mathscr{B}_{R}$ given in Example 16.2.6. Again, our bicategory of parametrized spectra will have analogous structure.

EXAMPLE 16.3.9. The $\odot$-closed symmetric bicategory $\mathscr{B}_{R}$ is also a $\otimes$-closed symmetric monoidal bicategory. Its left and right internal $\otimes$-hom objects are given by the $(B, A)$-bimodules and $(D, C)$-bimodules

$$
\operatorname{Hom}_{(D, C)}(Y, Z) \text { and } \operatorname{Hom}_{(B, A)}(X, Z) \text {, }
$$

where $X$ is a $(B, A)$-bimodule, $Y$ is a $(D, C)$-bimodule, and $Z$ is a $(B \otimes D, A \otimes C)$ bimodule. These give internal adjunctions

$$
\mathscr{B}_{R}\left(Y, \operatorname{Hom}_{(B, A)}(X, Z)\right) \cong \mathscr{B}_{R}(X \otimes Y, Z) \cong \mathscr{B}_{R}\left(X, \operatorname{Hom}_{(D, C)}(Y, Z)\right)
$$

relating the morphism categories $\mathscr{B}_{R}(A, B), \mathscr{B}_{R}(A \otimes C, B \otimes D)$, and $\mathscr{B}_{R}(C, D)$.

The adjunctions (16.3.2) imply various isomorphisms and natural transformations. Most of them would be familiar in $\otimes$ and Hom notation. Even in the symmetric case, it is most efficient to carefully distinguish between $\triangleleft$ and $\triangleright$. Here we could instead systematically collapse our dichotomy into statements concerning only one of these functors, using the canonical isomorphisms $X \cong t t X$ and $t(X \triangleright Z) \cong t Z \triangleleft t X$. Spelling this out is essential for purposes of calculation. In particular, this is where the symmetry isomorphism $\gamma$ and attendant signs in graded situations come into play. However, this is a routine categorical exercise. It is used implicitly throughout the theory of symmetric monoidal categories $\mathscr{M}$, where one defines only a single hom functor and regards it via $\gamma$ as giving both of the internal adjunctions displayed in (16.3.2). That is, one uses $\gamma$ to verify one of the two isomorphisms

$$
\mathscr{M}(X, \operatorname{Hom}(Y, Z)) \cong \mathscr{M}(X \otimes Y, Z) \cong \mathscr{M}(Y, \operatorname{Hom}(X, Z)) .
$$

This implicit use of $\gamma$ forces explicit use of it elsewhere in that theory.

We have natural isomorphisms

$$
(Y \odot X) \triangleright Z \cong Y \triangleright(X \triangleright Z)
$$

for $X: A \longrightarrow B, Y: B \longrightarrow C$, and $Z: A \longrightarrow D$,

$$
Z \triangleleft(Y \odot X) \cong(Z \triangleleft Y) \triangleleft X
$$

for $X: A \longrightarrow B, Y: B \longrightarrow C$, and $Z: D \longrightarrow C$, and

$$
(X \triangleright Z) \triangleleft Y \cong X \triangleright(Z \triangleleft Y)
$$

for $X: A \longrightarrow B, Y: C \longrightarrow D$, and $Z: A \longrightarrow D$. The proofs are exercises in adjunctions and the Yoneda lemma. For example, the last is a direct consequence of the associativity (up to natural isomorphism) of composition since

$$
\mathscr{C}(W,(X \triangleright Z) \triangleleft Y) \cong \mathscr{C}((Y \odot W) \odot X, Z)
$$

and

for $W: B \longrightarrow C$.

$$
\mathscr{C}(W, X \triangleright(Z \triangleleft Y)) \cong \mathscr{C}(Y \odot(W \odot X), Z)
$$


By defining their adjoints in terms of evaluation maps $\varepsilon$ of (16.3.3) and (16.3.4), we obtain natural maps

$$
\mu: Z \odot(X \triangleright Y) \longrightarrow X \triangleright(Z \odot Y)
$$

for $X: A \longrightarrow B, Y: A \longrightarrow C$, and $Z: C \longrightarrow D$,

$$
\nu:(Z \triangleleft Y) \odot W \longrightarrow(Z \odot W) \triangleleft Y
$$

for $Y: B \longrightarrow C, Z: A \longrightarrow C$, and $W: D \longrightarrow A$, and

$$
\omega:(Z \triangleleft Y) \odot(X \triangleright W) \longrightarrow X \triangleright(Z \odot W) \triangleleft Y
$$

for $X: A \longrightarrow B, W: A \longrightarrow C, Y: D \longrightarrow E$, and $Z: C \longrightarrow E$; we have omitted parentheses on the right since the two ways of parenthesizing the target give isomorphic results, by (16.3.12). When $X=U_{A}$ or $Y=U_{E}, \omega$ specializes to $\nu$ or $\mu$. Conversely, parenthesizing in the two possible ways, $\omega$ is the composite

$$
(Z \triangleleft Y) \odot(X \triangleright W) \stackrel{\mu}{\longrightarrow} X \triangleright((Z \triangleleft Y) \odot W) \stackrel{\operatorname{id} \triangleright \nu}{\longrightarrow} X \triangleright((Z \odot W) \triangleleft Y)
$$

or

$$
(Z \triangleleft Y) \odot(X \triangleright W) \stackrel{\nu}{\longrightarrow}(Z \odot(X \triangleright W)) \triangleleft Y \stackrel{\mu \triangleleft \mathrm{id}}{\longrightarrow}(X \triangleright(Z \odot W)) \triangleleft Y .
$$

\subsection{Duality in closed symmetric bicategories}

We develop duality theory in closed bicategories. We could focus just on left closed or right closed $\mathscr{C}$ using $\mathscr{C}^{\mathrm{op}}$ to translate from one to the other. However, we are interested in the closed symmetric case, where both versions are available. If we were writing for the categorical cognoscenti, we would use single arrows for 1-cells and double arrows for 2-cells, drawing pasting diagrams to illustrate our concepts. We prefer to be more down to earth. As in the bimodule examples in the previous sections, which illustrate our philosophy that a symmetric bicategory is to be thought of as a symmetric monoidal category with many objects, we want to consider the 1-cells as the fundamental objects of study, and we thus want to understand duality theory for 1-cells. For example, a module over a commutative $R$-algebra $B$ is "dualizable" if and only if it is finitely generated and projective, and it is natural to formulate a related categorical concept of dualizability for bimodules. We have not yet studied even such elementary algebraic examples, but it is clear that they are relevant to Morita theory.

We shall write $B$ for $U_{B}$ for simplicity of notation. It should be clear from context when $B$ is being viewed as a 1-cell (like an $R$-algebra $B$ viewed as a $(B, B)$ bimodule) rather than as a 0-cell. Since our internal homs exhibit an asymmetry, we start with a more symmetric version of duality that makes no reference to them and applies in any bicategory, not necessarily symmetric or closed. We shall omit most of the categorical proofs. They are diagram chases similar, but not always identical, to those in the one object symmetric monoidal case as given, for example, in [98, III $\S 1]$. We follow part of the treatment there (where dualizable objects are called "finite"), except that we reorder its definitions and results.

Definition 16.4.1. Let $X: B \longrightarrow A$ and $Y: A \longrightarrow B$ be 1-cells in a bicategory $\mathscr{C}$. Then $(X, Y)$ is said to be a dual pair if there are 2-cells

$$
\eta: A \longrightarrow X \odot Y \text { and } \varepsilon: Y \odot X \longrightarrow B
$$


called coevaluation and evaluation maps, such that the following diagrams commute in $\mathscr{C}(B, A)$ and $\mathscr{C}(A, B)$, respectively.
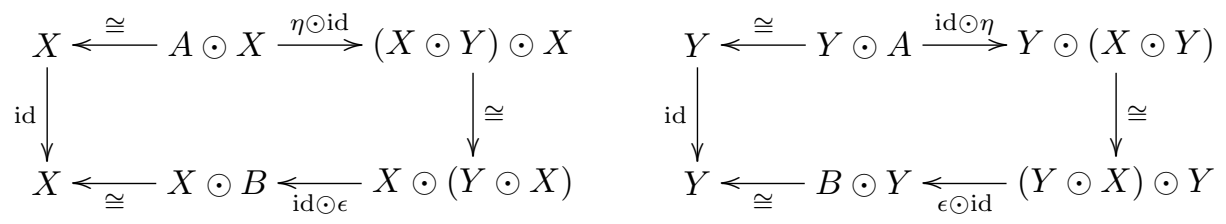

We say that $X$ is right dualizable if it is part of such a dual pair, and we say that $Y$ is left dualizable if it is part of such a dual pair. We say that $X$ is left dual to $Y$ and $Y$ is right dual to $X$.

Note that the right/left symmetry is forced on us by the directionality of 1-cells even when $\mathscr{C}$ is symmetric. One must not imagine that a right dualizable 1-cell is also left dualizable. The following examples show that this is not so.

EXAmple 16.4.2. Let $f: B \longrightarrow A$ be a map of $R$-algebras and recall the base change bimodules $A_{f}: B \longrightarrow A$ and ${ }_{f} A: A \longrightarrow B$ from Example 16.3.8. We may identify ${ }_{f} A \odot A_{f}: B \longrightarrow B$ with $A$ regarded as a $(B, B)$-bimodule by pullback along $f$, and we let $\eta=f: B \longrightarrow{ }_{f} A \odot A_{f}$. We have $A_{f} \odot{ }_{f} A=A \otimes_{B} A$, and we let $\varepsilon: A_{f} \odot{ }_{f} A \longrightarrow A$ be given by the product on $A$. Then $\eta$ and $\varepsilon$ display $\left({ }_{f} A, A_{f}\right)$ as a dual pair; the left and right unit laws for $A$ induce the required commutative diagrams. Observe that $\left(A_{f},{ }_{f} A\right)$ is not a dual pair in general.

Example 16.4.3. We specialize the previous example to the unit $\iota: R \longrightarrow A$ of an $R$-algebra with product $\phi: A \otimes_{R} A \longrightarrow A$. Here the 1-cells $A_{\iota}: R \longrightarrow A$ and ${ }_{\iota} A: A \longrightarrow R$ are $A$ regarded as an $(A, R)$-bimodule (= left $A$-module) and as an $(R, A)$-bimodule (= right $A$-module), and $\iota$ and $\phi$ display $\left({ }_{\iota} A, A_{\iota}\right)$ as a dual pair. For $\left(A_{\iota},{ }_{\iota} A\right)$ to be a dual pair we would have to have maps

$$
\eta: A \longrightarrow A \otimes_{R} A \quad \text { and } \quad \varepsilon: A \longrightarrow R
$$

of $(A, A)$ and $(R, R)$-bimodules, a coproduct and a counit, such that the left and right counit laws hold.

We shall have a topological analogue where the situation is precisely the reverse: the relevant objects will have a coproduct and a counit, but they will not have a product and a unit.

Duality is characterized by a duality adjunction. For 1-cells $X: B \longrightarrow A$ and $Y: A \longrightarrow B$ and a 2-cell $\varepsilon: Y \odot X \longrightarrow B$, define

$$
\varepsilon_{\#}: \mathscr{C}(W, Z \odot Y) \longrightarrow \mathscr{C}(W \odot X, Z)
$$

by letting $\varepsilon_{\#}(\alpha)$ be the composite 2 -cell

$$
W \odot X \stackrel{\alpha \odot \mathrm{id}}{\longrightarrow}(Z \odot Y) \odot X \cong Z \odot(Y \odot X) \stackrel{\mathrm{id} \odot \varepsilon}{\longrightarrow} Z \odot B \cong Z .
$$

Dually, for a 2-cell $\eta: A \longrightarrow X \odot Y$, define

$$
\eta_{\#}: \mathscr{C}(W \odot X, Z) \longrightarrow \mathscr{C}(W, Z \odot Y)
$$

by letting $\eta_{\#}(\beta)$ be the composite 2 -cell

$$
W \cong W \odot A \stackrel{\text { id } \odot \eta}{\longrightarrow} W \odot(X \odot Y) \cong(W \odot X) \odot Y \stackrel{\beta \odot \text { id }}{\longrightarrow} Z \odot Y .
$$


Both maps are defined for 1-cells $W: A \longrightarrow C$ and $Z: B \longrightarrow C$ for any 0-cell $C$. Duality says that these are inverse isomorphisms.

Proposition 16.4.6. The following conditions are equivalent, where $X: B \longrightarrow$ $A$ and $Y: A \longrightarrow B$ are 1-cells in a bicategory $\mathscr{C}$ and $\varepsilon: Y \odot X \longrightarrow B$ is a given 2 -cell.

(i) $(X, Y)$ is a dual pair with evaluation map $\varepsilon$.

(ii) $\varepsilon_{\#}$ is a bijection for all $W$ and $Z$.

(iii) $\varepsilon_{\#}$ is a bijection when $W=A$ and $Z=X$ and when $W=Y$ and $Z=B$.

Dually, the following conditions are equivalent when $\eta: A \longrightarrow X \odot Y$ is given.

(i') $(X, Y)$ is a dual pair with coevaluation map $\eta$.

(ii') $\eta_{\#}$ is a bijection for all $W$ and $Z$.

(iii') $\eta_{\#}$ is a bijection when $W=A$ and $Z=X$ and when $W=Y$ and $Z=B$.

If $\mathscr{C}$ is symmetric, then $\eta$ and $\varepsilon$ exhibit $(X, Y)$ as a dual pair if and only if

$$
\gamma^{-1} t(\eta): t A \longrightarrow t Y \odot t X \quad \text { and } \quad t(\varepsilon) \gamma: t X \odot t Y \longrightarrow t B
$$

exhibit $(t Y, t X)$ as a dual pair.

Proof. Trivially, (ii) implies (iii), and (i) implies (ii) since diagram chases show that $\varepsilon_{\#}$ and $\eta_{\#}$ are inverse bijections when $\varepsilon$ and $\eta$ display $(X, Y)$ as a dual pair. To see that (iii) implies (i), we construct $\eta$ by $\varepsilon_{\#}(\eta)=$ id, using the case $W=A$ and $Z=X$. This already gives one of the required diagrams. For the other diagram, we check that $\varepsilon_{\#}$ takes both the identity map and the composite $(\varepsilon \odot$ id $)($ id $\odot \eta)$ to $\varepsilon$ when $W=Y$ and $Z=B$.

Duals of 2-cells are characterized in the following result.

Proposition 16.4.7. Let $X, X^{\prime}: B \longrightarrow A$ and $Y, Y^{\prime}: A \longrightarrow B$ be 1-cells such that $(X, Y)$ and $\left(X^{\prime}, Y^{\prime}\right)$ are dual pairs and let $\alpha: X \longrightarrow X^{\prime}$ and $\beta: Y \longrightarrow Y^{\prime}$ be given 2-cells.

(i) There is a unique 2-cell $\alpha^{*}: Y^{\prime} \longrightarrow Y$ that makes either of the following diagrams commute, and then the other diagram also commutes.

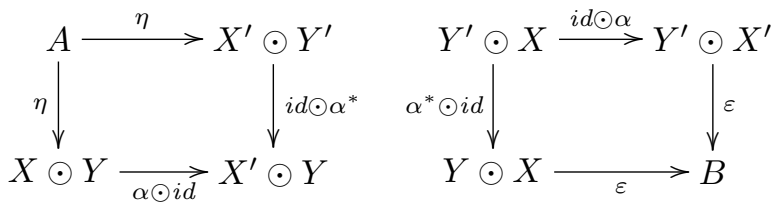

(ii) There is a unique 2-cell $\beta^{*}: X^{\prime} \longrightarrow X$ that makes either of the following diagrams commute, and then the other diagram also commutes.

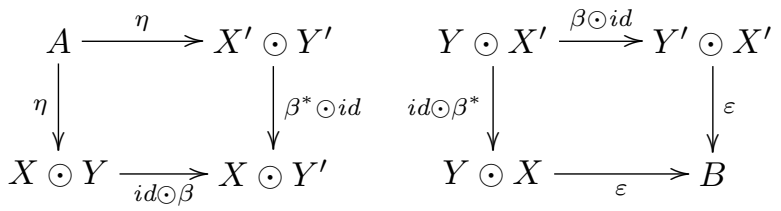

The definitions just given make sense whether or not $\mathscr{C}$ is closed. We assume from now on that $\mathscr{C}$ is closed, and we then have canonical candidates for right and left duals that are defined for any object, whether or not it is right or left dualizable. It is convenient to introduce notations for them. 
Definition 16.4.8. For a 1-cell $X: B \longrightarrow A$, define $D_{r} X=X \triangleright B: A \longrightarrow B$. For a 1-cell $Y: A \longrightarrow B$, define $D_{\ell} Y=B \triangleleft Y: B \longrightarrow A$.

A 2-cell $\varepsilon: Y \odot X \longrightarrow B$ has a pair of adjoint maps

$$
\tilde{\varepsilon}: X \longrightarrow D_{\ell} Y \text { and } \tilde{\varepsilon}: Y \longrightarrow D_{r} X \text {. }
$$

They are related to the canonical evaluation maps $\varepsilon$ of (16.3.3) and (16.3.4) by the commutative diagram

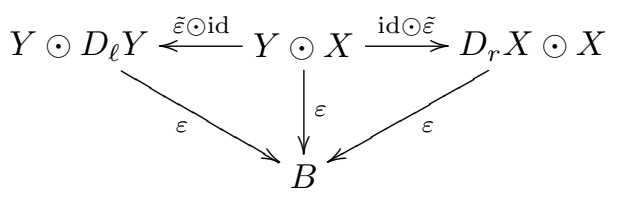

The following result justifies the left and right nomenclature of Definition 16.4.1.

Proposition 16.4.9. If $\varepsilon: Y \odot X \longrightarrow B$ is the evaluation map of a dual pair $(X, Y)$, then the adjoint 2-cells

$$
\tilde{\varepsilon}: X \longrightarrow D_{\ell} Y \quad \text { and } \tilde{\varepsilon}: Y \longrightarrow D_{r} X
$$

are isomorphisms.

We now change our point of view and focus on the canonical duals. The maps $\mu$ and $\nu$ of (16.3.13) and (16.3.14) specialize to maps

$$
\mu: Z \odot D_{r} X \longrightarrow X \triangleright Z
$$

and

$$
\nu: D_{\ell} Y \odot W \longrightarrow W \triangleleft Y .
$$

Clearly $\mu$ is an isomorphism 2-cell when $Z=B$ and $\nu$ is an isomorphism 2-cell when $W=B$.

Proposition 16.4.12. The following are equivalent for a 1-cell $X: B \longrightarrow A$.

(i) $X$ is right dualizable.

(ii) $\mu$ is an isomorphism when $Z=X$. Then the canonical evaluation map and composite

$$
\varepsilon: D_{r} X \odot X \longrightarrow B \quad \text { and } \quad \eta: A \stackrel{\zeta}{\longrightarrow} X \triangleright X \stackrel{\mu^{-1}}{\longrightarrow} X \odot D_{r} X
$$

display $\left(X, D_{r} X\right)$ as a dual pair, where $\zeta$ is adjoint to the unit $A \odot X \cong X$.

(iii) $\mu$ is an isomorphism for all $Z$.

When these hold, the adjoint of $\varepsilon$ is an isomorphism $X \longrightarrow D_{\ell} D_{r} X$.

Dually, the following are equivalent for a 1-cell $Y: A \longrightarrow B$.

(i') $Y$ is left dualizable.

$\left(i i^{\prime}\right) \nu$ is an isomorphism when $W=Y$. Then the canonical evaluation map and composite

$$
\varepsilon: Y \odot D_{\ell} Y \longrightarrow B \quad \text { and } \quad \eta: A \stackrel{\zeta}{\longrightarrow} Y \triangleleft Y \stackrel{\nu^{-1}}{\longrightarrow} D_{\ell} Y \odot Y
$$

display $\left(D_{\ell} Y, Y\right)$ as a dual pair, where $\zeta$ is adjoint to the unit $Y \odot A \cong Y$.

(iii') $\nu$ is an isomorphism for all $W$.

When these hold, the adjoint of $\varepsilon$ is an isomorphism $Y \longrightarrow D_{r} D_{\ell} Y$. 
In these results, the last statements give two versions of the usual isomorphism between a dualizable object and its double dual in a symmetric monoidal category. Similarly, we have the following analogues of results about dualizable objects in symmetric monoidal categories. They generalize (iii) in the previous results.

Proposition 16.4.13. Consider the maps $\mu, \nu$, and $\omega$.

(i) If either $X$ or $Z$ is right dualizable, then

$$
\mu: Z \odot(X \triangleright Y) \longrightarrow X \triangleright(Z \odot Y)
$$

is an isomorphism.

(ii) If either $W$ or $Y$ is left dualizable, then

$$
\nu:(Z \triangleleft Y) \odot W \longrightarrow(Z \odot W) \triangleleft Y
$$

is an isomorphism.

(iii) If $X$ is right and $Y$ is left dualizable, then

$$
\omega:(Z \triangleleft Y) \odot(X \triangleright W) \longrightarrow X \triangleright(Z \odot W) \triangleleft Y
$$

is an isomorphism.

PROOF. The proofs are modifications of those of the symmetric monoidal analogues given in [98, III.1.3]. In (i), if $X$ is right dualizable, then diagram chases show that the composite around the right in the following diagram is inverse to $\mu$.

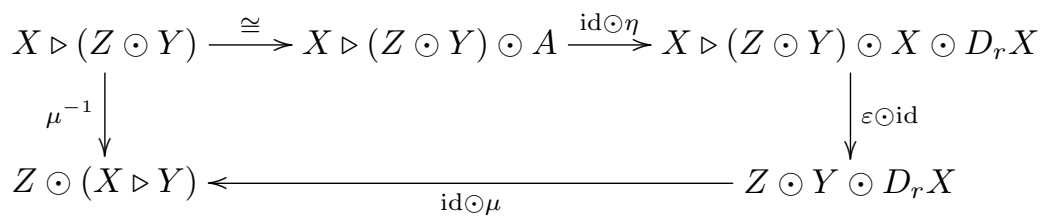

A similarly explicit inverse gives (ii) when $Y$ is left dualizable. These two parts imply (iii) by virtue of the description of $\omega$ as a composite of maps $\mu$ and $\nu$. To see that $\mu$ is an isomorphism when $Z$ is left dualizable, we use a naturality diagram to show that we may as well replace $Z$ by $D_{\ell} D_{r} Z$, and we then check that $\mu$ factors as displayed in the following diagram.

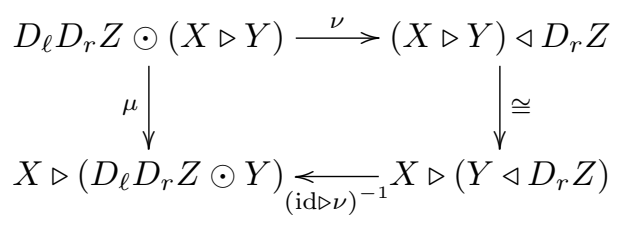

This concludes the proof.

\subsection{Composites and naturality of dualities}

The following result is trivial. We call it a theorem because more direct proofs of important special cases originally seemed to be non-trivial. While we will be using it in topology, it is also of interest in algebra.

Theorem 16.5.1. Consider 1-cells $W, X, Y, Z$ as in the diagram

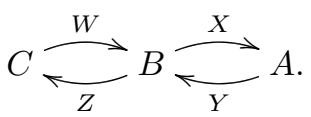


Let $(\eta, \varepsilon)$ be coevaluation and evaluation maps that exhibit $(X, Y)$ as a dual pair, and let $(\zeta, \sigma)$ be coevaluation and evaluation maps that exhibit $(W, Z)$ as a dual pair. Then the composites

$$
A \stackrel{\eta}{\longrightarrow} X \odot Y \stackrel{\cong}{\longrightarrow} X \odot B \odot Y \stackrel{i d \odot \zeta \odot i d}{\longrightarrow}(X \odot W) \odot(Z \odot Y)
$$

and

$$
(Z \odot Y) \odot(X \odot W) \stackrel{i d \odot \epsilon \odot i d}{\longrightarrow} Z \odot B \odot W \stackrel{\cong}{\longrightarrow} Z \odot W \stackrel{\sigma}{\longrightarrow} C
$$

are coevaluation and evaluation maps that exhibit $(X \odot W, Z \odot Y)$ as a dual pair of 1-cells.

Proof. We have put parentheses in the displayed composites in order to emphasize how to think about them, but otherwise we are ignoring associativity isomorphisms. Also ignoring insertion and deletion of unit objects via unit isomorphisms, we see that the square in the following diagram commutes by naturality, and the triangles commute by the given dualities.

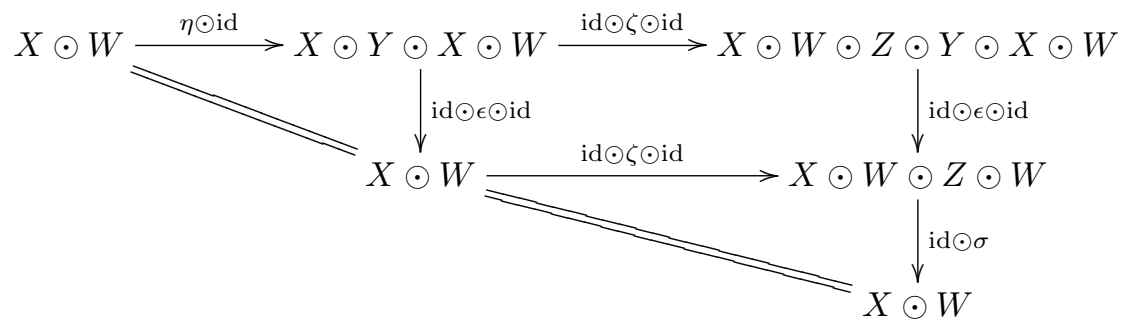

The commutativity of the other diagram required for the first claimed duality is proven similarly.

We have the following addendum.

Proposition 16.5.2. Assume further that $A=C$ and $X=Z$. Then $\eta$ is dual to $\sigma$ and $\epsilon$ is dual to $\zeta$ (with respect to the unit duality of $A$ or $B$ ).

Proof. With $X=Z$, we have the commutative diagram

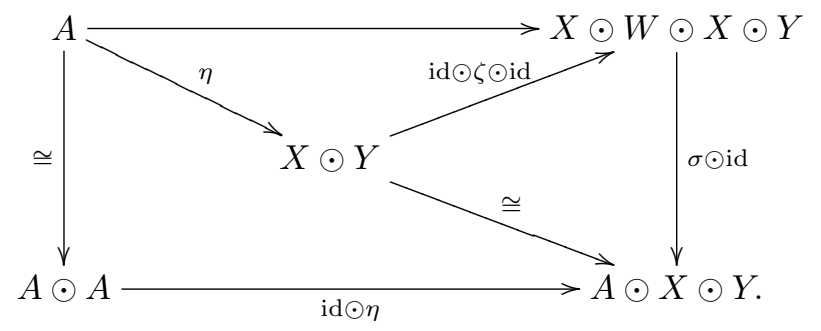

Since the unlabelled arrow is the unit for the composite adjunction, $\eta$ is dual to $\sigma$ by Proposition 16.4.7. A similar diagram shows that $\varepsilon$ is dual to $\zeta$.

We record a dual pair of naturality results. They admit several variants, such as the one given in [98, III.1.9] for an analogue in the context of closed symmetric monoidal categories. 
Proposition 16.5.3. Let $F: \mathscr{B} \longrightarrow \mathscr{C}$ be a lax functor between symmetric bicategories. Let $(X, Y)$ be a dual pair in $\mathscr{B}, X: B \longrightarrow A$ and $Y: A \longrightarrow B$, and assume that the unit and composition coherence 2-cells $U_{F B} \longrightarrow F U_{B}$ and $F X \odot F Y \longrightarrow F(X \odot Y)$ are isomorphisms. Then $(F X, F Y)$ is a dual pair in $\mathscr{C}$.

Proof. Let $\eta$ and $\varepsilon$ be coevaluation and evaluation maps that represent $(X, Y)$ as a dual pair. Then the 2-cells

$$
U_{F A} \stackrel{\text { unit }}{\longrightarrow} F U_{A} \stackrel{F \eta}{\longrightarrow} F(X \odot Y) \stackrel{\text { comp }}{\longleftarrow} F X \odot F Y
$$

and

$$
F Y \odot F X \stackrel{\text { comp }}{\longrightarrow} F(Y \odot X) \stackrel{F \varepsilon}{\longrightarrow} F U_{B} \stackrel{\text { unit }}{\longleftarrow} U_{F B}
$$

give coevaluation and evaluation maps that represent $(F X, F Y)$ as a dual pair.

Reversing the direction of the unit and composition 2-cells, we obtain the dual.

Proposition 16.5.4. Let $F: \mathscr{B} \longrightarrow \mathscr{C}$ be an oplax functor between symmetric bicategories. Let $(X, Y)$ be a dual pair in $\mathscr{B}, X: B \longrightarrow A$ and $Y: A \longrightarrow B$, and assume that the unit and composition coherence 2-cells $F U_{A} \longrightarrow U_{F A}$ and $F(Y \odot X) \longrightarrow F Y \odot F X$ are isomorphisms. Then $(F X, F Y)$ is a dual pair in $\mathscr{C}$.

\subsection{A quick review of triangulated categories}

We recall our preferred definition of a triangulated category from [124]. It is equivalent to Verdier's original definition. That and other basic consequences of the definition are proven in [124].

Definition 16.6.1. A triangulation on an additive category $\mathscr{C}$ is an additive self-equivalence $\Sigma: \mathscr{C} \longrightarrow \mathscr{C}$ together with a collection of "triangles"

$$
X \stackrel{f}{\longrightarrow} Y \stackrel{g}{\longrightarrow} Z \stackrel{h}{\longrightarrow} \Sigma X,
$$

called the distinguished triangles, such that the following axioms hold.

(T1) Let $X$ be any object and $f: X \longrightarrow Y$ be any map in $\mathscr{C}$.

(a) The triangle $X \stackrel{\mathrm{id}}{\longrightarrow} X \longrightarrow * \longrightarrow \Sigma X$ is distinguished.

(b) The map $f: X \longrightarrow Y$ is part of a distinguished triangle $(f, g, h)$.

(c) Any triangle isomorphic to a distinguished triangle is distinguished.

(T2) If $(f, g, h)$ is distinguished, then so is $(g, h,-\Sigma f)$.

(T3) (Verdier's axiom) Consider the following diagram.

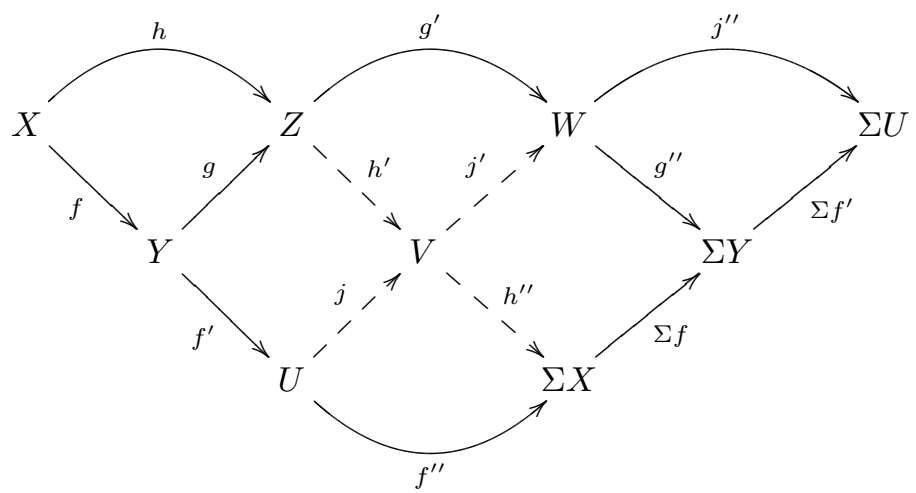


Assume that $h=g \circ f, j^{\prime \prime}=\Sigma f^{\prime} \circ g^{\prime \prime}$, and $\left(f, f^{\prime}, f^{\prime \prime}\right)$ and $\left(g, g^{\prime}, g^{\prime \prime}\right)$ are distinguished. If $h^{\prime}$ and $h^{\prime \prime}$ are given such that $\left(h, h^{\prime}, h^{\prime \prime}\right)$ is distinguished, then there are maps $j$ and $j^{\prime}$ such that the diagram commutes and $\left(j, j^{\prime}, j^{\prime \prime}\right)$ is distinguished. We call the diagram a braid of distinguished triangles generated by $h=g \circ f$ or a braid cogenerated by $j^{\prime \prime}=\Sigma f^{\prime} \circ g^{\prime \prime}$.

We record the implied converse versions of two of the axioms.

Lemma 16.6.2 (T2'). If $(g, h,-\Sigma f)$ is distinguished, then so is $(f, g, h)$.

LEMma 16.6.3 (T3'). In the diagram of (T3), if $j$ and $j^{\prime}$ are given such that $\left(j, j^{\prime}, j^{\prime \prime}\right)$ is distinguished, then there are maps $h^{\prime}$ and $h^{\prime \prime}$ such that the diagram commutes and $\left(h, h^{\prime}, h^{\prime \prime}\right)$ is distinguished.

We have labeled our axioms (T?), while Verdier's original axioms are labelled (TR?) [165]. Our (T1) is his (TR1), our (T2) and (T2') together are his (TR2), and our (T3) is his (TR4). We have omitted his (TR3), since it is implied.

LEMMA 16.6.4 (TR3). If the rows are distinguished and the left square commutes in the following diagram, then there is a map $k$ that makes the remaining squares commute.

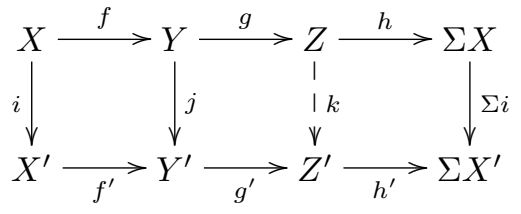

The maps asserted to exist in these axioms are not unique, and some choices are better than others. This point is important for the full force of the compatibility axioms relating triangulations to symmetric monoidal structures, but we refer the reader to $[124,134]$ for that. We recall some standard language.

Definition 16.6.5. Let $\mathscr{C}$ be a triangulated category. Any $Z$ that fits into a distinguished triangle starting with $f: X \longrightarrow Y$ is called a cofiber of $f$ and denoted $C f$; it is unique up to non-unique isomorphism by (TR3). The homotopy coequalizer $C(f, g)$ of maps $f, g: X \longrightarrow Y$ is $C(f-g)$; it is a weak coequalizer. The homotopy colimit, hocolim $X_{i}$, of a sequence of maps $f_{i}: X_{i} \longrightarrow X_{i+1}$ is the homotopy coequalizer of $\amalg$ id, $\amalg f_{i}$ : $\amalg X_{i} \longrightarrow \amalg X_{i}$. It is a weak sequential colimit.

DeFinition 16.6.6. An additive functor between triangulated categories is exact if it commutes with $\Sigma$ up to natural isomorphism and preserves distinguished triangles.

\subsection{Compatibly triangulated symmetric bicategories}

We are concerned with compatibility relations. Thus we now change our starting point, and we assume from now on that $\mathscr{C}$ is a closed symmetric bicategory.

Definition 16.7.1. $\mathscr{C}$ is triangulated if each category $\mathscr{C}(A, B)$ is triangulated.

We write $\Sigma$ rather than $\Sigma_{A, B}$ for the translation automorphism in $\mathscr{C}(A, B)$, and we write $\Sigma^{n}$ for the $n$-fold iterate of $\Sigma$, allowing negative $n$ by use of $\Sigma^{-1}$. Each $\mathscr{C}(A, B)$ is additive, and we write $\oplus$ for the biproduct.

The definition is of no interest without compatibility relations relating the triangulations to the compositions. We must relate the triangulation to $\odot$ and the 
identity 1-cells $U_{A}: A \longrightarrow A$ (abbreviated to $A$ as before) and to $\triangleright$ and $\triangleleft$. We may focus on $\triangleright$ since we assume that $\mathscr{C}$ is symmetric. Throughout, we do not specify sources and targets of 1-cells, assuming tacitly that their sources and targets match up appropriately.

REMARK 16.7.2. Our analogy between algebra and topology weakens at this point. In the derived bicategories of bimodules, the $\mathscr{C}(A, B)$ are not themselves symmetric monoidal categories. However, each $\mathscr{C}(A, B)$ in our bicategory of parametrized spectra is itself a symmetric monoidal category with a compatible triangulation. Pullbacks along diagonal maps of the cartesian products $B \times A$ lead to the internal products in the $\mathscr{C}(A, B)$, and this has no algebraic analogue in general.

There are five compatibility axioms, (TC1)-(TC5), for a triangulation to be compatible with a closed symmetric monoidal structure on a category $\mathscr{C}$. We define precise analogues of the first three and a weak form of the fourth for a closed symmetric bicategory $\mathscr{C}$ by direct comparison, briefly discussing the omitted axiom at the end. We call the result "weak compatibility" because of the omitted axiom. Note that the maps involved in exact triangles are composable 2-cells, so that each such triangle is in one of the categories $\mathscr{C}(A, B)$. Recall Definition 16.2.1.

Definition 16.7.3. The triangulation on $\mathscr{C}$ is weakly compatible with its closed symmetric structure if $\Sigma \mathscr{C} \longrightarrow \mathscr{C}$ is a pseudo-functor (in particular, $t \circ \Sigma \cong \Sigma \circ t$ ) and axioms (TC1)-(TC4) are satisfied.

(TC1) For $X: A \longrightarrow B$, there is a natural isomorphism

$$
\alpha: X \odot \Sigma A \longrightarrow \Sigma X
$$

such that the composite

$$
\Sigma^{2} A=\Sigma(\Sigma A) \stackrel{\alpha^{-1}}{\longrightarrow} \Sigma A \odot \Sigma A \stackrel{\gamma}{\longrightarrow} \Sigma A \odot \Sigma A \stackrel{\alpha}{\longrightarrow} \Sigma(\Sigma A)=\Sigma^{2} A
$$

is multiplication by -1 . There is also a natural isomorphism $t \circ \Sigma \cong \Sigma \circ t$.

(TC2) For a distinguished triangle $X \stackrel{f}{\longrightarrow} Y \stackrel{g}{\longrightarrow} Z \stackrel{h}{\longrightarrow} \Sigma X$

and an object $W$, each of the following triangles is distinguished.

$$
\begin{gathered}
X \odot W \stackrel{f \odot \mathrm{id}}{\longrightarrow} Y \odot W \stackrel{g \odot \mathrm{id}}{\longrightarrow} Z \odot W \stackrel{h \odot \mathrm{id}}{\longrightarrow} \Sigma(X \odot W) \\
W \odot X \stackrel{\mathrm{id} \odot f}{\longrightarrow} W \odot Y \stackrel{\mathrm{id} \odot g}{\longrightarrow} W \odot Z \stackrel{\mathrm{id} \odot h}{\longrightarrow} \Sigma(W \odot X) \\
W \triangleright X \stackrel{\mathrm{id} \triangleright f}{\longrightarrow} W \triangleright Y \stackrel{\mathrm{id} \triangleright g}{\longrightarrow} W \triangleright Z \stackrel{\mathrm{id} \triangleright h}{\longrightarrow} \Sigma W \triangleright X \\
\Sigma^{-1} X \triangleright W \stackrel{-h \triangleright \mathrm{id}}{\longrightarrow} Z \triangleright W \stackrel{g \triangleright \mathrm{id}}{\longrightarrow} Y \triangleright W \stackrel{f \triangleright \mathrm{id}}{\longrightarrow} X \triangleright W
\end{gathered}
$$

Moreover, the involution $t$ is an exact functor of $X$ and $Y$ such that $t \circ \Sigma$ is naturally isomorphic to $\Sigma \circ t$.

REMARK 16.7.4. In (TC2) and in (TC3) below, we implicitly use isomorphisms such as

$(\Sigma X) \odot Y \cong \Sigma(X \odot Y) \cong X \odot(\Sigma Y) \quad$ and $\quad\left(\Sigma^{-1} X\right) \triangleright Y \cong \Sigma(X \triangleright Y) \cong X \triangleright(\Sigma Y)$ that are implied by (TC1). Here use of $\alpha$ directly implies that $\Sigma(X \odot Y) \cong X \odot(\Sigma Y)$, and $\Sigma(X \odot Y) \cong(\Sigma X) \odot Y$ follows by use of the isomorphism $t \circ \Sigma \cong \Sigma \circ t$. 
We view axioms (TC1) and (TC2) as analogues of the elementary axioms (T1) and (T2) for a triangulated category. The completely expected nature of the following frightening looking diagrams is explained in [124], to which we refer the reader for explanations and intuition. It gives the basic relationship between (T3) and $\odot$.

(TC3) (The braid axiom for composites of triangles.) Suppose given distinguished triangles

$$
X \stackrel{f}{\longrightarrow} Y \stackrel{g}{\longrightarrow} Z \stackrel{h}{\longrightarrow} \Sigma X
$$

and

$$
X^{\prime} \stackrel{f^{\prime}}{\longrightarrow} Y^{\prime} \stackrel{g^{\prime}}{\longrightarrow} Z^{\prime} \stackrel{h^{\prime}}{\longrightarrow} \Sigma X^{\prime} .
$$

Then there are distinguished triangles

$$
\begin{gathered}
Y \odot X^{\prime} \stackrel{p_{1}}{\longrightarrow} V \stackrel{j_{1}}{\longrightarrow} X \odot Z^{\prime} \stackrel{f \odot h^{\prime}}{\longrightarrow} \Sigma\left(Y \odot X^{\prime}\right), \\
\Sigma^{-1}\left(Z \odot Z^{\prime}\right) \stackrel{p_{2}}{\longrightarrow} V \stackrel{j_{2}}{\longrightarrow} Y \odot Y^{\prime} \stackrel{-g \odot g^{\prime}}{\longrightarrow} Z \odot Z^{\prime}, \\
X \odot Y^{\prime} \stackrel{p_{3}}{\longrightarrow} V \stackrel{j_{3}}{\longrightarrow} Z \odot X^{\prime} \stackrel{h \odot f^{\prime}}{\longrightarrow} \Sigma\left(X \odot Y^{\prime}\right),
\end{gathered}
$$

such that the following diagram commutes.

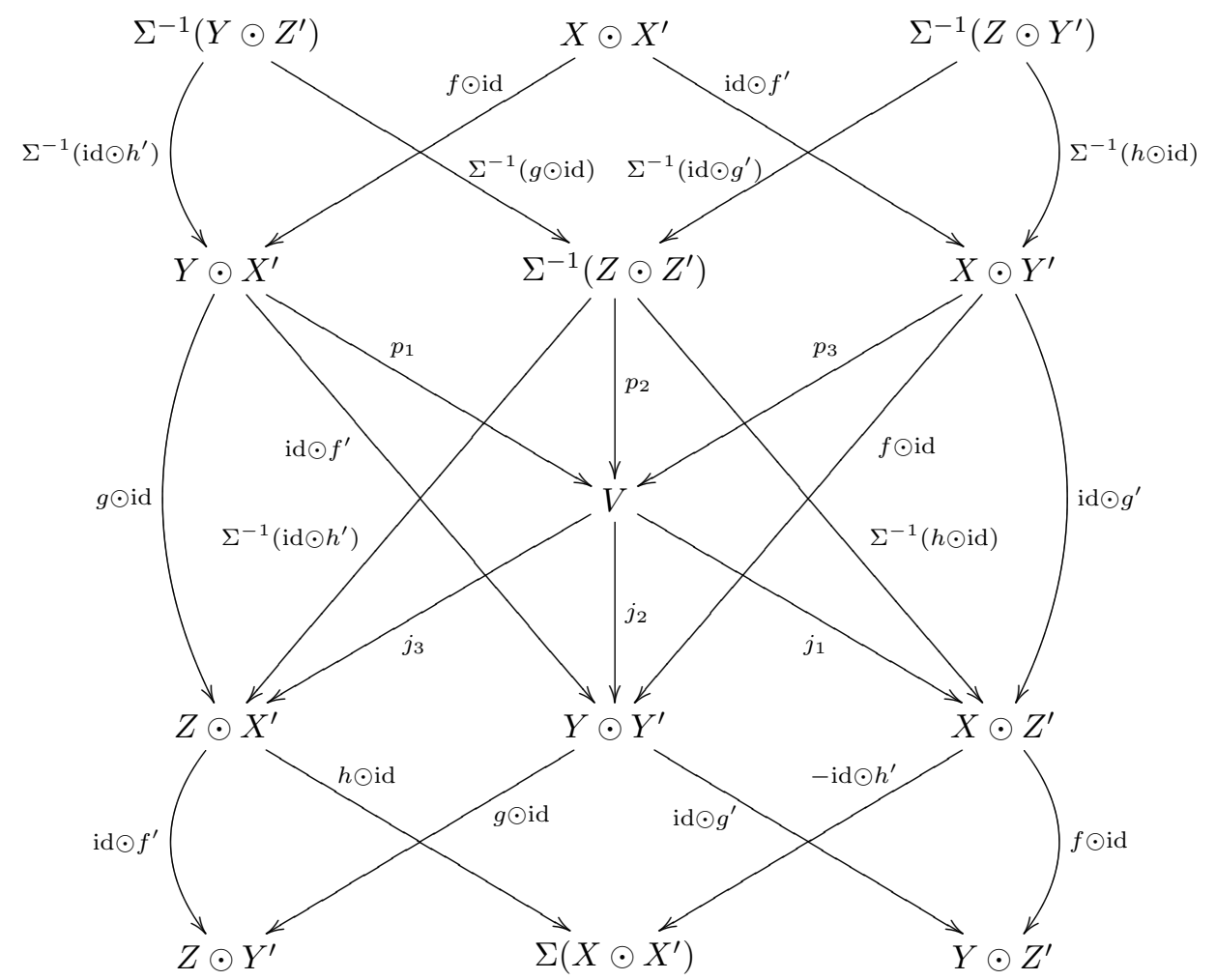

The point of (TC3) is that it intertwines three braids given by Verdier's axiom, saying that we can use the same maps in a priori different braids with the 
same objects. Applying (TC3) to the distinguished triangles $\left(-\Sigma^{-1} h, f, g\right)$ and $\left(-\Sigma^{-1} h^{\prime}, f^{\prime}, g^{\prime}\right)$, we obtain the following equivalent form of (TC3).

Lemma 16.7.5 $\left(\mathrm{TC} 3^{\prime}\right)$. For the distinguished triangles $(f, g, h)$ and $\left(f^{\prime}, g^{\prime}, h^{\prime}\right)$ displayed in (TC3), there are distinguished triangles

$$
\begin{aligned}
& X \odot Z^{\prime} \stackrel{k_{1}}{\longrightarrow} W \stackrel{q_{1}}{\longrightarrow} Z \odot Y^{\prime} \stackrel{h \odot g^{\prime}}{\longrightarrow} \Sigma\left(X \odot Z^{\prime}\right), \\
& Y \odot Y^{\prime} \stackrel{k_{2}}{\longrightarrow} W \stackrel{q_{2}}{\longrightarrow} \Sigma\left(X \odot X^{\prime} \stackrel{-\Sigma\left(f \odot f^{\prime}\right)}{\longrightarrow} \Sigma\left(Y \odot Y^{\prime}\right),\right. \\
& Z \odot X^{\prime} \stackrel{k_{3}}{\longrightarrow} W \stackrel{q_{3}}{\longrightarrow} Y \odot Z^{\prime} \stackrel{g \odot h^{\prime}}{\longrightarrow} \Sigma\left(Z \odot X^{\prime}\right),
\end{aligned}
$$

such that the following diagram commutes.

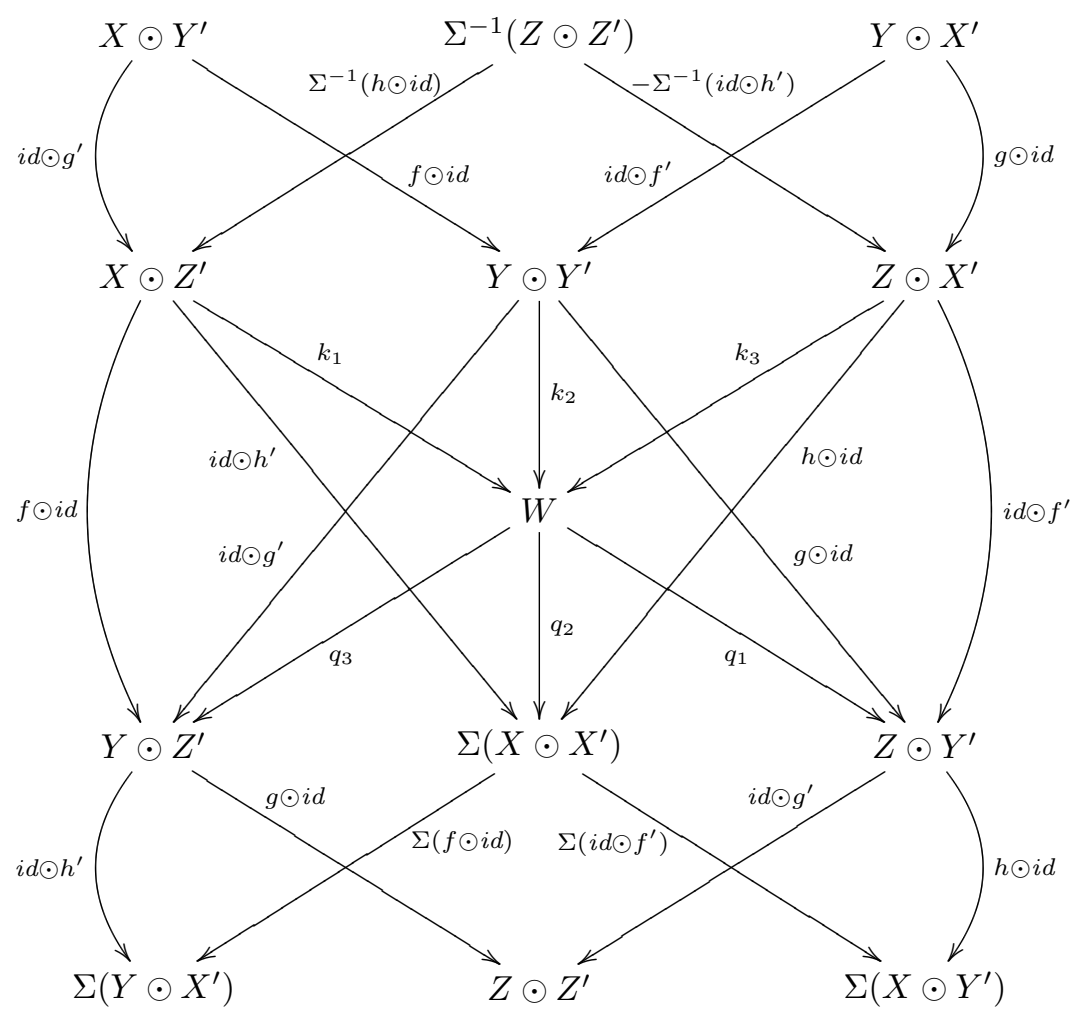

Axiom (TC4) relates the diagrams displayed in (TC3) and (TC3 ${ }^{\prime}$. It is not hard to formulate it in full by comparison with [124], but that would involve some recollections of definitions about well-behaved choices of maps in our axioms and would be digressive here. We state a weak form that gives the idea.

(TC4) (The additivity axiom - weak form.) The maps $j_{i}$ and $k_{i}$ in (TC3) and $\left(\mathrm{TC} 3^{\prime}\right)$ can be so chosen that $k_{2} \circ j_{2}=k_{1} \circ j_{1}+k_{3} \circ j_{3}$.

REMARK 16.7.6. In an interesting follow up to [124], Keller and Neeman claim that (TC4) is a consequence of (TC3) and Verdier's axiom [86, 4.1]. However, they assume that their triangulated categories all come from bounded derived categories of Abelian categories, which fails in the interesting topological examples. What 
is true is that in any reasonable context in which one can prove the axioms, one obtains a strong form of Verdier's axiom, made precise in [124, 3.6,3.8], in the presence of which (TC4) does follow from (TC3).

The omitted axiom (TC5), the "braid duality axiom", relates the diagrams (TC3) and (TC3') when $\left(f^{\prime}, g^{\prime}, h^{\prime}\right)=\left(D_{r} g, D_{r} f, D_{r} \Sigma^{-1} h\right)$. A precise formulation is subtle and beyond the scope of this book. The idea will become clear in $\S 17.5$, where the analogue for closed symmetric monoidal categories is discussed. The axiom (TC5) in closed symmetric monoidal categories is motivated by the importance of the additivity of transfer maps, such as those discussed in the previous chapter, and trace maps [124]. The relevant theory for closed symmetric bicategories has not yet been worked out. It is an observation of Kate Ponto that the relationship between the bicategory composition $\odot$ and the monoidal structure $\otimes$ that are both present in the motivating examples will play a key role in developing such a theory.

\subsection{Duality in triangulated symmetric bicategories}

We let $\mathscr{C}$ be a closed symmetric bicategory with a compatible triangulation throughout this section. Actually, we shall only use the elementary compatibility axioms (TC1) and (TC2). In [76], Hovey, Palmieri, and Strickland give an axiomatic study of stable homotopy theory, starting from a closed symmetric monoidal category, $\mathscr{H}$ say, with a compatible triangulation (again, with only the elementary compatibility axioms since [124] was not yet available). In [76, 1.1.5(c)], they asked how much of their theory works in derived categories of bimodules. With the new context provided here, the quick answer seems to be "nearly everything".

They define a stable homotopy category by requiring $\mathscr{H}$ to have a set of dualizable objects $\mathscr{D}$ such that the smallest localizing subcategory containing $\mathscr{D}$ is $\mathscr{H}$ itself. In our context, we can define a stable homotopy bicategory similarly, requiring each $\mathscr{C}(A, B)$ to have a set of (left or right) dualizable objects $\mathscr{D}(A, B)$ such that the smallest localizing subcategory containing $\mathscr{D}(A, B)$ is $\mathscr{C}(A, B)$ itself. We shall not pursue that line of thought here, since that would lose focus on what is relevant to parametrized stable homotopy theory. However, an understanding of the relationship between compact and dualizable objects in $\mathscr{C}(A, B)$ is illuminating in the topological theory and is also of interest in algebra. We illustrate the process of translation from the symmetric monoidal context of [76] to our symmetric bicategorical context by mimicking that part of [76] which discusses this issue.

Recall that an object $X$ of a triangulated category $\mathscr{C}$, is compact if the Abelian group valued functor $\mathscr{C}(X,-)$ commutes with coproducts. The full subcategory of compact objects in any triangulated category (understood to have coproducts) is clearly a thick subcategory. The subcategory of dualizable objects in a symmetric monoidal category is thick, and the analogue holds in a symmetric bicategory.

Proposition 16.8.1. The full subcategory of right (or left) dualizable objects is a thick subcategory of $\mathscr{C}(B, A)$.

Proof. One characterization of what it means for $X: B \longrightarrow A$ to be right dualizable is that the natural map

$$
\varepsilon_{\#}: \mathscr{C}\left(W, Z \odot D_{r} X\right) \longrightarrow \mathscr{C}(W \odot X, Z)
$$

defined in Proposition 16.4.6(ii) must be a bijection for all 1-cells $W: A \longrightarrow C$ and $Z: B \longrightarrow C$, where $C$ is any 0-cell. The functors of $X$ on the two sides of 
(16.8.2) send distinguished triangles to long exact sequences. By the naturality of $\varepsilon_{\#}$ and the five lemma, if (16.8.2) is an isomorphism for two 1-cells appearing in a distinguished triangle, then it is an isomorphism for the third one. Similarly, if $\varepsilon_{\#}$ is an isomorphism for $X$, then it is an isomorphism for any direct summand of $X$.

Proposition 16.8.3. If $\mathscr{C}(B, A)$ has a generating set $\mathscr{D}(B, A)$ of compact and right dualizable objects, then every compact object is right dualizable, and similarly for left dualizability.

Proof. The thick subcategory generated by $\mathscr{D}(B, A)$ is the subcategory of compact objects in $\mathscr{C}(B, A)$, by Theorem 13.1.14, and all of its objects are right dualizable.

Motivated by the symmetric monoidal analogue, a stable homotopy category is said to be algebraic in [76] if its dualizable generating objects are also compact. Heading towards a determination of when, conversely, right dualizable objects are necessarily compact, we shall give a characterization of dualizability in terms of a different notion of compactness, following [76, 2.1.2].

Definition 16.8.4. A 1 -cell $X: B \longrightarrow A$ is $\triangleright$-compact in a 0 -cell $C$ if the natural 2-cell

$$
\amalg\left(X \triangleright Y_{i}\right) \longrightarrow X \triangleright\left(\amalg Y_{i}\right)
$$

is an isomorphism for any set of 1-cells $Y_{i}: B \longrightarrow C$; it is $\triangleright$-compact if it is $\triangleright$-compact in all $C$. Dually, $X$ is $\triangleleft$-compact from $C$ if the natural 2-cell

$$
\amalg\left(Y_{i} \triangleleft X\right) \longrightarrow\left(\amalg Y_{i}\right) \triangleleft X
$$

is an isomorphism for any set of 1-cells $Y_{i}: C \longrightarrow A$.

The previous definition makes sense in any closed bicategory such that each $\mathscr{C}(B, A)$ has coproducts, but in our triangulated bicategory context it relates as follows to the notion of a compact object in a triangulated category.

LEMma 16.8.5. If $X: B \longrightarrow A$ is $\triangleright$-compact in $A$ and the unit 1-cell $A$ is compact in the triangulated category $\mathscr{C}(A, A)$, then $X$ is compact in the triangulated category $\mathscr{C}(B, A)$. Dually, if $X$ is $\triangleleft$-compact from $B$ and the unit 1-cell $B$ is compact, then $X$ is compact.

Proof. For $Y_{i}: B \longrightarrow A$, we have $\mathscr{C}\left(X, \amalg Y_{i}\right) \cong \mathscr{C}\left(A, X \triangleright\left(\amalg Y_{i}\right)\right) \cong \mathscr{C}\left(A, \amalg\left(X \triangleright Y_{i}\right)\right) \cong \oplus \mathscr{C}\left(A, X \triangleright Y_{i}\right) \cong \oplus \mathscr{C}\left(X, Y_{i}\right)$.

Motivated by the symmetric monoidal analogue, an algebraic stable homotopy category is said to be unital in [76] if its unit object is compact.

Proposition 16.8.6. If $X: B \longrightarrow A$ is right dualizable, then $X$ is $\triangleright$-compact. Conversely, if $\mathscr{C}(B, A)$ has a generating set of right dualizable objects and $X$ is $\triangleright$-compact in $A$, then $X$ is right dualizable.

Proof. For the first statement,

$$
X \triangleright\left(\amalg Z_{i}\right) \cong\left(\amalg Z_{i}\right) \odot D_{r} X \cong \amalg\left(Z_{i} \odot D_{r} X\right) \cong \amalg\left(X \triangleright Z_{i}\right) .
$$

For the second statement, consider the full subcategory of $\mathscr{C}(B, A)$ consisting of those 1-cells $Z$ such that

$$
\mu: Z \odot D_{r} X \longrightarrow X \triangleright Z
$$


is an isomorphism. It is clearly a thick subcategory, and, if $X$ is $\triangleright$-compact in $A$, it is a localizing subcategory. It is an isomorphism when $Z$ is right dualizable by Proposition 16.4.13(i), and it is therefore an isomorphism for all $Z$ when $\mathscr{C}(B, A)$ has a generating set of right dualizable objects. Taking $Z=X$ and using Proposition 16.4.12, we conclude that $X$ is right dualizable.

We combine the previous results to compare thick subcategories of $\mathscr{C}(B, A)$.

TheOREM 16.8.7. Assume that $\mathscr{C}(B, A)$ has a generating set of right dualizable objects. Then its thick subcategories of right dualizable objects, objects that are $\triangleright-$ compact in $A$, and $\triangleright$-compact objects all coincide. If, further, the unit 1-cell $A$ is compact, then this category also coincides with the thick subcategory of compact objects in $\mathscr{C}(B, A)$.

Finally, we record some relationships between the composition product $\odot$ and our notions of compactness and dualizability.

Proposition 16.8.8. Consider $X: B \longrightarrow A$ and $Y: A \longrightarrow C$.

(i) If $Y$ is compact and $X$ is $\triangleright$-compact in $C$, then $Y \odot X$ is compact.

(ii) If $X$ is compact and $Y$ is $\triangleleft$-compact from $A$, then $Y \odot X$ is compact.

(iii) If $X$ and $Y$ are $\triangleright$-compact in $D$, then $Y \odot X$ is $\triangleright$-compact in $D$.

(iv) If $X$ and $Y$ are $\triangleleft$-compact from $D$, then $Y \odot X$ is $\triangleleft$-compact from $D$.

Proof. These follow directly from (16.3.2), (16.3.10), (16.3.11) and the definitions.

Observe that we have already proven in Theorem 16.5.1 that $\odot$-composites of right (or left) dualizable objects are right (or left) dualizable. As in [76, 2.1.1(a)], we have further results of the same nature that mix compactness with dualizability.

Proposition 16.8.9. Consider $X: B \longrightarrow A$ and $Y: A \longrightarrow C$.

(i) If $X$ is right dualizable and $Y$ is compact (or $\triangleright$-compact in $D$ ), then $Y \odot X$ is compact (or $\triangleright$-compact in $D$ ).

(ii) If $Y$ is left dualizable and $X$ is compact (or $\triangleleft$-compact from $D$ ), then $Y \odot X$ is compact (or $\triangleleft$-compact from $D$ ).

Proof. As left adjoints, the functors, $Y \odot(-)$ and $(-) \odot X$ commute with coproducts. The conclusions follow formally from this, the duality adjunctions of Proposition 16.4.6, the isomorphisms (16.3.10) and (16.3.11), and the isomorphisms $\mu$ and $\nu$ of (16.3.13), and (16.3.14). 


\section{CHAPTER 17}

\section{The closed symmetric bicategory of parametrized spectra}

\section{Introduction}

We change our point of view on parametrized categories by letting the base space vary and putting the resulting categories together into a single bicategory. While we could work on the point-set level, the interest lies on the level of derived homotopy categories. We can work either with ex-spaces or with parametrized spectra, with identical formal structure and with compatibility via the suspension spectrum functors. For definiteness, and since the interest is in duality phenomena that only appear stably, we focus on parametrized spectra. As usual, we generally write $\simeq$ for isomorphisms in derived homotopy categories.

Spectra mean $G$-spectra in this chapter. Equivariance plays no special role. Fixing $G$ and omitting it from the notation, we construct the bicategory $\mathscr{E} x$ of parametrized spectra in $\S 17.1$. We then encode structure relating the various categories HoG $\mathscr{S}_{B}$ in terms of the structure of $\mathscr{E} x$. For this purpose, we introduce "base change spectra" associated to maps of base spaces in $\S 17.2$. These are analogues of the elementary base change bimodules defined in Example 16.3.8. They allow us to encode all of our base change functors in terms of the bicategory operations $\odot, \triangleleft$, and $\triangleright$. As we explain in $\S 17.3$, the base change spectra come in dual pairs, and compositions of dualities show that certain base change functors preserve dual pairs. This is essential to the applications of $\mathscr{E} x$ to the study of duality in parametrized homology and cohomology. Heading towards this, we explain in $\S 17.4$ how structure in the ambient bicategory $\mathscr{E} x$ informs us about the internal structure in the individual categories $\mathrm{Ho} G \mathscr{S}_{B}$.

We return to unfinished business in $\S 17.5$, explaining how to prove that the triangulation of $\mathrm{HoG} \mathscr{S}_{B}$ is compatible with its symmetric monoidal structure and showing how that implies the corresponding weak compatibility of the triangulation of $\mathscr{E} x$ with its bicategory composition.

\subsection{The definition of the bicategory $\mathscr{E} x$}

We shall arrive at structure that is formally similar to that in the bicategory of bimodules. Although the details work differently, it is helpful to keep the analogy in mind. We have the external smash product

$$
\bar{\wedge}: \operatorname{Ho} \mathscr{S}_{A} \times \operatorname{Ho} \mathscr{S}_{B} \longrightarrow \operatorname{Ho} \mathscr{S}_{A \times B} .
$$

It should be viewed as the analogue of the tensor product over $R$ of a left $A$-module with a left $B$-module to give a left $A \otimes_{R} B$-module. That external tensor product implicitly underlies the construction of the category $\mathscr{B}_{R}$ of bimodules. Similarly, 
the external function spectrum functor

$$
\bar{F}: \operatorname{Ho} \mathscr{S}_{B}^{\mathrm{op}} \times \operatorname{Ho} \mathscr{S}_{A \times B} \longrightarrow \operatorname{Ho} \mathscr{S}_{A}
$$

is the analogue of the functor $\operatorname{Hom}_{R}$ taking a right $B$-module and an $(A, B)$ bimodule to a left $A$-module.

Construction 17.1.3. We construct the closed bicategory $\mathscr{E} x$ of parametrized spectra over varying base spaces. The 0 -cells of $\mathscr{E} x$ are the spaces $B \in \mathscr{U}$. The category $\mathscr{E} x(A, B)$ of 1 -cells and 2-cells from $A$ to $B$ is $\operatorname{Ho} \mathscr{S}_{B \times A}$. (The order of the factors is dictated by the order in which we write the composition $\odot$ ). Thus the 1-cells are spectra $X$ over $B \times A$ and the morphism set $\mathscr{E} x(X, Y)$ is the set $[X, Y]_{B \times A}$ of maps in the stable category of spectra over $B \times A$. The essential point is the construction of the functors

$$
\odot: \mathscr{E} x(B, C) \times \mathscr{E} x(A, B) \longrightarrow \mathscr{E} x(A, C),
$$

and for that we need an analogue of the functor $\otimes_{B}$ in the bimodule context. Define

$$
\theta_{B}: \operatorname{Ho} \mathscr{S}_{C \times B \times B \times A} \longrightarrow \operatorname{Ho} \mathscr{S}_{C \times A}
$$

to be the composite $\pi_{C \times A} \Delta_{B}^{*}$ derived from the maps

$$
C \times A<\stackrel{\pi}{\longrightarrow} C \times B \times A \stackrel{\Delta}{\longrightarrow} C \times B \times B \times A
$$

induced by the projection $C \times B \times A \longrightarrow C \times A$ and the diagonal map of $B$; observe that $\theta_{B}$ is the identity functor when $B$ is a point. Then define $\odot$ to be the composite $\theta_{B} \circ \bar{\wedge}$, where the relevant external smash product is

$$
\bar{\wedge}: \operatorname{Ho} \mathscr{S}_{C \times B} \times \operatorname{Ho} \mathscr{S}_{B \times A} \longrightarrow \operatorname{Ho} \mathscr{S}_{C \times B \times B \times A} .
$$

The unit $U_{A}$ in $\mathscr{E} x(A, A)=\operatorname{Ho} \mathscr{S}_{A \times A}$ is defined to be $\Delta_{A !} S_{A}$; it is usually written $A$, with the interpretation as a 1 -cell being dictated by context. The functor $\theta_{B}$ has right adjoint $\theta_{B}^{*}=\Delta_{B *} \pi_{C \times A}^{*}$, and the left and right internal homs are

$$
Z \triangleleft Y=\bar{F}\left(Y, \theta_{B}^{*} Z\right): A \longrightarrow B \quad \text { and } \quad X \triangleright Z=\bar{F}\left(X, \theta_{B}^{*} Z\right): B \longrightarrow C,
$$

where $X: A \longrightarrow B, Y: B \longrightarrow C$, and $Z: A \longrightarrow C$.

It is not obvious that $\odot$ is unital and associative, but these are not hard to show. For this and other verifications, we make repeated use of the commutation relations proven in Theorems 13.7.6 and 13.7.7. The former gives the derived projection formula, among other things, and the latter gives the equality of composite change of base functors associated to pullback squares in which one arrow being pulled back is a $q$-fibration. The triviality that projection maps of cartesian products are fibrations often makes the fibration hypothesis obvious, but we must be wary of diagonal maps. The following observation expresses $\odot$ in terms of internal rather than external smash products. Our proofs and applications will go back and forth between the two interpretations. Nothing like this holds in the context of bimodules, and it is what makes the bicategory $\mathscr{E} x$ a useful tool for the study of homology.

Proposition 17.1.4. For $X: A \longrightarrow B$ and $Y: B \longrightarrow C$,

$$
Y \odot X \simeq \pi_{C \times A !}\left(\pi_{C \times B}^{*} Y \wedge_{C \times B \times A} \pi_{B \times A}^{*} X\right): A \longrightarrow C,
$$

where all indicated projections have source $C \times B \times A$. For $Z: A \longrightarrow C$,

$$
Z \triangleleft Y \simeq \pi_{B \times A_{*}} F_{C \times B \times A}\left(\pi_{C \times B}^{*} Y, \pi_{C \times A}^{*} Z\right): A \longrightarrow B
$$


and

$$
X \triangleright Z \simeq \pi_{C \times B_{*}} F_{C \times B \times A}\left(\pi_{B \times A}^{*} X, \pi_{C \times A}^{*} Z\right): B \longrightarrow C .
$$

Proof. Clearly $\Delta_{B}: C \times B \times A \longrightarrow C \times B \times B \times A$ factors as the composite of $\pi_{C \times B \times B \times A}$ and $\Delta_{C \times B \times A}$, and $\Delta_{B}^{*}$ factors accordingly. The second statement follows from the first by inspection of adjunctions.

Among other things, the following result implies that $\odot$ is unital. We indicate the target of all projection maps and the variable to which diagonal maps are applied in the statements of this and later results, but to simplify notations we just write $\pi$ and $\Delta$ in proofs, relying on context to determine the source and target of projection maps and the variable to which a diagonal map is being applied.

Proposition 17.1.5. For a spectrum $X$ over $A$ and spectra $Y, Z$ over $B \times A$,

$$
Y \odot \Delta_{A !} X \simeq Y \wedge_{B \times A} \pi_{A}^{*} X,
$$

$$
\Delta_{A}^{*}(Z \triangleleft Y) \simeq \pi_{A *} F_{B \times A}(Y, Z) \quad \text { and } \quad\left(\Delta_{A !} X\right) \triangleright Z \simeq F_{B \times A}\left(\pi_{A}^{*} X, Z\right) .
$$

Symmetrically, for spectra $X, W$ over $A \times B$ and a spectrum $Y$ over $B$,

$$
\Delta_{B !} Y \odot X \simeq \pi_{B}^{*} Y \wedge_{A \times B} X,
$$

$W \triangleleft\left(\Delta_{B !} Y\right) \simeq F_{A \times B}\left(\pi_{B}^{*} Y, W\right) \quad$ and $\quad \Delta_{B}^{*}(X \triangleright W) \simeq \pi_{B *} F_{A \times B}(X, W)$.

Proof. By Proposition 17.1.4 and Theorems 13.7.7 and 13.7.6, we have

$$
\begin{aligned}
Y \odot \Delta_{!} X & \simeq \pi_{!}\left(\pi^{*} Y \wedge_{B \times A \times A} \pi^{*} \Delta_{!} X\right) \\
& \simeq \pi_{!}\left(\pi^{*} Y \wedge_{B \times A \times A} \Delta_{!} \pi^{*} X\right) \\
& \simeq \pi_{!} \Delta_{!}\left(\left(\Delta^{*} \pi^{*} Y\right) \wedge_{B \times A} \pi^{*} X\right) \\
& \simeq Y \wedge_{B \times A} \pi^{*} X .
\end{aligned}
$$

The last isomorphism holds since the composite of id $\times \Delta_{A}: B \times A \longrightarrow B \times A \times A$ with either of the projections to $B \times A$ is the identity map, so that both $\pi_{!} \Delta_{!} \simeq$ id and $\Delta^{*} \pi^{*} \simeq$ id. Passage to adjoints and symmetry give the remaining conclusions.

Since $\pi_{A}^{*} S_{A} \simeq S_{B \times A}$, the right unit isomorphism $Y \odot A \simeq Y$, where $A$ denotes the unit $\Delta_{A !} S_{A}$, is now clear from the previous result and the fact that $S_{B \times A}$ is the unit for $\wedge_{B \times A}$. Symmetry gives the left unit isomorphism $B \odot X \simeq X$. For associativity, we check that for $X: A \longrightarrow B, Y: B \longrightarrow C$ and $Z: C \longrightarrow D$, both $Z \odot(Y \odot X)$ and $(Z \odot Y) \odot X$ are isomorphic to

$$
\pi_{D \times A_{!}}\left(\Delta_{C} \times \Delta_{B}\right)^{*}(Z \bar{\wedge} Y \bar{\wedge} X) .
$$

For the second of these isomorphisms, we use the following commutative diagram, in which the diamond is a pullback.

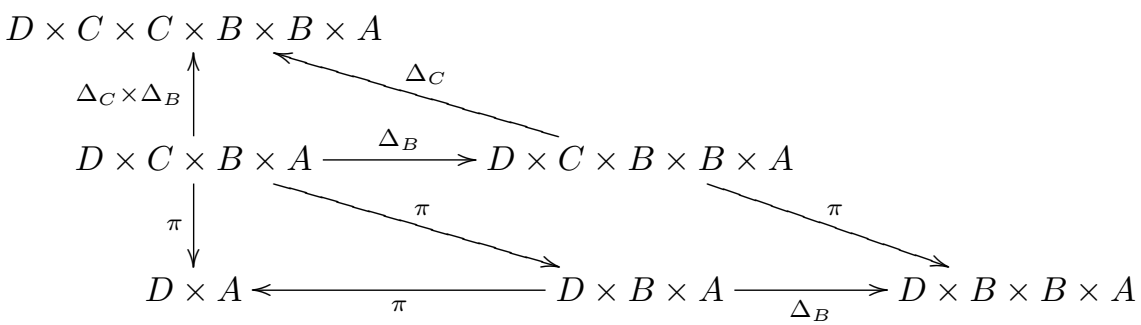


We obtain $(Z \odot Y) \odot X$ by applying base change functors coming from the zig-zag along the hypotenuse and the bottom side of the outer triangle to $Z \bar{\wedge} Y \bar{\wedge} X$, and commutation relations derived from the diagram give the required isomorphism.

Turning to the analogue for symmetry, we next construct the symmetric structure on $\mathscr{E} x$. Since there is no "opposite" to a space, $t$ is the identity on 0-cells, but it is not the identity on the morphism categories between them. Recall from Remark 2.5.4, extended to spectra, that $\bar{\wedge}$ in (17.1.1) has a natural symmetry isomorphism

$$
\gamma: Y \bar{\wedge} X \longrightarrow t^{*}(X \bar{\wedge} Y)
$$

where $t: B \times A \longrightarrow A \times B$ denotes the interchange map, which is not to be confused with the commutativity isomorphism $\bar{\gamma}$ for the external smash product.

Construction 17.1.6. Define $t B=B$ on 0-cells. Using the interchange map $t$, define

$$
t=t^{*}: \mathscr{E} x(A, B)=\operatorname{Ho} \mathscr{S}_{B \times A} \longrightarrow \operatorname{Ho} \mathscr{S}_{A \times B}=\mathscr{E} x(B, A) .
$$

For 1-cells $Y: B \longrightarrow C$ and $X: A \longrightarrow B$, we must define an isomorphism

$$
\gamma: t^{*} X \odot t^{*} Y \longrightarrow t^{*}(Y \odot X)
$$

of spectra over $A \times C$. We have a pair of pullback squares

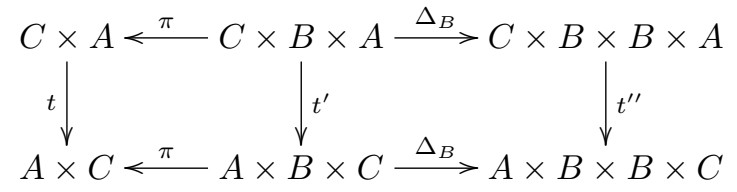

where $t^{\prime}(c, b, a)=(a, b, c)$ and $t^{\prime \prime}\left(c, b, b^{\prime}, a\right)=\left(a, b^{\prime}, b, c\right)=t(t \times t)\left(c, b, b^{\prime}, a\right)$. Commuting derived functors $(-)^{*}$ and $(-)$ ! and commuting functors both of the form $(-)^{*}$, we obtain the composite isomorphism

$$
\begin{aligned}
t^{*} X \odot t^{*} Y & =\theta_{B}\left(t^{*} X \bar{\wedge} t^{*} Y\right) \\
& \simeq \theta_{B}(t \times t)^{*}(X \bar{\wedge} Y) \\
& \simeq \theta_{B}(t \times t)^{*} t^{*}(Y \bar{\wedge} X) \\
& \simeq \theta_{B} t^{\prime \prime *}(Y \bar{\wedge} X) \\
& \simeq t^{*} \theta_{B}(Y \bar{\wedge} X) \\
& =t^{*}(Y \odot X) .
\end{aligned}
$$

REMARK 17.1.7. Just as the tensor product and Hom over the ground ring $R$ give $\mathscr{B}_{R}$ a structure of $\otimes$-closed symmetric monoidal bicategory, so the external smash product of (17.1.1) and the external function spectrum functor $\bar{F}$ gives rise to a $\bar{\wedge}$-closed symmetric monoidal structure on the bicategory $\mathscr{E} x$. The unit $* \longrightarrow \mathscr{E} x$ is given by the sphere spectrum over a point and its identity map, the product on 0 -cells is just the Cartesian product, and

$$
\bar{\wedge}: \mathscr{E}(A, B) \times \mathscr{E}(C, D) \longrightarrow \mathscr{E}(A \times C, B \times D)
$$

sends $(X, Y)$, where $X: A \longrightarrow B$ and $Y: B \longrightarrow Z$, to $(\mathrm{id} \times t \times \text { id })^{*}(X \wedge Y)$. The twist in the middle is needed since $X \bar{\wedge} Y$ is a spectrum over $B \times A \times D \times C$ and we require a spectrum over $B \times D \times A \times C$. The associativity, unity, and commutativity isomorphisms are all evident. What requires a little work is the verification that $\bar{\wedge}$ is indeed a morphism of bicategories. For $Z: A \times C \longrightarrow B \times D, \bar{F}(X, Z)$ and $\bar{F}(Y, Z)$ give the right and left internal $\bar{\wedge}$-hom functors. 


\subsection{Base change spectra}

The bicategory $\mathscr{E} x$ has still more structure. The 0-cells of $\mathscr{E} x$ are the objects of $\mathscr{U}$, and the morphisms of $\mathscr{U}$ induce base change functors that should be viewed from the perspective of $\mathscr{E} x$ as giving change of source and target functors. Here the context of Example 16.2.5 would be needed to build maps of base spaces themselves into an enlargement of the bicategory $\mathscr{E} x$. Remember that $\mathscr{E} x(A, B)=\operatorname{Ho} G \mathscr{S}_{B \times A}$. For a map $f: A \longrightarrow A^{\prime}$, the base change functor

$$
\left(\operatorname{id}_{B} \times f\right)^{*}: \operatorname{Ho} G \mathscr{S}_{B \times A^{\prime}} \longrightarrow \operatorname{HoG} \mathscr{S}_{B \times A}
$$

should be thought of as a change of source functor in $\mathscr{E} x$ :

$$
\mathscr{E} x\left(f^{*}, \mathrm{id}\right): \mathscr{E} x\left(A^{\prime}, B\right) \longrightarrow \mathscr{E} x(A, B) .
$$

This change of source functor has left and right adjoint change of source functors

$$
\left(\operatorname{id}_{B} \times f\right)_{!}=\mathscr{E} x\left(f_{!}, \mathrm{id}\right) \text { and }\left(\operatorname{id}_{B} \times f\right)_{*}=\mathscr{E} x\left(f_{*}, \mathrm{id}\right) \text {. }
$$

Similarly, for a map $g: B \longrightarrow B^{\prime}$, the base change functor

$$
\left(g \times \operatorname{id}_{A}\right)^{*}: \operatorname{HoG} \mathscr{S}_{B^{\prime} \times A} \longrightarrow \operatorname{HoG} \mathscr{S}_{B \times A}
$$

should be thought of as a change of target functor in $\mathscr{E} x$ :

$$
\mathscr{E} x\left(\mathrm{id}, g^{*}\right): \mathscr{E} x\left(A, B^{\prime}\right) \longrightarrow \mathscr{E} x(A, B) .
$$

It has left and right adjoint change of target functors

$$
\left(g \times \mathrm{id}_{A}\right) !=\mathscr{E} x\left(\mathrm{id}, g_{!}\right) \quad \text { and } \quad\left(g \times \mathrm{id}_{A}\right)_{*}=\mathscr{E} x\left(\mathrm{id}, g_{*}\right) \text {. }
$$

We shall use the notations in terms of id $\times f$ and $g \times$ id to limit the problem of incessant translation between two notations for the same functor, but we are thinking in terms of change of source and target in $\mathscr{E} x$. As we now explain, this structure is actually internal to the bicategory $\mathscr{E} x$. If we regard $\mathscr{U}$ as a bicategory (in fact 2-category) with only identity 2 -cells, then there is a bifunctor $\mathscr{U} \longrightarrow \mathscr{E} x$ which is the identity on 0-cells but sends a 1-cell $f: B \longrightarrow A$ to a 1-cell $S_{f}: B \longrightarrow A$. It comes with a companion twisted 1-cell $t S_{f}: A \longrightarrow B$, and we call both $S_{f}$ and $t S_{f}$ "base change spectra". When $f=\operatorname{id}_{A}$, these are both the unit 1-cell $\Delta_{!} S_{A}=A$. We are particularly interested in the case $r: B \longrightarrow *$, when $S_{r}$ is just the sphere spectrum $S_{B}$ viewed as a 1-cell $B \longrightarrow *$. The change of source and target functors are given by operations $\odot, \triangleleft$, and $\triangleright$ with base change spectra. In the next section, we will see that the pairs $\left(t S_{f}, S_{f}\right)$ play an important role in duality theory in $\mathscr{E} x$.

Definition 17.2.1. For a map $f: B \longrightarrow A$, define the base change spectrum $S_{f}: B \longrightarrow A$ by

$$
S_{f}=\Sigma_{A \times B}^{\infty}(B,(f, \mathrm{id}))_{+} \simeq(f, \mathrm{id}) ! S_{B} .
$$

Recall that $(B,(f, \text { id }))_{+}$denotes the ex-space over $A \times B$ obtained by adjoining a disjoint section to $(f$, id $): B \longrightarrow A \times B$. Define the (left dual) base change spectrum $t S_{f}: A \longrightarrow B$ by

$$
t S_{f}=\Sigma_{B \times A}^{\infty}(B,(\mathrm{id}, f))_{+} \simeq(\mathrm{id}, f) ! S_{B} .
$$

It is vitally important to remember the source and target of these 1-cells. However, ignoring the source and target, $S_{r} \simeq S_{B} \simeq t S_{r}$ as spectra over $B$. These base change spectra are obtained by change of source and target from unit 1-cells in $\mathscr{E} x$.

Proposition 17.2.2. For $f: B \longrightarrow A$, there are canonical equivalences

$$
(f \times i d) ! B \simeq S_{f} \simeq(i d \times f)^{*} A \quad \text { and } \quad(i d \times f) ! B \simeq t S_{f} \simeq(f \times i d)^{*} A .
$$


Proof. Since $B=\Delta_{!} S_{B},(f \times$ id $) \Delta=(f$, id $)$, and $($ id $\times f) \Delta=($ id, $f)$, the left hand equivalences of each displayed pair are clear. Passage to homotopy categories is immediate since the functors involved are Quillen left adjoints. On the pointset level of ex-spaces, we obtain right hand equivalences in the displayed pairs by inspection of the pushouts and pullbacks that define the relevant base change functors. The verifications are easy since all sections in sight are adjoined disjointly. However, passage to homotopy categories is less immediate, and we switch to our direct means of passage to homotopy categories. If $f$ is an $h$-fibration, we apply the ex-fibrant approximation functor $P$ to the ex-space $S_{f} \cong(\mathrm{id} \times f)^{*} \Delta_{!} S_{B}^{0}$. We may commute $P$ past $(\mathrm{id} \times f)^{*}$ since id $\times f$ is an $h$-fibration. Therefore $P S_{f} \simeq$ $(\text { id } \times f)^{*} P \Delta_{!} S_{A}^{0}$, which gives the derived identity in $\mathrm{Ho} G \mathscr{W}_{A \times B} \simeq$ Ho $G \mathscr{K}_{A \times B}$. We factor a general map $f$ as the composite $f=g \circ h$, where $h$ is a homotopy equivalence and $g$ is an $h$-fibration. We then have the chain of $f p$-equivalences

$$
S_{g} \simeq P S_{g} \cong P(\mathrm{id} \times g)^{*} \Delta ! S_{A} \simeq(\mathrm{id} \times g)^{*} P \Delta_{!} S_{A}
$$

of ex-fibrations. We apply the equivalence $(\mathrm{id} \times h)^{*}$, which preserves ex-fibrations, to obtain the general case in Ho $G \mathscr{K}_{A \times B}$. Finally, we apply $\Sigma_{A \times B}^{\infty}$ on the derived level, which commutes with all the relevant functors.

We next investigate how change of source or target interacts with horizontal composition. For composites $A \longrightarrow B \longrightarrow C$, we show that change of the source $A$ or target $C$ commutes with horizontal composition. We will then use this result to prove the promised encoding of changes of source and target as operations with base change spectra. In turn, that will allow us to reinterpret the following result as a collection of associativity relations, and then further associativity relations will relate change of the 0 -cell $B$ to horizontal composition.

Proposition 17.2.3. Suppose given 1-cells

$$
A \stackrel{X}{\longrightarrow} B \stackrel{Y}{\longrightarrow} C .
$$

(i) For maps of base spaces $f: A \longrightarrow A^{\prime}$ and $h: C \longrightarrow C^{\prime}$,

$$
(h \times i d) ! Y \odot(i d \times f) ! X \simeq(h \times f) !(Y \odot X): A^{\prime} \longrightarrow C^{\prime} .
$$

(ii) For maps of base spaces $f: A^{\prime} \longrightarrow A$ and $h: C^{\prime} \longrightarrow C$,

$$
(h \times i d)^{*} Y \odot(i d \times f)^{*} X \simeq(h \times f)^{*}(X \odot Y): A^{\prime} \longrightarrow C^{\prime} .
$$

Proof. Both (i) and (ii) can be proven in two ways, either using the definition of $\odot$ in terms of external smash products or its description in terms of internal smash products. For the first way, we start the proof by using the evident relation

$$
(h \times \operatorname{id} \times \operatorname{id} \times f) !(Y \bar{\wedge} X) \simeq(h \times \operatorname{id}) ! Y \bar{\wedge}(\mathrm{id} \times f) ! X
$$

or

$$
(h \times \mathrm{id} \times \mathrm{id} \times f)^{*}(Y \bar{\wedge} X) \simeq(h \times \mathrm{id})^{*} Y \bar{\wedge}(\mathrm{id} \times f)^{*} X .
$$

By composition, it suffices to prove the result when either $f=\mathrm{id}$ or $h=\mathrm{id}$. We can commute functors that are both of the form $(-)$ ! or both of the form $(-)^{*}$ associated to a commutative diagram, and we can commute functors $(-)$ ! and $(-)^{*}$ associated to a pullback diagram in which one pair of parallel arrows is given by $q$-fibrations. Using the first way and taking $f$ or $h$ to be the identity map, such 
commutation relations already imply (i). For (ii), we use the second way. In the case $h=\mathrm{id}$, the proof then goes as follows.

$$
\begin{aligned}
Y \odot(\mathrm{id} \times f) ! X & \simeq \pi_{!}\left(\pi^{*} Y \wedge_{C \times B \times A} \pi^{*}(\mathrm{id} \times f) ! X\right) \\
& \simeq \pi_{!}\left(\pi^{*} Y \wedge_{C \times B \times A}(\mathrm{id} \times \mathrm{id} \times f) ! \pi^{*} X\right) \\
& \simeq \pi_{!}(\mathrm{id} \times \mathrm{id} \times f) !\left((\mathrm{id} \times \mathrm{id} \times f)^{*} \pi^{*} Y \wedge_{C \times B \times A^{\prime}} \pi^{*} X\right) \\
& \simeq(\mathrm{id} \times f) ! \pi_{!}\left(\pi^{*} Y \wedge_{C \times B \times A^{\prime}} \pi^{*} X\right) \\
& \simeq(\mathrm{id} \times f) !(Y \odot X)
\end{aligned}
$$

Here the second and fourth equivalences use evident pullback squares of base spaces, and the third equivalence is an application of the projection formula.

Proposition 17.2.4. For a map $f: A \longrightarrow A^{\prime}$ of 0-cells, change of source is given by

$$
\begin{gathered}
Y \odot S_{f} \simeq(i d \times f)^{*} Y \simeq t S_{f} \triangleright Y: A \longrightarrow B \\
X \odot t S_{f} \simeq(i d \times f) ! X: A^{\prime} \longrightarrow B \quad \text { and } \quad S_{f} \triangleright X \simeq(i d \times f)_{*} X: A^{\prime} \longrightarrow B
\end{gathered}
$$

for 1-cells $X: A \longrightarrow B$ and $Y: A^{\prime} \longrightarrow B$. Similarly, for a map $g: B \longrightarrow B^{\prime}$ of 0 -cells, change of target is given by

$$
\begin{gathered}
t S_{g} \odot Z \simeq(g \times i d)^{*} Z \simeq Z \triangleleft S_{g}: A \longrightarrow B, \\
S_{g} \odot X \simeq(g \times i d)_{!} X: A \longrightarrow B^{\prime} \quad \text { and } \quad X \triangleleft t S_{g} \simeq(g \times i d)_{*} X: A \longrightarrow B^{\prime}
\end{gathered}
$$

for 1-cells $X: A \longrightarrow B$ and $Z: A \longrightarrow B^{\prime}$.

Proof. The four equivalences involving the horizontal composition $\odot$ are immediate from Proposition 17.2.2 and the cases $h=$ id and $f=$ id of Proposition 17.2.3, with $X$ or $Y$ taken to be a unit 1-cell. For example,

$$
(g \times \mathrm{id}) ! X \simeq(g \times \text { id })_{!}(B \odot X) \simeq(g \times \text { id }) ! B \odot X \simeq S_{g} \odot X .
$$

The four equivalences involving the left or right hom functors $\triangleleft$ or $\triangleright$ follow formally by use of the Yoneda lemma. For example,

$$
\mathscr{E} x\left(Y, S_{f} \triangleright X\right) \cong \mathscr{E} x\left(Y \odot S_{f}, X\right) \cong \mathscr{E} x\left((\mathrm{id} \times f)^{*} Y, X\right) \cong \mathscr{E} x\left(Y,(\mathrm{id} \times f)_{*} X\right) .
$$

REMARK 17.2.5. With notations for dual spectra to be introduced in the next section, the first and third display of the proposition gives that $D_{r} t S_{f} \simeq S_{f}$ when $Y$ is the unit 1-cell $A^{\prime}$ and that $D_{\ell} S_{g} \simeq t S_{g}$ when $Z$ is the unit 1-cell $B^{\prime}$.

The functoriality of the base change spectra is an immediate consequence.

Corollary 17.2.6. If $f: B \longrightarrow A$ and $g: C \longrightarrow B$ are maps of 0 -cells, then

$$
S_{f} \odot S_{g} \simeq S_{f g} \quad \text { and } \quad t S_{g} \odot t S_{f} \simeq t S_{f g} .
$$

Proof. For the first, we have

$$
S_{f} \odot S_{g} \simeq(f \times \mathrm{id}) ! S_{g} \simeq(f \times \mathrm{id}) !(g \times \mathrm{id})_{!} C \simeq(f g \times \mathrm{id}) ! C \simeq S_{f g} .
$$

The second follows by applying $t$.

The first equivalence of Proposition 17.2.3 can now be reinterpreted as the associativity equivalence

$$
\left(S_{h} \odot Y\right) \odot\left(X \odot t S_{f}\right) \simeq S_{h} \odot(Y \odot X) \odot t S_{f},
$$


and similarly for the second. The further associativity relations

$$
Y \odot\left(S_{g} \odot X\right) \simeq\left(Y \odot S_{g}\right) \odot X \quad \text { and } \quad\left(Y \odot t S_{g}\right) \odot X \simeq Y \odot\left(t S_{g} \odot X\right)
$$

give us the promised relationship between horizontal composition and base change in the 0-cell at which we are composing.

Proposition 17.2.7. Suppose given 1-cells $X: A \longrightarrow B$ and $Y: B^{\prime} \longrightarrow C$.

(i) For a map of base spaces $g: B \longrightarrow B^{\prime}$,

$$
Y \odot(g \times i d)_{!} X \simeq(i d \times g)^{*} Y \odot X
$$

(ii) For a map of base spaces $g: B^{\prime} \longrightarrow B$,

$$
(i d \times g) ! Y \odot X \simeq Y \odot(g \times i d)^{*} X
$$

By conjugating adjunctions, we can obtain still more such relationships.

REMARK 17.2.8. There are alternative lines of development here, and the details of one of them will become relevant later. Instead of first proving Proposition 17.2.3, we could first prove the three equivalences involving $\odot$ of Proposition 17.2.4 and then deduce Proposition 17.2.3 and the rest of the results of this section. To show directly that $Y \odot S_{f} \simeq(\mathrm{id} \times f)^{*} Y: A^{\prime} \longrightarrow B$ for a map $f: A \longrightarrow A^{\prime}$ and a 1-cell $Y: A^{\prime} \longrightarrow B$, consider the following diagram.

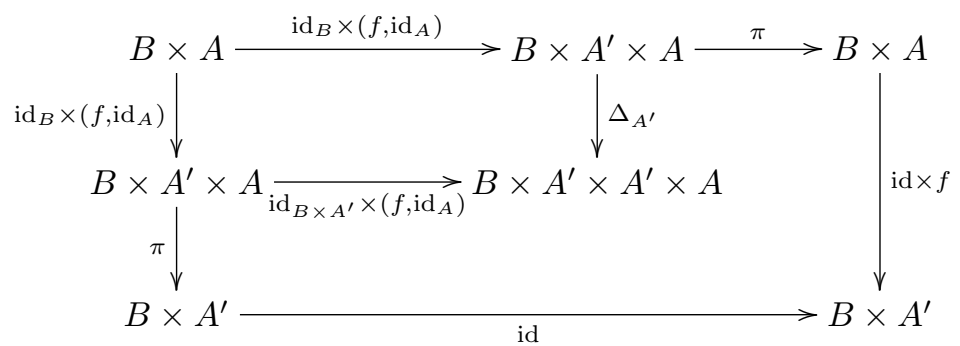

We start with $Y$ over $B \times A^{\prime}$ in the lower left corner. We observe that

$$
\pi^{*} Y \simeq \pi_{B \times A^{\prime}}^{*} Y \wedge_{B \times A^{\prime} \times A} S_{B \times A^{\prime} \times A} \simeq \pi_{B \times A^{\prime}}^{*} Y \wedge_{B \times A^{\prime} \times A} \pi_{A}^{*} S_{A} \simeq Y \bar{\wedge} S_{A}
$$

by the unit isomorphism for $\wedge_{B \times A^{\prime} \times A}$, Theorem 13.7.6, and the identification of external smash products of Lemma 2.5.5. By the definition of $\odot$ and $S_{f}$, we obtain $Y \odot S_{f}$ by starting with $Y$, pulling up along $\pi$, pushing right, pulling up, and pushing right. Of course, we obtain $(\mathrm{id} \times f)^{*} Y$ by pushing right and pulling up and, since the composite on the top row is the identity map, this is equivalent to pulling up along the left column and pushing forward along the top row. Thus it suffices to show that if we start with $\pi^{*} Y$, then the canonical map from pulling up and then pushing forward to pushing forward and then pulling up (as in the proof of Proposition 2.2.11) is an equivalence. To see this, we expand the upper left square by factoring its vertical maps as the vertical composites in the following 
diagram. To simplify notation, let $g=\mathrm{id}_{B} \times\left(f, \mathrm{id}_{A}\right)$.

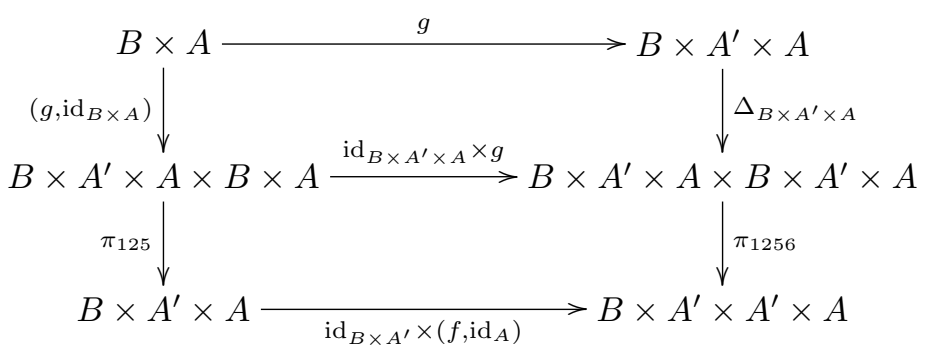

The upper pullback gives the projection formula for $g$ when one starts in the lower left corner with $X \wedge Y$ where $X$ is over $B \times A^{\prime} \times A$ and $Y$ is over $B \times A$. Pushing right and then pulling up gives $X \wedge_{B \times A^{\prime} \times A} g ! Y$ whereas pulling up and pushing right gives $g_{!}\left(g^{*} X \wedge_{B \times A} Y\right)$. The natural map from the latter to the former induces the equivalence of the projection formula. The lower pullback involves projections and therefore gives an analogous equivalence.

\subsection{Duality of base change spectra}

In analogy with Example 16.4.2, we prove that $\left(t S_{f}, S_{f}\right)$ is a dual pair, where $f$ is a map $B \longrightarrow A$. Note the order. We are particularly interested in the case $r: B \longrightarrow *$, and it would be awkward to have to translate $A$ to $B$ rather than $A$ to *. Recall that we have a 1-cell $t X: A \longrightarrow B$ associated to a 1-cell $X: B \longrightarrow A$.

Theorem 17.3.1. For any map $f: B \longrightarrow A,\left(t S_{f}, S_{f}\right)$ is a dual pair.

Proof. We emphasize that this does not imply that $\left(S_{f}, t S_{f}\right)$ is a dual pair. We shall explain why in the course of the proof. Let us begin by working on the ex-space level. All of our definitions, including the composition $\odot$, make sense on the point-set level, and here we have the elementary relation

$$
(A, p)_{+} \bar{\wedge}(B, q)_{+} \cong(A \times B, p \times q)_{+} .
$$

We use this to calculate:

$$
t S_{f}^{0} \odot S_{f}^{0}=\pi_{B \times B !}\left(\operatorname{id}_{B} \times \Delta_{A} \times \operatorname{id}_{B}\right)^{*}\left((B,(\mathrm{id}, f))_{+} \bar{\wedge}(B,(f, \mathrm{id}))_{+} .\right.
$$

We find that this is $(f \times f)^{*}(A, \Delta)_{+}$, and the diagonal map of $B$ gives

$$
\eta: \Delta_{!} S_{B}^{0}=(B, \Delta)_{+} \longrightarrow(f \times f)^{*}(A, \Delta)_{+} \cong t S_{f}^{0} \odot S_{f}^{0} .
$$

When $A=*$ and $f=r,(r \times r)^{*}(*, \Delta)=B \times B$ and the map is

$$
\eta=\Delta_{+}:(B, \Delta)_{+} \longrightarrow(B \times B, \text { id })_{+} .
$$

Observe that one cannot expect to have a map pointing the other way. When $f=r$, such a map would require a product $B \times B \longrightarrow B$ over $B \times B$, which is absurd. Similarly,

$$
S_{f}^{0} \odot t S_{f}^{0}=\pi_{A \times A !}\left(\operatorname{id}_{A} \times \Delta_{B} \times \mathrm{id}_{A}\right)^{*}\left((B,(f, \mathrm{id}))_{+} \bar{\wedge}(B,(\mathrm{id}, f))_{+}\right) .
$$

We find that this is $(B,(f, f))_{+}$over $A \times A$. Clearly $f$ itself gives a map

$$
\varepsilon: S_{f}^{0} \odot t S_{f}^{0} \cong(B,(f, f))_{+} \longrightarrow(A, \Delta)_{+}=\Delta_{!} S_{A}^{0} .
$$

When $A=*$ and $f=r$,

$$
\varepsilon=r_{+}: B_{+}=(B, r)_{+} \longrightarrow(*, \mathrm{id})_{+}=S^{0} .
$$


Observe again that, for a general map $f$, there is no map pointing the other way. When $f=r$, such a map would be a choice of basepoint in $B$. The commutativity of the space level diagrams, on the point-set level, is proven by a tedious, but straightforward, expansion of terms and inspection of definitions, which is especially simple when $f=r$, where it reduces to the fact that $r: B \longrightarrow *$ is a counit for the diagonal $\Delta: B \longrightarrow B \times B$. Applying parametrized suspension spectrum functors, which commute with functors of the form $(-) !,(-)^{*}$, and $(-) \bar{\wedge}(-)$, and noting that all functors in sight are Quillen left adjoints (at least if $B$ and $A$ are CW complexes), we see that this point-set level duality gives rise to a corresponding duality after passage to parametrized spectra and derived homotopy categories.

REMARK 17.3.2. For concreteness, we extract the following observations from the proof. Remember that $S_{r}$ and $t S_{r}$ denote $S_{B}$ regarded as a 1-cell $B \longrightarrow *$ or $* \longrightarrow B$. The coevaluation map

$$
\eta: \Delta_{!} S_{B} \simeq \Sigma_{B \times B}^{\infty}(B, \Delta)_{+} \longrightarrow \Sigma_{B \times B}^{\infty}(B \times B, \mathrm{id})_{+} \simeq t S_{r} \odot S_{r}
$$

is induced by $\Delta: B \longrightarrow B \times B$, viewed as a map $(B, \Delta)_{+} \longrightarrow(B \times B \text {, id })_{+}$of ex-spaces over $B \times B$, while the evaluation map

$$
\varepsilon: S_{r} \odot t S_{r} \cong \Sigma^{\infty} B_{+} \longrightarrow \Sigma^{\infty} *_{+}=S
$$

is induced by $r: B \longrightarrow *$, viewed as a map $(B, r)_{+} \longrightarrow(* \text {, id })_{+}$of ex-spaces over $*$.

The following result is fundamental, but its proof is now very easy.

TheOREM 17.3.3. If $X: B \longrightarrow A$ is right dualizable with right dual $Y: A \longrightarrow B$ and $f: B \longrightarrow C$ is any map, then $(i d \times f) ! X: C \longrightarrow A$ is right dualizable with right dual $(f \times i d) ! Y: A \longrightarrow C$.

PROOF. We just reinterpret the base change functors in terms of composition with base change spectra and apply Theorem 16.5.1. Precisely, translating along Theorem 17.3.1, the conclusion is that $\left(X \odot t S_{f}, S_{f} \odot Y\right)$ is a dual pair. Since $\left(t S_{f}, S_{f}\right)$ is a dual pair by Theorem 17.3.1, our formal result Theorem 16.5.1 on composites of duals shows how to define the required coevaluation and evaluation maps in terms of the given ones.

Although it is much less useful to us, we have another result of the same nature.

TheOREM 17.3.4. If $X: B \longrightarrow A$ is right dualizable with right dual $Y: A \longrightarrow B$ and $f: C \longrightarrow A$ is any map, then $(f \times i d)^{*} X: B \longrightarrow C$ is right dualizable with right dual $(i d \times f)^{*} Y: C \longrightarrow B$.

Proof. This just says that $\left(t S_{f} \odot X, Y \odot S_{f}\right)$ is a dual pair.

\subsection{Using $\mathscr{E} x$ to encode relations between HoG $\mathscr{S}_{B}$ and HoG $\mathscr{S}$}

We are interested primarily in the relationships between the two isomorphic copies $\mathscr{E} x(*, B)$ and $\mathscr{E} x(B, *)$ of $\mathrm{Ho} \mathscr{S}_{B}$ in $\mathscr{E} x$ and the copy $\mathscr{E} x(*, *)$ of HoG $\mathscr{S}$. These relationships are clarified conceptually by working in the ambient bicategory $\mathscr{E} x$, where it is natural to bring $\mathscr{E} x(B, B)$ into play. We will explain the role that $\mathscr{E} x(B, B)$ plays in its own right at the end of the section. To avoid ambiguity in the meaning of $\odot$, we adopt the following notational convention, which agrees with our categorical formalism concerning interchange maps such as $t: * \times B \longrightarrow B \times *$. 
Notations 17.4.1. A spectrum can and must be viewed as a 1-cell $* \longrightarrow *$. By default, we view a spectrum $X$ over $B$ as a 1-cell $B \longrightarrow *$. We write $t X$ for the same spectrum over $B$ viewed as a 1-cell $* \longrightarrow B$. Thus, for spectra $X$ and $Y$ over $B, X \odot t Y: * \longrightarrow *$ is a spectrum, but $t Y \odot X: B \longrightarrow B$ is a spectrum over $B \times B$.

The point is that, when working in $\mathscr{E} x$, we must always remember the source and target of our 1-cells. However, when we draw conclusions in $\operatorname{HoG} \mathscr{S}_{B}$, we can forget the distinction since that category, by itself, is unaware of the ambient bicategory $\mathscr{E} x$ that is informing us about its internal structure.

Taking two of $A, B$, and $C$ to be a point, Proposition 17.1.4 specializes to give a dictionary relating smash product and function spectra to $\odot, \triangleleft$, and $\triangleright$.

Proposition 17.4.2. Let $X$ and $Y$ be spectra over $B$ and let $Z$ be a spectrum. As spectra,

$$
Y \odot t X \simeq r_{!}\left(Y \wedge_{B} X\right) \quad \text { and } \quad t Y \triangleleft t X \simeq r_{*} F_{B}(X, Y) \simeq X \triangleright Y .
$$

As spectra over $B$,

$$
t X \odot Z \simeq X \bar{\wedge} Z, \quad Z \odot X \simeq Z \bar{\wedge} X \quad \text { and } \quad Z \triangleleft X \simeq F_{B}\left(X, r^{*} Z\right) \simeq t X \triangleright Z .
$$

As spectra over $B \times B$,

$$
t Y \odot X \simeq Y \bar{\wedge} X
$$

Here we have used Lemma 2.5.5 to rewrite some of these in simpler external form than the specialization of Proposition 17.1.4 gives directly. Similarly, taking $A$ or $B$ to be a point, Proposition 17.1.5 specializes to give the following description of smash products and function spectra over $B$ in terms of the bicategory structure.

Proposition 17.4.3. Let $X$ and $Y$ be spectra over B. Then

$$
\begin{gathered}
\Delta_{!} X \odot t Y \simeq X \wedge_{B} Y \simeq X \odot \Delta_{!} Y \\
\Delta_{!} X \triangleright Y \simeq F_{B}(X, Y) \simeq \Delta^{*}(X \triangleright Y) \quad \text { and } \quad X \triangleleft \Delta_{!} Y \simeq F_{B}(Y, X) \simeq \Delta^{*}(X \triangleleft Y) .
\end{gathered}
$$

Specialization of Proposition 17.2.4 gives descriptions of all of our base change functors as bicategory operations with base change spectra.

Corollary 17.4.4. Let $f: B \longrightarrow A$ be a map and let $X$ be a spectrum over $A$ and $Y$ be a spectrum over $B$, thought of as 1-cells $A \longrightarrow *$ and $B \longrightarrow *$. Then

$$
\begin{aligned}
f_{!} Y \simeq Y \odot t S_{f} & \text { and } \quad t f_{!} Y \simeq S_{f} \odot t Y \\
X \odot S_{f} \simeq f^{*} X \simeq t S_{f} \triangleright X & \text { and } t S_{f} \odot t X \simeq t f^{*} X \simeq t X \triangleleft S_{f} \\
f_{*} Y \simeq S_{f} \triangleright Y & \text { and } t f_{*} Y \simeq t Y \triangleleft t S_{f} .
\end{aligned}
$$

Using this dictionary, we can translate results on base change to results about compositions with base change spectra. For example, Theorem 13.7.7 can be interpreted in several ways as a statement about such bicategory operations. We can view the given 1-cells as having target $*$. Applying $t$ to the relations so obtained gives relations in which we view the given 1-cells as having source $*$. Moreover, each relation is an equivalence with one pullback $(-)^{*}$ on each side, and each pullback can be expressed in two ways. By symmetry and application of $t$, all such relations come from the following ones. 
THEOREM 17.4.5. Suppose given a pullback diagram of 0-cells

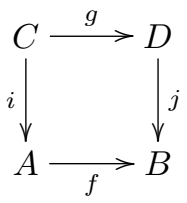

in which $f$ or $j$ is a q-fibration. Let $X$ be a spectrum over $A$ and $Y$ be a spectrum over D. Then

and

$$
t S_{j} \triangleright\left(X \odot t S_{f}\right) \simeq X \odot t S_{f} \odot S_{j} \simeq X \odot S_{i} \odot t S_{g} \simeq\left(t S_{i} \triangleright X\right) \odot t S_{g}
$$

$$
t S_{f} \triangleright\left(S_{j} \triangleright t Y\right) \simeq\left(S_{j} \triangleright t Y\right) \odot S_{f} \simeq S_{i} \triangleright\left(Y \odot S_{g}\right) \simeq S_{i} \triangleright\left(t S_{g} \triangleright Y\right) .
$$

Remarkably, although we view $\mathscr{E} x(B, B)$ primarily as a part of $\mathscr{E} x$ that is especially helpful for the study of the two copies of $\mathrm{HoG} \mathscr{S}_{B}$ embedded as the 1-cells $B \longrightarrow *$ and $* \longrightarrow B$, it bears its own intrinsically close relationship with HoG $\mathscr{S}_{B}$. We may view $\mathscr{E} x(B, B)$ as a monoidal category under $\odot$, with unit object $B=\Delta_{!} S_{B}$, or we may view both it and the symmetric monoidal category HoG $\mathscr{S}_{B}$ as symmetric bicategories with a single object. Taking the latter point of view, we have the following observation.

Proposition 17.4.6. The functor $\Delta_{!}: H o G \mathscr{S}_{B} \longrightarrow \mathscr{E} x(B, B)$ is a map of symmetric bicategories.

Proof. By the first display in Proposition 17.1.5, the projection formula, and the fact that $\Delta^{*} \pi^{*} \simeq$ id since $\pi \circ \Delta=\mathrm{id}$, we have

$$
\Delta_{!} X \odot \Delta_{!} Y \simeq \Delta_{!} X \wedge_{B \times B} \pi^{*} Y \simeq \Delta_{!}\left(X \wedge_{B} \Delta^{*} \pi^{*} Y\right) \simeq \Delta_{!}\left(X \wedge_{B} Y\right)
$$

It is easily checked that this equivalence is compatible with the unit, associativity, and symmetry constraints. We are not saying, because it is not true, that the category $\mathscr{E} x(B, B)$ is symmetric monoidal under $\odot$, but rather that $\mathrm{Ho}_{\mathcal{B}} \mathscr{S}_{B}$ is mapping into it in a symmetric way. With the language of symmetric bicategories, the meaning is clear.

REMARK 17.4.7. Continuing with the analogy and contrast between bimodules and parametrized spectra of Examples 16.2.2 and 16.4.3, recall that a commutive $R$-algebra $A$ is a 0 -cell such that $t A=A$, whereas $t B=B$ for every space $B$. The functor $\Delta_{\text {! }}$ can be viewed as an analogue of the embedding of the symmetric monoidal category of $A$-modules as a symmetric monoidal subcategory of the monoidal category of $(A, A)$-bimodules. Here again, diagonals in topology are replaced by multiplications in algebra, since the latter embedding is given by $\phi^{*}: \mathscr{M}_{A} \longrightarrow \mathscr{B}_{R}(A, A)$, where $\phi: A \otimes A \longrightarrow A$ is the product of $A$.

\subsection{Sketch proofs of the compatible triangulation axioms}

We return to the proof Theorem 15.2.6, which says that HoG $\mathscr{S}_{B}$ is a closed symmetric monoidal category with a compatible triangulation. We have the closed symmetric monoidal structure and the triangulation, the latter by Lemma 13.1.5. There are five axioms to prove, (TC1)-(TC5). The statements of the first three are exactly the same as given in $\S 16.7$, except that $\odot$ and $\triangleright$ must be replaced by $\wedge_{B}$ and $F_{B}$ and the unit 1-cells in (TC1) must be replaced by $S_{B}$; (TC4) is a strengthened version of the axiom (TC4) as stated in $\$ 16.7$. 
The model theoretic method of proof described in [124] assumes the usual model theoretic compatibilities, such as the pushout-product axiom of [146], and these fail to hold in the present context. The details of the modified arguments only make sense by close comparison with the proofs in [124], but we shall at least explain what is involved. The essential idea is to first verify versions of the axioms using external smash products and function objects and then pull back along diagonal maps to obtain the conclusions. Note that the base change functors associated to a map $f$ of base spaces are exact. We see this for $f$ ! since it preserves cofibration sequences, and it follows for $f^{*}$ and $f_{*}$ since (left or right) adjoints of exact functors between triangulated categories are exact.

The axiom (TC1) only involves suspension, in our case $\Sigma_{B}$, and is thus easily checked using Proposition 12.6.4. For (TC2), we must show that the functors $X \wedge_{B}(-), F_{B}(X,-)$, and $F_{B}(-, Y)$ preserve distinquished triangles, where $X$ and $Y$ are $G$-spectra over $B$. Either model theoretically or by standard topological arguments with cofiber sequences and fiber sequences, it is easy to see that these conclusions hold with $\wedge_{B}$ and $F_{B}$ replaced by the external functors $\bar{\wedge}$ and $\bar{F}$. For $\bar{\wedge}$, we use the proof of Theorem 12.4.2 to arrange level quasi-fibrant cofibers. For $F_{B}$, we remember that the triangulation by fibrations is the negative of the triangulation by fibrations, as a consequence of Proposition 12.4.5. Since $\Delta^{*}$ and $\Delta_{*}$ are exact, the conclusion internalizes directly.

Similarly, the braid axiom (TC3) and the additivity axiom (TC4) hold for $\bar{\wedge}$ by the arguments explained in $[124, \S 6]$. These arguments work with cofiber sequences in which one uses actual quotients instead of cofibers, but one can transfer the conclusions to standard cofiber sequences by using the usual equivalence between quotients and cofibers of $C y l$-cofibrations. This works since our $s$-cofibrations are Cyl-cofibrations. The essential point is that, on the external level, smash products of cofibrant objects are cofibrant and one can work with actual cones and cofibers, modulo the replacement argument of Theorem 12.4.2, just as if we were in a more naive space level context. These results for the external products pull back along $\Delta^{*}$ to give the axioms internally in $\mathrm{Ho} G \mathscr{S}_{B}$. In particular, the constructed central terms $V$ and $W$ that one obtains in (TC3) and (TC3') are of the form $\Delta^{*} \mathbb{V}$ and $\Delta^{*} \mathbb{W}$ for objects $\mathbb{V}$ and $\mathbb{W}$ in $G \mathscr{S}_{B \times B}$.

Before turning to the last axiom, we explain how the weak compatibility axioms for $\mathscr{E} x$ follow from the arguments just given. The morphism category $\mathscr{E} x(A, B)$ is Ho $\mathscr{S}_{B \times A}$, which is a symmetric monoidal category with a compatible triangulation. We have a dictionary, Proposition 17.1.4, that translates $\odot$, $\triangleright$, and $\triangleleft$ into the language of base change functors and smash product and function spectra that are internal to one or another of the categories $\operatorname{Ho} \mathscr{S}_{B \times A}$, and the axioms involve distinguished triangles in one such category at a time. This allows us to deduce the axioms (TC1)-(TC4) for $\mathscr{E} x$ directly from the corresponding axioms for the various categories $\operatorname{Ho} \mathscr{S}_{B}$ and the exactness of base change functors.

The last axiom is the braid duality axiom (TC5) of $[124, \S 4]$. It is more subtle because it involves simultaneous use of $\wedge_{B}$ and $F_{B}$. We describe what it says. We fix a distinguished triangle

$$
X \stackrel{f}{\longrightarrow} Y \stackrel{g}{\longrightarrow} Z \stackrel{h}{\longrightarrow} \Sigma_{B} X
$$


and we have a dual distinguished triangle

$$
D_{B} Z \stackrel{D_{B} g}{\longrightarrow} D_{B} Y \stackrel{D_{B} f}{\longrightarrow} D_{B} X \stackrel{D_{B}\left(\Sigma_{B}^{-1} h\right)}{\longrightarrow} \Sigma_{B} D_{B} Z .
$$

For simplicity, we assume right away that the given spectra $X, Y$, and $Z$ over $B$ are fiberwise dualizable. We can apply (TC3) and $\left(\mathrm{TC} 3^{\prime}\right)$ to both the pair

$$
\left((f, g, h),\left(D_{B} g, D_{B} f, D_{B} \Sigma_{B}^{-1} h\right)\right)
$$

and the reverse pair

$$
\left(\left(D_{B} g, D_{B} f, D_{B} \Sigma_{B}^{-1} h\right),(f, g, h)\right) .
$$

The dual of a diagram (TC3) for the first pair is a diagram (TC3') for the second pair $[124,4.1 .3]$. A weakened form of the axiom that suppresses some further information relating to (TC4) reads as follows. We write the central entry in a diagram as in (TC3 ${ }^{\prime}$ ) for the second pair as $\bar{W}$ to avoid confusion with (TC3') for the first pair, and similarly for the maps that appear in such a diagram.

(TC5) (The braid duality axiom.) There is a diagram as in (TC3') for the pair of distinguished triangles $\left(\left(D_{B} g, D_{B} f, D_{B} \Sigma_{B}^{-1} h\right),(f, g, h)\right)$ which satisfies the following properties.

(a) There is a map $\bar{\varepsilon}: \bar{W} \longrightarrow S_{B}$ such that the following diagram commutes.

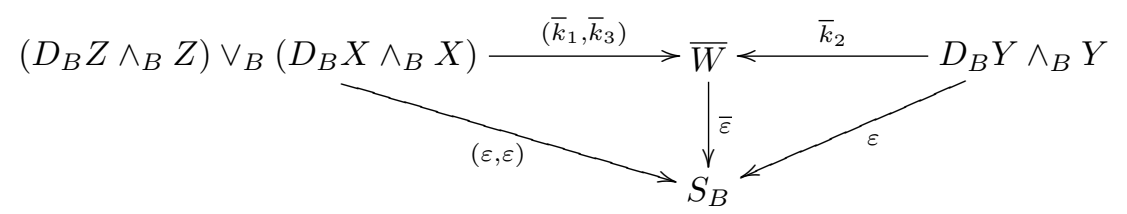

(b) The chosen diagram as in $\left(\mathrm{TC}^{\prime}\right)$ is isomorphic to the dual of a diagram as in (TC3) for the pair of distinguished triangles

$$
\left((f, g, h),\left(D_{B} g, D_{B} f, D_{B} \Sigma_{B}^{-1} h\right)\right) .
$$

The dual of (a) for the coevaluation maps is implied.

LEMMA 17.5.3 (TC5a'). For a diagram as in (TC3) for the pair of triangles $\left((f, g, h),\left(D_{B} g, D_{B} f, D_{B} \Sigma_{B}^{-1} h\right)\right)$ which satisfies (TC5b), there is a map $\bar{\eta}: S_{B} \rightarrow V$ such that the following diagram commutes.

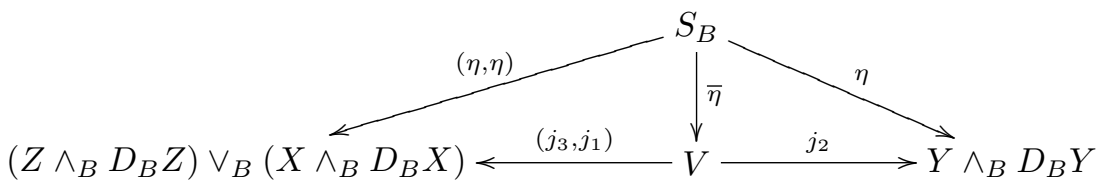

This gives precisely the information that is needed to prove the additivity of Euler characteristics and trace maps. The observant reader will recognize that it is not clear how to formulate an analogous axiom for closed symmetric bicategories.

The rest of the section is for the diligent reader who is willing to compare line by line with $[124, \S 7]$. The argument for (TC5) there takes five dense pages, and we do not want to reproduce it here. Rather, we describe what needs to be changed in the proof to deal with our more complicated situation.

To have well-behaved point-set level smash products, we must work externally, using $\bar{\wedge}$ and the $s$-model structure. We begin the argument for (TC5a) by letting $T \in G \mathscr{S}_{B \times B}$ be a fibrant and cofibrant model of the derived $\Delta_{*} S_{B}$. We take our cofiber sequence $(f, g, h)$ in canonical form in $G \mathscr{S}_{B}$, with $X, Y$, and $Z$ all cofibrant. 
Then $\bar{F}(X, T)$ is fibrant, and a cofibrant approximation $D_{B} X \longrightarrow \bar{F}(X, T)$ gives a fibrant and cofibrant point-set level model for the derived $D_{B} X=F_{B}\left(X, S_{B}\right)$ in Ho $G \mathscr{S}_{B}$. Here we are implicitly using that $F_{B}\left(X, S_{B}\right) \simeq \bar{F}\left(X, \Delta_{*} S_{B}\right)$ in $\operatorname{Ho} G \mathscr{S}_{B}$. Arguing as in $[124, \S 7]$, we construct a good point-set level model for the cofibration sequence (17.5.2) in $G \mathscr{S}_{B}$, using the same notation for its terms. That leads to a diagram exactly like $[124,(7.5)]$, hence to a diagram like $[124,(7.3)]$, except that it lies in $G \mathscr{S}_{B \times B}$ and features an object $\overline{\mathbb{W}}$ there. All of the rest of its terms are of the form $(-) \bar{\wedge}(-)$, except for its target, which is $T$. The diagram passes to the homotopy category $\mathrm{HoG} \mathscr{S}_{B \times B}$, where its target is $\Delta_{*} S_{B}$. The adjoint of the resulting diagram, which is obtained by applying $\Delta^{*}$ and then the evaluation map $\Delta^{*} \Delta_{*} S_{B} \longrightarrow S_{B}$, is the diagram called for in (TC5a).

Following the proof of (TC5b) in $[124, \S 7]$, the next step would be to obtain a good point-set level model for the natural duality map

$$
\xi: D_{B} X \wedge_{B} Y \longrightarrow D_{B}\left(X \wedge_{B} D_{B} Y\right),
$$

starting from good point-set level models for $D_{B} X$ and $D_{B} Y$ as in the previous paragraph. The argument there begins by constructing a map $r: T \wedge T \longrightarrow T$ and using it to obtain a point-set level map $F(X, T) \wedge F(Y, T) \longrightarrow F(X \wedge Y, T)$ of models for duals in the relevant point-set level category. This makes no sense in our external context. Specializing the general categorical definition, $\xi$ in $\mathrm{HoG}_{B} \mathscr{S}_{B}$ is the adjoint of the map

$$
D_{B} X \wedge_{B} Y \wedge_{B} X \wedge_{B} D_{B} Y \simeq D_{B} X \wedge_{B} X \wedge_{B} D_{B} Y \wedge_{B} Y \stackrel{\varepsilon \wedge_{B} \varepsilon}{\longrightarrow} S_{B} \wedge_{B} S_{B} \cong S_{B},
$$

where the equivalence is given by commutation equivalences $\gamma$. A Yoneda lemma argument shows that we have an equivalence

$$
\Delta_{B}^{*} \bar{F}\left(\mathbb{V}, \Delta_{*}^{B \times B} S_{B \times B}\right) \simeq \bar{F}\left(\Delta_{B}^{*} \mathbb{V}, \Delta_{*}^{B} S_{B}\right) \simeq D_{B}\left(\Delta_{B}^{*} \mathbb{V}\right)
$$

in $\mathrm{HoG} \mathscr{S}_{B}$ for $\mathbb{V} \in G \mathscr{S}_{B \times B}$, where we use subscript and superscript $B$ and $B \times B$ to indicate the space whose diagonal map is intended.

Working in $\mathrm{Ho} G \mathscr{S}_{B \times B}$, with $X, Y$ in $G \mathscr{S}_{B}$, we have a natural map

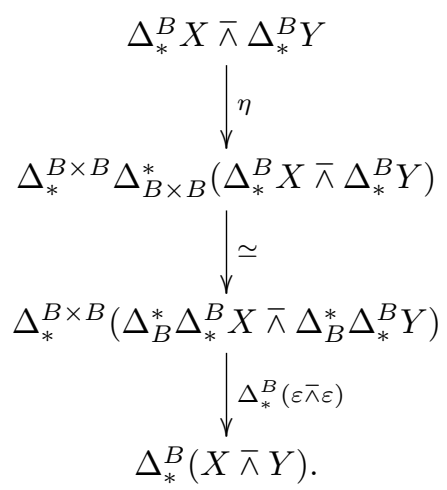

The equivalence in the middle is a base change equivalence coming from the trivial observation that $(\mathrm{id} \wedge \gamma \wedge \mathrm{id}) \circ\left(\Delta_{B} \times \Delta_{B}\right)=\Delta_{B \times B}$. Taking $X=Y=S_{B}$ and using that $S_{B} \bar{\wedge} S_{B}$ is isomorphic to $S_{B \times B}$, we obtain a map

$$
\Delta_{*} S_{B} \bar{\wedge} \Delta_{*} S_{B} \longrightarrow \Delta_{*} S_{B \times B}
$$

Letting $U \in G \mathscr{S}_{B \times B \times B \times B}$ be a fibrant and cofibrant model for $\Delta_{*} S_{B \times B}$, we can represent the displayed map in $\mathrm{HoG}_{B \times B}$ by a map $r: T \wedge T \longrightarrow U$ in 
$G \mathscr{S}_{B \times B \times B \times B}$. We have a natural point set level pairing

$$
\bar{F}(X, T) \bar{\wedge}(Y, T) \longrightarrow \bar{F}(X \bar{\wedge} Y, T \bar{\wedge} T)
$$

of spectra over $B \times B$. Composing with $r$, we obtain a pairing

$$
\bar{F}(X, T) \bar{\wedge} \bar{F}(Y, T) \longrightarrow \bar{F}(X \bar{\wedge} Y, U) .
$$

Using a cofibrant approximation $D_{B} Y \longrightarrow \bar{F}(Y, T)$ and the evaluation map for $Y$, we obtain a composite map $D_{B} Y \bar{\wedge} Y \longrightarrow \bar{F}(Y, T) \bar{\wedge} Y \longrightarrow T$ with adjoint map $Y \longrightarrow \bar{F}\left(D_{B} Y, T\right)$. The required point-set level model for $\xi$ is obtained by applying $\Delta^{*}$ to the composite

$$
D_{B} X \bar{\wedge} \longrightarrow \bar{F}(X, T) \bar{\wedge} \bar{F}\left(D_{B} Y, T\right) \longrightarrow \bar{F}\left(X \bar{\wedge} D_{B} Y, U\right) .
$$

At precisely this point in the cited proof of $[124, \S 7]$, where a model for $\xi$ has just been obtained, the notation $D^{\prime} X$ is introduced for $F(X, T)$. We instead define $D^{\prime}(\mathbb{V})=\bar{F}(\mathbb{V}, U)$ for $\mathbb{V} \in G \mathscr{S}_{B \times B}$. Now we carry out our diagram chasing exactly as in $[124, \S 7]$, but working in $G \mathscr{S}_{B \times B}$ with our new functor $D^{\prime}$. After constructing all of the diagrams of the proof on the point-set level there and passing to homotopy categories, we apply $\Delta^{*}$, remembering that it is an exact functor. The diligent reader will see that the argument then goes through verbatim to give (TC5b). 


\section{CHAPTER 18}

\section{Costenoble-Waner duality}

\section{Introduction}

We specialize the theory of duality from Chapters 16 and 17 to the theory of Costenoble-Waner duality in the category of $G$-spectra over $B$ in $\S 18.1$. The similarity with Spanier-Whitehead duality will be evident. The essential point is to identify dual parametrized spectra in the Costenoble-Waner sense. We show in $\S 18.2$ that retracts of finite cell spectra over $B$ (in the $s$-model structure) are dualizable and that the compact spectra over $B$ are precisely the dualizable spectra over $B$. As we shall explain, we do not know how to prove conversely that a dualizable spectrum is a retract of a finite cell spectrum.

We also do not have a direct proof that the single-cell spectra over $B$ are Costenoble-Waner dualizable, which of course is the starting point for the inductive proof that all finite cell spectra over $B$ are dualizable. However, parametrized Atiyah duality implies that they are. We recall that single-cell spectra arise from (non-fibrant) sphere spaces over $B$ and that the (invertible) sphere spectrum $S_{B}$ over $B$ is not among them. For this reason, intuition from the classical case is misleading. Equivariantly, duality of single-cell spectra is in any case non-trivial since Atiyah duality specializes to give the dualizability of orbit spectra.

We prove parametrized Atiyah duality for smooth $G$-manifolds in $\S 18.6$. This reproves the classical Atiyah duality theorem since the functor $r_{\text {! }}$ preserves dual pairs. In fact, as advertised in Example 0.0.3, we give a relative version that appears to be new even in the non-parametrized case. It gives an explicit description of the Spanier-Whitehead dual of $M / L$, where $M$ is a smooth closed manifold and $L$ is a smooth closed submanifold. We treat the more usual relative case of manifolds with boundary in $\S 18.7$. In both versions, Atiyah duality is a special case of a general space level Costenoble-Waner duality theorem for $G$-ENR pairs that we explain in $\S 18.5$ and prove in $\S 18.8$, following Costenoble and Waner [41, 4.2.8]. Their proof relies for details on the non-parametrized treatment given in [98, III.§4], and we give a more self-contained account. We begin work in $\$ 18.3$ by showing how to deduce spectrum level Costenoble-Waner duality from space level CostenobleWaner duality, and we give preliminaries on relative mapping cones in $\S 18.4$.

Equivariance adds no extra difficulties in this chapter. We assume that a given compact Lie group $G$ acts on all of our spaces and spectra, and we generally omit $G$ from the notations except for occasional emphasis. We assume that all base $G$-spaces have the homotopy types of $G$-CW complexes. Here again, "equivalence" means isomorphism in the relevant stable homotopy category and is denoted by $\simeq$. 


\subsection{The two notions of duality in $\operatorname{Ho} G \mathscr{S}_{B}$}

In $\S 15.1$ we discussed fiberwise duality for parametrized spectra $X$ in $\operatorname{Ho} G \mathscr{S}_{B}$ and defined the fiberwise dual of $X$ to be

$$
D_{B} X=F_{B}\left(X, S_{B}\right) \simeq \bar{F}\left(X, \Delta_{*} S_{B}\right) .
$$

There is another notion of duality in $\mathrm{Ho}_{\boldsymbol{B}} \mathscr{S}_{B}$. Costenoble and Waner discovered it in [41], and we therefore name it after them. The Costenoble-Waner dual of $X$ in $\mathrm{Ho} G \mathscr{S}_{B}$ is

$$
D_{B}^{C W} X=\bar{F}\left(X, \Delta_{!} S_{B}\right) .
$$

In this section, we describe both notions of duality as special cases of duality in the bicategory $\mathscr{E} x$ of parametrized spectra.

Recall that, by the conventions of Notations 17.4.1, we implicitly view a parametrized spectrum $X$ over $B$ as a 1-cell $X: B \longrightarrow *$ in the bicategory $\mathscr{E} x$, writing $t X$ for the opposite 1-cell $t X: * \longrightarrow B$. Here the spaces $*$ and $B$ are 0-cells, but we also write $*$ and $B$ for the unit 1-cells $S$ in $G \mathscr{S}=\mathscr{E} x(*, *)$ and $\Delta_{!} S_{B}$ in $G \mathscr{S}_{B \times B}=\mathscr{E} x(B, B)$. We have four duals with respect to these 1-cells. The ones with respect to $*$ are

$$
D_{\ell} X=* \triangleleft X: * \longrightarrow B \quad \text { and } \quad D_{r} t X=t X \triangleright *: B \longrightarrow *,
$$

and the ones with respect to $B$ are

$$
D_{\ell} t X=B \triangleleft t X: B \longrightarrow * \text { and } \quad D_{r} X=X \triangleright B: * \longrightarrow B .
$$

First we consider the duals with respect to $*$. We have that $t X$ is right dualizable if and only if $X$ is left dualizable, and $t D_{\ell} X \simeq D_{r} t X$. Therefore, these duals have the same underlying spectrum over $B$, which by Proposition 17.1.4 is

$$
F_{B}\left(X, r^{*} S\right) \simeq F_{B}\left(X, S_{B}\right) .
$$

Said another way, $(t X, Y)$ is a dual pair if and only if $(t Y, X)$ is a dual pair, and then the underlying spectrum of $Y$ is equivalent to $F_{B}\left(X, S_{B}\right)$. The evaluation and coevaluation maps of the two pairs correspond under application of the involution $t$ in our symmetric bicategory. By the following result, this notion of duality in $\mathscr{E} x$ is equivalent to fiberwise duality.

Proposition 18.1.1. Let $X$ and $Y$ be spectra over B. Then a pair of maps

$$
\eta: \Delta ! S_{B} \longrightarrow t X \odot Y \quad \text { and } \quad \varepsilon: Y \odot t X \longrightarrow S,
$$

represent $(t X, Y)$ as a dual pair if and only if their adjoint maps

$$
\bar{\eta}: S_{B} \longrightarrow X \wedge_{B} Y \quad \text { and } \quad \bar{\varepsilon}: Y \wedge_{B} X \longrightarrow S_{B},
$$

represent $(X, Y)$ as a fiberwise dual pair.

Proof. The respective adjoints are taken with respect to the adjunctions $\left(\Delta_{!}, \Delta^{*}\right)$ and $\left(r_{!}, r^{*}\right)$. This makes sense since $t X \odot Y=X \bar{\wedge} Y$ and thus $\Delta^{*}(t X \odot Y)=$ $X \wedge_{B} Y$ and since $Y \odot t X=r_{!}\left(Y \wedge_{B} X\right)$ and $S_{B}=r^{*} S$. Expanding everything out in terms of external smash products and base change maps associated to $r$ and $\Delta$, we find by easy but laborious diagram chases that the defining triangle identities are equivalent. Alternatively, we can check that $\varepsilon$ satisfies the condition required of a coevaluation map if and only if $\bar{\varepsilon}$ does, and similarly for $\eta$ and $\bar{\eta}$. For the former, let $W$ be a spectrum over $B$, let $Z$ be a spectrum, and consider the following diagram, in which $\mathscr{C}$ stands for Ho $G \mathscr{S}$ and $\mathscr{C}_{B}$ stands for Ho $G \mathscr{S}_{B}$. 


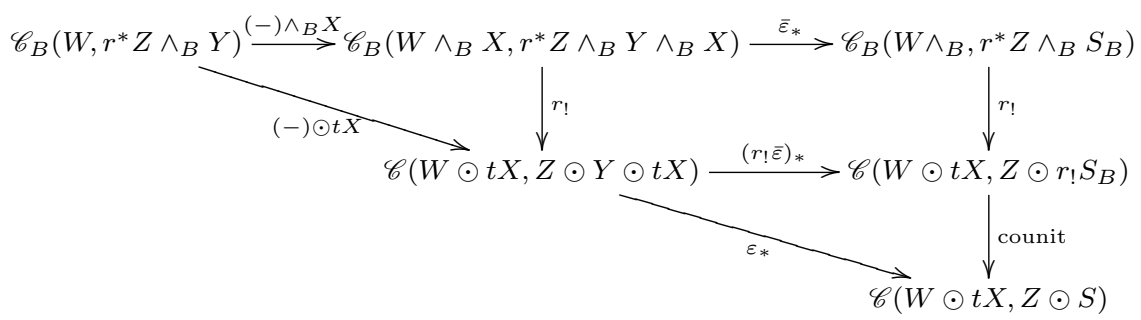

The composite on the top row is the map $\bar{\varepsilon}_{\sharp}$. It is an isomorphism if $X$ is fiberwise dualizable with evaluation $\bar{\varepsilon}$. We have $r^{*} Z \wedge_{B} Y \cong Z \odot Y$, by Proposition 17.1.5, and the composite on the hypotenuse is the map $\varepsilon_{\sharp}$ of Proposition 16.4.6(ii). It is an isomorphism if $(t X, Y)$ is a dual pair with evaluation map $\varepsilon$. The upper vertical arrows are obtained by applying $r$ ! to maps and using the projection formula and Proposition 17.1.4 to identify the target objects. The lower vertical arrow is induced by the counit $r ! S_{B}=r ! r^{*} S \longrightarrow S$ of the adjunction $\left(r_{!}, r^{*}\right)$, and the vertical composite on the right is the adjunction isomorphism.

For the latter, let both $W$ and $Z$ be spectra over $B$ and consider the following diagram, in which $\mathscr{C}_{B}$ and $\mathscr{C}_{B \times B}$ stand for Ho $G \mathscr{S}_{B}$ and HoG $\mathscr{S}_{B \times B}$.

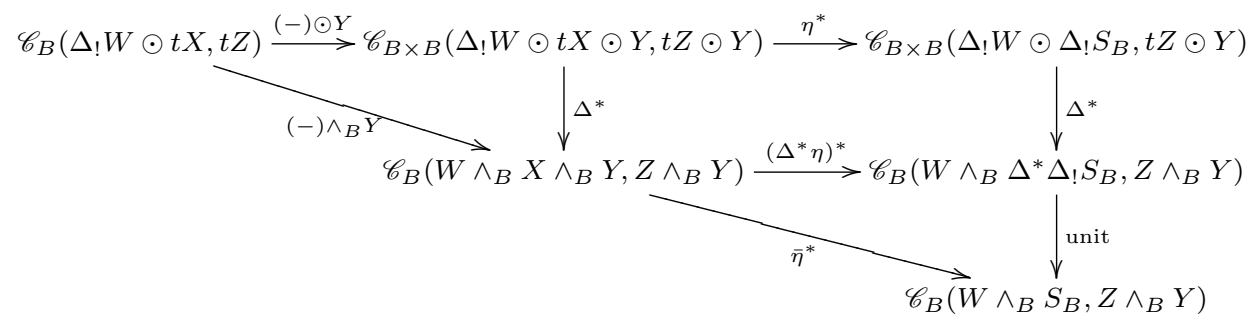

The composite on the top row is the map $\eta_{\sharp}$ of Proposition 16.4.6(iii). It is an isomorphism if $t X$ is right dualizable with coevaluation $\eta$. We have $\Delta_{!} W \odot t X \simeq$ $W \wedge_{B} X$, by Proposition 17.4.3, and the composite on the hypotenuse is the map $\bar{\eta}_{\sharp}$. It is an isomorphism if $X$ is fiberwise dualizable with coevaluation map $\bar{\eta}$. If $U: B \longrightarrow B$ is a 1-cell, then $\Delta_{!} W \odot U \simeq \pi^{*} W \wedge_{B \times B} U$, by Proposition 17.1.4, and therefore $\Delta^{*}\left(\Delta_{!} W \odot U\right) \simeq W \wedge_{B} \Delta^{*} U$. Also note that $t Z \odot Y \simeq Z \bar{\wedge} Y$. Using the last two observations, the upper vertical arrows are obtained by applying $\Delta^{*}$ to maps. The lower vertical arrow is induced by the unit of the $\left(\Delta_{!}, \Delta^{*}\right)$ adjunction, and the vertical composite on the right is the adjunction isomorphism.

We now turn to the duals with respect to $B$. We have that $X$ is right dualizable if and only if $t X$ is left dualizable, and $D_{\ell} t X \simeq t D_{r} X$. Therefore, the two duals have the same underlying spectrum over $B$ which we identify using Proposition 17.1.4.

Definition 18.1.2. Let $X$ be a spectrum over B. Define the Costenoble-Waner dual spectrum of $X$ in Ho $G \mathscr{S}_{B}$ to be

$$
D_{B}^{C W} X=\bar{F}\left(X, \Delta_{!} S_{B}\right) .
$$

Viewed in two ways as a 1 -cell in $\mathscr{E} x$,

$$
D_{B}^{C W} X \simeq B \triangleleft t X=D_{\ell} t X: B \longrightarrow * \text { and } t D_{B}^{C W} X \simeq X \triangleright B=D_{r} X: * \longrightarrow B .
$$


We say that $X$ is Costenoble-Waner dualizable with dual $Y$ if $(X, t Y)$, or equivalently $(Y, t X)$, is a dual pair in $\mathscr{E} x$, and then the underlying spectrum of $Y$ is equivalent to $D_{B}^{C W} X$.

Note for consistency with Proposition 17.1.4 that Lemma 2.5.5 gives

$$
\pi_{1 *} F_{B \times B}\left(\pi_{2}^{*} X, \Delta_{!} S_{B}\right) \simeq \bar{F}\left(X, \Delta_{!} S_{B}\right) \simeq \pi_{2 *} F_{B \times B}\left(\pi_{1}^{*} X, \Delta_{!} S_{B}\right) .
$$

REMARK 18.1.3. To reiterate, let us be precise about the specializations of right and left duality that are visible to us now. For two spectra $X$ and $Y$ over $B$ to be Costenoble-Waner duals, or equivalently for $(X, t Y)$ to be a dual pair of 1-cells, we must have maps

$$
\eta: S \longrightarrow X \odot t Y \quad \text { and } \quad \varepsilon: t Y \odot X \longrightarrow \Delta_{!} S_{B}
$$

such that the appropriate diagrams commute. However, for two spectra $X$ and $Y$ to be fiberwise duals, or equivalently for $(t X, Y)$ to be a dual pair of 1-cells, we must have maps

$$
\eta: \Delta_{!} S_{B} \longrightarrow t X \odot Y \quad \text { and } \quad \varepsilon: Y \odot t X \longrightarrow S
$$

such that the appropriate diagrams commute. As we have seen in the case of $S_{B}$ and $t S_{B}$, these are wholly different conditions.

Propositions 16.4.6 and 16.4.12 give three ways of thinking about the following formal consequence of the definition.

Proposition 18.1.4. A spectrum $X$ over $B$ is Costenoble-Waner dualizable with dual $Y$ if and only if $Y$ is Costenoble-Waner dualizable with dual $X$, and then $D_{B}^{C W} D_{B}^{C W} X$ is equivalent to $X$.

Of course, the analogue for fiberwise duality also holds. We record the most important consequence of Costenoble-Waner duality. In view of Proposition 17.4.2, it is an immediate reinterpretation of Proposition 16.4.12.

Proposition 18.1.5. If $X$ is a Costenoble-Waner dualizable spectrum over $B$ and $J$ is any spectrum over $B$, then

$$
\mu: r_{!}\left(J \wedge_{B} D_{B}^{C W} X\right) \simeq J \odot t D_{B}^{C W} X \longrightarrow X \triangleright J \simeq r_{*} F_{B}(X, J)
$$

is an equivalence of spectra.

Our general duality theory in $\mathscr{E} x$ reduces in the case $A=B=*$ to SpanierWhitehead duality theory in the stable homotopy category HoGS $\mathscr{S}$, and Theorem 17.3.3 specializes to give the following result.

Corollary 18.1.6. If the sphere spectrum $S_{B}$ over $B$ is Costenoble-Waner dualizable, then $\Sigma^{\infty} B_{+}$is dualizable in the classical sense.

Proof. As noted in Lemma 15.3.1, $r ! S_{B} \simeq \Sigma^{\infty} B_{+}$.

\subsection{Costenoble-Waner dualizability of finite cell spectra}

We explain the proof of the following two results in this section.

THEOREM 18.2.1. If a spectrum $X$ over $B$ is a wedge summand in $H o G \mathscr{S}_{B}$ of a finite cell spectrum, then $X$ is Costenoble-Waner dualizable.

Recall the notion of a compact object in a triangulated category from Definition 13.1.8. 
TheOrem 18.2.2. A spectrum $X$ over $B$ is compact if and only if it is Costenoble-Waner dualizable.

Putting these results together, we obtain the following immediate consequence.

Corollary 18.2.3. A retract of a finite cell spectrum over $B$ is a compact object of $\mathrm{Ho} G \mathscr{S}_{B}$.

This result may seem obvious at first sight, but in view of Warning 7.5.16 it is actually rather surprising. We would not know how to prove it directly, without use of the theory of dualizable objects. One might ask whether the converse of Theorem 18.2.1 (or equivalently Corollary 18.2.3) is true, and we will return to that question after proving the theorems. It is convenient to use the following shorthand language.

Definition 18.2.4. An ex-space $K$ over $B$ is said to be Costenoble-Waner dualizable if $\Sigma_{B}^{\infty} K$ is Costenoble-Waner dualizable in Ho $G \mathscr{S}_{B}$.

The first and main step in the proof of Theorem 18.2.1 is a special case of the parametrized Atiyah duality theorem, which we will explain in a more precise form than is needed here in $\S 18.5$ below.

THEOREM 18.2.5. If $M$ is a smooth compact $G$-manifold, then the sphere exspace $S_{M}^{0}$ over $M$ is Costenoble-Waner dualizable.

Corollary 18.2.6. For $H \subset G$ and $n \geq 0, S_{G / H \times S^{n}}^{0}$ is Costenoble-Waner dualizable.

To generalize from spheres $S_{M}^{0}$ to ex-spaces over general base spaces, we use a quick trick that we learned from Costenoble and Waner [41]. It is based on the following elementary observation.

Lemma 18.2.7. For a space $(K, p)$ over $B,(K, p)_{+}$is isomorphic to $p_{!} S_{K}^{0}$.

Proposition 18.2.8. If $S_{K}^{0}$ is Costenoble-Waner dualizable and $(K, p)$ is a space over $B$, then $(K, p)_{+}$is Costenoble-Waner dualizable.

Proof. We apply Theorem 17.3.3 with $A=*$ and $f=p$.

Since the domains and targets of our generating $s$-cofibrations are of the form $\left(G / H \times S^{n}, p\right)_{+}$and $\left(G / H \times D^{n}, p\right)_{+}$, they are Costenoble-Waner dualizable.

Proposition 18.2.9. The cofiber of a map of Costenoble-Waner dualizable spectra over $B$ is Costenoble-Waner dualizable, and a retract of a Costenoble-Waner dualizable spectrum over $B$ is Costenoble-Waner dualizable.

Proof. This is implied by Proposition 16.8.1, which says that the full subcategory of right dualizable objects in a triangulated symmetric bicategory is thick, but of course it could also be proven directly by specializing the proof there.

Applying this to generating cofibrations and then proceeding by induction on the number of cells, this implies Theorem 18.2.1. Since the compact spectra over $B$ are the objects of the thick subcategory generated by a subset $\mathscr{D}_{B}$ of the collection of compact single-cell spectra over $B$, by Lemma 13.1.11 and Theorem 13.1.14, Theorem 18.2.2 is a special case of the general result Proposition 16.8.3. That result gives a further characterization of the thick subcategory of Costenoble-Waner 
dualizable spectra over $B$ as the category of $\triangleright$-compact spectra over $B$. Since the generating set $\mathscr{D}_{B}$ consists of single-cell spectra over $B$, another characterization is that this is the thick subcategory generated by the finite cell spectra over $B$.

REMARK 18.2.10. The converse of Theorem 18.2.1 is equivalent to the assertion that the retracts up to isomorphism of finite cell spectra over $B$ are themselves the objects of a thick subcategory of Ho $G \mathscr{S}_{B}$. However, a map $f: X \longrightarrow Y$ in Ho $G \mathscr{S}_{B}$ between finite cell spectra over $B$ can only be realized on the point set level after fibrant approximation of its target, using $[X, Y] \cong \pi(X, R Y)$. While $R Y$ is a cell spectrum over $B$, it is hardly ever a finite one, and we see no reason to believe that the cofiber of $f$ is isomorphic in $\mathrm{HoG} \mathscr{S}_{B}$ to a finite cell spectrum.

We can see the same problem differently by trying to adapt the proof that a dualizable spectrum is a retract of a finite CW spectrum, [118, XVI.7.4], to the parametrized context. Thus suppose that $X$ is Costenoble-Waner dualizable with right dual $Y$ and coevaluation map $\eta: S \longrightarrow X \odot Y$. We may assume that $X$ and $Y$ are $s$-fibrant cell spectra over $B$. We ask whether $X$ is a retract in $\operatorname{Ho} G \mathscr{S}_{B}$ of a finite cell spectrum over $B$. Since $X$ is the colimit of its finite subcomplexes $W$ (although these need not be $s$-fibrant) and $S$ is compact, it might seem clear that $\eta$ factors through $W \odot t Y$ for some finite subcomplex $W$ of $X$. Assuming that it does, we have the following commutative diagram whose bottom composite is the identity map.

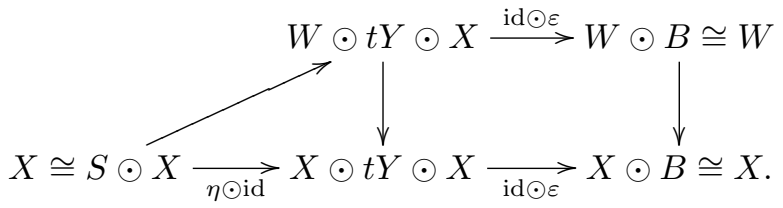

Therefore $X$ is a retract up to homotopy and thus a wedge summand up to homotopy of $W$. However, $\eta$ is only given in $\mathrm{HoG}_{\mathscr{S}_{B}}$. Its target there is $X \odot t Y=$ $r ! \Delta^{*}(X \bar{\wedge} Y)$, and this must be understood in the derived sense. Now $\Delta^{*}$ is a Quillen right adjoint, $r_{\text {! }}$ is a Quillen left adjoint, and $S$ is cofibrant, so $\eta$ is represented by a point-set level map $S \longrightarrow R r_{!} Q \Delta^{*} R(X \bar{\wedge} Y)$, where $Q$ and $R$ denote cofibrant and fibrant approximations (in $G \mathscr{S}_{B}$ or $G \mathscr{S}$ ). The fibrant approximations throw a monkey wrench into the proposed use of the compactness of $S$ to prove the desired factorization.

One interest of this discussion is that we do not yet know the answer to the following question.

Question 18.2.11. Let $M$ be a smooth $G$-manifold. Is $S_{M}$ equivalent in HoG $\mathscr{S}_{M}$ to a retract of a finite cell spectrum over $M$ ?

A general conclusion of this discussion is that, in a compactly generated topological model category whose objects are not fibrant, one cannot expect to work with finite complexes with any degree of facility. The relevant fibrant approximations are blind to finite cell structures. We shall discuss other problems of this nature in Chapter 24.

\subsection{Costenoble-Waner $V$-duality}

As in the study of Spanier-Whitehead duality, we can work on the ex- $G$-space level and define Costenoble-Waner $V$-duality for a representation $V$ of $G$. We adopt 
the same notational conventions for the bicategory of ex- $G$-spaces over $B$ that we adopted for the bicategory of parametrized $G$-spectra in Notations 17.4.1.

Definition 18.3.1. Let $K$ and $L$ be in Ho $G \mathscr{K}_{B}$. We say that $(K, t L)$ is a $V$-dual pair if there are maps

$$
\eta: S^{V} \longrightarrow K \odot t L \quad \text { and } \quad \varepsilon: t L \odot K \longrightarrow \Delta_{!} S_{B}^{V}
$$

such that the following two diagrams commute.
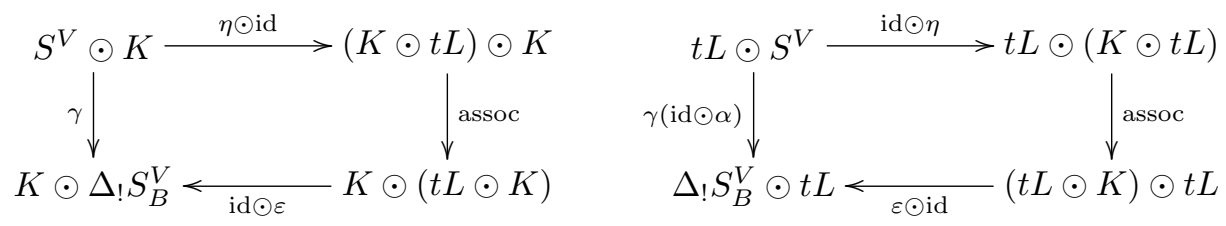

We must explain the two left vertical maps. Rewriting the source and target, $\gamma: S^{V} \wedge K \longrightarrow K \wedge S^{V}$ is the obvious interchange map, and similarly for $L$, while $\alpha: S^{V} \longrightarrow S^{V}$ is the antipode map sending $v$ to $-v$.

Recall that the Lefschetz constant $\chi(\alpha)$, namely the map of $G$-spectra

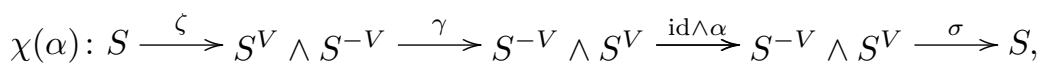

is the identity [98, II.5.11]. Here $S^{-V}=\Sigma_{V}^{\infty} S^{0}$ and $\zeta$ and $\sigma$ are the coevaluation and evaluation maps of the dual pair $\left(S^{V}, S^{-V}\right)$ of $G$-spectra, where we have suppressed the suspension spectrum functor $\Sigma^{\infty}$ from the notation. We use this to prove the following expected result.

Proposition 18.3.2. If $(K, t L)$ is a $V$-dual pair, then $\left(\Sigma_{B}^{\infty} K, t \Sigma_{B}^{-V} \Sigma_{B}^{\infty} L\right)$ is a dual pair of $G$-spectra over $B$.

Proof. In what follows, we suppress the suspension functor $\Sigma_{B}^{\infty}$, implicitly applying it to $K$ and $L$, and we write $(-)_{B}$ for $r^{*}$ on both $G$-spaces (as in Remark 10.1.6) and $G$-spectra. Define $\bar{\eta}$ to be the composite

$$
S \stackrel{\zeta}{\longrightarrow} S^{V} \odot S^{-V} \stackrel{\eta \odot \text { id }}{\longrightarrow}(K \odot t L) \odot S^{-V} \stackrel{\text { assoc }}{\longrightarrow} K \odot\left(t L \odot S^{-V}\right)
$$

and define $\bar{\varepsilon}$ to be the composite displayed in the diagram

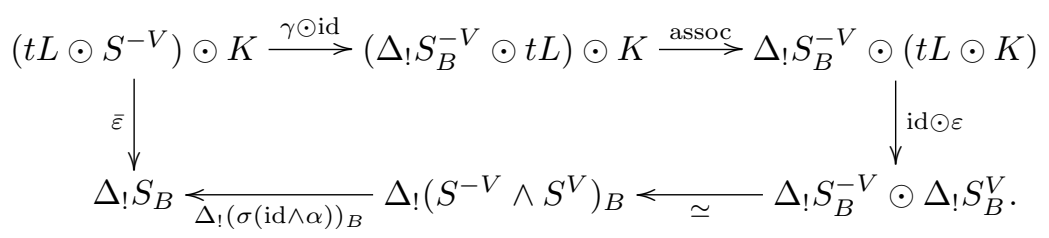

Here, modulo unit isomorphisms, $\gamma$ permutes smashing with $S^{-V}$ from the right to the left. We must verify the commutativity of the diagrams required of $(\bar{\eta}, \bar{\varepsilon})$. They are given by the perimeters of the following two diagrams, in which we abbreviate $\odot$ to $\cdot$ and omit parentheses and uses of associativity isomorphisms. 


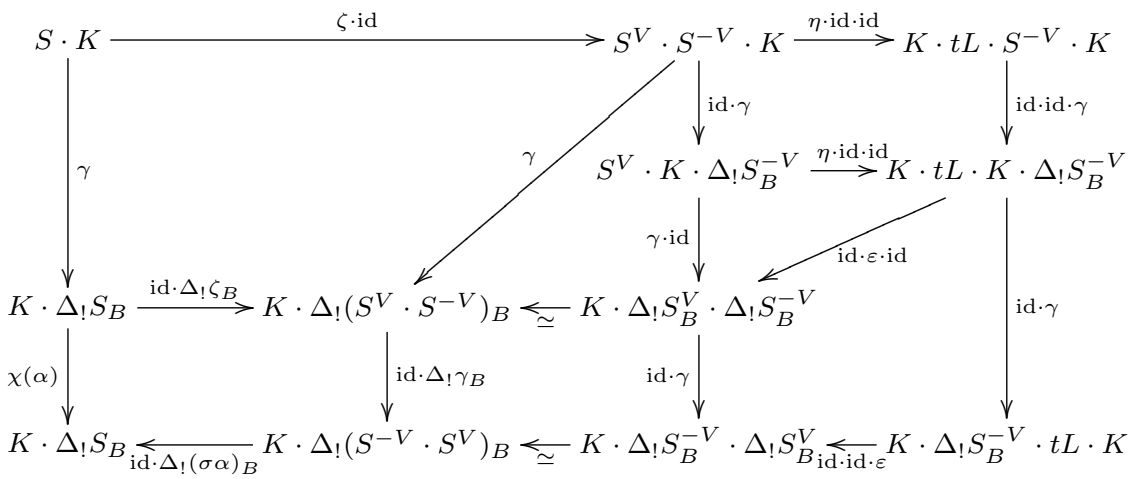

Here the top row is $\bar{\eta} \odot$ id. The middle triangle is a transitivity relation among maps $\gamma$ and, using a similar implicit triangle at the right, we see that the composite down the right column and along the bottom row is id $\odot \bar{\varepsilon}$. The triangle on the right is the first $V$-duality diagram. The two trapezoids, the top right square, and the bottom middle square are naturality diagrams, from which uses of associativity isomorphisms and the associativity pentagon have been suppressed. The bottom left square commutes by the definition of $\chi(\alpha)$. Since $\chi(\alpha)=$ id, the left vertical composite is $\gamma$, and it therefore becomes the identity after using unit equivalences to identify its source and target with $K$.

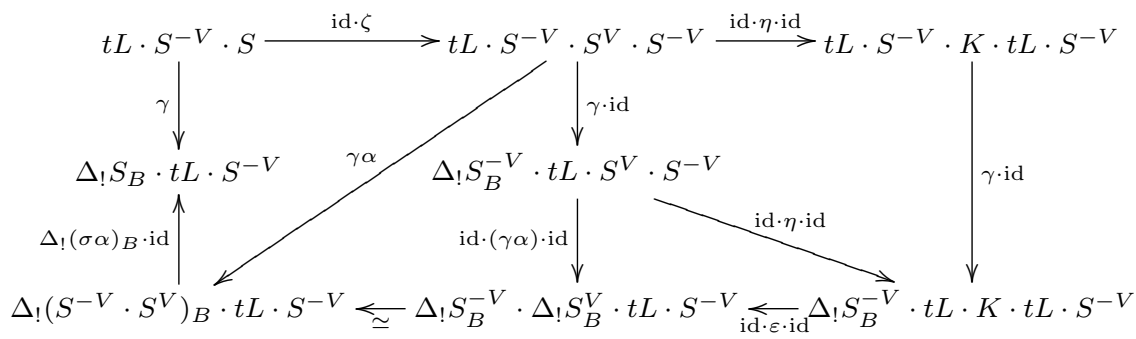

Here the top row is id $\odot \eta$ and the composite down the right, along the bottom, and up to the middle entry in the first column is $\varepsilon \odot$ id. The triangle at the right is the second $V$-dual pair diagram. The trapezoid and the lower left triangle are naturality diagrams. The upper left triangle commutes since the maps $(\zeta, \sigma)$ are the structure maps of the dual pair $\left(S^{V}, S^{-V}\right)$.

\subsection{Preliminaries on unreduced relative mapping cones}

There are several constructions on unsectioned parametrized spaces that naturally give rise to ex-spaces and that we make heavy use of in this chapter. We collect the main results here, following [51] and [98, III $\$ 4]$ in the nonparametrized context. Recall from Remark 2.1.9 that we still have base change adjunctions in this setting. We are interested in the relative theory, and we let $G \mathscr{U}^{2} / B$ denote the category of pairs $(K, L)$ over $B$, where $L$ is a closed subspace of a compactly generated space $K$. We restrict to $\mathscr{U}$ and assume that $L$ is closed in $K$ to avoid irrelevant point-set pathology and to ensure that the inclusion $i: L \longrightarrow K$ is an $f$-cofibration if and only if it is an $\bar{f}$-cofibration.

Construction 18.4.1. We describe quotients and cofibers in $G \mathscr{U}^{2} / B$. 
(i) Let $K /{ }_{B} L$ be the fiberwise quotient over $B$. It is constructed as the pushout in $G \mathscr{U} / B$ of the diagram

$$
B<L \longrightarrow K \text {. }
$$

The map from $B$ into the pushout gives a canonical section; explicitly, the fiber over $b$ is the based space $K_{b} / L_{b}$, and the base points give the section.

(ii) Let $C_{B}(K, L)$ be the (unreduced) fiberwise cofiber of $i$. It is the fiberwise double mapping cylinder of $i$ and the projection $p: L \longrightarrow B$ and can be constructed as the pushout in $G \mathscr{U} / B$ of the evident diagram

$$
K \amalg B<L \amalg L \longrightarrow L \times I .
$$

Equivalently, it is the quotient $M_{B}(K, L) /{ }_{B} L$ of the (unreduced) fiberwise mapping cylinder of $i$. The cone points specify a section, allowing us to regard $C_{B}$ as a functor $G \mathscr{U}^{2} / B \longrightarrow G \mathscr{U}_{B}$.

(iii) We write $C_{B}(K)=C_{B}(K, \emptyset)$. It can be identified with $(k, p)_{+}$, where $p$ is the projection of $K$.

Standard arguments, Proposition 8.2.1, and inspection of definitions give the following three results.

Proposition 18.4.2. If $i: L \longrightarrow K$ is an $f$-cofibration over $B$ or, equivalently, $(K, L)$ is an $f$-NDR pair over $B$, then the fiberwise collapse of $C_{B} L$ is an $f p$ equivalence

$$
C_{B}(K, L) \longrightarrow K / B L
$$

Proposition 18.4.3. The ex-space $C_{B}(K, L)$ is always well-sectioned, and it is an ex-fibration if both $K$ and $L$ are $h$-fibrant. If $(K, L)$ is an $f-N D R$ pair, then $K /{ }_{B} L$ is well-sectioned.

Proposition 18.4.4. Let $f: A \longrightarrow B$ be a map of base spaces, let $(K, L)$ be a pair over $A$, and let $(M, N)$ be a pair over $B$. Then

$$
f_{!} C_{A}(K, L) \cong C_{B}\left(f_{!} K, f_{!} L\right) \quad \text { and } \quad f^{*} C_{B}(M, N) \cong C_{A}\left(f^{*} M, f^{*} N\right) .
$$

We emphasize that, here in the unsectioned context, $f_{!} K$ is just $K$ with projection $f p$, where $p$ is the projection of $K$. Taking $f$ to be the inclusion of a point $b: * \longrightarrow B$, the second part recovers that the fiber $C_{B}(K, L)_{b}$ is $C\left(K_{b}, L_{b}\right)$. If $L_{b}$ is empty, this is $K_{b}$ with a disjoint basepoint.

Proposition 18.4.5 (Excision). Let $U \subset L \subset K$ be spaces over $B$. The inclusion $K-U \longrightarrow K$ induces a homeomorphism

$$
(K-U){ }_{B}(L-U) \longrightarrow K /{ }_{B} L .
$$

Therefore, if $(K-U, L-U)$ and $(K, L)$ are $f$-NDR pairs, the inclusion induces an fp-equivalence

$$
C_{B}(K-U, L-U) \simeq C_{B}(K, L) .
$$

Proof. For the first statement, the displayed quotient map is a continuous bijection. By examining the relevant subspace and quotient topologies we see that the map is in fact a homeomorphism. When we have $f$-NDR pairs, the fiberwise cofibers are $f p$-equivalent to the corresponding fiberwise quotients by Proposition 18.4.2. 
Proposition 18.4.6. Let $(K, L)$ and $(M, N)$ be pairs over $A$ and $B$. Then there is a natural homeomorphism

$$
K /{ }_{A} L \bar{\wedge} M /{ }_{A} N \cong(K \times M) / A \times B(L \times M \cup K \times N) .
$$

Therefore, if $(K, L)$ and $(M, N)$ are $f-N D R$ pairs over $A$ and $B$, there is a canonical fp-equivalence

$$
C_{A}(K, L) \bar{\wedge} C_{B}(M, N) \simeq C_{A \times B}(K \times M, L \times M \cup K \times N) .
$$

Proof. The fiberwise quotient on the right is the pushout displayed in the outer rectangle of the diagram

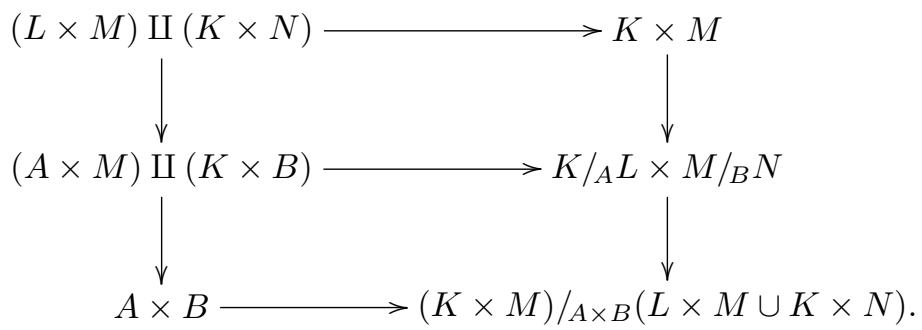

The composite construction implied by the diagram gives the first statement. The second statement follows from Proposition 18.4.2.

In view of the previous lemma, the notation

$$
(K, L) \times(M, N)=(K \times M, L \times M \cup K \times N)
$$

is as convenient as it is categorically incorrect. Using this notation, we endow the categories of parametrized pairs $G \mathscr{U}^{2} /(B \times A)$ with the horizontal composition specified by

$$
(M, N) \odot(K, L)=\pi_{C \times A !} \Delta_{B}^{*}((M, N) \times(K, L)),
$$

where $(K, L)$ is a pair over $B \times A$ and $(M, N)$ is a pair over $C \times B$. The fiberwise cone construction preserves this composition.

Proposition 18.4.7. For $f$-NDR pairs $(K, L)$ and $(M, N)$ of $G$-spaces over $B \times A$ and $C \times B$, respectively, there is a canonical fp-equivalence

$$
C_{C \times A}((M, N) \odot(K, L)) \simeq C_{C \times B}(M, N) \odot C_{B \times A}(K, L) .
$$

Proof. This follows directly from Propositions 18.4.4 and 18.4.6.

Proposition 18.4.8. The functor $C_{B}: G \mathscr{U}^{2} / B \longrightarrow G \mathscr{U}_{B}$ preserves q-equivalences.

Proof. This is immediate from the gluing lemma and the definition of $C_{B}$ as a double mapping cylinder.

Of course, pushforward functors $f_{\text {! }}$ do not change total spaces in the unsectioned context, so they also preserve $q$-equivalences. These facts give the information we need to pass from the point-set level to the derived homotopy category level in our use of the functor $C_{B}$ in the arguments to follow. 


\section{5. $V$-duality of $G$-ENRs}

In this section we provide a large class of Costenoble-Waner $V$-dualizable parametrized ex-spaces $(K, p)_{+}$over $B$. By Proposition 18.2.8, if $S_{K}^{0}$ is CostenobleWaner dualizable, then so is $p_{!} S_{K}^{0} \simeq(K, p)_{+}$, and we therefore focus on $S_{K}^{0}$.

Throughout this section, $(K, L)$ will be a compact $G$-ENR pair. This means that $(K, L)$ is an $h$-NDR pair such that $K$ can be embedded as a $G$-subspace of a representation $V$ in such a way that there is a $G$-retraction $q: N \longrightarrow K$ of an open neighborhood $N$ of $K$ in $V$. We view $(N, q)$ as a space over $K$; the example to keep in mind is that of a tubular neighborhood of a $G$-manifold.

TheOREM 18.5.1 (Costenoble-Waner duality theorem). If $(K, L)$ is a compact $G$-ENR pair, then the ex-space $C_{K}(K, L)$ over $K$ is Costenoble-Waner $V$-dualizable with dual $C_{K}(N-L, N-K)$.

The proof will be given in $\S 18.6$. This implies the general result that we are really after. For the absolute case, recall that $(K, p)_{+}=C_{B}(K, \emptyset) \equiv C_{B}(K)$.

THEOREM 18.5.2. If $(K, p)$ is a $G$-space over $B$ and $(K, L)$ is a compact $G$-ENR pair, then $C_{B}(K, L)$ is Costenoble-Waner $V$-dualizable with dual $C_{B}(N-L, N-K)$. In particular, $(K, p)_{+}$is $V$-dual to $C_{B}(N, N-K)$.

Proof. In the statement, we view $N$ and $K$ as spaces over $B$ with projections $p q$ and $p$, which means that we first consider them as spaces over $K$ with projections $q$ and $\operatorname{id}_{K}$ and then apply $p_{!}$. Theorem 18.5.1 gives that $C_{K}(K, L)$ and $C_{K}(N-L, N-K)$ are $V$-duals over $K$. Using Proposition 18.4.4, it follows from Proposition 18.2.8 that $p_{!} C_{K}(K, L) \cong C_{B}(K, L)$ and $p_{!} C_{K}(N-L, N-K) \cong$ $C_{B}(N-L, N-K)$ are $V$-duals over $B$.

REMARK 18.5.3. In the original nonparametrized version of Dold and Puppe [51] and its equivariant generalization of [98, III§4], $C(N-L, N-K)$ is replaced by its excisive equivalent $C(V-L, V-K)$. However, the parametrization forces us to focus on $N$ rather than $V$.

Returning to Theorem 18.5.1, we compare its absolute and relative cases, but first we introduce some notation. Let $i: L \longrightarrow K$ be the inclusion. Since $i$ is an $h$-cofibration, we have a neighborhood deformation retraction $d: K \longrightarrow K$ which restricts to a retraction $j: U \longrightarrow L$ defined on some open neighborhood $U$ of $L$ in $K$. Let $\iota: L \longrightarrow U$ and $\kappa: U \longrightarrow K$ be the inclusions, so that $i=\kappa \iota$ and $d \kappa=i j$. We display these maps in the commutative diagram

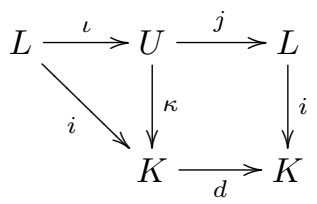

where the composite along the top row is the identity.

Since we are working on the space and not the spectrum level in this section, the desuspension in the following result should be interpreted as appearing after stabilization. We have written the result in the form most convenient for our later applications. 
THEOREM 18.5.5. The cofiber sequences

$$
(L, i)_{+} \longrightarrow(K, i d)_{+} \longrightarrow C_{K}(K, L) \longrightarrow \Sigma_{K}(L, i)_{+}
$$

and

$\Sigma_{K}^{-1} C_{K}(N, N-L) \longrightarrow C_{K}(N-L, N-K) \longrightarrow C_{K}(N, N-K) \longrightarrow C_{K}(N, N-L)$

are $V$-dual, where $i: L \longrightarrow K$ is the inclusion.

ProOF. The first cofiber sequence is

$$
C_{K}(L) \longrightarrow C_{K}(K) \longrightarrow C_{K}(K, L) \longrightarrow \Sigma_{K} C_{K}(L) .
$$

We recognize from Theorem 18.5.1 that the second and third terms in the second sequence are $V$-dual to the third and second terms, respectively, in the first sequence. Recall the maps displayed in (18.5.4). We define

$$
N_{L}=j ! j^{*} i^{*} N=j ! \kappa^{*} d^{*} N .
$$

This is a space over $L$ whose projection is a retraction of an open neighborhood of $L$ in $V$. In the next section, we will take $L$ to be a closed submanifold of a closed manifold, and in that context $N_{L}$ will be the normal bundle $\nu_{L}$ of $L$. We have a canonical inclusion

$$
i_{!} N_{L}=i_{!} j_{!} \kappa^{*} d^{*} N=i_{!} j_{!} j^{*} i^{*} N \longrightarrow N
$$

over $K$. By excision, Proposition 18.4.5, it induces an equivalence

$$
i_{!} C_{L}\left(N_{L}, N_{L}-L\right) \cong i_{!} C_{L}\left(j_{!} \kappa^{*} d^{*} N, j_{!} \kappa^{*} d^{*} N-L\right) \longrightarrow C_{K}(N, N-L) .
$$

We conclude that the fourth (and first) term in the second sequence is $V$-dual to the first (and fourth) term in the first sequence since $C_{K}(L) \simeq i_{!} C_{L}(L)$ and $i_{\text {! }}$ preserves dual pairs. That the maps in the second sequence are the $V$-duals of the maps in the first sequence will follow from Proposition 16.4.7 and the definition of the coevaluation maps $\bar{\eta}$ in (18.8.1). In fact, the direction of argument will be reversed in the last subsection of $\$ 18.7$, where we use this comparison of cofibration sequences to deduce the relative case of Theorem 18.5.1 from the absolute case.

\subsection{Parametrized Atiyah duality for closed manifolds}

The results of the previous section specialize to lift the familiar Atiyah duality theorem to a statement in the parametrized world. In the absolute case, the conclusion reads as follows. We remind the reader that everything is equivariant.

THEOREM 18.6.1 (Parametrized Atiyah duality theorem). Let $M$ be a smooth closed manifold embedded in a representation $V$. Then $\left(S_{M}, S^{\nu_{M}}\right)$ is a CostenobleWaner $V$-dual pair.

We have two natural relative versions of Theorem 18.6.1. The first concerns a closed submanifold $L$ of a smooth closed manifold $M$. The second concerns a smooth compact manifold $M$ with non-empty boundary. There presumably is a more general context giving both of these as special cases, where $L$ is allowed to be a submanifold of a smooth compact manifold $M$ such that the boundary of $L$ is the intersection, assumed transverse, of $L$ with the boundary of $M$. However, we have not pursued that idea.

We deal with the relative case for closed manifolds in this section. As we have mentioned, the non-parametrized implication of this relative version does not seem to appear in the literature. In fact, it is harder to see the non-parametrized version 
directly than to deduce it from the parametrized version. The non-parametrized implication in the case when $M$ is a manifold with boundary, which we deal with in the next section, is standard.

Thus, specializing the notations of the previous section, let $K=M$ be a smooth closed manifold embedded in a representation $V$ and let $L$ be a closed submanifold of $M$ with inclusion $i: L \longrightarrow M$. We take $U$ to be the normal bundle $\nu_{M, L}$ of $L$ embedded as a tubular neighborhood in $M, N$ to be the normal bundle $\nu_{M}$ of $M$ in $V$, and $N_{L}$ to be the normal bundle $\nu_{L}=i^{*} \nu_{M} \oplus \nu_{M, L}$ of $L$ in $V$. Then (18.5.4) takes the form

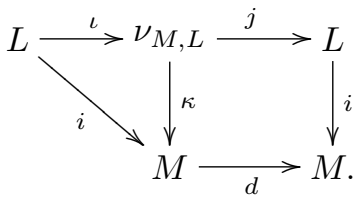

Here $\iota$ is the inclusion of the zero section in $\nu_{M, L}, \kappa$ is the embedding of $\nu_{M, L}$ in $M$, and $d$ is a deformation of $M$ that restricts on $\nu_{M, L}$ to the projection $j$ of this relative normal bundle. More explicitly, we may take $U$ to be the open disc bundle in a larger tubular neighborhood $W$. Then $j$ is the projection of the disk bundle, and we can take $d$ to agree with $j$ on $U$, to be the identity on $M-W$, and to send $w$ to $\phi(|w|) w$ for $|w| \geq 1$ in $W$, where $\phi$ is any continuous function on $[1, \infty)$ such that $\phi(1)=0$ and $\phi(t) \rightarrow 1$ as $t \rightarrow \infty$.

As usual, if $\xi$ is a bundle, we let $S^{\xi}$ denote the fiberwise one-point compactification of $\xi$ with section given by the points at infinity.

Construction 18.6.3. We construct a map $t_{M}: S^{\nu_{M}} \longrightarrow i_{!} S^{\nu_{L}}$ over $M$ which is a parametrized precursor of the $V$-dual of the inclusion $L_{+} \longrightarrow M_{+}$and is closely related to the Pontryagin-Thom map $t: M_{+} \longrightarrow T \nu_{M, L}$. We define an exmap $\alpha: d_{!} S_{M}^{0} \longrightarrow i_{!} S^{\nu_{M, L}}$ such that $r_{!} \alpha$ is homotopic to $t$ and define $t_{M}$ in terms of $\alpha$. To define $\alpha$, it suffices to specify a map $(M, d) \longrightarrow i_{!} S^{\nu_{M, L}}$ over $M$. Note that $i_{!} S^{\nu_{M, L}}$ is obtained from $S^{\nu_{M, L}}$ by gluing $M-i(L)$ to the section. Let $\bar{\nu}_{M, L}$ be the closure of $\nu_{M, L}$ in $M$. The identity map of $\nu_{M, L}$ extends to give a continuous map $\bar{\nu}_{M, L} \longrightarrow S^{\nu_{M, L}}$ over $L$ that sends a point on the boundary to the corresponding point in the section of $S^{\nu_{M, L}}$. Identifying $i(L)$ with the section, this map agrees on the boundary with the restriction of $d$ to $M-\nu_{M, L}$. These two maps glue together to give the required map $\alpha$.

The desired map $t_{M}$ is now defined up to homotopy by the following zig-zag.

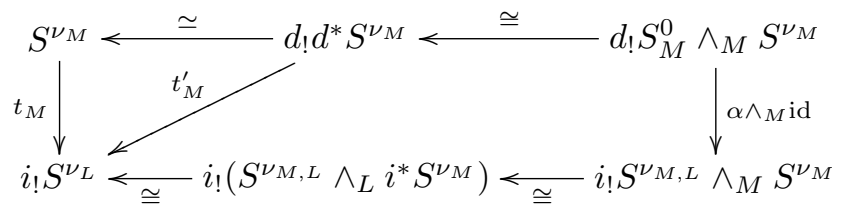

The upper left wrong way pointing map is the counit of the $\left(d_{!}, d^{*}\right)$ adjunction and is an equivalence because $d$ is a homotopy equivalence. The two isomorphisms on the right are given by the projection formula, and the bottom left isomorphism comes from $\nu_{L} \cong \nu_{M, L} \oplus i^{*} \nu_{M}$. The map $t_{M}^{\prime}$ is defined by commutativity of the trapezoid on the right. By the diagram, the cofiber $C_{M}\left(t_{M}\right)$ of $t_{M}$ is equivalent to $C_{M}(\alpha) \wedge_{M} S^{\nu_{M}}$. 
Theorem 18.6.5 (Parametrized relative Atiyah duality theorem). Let $L$ be a closed submanifold of a smooth closed manifold $M$. The Pontryagin-Thom map

$$
t_{M}: S^{\nu_{M}} \longrightarrow i_{!} S^{\nu_{L}}
$$

of Construction 18.6.3 is $V$-dual to the inclusion $i_{!} S_{L}^{0} \longrightarrow S_{M}^{0}$, and these maps give rise to Costenoble-Waner dual cofiber sequences

$$
\Sigma_{M}^{-1} \Sigma_{M}^{\infty} M /{ }_{M} L \longrightarrow i_{!} S_{L} \longrightarrow S_{M} \longrightarrow \Sigma_{M}^{\infty} M /{ }_{M} L
$$

and

$$
\Sigma_{M}^{-V-1} C_{M}\left(t_{M}\right) \longrightarrow \Sigma_{M}^{-V} S^{\nu_{M}} \longrightarrow \Sigma_{M}^{-V} i_{!} S^{\nu_{L}} \longrightarrow \Sigma_{M}^{-V} C_{M}\left(t_{M}\right)
$$

of $G$-spectra over $M$. In particular, the dual of $\Sigma_{M}^{\infty} M /{ }_{M} L$ is $\Sigma_{M}^{-V-1} C_{M}\left(t_{M}\right)$.

Proof. We observe first that the quotient map $C_{M}(M, L) \longrightarrow M /{ }_{M} L$ is an equivalence. This does not follow from Proposition 18.4.2 since $(M, L)$ is not an $f$ NDR pair over $M$. However, it is an NDR-pair, and the gluing lemma gives that the quotient map is a $q$-equivalence. Therefore Theorem 18.5.5 and Proposition 18.3.2 identify the dual of the first cofiber sequence as the cofiber sequence obtained by applying $\Sigma^{-V} \Sigma_{M}^{\infty}$ to the evident cofiber sequence

$C_{M}(N-L, N-M) \rightarrow C_{M}(N, N-M) \rightarrow C_{M}(N, N-L) \rightarrow \Sigma_{M} C_{M}(N-L, N-M)$.

We must obtain an equivalence between this cofiber sequence and the second cofiber sequence displayed in the statement of the theorem. Focus on the second maps of both sequences. By a standard comparison of cofiber sequences argument, it suffices to obtain horizontal equivalences that make the following diagram commute (up to stable homotopy).

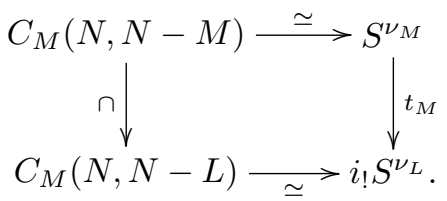

With $N=\nu_{M}$, inclusion and collapse induce a zig-zag of equivalences

$$
C_{M}(N, N-M) \longleftarrow C_{M}\left(N, N-D_{M}\right) \longrightarrow S^{\nu_{M}},
$$

where $D_{M}$ is a small disk bundle around $M$ in $N$. This gives the top equivalence. Similarly, inclusion and collapse induce a zig-zag of equivalences

$$
C_{L}\left(N_{L}, N_{L}-L\right) \longleftarrow C_{L}\left(N_{L}, N_{L}-D_{L}\right) \longrightarrow S^{\nu_{L}},
$$

where $D_{L}$ is a small disk bundle around $L$ in $N_{L}$. Applying $i_{\text {! }}$, this gives an equivalence between $i_{!} C_{L}\left(N_{L}, N_{L}-L\right)$ and $i_{!} S^{\nu_{L}}$. The composite along the bottom row in the diagram that follows displays an equivalence between $C_{M}(N, N-L)$ and $i_{1} C_{L}\left(N_{L}, N_{L}-L\right)$; it is a specialization of a general equivalence that plays a role in the proof of Theorem 18.5.5 given in $\S 18.7$ below. The composite gives the bottom equivalence. We have still another such zig-zag of equivalences

$$
C_{L}(U, U-L) \longleftarrow C_{L}\left(U, U-D_{M, L}\right) \longrightarrow S^{\nu_{M, L}},
$$

where $D_{M, L}$ is a (compatibly chosen) small disk bundle around $L$ in $U$. Via this zig-zag, the map $\alpha: d_{!} S_{M}^{0} \longrightarrow i_{!} S^{\nu_{M, L}}$ of Construction 18.6.3 corresponds to the map $\bar{\alpha}: d_{!} S_{M}^{0} \cong(M, d)_{+} \longrightarrow i_{!} C_{L}(U, U-L)$ that is given by the identity on the closure of $U \cong \nu_{M, L}$ in $M$ and that sends $M-U$ to the section via the map $d$. 
Now consider the following diagram, in which we have used more compact notations, such as $C_{M}\left(\begin{array}{c}N \\ N-M\end{array}\right)$ for $C_{M}(N, N-M)$. The left vertical arrow is the inclusion and, under the equivalences just given, we find that the zig-zag along the top row and the rightmost column is equivalent to the zig-zag of (18.6.4) that defines $t_{M}$. Therefore, it suffices to prove that the diagram commutes.

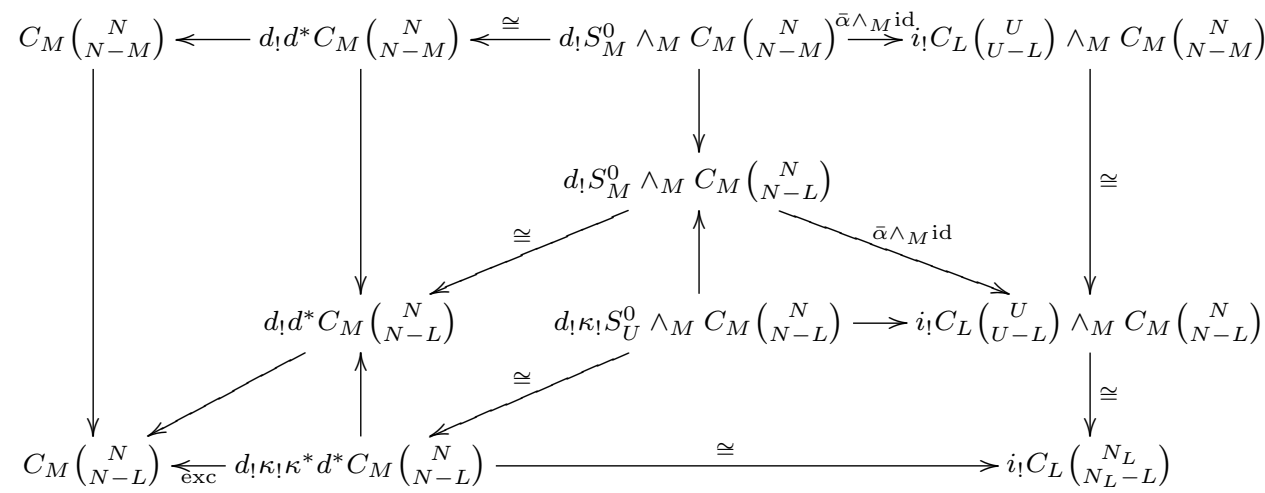

We freely use Proposition 18.4.4 to commute the fiberwise cone construction with base change functors. The isomorphisms relating the second and third columns are given by projection formulas. The maps relating the first and second column, and the vertical maps pointing upwards in the second and third columns are all given by the counit of either the $\left(d_{!}, d^{*}\right)$ adjunction or the $\left(\kappa !, \kappa^{*}\right)$ adjunction. The projection formula shows that the upper vertical arrow in the last column is an isomorphism since $i^{*}(N, N-M) \cong i^{*}(N, N-L)$. The lower vertical arrow in the last column is the isomorphism induced by the isomorphism

$$
(U, U-L) \times{ }_{L} i^{*}(N, N-L) \cong j ! j^{*} i^{*}(N, N-L)=\left(N_{L}, N_{L}-L\right)
$$

by use of the projection formula. The upper three trapezoids commute by naturality since their vertical maps are all induced from the inclusion of $N-M$ in $N-L$. The bottom trapezoid commutes by inspection since the bottom map identifies $d_{!} \kappa_{!} \kappa^{*} d^{*} N$ as $i_{!} N_{L}$, the top map is the inclusion of $d_{!} \kappa_{!} S_{U}^{0} \cong i_{!} j_{!} S_{U}^{0} \cong i_{!} C_{L}(U)$, and the other two maps are given by the projection formula. The triangle on the left and the parallelogram commute by simpler inspections, leaving only the triangle in the middle right. For that, we note that $d_{!} \kappa_{!} \kappa^{*} S_{M}^{0} \cong d_{!} \kappa_{!} S_{U}^{0} \cong j_{!} i_{!} S_{U}^{0}$ and the restriction of $\bar{\alpha}$ to $S_{U}^{0}$ is the identity map.

We can apply base change functors $p_{\text {! }}$ to these $V$-dual pairs to obtain new ones. In particular, taking $p=r: M \longrightarrow *$, this gives the $V$-dual pairs $\left(M_{+}, T \nu_{M}\right)$, $\left(L_{+}, T \nu_{L}\right)$ and $(M / L, C(t))$ where $T \xi$ denotes the Thom complex of a bundle $\xi$ and $t: T \nu_{M} \longrightarrow T \nu_{L}$ is the Pontryagin-Thom construction in Construction 18.6.3.

Corollary 18.6.7. Let $L$ be a closed submanifold of a smooth closed manifold M. The Pontryagin-Thom map

$$
t: T \nu_{M} \longrightarrow T \nu_{L}
$$

of Construction 18.6.3 is $V$-dual to the inclusion $L_{+} \longrightarrow M_{+}$, and these maps give rise to Spanier-Whitehead dual cofiber sequences

$$
\Sigma^{-1} \Sigma^{\infty} M / L \longrightarrow \Sigma^{\infty} L_{+} \longrightarrow \Sigma^{\infty} M_{+} \longrightarrow \Sigma^{\infty} M / L
$$


and

$$
\Sigma^{-V-1} C(t) \longrightarrow \Sigma^{-V} T \nu_{M} \longrightarrow \Sigma^{-V} T \nu_{L} \longrightarrow \Sigma^{-V} C(t) .
$$

In particular, the dual of $\Sigma^{\infty} M / L$ is $\Sigma^{-V-1} C(t)$.

\subsection{Parametrized Atiyah duality for manifolds with boundary}

We now turn to the case when the smooth manifold $M$ is compact but has a non-empty boundary $\partial M$. Let $i: \partial M \longrightarrow M$ be the inclusion and embed $M$ in a representation $V$. We can arrange that $V=V^{\prime} \oplus \mathbb{R}$ and that $M$ embeds in the half space $V^{\prime} \times[0, \infty)$ in such a way that $\partial M$ embeds in $V^{\prime}$ and $M-\partial M$ embeds in $V^{\prime} \times(0, \infty)$. The manifold $M$ has a normal bundle $\nu_{M}$ which one can think of as a neighborhood of $M$ in $V^{\prime} \times[0, \infty)$ (but not as a neighborhood of $M$ in all of $V$ ). Its restriction to $\partial M$ is the normal bundle $\nu_{\partial M}$ of $\partial M$ in $V^{\prime}$ (but not in $V$ ). Note in particular that if $\partial M \neq \emptyset$, then the normal bundle of $M$ cannot be identified with a tubular neighborhood of $M$ (considered as embedded in all of $V$ ). A simple example to keep in mind is $M=\left\{(x, y) \in \mathbb{R}^{2} \mid x^{2}+y^{2}=1, y \geq 0\right\}$, the upper half of the unit circle in the plane, with $V^{\prime}$ the $x$-axis.

WARNING 18.7.1. We emphasize the difference in the meaning of $\nu_{\partial M}$ here and of $\nu_{L}$ in the previous section. In both cases, we have submanifolds, $\partial M$ and $L$, of a manifold $M$ embedded in a representation $V$. In the previous section, $\nu_{L}$ referred to the normal bundle of $L$ in $V$. In this section, $\nu_{\partial M}$ refers to the normal bundle of $\partial M$ in $V^{\prime}$. The relation with $\nu_{M}$ is therefore quite different in the two cases. Here we have that $i^{*} S^{\nu_{M}} \cong S^{\nu_{\partial M}}$ and we have an inclusion $i_{!} S^{\nu_{\partial M}} \longrightarrow S^{\nu_{M}}$, whereas there we had $i^{*} S^{\nu_{M}} \wedge_{L} S^{\nu_{M, L}} \cong S^{\nu_{L}}$ and we had a map $t_{M}: S^{\nu_{M}} \longrightarrow i_{!} S^{\nu_{L}}$.

Theorem 18.7.2 (Parametrized Atiyah duality theorem-boundary version). Let $M$ be a smooth compact manifold with boundary $\partial M$. The quotient map

$$
S^{\nu_{M}} \longrightarrow S^{\nu_{M}} /{ }_{M} S^{\nu_{\partial M}}
$$

is $V$-dual to the quotient map $S_{M}^{0} \longrightarrow M /{ }_{M} \partial M$, and these maps give rise to Costenoble-Waner dual cofiber sequences

$$
i_{!} S_{\partial M} \longrightarrow S_{M} \longrightarrow \Sigma_{M}^{\infty} M /{ }_{M} \partial M \longrightarrow \Sigma_{M} i_{!} S_{\partial M}
$$

and

$$
\Sigma_{M}^{-V} i_{!} S^{\nu_{\partial M}} \longrightarrow \Sigma_{M}^{-V} S^{\nu_{M}} \longrightarrow \Sigma_{M}^{-V} S^{\nu_{M}} /{ }_{M} S^{\nu_{\partial M}} \longrightarrow \Sigma_{M}^{-V+1} i_{!} S^{\nu_{\partial M}}
$$

of $G$-spectra over $M$.

Proof. As we have already remarked, the normal bundle of $M$ cannot be identified with a tubular neighborhood of $M$ in $V$ when $\partial M \neq \emptyset$. We begin by making the relation precise in a way that will be convenient for the proof of the theorem. We glue an exterior boundary collar $\partial M \times[-2,0]$ to $\partial M=M \cap\left(V^{\prime} \times 0\right)$ to obtain a manifold $M_{2}$ with boundary $\partial M_{2}$ embedded in $V^{\prime} \times[-2, \infty)$. We let $M_{1}=M_{2} \cap\left(V^{\prime} \times[-1, \infty)\right)$, with boundary $\partial M_{1}$. We sometimes also write $\left(M_{0}, \partial M_{0}\right)$ for $(M, \partial M)$. Let $q_{2}: M_{2} \longrightarrow M$ be the identity on $M$ and retract $\partial M \times[-2,0]$ to $\partial M$. Let $q_{i}: M_{i} \longrightarrow M, i=0$ and $i=1$ be the restrction of $q_{2}$ to $M_{i}$, so that $q_{0}=$ id. Let $N$ be a tubular neighborhood of $M_{2}$ in $V$ and let $\rho: N \longrightarrow M_{2}$ be the retraction corresponding to the bundle projection. Let $N_{i} \subset N_{2}, i=0$ and $i=1$, be the restriction of $(N, \rho)$ to $M_{i} \subset M_{2}$. Although $N$ cannot be identified with the normal bundle of $M_{2}$, we can identify $N_{i}$ with the normal bundle of $M_{i}$, $i=0$ and $i=1$, and the restrictions $N_{\partial M_{i}}$ to the boundaries can be identified with 
the normal bundles $\nu_{\partial M_{i}}$. From now on, we view $N$ as a space over $M$ via the composite retraction $q_{2} \circ \rho$.

Observe that the evident identification of $[-2,0]$ with $[0,1]$ induces an identification of the quotient $M_{2} / M_{M}\left(\partial M_{2} \cup M_{0}\right)$ with $\Sigma_{M} \partial M_{+}$. That is, since $q_{2}$ is just the collar retraction, the definitions of these two spaces over $M$ are identical. We therefore have a cofiber sequence

$$
\left(\partial M_{2}, q_{2}\right)_{+} \longrightarrow\left(M_{2}, q_{2}\right)_{+} \longrightarrow C_{M}\left(M_{2}, \partial M_{2}\right) \longrightarrow C_{M}\left(M_{2}, \partial M_{2} \cup M_{0}\right)
$$

and Theorem 18.5.5 gives that its $V$-dual is

$$
C_{M}\left(\begin{array}{c}
N-\left(\partial M_{2} \cup M_{0}\right) \\
N-M_{2}
\end{array}\right) \longrightarrow C_{M}\left(\begin{array}{c}
N-\partial M_{2} \\
N-M_{2}
\end{array}\right) \longrightarrow C_{M}\left(\begin{array}{c}
N \\
N-M_{2}
\end{array}\right) \longrightarrow C_{M}\left(\begin{array}{c}
N \\
N-\partial M_{2}
\end{array}\right) .
$$

Using that $q_{2}: M_{2} \longrightarrow M$ is an equivalence, we see that the first cofiber sequence above is equivalent to the first displayed cofiber sequence in the statement of the theorem. We must therefore obtain an equivalence between the second cofiber sequence above and the second cofiber sequence in the statement of the theorem. We focus on the first maps of these two cofiber sequences. Although in this case we could easily elaborate our arguments to compare the full cofiber sequences, as in [98, III.5.4], we instead use that it suffices to obtain horizontal equivalences that make the following diagram commute.

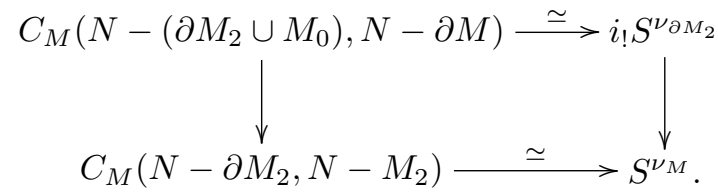

To obtain the horizontal maps in the above diagram, we begin by describing an equivalence

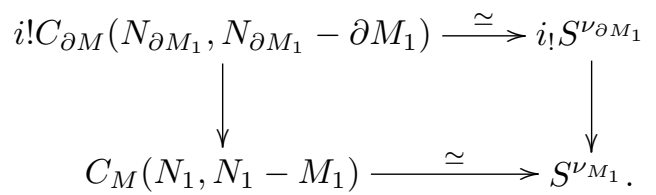

Recall that $N_{1}$ is the inverse image of $M_{1}$ in $N$ and $N_{\partial M_{1}}$ is its restriction to $\partial M_{1}$. Then $N_{1}$ and $N_{\partial M_{1}}$ are equivalent to the normal bundles of $M_{1}$ and $\partial M_{1}$ and the horizontal maps in the above square are given by the zig-zag where we replace $\left(M_{1}, \partial M_{1}\right)$ by the unit disk bundles in $\left(N_{1}, N_{\partial M_{1}}\right)$ and then use the quotient maps. The equivalence $q_{1}: M_{1} \longrightarrow M$ shows that the right vertical map in the above square is equivalent to the right vertical map in (18.7.5).

It now suffices to show that the horizontal inclusions in the following square are equivalences, since we have already seen that the left vertical map is equivalent to the inclusion $i_{!} S^{\nu_{\partial M}} \longrightarrow S^{\nu_{M}}$.

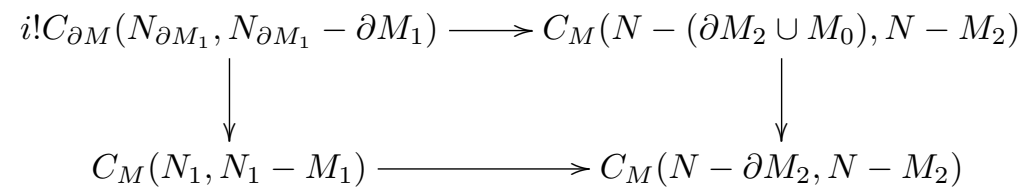

We would like to say that the horizontal inclusions are excision equivalences, but as they stand the pairs on the left and right hand side are not $f$-NDR pairs. We can work around that problem since, up to equivalence, we can replace $\left(M_{\varepsilon}, \partial M_{\varepsilon}\right)$ 
in the above square with the unit disk bundles $\left(D_{M_{\varepsilon}}, D_{\partial M_{\varepsilon}}\right)$ in the normal bundles $N_{\varepsilon}$ of $M_{\varepsilon}$ for $\varepsilon=1,2$. Then the second horizontal map becomes excision by $U=N-\left(N_{1} \cup D_{M_{2}}\right)$ since

$$
\left(N-D_{M_{2}}\right)-U=N_{1}-D_{M_{1}}, \quad \text { and } \quad\left(N-D_{\partial M_{2}}\right)-U=N_{1} \cup D_{M_{2}-\partial M_{2}} \simeq N_{1}
$$

and the first horizontal map becomes excision by $U=N-\left(N_{\partial M_{1}} \cup D_{M_{2}}\right)$ since

$$
\begin{gathered}
\left(N-D_{M_{2}}\right)-U=N_{\partial M_{1}}-D_{\partial M_{1}}, \\
\left(N-\left(D_{\partial M_{2} \cup M_{0}}\right)\right)-U=N_{\partial M_{1}} \cup D_{M \times(-2,0)} \simeq N_{\partial M_{1}} .
\end{gathered}
$$

This concludes the proof that (18.7.4) is equivalent to the second displayed cofiber sequence in the statement of the theorem.

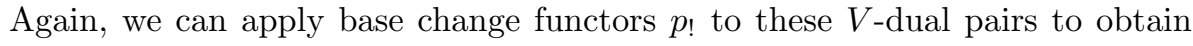
new ones. Taking $p=r: M \longrightarrow *$, this gives the $V$-dual pairs $\left(M / \partial M, T \nu_{M}\right)$, $\left(M_{+}, T \nu_{M} / T \nu_{\partial M}\right)$ and $\left(\partial M_{+}, \Sigma T \nu_{\partial M}\right)$. For consistency, note that the dual of $\partial M_{+}$is $\Sigma^{-V+1} T \nu_{\partial M} \simeq \Sigma^{V^{\prime}} T \nu_{\partial M}$.

COROLlarY 18.7.6. Let $M$ be a compact smooth manifold $M$ with boundary $\partial M$. The quotient map

$$
T \nu_{M} \longrightarrow T \nu_{M} / T \nu_{\partial M}
$$

is $V$-dual to the quotient map $M_{+} \longrightarrow M / \partial M$, and these maps give rise to SpanierWhitehead dual cofiber sequences

$$
\Sigma^{\infty} \partial M_{+} \longrightarrow \Sigma^{\infty} M_{+} \longrightarrow \Sigma^{\infty} M / \partial M \longrightarrow \Sigma^{\infty} \Sigma \partial M_{+}
$$

and

$$
\Sigma^{-V} T \nu_{\partial M} \longrightarrow \Sigma^{-V} T \nu_{M} \longrightarrow \Sigma^{-V} T \nu_{M} / T \nu_{\partial M} \longrightarrow \Sigma^{-V+1} T \nu_{\partial M}
$$

\subsection{The proof of the Costenoble-Waner duality theorem}

This section is devoted to the proof of Theorem 18.5.1 and we retain the notation introduced at the beginning of $\S 18.5$. We begin with an explicit description of the structure maps of the $V$-dual pair when $K=M$ is a compact $G$-manifold and $L$ is empty. Keeping this description in mind will aid in understanding the proof that follows.

We then define the structure maps in the general case and proceed with the proof of Theorem 18.5.1. To begin with, we work on the point-set level in categories of pairs of $G$-spaces over the base $G$-spaces *, $K$ and $K \times K$ and the corresponding categories of ex- $G$-spaces. To fit diagrams on the page, instead of $(X, W)$ we will often use the more compact notation $\left(\begin{array}{l}X \\ W\end{array}\right)$ for objects in categories of pairs. We will make frequent use of the properties of the unreduced cone construction that we discussed in $\$ 18.4$. Since $K \subset V$ is compact, we can assume that its neighborhood $N$ is contained in a closed disc $D$ about the origin in $V$.

The geometric structure maps. As usual, $M$ is embedded with tubular neighborhood $N$ in a $G$-representation $V$. We identify $N$ with the normal bundle $\nu$ of $M$. Then $C_{M}(M)=S_{M}^{0}$ and $C_{M}(N, N-M) \simeq S^{\nu}$. Under this equivalence, the coevaluation map is given by the Pontryagin-Thom construction

$$
\eta: S^{V} \longrightarrow T \nu=r_{!} S^{\nu} \cong r_{!}\left(S_{M}^{0} \wedge_{M} S^{\nu}\right) \simeq S_{M}^{0} \odot t S^{\nu} .
$$

To construct the evaluation map we use the zero section of $\nu$ to embed $M$ as the diagonal in $\nu \times M \cong N \times M$, which we view as a space over $M \times M$ with projection 
$q \times$ id. A normal bundle $\Gamma$ of this embedding is given by the subspace $(q \times \text { id })^{-1}(U)$ sitting over a tubular neighborhood $U$ of the diagonal in $M \times M$. Now $U$ can be identified with the tangent bundle of $M$. Such an identification can be given by shrinking $\tau$ to a neighborhood of the zero-section and then using an exponential function so that the point $(x, m)$ determines a unique geodesic from $x$ to $m$. This geodesic determines a tangent vector based at $x$. Thus a point of $U$ is thought of both as a point $(x, m) \in M \times M$ and a tangent vector at $x$. A point $(n, m)$ in $\Gamma$ can thus be thought of as specifying the normal vector $n$ at $q(n)$ together with the tangent vector $(q(n), m)$ at $q(n)$. This identifies $\Gamma$ with the sum of the normal and tangent bundles and therefore with the trivial bundle $M \times V$. Note, however, that when we think of $\Gamma$ as a space over $M \times M$, then we are remembering how $(n, m)$ splits into the normal and tangent vectors, but when we look at $\pi_{1 !} \Gamma$, then we have forgotten about the splitting.

In essence, we can now define $\varepsilon$ to be the Pontryagin-Thom construction of the embedding $M \longrightarrow \nu \times M$. However, since this must be a map over $M \times M$, we specify it more precisely as the zig-zag

$$
t S^{\nu} \odot S_{M}^{0} \simeq S^{\nu} \times M \longrightarrow \bar{\Gamma} / M \times M \partial \Gamma \longrightarrow\left(M^{I}, p\right)_{+} \wedge S^{V} \longleftarrow \Delta_{!} S_{M}^{V}
$$

Here the first map is given by the Pontryagin-Thom construction and $\bar{\Gamma}$ is the closure of $\Gamma$ in $V$. The projection $p: M^{I} \longrightarrow M \times M$ is given by evaluation at 0 and 1. The second map sends $(n, m)$ to $\left(\omega_{q(n), m}, v\right)$ where $\omega_{q(n), m}$ is a geodesic from $q(n)$ to $m$ and $v$ is the sum of the normal vector $n$ and the tangent vector $(q(n), m)$. The third (wrong way pointing) map is the equivalence induced from the constant path map $c: M \longrightarrow\left(M^{I}, p\right)$ over the diagonal $\Delta: M \longrightarrow M \times M$ by adjoining the identity map on the disjoint sections and taking the fiberwise smash product with the identity map of $S^{V}$. Thus it sends a point $(m, v) \in \Delta_{!} S_{M}^{V}$ that is not in the section to $\left(c_{m}, v\right)$.

The coevaluation map. We apply the unreduced cone construction $C$ to the zig-zag of inclusions

$$
\bar{\eta}:\left(\begin{array}{c}
V \\
V-D
\end{array}\right) \longrightarrow\left(\begin{array}{c}
V \\
(V-K) \cup L
\end{array}\right) \stackrel{\mathrm{exc}}{\longleftarrow} r_{!}\left(\begin{array}{c}
N-L \\
N-K
\end{array}\right)
$$

where the map on the right is excision by $(V-N) \cup L$. Identifying the source and using that $r_{!} C_{K} \cong C_{*} r_{\text {! }}$, by Proposition 18.4.4, we obtain

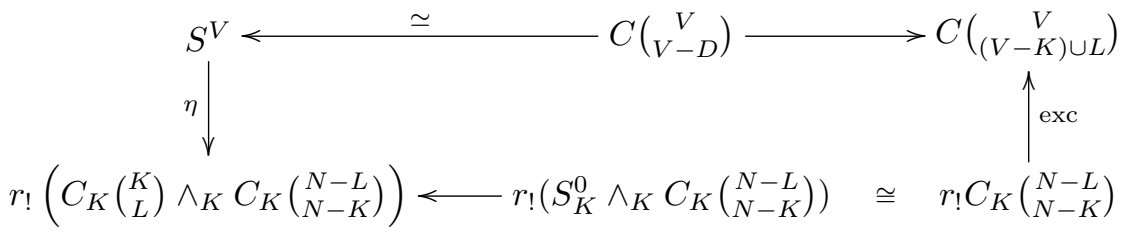

where the map on the bottom is induced from $S_{K}^{0}=C_{K}(K, \emptyset) \longrightarrow C_{K}(K, L)$. This is of course a homeomorphism when $L=\emptyset$.

The evaluation map. To define the evaluation map, we need the analogue of the tubular neighborhood $\Gamma$ we described in the manifold case at the end of $\S 18.5$. By the compactness of $K$, there is a Lebesgue number $\varepsilon>0$ such that any $\varepsilon$-ball centered on a point of $K$ is contained in $N$. We agree to replace $N$ with the smaller 
neighborhood of $K$ that is the union of all such $\varepsilon$-balls. We define

$$
\Gamma=\{(n, x) \in(N-L) \times K \mid\|q(n)-x\|<\varepsilon\} .
$$

For points $x, y \in V$, let $\omega_{(x, y)}(t)=(1-t) x+t y$ be the linear path from $x$ to $y$. Note that the parametrized line segment $\omega_{(q(n), x)}$ is contained in $N$ if $(n, x) \in \Gamma$. Moreover, $\Gamma$ is a neighborhood of the diagonal $\Delta_{K-L}$ inside $(N-L) \times K$, and the image of the projection is a neighborhood of $\Delta_{K-L}$ in $K \times K$. By analogy with the manifold case, we think of the image of the projection of $\Gamma$ as a tubular neighborhood of the diagonal in $K \times K$, and we think of points $(n, x) \in \Gamma$ as specifying a "normal vector" $n$ and a "tangent vector" $(q(n), x)$, both based at the point $q(n)$.

The evaluation map is now obtained from the following zig-zag of spaces over $K \times K$.

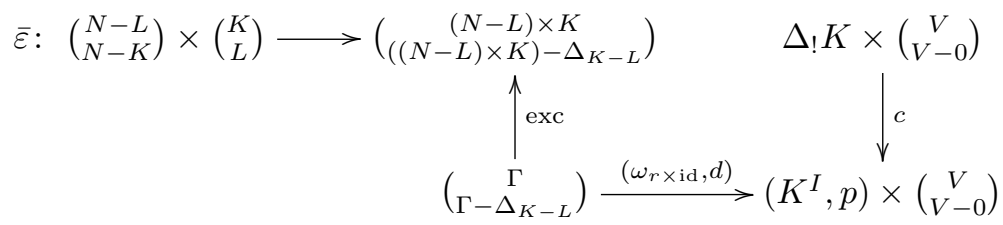

The first two maps are inclusions, the second being obtained by excising the complement of $\Gamma$ in $(N-L) \times K$. The third map sends $(n, x) \in \Gamma$ to $\left(\omega_{(q(n), x)}, n-x\right)$ and the fourth sends $(x, v)$ to $\left(c_{x}, v\right)$ where $c_{x}$ is the constant path at $x$. The projection $p$ of $K^{I}$ is given by evaluation at the endpoints, $p(\omega)=(\omega(0), \omega(1))$. Applying $C_{K \times K}$ to the above zig-zag and identifying the source and target, we obtain the following composite.

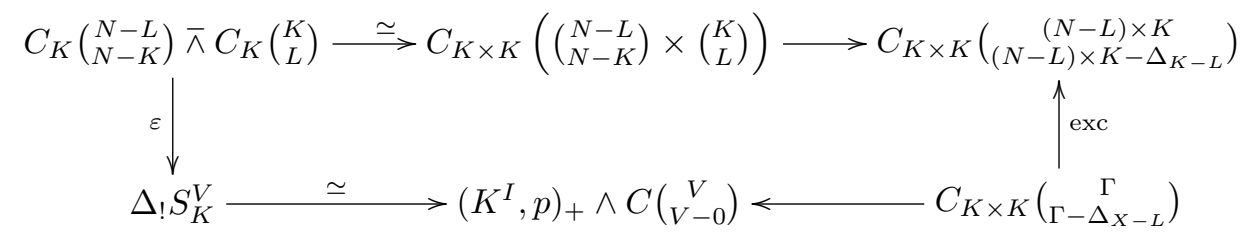

The first equivalence is given by Proposition 18.4.6 and the last one uses an inverse of a based homotopy equivalence $C(V, V-0) \longrightarrow S^{V}$.

The first $V$-duality diagram. First we consider the commutativity of the following diagram on the point-set level.

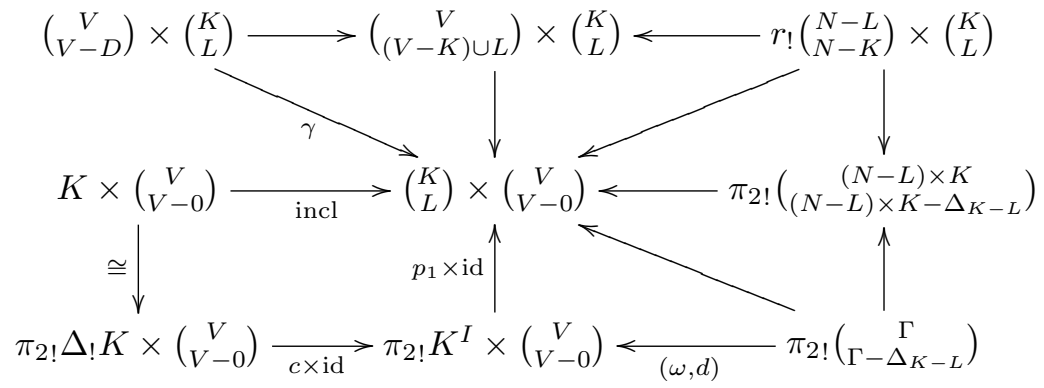

The top row is the product of the zig-zag $\bar{\eta}$ of (18.8.1) with $(K, L)$ and the zig-zag continuing clockwise along the perimeter and ending in the bottom left corner is obtained from the zig-zag $\bar{\varepsilon}$ of $(18.8 .2)$ by applying $\pi_{2 !}$. The unlabeled maps to 
the center are all given by $\left(\pi_{2}, d\right)$ which sends $(n, x)$ to $(x, n-x)$. The map $p_{1}$ is the endpoint projection. The bottom left square commutes, as do all of the small triangles with one vertex in the center, except the one in the upper left corner. That one only commutes up to the homotopy

$$
h(v, x, t)=(x, v-t x)
$$

which is easily seen to land inside the required target.

We now pass to homotopy categories. By Proposition 18.4.8, the unreduced cone construction passes directly to homotopy categories as it takes $q$-equivalences between parametrized pairs to $q$-equivalences of ex-spaces. Applying $C_{K}$ to the above diagram and commuting it past base change functors we see that the composite of the left column with the bottom horizontal map in the following diagram in $\mathrm{Ho} G \mathscr{K}_{K}$ is given by $\gamma$.

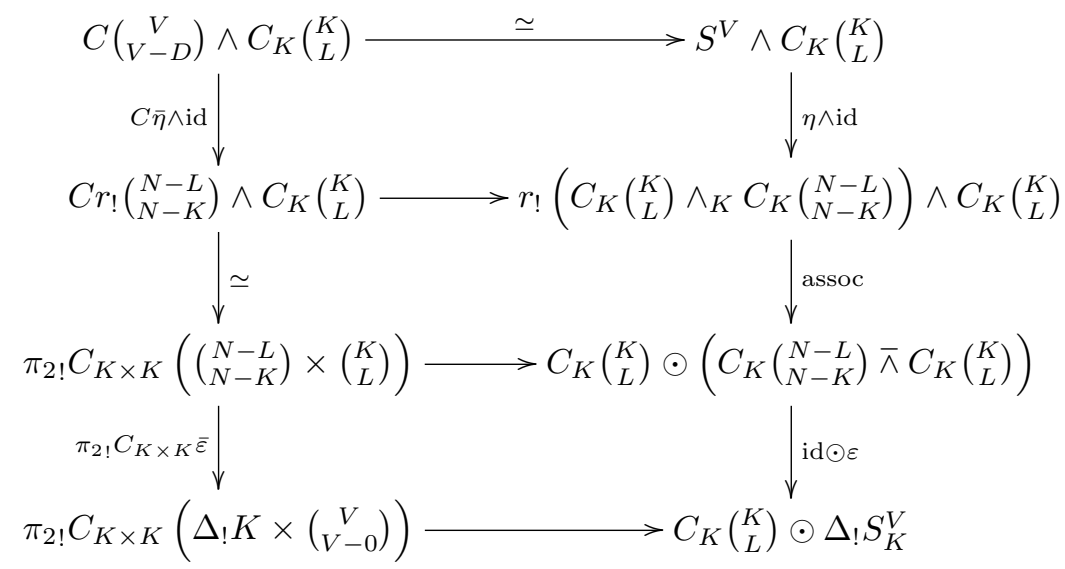

The top square commutes by the definition of $\eta$ and the bottom square commutes by the definition of $\varepsilon$. The unlabeled horizontal maps are all induced by the inclusion $S_{K}^{0}=C_{K}(K, \emptyset) \longrightarrow C_{K}(K, L)$. In particular, letting $(X, W)$ denote any pair over $K \times K$, the middle two horizontal maps are given as follows, where we use Proposition 18.4.6 to identify the target.

$$
\pi_{2 !} C_{K \times K}\left(\begin{array}{c}
X \\
W
\end{array}\right) \simeq \pi_{2 !}(\Delta \times \mathrm{id})^{*}\left(S_{K}^{0} \bar{\wedge} C_{K \times K}\left(\begin{array}{c}
X \\
W
\end{array}\right)\right) \longrightarrow C_{K}\left(\begin{array}{c}
K \\
L
\end{array}\right) \odot C_{K \times K}\left(\begin{array}{c}
X \\
W
\end{array}\right)
$$

We conclude that the composite of the right hand column is the twist map. This verifies the first $V$-duality diagram.

The second $V$-duality diagram, absolute case. We consider first the absolute case $L=\emptyset$. We have the diagram

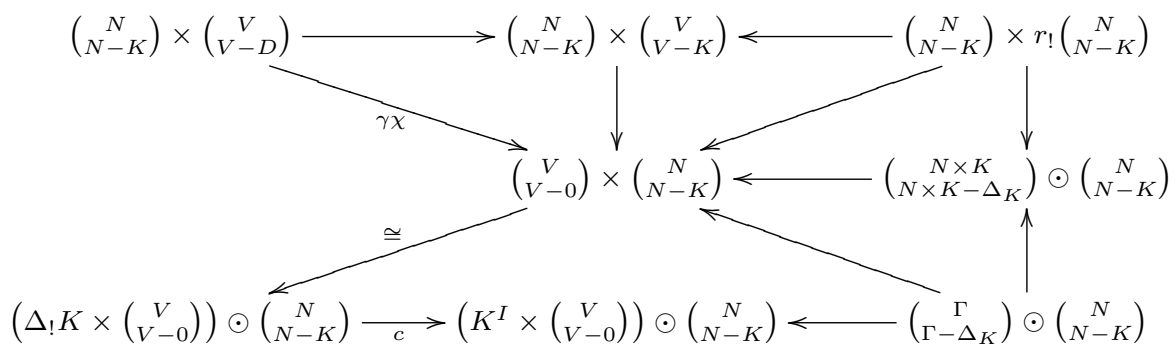


where the top row is the product of $(N, N-K)$ with the zig-zag $\bar{\eta}$ of (18.8.1). For a space or pair $X$ over $K$ we have that

$$
X \times\left(\begin{array}{c}
N \\
N-K
\end{array}\right) \cong X \times \Delta^{*} \pi_{2}^{*}\left(\begin{array}{c}
N \\
N-K
\end{array}\right) \cong(\mathrm{id} \times \Delta)^{*}\left(X \times K \times\left(\begin{array}{c}
N \\
N-K
\end{array}\right)\right) .
$$

Taking $X=(N, N-K)$, this identifies the space in the upper right hand corner of the above diagram as

$$
\pi_{1 !}\left(\left(\begin{array}{c}
N \\
N-K
\end{array}\right) \times\left(\begin{array}{c}
N \\
N-K
\end{array}\right)\right) \simeq\left(\left(\begin{array}{c}
N \\
N-K
\end{array}\right) \times K\right) \odot\left(\begin{array}{c}
N \\
N-K
\end{array}\right) .
$$

The zig-zag going clockwise along the perimeter from the upper right hand corner to the lower left hand corner is $\bar{\varepsilon} \odot(N, N-K)$. The four unlabeled maps to the center are given by $\left(d, \pi_{1}\right)$, sending $(n, v)$ to $(n-v, n)$. The only map out of the center is the the unit isomorphism for $\odot$. All triangles except the first and last commute. For the first we have the homotopy

$$
h(n, v, t)=(t n-v, n)
$$

and for the last we need a homotopy

$$
k:\left(\begin{array}{c}
\Gamma \\
\Gamma-\Delta_{K}
\end{array}\right) \odot\left(\begin{array}{c}
N \\
N-K
\end{array}\right) \longrightarrow\left(K^{I} \times\left(\begin{array}{c}
V \\
V-0
\end{array}\right)\right) \odot\left(\begin{array}{c}
N \\
N-K
\end{array}\right)
$$

between the two maps around the bottom triangle. We identify the source of $k$ as the pair $(X, W)$ over $K$, where

$$
X=\{(n, m) \in N \times N \mid(n, q(m)) \in \Gamma\},
$$

with projection sending $(n, m)$ to $q(n)$, and $W$ consists of all $(n, m) \in X$ such that either $q(n) \neq q(m)$ or $m \neq q(m)$. The target of $k$ is the pair $(Y, Z)$ over $K$, where

$$
Y=\left\{(\omega, v, m) \in K^{I} \times V \times N \mid q(m)=\omega(1)\right\},
$$

with projection sending $(\omega, v, m)$ to $\omega(0)$, and $Z$ consists of all $(\omega, v, m) \in Y$ such that either $v \neq 0$ or $q(m) \neq m$. With these identifications, we must define $k$ to be a homotopy between

$$
k_{0}(n, m)=\left(\omega_{(q(n), q(m))}, n-q(m), m\right) \quad \text { and } \quad k_{1}(n, m)=\left(c_{q(n)}, n-m, n\right) .
$$

Since $(n, q(m)) \in \Gamma$ we have that $\|n-q(m)\|<\varepsilon$, and the line segment between $n$ and $m$ is therefore contained in $N$. We let $m_{t}=\omega_{(n, m)}(1-t)$ and define

$$
k(n, m, t)=\left(\omega_{\left(q(n), q\left(m_{t}\right)\right)}, n-\omega_{(q(m), m)}(t), m_{t}\right) .
$$

We apply $C_{K}$ to the above diagram, commute it past base change functors, and get the following diagram in Ho $G \mathscr{K}_{B}$, where the composite of the left hand column with the bottom map is given by $\gamma(\mathrm{id} \wedge \alpha)$. 


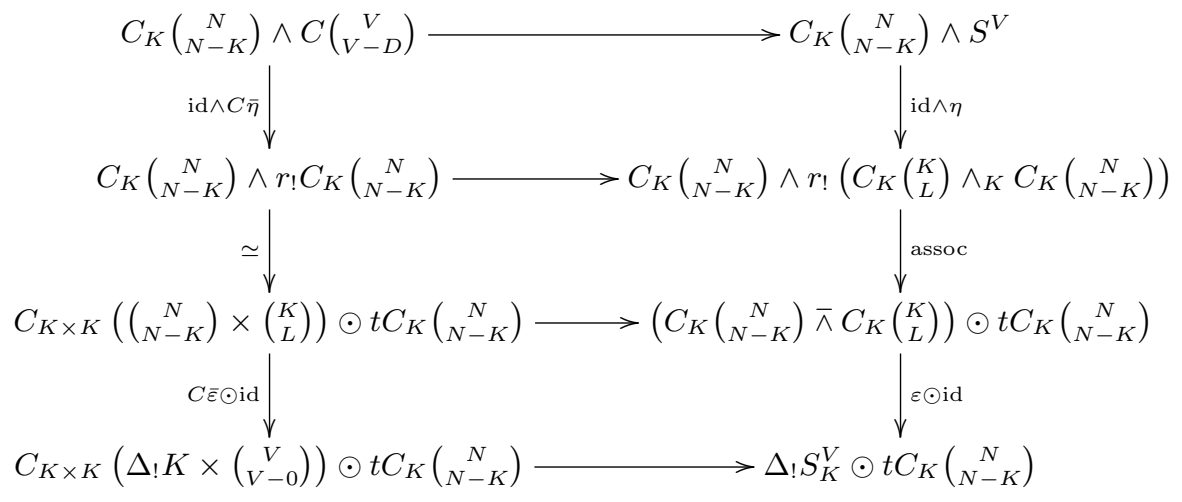

Here the top and bottom squares commute by the definition of $\eta$ and $\varepsilon$, and for the middle square we have used the following equivalence from Proposition 18.4.2:

$$
C_{K}\left(W \odot\left(\begin{array}{c}
N \\
N-K
\end{array}\right)\right) \simeq C_{K \times K} W \odot t C_{K}\left(\begin{array}{c}
N \\
N-K
\end{array}\right)
$$

The second $V$-duality diagram, relative case. We derive the relative case from the absolute case by using the cofiber sequences

$$
i_{!} S_{L}^{0} \longrightarrow S_{K}^{0} \longrightarrow C_{K}(K, L)
$$

and

$$
C_{K}\left(\begin{array}{l}
N-L \\
N-K
\end{array}\right) \longrightarrow C_{K}\left(\begin{array}{c}
N \\
N-K
\end{array}\right) \longrightarrow C_{K}\left(\begin{array}{c}
N \\
N-L
\end{array}\right)
$$

of Theorem 18.5.5. We have $i_{!} C_{L}\left(N_{L}, N_{L}-L\right) \simeq C_{K}(N, N-L)$, as in the proof of Theorem 18.5.5, and we see from the absolute case we have already proven that the second and third terms of the second sequence are the $V$-duals of the second and first terms of the first sequence.

To complete our proof that $\left(C_{K}(K, L), C_{K}(N-K, N-L)\right)$ is a dual pair with structure maps $(\eta, \varepsilon)$, it suffices to show that $\eta$ is the coevaluation map of a dual pair. Indeed, since we have already shown that $(\eta, \varepsilon)$ satisfies one of the $V$-duality diagrams, it will follow that $\varepsilon$ must be the evaluation map of the duality. By Proposition 16.4.6(iii'), it suffices to show that

$$
\eta_{\#}: \mathscr{E} x\left(W \odot C_{K}(K, L), Z\right) \longrightarrow \mathscr{E} x\left(W, Z \odot C_{K}(N-K, N-L)\right)
$$

is a bijection for all 1-cells $W: * \longrightarrow C$ and $Z: B \longrightarrow C$, where $C$ is any target space. We have corresponding maps for the pairs $\left(S_{K}^{0}, C(N, N-K)\right)$ and $\left(i_{!} S_{L}^{0}, C_{K}(N, N-L)\right)$, which by the absolute case we already know to be isomorphisms. Since both sides of the maps $\eta_{\#}$ are exact in the relevant variables, the result will follow from the five-lemma once we show that the maps $\eta_{\#}$ are compatible with the cofiber sequences (18.8.3) and (18.8.4), so that (18.8.4) is the $V$-dual of (18.8.3). Thus the conclusion will follow by Proposition 16.4.7 once we verify the commutativity of the following three diagrams. 


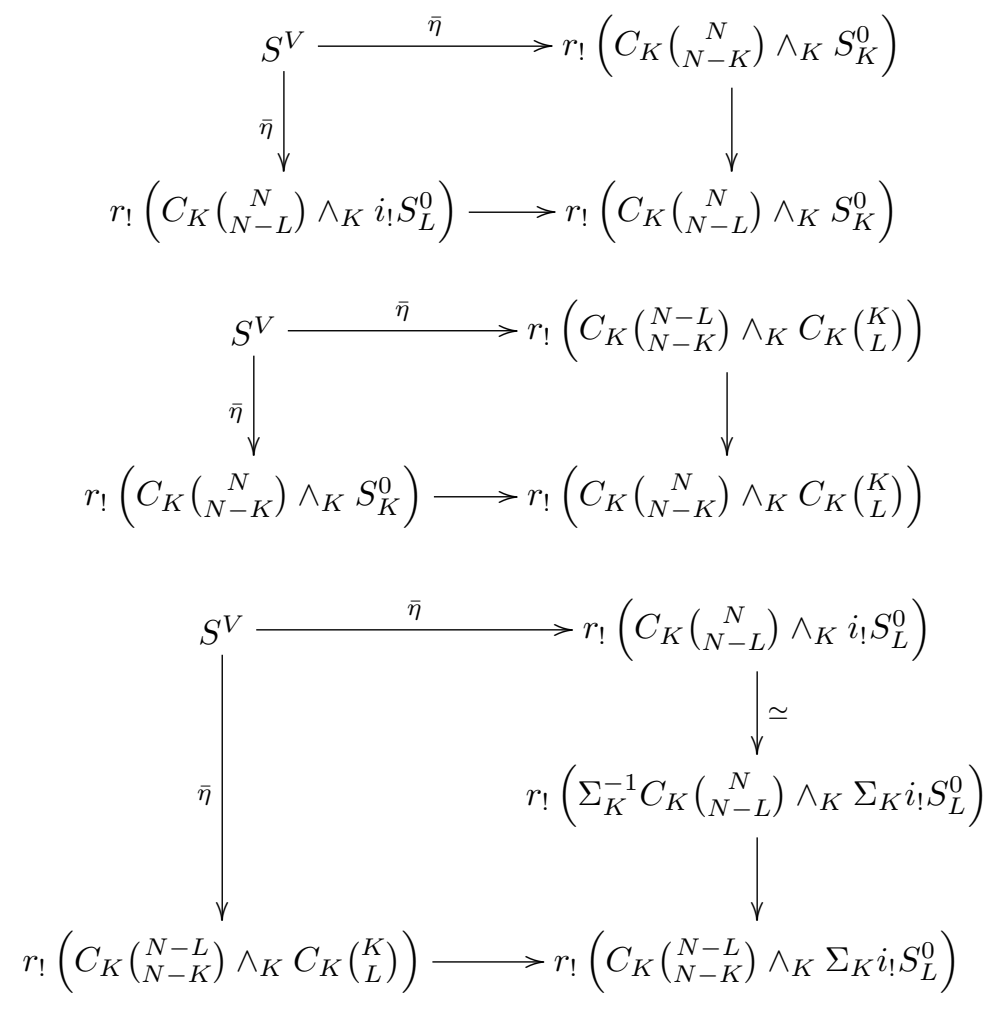

These are elementary diagram chases using the definition of the coevaluation maps $\bar{\eta}$ given in (18.8.1). They follow from the following two and a desuspension of the third.
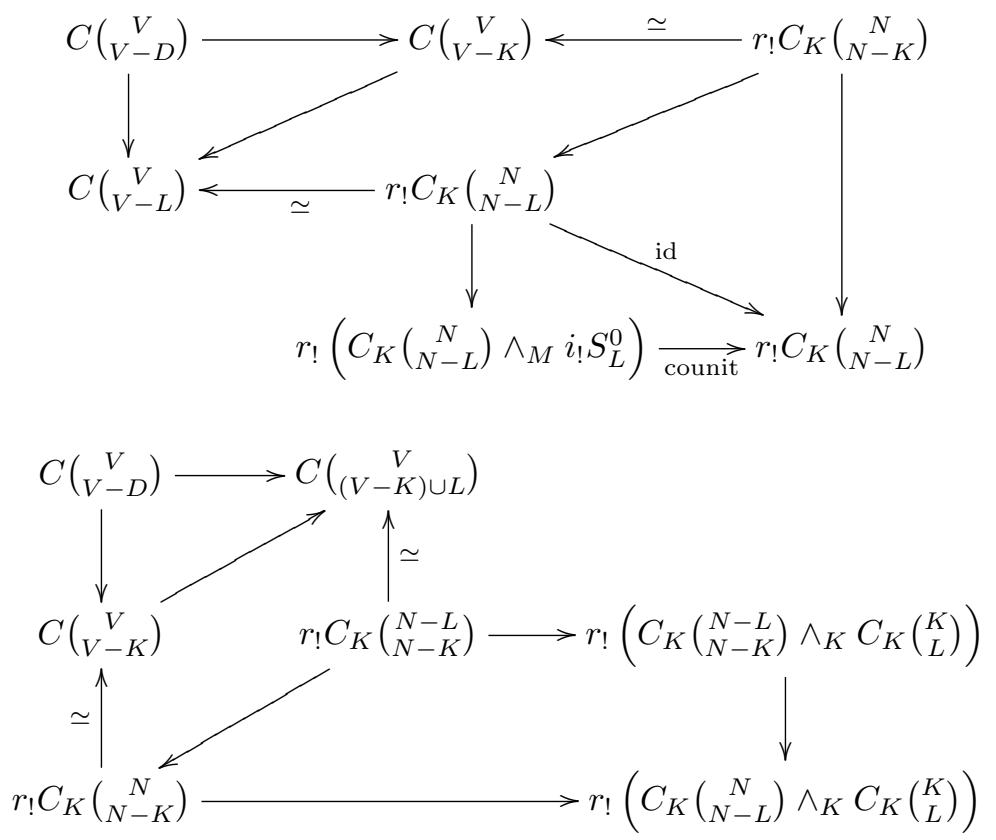


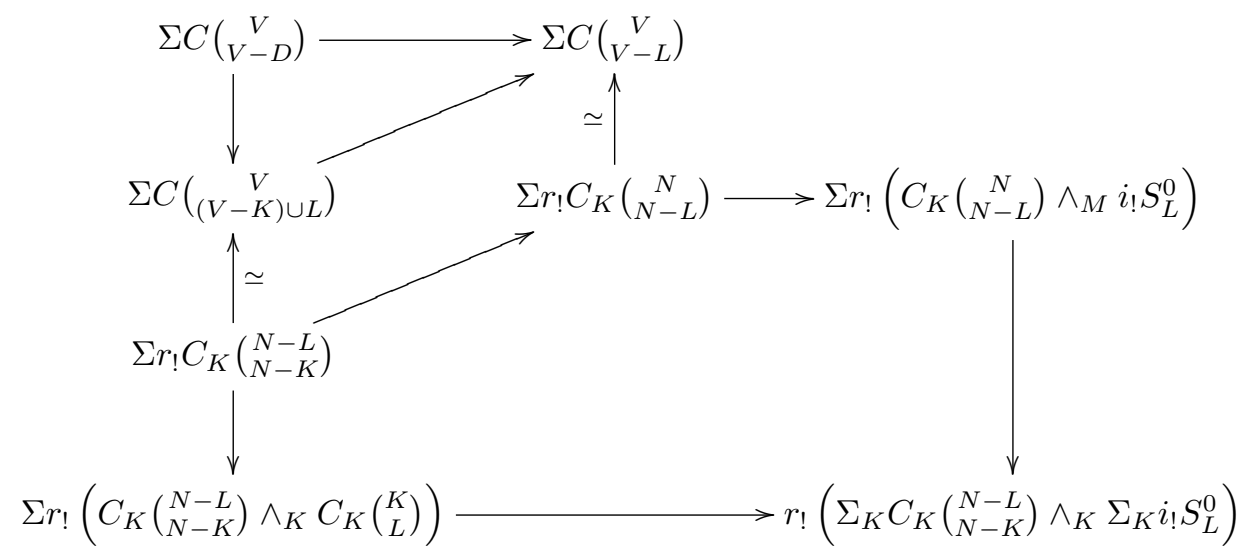





\section{CHAPTER 19}

\section{Fiberwise Costenoble-Waner duality}

\section{Introduction}

It is often the case that a given functor will have a relatively familiar left adjoint, like induction, and a relatively mysterious right adjoint, like coinduction. Results that prove an equivalence of the right adjoint with a shift of the left adjoint are common and are studied categorically in [62].

Let $F$ be a $G$-space such that $S_{F}$ is a Costenoble-Waner dualizable $G$-spectrum over $F$ and let $T_{F}$ be dual to $S_{F}$. For example, $F$ could be a smooth $G$-manifold. We show in $\S 19.1$ that Costenoble-Waner duality for the pair $\left(S_{F}, T_{F}\right)$ specializes to such a comparison between the left adjoint $r$ ! (quotient out sections) and the right adjoint $r_{*}$ (take global sections) of the pullback functor $r^{*}$ associated to $r: F \longrightarrow *$. Specialized to orbits $G / H$, this result translates under the equivalence between the category of $G$-spectra over $G / H$ and the category of $H$-spectra to the Wirthmüller isomorphism in the form proven by Lewis and May in [98, II $\S 6$. However, the general result is already of fundamental importance nonequivariantly. In particular, specialization of this result to smooth manifolds will give the parametrized homotopical version of Poincaré duality that we advertised in Example 0.0.2.

In $\S 19.5$, we show that the comparison between $r_{\text {! }}$ and $r_{*}$ in $\S 19.1$ is a special case of a general fiberwise comparison between left and right adjoints $p_{\text {! }}$ and $p_{*}$ associated to bundles $p: E \longrightarrow B$ with fiber $F$. The bundles we consider are of the type introduced in $\S 3.2$ and studied in $\S 15.3$. The fiber $F$ is now a $\Gamma$-space, where $\Gamma$ is an extension of the ambient group $G$ by the structural group $\Pi$. When $\Gamma=\Pi, G$ is trivial and we are considering nonequivariant bundles. Fiberwise Costenoble-Waner duality computes the right adjoint $p_{*}: \mathrm{Ho} \mathscr{S}_{E} \longrightarrow \mathrm{HoG} \mathscr{S}_{B}$ as a suitable shift of the left adjoint $p_{!}$. As we observe in $\S 19.6$, this result specializes to a fiberwise variant of our homotopical Poincaré duality theorem.

The proofs are based on a generalization of the foundations. In $\S 19.2$, we construct a symmetric bicategory $G \mathscr{E} x_{B}$ for any base $G$-space $B$. The bicategory $\mathscr{E} x$, or $G \mathscr{E} x$ to indicate the ambient group, is the case when $B$ is a point. In $\S 19.3$, we obtain an oplax functor $\iota_{!}: G \mathscr{E} x_{B} \longrightarrow G \mathscr{E} x$ by use of pushforward functors associated to inclusions of the form $\iota: E_{2} \times_{B} E_{1} \longrightarrow E_{1} \times E_{2}$. We also show how the bicategories $G \mathscr{E} x_{B}$ behave with respect to base change and change of groups. In $\S 19.4$, we show that the bundle construction gives rise to a pseudofunctor $\mathbb{P}: \Gamma \mathscr{E} x \longrightarrow G \mathscr{E} x_{B}$. The composite $\iota ! \mathbb{P}$ carries the dual pair $\left(S_{F}, t T_{F}\right)$ to a dual pair $\left(S_{p}, t T_{p}\right)$. Costenoble-Waner duality for this pair specializes to give the claimed comparison between $p_{\text {! }}$ and $p_{*}$. Moreover, this comparison restricts on fibers to a comparison of the form given in $\S 19.1$ relating $r_{\text {! }}$ and $r_{*}$. In earlier drafts, we called fiberwise Costenoble-Waner duality the fiberwise Wirthmüller isomorphism, but the new point of view gives more information. It is also reasonable to call the 
result $\mathrm{Hu}$ duality since, when $\Gamma=G \times \Pi$ and $M$ is a smooth manifold, it specializes to a version of the main result of Po Hu's monograph [78].

In $\$ 19.7$, we show that the Adams isomorphism relating orbit spectra and fixed point spectra in the form given by Lewis and May in [98, II $\S 8]$ is a virtually immediate special case of fiberwise Costenoble-Waner duality.

Since the results of this chapter subsume a number of results with a substantial history and since earlier versions were proven quite differently, we give some background discussion and comparison of methods in $\S 19.8$. In particular, we show that a general map from right adjoints to left adjoints that featured prominently in earlier proofs of some of our results gives inverse equivalences to the natural duality maps that appear in our framework.

\subsection{Costenoble-Waner duality and homotopical Poincaré duality}

We assume that $F$ is a $G$-space such that $S_{F}$ is a Costenoble-Waner dualizable $G$-spectrum over $F$ with (right) dual $T_{F}$, so that we have a dual pair $\left(S_{F}, t T_{F}\right)$ in $G \mathscr{E} x$. Thus $t T_{F}$ is equivalent to the right dual $D_{r} S_{F}$. Therefore the map

$$
\mu_{X}: X \odot t T_{F} \longrightarrow S_{F} \triangleright X
$$

of Proposition 18.1.5 is an equivalence for all 1-cells $X: F \longrightarrow A$, where $A$ is any $G$ space. Taking $A=*$, so that $X$ is a $G$-spectrum over $F$, and using the dictionaries Proposition 17.4.2 and Corollary 17.4.4 to translate from the bicategory notation, this equivalence takes the form

$$
r_{!}\left(X \wedge_{F} T_{F}\right) \simeq r_{*} X .
$$

Thus it calculates the right adjoint $r_{*}$ in terms of a shift of the left adjoint $r_{!}$.

Taking $F$ to be the simplest kind of $G$-manifold, an orbit $G / H$, recall from Corollary 11.5.4 that the category of $H$-spectra is equivalent to the category of $G$-spectra over $G / H$. The equivalence is given in one direction by applying the functor $G \times{ }_{H}(-)$, and in the other by taking the fiber over the identity coset. This equivalence preserves all structure in sight, including the symmetric monoidal and model structures. Under this equivalence, $r^{*}$ corresponds to the restriction of group action functor associated to the inclusion $\iota: H \longrightarrow G$, and its left and right adjoints $r_{!}$and $r_{*}$ therefore correspond to induction and coinduction. By Proposition 11.5.2, $T_{G / H}=\iota ! S^{-L}$, where $L$ is the tangent representation at the identity coset in $G / H$. Translated to the nonparametrized context, (19.1.2) gives a natural equivalence

$$
G_{+} \wedge_{H}\left(X \wedge S^{-L}\right) \simeq F_{H}\left(G_{+}, X\right)
$$

for all $H$-spectra $X$. In this form, the result is known as the Wirthmüller isomorphism. Previous proofs were quite different.

Heading towards Poincaré duality, but still working equivariantly, take $F$ to be a smooth closed $G$-manifold and write it $M$. Then $T_{M}=\Sigma_{M}^{-V} S^{\nu}$ is not just dualizable in the symmetric monoidal category $\operatorname{Ho} G \mathscr{S}_{M}$, it is invertible with inverse $\Sigma_{M}^{\infty} S^{\tau}$. Precisely,

$$
\Sigma_{M}^{\infty} S^{\tau} \wedge_{M} T_{M} \simeq \Sigma^{-V}\left(S^{\tau} \wedge_{M} S^{\nu}\right) \simeq S_{M}
$$

since $\tau \oplus \nu$ is the trivial bundle and so $S^{\tau} \wedge_{M} S^{\nu} \cong S_{M}^{V}$. This leads to the following result, which in turn will lead to the usual homological Poincaré duality theorem in $\S 20.5$ and, equivariantly, in $\S 21.4$. 
Theorem 19.1.5 (Homotopical Poincaré duality). Let $M$ be a smooth closed $G$-manifold and let $k$ be a non-parametrized $G$-spectrum. Then there is a canonical equivalence of $G$-spectra

$$
k \wedge M_{+} \simeq S_{M} \triangleright\left(k \wedge S^{\tau}\right) .
$$

Proof. The smash product on the left is just the nonparametrized tensor of a spectrum and a space. That on the right is the (external) smash product of a spectrum and an ex-space over $M$, and can be written in several equivalent ways:

$$
k \wedge S^{\tau} \simeq k \wedge \Sigma_{M}^{\infty} S^{\tau} \simeq r^{*} k \wedge_{M} \Sigma_{M}^{\infty} S^{\tau},
$$

where the middle term is the (external) smash product of a spectrum and a spectrum over $M$, and the right term is a smash product of spectra over $M$. We have the chain of equivalences

$$
\begin{array}{rlrl}
k \wedge M_{+} & \simeq r_{!}\left(k \wedge_{M} S_{M}\right) & \text { by } r_{!} S_{M}=M_{+} \text {and the projection formula } \\
& \simeq r_{!}\left(k \wedge_{M} S^{\tau} \wedge_{M} T_{M}\right) & & \text { since } T_{M} \text { is invertible with inverse } S^{\tau}, \\
& \simeq r_{*}\left(k \wedge_{M} S^{\tau}\right) & & \text { by Costenoble-Waner duality (19.1.2) } \\
& \simeq S_{M} \triangleright\left(k \wedge_{M} S^{\tau}\right) & & \text { by } S_{M}=S_{r} \text { and Corollary 17.4.4. }
\end{array}
$$

We have relative versions of this result, both for compact manifolds with boundary and for pairs of closed manifolds.

Theorem 19.1.6 (Homotopical Poincaré duality—boundary version). Let $M$ be a smooth compact manifold with boundary $\partial M$. Let $k$ be a non-parametrized $G$-spectrum and define $J=k \wedge S^{\tau_{M}}$. Then there is a canonical equivalence

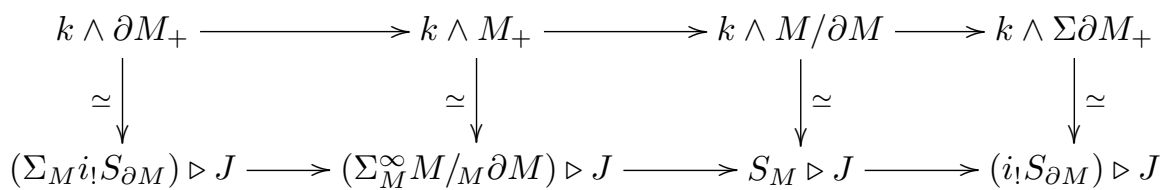

of cofiber sequences of $G$-spectra.

Proof. Recall from Proposition 16.4.12 that if $(X, t Y)$ is a dual pair of spectra over $M$, then there is a natural equivalence

$$
\mu_{J}: J \odot t Y \longrightarrow X \triangleright J
$$

We insert the cofiber sequence of right dualizable parametrized spectra

$$
i_{!} S_{\partial M} \longrightarrow S_{M} \longrightarrow \Sigma_{M}^{\infty} M / M_{M} \partial M \longrightarrow \Sigma_{M} i_{!} S_{\partial M}
$$

into the right hand side of (19.1.7) in place of $X$. This gives us the bottom row of the claimed diagram. We showed in Theorem 18.7.2 that the dual of the cofiber sequence in the previous display is

$$
\Sigma_{M}^{-V} i_{!} S^{\nu_{\partial M}} \longrightarrow \Sigma_{M}^{-V} S^{\nu_{M}} \longrightarrow \Sigma_{M}^{-V} S^{\nu_{M}} /{ }_{M} S^{\nu_{\partial M}} \longrightarrow \Sigma_{M}^{-V+1} i_{!} S^{\nu_{\partial M}} .
$$

As explained in $\S 18.7, \nu_{\partial M}$ is the normal bundle of $\partial M$ in $V^{\prime}$, where $V=V^{\prime} \oplus \mathbb{R}$. We can insert this sequence into the left hand side of (19.1.7) in place of $t Y$, and that gives us a cofiber sequence and an equivalence with the cofiber sequence in the bottom row. It remains to identify the resulting sequence with the top row of the claimed diagram. Using Proposition 17.4.2, we see that the first map in (19.1.8) becomes

$$
r_{!}\left(k \wedge S^{\tau_{M}} \wedge_{M} \Sigma_{M}^{-V} i_{!} S^{\nu_{\partial M}}\right) \longrightarrow r_{!}\left(k \wedge S^{\tau_{M}} \wedge_{M} \Sigma_{M}^{-V} S^{\nu_{M}}\right) .
$$


Using that $\tau_{M} \oplus \nu_{M}=M \times V$ and $\nu_{\partial M}=i^{*} \nu_{M}$, together with the projection formula and the commutation of $i^{*}$ with smash products, we see that this map is equivalent to the map $k \wedge \partial M_{+} \longrightarrow k \wedge M_{+}$induced by the inclusion.

TheOrem 19.1.9 (Homotopical Poincaré duality-relative version). Let $M$ be a smooth closed manifold with a closed submanifold $L$. Let $k$ be a non-parametrized $G$-spectrum and define $J=k \wedge S^{\tau_{M}}$. Then there is a canonical equivalence

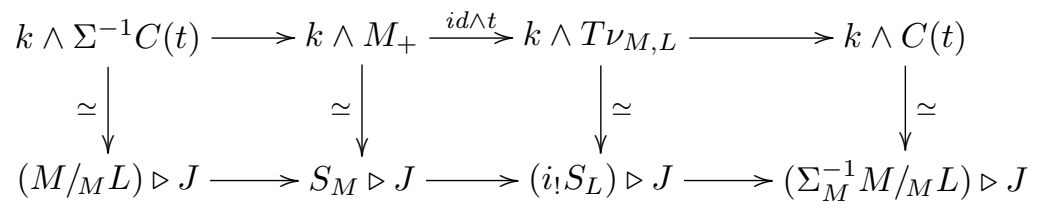

of cofiber sequences of $G$-spectra, where $t: M_{+} \longrightarrow T \nu_{M, L}$ is the Pontryagin-Thom map that collapses the complement of $\nu_{M, L} \subset M$ to a point.

Proof. We begin exactly as in the proof of Theorem 19.1.6 and insert the cofiber sequence of right dualizable parametrized spectra

$$
\Sigma_{M}^{-1} \Sigma_{M}^{\infty} M /{ }_{M} L \longrightarrow i_{!} S_{L} \longrightarrow S_{M} \longrightarrow \Sigma_{M}^{\infty} M / M_{M} L
$$

into the right hand side of (19.1.7) in place of $X$. This gives us the bottom row of the claimed diagram. We showed in Theorem 18.6.5 that the dual sequence of the cofiber sequence in the previous display is

$$
\Sigma_{M}^{-V-1} C_{M}\left(t_{M}\right) \longrightarrow \Sigma_{M}^{-V} S^{\nu_{M}} \longrightarrow \Sigma_{M}^{-V} i_{!} S^{\nu_{L}} \longrightarrow \Sigma_{M}^{-V} C_{M}\left(t_{M}\right) .
$$

We can insert this sequence into the left hand side of (19.1.7) in place of $t Y$, and that gives us a cofiber sequence and an equivalence with the cofiber sequence in

the bottom row. It remains to identify the resulting sequence with the top row of the claimed diagram. Using Proposition 17.4.2, we see that the second map in (19.1.10) becomes

$$
r_{!}\left(k \wedge S^{\tau_{M}} \wedge_{M} \Sigma_{M}^{-V} S^{\nu_{M}}\right) \stackrel{r_{!}\left(\mathrm{id} \wedge_{M} \Sigma_{M}^{-V} t_{M}\right)}{\longrightarrow} r_{!}\left(k \wedge S^{\tau_{M}} \wedge_{M} \Sigma_{M}^{-V} i_{!} S^{\nu_{L}}\right)
$$

where $t_{M}$ is the left vertical map in the diagram (18.6.4). Using the equivalence $S^{\tau_{M}} \wedge_{M} S^{\nu_{M}} \simeq \Sigma_{M}^{V} S_{M}$, it follows from that diagram that this map is equivalent to the map

$$
k \wedge M_{+} \simeq r_{!}\left(k \wedge_{M} d_{!} S_{M}^{0}\right) \stackrel{r_{!}\left(\operatorname{id} \wedge_{M} \alpha\right)}{\longrightarrow} r_{!}\left(k \wedge_{M} i_{!} S^{\nu_{M, L}}\right) \simeq k \wedge T \nu_{M, L}
$$

which is id $\wedge r_{!} \alpha$. It was shown in Construction 18.6.3 that $r_{!} \alpha$ is equivalent to the Pontryagin-Thom map $t: M_{+} \longrightarrow T \nu_{M, L}$.

\subsection{The bicategories $\mathscr{E} x_{B}$}

At the end of $\S 16.2$, we mentioned that there is a categorical structure, not a tricategory but similar in flavor, of commutative rings, algebras, bimodules, and maps of bimodules that better encodes the full structure present in the algebraic situation. Analogously, we have the same categorical structure made up of spaces, spaces over spaces, spectra over spaces over spaces, and maps of such parametrized spectra. This idea might seem esoteric, were it not that, at least implicitly, it plays a fundamental role in our work. We shall not make the full structure explicit. However, in analogy with the bicategories $\mathscr{B}_{R}$ parametrized by commutative rings 
$R$, we shall construct and use a collection of closed symmetric bicategories $\mathscr{E} x_{B}$ parametrized by $G$-spaces $B$. The idea is that the implicit use of a point (the analogue of the integers) in the definition of $\mathscr{E} x$ can be replaced by use of $B$ throughout chapter 17 .

Instead of the external smash product and function spectra functors of (17.1.1) and (17.1.2), the starting point for the definition is given by external smash product and function spectra functors

$$
\bar{\wedge}_{B}: \operatorname{Ho} \mathscr{S}_{K} \times \operatorname{Ho} \mathscr{S}_{L} \longrightarrow \operatorname{Ho} \mathscr{S}_{K \times_{B} L}
$$

and

$$
\bar{F}_{B}: \operatorname{Ho} \mathscr{S}_{L}^{\mathrm{op}} \times \operatorname{Ho} \mathscr{S}_{K \times{ }_{B} L} \longrightarrow \operatorname{Ho} \mathscr{S}_{K}
$$

for (unsectioned) $G$-spaces $K$ and $L$ over $B$. Letting

$$
\iota: K \times{ }_{B} L \longrightarrow K \times L
$$

be the inclusion, these functors are specified by

$$
X \bar{\wedge}_{B} Y=\iota^{*}(X \bar{\wedge} Y) \text { and } \bar{F}_{B}(Y, Z)=\bar{F}\left(Y, \iota_{*} Z\right) .
$$

With this definition, we have the expected equivalence

$$
\bar{F}_{B}\left(X \bar{\wedge}_{B} Y, Z\right) \simeq \bar{F}_{B}\left(X, \bar{F}_{B}(Y, Z)\right)
$$

and adjunction

$$
\operatorname{Ho} \mathscr{S}_{K \times_{B} L}\left(X \bar{\wedge}_{B} Y, Z\right) \cong \operatorname{Ho} \mathscr{S}_{K}\left(X, \bar{F}_{B}(Y, Z)\right) .
$$

Definition 19.2.6. Define a bicategory $\mathscr{E} x_{B}$ as follows. The 0-cells are the $G$-spaces $K=(K, p)$ over $B$. The category $\mathscr{E} x_{B}(K, L)$ is the category HoG $\mathscr{S}_{L \times{ }_{B} K}$ of $G$-spectra over the pullback $L \times_{B} K$ over $B$. We denote its objects, that is the 1-cells, by $X: K-B \rightarrow L$. The composition

$$
\odot_{B}: \operatorname{Ho} G \mathscr{S}_{M \times_{B} L} \times \mathrm{HoG} \mathscr{S}_{L \times{ }_{B} K} \longrightarrow \operatorname{Ho} G \mathscr{S}_{M \times_{B} K}
$$

is $\theta \circ \bar{\wedge}_{B}$, where

$$
\theta: \operatorname{Ho} \mathscr{S}_{M \times_{B} L \times_{B} L \times_{B} K} \longrightarrow \operatorname{Ho} \mathscr{S}_{M \times_{B} K}
$$

is given by pullback along the diagonal and pushforward along the projection displayed in the diagram

$$
M \times_{B} L \times_{B} L \times_{B} K \stackrel{\delta_{L}}{\longleftarrow} M \times_{B} L \times_{B} K \stackrel{\pi}{\longrightarrow} M \times_{B} K .
$$

For a 0 -cell $K=(K, p)$, the unit 1-cell $U_{K}$ in $\mathscr{E} x(K, K)=\operatorname{Ho}_{K} \mathscr{S}_{K}{ }_{B}$ is $\delta_{K !} S_{K}$, $\delta_{K}: K \longrightarrow K \times_{B} K$, and we usually write $K$ instead of $U_{K}$. The functor $\theta$ has a right adjoint $\theta^{*}$, and the left and right internal homs are

$$
Z \triangleleft_{B} Y=\bar{F}_{B}\left(Y, \theta^{*} Z\right): K-B \rightarrow L \quad \text { and } \quad X \triangleright_{B} Z=\bar{F}_{B}\left(X, \theta^{*} Z\right): L-B \rightarrow M
$$

for 1-cells $X: K-B \rightarrow L, Y: L-B \rightarrow M$, and $Z: K-B \rightarrow M$.

The commutative diagram

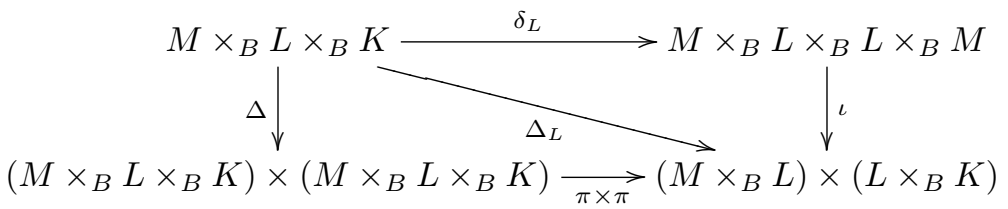


of spaces over $B$ gives rise to a description of the horizontal composition and internal hom functors parallel to that given for $\mathscr{E} x$ in Proposition 17.1.4.

Proposition 19.2.8. For 1-cells $X: K-B \rightarrow L$ and $Y: L-B \rightarrow M$,

$$
Y \odot_{B} X \simeq \pi_{M \times_{B} K !}\left(\pi_{M \times_{B} L}^{*} Y \wedge_{M \times_{B} L \times_{B} K} \pi_{L \times_{B} K}^{*} X\right): K-B \rightarrow M,
$$

where all indicated projections have source $M \times_{B} L \times_{B} K$. For $Z: K-B \rightarrow M$,

$$
Z \triangleleft_{B} Y \simeq \pi_{L \times_{B} K_{*}} F_{M \times_{B} L \times_{B} K}\left(\pi_{M \times_{B} L}^{*} Y, \pi_{M \times_{B} K}^{*} Z\right): K-B \rightarrow L
$$

and

$$
X \triangleright_{B} Z \simeq \pi_{M \times_{B} L_{*}} F_{M \times_{B} L \times_{B} K}\left(\pi_{L \times_{B} K}^{*} X, \pi_{M \times_{B} K}^{*} Z\right): L-B \rightarrow M .
$$

From here, generalizations of other results in $\$ 17.1$ hold with nearly identical proofs. The results of $\S \S 17.2-17.5$ also generalize, but we shall not go into detail.

\subsection{Comparisons of bicategories}

We shall use $G \mathscr{E} x_{B}$ as a tool for studying $G \mathscr{E} x$, and for that we need functors that relate these bicategories as $B$ and $G$ vary. We especially need the following comparison functor.

Proposition 19.3.1. For each $B$, there is a pushforward oplax functor

$$
\iota !: \mathscr{E} x_{B} \longrightarrow \mathscr{E} x
$$

that sends a 0 -cell $(K, p)$ to the total space 0 -cell $K$ and sends a 1 -cell $X: K-B \rightarrow L$ to the 1-cell $\iota$ ! $X: K \longrightarrow L$, where $\iota: L \times_{B} K \longrightarrow L \times K$ is the inclusion. The unit coherence 2 -cell is an equivalence.

ProOF. The factorization $\Delta=\iota \circ \delta$ induces the required unit equivalence

$$
\iota_{!} U_{(K, p)}=\iota_{!} \delta_{!} S_{K} \simeq \Delta_{!} S_{K}=U_{K}
$$

We have a commutative diagram of base spaces

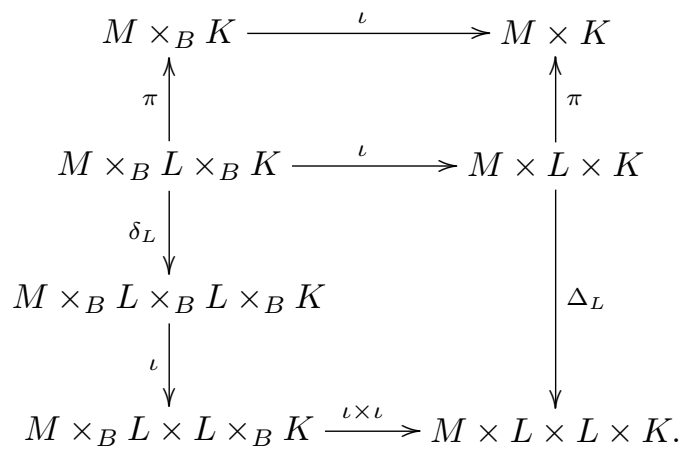

The top square gives rise to an equivalence $\iota_{!} \pi_{!} \simeq \pi_{!} \iota$ ! . As in the proof of Proposition 2.2.11, the bottom square gives rise to a natural map

$$
\alpha: \iota_{!} \delta_{L}^{*} \iota^{*} \longrightarrow \Delta_{L}^{*}(\iota \times \iota) !
$$

namely the adjoint of the map

$$
\delta_{L}^{*} \iota^{*} \longrightarrow \delta_{L}^{*} \iota^{*}(\iota \times \iota)^{*}(\iota \times \iota) ! \simeq \iota^{*} \Delta_{L}^{*}(\iota \times \iota) !
$$


given by the unit of the adjunction $\left((\iota \times \iota) !,(\iota \times \iota)^{*}\right)$. Note that although the bottom square is a pullback, there is no reason to expect $\alpha$ to be an equivalence. Applying $\pi$ ! to $\alpha$, we obtain a natural map

$$
\iota ! \pi ! \delta_{L}^{*} \iota^{*} \simeq \pi_{!} \iota ! \delta_{L}^{*} \iota^{*} \longrightarrow \pi_{!} \Delta_{L}^{*}(\iota \times \iota) !
$$

Applying this construction to $Y \wedge X$ for $G$-spectra $Y$ over $M \times_{B} L$ and $X$ over $L \times_{B} K$, this gives the required coherence 2-cell

$$
\psi: \iota_{!}\left(Y \odot_{B} X\right) \longrightarrow \iota_{!} Y \odot \iota_{!} X .
$$

By Proposition 16.5.4, we have the following immediate consequence.

Corollary 19.3.2. If $(X, Y)$ is a dual pair in $\mathscr{E} x_{B}$ and the coherence 2-cell

$$
\psi: \iota_{!}(Y \odot X) \longrightarrow \iota_{!} Y \odot \iota_{!} X
$$

is an equivalence, then $(\iota ! X, \iota ! Y)$ is a dual pair in $\mathscr{E} x$.

We exhibit a key example where $\psi$ is an equivalence.

Proposition 19.3.3. Taking $L=B$, let $Y$ be a $G$-spectrum over $M \cong M \times_{B} B$ and regard $S_{K}$ as a $G$-spectrum over $K \cong B \times{ }_{B} K$. Then the coherence 2-cell

$$
\psi: \iota_{!}\left(Y \odot_{B} S_{K}\right) \longrightarrow \iota_{!} Y \odot \iota_{!} S_{K}
$$

is an equivalence.

Proof. By the definition of $\odot_{B}$ and the observation that $\delta_{B}: B \longrightarrow B \times_{B} B$ and $\pi: M \times_{B} B \times{ }_{B} K \longrightarrow M \times{ }_{B} K$ are both isomorphic to identity maps, the source of $\psi$ is just $\iota \iota^{*}\left(Y \bar{\wedge} S_{K}\right), \iota: M \times_{B} K \longrightarrow M \times K$. As in (17.2.9), $Y \bar{\wedge} S_{K} \simeq \pi_{1}^{*} Y$. Therefore the source and target of $\psi$ are equivalent to

$$
\iota ! \iota^{*} \pi_{1}^{*} Y \text { and } \pi_{!} \Delta_{B}^{*}(\iota \times \iota) ! \pi_{1}^{*} Y .
$$

When $L=B$, the lower square of the diagram in the previous proof can be rewritten as the upper left square in the following diagram.

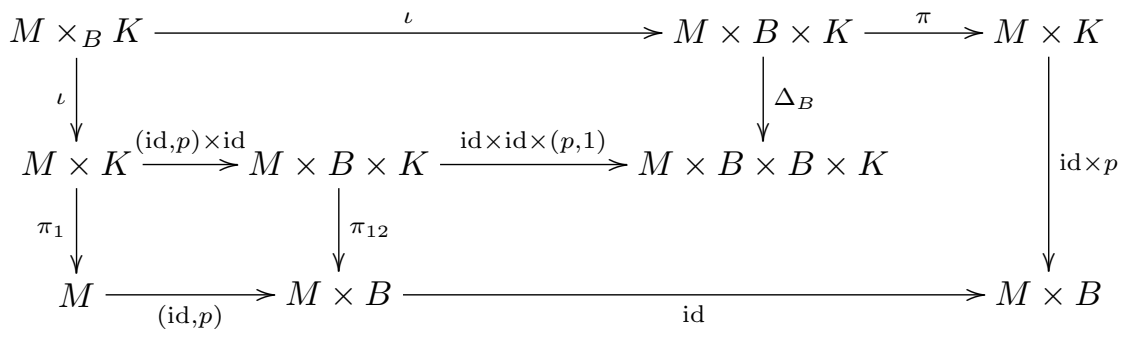

The top square in the diagram of the previous proof reduces to the identification of the composite $\pi \circ \iota$ on the top row as $\iota: M \times{ }_{B} K \longrightarrow M \times K$. The composite on the middle row is $\iota \times \iota$, and the left vertical composite $\pi_{1} \circ \iota$ is the projection $\pi_{1}: M \times_{B} K \longrightarrow M$. Starting with $Y$ over $M$ in the lower left corner, pulling up along the left column and pushing forward along the top row calculates the source of $\psi$, while pulling half way up, pushing right twice, pulling up and pushing right calculates the target of $\psi$. The outer rectangle and the lower left square are pullbacks of bundles and so induce equivalences from lower left to upper right vertices of the two pushforward and pullback composites. In the remaining (noncommutative) part of the diagram, pulling back along id $\times p$ is equivalent to pulling back along $\pi_{12}$, pushing right, pulling up, and pushing right, by the argument in 
Remark 17.2.8. It follows that the source and target of $\psi$ are equivalent via the canonical transformation associated to the upper left rectangle which maps the composite functor obtained by pushing right twice and pulling up to the composite functor obtained by pulling up and pushing right. By definition, this map gives $\psi$ after composition with $\pi_{\text {! }}$.

In our applications, we shall only be concerned with the full sub-bicategory $G \mathscr{E} x_{B}^{\text {fib }}$ of $G \mathscr{E} x_{B}$ whose 0-cells $(K, p)$ are (Hurewicz) fibrations. We then have the following observation about base change functors.

Proposition 19.3.4. Let $f: A \longrightarrow B$ be a map of $G$-spaces. Then pullback along $f$ induces a pseudofunctor $f^{*}: G \mathscr{E} x_{B}^{f i b} \longrightarrow G \mathscr{E} x_{A}^{f i b}$.

Proof. On 0-cells, we define $f^{*}(K, p)=\left(A \times_{B} K\right.$, id $\left.\times_{B} q\right)$, The functor

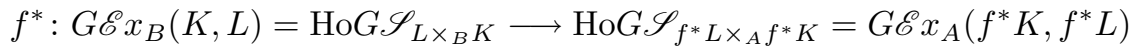

is defined by observing that $f^{*}\left(L \times_{B} K\right) \cong f^{*} L \times_{A} f^{*} K$ and that one arrow of the evident pullback diagram is a map

$$
f^{*} L \times_{A} f^{*} K \longrightarrow L \times_{B} K .
$$

By abuse of notation, we also write this map as $f$, and then the required functor is indeed the pullback $f^{*}$. For triples $(M, L, K)$ of 0 -cells of $G \mathscr{E} x_{B}$, we have a commutative diagram

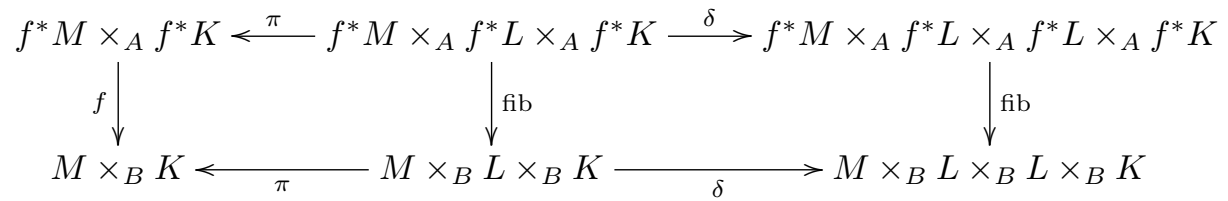

in which the left square is isomorphic to the first of the following pair of pullbacks.

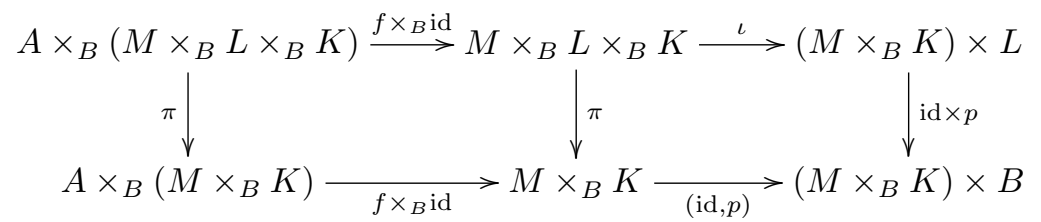

Here $p$ is used generically for projections to $B$. Since $p: L \longrightarrow B$ is a fibration, so are the left two vertical maps. This ensures that $f^{*} \pi_{!} \simeq \pi_{!} f^{*}$. Commutation isomorphisms relating $f^{*}$ to the remaining constituents in the definition of $\odot$ and its unit 1-cells are evident, using (19.2.7), and the verification that $f^{*}$ is a pseudofunctor is straightforward.

We use this together with the following remark to consider passage to fibers.

REMARK 19.3.5. We would like to say that change of groups induces an equivalence $G \mathscr{E} x_{G / H}^{\mathrm{fib}} \cong H \mathscr{E} x$. However, we have not introduced enough categorical language to express this. With the language of Example 16.2.5, the point is that the equivalence is given in terms of vertical 1-cells rather than the horizontal 1-cells that appear in the definition of a biequivalence of bicategories. We will develop the relevant category theory elsewhere, but we can explain the proof without giving the categorical specification of what it is proving. On 0 -cells, we send an $H$-space 
$K$ to the $G$-space $G \times_{H} K$ over $G / H$ and send a $G$-space $(K, p)$ over $G / H$ to the fiber $K_{e}$ over $e H$. We have

$$
G \mathscr{E} x_{G / H}(K, L)=\operatorname{Ho} G \mathscr{S}\left(L \times_{G / H} K\right),
$$

and $\left(L \times_{G / H} K\right)_{e} \cong L_{e} \times K_{e}$. Restriction to fibers and extension of scalars give inverse equivalences which are easily seen to commute with $\odot$ and unit objects to give the kind of equivalence we have in mind; compare $§ 14.3$.

COROLlary 19.3.6. Restriction to fibers defines a pseudo-functor $G \mathscr{E} x_{B}^{f i b} \longrightarrow$ $G_{b} \mathscr{E} x$ for each $b \in B$.

Proof. We apply Proposition 19.3.4 to $\tilde{b}: G / G_{b} \longrightarrow B$ and then apply Remark 19.3.5 with $H=G_{b}$.

\subsection{The bundle construction pseudo-functor}

We fix notations as in $\S 3.3$ and $\S 15.4$, letting $P$ be a $\Pi$-free $\Gamma$-space for an extension $\Gamma$ of $G$ by $\Pi$, these being compact Lie groups. We let $B=P / \Pi$. For a $\Gamma$-space $F$, we defined the bundle construction $P_{F}$ on ex- $\Gamma$-spaces over $F$ in $\S 3.3$. We extended the construction to $\Gamma$-spectra over $F$ in $\$ 15.4$. There we have two variants, $P_{F}$ and $\mathbb{P}_{F}$, the latter being needed to deal with $\Gamma$-spectra over $F$ indexed on complete universes. In $\S 15.6$, we showed that $\mathbb{P}_{F}$ carries fiberwise dual pairs of spectra over $F$ to fiberwise dual pairs of spectra over $E$, where $E$ is the associated bundle $P \times_{\Pi} F$. Analogously, we prove here that $\mathbb{P}_{F}$ carries Costenoble-Waner dual pairs of spectra over $F$ to Costenoble-Waner dual pairs of spectra over $E$.

The essential point is to switch focus from the bundle construction $\mathbb{P}_{F}$ for a fixed fiber $\Gamma$-space $F$ to a pseudo-functor $\mathbb{P}$ between symmetric bicategories that is obtained from such $\mathbb{P}_{F}$ by letting $F$ run over cartesian products $F_{1} \times F_{2}$ of $\Gamma$-spaces and $E$ run over cartesian products $E_{1} \times{ }_{B} E_{2}$ in the category $\mathscr{K} / B$ of spaces over $B$. Recall from Lemma 3.3.4 that the functor $P \times_{\Pi}(-)$ from spaces to spaces over $B$ is cartesian.

Thus, to begin with, we have the functors

$$
\mathbb{P}_{F}: \operatorname{Ho\Gamma } \mathscr{S}_{F} \longrightarrow \operatorname{HoG} \mathscr{S}_{E} .
$$

Here we are regarding $E=P \times_{\Pi} F$ just as a $G$-space, ignoring the fact that it has the projection $p=P \times_{\Pi} r: E \longrightarrow B$. The key point in assembling such functors into a pseudo-functor between bicategories is to remember the implicit projections.

As in $\S 15.6$, there are two layers of structure to consider, and we shall proceed in two stages. The description given above refers to the more elementary first stage.

Construction 19.4.2. We define a pseudo-functor

$$
\mathbb{P}: \Gamma \mathscr{E} x \longrightarrow G \mathscr{E} x_{B}
$$

between symmetric bicategories. Write $F$ for a typical 0 -cell of $\Gamma \mathscr{E} x$, so that $F$ is a $\Gamma$-space. We define $\mathbb{P} F=(E, p)$, where $E=P \times_{\Pi} F$ and $p=P \times_{\Pi} r: E \longrightarrow B$, $r: F \longrightarrow *$. We define $\mathbb{P}$ on 1-cells and 2-cells by letting

$$
\mathbb{P}: \Gamma \mathscr{E} x\left(F_{1}, F_{2}\right) \longrightarrow G \mathscr{E} x_{B}\left(E_{1}, E_{2}\right),
$$

$E_{i}=P \times_{\Pi} F_{i}$, be the functor

$$
\mathbb{P}_{F_{2} \times F_{1}}: \operatorname{Ho\Gamma } \mathscr{S}_{F_{2} \times F_{1}} \longrightarrow \mathrm{HoG} \mathscr{S}_{E_{2} \times B_{B} E_{1}} .
$$


The unit isomorphism 2-cell

$$
U_{\mathbb{P} F}=\delta_{!} S_{\mathbb{P} F} \longrightarrow \mathbb{P} \Delta_{!} S_{F}=\mathbb{P} U_{F}
$$

is obtained by specialization of equivalences in Propositions 15.4.4 and 15.6.1. The horizontal composition isomorphism 2-cell

$$
\mathbb{P} Y \odot \mathbb{P} X \longrightarrow \mathbb{P}(Y \odot X)
$$

is obtained by using Propositions 3.3.8 and 15.6.1 to commute $\mathbb{P}$ past the functors that define the horizontal composition in $\Gamma \mathscr{E} x$.

In the previous construction, $F$ was a variable. We now change our point of view and generalize the previous construction to one whose starting point is some fixed $F$; the previous construction is the case $F=*$ of the generalized verion.

Construction 19.4.3. Fix a base $\Gamma$-space $F$ with associated $G$-bundle $E=$ $P \times_{\Pi} F$. We define a pseudo-functor

$$
\mathbb{P} / F: \Gamma \mathscr{E} x_{F} \longrightarrow G \mathscr{E} x_{E} .
$$

Write $(L, q)$ for a 0 -cell of $\Gamma \mathscr{E} x_{F}$; thus $(L, q)$ is a $\Gamma$-space over $F$. We define

$$
\mathbb{P} / F(L, q)=\left(P \times_{\Pi} L, P \times_{\Pi} q\right),
$$

which is a $G$-space over $E$. We define

$$
\mathbb{P} /_{F}: \Gamma \mathscr{E} x_{F}\left(L_{1}, L_{2}\right) \longrightarrow G \mathscr{E} x_{E}\left(N_{1}, N_{2}\right),
$$

$N_{i}=P \times_{\Pi} L_{i}$, to be the functor

$$
\mathbb{P}_{L_{2} \times_{F} L_{1}}: \operatorname{Ho\Gamma } \mathscr{S}_{L_{2} \times_{F} L_{1}} \longrightarrow \operatorname{HoG} \mathscr{S}_{N_{2} \times_{E} N_{1}} .
$$

The coherence isomorphism 2-cells are defined as in the previous construction.

Here we use the notation $\mathbb{P} / F$ rather than $\mathbb{P}_{F}$ to avoid confusion. The symmetric monoidal functor $\mathbb{P}_{F}$ of (19.4.1) preserves fiberwise dual pairs, whereas the pseudofunctor $\mathbb{P} / F$ preserves all Costenoble-Waner dualizable pairs.

Corollary 19.4.4. If $(X, Y)$ is a dual pair in $\Gamma \mathscr{E} x$, then $(\mathbb{P} X, \mathbb{P} Y)$ is a dual pair in $G \mathscr{E} x_{B}$. More generally, if $(X, Y)$ is a dual pair in $\Gamma \mathscr{E} x_{F}$, then $(\mathbb{P} / F, \mathbb{P} / F Y)$ is a dual pair in $G \mathscr{E} x_{E}$.

\subsection{The fiberwise Costenoble-Waner duality theorem}

Again, fix an extension $\Gamma$ of $G$ by $\Pi$ and a $\Pi$-free $\Gamma$-space $P$ with base space $B=P / \Pi$. Fix a $\Gamma$-space $F$ and let $E=P \times_{\Pi} F$. We assume that $S_{F}$ is a CostenobleWaner dualizable $\Gamma$-spectrum over $F$ with (right) dual $T_{F}$, so that $\left(S_{F}, t T_{F}\right)$ is a dual pair. We fix this context throughout the section. With these hypotheses, we now generalize (19.1.2) from $r: F \longrightarrow *$ to the $G$-bundle $p: E \longrightarrow B$ with fiber $F$. Note that we will use Construction 19.4.2 here, not Construction 19.4.3. That is, we will use the ambient bicategory $G \mathscr{E} x_{B}$ to inform us about $G \mathscr{E} x$. A more general analogue can be obtained using Construction 19.4.3.

Theorem 19.5.1. Let $T_{E}$ be the $G$-spectrum $\mathbb{P}_{F} T_{F}$ over $E$. Then $\left(S_{p}, t T_{p}\right)$ is a dual pair in $G \mathscr{E} x$, where

$$
S_{p} \equiv \iota ! \mathbb{P} S_{F} \simeq(p, i d) ! S_{E} \quad \text { and } \quad T_{p} \equiv \iota ! \mathbb{P} T_{F}=(p, i d) ! T_{E} .
$$


Proof. Here $S_{F}$ and $t T_{F}$ are 1-cells $F \longrightarrow *$ and $* \longrightarrow F, \mathbb{P} S_{F}$ and $t \mathbb{P} T_{F}$ are 1-cells $E-B \rightarrow B$ and $B-B \rightarrow E$, and $S_{p}$ and $t T_{p}$ are 1-cells $E \longrightarrow B$ and $B \longrightarrow E$. The relevant parts of the oplax functor $\iota !: G \mathscr{E}_{B} \longrightarrow G \mathscr{E} x$ of Proposition 19.3.1 are given by pushforward along

$$
\iota=(p, \text { id }): E \cong B \times{ }_{B} E \longrightarrow B \times E \quad \text { and } \quad \iota=(\text { id, } p): E \cong E \times_{B} B \longrightarrow E \times B .
$$

The conclusion follows immediately from Proposition 19.3.3 and Corollaries 19.3.2 and Corollary 19.4.4.

With the notations of the previous theorem, we have the following consequence. Let $j_{b}: E_{b} \longrightarrow E$ be the inclusion and let $\rho_{b}: G_{b} \longrightarrow \Gamma$ be the fiber representation specified in Lemma 3.2.1. Recall the description of $P_{F}$ on fibers from Lemma 15.4.3.

Theorem 19.5.2. For 1-cell G-spectra $X: E \longrightarrow A$, the dual pair $\left(S_{p}, t T_{p}\right)$ gives rise to a natural duality equivalence

$$
\mu_{X}: X \odot t T_{p} \longrightarrow S_{p} \triangleright X
$$

of 1-cell $G$-spectra $B \longrightarrow A$. Restricting the source along $b: * \longrightarrow B$ by applying $(i d \times b)^{*}(-) \simeq(-) \odot S_{b}, \mu$ gives rise to the corresponding equivalence

$$
\mu_{X \odot S_{j}}:\left(X \odot S_{j}\right) \odot t \rho_{b}^{*} T_{F} \longrightarrow \rho_{b}^{*} S_{F} \triangleright\left(X \odot S_{j}\right)
$$

of (19.1.1). When $A$ is a point, so that $X$ is a $G$-spectrum over $E, \mu_{X}$ is an equivalence

$$
p_{!}\left(X \wedge_{E} T_{E}\right) \simeq p_{*} X
$$

of $G$-spectra over $B$ that restricts on fibers to the corresponding equivalences

$$
r_{!}\left(j_{b}^{*} X \wedge_{\rho_{b}^{*} F} \rho_{b}^{*} T_{F}\right) \simeq r_{*} X
$$

of $G_{b}$-spectra given in (19.1.2).

Proof. The first statement is immediate. For the second, we fix $b$ and we agree to restrict group actions to $G_{b}$ throughout the proof. We also abbreviate $j=j_{b}$. Identifying $\rho_{b}^{*} F$ with $E_{b}$ and using change of universe and change of groups, Lemma 15.4.3 tells us that the functors $\rho_{b}^{*}$ and $j^{*} \mathbb{P}$ from $\mathrm{Ho} \Gamma \mathscr{S}_{F}$ to $\mathrm{Ho}_{b} \mathscr{S}_{E_{b}}$ are isomorphic. In particular, $\rho_{b}^{*} T_{F} \simeq j^{*} \mathbb{P} T_{F}=j^{*} T_{E}$. Thinking of $r: E_{b} \longrightarrow *$, we write the latter as $T_{r}$, so that $\left(S_{r}, t T_{r}\right)$ is a dual pair of $G_{b}$-spectra over $E_{b}$. It is essentially just the original dual pair $\left(S_{F}, t T_{F}\right)$, pulled back to $G_{b}$ along $\rho_{b}$. Let $\bar{\varepsilon}$ and $\varepsilon$ denote the evaluation maps of the pairs $\left(S_{p}, t T_{p}\right)$ and $\left(S_{r}, t T_{r}\right)$. We first obtain a diagram relating $\varepsilon$ to $\bar{\varepsilon}$ and then use it to prove the second statement of the theorem. The comparison relies on the following commutative diagram of base 
spaces.

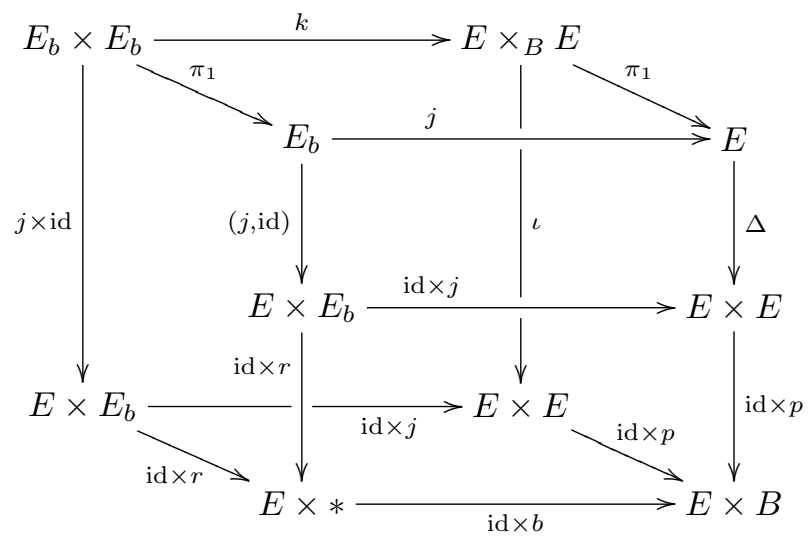

The left, right, and bottom faces are pullbacks, and the lower square in the front face is the same as the bottom face. The vertical composites in the front face may be identified with $j$ and (id, $p$ ). We construct the following commutative diagram.

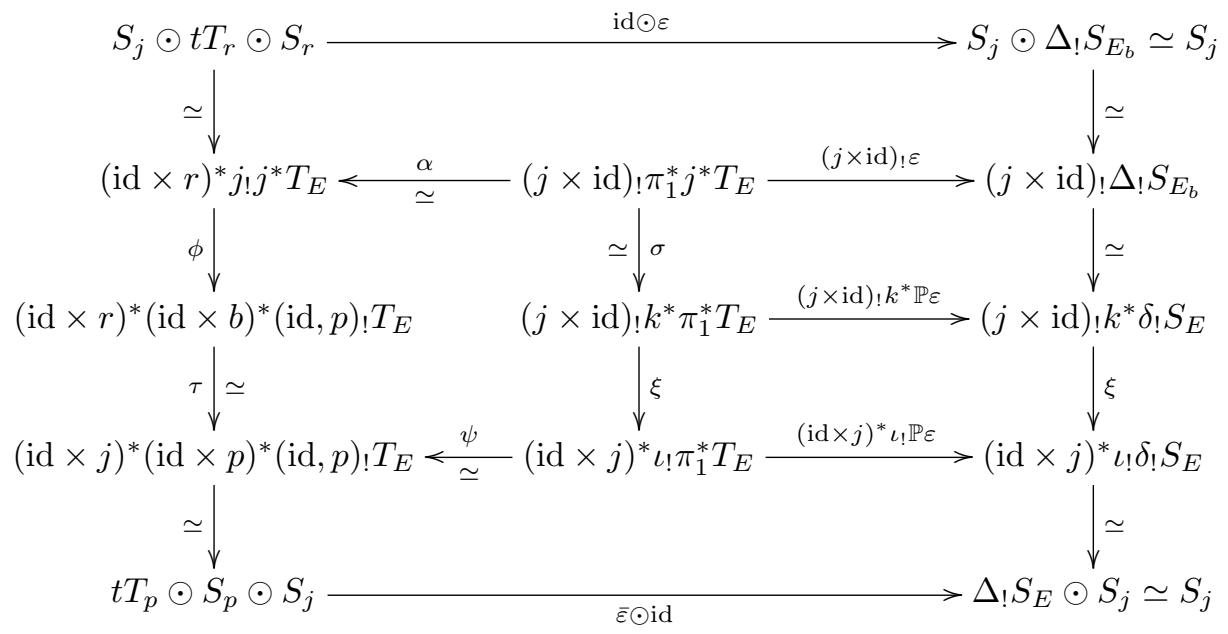

The unlabelled equivalences in the top and bottom rectangle are given by the dictionary Proposition 17.2.4. The equivalences $\alpha$ and $\psi$ are obtained from the left and right pullback faces of (19.5.5). The equivalences $\sigma$ and $\tau$ are commutation relations induced from the top and bottom faces. The map $\phi$ is obtained from the front face (as in Proposition 2.2.11), starting with $T_{E}$ over the $E$ in the upper right corner, and the maps $\xi$ are obtained from the back face. We shall prove shortly that $\phi$ and therefore also the left map $\xi$ is an equivalence, and the right map $\xi$ is an equivalence since the right vertical composite agrees with the evident composite of unit equivalences. The middle left hexagon commutes by a diagram chase from the commutative diagram (19.5.5), and the lower middle rectangle is a naturality diagram. We must explain the remaining three rectangles.

As observed more generally in (17.2.9), the domain $t T_{F} \odot S_{F}$ of the evaluation map for the dual pair $\left(S_{F}, t T_{F}\right)$ may be identified with $\pi_{1}^{*} T_{F}$, and similarly for 
the equivalent dual pair $\left(S_{r}, t T_{r}\right)$. Instead of starting with the top left vertical equivalence, we could have identified $S_{j} \odot t T_{r} \odot S_{r}$ with $(j \times \text { id })_{!}(\mathrm{id} \times r)^{*} j^{*} T_{E}$. The map $\alpha$ in the diagram specifies the associativity isomorphism relating these two identifications, and the top rectangle amounts to the diagram obtained by applying $(j \times$ id $)$ ! to the specification of $\varepsilon$ as a map with domain $\pi_{1}^{*} T_{r}=\pi_{1}^{*} j^{*} T_{E}$. The map $k$ at the top of (19.5.5) is the inclusion $E_{b} \times E_{b}=\left(E \times{ }_{B} E\right)_{b} \longrightarrow E \times_{B} E$ of the fiber over $b$. Recall from Corollary 19.3.6 that base change functors along inclusions of fibers give a pseudofunctor $G \mathscr{E} x_{B}^{\mathrm{fib}} \longrightarrow G_{b} \mathscr{E} x$. We have the dual pair $\left(\mathbb{P} S_{F}, \mathbb{P} t T_{F}\right)$, and applying $k$ to its evaluation map $\mathbb{P} \varepsilon$ recovers the evaluation map for the fiber dual pair $\left(j^{*} S_{r}, j^{*} t T_{r}\right)$, modulo the identification of domains given by the map $\sigma$ in the diagram. This gives the upper middle rectangle. Finally, the equivalence $\psi$ in the bottom rectangle is obtained by applying $(\mathrm{id} \times j)^{*}$ to the equivalence $\psi$ of Proposition 19.3.3 (which simplifies considerably in our case $M=K=E$ ). The commutativity of the bottom rectangle is obtained by applying (id $\times j)^{*}$ to the definition of $\bar{\varepsilon}$ in terms of $\mathbb{P} \varepsilon$; compare Proposition 16.5.4.

Now return to the second statement of the theorem. We are given a 1-cell $X \odot S_{j}: E_{b} \longrightarrow A$, and our claim is that

$$
\mu_{X} \odot S_{b} \simeq \mu_{X \odot S_{j}}
$$

up to identifications of their source and target 1-cells. Once we prove that, this will also give us an alternative proof of Theorem 19.5.2 as a consequence of equivalences of the form (19.1.1). Indeed, $\mu_{X}$ is an equivalence if and only if its restriction to fibers is an equivalence, and we can restrict to the fiber over $(a, b)$ by first restricting to $A \times\{b\}$ and then restricting to $(a, b)$.

We define equivalences $\phi$ and $\omega$ that make the following diagram commute up to natural equivalence (an isomorphism 2-cell in $\mathscr{E} x$ ).

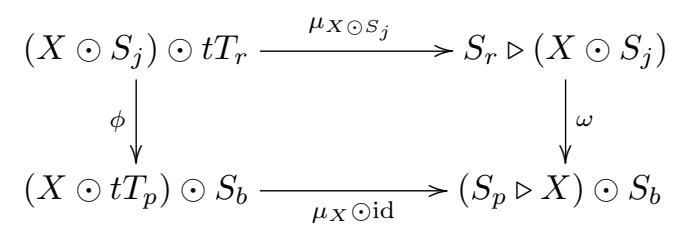

To obtain $\omega$, we apply the dictionary Proposition 17.2.4 to interpret the source and target as base change functors and use a commutation relation derived from the product of $A \times(-)$ with the pullback diagram

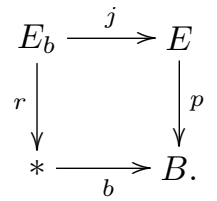

Explicitly, with $\mathrm{id}=\mathrm{id}_{A}, \omega$ is the equivalence

$$
S_{r} \triangleright\left(X \odot S_{j}\right) \simeq(\mathrm{id} \times r)_{*}(\mathrm{id} \times j)^{*} X \simeq(\mathrm{id} \times b)^{*}(\mathrm{id} \times p)_{*} X \simeq\left(S_{p} \triangleright X\right) \odot S_{b} .
$$

To obtain $\phi$, we apply the funtor $X \odot(-)$ to the following map derived (as before) from the front face of (19.5.5), where id $=\mathrm{id}_{E}$ and the dictionary Proposition 17.2.4 gives the first and last equivalences

$$
S_{j} \odot t T_{r} \simeq j_{!} t T_{r}=t j_{!} j^{*} T_{E} \simeq t(\mathrm{id} \times b)^{*}(\mathrm{id}, p) ! T_{E}=t(\mathrm{id} \times b)^{*} T_{p} \simeq t T_{p} \odot S_{b} .
$$


The prefixed letter $t$ in the middle terms just indicates that we are viewing these $G_{b^{-}}$ spectra over $E$ as 1-cells $* \longrightarrow E$, as dictated by consistency with the outermost terms. We claim that this map is an equivalence. Logically, the verification is only necessary to obtain the second proof that $\mu_{X}$ is an equivalence, since the commutativity of (19.5.8) will imply that $\phi$ must be an equivalence. However, we can see this directly by checking that the map just displayed agrees with the following composite equivalence of 1 -cells $* \longrightarrow E$.

$$
\begin{array}{rlr}
t j_{!} j^{*} T_{E} & \simeq t j_{!}\left(j^{*} T_{E} \wedge_{E_{b}} S_{r}\right) & \text { by a unit isomorphism } \\
& \simeq t\left(T_{E} \wedge_{E} j_{!} r^{*} S\right) & \text { by the projection formula, } \\
& \simeq \Delta_{!} T_{E} \odot S_{j} \odot t S_{r} & \text { by Proposition } 17.4 .3 \\
& \simeq \Delta_{!} T_{E} \odot t S_{p} \odot S_{b} & \text { by Theorem } 17.4 .5 \\
& \simeq t(\mathrm{id} \times b)^{*}(\mathrm{id}, p) ! T_{E} &
\end{array}
$$

Recall that $\mu_{X}$ is defined as the adjoint of $\bar{\varepsilon}: X \odot t T_{p} \odot S_{p} \longrightarrow X \odot E \simeq X$, and similarly for $\mu_{X \odot S_{j}}$. We expand (19.5.8) as follows, abbreviating some labels of arrows by deleting functors applied to maps. For example, the map $\phi$ on the second row is shorthand for $S_{r} \triangleright\left(\phi \odot S_{r}\right)$.

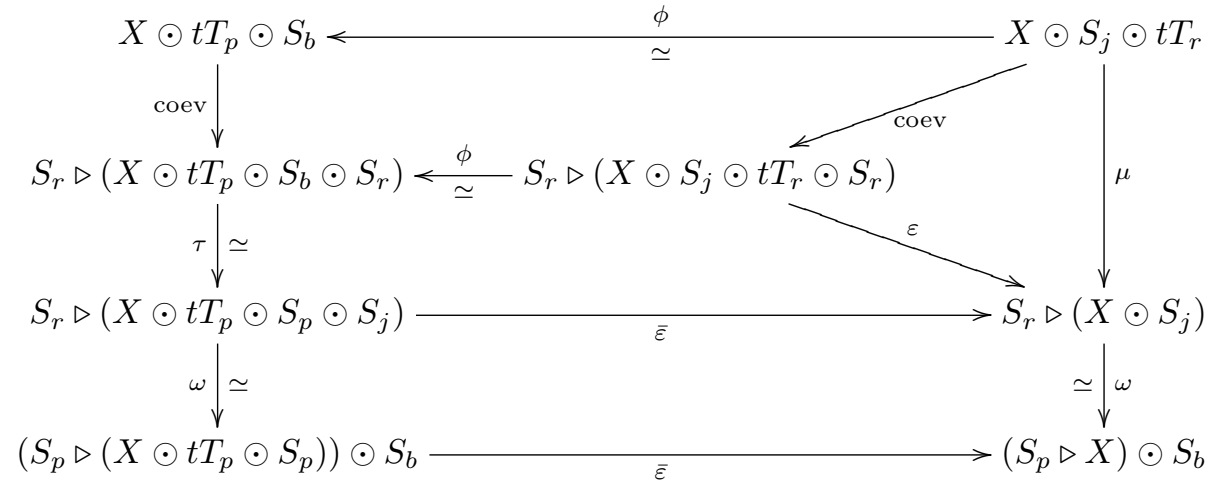

The composite of the maps in the left column is coev $\odot$ id, hence the composite down the left column and along the bottom row is $\mu_{X} \odot$ id, so that the diagram is indeed an expansion of (19.5.8). The top trapezoid and bottom rectangle are naturality diagrams, and the triangle commutes by the definition of $\mu_{X \odot S_{j}}$ as the adjoint of $\varepsilon$. The middle trapezoid commutes since it is obtained by applying the functor $S_{r} \triangleright(X \odot(-))$ to the commutative diagram (19.5.6).

\subsection{Fiberwise Poincaré duality}

Just as (19.1.2) led to the homotopical version of Poincaré duality formulated in (Theorem 19.1.5), so 19.5.4 leads to a fiberwise homotopical Poincaré duality theorem. We retain the context of the previous section, except that we now take $F$ to be a smooth closed $\Gamma$-manifold and rename it $M$. Thus we have the ex- $G$ space $(E, p)_{+}$over $B$, where $B=P / \Pi$ and $E=P \times_{\Pi} M$. Since the $\Gamma$-spectrum $T_{M}=\Sigma_{M}^{-V} S^{\nu}$ over $M$ is invertible with inverse $\Sigma_{M}^{\infty} S^{\tau}$ in $Н$ Н $\Gamma \mathscr{S}_{M}$ and the functor $\mathbb{P}_{M}: \operatorname{Ho\Gamma } \mathscr{S}_{M} \longrightarrow \operatorname{HoG} \mathscr{S}_{B}$ is symmetric monoidal, by Proposition 15.6.1, the $G$-spectrum $T_{E}=\mathbb{P}_{M} T_{M}$ over $B$ is invertible in HoG $\mathscr{S}_{B}$. Its inverse is the spectrum level $G$-bundle of spherical tangents along the fiber associated to $p$, namely 
$\Sigma_{E}^{\infty} P_{M} S^{\tau} \simeq \mathbb{P}_{M} \Sigma_{E}^{\infty} S^{\tau}$, which we will denote $\mathbb{P}_{M} S^{\tau}$. We have two versions of fiberwise homotopical Poincaré duality, one starting with a $G$-spectrum $J$ over $B$ and the other with a $G$-spectrum $k$. It is convenient below to write $\bar{r}: B \longrightarrow *$ and $r: E \longrightarrow *$, so that $\bar{r} \circ p=r$.

Theorem 19.6.1 (Homotopical Poincaré duality for bundles). Let $M$ be a smooth closed $\Gamma$-manifold and let $J$ be a $G$-spectrum over $B$. Then there is a canonical equivalence of $G$-spectra over $B$

$$
J \wedge_{B}(E, p)_{+} \simeq S_{p} \triangleright\left(p^{*} J \wedge_{E} \mathbb{P}_{M} S^{\tau}\right) .
$$

Therefore, taking $J=\bar{r}^{*} k$ for a $G$-spectrum $k$,

$$
k \wedge(E, p)_{+} \simeq S_{p} \triangleright\left(k \wedge \mathbb{P}_{M} S^{\tau}\right) .
$$

Proof. In the first statement, the smash product on the left is the tensor of a spectrum over $B$ and an ex-space over $B$. That on the right is the smash product of spectra over $E$ and is equivalent to the tensor $p^{*} J \wedge_{E} P_{M} S^{\tau}$. The following chain of equivalences gives the conclusion.

$$
\begin{array}{rlr}
J \wedge_{B} \Sigma_{B}^{\infty}(E, p)_{+} & \simeq J \wedge_{B} p_{!} S_{E} & \text { since } p_{!} S_{E} \simeq \Sigma_{B}^{\infty}(E, p)_{+}, \\
& \simeq p_{!}\left(p^{*} J \wedge_{E} S_{E}\right) & \text { by the projection formula, } \\
& \simeq p_{!}\left(p^{*} J \wedge_{E} \mathbb{P}_{M} S^{\tau} \wedge_{E} T_{E}\right) & \text { since } S_{E} \simeq \mathbb{P}_{M} S^{\tau} \wedge_{E} T_{E}, \\
& \simeq p_{*}\left(p^{*} J \wedge_{E} \mathbb{P}_{M} S^{\tau}\right) & \text { by }(19.5 .4), \\
& \simeq S_{p} \triangleright\left(p^{*} J \wedge_{E} \mathbb{P}_{M} S^{\tau}\right) & \text { by Corollary 17.4.4. }
\end{array}
$$

In the second statement, the smash product on the left pairs a spectrum with an ex-space over $B$ to obtain a spectrum over $B$ and is equivalent to $J \wedge_{B}(E, p)_{+}$; that on the right pairs a spectrum with a spectrum over $E$ to obtain a spectrum over $E$ and is equivalent to $p^{*} J \wedge_{E} P_{M} S^{\tau}$.

If we apply $\bar{r}_{\text {! to the }}$ above result, taking $J=\bar{r}^{*} k$, the left side becomes $k \wedge \Sigma^{\infty} E_{+}$, whose homotopy groups are $k_{*}(E)$. We would like to understand the right side in cohomological terms. In general, the right side is hard to interpret since

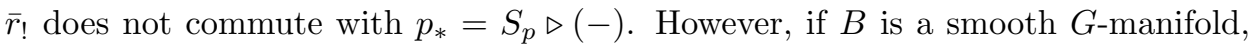
then $\bar{r}_{!}(X) \simeq \bar{r}_{*}\left(X \wedge_{B} S^{\tau_{B}}\right)$ for any $G$-spectrum $X$ over $B$, since $S^{\tau_{B}}$ is inverse to $T_{B}$, and the right side begins to look cohomological. We still need to commute $p_{*}$ past smash products, but the following general observation achieves that.

Remark 19.6.2. Let $f: A \longrightarrow B$ be a map such that there is a natural isomorphism $f_{*} X \simeq f_{!}\left(X \wedge_{A} I\right)$, where $I$ is invertible with (homotopical) inverse $I^{-1}$ so that we also have $f_{!} X \simeq f_{*}\left(X \wedge_{A} I^{-1}\right)$. Then the projection formula for $f_{!}$formally implies the projection formula for $f_{*}$. That is, $f_{*}\left(f^{*} Y \wedge_{A} X\right) \simeq Y \wedge_{B} f_{*} X$ for $G$-spectra $X$ over $A$ and $Y$ over $B$.

TheOrem 19.6.3. Let $M$ be a smooth closed $\Gamma$-manifold and $B$ be a smooth closed $G$-manifold, and let $J$ be a $G$-spectrum over $B$. Then there is a canonical equivalence of $G$-spectra over $B$

$$
\bar{r}_{!}\left(J \wedge_{B}(E, p)_{+}\right) \simeq S_{E} \triangleright\left(p^{*} J \wedge_{E} \mathbb{P}_{M} S^{\tau_{M}} \wedge_{E} p^{*} S^{\tau_{B}}\right) .
$$

Therefore, taking $J=\bar{r}^{*} k$ for a $G$-spectrum $k$,

$$
k \wedge E_{+} \simeq S_{E} \triangleright\left(k \wedge\left(\mathbb{P}_{M} S^{\tau_{M}} \wedge_{E} p^{*} S^{\tau_{B}}\right)\right) .
$$


Proof. Applying $\bar{r}_{\text {! }}$ to the first equivalence of Theorem 19.6.1, the left side becomes $\bar{r}_{!}\left(J \wedge_{B}(E, p)_{+}\right)$and we must evaluate the right side. The first and last of the following equivalences are immediate, the second is given by Remark 19.6.2 and the third by $r_{*} \simeq \bar{r}_{*} p_{*}$.

$$
\begin{aligned}
\bar{r}_{!}\left(S_{p} \triangleright\left(p^{*} J \wedge_{E} \mathbb{P}_{M} S^{\tau_{M}}\right)\right) & \simeq \bar{r}_{*}\left(p_{*}\left(p^{*} J \wedge_{E} \mathbb{P}_{M} S^{\tau_{M}}\right) \wedge_{B} S^{\tau_{B}}\right) \\
& \simeq \bar{r}_{*} p_{*}\left(p^{*} J \wedge_{E} \mathbb{P}_{M} S^{\tau_{M}} \wedge_{E} p^{*} S^{\tau_{B}}\right) \\
& \simeq r_{*}\left(p^{*} J \wedge_{E} \mathbb{P}_{M} S^{\tau_{M}} \wedge_{E} p^{*} S^{\tau_{B}}\right) \\
& \simeq S_{E} \triangleright\left(p^{*} J \wedge_{E} \mathbb{P}_{M} S^{\tau_{M}} \wedge_{E} p^{*} S^{\tau_{B}}\right)
\end{aligned}
$$

When $J=\bar{r}^{*} k$, the left side reduces to $k \wedge E_{+}$by the projection formula and the right side reduces to $S_{E} \triangleright\left(k \wedge\left(\mathbb{P}_{M} S^{\tau_{M}} \wedge_{E} p^{*} S^{\tau_{B}}\right)\right)$ since $p^{*} J \simeq r^{*} k$.

In fact, the previous result is a rather elaborate consistency check on our general theory. There is a bundle theoretic reinterpretation that allows it to be viewed as a special case of Theorem 19.1.5. Assuming that $P$ is completely regular, so that $P \longrightarrow B$ is locally trivial in the appropriate equivariant sense [92, p. 267], the smooth structures on $B, \Gamma$, and $M$ induce smooth structures on $P$ and $E$. Therefore both ordinary and fiberwise Costenoble-Waner duality apply to $E$. It is clear by inspection that the tangent $G$-bundle of $E$ is isomorphic to the Whitney sum of the bundle of tangents along the fibers and the pullback of the tangent bundle of $B$ :

$$
\tau_{E} \cong\left(P \times_{\Pi} \tau_{M}\right) \oplus p^{*} \tau_{B}
$$

Therefore, the sphere $G$-bundle $S^{\tau_{E}}$ is isomorphic to the fiberwise smash product $P_{M} S^{\tau} \wedge_{E} p^{*} S^{\tau_{B}}$. Applying $\Sigma_{E}^{\infty}$, which commutes with smash products, we see that

$$
\Sigma_{E}^{\infty} S^{\tau_{E}} \simeq \mathbb{P}_{M} S^{\tau_{M}} \wedge_{E} p^{*} S^{\tau_{B}} .
$$

If we insert this into Theorem 19.6.3, taking $J=\bar{r}^{*} k$, we obtain

$$
k \wedge E_{+} \simeq S_{E} \triangleright\left(k \wedge S^{\tau_{E}}\right) .
$$

which is the homotopical Poincaré duality of Theorem 19.1.5.

\subsection{The Adams isomorphism}

Let $N$ be a normal subgroup of $G$ and let $\varepsilon: G \longrightarrow J$ be the quotient by $N$. The conjugation action of $G$ on $N$ induces an action of $G$ on the tangent space of $N$ at the identity element, giving us the adjoint representation $A=A(N ; G)$. Let $\left(i_{*}, i^{*}\right)$ be the change of universe adjunction associated to the inclusion $i: q^{*} \mathscr{V}_{J} \longrightarrow \mathscr{V}_{G}$ of the complete $J$-universe $\mathscr{V}_{J}$ as the universe of $N$-trivial representations in the complete $G$-universe $\mathscr{V}_{G}$.

Recall the discussion of $N$-free $G$-spectra from $\S 15.5$, where $\Pi$ and $\Gamma$ played the roles of $N$ and $G$.

Theorem 19.7.1 (Adams isomorphism). For $N$-free $G$-spectra $X$ in $G \mathscr{S}^{N \text {-triv }}$, there is a natural equivalence

$$
X / N \simeq\left(i^{*} \Sigma^{-A} i_{*} X\right)^{N}
$$

in Ho J $\mathscr{S}^{N-\text { triv }}$.

We shall derive this by applying Theorem 19.5.2 to the quotient $G$-map

$$
p: E(N ; G) \longrightarrow B(N ; G),
$$


where $E(N ; G)$ is the universal $N$-free $G$-space and $B(N ; G)=E(N ; G) / N$. To place ourselves in the required bundle theoretic context, we give another description of $p$, following [118, II $\S 7]$. It is formal and would similarly identify $p: E \longrightarrow E / N$ for any $N$-free $G$-space $E$. Let $\Gamma=G \ltimes N$ be the semi-direct product of $G$ and $N$, where $G$ acts by conjugation on $N$. Write $\Pi$ for the normal subgroup $\{e\} \ltimes N$ of $\Gamma$. We then have an extension

$$
1 \longrightarrow \Pi \longrightarrow \Gamma \stackrel{\theta}{\longrightarrow} G \longrightarrow 1
$$

where $\theta(g, n)=g n$. Give $N$ the $\Gamma$-action $(g, n) \cdot m=g n m g^{-1}$. Then $N \cong \Gamma / G$ as $\Gamma$-spaces, where we view $G$ as the subgroup $G \ltimes\{e\}$ of $\Gamma$. The composite

$$
E(N ; G) \cong \theta^{*} E(N ; G) \times_{\Pi}(\Gamma / G) \longrightarrow \theta^{*} E(N ; G) \times_{\Pi} * \cong B(N ; G)
$$

induced by $\Gamma / G \longrightarrow *$ is $p$. Since $\theta^{*} E(N ; G)$ is a $\Pi$-free $\Gamma$-space, we see that $p$ is a bundle with fiber $\Gamma / G \cong N$ to which Theorem 19.5.2 applies. We must identify the $G$-spectrum $T_{p}$ over $E(N, G)$ of Theorem 19.5 .1 when $F=\Gamma / G$. We write $r$ for the map $E(N ; G) \longrightarrow *$.

Proposition 19.7.2. The $G$-spectrum $T_{p}$ over $E(N, G)$ is equivalent to $r^{*} S^{-A}$.

Proof. The tangent bundle of $\Gamma / G \cong N$ is the trivial bundle $N \times A$ [98, p. 99]. Indeed, let $\Gamma$ act on $A$ via the projection $\varepsilon: \Gamma \longrightarrow G, \varepsilon(n, g)=g$. We obtain a $\Gamma$-trivialization of the tangent bundle of $\Gamma / G$ by sending $(n, a) \in N \times A$ to $d_{e} L_{n}(a)$, where $d_{e} L_{n}$ is the differential at $e$ of left translation by $n$. It follows that the tangent bundle along the fibers of $p$ is also trivial:

$$
\left.\theta^{*} E(N ; G) \times{ }_{N}(\Gamma / G \times A) \cong\left(\theta^{*} E(N ; G) \times{ }_{N} \Gamma / G\right)\right) \times A \cong E(N ; G) \times A .
$$

Thus the spherical bundle of tangents along the fiber is $E(N ; G) \times S^{A}=r^{*} S^{A}$. Its inverse is the bundle construction on the spherical fibration of the normal bundle of $\Gamma / G$, which of course is also trivial. Its $G$-spectrum over $E(N ; G)$ is $r^{*} S^{-A}$.

Proof of the Adams isomorphism. Let $X \in G \mathscr{S}^{N \text {-triv }}$ be $N$-free. Applying Theorem 19.5.2 to the $G$-spectrum $r^{*} i_{*} X$ over $E(N ; G)$ and using that $T_{p}$ is $r^{*} S^{-A}$, we obtain a natural equivalence

$$
p_{!}\left(r^{*} i_{*} X \wedge_{E(N ; G)} r^{*} S^{-A}\right) \simeq p_{*} r^{*} i_{*} X
$$

of $G$-spectra over $B(N ; G)$. Write $\bar{r}$ for the map $B(N ; G) \longrightarrow *$, so that $\bar{r} \circ p=r$. Applying the functor $\bar{r}_{!}\left(\left(i^{*}(-)\right)^{N}\right)$ to the displayed equivalence, we obtain a natural equivalence

$$
\bar{r}_{!}\left(\left(i^{*} p_{!}\left(r^{*} i_{*} X \wedge_{E(N ; G)} r^{*} S^{-A}\right)\right)^{N}\right) \simeq \bar{r}_{!}\left(\left(i^{*} p_{*} r^{*} i_{*} X\right)^{N}\right)
$$

in Ho $J \mathscr{S}^{N \text {-triv }}$. We proceed to identify both sides. The source is

$$
\begin{aligned}
\bar{r}_{!}\left(\left(i ^ { * } p _ { ! } \left(r^{*} i_{*} X\right.\right.\right. & \left.\left.\left.\wedge E(N ; G) r^{*} S^{-A}\right)\right)^{N}\right) & & \\
& \simeq \bar{r}_{!}\left(\left(i^{*} p_{!} r^{*} \Sigma^{-A} i_{*} X\right)^{N}\right) & & \text { by Theorem } 13.7 .3 \\
& \simeq\left(\bar{r}_{!} i^{*} p ! r^{*} \Sigma^{-A} i_{*} X\right)^{N} & & \text { by Proposition } 14.4 .4 \\
& \simeq\left(i^{*} \bar{r}_{!} p ! r^{*} \Sigma^{-A} i_{*} X\right)^{N} & & \text { by Propositions } 14.2 .8 \text { and } 15.5 .5 \\
& \simeq\left(i^{*} r ! r^{*} \Sigma^{-A} i_{*} X\right)^{N} & & \text { by functoriality } \\
& \simeq\left(i^{*} \Sigma^{-A} i_{*} X\right)^{N} & & \text { by Definition } 15.5 .1 .
\end{aligned}
$$


The target is

$$
\begin{aligned}
\bar{r}_{!}\left(\left(i^{*} p_{*} r^{*} i_{*} X\right)^{N}\right) & \simeq \bar{r}_{!}\left(\left(p_{*} r^{*} i^{*} i_{*} X\right)^{N}\right) & & \text { by Proposition 14.2.8 } \\
& \simeq \bar{r}_{!}\left(\left(p_{*} r^{*} X\right)^{N}\right) & & \text { by Proposition 15.5.5 } \\
& \simeq \bar{r}_{!}\left(\left(p_{!} r^{*} X\right) / N\right) & & \text { by Proposition 14.4.5 } \\
& \simeq\left(\bar{r}_{!} p_{!} r^{*} X\right) / N & & \text { by Proposition 14.4.4 } \\
& \simeq\left(r_{!} r^{*} X\right) / N & & \text { by functoriality } \\
& \simeq X / N . & & \text { by Definition } 15.5 .1 .
\end{aligned}
$$

\subsection{Some background and comparisons}

Aside from acknowledgment of the essential precursor [41] of Costenoble and Waner, it is hard for us to give precise antecedents for our homotopical versions of Poincaré duality in $\S 19.1$ and $\S 19.5$. There are various hints in the literature that something like our results should be true but, to the best of our knowledge, the present formulations are new.

The Wirthmüller and Adams isomorphisms, in their appropriately general versions as isomorphisms between certain left and right adjoint functors on $G$-spectra, were the most difficult results in Lewis and May's work on the foundations of equivariant stable homotopy theory [98]. The proofs there always seemed unsatisfactory, but the results have since been used in many applications. With our new perspective, these results are just very special cases of parametrized Atiyah duality and its fiberwise generalization. The new proofs complete the program begun in [105] of reproving conceptually all of the basic foundational results that were first proven in a less satisfactory ad hoc way in [98].

Historically, the Wirthmüller isomorphism, viewed as a statement about the behavior of equivariant homology theories on orbit $G$-spaces, is a key result in Wirthmüller's early paper [171]. Shortly afterwards, he recognized it both as a duality theorem on orbits and as a special case of equivariant Atiyah duality [172]. The Adams isomorphism was first proven in $[3,5.4]$, but only for finite groups and only in the equivariant Spanier-Whitehead suspension category. Adams knew and cited the Lewis-May result for $G$-free spectra, which had not yet appeared, but Lewis and May called the result the Adams isomorphism in their published version [98] because Adams was the first to formulate the result in proper generality as a statement about general normal subgroups, rather than just the trivial subgroup.

The basic idea that parametrized $G$-spectra should clarify and simplify the Wirthmüller and Adams isomorphisms was explained in an e-mail from Gaunce Lewis to $\mathrm{Po} \mathrm{Hu}$ [97]. In her monograph [78], $\mathrm{Hu}$ first formulated and proved a fiberwise version of the Wirthmüller isomorphism, and she used it to prove the Adams isomorphism. Our results in this chapter include variant versions of all of her results, and our original arguments were inspired by those in her work.

REMARK 19.8.1. Taking the fiber $F$ in $\S 19.5$ to be a smooth manifold $M$ and taking $\Gamma=G \times \Pi$ with only $\Pi$ acting on $M$, one can think of $p: E \longrightarrow B$ as a topological $G$-bundle with a reduction of its structural group to a suitably large compact subgroup $\Pi$ of the group of diffeomorphisms of $M$. That case of Theorem 19.5.2 is a variant of the main theorem, $[78,4.8]$, of Hu's monograph. She worked with $\operatorname{Diff}(M)$ itself as an implicit structure group, without use of an auxiliary group $\Pi$ and without an ambient group $\Gamma$. That bundle theoretic framework leads to 
formidable complications, hence her arguments are much more difficult than ours. Her result is both more and less general than the specialization of ours to the case $\Gamma=G \times \Pi$ : it allows bundles that might not admit a single compact structure group $\Pi$, but it requires the base spaces to be $G$-CW complexes with countably many cells, requires smooth manifolds as fibers, and does not handle more general group extensions.

REMARK 19.8.2. In outline, our proof of the Adams isomorphism is an alternative implementation of the argument that Hu gave in [78, pp 81-99]. However, in her restricted bundle theoretic context, the argument, although more conceptual, is technically more difficult than the original proof in [98, pp 96-102]. The context supplied by our bundle construction eliminates the need for most of her work.

A general categorical study of isomorphisms between left and right adjoints was given by Fausk, Hu, and May in [62], and a simplified proof of the Wirthmüller isomorphism from that perspective was given in [125]. It is a curious feature of those papers, and the earlier work cited above, that they start with a general natural map from the right adjoint to the left adjoint and then explain when that map is an equivalence. Specializing their categorical context, take $p$ and the spectrum $T_{E}$ as in $\S 19.5$, so that there is an equivalence

$$
\alpha_{p}: p_{!} T_{E} \longrightarrow D_{B}\left(p_{!} S_{E}\right) .
$$

For a $G$-spectrum $X$ over $E$, their definitions specialize to give a canonical candidate map

$$
\omega_{p}: p_{*} X \longrightarrow p_{!}\left(X \wedge_{E} T_{E}\right)
$$

for an equivalence, namely the composite displayed in the commutative diagram

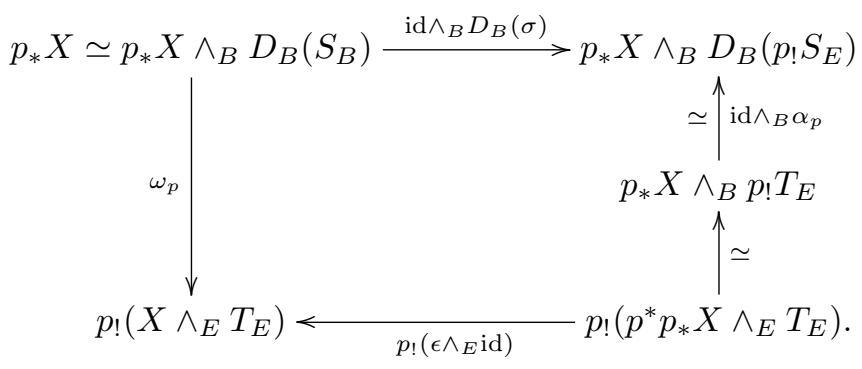

The maps $\sigma: p_{!} S_{E} \simeq p_{!} p^{*} S_{B} \longrightarrow S_{B}$ and $\epsilon: p_{*} p^{*} X \longrightarrow X$ are given by the counits of the adjunctions $\left(p_{!}, p^{*}\right)$ and $\left(p^{*}, p_{*}\right)$. The lower right equivalence is given by the projection formula. We took the same perspective in earlier versions of this book. Note however that the definition of $\omega_{p}$ relies on having the equivalence $\alpha_{p}$, which from our present perspective already depends on knowing that $S_{F}$ is CostenobleWaner dualizable. In contrast, we now start from a canonical duality map from the left adjoint to the right adjoint that always exists, without any further assumptions.

The following comparison is only to be expected. Specialize the definition of $\omega_{p}$ to the case when $p$ is $r: F \longrightarrow *$ for a $G$-space $F$ and write $\omega_{X}$ instead of $\omega_{r}$ to emphasize the dependence on $X$, where $X$ is a $G$-spectrum over $F$. The following result shows that $\omega_{X}$ is a left inverse of the map $\mu_{X}$ of (19.1.1). The analogue for the map $\mu_{X}$ used in Theorem 19.5.2 follows fiberwise. 
Proposition 19.8.4. Let $\left(S_{F}, t T_{F}\right)$ be a dual pair of $G$-spectra over $F$. Then the equivalence

$$
\mu_{X}: X \odot t T_{F} \longrightarrow S_{F} \triangleright X
$$

is right inverse to $\omega_{X}$; that is $\omega_{X} \circ \mu_{X} \simeq i d$.

PRoOF. We begin by reformulating the definition of

$$
\omega_{X}: r_{*}(X) \longrightarrow r_{!}\left(X \wedge_{F} T_{F}\right)
$$

in terms of the bicategory $\mathscr{E} x$. Let $(\eta, \varepsilon)$ be the structure maps of the dual pair $\left(S_{F}, t T_{F}\right)$ and recall that $\varepsilon$ induces a map

$$
\xi_{X}:\left(X \odot t T_{F}\right) \odot S_{F} \stackrel{\text { assoc }}{\longrightarrow} X \odot\left(t T_{F} \odot S_{F}\right) \stackrel{\text { id } \odot \varepsilon}{\longrightarrow} X \odot F \stackrel{\simeq}{\longrightarrow} X
$$

whose adjoint is the equivalence $\mu_{X}$. Let $(\sigma, \zeta)$ be the structure maps of the dual pair $\left(t S_{F}, S_{F}\right)$. We have the composite duality $\left(S_{F} \odot t S_{F}, S_{F} \odot t T_{F}\right)$ of 1-cells $* \longrightarrow *$, and $\eta$ is the dual of $\sigma$; see Proposition 16.5.2. We obtain the following diagram, in which $*$ denotes the unit 1-cell $\Delta_{!} S=S$ of the 0 -cell $*$.

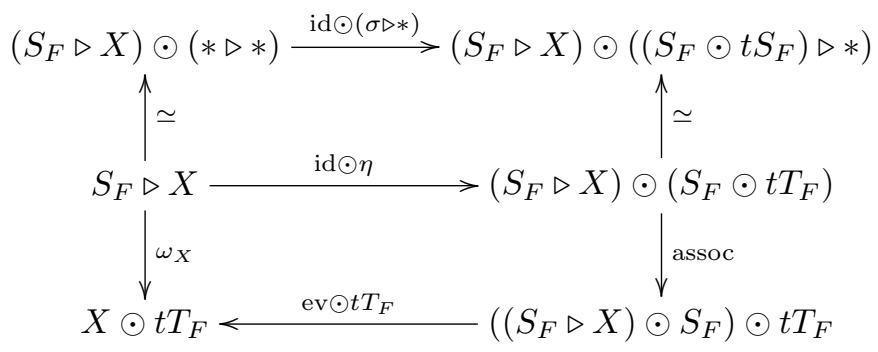

Translating it in terms of the category Ho $G \mathscr{S}_{F}$ and base change functors associated to $r: F \longrightarrow *$, we see that the perimeter is exactly the definition of $\omega$. For example, the associativity isomorphism translates into the projection formula, the spectrum in the lower right hand corner is $r_{!}\left(r^{*} r_{*} X \wedge_{F} T_{F}\right)$, and so on. Note that $\omega_{X}$ is clearly functorial in $X$.

Now consider the following diagram.

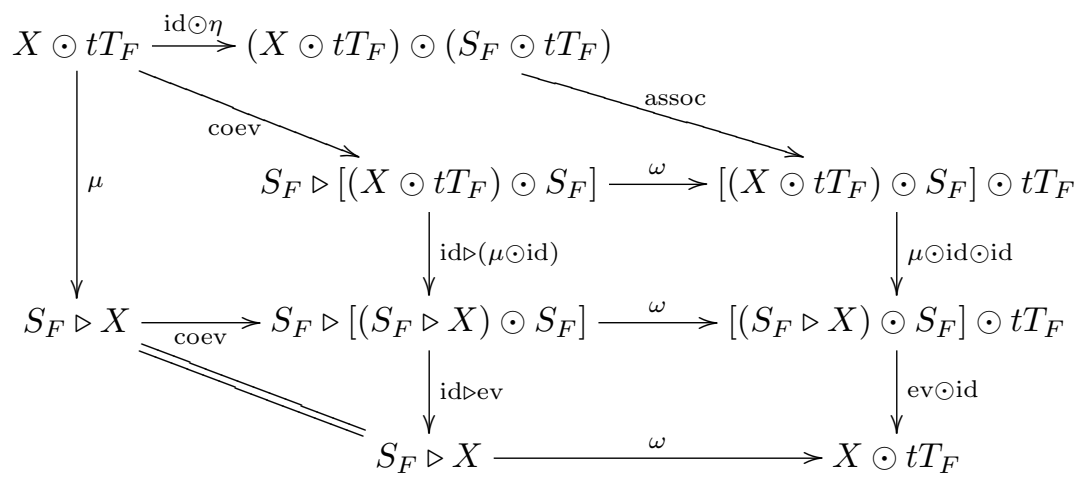


The top face commutes since $\omega_{Z}$ factors as the bottom row in the following diagram, where we write $Z$ for $X \odot T_{F}$.

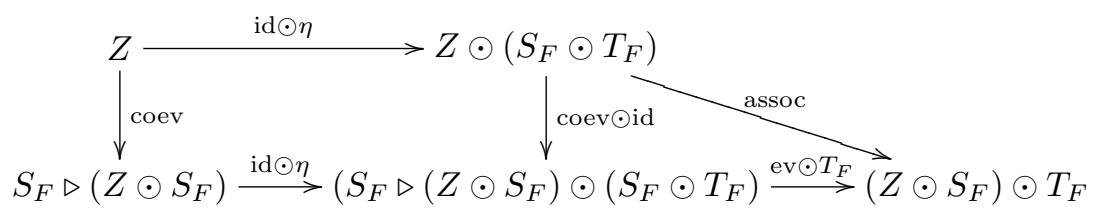

Since $\mu$ is the adjoint of $\xi$, the right vertical composite in our large diagram is $\xi \odot$ id. In view of the definition of $\xi$ and the defining property of the duality $(\eta, \varepsilon)$, the composite from the top left hand corner to the bottom right hand corner, through the top right hand corner, is the identity. Since the composite through the lower left hand corner is $\omega \circ \mu$, this proves that $\omega \circ \mu \simeq$ id. 



\section{Part V}

\section{Homology and cohomology, Thom spectra, and addenda}





\section{Introduction}

Parametrized homology and cohomology theories have a surprisingly scanty history, perhaps due to the lack of rigorous foundations. An early paper of Dold [49] gave an axiomatization. Several later papers, notably [74,153,154] of Hodgkins and Smith, studied the Eilenberg-Moore spectral sequence, viewed as a Künneth spectral sequence for homology on parametrized spaces. The book [42] of Crabb and James ends with some sample applications of such theories.

However, from our point of view, the parametrized theories studied in these references are usually just those obtained by applying classical homology and cohomology theories on based spaces, most often ordinary theories, to the based spaces $r ! X$ derived from ex-spaces $X$. This elementary construction is already very useful, but we are interested in the study of parametrized theories that are represented by general parametrized spectra, not just those that are represented by $r^{*} k$ for a nonparametrized spectrum $k$. As illustrated in Example 0.0.2, the more general theories illuminate even very basic facets of the classical theories.

We introduce such parametrized theories in Chapter 20, describing them in both axiomatic and represented versions. It is usual to give equivalent axiomatizations of classical theories on general spaces and on CW complexes. We will show how to axiomatize parametrized theories on cell complexes, but we do not have a theory of CW complexes that is adequate for this purpose. We will return to this question in Chapter 24. Another twist is that Adams' variant of Brown representability to the effect that a cohomology theory defined only on finite complexes is representable does not always apply, so that the representability of homology theories requires

some new arguments. An interesting new feature, not fully explored here, concerns the relevant notion of coefficients for parametrized theories, which involves the fundamental groupoid of the base space. However, such technicalities and new features aside, we show that the whole panoply of structure familiar from classical algebraic topology is now available in the parametrized context. In particular, we have all of the usual machinery of products and duality.

For readability, we have separated out the equivariant theory for a separate treatment in Chapter 21. One basic motivation for the equivariant parametrized theory is that it gives a context in which to better understand equivariant orientations, Thom isomorphisms, and Poincaré duality. There is no problem for $G$-simply connected manifolds $M[98$, III $\S 6]$, but restriction to such $M$ is clearly inadequate for applications to transformation group theory. Despite a great deal of work on the subject by Costenoble and Waner, and some by May, [37-40,119], this circle of ideas is not yet fully understood. Costenoble and Waner [41] use our work to study this problem for ordinary equivariant theories, but there is much more to be done.

Recently, twisted $K$-theory has entered mathematics through string theory and has been studied heuristically and calculationally in such papers as $[5,6,52$, 
64-66,107,141,164]. There are many points of view that one can take. From our perspective, as we explain in Chapter 22, we see the full force of parametrized theory coming into play. Twisted $K$-theory is an example of a general type of represented parametrized cohomology theory. Starting with a spectrum $k$ and a subgroup $\Pi$ of its automorphism monoid, one constructs a spectrum $k_{\Pi}=E \Pi \times_{\Pi} k$ over $B \Pi$. For a space $X$, a "twisting" is a $\Pi$-bundle classified by a map $p: X \longrightarrow$

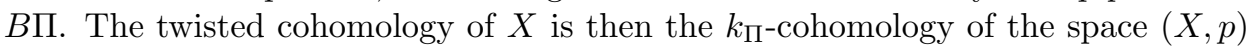

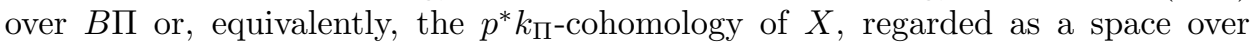
itself. The definition works equally well equivariantly. The bundle construction that we emphasized in Chapter 15 is exactly right for applications like this. We also explain various parametrized spectral sequences, including a Čech type local to global spectral sequence, a twisted Rothenberg-Steenrod spectral sequence, and the parametrized Künneth (or Eilenberg-Moore) spectral sequence.

Chapter 23 gives new perspectives on Thom spectra. It gives a new way of constructing commutative algebras over commutative orthogonal ring spectra via a conceptual axiomatization of exactly how Thom spectra appear "in nature". There is a longstanding notion of a functor with smash product, or FSP. There are several variants of this notion, including one based on orthogonal spaces, which we call an $\mathscr{I}$-FSP. There is an analogous notion of an $\mathscr{I}$-functor with Cartesian products, or $\mathscr{I}$-FCP. We introduce the notion of a parametrized $\mathscr{I}$-functor with smash product, or $\mathscr{I}$-PFSP, over an $\mathscr{I}$-FSP. This is an elementary ex-space level notion, making no use of parametrized spectra. An $\mathscr{I}$-PFSP $E$ over an $\mathscr{I}$-FSP $R$ has a base space $\mathscr{I}$-FCP $B$, and it gives a commutative orthogonal $R$-algebra $r_{!} E$ on collapsing out sections. Starting with a suitable monoid or group-valued $\mathscr{I}$-FCP $\Pi$ that acts from the right on an $\mathscr{I}$-FCP $D$ and from the left on an $\mathscr{I}$-FSP $R$, there is a twosided bar construction $B(D, \Pi, R)$ that gives many examples. In particular, with $D=*$, we construct a "Thom Thom spectrum" $M M U$, which is a commutative $M U$-algebra. The construction iterates to give a Thom spectrum $M^{q} U$, which is an $M^{q-1} U$-algebra. Allowing general $D$ allows examples like $D=G L_{1} Q$, the $\mathscr{I}$-FCP of units of a commutative ring spectrum $Q$. At this writing, we have not yet begun the serious study of these new spectra, but their mere existence is tantalizing.

We also describe a conceptual construction of the Thom spectrum $M f$ associated to a map $f: X \longrightarrow B F$, where $B F$ is the classifying space for stable spherical fibrations. There is a universal spherical fibration spectrum $B(F, S)$ over $B F$. The Thom spectrum of $f$ is just $r_{!} f^{*} B(F, S)$. The construction generalizes. We can replace $B F$ by a classifying space obtained from any base $\mathscr{I}$-FCP $B(D, \Pi)$. This is the starting point for work in progress with Blumberg. As an aside, we give insights into the nature of the unit space $G L_{1}(R)$ of a ring spectrum $R$ in $\S \S 22.2$ and 23.6.

The epilogue, Chapter 24, gives some odds and ends. It discusses the blindness of model category theory to CW theory, as opposed to cell theory, and it describes as much of CW theory as seems to apply to parametrized homotopy theory. It contrasts the diagram spectra utilized in this book to the more rigidly structured spectra utilized in such sources as $[61,98]$. It does not go deeply into such alternative foundations, but it does give a proof that our parametrized stable category is equivalent to a category defined in terms of such structured parametrized spectra. 


\section{CHAPTER 20}

\section{Parametrized homology and cohomology theories}

\section{Introduction}

We set up the foundations for parametrized homology and cohomology theory in this chapter, focusing on the nonequivariant case. We discuss axiomatizations of homology and cohomology theories on ex-spaces in $\S 20.1$, describing four equivalent variants: theories on pairs of spaces over $B$, reduced theories on ex-spaces over $B$, and cellular versions of both. The cellular theory is based on model categorical cellular spaces over $B$, rather than on $\mathrm{CW}$ spaces over $B$ as one would expect from classical homology theory. We defer discussion of this difference to Chapter 24 . We discuss represented theories on ex-spaces and on spectra over $B$ in $\S 20.2$, and we prove there that all parametrized cohomology theories are representable.

We discuss features special to the parametrized world in the rather sketchy $\S 20.3$. There is much more to be said about this subject. We just set out the basic definitions. In particular, we describe the behavior of theories with respect to base change and construct coefficient systems of represented theories as functors from the fundamental groupoid of the base space to graded Abelian groups. We describe parametrized Serre spectral sequences in $\S 20.4$. Other spectral sequences will be discussed in Chapter 22.

We describe the homological implications of Costenoble-Waner and parametrized Atiyah duality in $\S 20.5$, where we flesh out the discusion of Poincaré duality given in Example 0.0.2 and describe the Thom isomorphism. We describe relative Poincaré duality separately in $\S 20.6$. Even in the nonparametrized case, we have not seen our relative Poincaré duality theorem for a closed submanifold of a smooth closed manifold in the literature.

We use the bicategory approach to parametrized theory to explain products in homology and cohomology in $\S 20.7$. Finally, we turn to the representability of parametrized homology theories in $\S 20.8$. The classical method for proving representability fails, and we explain an alternative route to the conclusion.

One point of notation should be mentioned. In Example 0.0.2, we used the notations $k_{*}^{B}$ and $k_{B}^{*}$ for homology and cohomology theories represented by a spectrum $k_{B}$ over $B$. In applications, this is likely to be a useful distinguishing convention. However, it is more logical and nearly consistent with the examples used there to reserve this notational convention for the case when $k_{B}$ is $r^{*} k \cong k \wedge S_{B}$ for a nonparametrized spectrum $k$. The standard generic notation for a homology theory is $k_{*}$ or $E_{*}$. We shall use $k_{*}$, and we shall understand this notation to indicate that a nonparametrized theory represented by a spectrum $k$ is intended. Use of the generic notation $E_{*}$ for a homology theory represented by a (parametrized or nonparametrized) spectrum $E$ would be awkward since it would later conflict with our use of $E$ for total spaces of bundles. We shall instead use the generic notations $J_{*}$ 
and $J^{*}$ for parametrized homology and cohomology theories, thought of as represented by a parametrized spectrum $J$. Thus we omit $B$ from the notations and take the parametrized context for granted. This is most convenient when everything is parametrized, as it is in most of this chapter.

We should also comment on a path that we have chosen not to take. In analogy with grading by representations equivariantly, one might consider grading by vector bundles over $B$ in the parametrized setting. However this is done, it is basically a notational device for incorporating smash products with sphere bundles. We find it best to use such smash products explicitly. An indexing that depends on the base space makes it considerably more awkward to formulate the relationship between homology and base change functors. Moreover, for non-compact base spaces, not all sphere bundles are invertible, and the grading would only be useful upon restriction to the invertible ones.

\subsection{Axioms for parametrized homology and cohomology theories}

Consider the category $\mathscr{K}^{2} / B$ of pairs $(X, Y)$ of spaces over $B$. We describe homology and cohomology theories on this category axiomatically. The details work out exactly as in the classical case when $B$ is a point, and we follow the elementary textbook treatment of [121]. A map $f:(X, Y) \longrightarrow\left(X^{\prime}, Y^{\prime}\right)$ of pairs is a weak equivalence if both $f: X \longrightarrow X^{\prime}$ and $f: Y \longrightarrow Y^{\prime}$ are weak equivalences. A triad $(X ; Y, Z)$ of spaces over $B$ is excisive if it is excisive on total spaces, so that $X$ is the union of the interiors of $Y$ and $Z$.

Definition 20.1.1. A homology theory $J_{*}$ on $\mathscr{K}^{2} / B$ consists of functors $J_{q}$ from $\mathscr{K}^{2} / B$ to the category of Abelian groups (or any other Abelian category) together with natural transformations $\partial: J_{q}(X, Y) \longrightarrow J_{q-1}(Y)$, where $J_{q}(X)$ is defined to be $J_{q}(X, \emptyset)$. These functors and natural transformations must satisfy the following axioms.

- ExACtness. The following sequence is exact, where the arrows are $\partial$ and maps induced by the inclusions $Y \longrightarrow X$ and $(X, \emptyset) \longrightarrow(X, Y)$ :

$$
\cdots \longrightarrow J_{q}(Y) \longrightarrow J_{q}(X) \longrightarrow J_{q}(X, Y) \longrightarrow J_{q-1}(Y) \longrightarrow \cdots .
$$

- Excision. If $(X ; Y, Z)$ is excisive, then the inclusion $(Y, Y \cap Z) \longrightarrow(X, Z)$ induces an isomorphism

$$
J_{*}(Y, Y \cap Z) \longrightarrow J_{*}(X, Z) .
$$

- Additivity. If $(X, Y)$ is the disjoint union of a set of pairs $\left(X_{i}, Y_{i}\right)$, then the inclusions $\left(X_{i}, Y_{i}\right) \longrightarrow(X, Y)$ induce an isomorphism

$$
\bigoplus_{i} J_{*}\left(X_{i}, Y_{i}\right) \longrightarrow J_{*}(X, Y) \text {. }
$$

- WeAK Equivalence. If $f:(X, Y) \longrightarrow\left(X^{\prime}, Y^{\prime}\right)$ is a weak equivalence, then

$$
f_{*}: J_{*}(X, Y) \longrightarrow J_{*}\left(X^{\prime}, Y^{\prime}\right)
$$

is an isomorphism.

Cohomology theories $J^{*}$ are defined via the dual axioms.

The weak equivalence axiom implies that the theory is defined on the homotopy category Ho $\mathscr{K}^{2} / B$ obtained by inverting the weak equivalences. We say that a theory is "ordinary" if it satisfies the dimension axiom $J_{q}(B)=0$ for $q \neq 0$, where $B$ is regarded as a space over itself via the identity map. (Recall that we write $*_{B}$ 
for the sectioned analogue, but we are working in $\mathscr{K} / B$ here). Such theories exist, but are not particularly interesting; see Remark 20.3.7.

Similarly, we have reduced homology theories defined on ex-spaces over $B$, which are the analogues of reduced homology theories on based spaces.

Definition 20.1.2. A reduced homology theory $\tilde{J}_{*}$ consists of functors $\tilde{J}_{q}$ from the category of well-grounded ex-spaces over $B$ to the category of Abelian groups together with natural suspension isomorphisms

$$
\sigma: \tilde{J}_{q}(X) \cong \tilde{J}_{q+1}\left(\Sigma_{B} X\right)
$$

that satisfy the following axioms.

- Exactness. If $i: Y \longrightarrow X$ is an $f p$-cofibration, then the sequence

$$
\tilde{J}_{q}(Y) \longrightarrow \tilde{J}_{q}(X) \longrightarrow \tilde{J}_{q}\left(X /{ }_{B} Y\right)
$$

is exact.

- Additivity. If $X$ is the wedge over $B$ of a set of well-grounded ex-spaces $X_{i}$, then the inclusions $X_{i} \longrightarrow X$ induce an isomorphism

$$
\bigoplus_{i} \tilde{J}_{*}\left(X_{i}\right) \longrightarrow \tilde{J}_{*}(X)
$$

- WEAK Equivalence. If $f: X \longrightarrow X^{\prime}$ is a weak equivalence, then

$$
f_{*}: \tilde{J}_{*}(X) \longrightarrow \tilde{J}_{*}\left(X^{\prime}\right)
$$

is an isomorphism.

Reduced cohomology theories $\tilde{J}^{*}$ are defined via the dual axioms.

As in the classical case, the exactness, additivity, and weak equivalence axioms work one degree at a time and do not require mention of the suspension isomorphism. A reduced theory is ordinary if $\tilde{J}_{q}\left(S_{B}^{0}\right)=0$ for $q \neq 0$.

Using the foundations of $\$ 5.6$, the long exact sequences associated to cofiber sequences, the Mayer-Vietoris theorem, the commutation of homology with directed colimits, the $\lim ^{1}$ exact sequence in cohomology, and other standard properties can be deduced from the axioms exactly as in the classical case [121, Chapters 14 and 19]. Their statements are essentially identical to those given there.

It is convenient to have an alternative axiomatization on cellular pairs, by which we mean a $q f$-cell complex $X$ and a subcomplex $Y$. We let $\mathscr{C}^{2} / B$ denote the category of such pairs and all continuous maps between them and let $\mathrm{Ho}_{\mathscr{C}}{ }^{2} / B$ denote its homotopy category. We insist on using the $q f$-model structure and not the $q$-model structure, especially when considering reduced theories. As we have seen in Counterexample 6.1.5, $q$-cell complexes over $B$ need not be well-grounded. We restricted to well-grounded ex-spaces when defining reduced homology theories for the same compelling reasons that dictate restriction to well-based spaces in defining reduced homology theories on based spaces; compare Lemmas 5.6.5 and 5.6 .7 .

Definition 20.1.3. A homology theory $J_{*}$ on $\mathscr{C}^{2} / B$ consists of functors $J_{q}$ from $\mathrm{Ho}_{\mathscr{C}} \mathscr{C}^{2} B$ to the category of Abelian groups together with natural transformations $\partial: J_{q}(X, Y) \longrightarrow J_{q-1}(Y)$, where $J_{q}(X)=J_{q}(X, \emptyset)$. These functors and natural transformations must satisfy the exactness and additivity axioms as in Definition 20.1.1 and the following version of the excision axiom. 
- Excision. If $(X ; Y, Z)$ is a cellular triad, in the sense that $X$ is the union of subcomplexes $Y$ and $Z$, then the inclusion $(Y, Y \cap Z) \longrightarrow(X, Z)$ induces an isomorphism

$$
J_{*}(Y, Y \cap Z) \longrightarrow J_{*}(X, Z) .
$$

Similarly, let $\mathscr{C}_{B}$ denote the category of $q f$-cell complexes in $\mathscr{K}_{B}$ and let Ho $\mathscr{C}_{B}$ denote its homotopy category.

Definition 20.1.4. A reduced homology theory $\tilde{J}_{*}$ on $\mathscr{C}_{B}$ consists of functors $\tilde{J}_{q}$ from $\mathrm{Ho}_{B}$ to the category of Abelian groups together with natural suspension isomorphisms

$$
\sigma: \tilde{J}_{q}(X) \cong \tilde{J}_{q+1}\left(\Sigma_{B} X\right)
$$

that satisfy the exactness axiom for the inclusion of a subcomplex and the additivity axiom as stated in Definition 20.1.2.

Again, cohomology theories are defined dually.

THEOREM 20.1.5. The four notions of homology theories defined above are equivalent, in the sense that a theory of any one of the four types canonically determines and is determined by a theory of each of the other three types. The analogous statement holds for cohomology.

Proof. Given either type of absolute theory $J_{*}$, we define the corresponding reduced theory by $\tilde{J}_{*}(X)=J_{*}(X, B)$, where we regard the section as an inclusion $B \longrightarrow X$ in $\mathscr{K} / B$. Given a reduced theory $\tilde{J}_{*}$, we define the corresponding unreduced theory by letting $J_{*}(X)=\tilde{J}_{*}\left(X_{+}\right)$and $J_{*}(X, Y)=\tilde{J}_{*}\left(C_{B}\left(i_{+}\right)\right)$. Here the plus denotes addition of a disjoint section $B ; i: Y \longrightarrow X$ is the inclusion, and $C_{B}$ is the cofiber functor on maps. If $i$ is an $f$-cofibration, for example if $(X, Y)$ is a cellular pair, then $\tilde{J}\left(C_{B}\left(i_{+}\right)\right) \cong \tilde{J}\left(X_{+} /{ }_{B} Y_{+}\right)$. (When $B=*$, the quotient here is just $X / Y$ with basepoint the image of $Y$, but that makes no sense in the parametrized context). If $J_{*}$ or $\tilde{J}_{*}$ is a theory on general pairs or general ex-spaces, then restriction to cellular pairs or cellular ex-spaces gives the corresponding cellular theory. For a theory $J_{*}$ or $\tilde{J}_{*}$ on cellular pairs or cellular ex-spaces, we construct the corresponding theory on general pairs or general ex-spaces by using $q f$-cofibrant approximations given by the small object argument. This proceeds in three stages. We approximate spaces or ex-spaces over $B$ as usual. For pairs $(X, Y)$ of spaces over $B$, we first approximate $Y$, obtaining $Q Y \longrightarrow Y$, and then approximate $X$ by factoring $Q Y \longrightarrow Y \longrightarrow X$ though a $q f$-cofibration $Q Y \longrightarrow Q X$ and an acyclic $q f$-fibration $Q X \longrightarrow X$. For excisive triads $(X ; Y, Z)$, we approximate the pairs $(Y, Y \cap Z)$ and $(Z, Y \cap Z)$ in this fashion and then define $Q X=Q Y \cup_{Q(Y \cap Z)} Q Z$. This gives a cellular triad $(Q X ; Q Y, Q Z)$ and a map of triads from it to the excisive triad $(X ; Y, Z)$. The essential point is that $Q X \longrightarrow X$ is a weak equivalence, and this follows from the nonparametrized analogue proven in $[121, \S 10.7]$. In the cellular version of the passage from a reduced homology theory to a homology theory on pairs, excision is trivial since $Y_{+} /_{B}(Y \cap Z)_{+} \cong X_{+} /{ }_{B} Z_{+}$for a cellular triad $(X ; Y, Z)$. As in $[121, \S 14.4]$, in the non-cellular case we can deduce excision when passing from reduced to unreduced homology theories by an indirect argument. Indeed, unreduced general and unreduced cellular theories are equivalent, reduced general and reduced cellular theories are equivalent, and reduced and unreduced cellular theories are equivalent. Therefore, reduced and unreduced general theories 
are equivalent. Thus excision in the general context is derived from the cellular approximation of triads.

REMARK 20.1.6. In nonparametrized theory, one uses CW complexes rather than cell complexes in the axiomatizations. In the parametrized context, the foundational theory of CW complexes does not extend so far, for reasons explained in Chapter 24, notably the failure of the cellular approximation theorem in general. However, there are distinctions to be made here between the reduced and unreduced contexts. First, in the unreduced context we could just as well have used the $q$-cell complexes rather than $q f$-cell complexes since the $q f$-model structure was introduced solely to circumvent problems arising from the presence of sections. Second, and more interesting, if we are given a space $X$ over $B$, we can compose with $Q X \longrightarrow X$, where $Q X$ is a $\mathrm{CW}$ approximation to $X$, to obtain a $\mathrm{CW}$-approximation over $B$. Thus there is no loss of calculational generality if we restrict attention to $\mathrm{CW}$ complexes over $B$. Trivially, the skeletal filtration is given by spaces over $B$. In analogy with nonparametrized theory, it would seem that this should lead to a parametrized Atiyah-Hirzebruch spectral sequence. However, since the inclusions of skeleta are not fiberwise cofibrations and since the appropriate form of an $E_{2}$-term is unclear, we have not pursued this idea.

\subsection{Represented homology and cohomology theories}

From now on in this chapter, we focus on reduced homology and cohomology theories on ex-spaces, and we follow standard modern practice by omitting the tilde from the notation. We also generalize from ex-spaces to spectra over $B$.

There is an obvious first example of homology and cohomology theories, and we use it to guide us towards the right general notions of represented theories. Let $k_{*}$ and $k^{*}$ be (reduced) homology and cohomology theories on well-based spaces. Recall that the functor $r_{!}: \mathscr{K}_{B} \longrightarrow \mathscr{K}_{*}$ associated to the trivial map $r: B \longrightarrow *$ is given by $r ! X=X / B$. Since this functor is a left adjoint that preserves tensors with based spaces, suspension, and cofiber sequences, we see immediately that if we define

$$
k_{*}^{B}(X)=k_{*}\left(r_{!} X\right) \quad \text { and } \quad k_{B}^{*}(X)=k^{*}\left(r_{!} X\right),
$$

then these are (reduced) homology and cohomology theories on $\mathscr{K}_{B}$. We call these the parametrized theories induced by the theories $k_{*}$ and $k^{*}$ and refer to them as classical theories.

Now suppose that $k_{*}$ and $k^{*}$ are represented by an $\Omega$-prespectrum $k$. Then

$$
k_{*}^{B}(X)=\pi_{*}\left(k \wedge r_{!} X\right) \quad \text { and } \quad k_{B}^{*}(X)=\pi_{-*} F\left(r_{!} X, k\right) .
$$

We have the $\Omega$-prespectrum $r^{*} k$ over $B$, and we have the relations

$$
k \wedge r_{!} X \simeq r_{!}\left(r^{*} k \wedge_{B} X\right) \quad \text { and } \quad F\left(r_{!} X, k\right) \simeq r_{*} F_{B}\left(X, r^{*} k\right) .
$$

Here and later, we are working in derived homotopy categories and using the notation $\simeq$ for natural equivalences there. The displayed equivalences are special cases of the derived versions of (2.2.6) and (2.2.7) proven in Theorem 9.4.5. This suggests the following general definition.

Definition 20.2.4. Let $J$ and $X$ be spectra over $B$. For integers $n$, define the $n$-th $J$-homology and $J$-cohomology groups of $X$ by

$$
J_{n}(X)=\pi_{n}\left(r_{!}\left(J \wedge_{B} X\right)\right)
$$


and

$$
J^{n}(X)=\pi_{-n}\left(r_{*} F_{B}(X, J)\right) \cong\left[S_{B}^{-n}, F_{B}(X, J)\right]_{B} .
$$

The last isomorphism comes from the $\left(r^{*}, r_{*}\right)$ adjunction and the isomorphisms $r^{*} S^{n} \cong S_{B}^{n}$. It implies that $J^{0}(X)$ is just the represented functor $[X, J]_{B}$ of $X$. The same definitions specify the homology and cohomology groups of an ex-space $K$, and these are given equivalently by taking $X=\Sigma_{B}^{\infty} K$ in the definition above. These groups give homology and cohomology theories in $K$, as axiomatized in Definition 20.1.2. This innocuous statement depends on the full strength of our foundational work on the properties of our derived homotopy categories and the functors relating them. The exactness and wedge axioms go all the way back to the cofiber sequence and wedge lemmas, Lemmas 5.6.5 and 5.6.7. Those results motivate the requirement that ex-spaces be well-grounded in our definition of reduced homology and cohomology theories, and it is essential to the deduction of the axioms that our $s$-model structure is well-grounded. Note too that it is essential that we can work freely with the functors $\wedge_{B}$ and $F_{B}$, even though they are not related by Quillen adjunctions.

On ex-spaces $X$, homology and cohomology theories are determined by their values for non-negative $n$, since we can take

$$
J_{-n}(X) \cong J_{0}\left(\Sigma_{B}^{n} X\right) \text { and } J^{-n}(X) \cong J^{0}\left(\Sigma_{B}^{n} X\right)
$$

as definitions. On spectra $X$ over $B$, this still makes sense for negative $n$. Thus homology and cohomology theories on spectra over $B$ are determined by their values for $n=0$, where the appropriate axiomatization just requires an exact and additive contravariant homotopy functor. As usual, we can apply the Brown representability theorem in Ho $\mathscr{K}_{B}$ and Ho $\mathscr{S}_{B}$ to prove the following result.

TheOREM 20.2.5. A reduced cohomology theory on ex-spaces over $B$ is represented by an $\Omega$-prespectrum $J$, unique up to non-unique equivalence. A cohomology theory on spectra over $B$ is represented uniquely by an orthogonal spectrum over $B$ in $\operatorname{HoS}_{B}$.

The non-uniqueness works just as it does classically. The morphisms of $\Omega$ prespectra that correspond to morphisms of cohomology theories on ex-spaces are sequences of maps $J_{i} \longrightarrow J_{i}^{\prime}$ that are only compatible up to homotopy. There is a $\lim ^{1}$ exact sequence

$$
0 \longrightarrow \lim ^{1}\left[\Sigma_{B} J_{i}, J_{i}^{\prime}\right]_{B} \longrightarrow\left[J, J^{\prime}\right]_{B} \longrightarrow \lim \left[J_{i}, J_{i}^{\prime}\right]_{B} \longrightarrow 0
$$

that relates such "weak maps" of $\Omega$-prespectra to maps in the stable category and thus relates maps of cohomology theories on ex-spaces over $B$ to maps of cohomology theories on spectra over $B$.

The representability of homology is less obvious, and we shall return to this in $\S 20.8$. Represented parametrized theories behave as follows with respect to base change.

Proposition 20.2.6. Let $f: A \longrightarrow B$ be a map.

(i) For $J \in \mathscr{S}_{A}$ and $X \in \mathscr{S}_{B}$,

$$
\left(f_{!} J\right)_{n}(X) \cong J_{n}\left(f^{*} X\right) \quad \text { and } \quad\left(f_{*} J\right)^{n}(X) \cong J^{n}\left(f^{*} X\right) .
$$

(ii) For $X \in \mathscr{S}_{A}$ and $J \in \mathscr{S}_{B}$,

$$
\left(f^{*} J\right)_{n}(X) \cong J_{n}\left(f_{!} X\right) \quad \text { and } \quad\left(f^{*} J\right)^{n}(X) \cong J^{n}\left(f_{!} X\right) .
$$


Proof. These are immediate from the fact that $r_{B} \circ f=r_{A}$ and the derived versions of (11.4.5) and (11.4.6), which imply that all four isomorphisms are obtained by passage to homotopy groups from natural equivalences of spectra.

In particular, as our motivation dictates, we have the following special case, which shows that we recover the theories of (20.2.2) as represented theories.

Corollary 20.2.7. Let $k$ be a spectrum and $X$ be a spectrum over $B$. Then

$$
\left(r^{*} k\right)_{*}(X) \cong k_{*}\left(r_{!} X\right) \equiv k_{*}^{B}(X) \quad \text { and } \quad\left(r^{*} k\right)^{*}(X) \cong k^{*}\left(r_{!} X\right) \equiv k_{B}^{*}(X) \text {. }
$$

\subsection{Coefficient systems and restriction maps}

Another special case relates parametrized theories to classical theories fiberwise.

Corollary 20.3.1. Let $b: * \longrightarrow B$ be the inclusion of a point of $B$.

(i) For $k \in \mathscr{S}$ and $X \in \mathscr{S}_{B}$,

$$
\left(b_{!} k\right)_{n}(X) \cong k_{n}\left(X_{b}\right) \quad \text { and } \quad\left(b_{*} k\right)^{n}(X) \cong k^{n}\left(X_{b}\right) .
$$

(ii) For $X \in \mathscr{S}$ and $J \in \mathscr{S}_{B}$,

$$
\left(J_{b}\right)_{n}(X) \cong J_{n}\left(b_{!} X\right) \quad \text { and } \quad\left(J_{b}\right)^{n}(X) \cong J^{n}\left(b_{!} X\right) .
$$

In particular,

$$
J_{n}\left(b_{!} S\right) \cong \pi_{n}\left(J_{b}\right) \cong J^{-n}\left(b_{!} S\right)
$$

The homotopy groups $\pi_{*}\left(J_{b}\right)$ as $b$ varies should be viewed as a parametrized system of coefficient groups for the homology and cohomology theories represented by $J$. When $J=r^{*} k,\left(r^{*} k\right)_{b}=k$ and these groups are all just $\pi_{*}(k)$. In general, these coefficient groups are coherently twisted as we move around the base space, the coherence being given in terms of the fundamental groupoid $\Pi B$. We use the following geometric precursor of coefficient systems to prove this. It is convenient to write $b^{*} X$ rather than $X_{b}$ for the derived fiber of $X$ at $b$.

Proposition 20.3.2. For ex-spaces $X$ over $B$, there is a (covariant) functor $C(X)$ from $\Pi B$ to the homotopy category Ho $\mathscr{K}_{B} / X$ of ex-spaces over $B$ with augmentations to $X$ that sends $b \in B$ to $b_{!} b^{*} X$ with augmentation given by the counit $b_{!} b^{*} X \longrightarrow X$ of the $\left(b_{!}, b^{*}\right)$ adjunction. The corresponding result for spectra $J$ over $B$ also holds, and $C\left(\Sigma_{B}^{\infty} X\right)$ is naturally isomorphic to $\Sigma_{B}^{\infty} C(X)$.

Proof. For a path $h: I \longrightarrow B$ from $b$ to $b^{\prime}$, counits of the evident $q$-equivaleneces give weak equivalences

$$
i_{0 !} i_{0}{ }^{*} h^{*} X \longrightarrow h^{*} X \longleftarrow i_{1 !} i_{1}{ }^{*} h^{*} X
$$

of ex-spaces over $I$. Apply $h_{\text {! }}$ and observe that the factorizations $h i_{0}=b$ and $h i_{1}=b^{\prime}$ give corresponding factorizations of functors $(-)$ ! and $(-)^{*}$. We obtain weak equivalences

$$
b_{!} b^{*} X \longrightarrow h_{!} h^{*} X \longleftarrow b_{!}^{\prime} b^{\prime *} X
$$

of ex-spaces over $B$ augmented over $X$. If we have another path $h^{\prime}: b \longrightarrow b^{\prime}$ and a homotopy $k$ between them, we may view $k$ as defined on a disk $D$ with two base points connected by two boundary curves $h_{0}: I \longrightarrow D$ and $h_{1}: I \longrightarrow D$. We construct an analogous diagram of weak equivalences

$$
h_{!} h^{*} X \longrightarrow k_{!} k^{*} X \longleftarrow h_{!}^{\prime} h^{\prime *} X
$$


and a diagram chase shows that the weak equivalences between $b_{!} b^{*} X$ and $b_{!}^{\prime} b^{\prime *} X$ obtained from $h$ and $h^{\prime}$ are equal in the homotopy category. The compatibility with $\Sigma_{B}^{\infty}$ is easily checked.

Note that, by composition, a map $f: X \longrightarrow Y$ of ex-spaces over $B$ induces a functor Ho $\mathscr{K}_{B} / X \longrightarrow$ Ho $\mathscr{K}_{B} / Y$, hence a natural transformation $C(X) \longrightarrow C(Y)$. We have proven the previous result in a version convenient for equivariant generalization, where orbits replace points, but here in the nonequivariant world the following corollary may seem more natural.

Corollary 20.3.3. For ex-spaces $X$ over $B$, there is a canonical functor $\Pi B \longrightarrow$ Ho $\mathscr{K}$ that sends $b$ to $X_{b}$, and similarly for spectra over $B$.

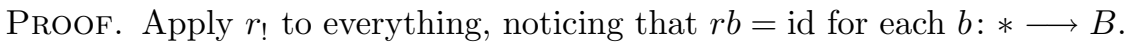

Definition 20.3.4. Let $J$ and $X$ be parametrized spectra over $B$.

(i) The coefficient system of $J$, denoted $\mathscr{L}_{*}(J)$, is the composite functor $\pi_{*} r_{!} C J$ from $\Pi B$ to graded Abelian groups that sends $b$ to $\pi_{*}\left(J_{b}\right)$.

(ii) The coefficient system of $J_{*}(X)$, denoted $\mathscr{L}_{*}(X, J)$, is the composite functor $\pi_{*} r_{!} C\left(J \wedge_{B} X\right)$ from $\Pi B$ to graded Abelian groups that sends $b$ to $J_{b *}\left(X_{b}\right)$.

(iii) The coefficient system of $J^{*}(X)$, denoted $\mathscr{L}^{*}(X, J)$, is the composite functor $\pi_{-*} r_{!} C\left(F_{B}(X, J)\right)$ from $\Pi B$ to graded Abelian groups that sends $b$ to $J_{b}^{*}\left(X_{b}\right)$.

In (i), the groups involved are just the homotopy groups of derived fibers in terms of which weak equivalences in the stable category were originally defined, but now their coherence as $b$ varies is relevant. There is another way of thinking about "coefficients". Since $S=b^{*} S_{B}$, the counit maps $b_{!} b^{*} S_{B} \longrightarrow S_{B}$ induce maps

$$
J_{*}\left(b_{!} S\right) \longrightarrow J_{*}\left(S_{B}\right) \text { and } J^{*}\left(S_{B}\right) \longrightarrow J^{*}\left(b_{!} S\right) .
$$

REMARK 20.3.6. By analogy with classical theories, the groups $J_{*}\left(S_{B}\right)$ and $J^{*}\left(S_{B}\right)$ should be called coefficient groups. Here we encounter a surprise: these groups are different. In fact,

$$
J_{*}\left(S_{B}\right)=\pi_{*}\left(r_{!} J\right) \quad \text { and } \quad J^{*}\left(S_{B}\right)=\pi_{*}\left(r_{*} J\right) .
$$

From this point of view, the map $r_{*} J \longrightarrow r_{*} b_{*} b^{*} J \cong J_{b}$ induced by the unit map id $\longrightarrow b_{*} b^{*}$ also gives rise to a natural map $J^{*}\left(S_{B}\right) \longrightarrow J^{*}\left(b_{!} S\right)$, but this agrees with the second map of (20.3.5) by Remark 2.2.9. The existence of both fiberwise coefficient systems and global coefficient groups is related to the fact that the duality of $\$ 15.1$ is not the right duality for the comparison of homology and cohomology theories.

REMARK 20.3.7. For a spectrum $k$ and a point $b \in B$, Corollary 20.3.1(i) gives

$$
\left(b_{!} k\right)_{*}\left(S_{B}\right)=k_{*} \text { and }\left(b_{*} k\right)^{*}\left(S_{B}\right)=k^{*} .
$$

In particular, with $k=H \pi$ for an Abelian group $\pi$, the parametrized homology and cohomology theories $\left(b_{!} k\right)_{*}$ and $\left(b_{*} k\right)^{*}$ are ordinary.

\subsection{The Serre spectral sequence}

Starting from the skeletal filtration of a CW base space, we give a parametrized interpretation of the Serre spectral sequence. The parametrized setting leads to a description of the $E_{2}$-terms in terms of the local systems that are specified in Definition 20.3.4. Remember that our homology and cohomology theories are understood 
to be reduced. We set up the desired spectral sequences without assuming that our theories are representable. This will allow us to use them in $\S 20.8$ to prove the representability of homology, which in turn allows us to assume that our theories are representable when identifying the $E_{2}$ terms. Therefore the hypotheses on $B$, $X$, and $J$ in the following result can always be arranged without loss of generality.

Theorem 20.4.1 (Serre spectral sequence). Let $B$ be a $C W$ complex with $p$ skeleton $B^{p}$ and let $X$ be an excellent spectrum over $B$. Let $j_{p}: B^{p} \longrightarrow B$ be the inclusion, let $X^{p}=j_{p !} j_{p}^{*} X$, and let $i_{p}: X^{p} \longrightarrow X^{p+1}$ be the induced inclusion of spectra over $B$. Let $J_{*}$ and $J^{*}$ be parametrized homology and cohomology theories over $B$.

(i) There is a strongly convergent spectral sequence

$$
E_{p, q}^{1}=\bigoplus_{p \text {-cells } e} J_{p+q}\left(e_{!} e^{*} X^{p}, \partial e_{!} \partial e^{*} X^{p-1}\right) \Longrightarrow J_{p+q}(X) .
$$

(ii) There is a conditionally convergent spectral sequence

$$
E_{1}^{p, q}=\prod_{p-\text { cells } e} J^{p+q}\left(e_{!} e^{*} X^{p}, \partial e_{!} \partial e^{*} X^{p-1}\right) \Longrightarrow J^{p+q}(X) .
$$

The sequence converges strongly if the derived $E_{\infty}$ terms $R E_{\infty}$ vanish.

If the theories are represented by an excellent spectrum $J$ over $B$, then

$$
E_{p, q}^{2}=H_{p}\left(B ; \mathscr{L}_{q}(X, J)\right) \quad \text { and } \quad E_{2}^{p, q}=H^{p}\left(B ; \mathscr{L}^{q}(X, J)\right) .
$$

Proof. The convergence statements are explained in Boardman's study [13, (5.1), 6.1, 7.1, and §13], and their proofs are direct application of his results. We shall say no more about them.

The spectral sequences are constructed from the exact couple associated to the long exact homology and cohomology sequences of the pairs $\left(X^{p}, X^{p-1}\right)$. Note that, since $X$ is excellent, it is levelwise ex-fibrant, and the same is true for all pullbacks of $X$. The inclusions $i_{p}$ are level $h$-cofibrations by Proposition 4.4.5, and it follows that $X$ is weakly equivalent to the telescope of the $X^{n}$. The $E_{1}$-terms are $E_{p, q}^{1}=J_{p+q}\left(X^{p}, X^{p-1}\right)$ and $E_{1}^{p, q}=J^{p+q}\left(X^{p}, X^{p-1}\right)$. To identify them, we must calculate the cofiber $C_{B}\left(i_{p-1}\right)$. The formal background is given by Theorem 12.4.2 and its proof. Consider the following diagram in $\mathrm{Ho}_{B_{B^{p}}}$, where we are implicitly extending the section of parametrized spectra on subspaces of $B^{p}$ to all of $B^{p}$ by pushforward along inclusions.

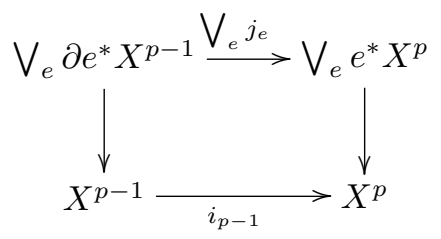

The cell boundary inclusions $j_{e}: \partial e^{*} X^{p} \longrightarrow e^{*} X^{p}$ are level $h$-cofibrations, by Proposition 4.4.5 again, and it follows that the square is a homotopy pushout.

However, the horizontal arrows will in general not be level $f$-cofibrations, and it is not obvious that their fiberwise cofibers are weakly equivalent. To see that they are, we look more closely at the comparison map of fiberwise cofibers, which 
is constructed as the induced map of pushouts from the front face to the back face in the following cube; again, everything is pushed forward to spectra over $B$

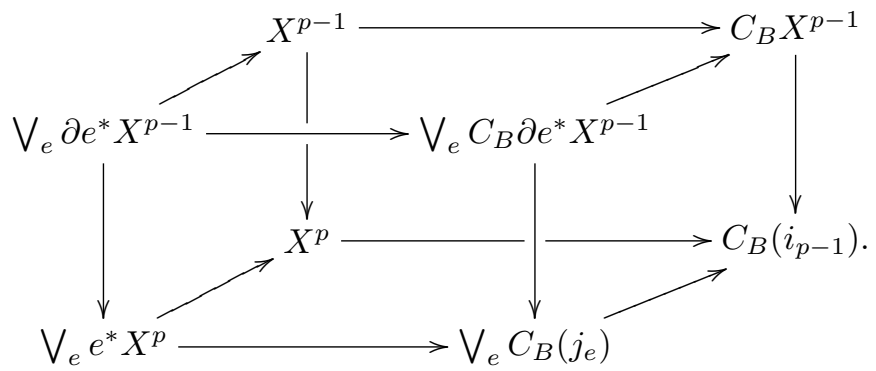

Since the front and back face are pushouts, it follows that the right side, like the left side, is a homotopy pushout. Looking at the right side, the map on top is a weak equivalence since it is a map between fiberwise contractible spectra over $B^{p}$, and the left map is a level $h$-cofibration. It follows that the bottom map is also a weak equivalence.

To identify the $E_{2}$-term, recall the definitions of the coefficient systems $\mathscr{L}_{*}(X, J)$ and $\mathscr{L}^{*}(X, J)$ from Definition 20.3.4. Since the proofs of the homology and cohomology statements are nearly identical, we only treat cohomology. We continue to work in the category of excellent prespectra over $B$. Thinking of $S^{p-1}$ and $D^{p}$ as the equator and upper hemisphere of $S^{p}$, a choice of basepoint in $S^{0}$ determines a basepoint in all spheres and disks, and we let $b(e)=e(*) \in B$ for each cell $e$. The inclusion of the full subcategory of $\Pi B$ with objects these basepoints of cells is an equivalence, and we can therefore restrict attention to these points of $\Pi B$ when defining and computing local cohomology. Consider $r: D^{p} \longrightarrow *$. Then Proposition 20.2.6(ii) gives the first and third of the following isomorphisms, while the second comes from the fact that the inclusion of the basepoint in $D^{p}$ is an inverse equivalence to $r$ that gives $b(e)$ when composed with $e$.

$$
\begin{aligned}
J^{p+q}\left(e_{!} e^{*} X^{p}, \partial e_{!} \partial e^{*} X^{p-1}\right) & \cong\left(e^{*} J\right)^{p+q}\left(e^{*} X^{p}, \partial e^{*} X^{p-1}\right) \\
& \cong\left(r^{*} J_{b(e)}\right)^{p+q}\left(e^{*} X^{p}, \partial e^{*} X^{p-1}\right) \\
& \cong J_{b(e)}^{p+q}\left(r_{!} e^{*} X^{p}, r ! \partial e^{*} X^{p-1}\right)
\end{aligned}
$$

Since $e\left(D^{p}\right)$ is contractible,

$$
r ! e^{*} X^{p} \simeq r ! r^{*} b(e)^{*} e^{*} X^{p} \simeq r ! r^{*} X_{b(e)} \simeq D_{+}^{p} \wedge X_{b(e)},
$$

and similarly $r_{!} \partial e^{*} X^{p-1} \simeq S_{+}^{p-1} \wedge X_{b(e)}$. The cofiber of the inclusion of the latter into the former is then $S^{p} \wedge X_{b(e)}$ and we have

$$
J_{b(e)}^{p+q}\left(r_{!} e^{*} X^{p}, r_{!} \partial e^{*} X^{p-1}\right) \cong J_{b(e)}^{p+q}\left(S^{p} \wedge X_{b(e)}\right) \cong J_{b(e)}^{q}\left(X_{b(e)}\right) .
$$

This recalculates the $E_{1}$ term as the relevant cellular chain group

$$
E_{1}^{p, q}=\prod_{p \text {-cells } e} J_{b(e)}^{q}\left(X_{b(e)}\right) \cong C_{\text {cell }}^{p}\left(B ; \mathscr{L}^{q}(X, J)\right) .
$$

Standard but careful arguments, paying close attention to signs, identify the canonical differentials on either side. Passing to cohomology, we obtain the desired identification of the $E_{2}$ term. 
Remark 20.4.2. The case $X=S_{B}$ should be regarded as an Atiyah-Hirzebruch type spectral sequence for the computation of the coefficients of parametrized homology and cohomology theories. By Lemma 18.2.7 and Proposition 20.2.6(ii), we can use this version of the Atiyah-Hirzebruch spectral sequence more generally than it appears at first. If $(K, p)$ is a space over $B$, where $K$ is a CW-complex, we obtain spectral sequences that converge from

$$
E_{p, q}^{2}=H_{p}\left(K ; \mathscr{L}_{q}\left(S_{K}, p^{*} J\right)\right) \quad \text { and } \quad E_{2}^{p, q}=H^{p}\left(K ; \mathscr{L}^{q}\left(S_{K}, p^{*} J\right)\right)
$$

to $J_{*}\left((K, p)_{+}\right)$and $J^{*}\left((K, p)_{+}\right)$, where $J$ is an excellent spectrum over $B$.

\subsection{Poincaré duality and the Thom isomorphism}

To study duality and products, we reinterpret represented parametrized homology and cohomology theories in terms of our bicategory of parametrized spectra. By Proposition 17.4.3, for spectra $X$ and $J$ over $B$ we have equivalences of spectra

$$
r_{!}\left(J \wedge_{B} X\right) \simeq J \odot t X \text { and } r_{*} F_{B}(X, J) \simeq X \triangleright J .
$$

Therefore Definition 20.2.4 admits the following direct reinterpretation.

Proposition 20.5.1. For spectra $J$ and $X$ over $B$,

$$
J_{*}(X)=\pi_{*}(J \odot t X) \quad \text { and } \quad J^{*}(X)=\pi_{-*}(X \triangleright J) .
$$

The Costenoble-Waner analogue of Spanier-Whitehead duality relating the homology and cohomology theories represented by a spectrum $J$ over $B$ is a direct consequence of Proposition 18.1.5, which gives that $J \odot t Y \simeq X \triangleright J$ if $(X, Y)$ is a dual pair of spectra over $B$.

Theorem 20.5.2 (Costenoble-Waner duality). Let $X$ be a Costenoble-Waner dualizable spectrum over B with Costenoble-Waner dual $Y$. Then

$$
J_{*}(Y) \cong J^{-*}(X) .
$$

Turning to Poincaré duality, let $k$ be a spectrum and $M$ be a smooth closed $n$-manifold. The fiberwise one-point compactification of the tangent bundle $\tau$ is denoted $S^{\tau}$, and Theorem 19.1.5 gives that $k \wedge M_{+} \simeq S_{M} \triangleright\left(k \wedge S^{\tau}\right)$. Taking $X=S_{M}$ and $J=k \wedge S^{\tau}$ in Proposition 20.5.1 and using a notation that emphasizes that the relevant cohomology is parametrized, we obtain the version of Poincaré duality stated in Example 0.0.2.

Theorem 20.5.3 (Poincaré duality-unoriented version). Let $k$ be a spectrum and $M$ be a smooth closed manifold. Then

$$
k_{*}\left(M_{+}\right) \cong\left(k \wedge S^{\tau}\right)^{-*}\left(S_{M}\right) .
$$

By introducing grading by bundles, one might arrive at the notation $k^{\tau-*}\left(M_{+}\right)$ for the right hand side. We have not chosen that route, but it gives the right idea. The purpose of an orientation is to allow one to replace $\tau$ by the dimension $n$ of $M$. We give a direct a homotopical interpretation. Recall that

$$
k \wedge X \simeq r^{*} k \wedge_{B} X
$$

for any space or spectrum $X$ over $B$.

Definition 20.5.4. Define a spherical fibration over $B$ to be an ex-fibration $(X, p, s)$ whose fibers $X_{b}$ are all equivalent to $S^{n}$ for some $n \geq 0$. The Thom complex of $X$ is $T X=r_{!} X=X / s(B)$. 
(i) A $k$-trivialization of $X$ is an equivalence

$$
k \wedge X \simeq k \wedge S_{B}^{n}
$$

of spectra over $B$.

(ii) Let $k$ be a commutative ring spectrum. A $k$-orientation of $X$ is a cohomology class $\mu \in k^{n}(T X)$ such that the restriction of $\mu$ to fibers,

$$
\mu_{b} \in k^{n}\left(T X_{b}\right) \cong k^{n}\left(S^{n}\right) \cong k^{0}\left(S^{0}\right)=\pi_{0}(k),
$$

is a unit in the ring $\pi_{0}(k)$ for each $b \in B$.

(iii) A $k$-trivialization or $k$-orientation of a smooth closed $n$-manifold $M$ is a $k$ trivialization or $k$-orientation of $S^{\tau}$.

Note that $n$ is fixed even if $B$ is not connected. This is not a serious restriction since we can work one component at a time. In contrast, the analogous restriction in the equivariant case presents serious mathematical problems, as we will discuss in $\S 21.4$. Students of algebraic topology often find the definition of an orientation mysterious and unenlightening. In the parametrized context, however, the following result gives it immediate intuitive content. It says that a $k$-oriented spherical fibration over $B$ is $k$-equivalent to the trivial spherical fibration $B \times S^{n}=S_{B}^{n}$ over $B$. This is a parametrized version of a standard observation on the Thom spectrum level that we believe was first noticed by Mahowald and Ray [103].

Proposition 20.5.5. A k-orientation $\mu$ of a spherical fibration $X$ specifies a $k$-trivialization of $X$.

Proof. Using suspension spectra implicitly, $\mu \in k^{n}(T X)$ is represented by a map

with adjoint

$$
\mu: r ! X=T X \longrightarrow \Sigma^{n} k=k \wedge S^{n}
$$

$$
\tilde{\mu}: X \longrightarrow r^{*}\left(k \wedge S^{n}\right) \simeq k \wedge S_{B}^{n} .
$$

Smashing on the left with $k$ and using the product $k \wedge k \longrightarrow k$, we obtain the map $\bar{\mu}$. It restricts on the fiber over $b \in B$ to a map of $k$-module spectra

$$
\bar{\mu}_{b}: k \wedge S^{n} \simeq k \wedge X_{b} \longrightarrow k \wedge\left(b \times S^{n}\right) \simeq k \wedge S^{n} .
$$

On passage to homotopy groups, this induces a map of free $\pi_{*}(k)$-modules on one generator, and to say that $\mu$ restricts to a unit on the fiber over $b$ is precisely to say that this restriction is an equivalence. This means that $\bar{\mu}$ is an equivalence.

Of course, $r ! S_{B}^{0}=B_{+}$. We have

$$
\left(k \wedge S_{B}^{n}\right)^{-*}\left(S_{B}\right) \cong\left(r^{*} \Sigma^{n} k\right)^{-*}\left(S_{B}^{0}\right) \cong k^{n-*}\left(B_{+}\right) .
$$

Taking $B=M$ and applying the equivalence $\bar{\mu}$ to the right side of the unoriented version of the Poincaré duality theorem we obtain the oriented version. Intuitively, the orientation untwists the parametrized twisting encoded by the tangent bundle.

Theorem 20.5.6 (Poincaré duality - oriented version). Let $k$ be a commutative ring spectrum and $M$ be a $k$-oriented smooth closed $n$-manifold. Then

$$
k_{*}\left(M_{+}\right) \cong k^{n-*}\left(M_{+}\right) .
$$

The homology Thom isomorphism is also induced by the equivalence $\bar{\mu}$, and the cohomology Thom isomorphism is induced by the equivalence $\hat{\mu}$ given in the following dual version of Proposition 20.5.5. 
Proposition 20.5.7. A k-orientation $\mu$ of a spherical fibration $X$ induces an equivalence of spectra over $B$

$$
\hat{\mu}: F_{B}\left(S_{B}^{n}, r^{*} k\right) \longrightarrow F_{B}\left(X, r^{*} k\right) .
$$

Proof. For spectra $Y$ and $Z$ over $B$, we have a natural map

$$
F_{B}(Y, k \wedge Z) \longrightarrow F_{B}(k \wedge Y, k \wedge Z)
$$

where $\wedge$ is the external smash product of a spectrum and a spectrum over $B$, namely the adjoint of the composite

$F_{B}(Y, k \wedge Z) \wedge_{B}(k \wedge Y) \simeq F_{B}(Y, k \wedge Z) \wedge_{B} Y \wedge k \longrightarrow k \wedge Z \wedge k \simeq k \wedge k \wedge Z \longrightarrow k \wedge Z$

induced by transpositions, the evaluation map, and the product on $k$. Taking $Y=S_{B}^{n}$ and $Z=S_{B}$, so that $k \wedge Z \simeq r^{*} k$, this gives

$$
F_{B}\left(S_{B}^{n}, r^{*} k\right) \longrightarrow F_{B}\left(k \wedge S_{B}^{n}, r^{*} k\right),
$$

and $\hat{\mu}$ is obtained by composing this map with $\tilde{\mu}^{*}$. On passage to fibers over $b \in B$ and then to $\pi_{*}, \hat{\mu}$ induces a map of free $\pi_{*}(k)$-modules on one generator that is an isomorphism by the defining property of an orientation. Thus $\hat{\mu}$ induces an equivalence on fibers and is therefore an equivalence.

THEOREM 20.5.8 (Thom isomorphism). If $X$ is a k-oriented spherical fibration over $B$, then there are canonical equivalences

$$
k \wedge T X \simeq k \wedge \Sigma^{n} B_{+} \quad \text { and } \quad F\left(\Sigma^{n} B_{+}, k\right) \simeq F(T X, k)
$$

and therefore canonical isomorphisms

$$
k_{*}\left(B_{+}\right) \cong k_{n+*}(T X) \quad \text { and } \quad k^{*}\left(B_{+}\right) \cong k^{n+*}(T X) .
$$

Proof. We obtain the first equivalence by applying $r$ ! to $\bar{\mu}$ and using that $r_{!}(k \wedge Y) \simeq k \wedge r_{!} Y$ for any spectrum $Y$ over $B$. We obtain the second equivalence by applying $r_{*}$ to $\hat{\mu}$ and using that $r_{*} F_{B}\left(Y, r^{*} k\right) \simeq F\left(r_{!} Y, k\right)$ for any $Y$.

Remark 20.5.9. Remembering that $r ! \eta: T X \longrightarrow B_{+} \wedge T X$ is the Thom diagonal $\Delta$, where $\eta: X \longrightarrow r^{*} r_{!} X$ is the unit of the adjunction $\left(r_{!}, r^{*}\right)$, a diagram chase shows that the second equivalence is the composite

$$
F\left(\Sigma^{n} B_{+}, k\right) \longrightarrow F\left(\Sigma^{n} k \wedge B_{+}, k\right) \stackrel{(\mu \wedge \text { id })^{*}}{\longrightarrow} F\left(T X \wedge B_{+}, k\right) \stackrel{\Delta^{*}}{\longrightarrow} F(T X, k),
$$

where the first arrow is induced from the product of $k$ as in the proof of Proposition 20.5.7. This is a standard description from which it follows directly that the cohomology Thom isomorphism sends $x \in k^{q}\left(B_{+}\right)$to the product $x \mu \in k^{q+n}(T X)$. Similarly, the cap product description of the Poincaré duality isomorphism follows directly from our proof. Observe that our proofs of these isomorphisms are purely homotopical, with no use of spectral sequences.

We shall use the results of $\S 19.6$ to give fiberwise versions of some of these results in $\S 21.7$. The results there give nonequivariant information and use little equivariant theory, but it seems best to defer their discussion until the equivariant theory is in place. 


\subsection{Relative Poincaré duality}

Using the arguments of the previous section, Theorems 19.1.6 and 19.1.9 directly imply two relative versions of Poincaré duality. The nonparametrized oriented version of the first is familiar, but this does not seem to be true of the second.

Theorem 20.6.1 (Boundary Poincaré duality). Let $M$ be a smooth compact manifold with boundary $\partial M$ and boundary inclusion $i: \partial M \longrightarrow M$. Let $k$ be a spectrum and define $J=k \wedge S^{\tau_{M}}$. Then there is an isomorphism of long exact sequences

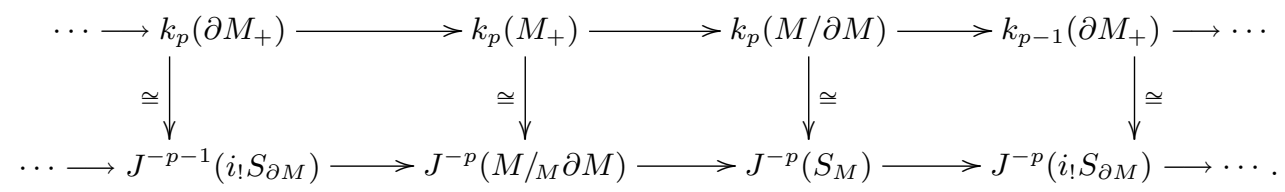

If $k$ is a commutative ring spectrum and $M$ is $k$-oriented, then the bottom sequence is isomorphic to the long exact sequence

$$
\cdots \longrightarrow k^{n-1-p}\left(\partial M_{+}\right) \longrightarrow k^{n-p}(M / \partial M) \longrightarrow k^{n-p}\left(M_{+}\right) \longrightarrow k^{n-p}\left(\partial M_{+}\right) \longrightarrow \cdots
$$

Theorem 20.6.2 (Relative Poincaré duality). Let $M$ be a smooth closed manifold with a closed submanifold $L$ and inclusion $i: L \longrightarrow M$. Let $t: M_{+} \longrightarrow T \nu_{M, L}$ be the Pontryagin-Thom map and let $C(t)$ be its cofiber. Let $k$ be a spectrum and define $J=k \wedge S^{\tau_{M}}$. Then there is an isomorphism of long exact sequences

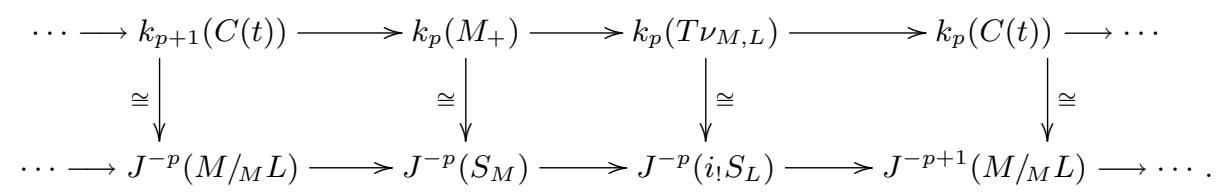

If $k$ is a commutative ring spectrum and $M$ is $k$-oriented, then there is also an isomorphism with the long exact sequence

$$
\cdots \longrightarrow k^{n-p}(M / L) \longrightarrow k^{n-p}\left(M_{+}\right) \longrightarrow k^{n-p}\left(L_{+}\right) \longrightarrow k^{n-p+1}(M / L) \longrightarrow \cdots
$$

EXAMPLE 20.6.3. Combining the first isomorphism in this last result with the absolute oriented version of Poincaré duality for the smooth closed $d$-manifold $L$, we see that

$$
k_{n-d+p}\left(T \nu_{M, L}\right) \cong k^{d-p}\left(L_{+}\right) \cong k_{p}\left(L_{+}\right) .
$$

\subsection{Products in parametrized homology and cohomology}

Products and slant products in parametrized homology and cohomology work in much the same way as in $[124, \S 9]$. There, these products in nonparametrized homology and cohomology are described as formal consequences of structure present in any triangulated category with a compatible closed symmetric monoidal structure. We have such structure in each category $\operatorname{Ho} G \mathscr{S}_{B}$, but it is more relevant that we have analogous structure in $\mathscr{E} x$, as explained in $\S 16.7$ and $\S 17.5$.

We have the evident pairing

$$
\pi_{m}(X) \otimes \pi_{n}(Y) \longrightarrow \pi_{m+n}(X \wedge Y),
$$


for ordinary spectra $X$ and $Y$. Remember that $\wedge$ on the right can be viewed as

$$
\odot: \mathscr{E} x(*, *) \times \mathscr{E} x(*, *) \longrightarrow \mathscr{E} x(*, *)
$$

Remember too that a spectrum $X$ over $B$ is regarded as a 1-cell $B \longrightarrow *$ when identified with a spectrum over $* \times B$ and as a 1 -cell $* \longrightarrow B$, denoted $t X$, when identified with a spectrum over $B \times *$. This notational convention fixes the meaning of the operation $\odot$, so that $J \odot t X$ means a 1 -cell $* \longrightarrow *$ and thus a spectrum. Similarly, in $t J \triangleleft t X$ we regard both $X$ and $J$ as 1-cells $* \longrightarrow B$ and in $X \triangleright J$ we regard both $X$ and $J$ as 1-cells $B \longrightarrow *$, so that we have a spectrum in either case. Since we insist that all of our pairings $\odot, \triangleleft$, and $\triangleright$ have ordinary spectra as output in what follows, there is no ambiguity if we omit the notational reminder " $t$ ", and we agree to do so in all of our homological and cohomological statements. However, we sometimes return $t$ to the notation when discussing the homotopical antecedents of our homological pairings.

Let $J, K, X$, and $Y$ be spectra over $B$. Then we have external pairings

$$
\begin{aligned}
& \wedge: J_{p}(X) \otimes K_{q}(Y) \longrightarrow(J \odot K)_{p+q}(X \odot Y) \\
& \cup: J^{p}(X) \otimes K^{q}(Y) \longrightarrow(J \odot K)^{p+q}(X \odot Y) .
\end{aligned}
$$

The slant products require a mixture of spectra and spectra over $B$, and there are several cases present. If either $J$ or $X$ is a spectrum and the other three variables are spectra over $B$, then we have a slant product

$$
/: J^{p}(X \odot Y) \otimes K_{q}(Y) \longrightarrow(J \odot K)^{p-q}(X) .
$$

If either $K$ or $Y$ is a spectrum and the other three variables are spectra over $B$, then we have a slant product

$$
\backslash: J^{p}(X) \otimes K_{q}(X \odot Y) \longrightarrow(J \odot K)_{q-p}(Y) .
$$

The naturality of slant products is better seen by rewriting them in adjoint form

$$
\begin{aligned}
& /: J^{p}(X \odot Y) \longrightarrow \operatorname{Hom}\left(K_{q}(X),(J \odot K)^{p-q}(X)\right), \\
& \backslash: K_{q}(X \odot Y) \longrightarrow \operatorname{Hom}\left(J^{p}(X),(J \odot K)_{q-p}(Y)\right) .
\end{aligned}
$$

Observe that all of these products mix nonparametrized and parametrized theories. They are obtained by passing to $\pi_{*}$ and applying the pairing (20.7.1) and functoriality to formally defined canonical maps of spectra. However, there is an important conceptual point to be made. As observed in Remark 16.2.7, the bicategorical context does not incorporate maps of 0 -cells, in our case maps of spaces. Said another way, it does not capture all of the structure implicit in the category of retracts of $\S 2.5$. We need the spectrum level version of the external commutativity isomorphism $\bar{\gamma}$ of Remark 2.5.4 to define the following maps, and it is not part of our mere bicategorical framework. We shall not go into categorical detail since the topological context should be clear.

The pairing (20.7.2) is induced by the following pairing of spectra. The isomorphisms on the left and right record that the relevant composition $\odot$ is just the ordinary smash product $\wedge$ and use the associativity isomorphism for $\odot$.

$(J \odot X) \wedge(K \odot Y) \simeq J \odot(X \odot K) \odot Y \longrightarrow J \odot(K \odot X) \odot Y \simeq(J \odot K) \wedge(X \odot Y)$ 
Here $X \odot K$ means $t X \odot K$, which is just the external smash product $X \bar{\wedge} K$ over $B \times B$. The middle arrow is given by the commutativity isomorphism

$$
\bar{\gamma}: X \odot K \longrightarrow K \odot X \text {. }
$$

Similarly, the pairing (20.7.3) is induced by a pairing

$(X \triangleright J) \wedge(Y \triangleright K) \simeq(X \triangleright J) \odot(Y \triangleright K) \longrightarrow(X \odot Y) \triangleright(J \odot K) \simeq F(X \odot Y, J \odot K)$.

Again, the isomorphisms on the left and right record that the relevant operations $\odot$ and $\triangleright$ in $\mathscr{E} x$ reduce to $\wedge$ and $F$. The middle arrow is the adjoint of the composite

$$
(X \triangleright J) \odot((Y \triangleright K) \odot X) \odot Y \longrightarrow(X \triangleright J) \odot(X \odot(Y \triangleright K)) \odot Y \stackrel{\varepsilon \odot \varepsilon}{\longrightarrow} J \odot K .
$$

The operation $\odot$ inside the middle parentheses of the first two terms is $\bar{\wedge}$, and the first arrow is a commutativity isomorphism $\bar{\gamma}$.

The pairing (20.7.4) depends for its details on which of $J$ or $X$ is a spectrum. In either case, it is induced by a pairing of the form

$$
((X \odot Y) \triangleright J) \odot K \odot Y \longrightarrow X \triangleright(J \odot K) .
$$

To describe this, recall from (16.3.10) that we have the natural isomorphism

$$
(X \odot Y) \triangleright J \simeq X \triangleright(Y \triangleright J),
$$

which is proven by the same Yoneda lemma argument as the isomorphism

$$
F(X \wedge Y, J) \simeq F(Y, F(X, J))
$$

when all variables are spectra. Then (20.7.10) is the composite

$$
\begin{aligned}
((X \odot Y) \triangleright J) \odot K \odot Y & \longrightarrow(Y \triangleright(X \triangleright J)) \odot Y \odot K \\
& \longrightarrow(X \triangleright J) \odot K \\
& \longrightarrow X \triangleright(J \odot K),
\end{aligned}
$$

where the first arrow uses $\bar{\gamma}$ and the isomorphism just cited, the second arrow is $\varepsilon \odot \mathrm{id}$, and the third is the adjoint of the composite

$$
(X \triangleright J) \odot K \odot X \stackrel{\text { id } \odot \bar{\gamma}}{\longrightarrow}(X \triangleright J) \odot X \odot K \stackrel{\varepsilon \odot \text { id }}{\longrightarrow} J \odot K .
$$

Finally, the pairing (20.7.5) is induced by a pairing of the form

$$
(X \triangleright J) \odot K \odot X \odot Y \longrightarrow J \odot K \odot Y
$$

obtained by first using $\bar{\gamma}$ to permute $K$ and $X$ and then using $\varepsilon:(X \triangleright J) \odot X \longrightarrow J$.

There are many unit, associativity, and commutativity relations relating the four products. In the nonparametrized setting these are catalogued in [2] and [162], and many of them follow from isomorphisms displayed explicitly in $§ 16.3$. These formulas in the parametrized setting would be direct consequences of the axioms for a closed symmetric bicategory with a weakly compatible triangulation, were it not for the use of $\bar{\gamma}$; that introduces the usual signs but no complications. Actually, the triangulation has not yet entered explicitly, but we have in mind the formulas and commutative diagrams given by Adams [2, pp. 235-244] and Switzer [162, pp. 276-283] that relate the four products to connecting homomorphisms in the homology and cohomology of pairs of spaces, and these now extend directly to pairs of ex-spaces. For spaces $(X, p)$ over $B$, we can internalize external products via the diagonal map

$$
\Sigma_{B}^{\infty}(X, p)_{+} \longrightarrow \Sigma_{B}^{\infty}(X, p)_{+} \wedge_{B} \Sigma_{B}^{\infty}(X, p)_{+}
$$


that is made precise in (15.3.2). The internalization of the product $\backslash$ is the cap product.

We can internalize the representing spectrum along any pairing $J \odot K \longrightarrow L$ of spectra, or of spectra over $B$ in the cases of (20.7.10) or (20.7.11) when $J$ or $K$ is a spectrum rather than a spectrum over $B$. If $J$ is a ring spectrum over $B$ with product $J \wedge_{B} J \longrightarrow J$, we have the induced product

$$
J \odot J \cong r_{!}\left(J \wedge_{B} J\right) \longrightarrow r_{!} J .
$$

In particular, if $J=r^{*} k$ for a ring spectrum $k$, we have the product

$$
r^{*} k \wedge_{B} r^{*} k \cong r^{*}(k \wedge k) \longrightarrow r^{*} k .
$$

Applying $r$ and using the counit $r ! r^{*} k \longrightarrow k$, we obtain a pairing

$$
r^{*} k \otimes r^{*} k \longrightarrow k \text {. }
$$

Here we also have that

$$
r^{*} k \odot k=r^{*} k \bar{\wedge} k \cong r^{*}(k \wedge k) \cong k \bar{\wedge} r^{*} k=k \odot r^{*} k .
$$

The isomorphisms are clear pointwise on the ex-space level, hence hold levelwise on the spectrum level, and they descend model categorically to homotopy categories. Thus, for spectra $X$ and $Y$ over $B$, we can internalize all four of our pairings so that they take values in $k_{*}$ or $k^{*}$ when we start with $J=K=r^{*} k$ in (20.7.4) and (20.7.5), with $J=k$ and $K=r^{*} k$ in (20.7.6), and with $J=r^{*} k$ and $K=k$ in (20.7.7).

We conclude that all of the products that we are accustomed to dealing with in nonparametrized theories are available to us in our classical parametrized homology and cohomology theories, where they continue to satisfy all of the usual properties.

\subsection{The representability of homology theories}

In classical homology theory, if one is given a homology theory $k_{*}$ defined on finite complexes, one defines a corresponding cohomology theory $k^{*}$ on finite complexes by applying $k_{*}$ to dual complexes. One then quotes Adams' variant [1] of Brown representability [25] to conclude that $k^{*}$ and therefore $k_{*}$ is represented.

Since all finite cell spectra over $B$ are Costenoble-Waner dualizable, one might expect to be able to apply the same arguments in our parametrized context. There are several problems. First, although we know that finite parametrized cell complexes are dualizable, we do not know that their duals are finite complexes since we do not know that the dual of a 1-cell complex is a finite cell complex. We can get around this, albeit not altogether satisfactorily, by assuming that the given parametrized theory $J_{*}$ is defined on all Costenoble-Waner dualizable spectra over $B$ rather than just on the finite complexes. Then the definition of $J^{*}$ makes sense. Since its domain of definition contains the finite cell spectra over $B$, we can represent it if we can represent appropriate functors defined on finite cell complexes.

Another problem concerns countability assumptions. Recall that Brown [25] originally proved the relevant representability theorem for those set-valued (contravariant) functors on finite $\mathrm{CW}$ complexes that satisfy the Mayer-Vietoris axiom and take countable values. Adams [1] assumed that the functor is group-valued and removed the countability hypothesis. However, countability is still central to his proof. He uses heavily that, up to equivalence, there are only countably many finite CW complexes and countably many maps between them. Neeman [133] gave 
a different proof applicable to general triangulated categories. Recall the definitions of detecting and generating sets from Definition 7.5.5 and the discussion of Brown representability in triangulated categories from $\S 13.1$.

TheOREM 20.8.1 (Neeman). Let $\mathscr{A}$ be a compactly generated triangulated category, let $\mathscr{B}$ be the full subcategory of compact objects in $\mathscr{A}$, and assume that $\mathscr{B}$ is equivalent to a countable category (countably many objects and countably many morphisms). If $h: \mathscr{B}^{o p} \longrightarrow \mathscr{A}$ b takes distinguished triangles to exact sequences, then $h$ is representable in the sense that $h$ is the restriction to $\mathscr{B}$ of the functor $\mathscr{A}(-, X)$ for some $X$ in $\mathscr{A}$. A natural transformation $h \longrightarrow j$ of functors represented by $X$ and $Y$ is represented by a map $X \longrightarrow Y$, not necessarily unique.

This is the concatenation of two results. Say that a functor $h$ as in the statement is "homological". First, the countability hypothesis ensures that the subcategory of homological functors in the category of functors $\mathscr{B}^{\text {op }} \longrightarrow \mathscr{A} b$ coincides with the subcategory of functors of projective dimension $\leq 1[133,5.1]$. Second, assuming that $\mathscr{B}$ is essentially small, Brown representability holds in the form stated for all homological functors $h$ if and only if the homological functors coincide with the functors of projective dimension $\leq 1[133,4.1,4.11]$. Neeman $[133, \S 6]$ also gave a counterexample in the absence of the countability hypothesis.

Remark 20.8.2. It is essential to Theorem 20.8.1 that $\mathscr{B}$ be equivalent to a countable category rather than just have a countable set of compact generators. In the derived category of any commutative ring $R$, the complexes $R[n]$ are compact (since $R[n]$ is $R$-free on one generator) and detect homology, hence they give a countable detecting set. However Neeman's counterexample is the derived category of $K[x, y]$ for any uncountable field $K$.

We need a criterion for verifying the countability assumption on $\mathscr{B}$.

LEMma 20.8.3. Let $\mathscr{A}$ be a triangulated category with a detecting set $\mathscr{D}$ of compact objects such that the full subcategory of $\mathscr{A}$ with object set $\mathscr{D}$ is countable. Then the full subcategory $\mathscr{B}$ of all compact objects in $\mathscr{A}$ is countable.

By Theorem $13.1 .14, \mathscr{B}$ is the thick subcategory of $\mathscr{A}$ generated by $\mathscr{D}$. Thus the conclusion is a special case of the following general result. We are very grateful to Neeman, who gave us the proof in response to our asking if the previous lemma might be true.

Lemma 20.8.4. Let $\mathscr{C}$ be a countable subcategory of a triangulated category $\mathscr{A}$. Then the thick subcategory $\mathscr{T}$ generated by $\mathscr{C}$ is countable.

Proof. For an object $X$ of $\mathscr{A}$, let $\mathscr{S}[X]$ be the full subcategory of $\mathscr{A}$ whose objects are those $Y$ such that $\mathscr{A}(X, Y)$ and $\mathscr{A}(Y, X)$ are countable. Clearly $\mathscr{S}[X]$ is thick. In general, $\mathscr{S}[X]$ could only contain the zero object. However, if $X$ is in $\mathscr{C}$, then $\mathscr{C}$ is contained in $\mathscr{S}[X]$ and therefore $\mathscr{T}$ is contained in $\mathscr{S}[X]$. We conclude that if $X$ is in $\mathscr{C}$, then $\mathscr{A}(X, Y)$ and $\mathscr{A}(Y, X)$ are countable for every $Y$ in $\mathscr{T}$. This implies that if $X$ is in $\mathscr{T}$, then $\mathscr{S}[X]$ contains $\mathscr{C}$ and therefore also contains $\mathscr{T}$. Therefore $\mathscr{A}(X, Y)$ is countable for every pair of objects of $\mathscr{T}$.

It remains to show that $\mathscr{T}$ has only countably many isomorphism classes of objects. We use the usual inductive construction of the thick closure $\mathscr{T}$ of $\mathscr{C}$. For any full countable subcategory $\mathscr{F}$ of $\mathscr{A}$, define $\mathscr{S}[\mathscr{F}]$ to be the full subcategory of $\mathscr{A}$ whose objects are all direct summands of all objects $Z$ that occur in a distinguished 
triangle $X \longrightarrow Y \longrightarrow Z \longrightarrow \Sigma X$ generated by a map $X \longrightarrow Y$ in $\mathscr{F}$. Since there are only countably many such maps, there are only countably many isomorphism classes of such objects $Z$. Each such $Z$ is in the thick subcategory generated by $\mathscr{F}$, hence $\mathscr{A}(Z, Z)$ is countable by the first paragraph. Therefore there are countably many idempotents $Z \longrightarrow Z$, hence countably many direct summands of $Z$. Thus $\mathscr{S}[\mathscr{F}]$ has countably many objects. By the first paragraph, it also has countably many morphisms since it is contained in the thick subcategory generated by $\mathscr{F}$. Now define $\mathscr{T}_{0}=\mathscr{C}$, and, inductively, $\mathscr{T}_{n}=\mathscr{S}\left[\mathscr{T}_{n-1}\right]$. By induction, each $\mathscr{T}_{n}$ is countable, and $\mathscr{T}$ is the union of the $\mathscr{T}_{n}$.

Proposition 20.8.5. If $B$ has countably many path components and each homotopy group of each path component is countable, then the category of compact objects in $\mathrm{Ho}_{\mathrm{B}}$ is countable.

Proof. It suffices to find a detecting set $\mathscr{D}_{B}$ of compact objects such that the full subcategory of $\operatorname{Ho} \mathscr{S}_{B}$ with object set $\mathscr{D}_{B}$ is countable. We have the generating set of all $S^{n, b}$ specified in Definition 13.1.1, where $n$ runs over the integers and $b$ runs over the points of $B$. By Corollary 20.3.3, if $\pi_{*}\left(X_{b}\right)=0$, then $\pi_{*}\left(X_{b^{\prime}}\right)=0$ for all $b^{\prime}$ in the same path component as $b$. Thus we obtain a countable detecting subset by choosing one $b$ from each path component of $B$. We have

$$
\left[S^{m, a}, S^{n, b}\right]_{B} \cong \pi_{m}\left(S_{a}^{n, b}\right)
$$

and it remains to show that these homotopy groups of (derived) fibers are countable. Recall that $S^{n, b}=b_{!} S^{n}$. Spacewise, the homotopy groups of the total spaces of the point-set level spectra $b ! S^{n}$ over $B$ are countable because the homotopy groups of $B$ are countable. Therefore, after level fibrant approximation, the homotopy groups of fibers are countable. The spectrum level homotopy groups of fibers are countable colimits of these groups and are therefore also countable.

We conclude that Theorem 20.8.1 applies to prove the representability of cohomology theories defined on compact objects of $\operatorname{Ho} \mathscr{S}_{B}$ when $B$ satisfies the countability assumptions of Proposition 20.8.5. In the rest of this section, we sketch how to use a colimit argument to obtain the same conclusion more generally. Since the countability hypothesis of Proposition 20.8.5 is not unduly restrictive, we shall be brief.

THEOREM 20.8.6. Let h be a contravariant functor defined on the full subcategory of compact objects of Ho $\mathscr{S}_{B}$ and taking values in Abelian groups. If $h$ satisfies the wedge and Meyer-Vietoris axioms, then it is the restriction of a represented functor on $\mathrm{Ho}_{\mathrm{S}} \mathscr{S}_{B}$.

Sketch PROOF. Using a Quillen equivalence induced by an equivalence on base spaces, we may replace $B$ by the second barycentric subdivison of the geometric realization of its total singular complex and so assume that $B$ is a simplicial complex. Let $\Delta_{n}$ be the topological $n$-simplex and consider a simplex $e: \Delta_{n} \subset B$. Since $e_{\text {! }}$ preserves compact objects, $h \circ e_{!}$is a cohomological functor defined on the compact objects of Ho $\mathscr{S}_{\Delta_{n}}$. Since Ho $\mathscr{S}_{\Delta_{n}}$ is equivalent to Ho $\mathscr{S}_{*}$, this functor is represented by a spectrum $J_{e}$ over $\Delta_{n}$. If two $n$-simplices $e_{1}$ and $e_{2}$ intersect in an $(n-1)$-dimensional simplex $e$, let $i_{1}: e \longrightarrow e_{1}$ and $i_{2}: e \longrightarrow e_{2}$ be the inclusions. The two represented cohomological functors $\left[i_{1 !}(-), J_{e_{1}}\right]$ and $\left[i_{2 !}(-), J_{e_{2}}\right]$ both restrict to $h \circ e_{!}$, and we obtain non-canonical equivalences $i_{1}^{*} J_{e_{1}} \simeq J_{e} \simeq i_{2}^{*} J_{e_{2}}$ whose represented functors on compact objects are canonically isomorphic to $h \circ e_{!}$. 
Now we construct a spectrum $J$ over $B$ as the telescope of spectra $j_{n !} J^{n}$ over $B$, where the $J^{n}$ are spectra over the $n$-skeleta $B^{n}$ and $j_{n}: B^{n} \longrightarrow B$ is the inclusion. Let $J^{0}$ be the disjoint union of the pushforward over $B^{0}$ of the $J_{e}$ for all 0-cells $e$. Inductively, construct $J^{n}$ as the homotopy pushout

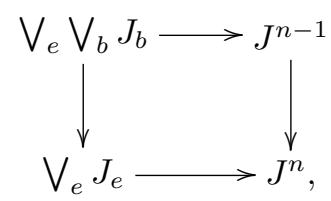

where $e$ runs over the $n$-simplices of $B, b$ runs over the faces of the boundary $\partial e$, and all spectra are pushed forward to be spectra over $B^{n}$. Then $e^{*} J$ is equivalent to $J_{e}$ for all $e$. Of course, $h$ and the functor represented by $J$ extend to cohomology theories by the suspension axiom. Using Theorem 20.4.1, we construct spectral sequences for the computation of $h^{*}$ and $J^{*}$ on compact spectra $X$ over $B$. The $E_{1}$-terms depend only on the restrictions of $X$ to simplices in $B$ and so can be identified. Therefore $h^{*}$ is the restriction of $J^{*}$ to compact spectra over $B$. The intuition is just that $h^{*}$ is built up from its restrictions to simplices, on which it agrees with $J^{*}$ by construction. The spectral sequence merely serves to formalize this intuition. 


\section{CHAPTER 21}

\section{Equivariant parametrized homology and cohomology}

\section{Introduction}

The material of the previous chapter generalizes readily to the equivariant context, as we show in $\S \S 21.1-21.5$. Homology and cohomology theories on $G$ spaces can be $\mathbb{Z}$-graded or $R O(G)$-graded. The $\mathbb{Z}$-graded theories are represented by naive $G$-spectra, which are indexed on trivial representations. Such theories do not admit Spanier-Whitehead or Poincaré duality. Their axioms read in precisely the same way as in the nonequivariant context of $\S 20.1$. The $R O(G)$-graded theories are the ones that are of interest to us, and they are indexed on all representations of $G$.

We restrict attention to reduced homology and cohomology theories, and we write them without a tilde. We define theories axiomatically in $\S 21.1$ and in represented form in $\S 21.2$, and we discuss coefficient systems in $\S 21.3$. We describe Costenoble-Waner and parametrized Poincaré duality in $\S 21.4$. At this point the theory diverges sharply from the nonequivariant theory since the evident equivariant analogue of a $k$-orientation is too restrictive, as we shall explain. We consider products and the representability of homology in $\S 21.5$.

The last two sections are not direct generalizations of the results of the previous chapter, but rather are two new beginnings, both of which are of interest nonequivariantly as well as equivariantly. The context of the bicategories $G \mathscr{E} x_{B}$ established in Chapter 19 suggests a generalization of our parametrized homology and cohomology theories in which the implicit base object $*$ that we have used so far is replaced by a general $G$-space $B$. We give the definitions because they are so natural, in $\S 21.6$, but we shall not pursue this point of view in any depth. A generalized version of Costenoble-Waner duality is immediate.

We turn to fiberwise versions of Poincaré duality in $§ 21.7$, emphasizing the nonequivariant case. While we have a version applicable to general base spaces, we lack calculational understanding of its cohomological side. We focus on bundles $E$ whose base $B$ and fiber $M$ are smooth closed manifolds. Clearly $E$ need not be $k$-orientable when $B$ and $M$ are $k$-orientable. For example, the Klein bottle is an $S^{1}$-bundle over $S^{1}$ but is not $H \mathbb{Z}$-orientable. It is a natural problem to determine when $E$ does inherit a $k$-orientation from $B$ and $M$, but we know of no general discussion in the literature. Somewhat digressively, since parametrized theory is not essential, we illustrate ideas by describing two ways of resolving this problem.

We only establish the barest beginnnings of the equivariant theory here, giving little more than parallels to the material of the previous chapter. The equivariant parametrized theory has many special features. The coefficient sytems that we describe are just the tip of the iceberg of a new theory that is in process of 
development. The paper of Costenoble and Waner [41] makes substantial progress. It focuses on "ordinary" $R O(G)$-graded homology and cohomology theories and builds on the equivariant geometric orientation theory of [37].

\subsection{Equivariant homology and cohomology theories}

The term " $R O(G)$-graded" is technically a misnomer for the theories that we are interested in since one cannot think of representations as isomorphism classes and still keep track of signs, which in equivariant theories are units in the Burnside ring $A(G)$. In the nonparametrized context, $R O(G)$-graded theories are given a precise axiomatization and are shown to be representable in [118, §XIII.1], although the exposition there is unnecessarily cumbersome. We show how to adapt that treatment to the parametrized context. Such an adaptation has also been carried out by Costenoble and Waner [41].

Recall that we started our stable work with the $G$-category $\left(\mathscr{I}_{G}, G \mathscr{I}\right)$ of finite dimensional $G$-inner product spaces and linear isometric isomorphisms. We now focus on the equivariant maps and we rename $G \mathscr{I}$, calling it $\mathscr{R} O(G)$ in order to indicate that we are now thinking of it as a forerunner of the real representation ring $R O(G)$. Say that two maps in $\mathscr{R} O(G)$ are homotopic if their associated based $G$-maps $S^{V} \longrightarrow S^{W}$ are stably homotopic. Let $h \mathscr{R} O(G)$ be the resulting homotopy category. Then $h \mathscr{R} O(G)(V, V)$ is a copy of the Burnside ring $A(G)$.

DEFINITION 21.1.1. An $R O(G)$-graded parametrized homology theory is a functor

$$
J_{*}^{G}: h \mathscr{R} O(G)^{\mathrm{op}} \times \mathrm{HoG} \mathscr{K}_{B} \longrightarrow \mathscr{A} b
$$

written $J_{V}^{G}(X)$ on objects $(V, X)$ and similarly on morphisms, together with natural suspension isomorphisms

$$
\sigma^{W}: J_{V}^{G}(X) \longrightarrow J_{V \oplus W}^{G}\left(\Sigma_{B}^{W} X\right)
$$

such that the following axioms are satisfied.

- Exactness And AdDitivity. For each representation $V$, the functor $J_{V}^{G}$ is exact on cofiber sequences and sends wedges to sums.

- Compatibility. The following diagram commutes, where $\alpha$ is map $W \longrightarrow W^{\prime}$ in $h \mathscr{R} O(G)$ :

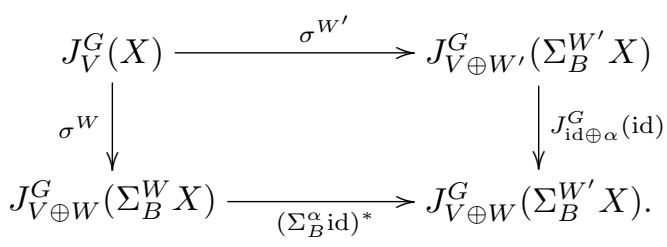

- Transitivity. $\sigma^{0}=\mathrm{id}$ and the $\sigma$ are transitive in the sense that the following diagram commutes for each pair of representations $(W, Z)$ :

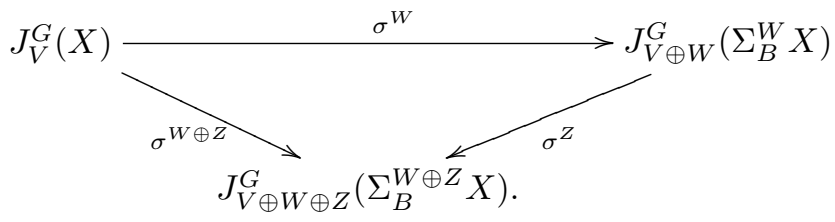


DeFINITIOn 21.1.2. An $R O(G)$-graded parametrized cohomology theory is a functor

$$
J_{G}^{*}: h \mathscr{R} O(G) \times\left(\mathrm{Ho} G \mathscr{K}_{B}\right)^{\text {op }} \longrightarrow \mathscr{A} b,
$$

written $J_{G}^{V}(X)$ on objects $(V, X)$ and similarly on morphisms, together with natural isomorphisms

$$
\sigma^{W}: J_{G}^{V}(X) \longrightarrow J_{G}^{V} \oplus W\left(\Sigma_{B}^{W} X\right)
$$

such that the evident duals of the axioms for homology are satisfied.

The following observation illuminates the functoriality in $h \mathscr{R} O(G)$, although we shall make little use of it. It is relevant to the proof that cohomology theories are representable. Thinking of cohomology theories on ex- $G$-spaces as represented one degree at a time, $J_{G}^{V}(X)=[X, J(V)]_{G, B}$, it describes the functoriality in $V$.

Lemma 21.1.3. Let $J$ be an orthogonal $\Omega$-G-spectrum over $B$. Then $J$ induces a functor $J: h \mathscr{R} O(G) \longrightarrow H o G \mathscr{K}_{B}$.

Proof. Since $J: \mathscr{I} \longrightarrow \mathscr{K}_{G, B}$ is a continuous $G$-functor, it induces homotopy preserving maps $G \mathscr{I}(V, W) \longrightarrow G \mathscr{K}_{B}(J(V), J(W))$ for all $V$ and $W$. Since $J$ is an $\Omega$ - $G$-spectrum over $B$, stably homotopic maps $V \longrightarrow W$ induce the same map $J(V) \longrightarrow J(W)$ in $\mathrm{HoG} \mathscr{K}_{B}$.

The axioms refer only to the "positive degree" part of the theory. We extend a theory so defined to "formal differences $V \ominus W$ " for any pair of representations $(V, W)$ by setting

$$
J_{V \ominus W}^{G}(X)=J_{V}^{G}\left(\Sigma_{B}^{W} X\right) \quad \text { and } \quad J_{G}^{V \ominus W}(X)=J_{G}^{V}\left(\Sigma_{B}^{W} X\right) .
$$

We use the symbol $\ominus$ to avoid confusion with either orthogonal complement or difference in the representation ring, but we write - instead of $\ominus$ when $V=0$. Rigorously, we are thinking of $V \ominus W$ as an object of the category $h \mathscr{R} O(G)^{o p} \times$ $h \mathscr{R} O(G)$. For each $X,(21.1 .4)$ defines functors from this category and from its opposite category to the category of Abelian groups.

The representation group $R O(G)$ is obtained by passage to equivalence classes from the set of formal differences $V \ominus W$, where $V \ominus W$ is equivalent to $V^{\prime} \ominus W^{\prime}$ if there is a $G$-linear isometric isomorphism $\alpha: V \oplus W^{\prime} \longrightarrow V^{\prime} \oplus W$. Of course, $R O(G)$ is a ring under $\otimes$, but the ring structure is not directly relevant to us. When interpreting $R O(G)$-graded homology theories, we must keep track of the choice of $\alpha$, and we see that a given $\alpha$ determines the explicit isomorphism displayed as the unlabelled arrow in the diagram of isomorphisms

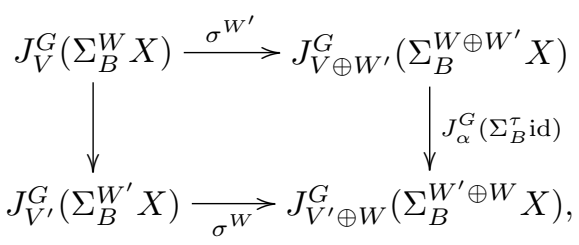

where $\tau: W \oplus W^{\prime} \longrightarrow W^{\prime} \oplus W$ is the transposition isomorphism. The purpose of the careful axiomatization is to give precision to signs arising from such permutations of representations.

If $V^{G}=0$, write $V+n=V \oplus \mathbb{R}^{n}$ and $V-n=V \ominus \mathbb{R}^{n}$. The first axiom ensures that, for each such $V$, the $J_{V+n}^{G}$ and $\sigma^{1}$ define a $\mathbb{Z}$-graded homology theory. The second axiom ensures that we retain complete information on suspensions if we 
restrict attention to one object in each isomorphism class of representations, that is, if we restrict to any skeleton of $\mathscr{R} O(G)$. We can restrict further to a skeleton of its homotopy category. The third axiom says that we can restrict further to irreducible representations, as is implicit in restricting to $\sigma=\sigma^{1}$ when $G=e$. We can replace $G \mathscr{K}_{B}$ by $G \mathscr{S}_{B}$ in the definitions just given and so define $R O(G)$-graded homology and cohomology theories on $G$-spectra over $B$.

\subsection{Represented equivariant theories}

The definition of represented theories is now the same as Definition 20.2.4, interpreted equivariantly, but we must first recall the relevant homotopy group functors.

Definition 21.2.1. For $G$-inner product spaces $V$, define the $V$ th and $(-V)$ th homotopy groups of a $G$-spectrum $Y$ by

$$
\pi_{V}^{G}(Y)=\left[S^{V}, Y\right]_{G} \text { and } \pi_{-V}^{G}(Y)=\left[S^{-V}, Y\right]_{G} .
$$

Observe that these give functors

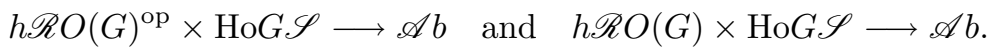

Indeed, for a map $\alpha: V \longrightarrow W$ in $h \mathscr{R} O(G)$, we have the map $\alpha: S^{V} \longrightarrow S^{W}$ with dual $D(\alpha): S^{-W} \longrightarrow S^{-V}$ characterized by commutativity of the diagram

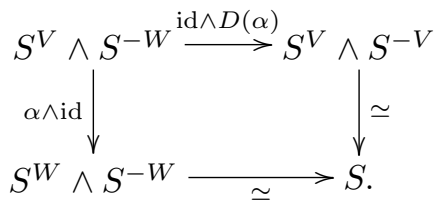

Applying $[-, Y]_{G}$ to the maps $\alpha$ and $D(\alpha)$ gives functoriality in the variable $V$.

Definition 21.2.2. For $G$-spectra $J$ and $X$ over $B$, define the $J$-homology and $J$-cohomology groups of $X$ by

$$
J_{V}^{G}(X)=\pi_{V}^{G}\left(r_{!}\left(J \wedge_{B} X\right)\right)
$$

and

$$
J_{G}^{V}(X)=\pi_{-V}^{G}\left(r_{*} F_{B}(X, J)\right) \cong\left[S_{B}^{-V}, F_{B}(X, J)\right]_{G, B} .
$$

The required functoriality is clear. The suspension isomorphisms $\sigma^{W}$ in homology and cohomology are given by

$$
\begin{aligned}
{\left[S^{V}, r_{!}\left(J \wedge_{B} X\right)\right]_{G} } & \cong\left[S^{V} \wedge S^{W}, r_{!}\left(J \wedge_{B} X\right) \wedge S^{W}\right]_{G} \\
& \cong\left[S^{V \oplus W}, r_{!}\left(J \wedge_{B} \Sigma_{B}^{W} X\right)\right]_{G}
\end{aligned}
$$

and

$$
\begin{aligned}
{\left[S^{-V}, r_{*} F_{B}(X, J)\right]_{G} } & \cong\left[S^{-V} \wedge S^{-W}, r_{*} F_{B}(X, J) \wedge S^{-W}\right]_{G} \\
& \cong\left[S^{-(V \oplus W)}, r_{*} F_{B}\left(\Sigma_{B}^{W} X, J\right)\right]_{G} .
\end{aligned}
$$

The first isomorphisms are given by smashing with $S^{W}$ or with $S^{-W}$. The second isomorphisms are obvious on the sources in our sets $[-,-]_{G}$ and are given on the targets by the canonical commutation of funtors equivalences

$$
r_{!}\left(J \wedge_{B} X\right) \wedge S^{W} \simeq r_{!}\left(J \wedge_{B} X \wedge_{B} S^{W}\right) \simeq r_{!}\left(J \wedge_{B} \Sigma_{B}^{W} X\right)
$$


and

$$
r_{*} F_{B}(X, J) \wedge S^{-W} \simeq r_{*} F_{B}\left(X \wedge_{B} S^{W}, J\right) \simeq r_{*} F_{B}\left(\Sigma_{B}^{W} X, J\right) .
$$

In the first composite, the left equivalence is given by commuting tensors with $r_{\text {! }}$. In the second composite, the left equivalence is obtained by passing to adjoints and using that $r^{*}$ commutes with tensors. In both, the right equivalence is given by $X \wedge_{B} S^{W} \cong \Sigma_{B}^{W} X$. The naturality in $V$ and $X$ is clear. The compatibility and transitivity axioms follow by direct inspection.

The exactness and wedge axioms work in exactly the same way as nonequivariantly. The same definitions specify the homology and cohomology groups of an ex- $G$-space $K$, and these are given equivalently by taking $X=\Sigma_{B}^{\infty} K$ in the definition above. Clearly these groups give (reduced) homology and cohomology theories in $K$, as axiomatized in Definition 21.1.1.

Since we are working with spectra and have desuspensions, the entire theory can be reconstructed from $J_{0}^{G}$ or $J_{G}^{0}$, turning the suspension isomorphisms and the axioms relating them into definitions. In particular, if we set $V=0$ in the second axiom, it turns into definitions of $J_{\alpha}$ and $J^{\alpha}$ on morphisms $\alpha: W \longrightarrow W^{\prime}$ in $h \mathscr{R} O(G)$. Of course, in cohomology, $J_{G}^{0}(X)$ is the represented functor $[X, J]_{G, B}$ of $X$. Brown's representability applies just as it does nonequivariantly.

TheOREM 21.2.3. A reduced cohomology theory on ex-G-spaces over $B$ is represented by an $\Omega-G$-prespectrum $J$, unique up to non-unique equivalence. A cohomology theory on $G$-spectra over $B$ is represented uniquely by an orthogonal $G$-spectrum over $B$ in $H_{o} G \mathscr{S}_{B}$.

Proof. For the ex- $G$-space level, we use Brown representability in $\operatorname{Ho} G \mathscr{K}_{B}$ to obtain $J(V)$ such that $J_{G}^{V}(X) \cong[X, J(V)]_{G, B}$. Suspension isomorphisms give weak equivalences $J(V) \longrightarrow \Omega^{W} J(V \oplus W)$. The axioms imply that we lose no information if we restrict attention to a cofinal sequence in a skeleton of $\mathscr{R} O(G)$, and we then have an $\Omega$ - $G$-prespectrum defined on an indexing sequence. By our general theory, it is weakly equivalent to the underlying prespectrum of an orthogonal $G$-spectrum. Now we can use Lemma 21.1.3 to obtain the functoriality in $V$ and (tediously) verify the compatibility and transitivity axioms. The spectrum level version is easier since we need only use Brown representability in $\operatorname{Ho} G \mathscr{S}_{B}$ to represent $J_{G}^{0}$.

The following corollary is standard, but powerful. Many $R O(G)$-graded theories on $G$-spectra, such as the ordinary theory associated to a Mackey functor, arise most naturally by applying it.

Corollary 21.2.4. A $\mathbb{Z}$-graded cohomology theory on $G$-spectra over $B$ is represented by a $G$-spectrum over $B$ and therefore extends to an $R O(G)$-graded cohomology theory on $G$-spectra over B.

\subsection{Change of base and equivariant cofficient systems}

Change of base in homology and cohomology behave in the same way equivariantly as nonequivariantly, giving generalizations of Proposition 20.2.6 and, with an equivariant reinterpretation of the $E_{2}$-term, Theorem 20.4.1. The first of these impliesgeneralizations of Corollary 20.2.7, to be discussed in the next section, and Corollary 20.3.1. However, the latter should be interpreted in terms of the $G$-maps $\tilde{b}: G / G_{b} \longrightarrow B$ rather than the $G_{b}$-maps $b: * \longrightarrow B$, since otherwise its last statement fails for general compact Lie groups in view of the Wirthmüller isomorphism. 
Looking at the last statement of Corollary 20.3.1, it seems reasonable to interpret the groups

$$
J_{V}^{G}\left(\tilde{b}_{!} S_{G / G_{b}}\right) \text { and } J_{G}^{V}\left(\tilde{b}_{!} S_{G / G_{b}}\right),
$$

where $\tilde{b}$ is thought of as a map $G / G_{b} \longrightarrow B$, as the coefficient groups of the homology and cohomology theories represented by $J$. However, it is notationally and conceptually preferable to work with general maps $p: G / H \longrightarrow B$, thought of both as maps which generate base change functors and as $G$-spaces $(G / H, p)$ over $B$. We then have the following equivariant analogue of the last statement of Corollary 20.3.1. Here $S_{G / H}$ is the sphere $G$-spectrum over $G / H$, that is $\Sigma_{G / H}^{\infty} S_{G / H}^{0}$.

Proposition 21.3.1. Let $p: G / H \longrightarrow B$ be a $G$-map with $p(e H)=b$ and let $V$ be a representation of $G$ regarded as a representation of $H$ by restriction. Then

$$
J_{V}^{G}\left(p_{!} S_{G / H}\right) \cong\left[S^{V}, r_{!} p^{*} J\right]_{G} \cong \pi_{V \ominus L(H)}^{H}\left(J_{b}\right),
$$

where $L(H)$ is the tangent $H$-representation of $G / H$ at $e H$, and

$$
J_{G}^{V}\left(p_{!} S_{G / H}\right) \cong\left[S^{-V}, r_{*} p^{*} J\right]_{G} \cong \pi_{-V}^{H}\left(J_{b}\right) .
$$

PROOF. For the first isomorphism, the equivariant version of Corollary 20.3.1(ii) gives

$$
J_{V}^{G}\left(p_{!} S_{G / H}\right) \cong\left(p^{*} J\right)_{V}^{G}\left(S_{G / H}\right)=\left[S^{V}, r_{!}\left(p^{*} J \wedge_{G / H} S_{G / H}\right)\right]_{G} \cong\left[S^{V}, r_{!} p^{*} J\right]_{G},
$$

where we have used that $S_{G / H}$ is the unit for the smash product over $G / H$. Since $J_{b} \simeq\left(p^{*} J\right)_{e H}$, we have $r_{!} p^{*} J \simeq \iota_{!} p^{*} J=G_{+} \wedge_{H} J_{b}$ by the change of group isomorphism described in $\S 2.3$ and proven in stable categories in Proposition 14.3.3. The Wirthmüller isomorphism (19.1.3) gives $G_{+} \wedge_{H} J_{b} \simeq F_{H}\left(G_{+}, \Sigma^{L(H)} J_{b}\right)$, and this implies that

$$
\left[S^{V}, r_{!} p^{*} J\right]_{G} \cong \pi_{V}^{H}\left(\Sigma^{L(H)} J_{b}\right)=\pi_{V \ominus L(H)}^{H}\left(J_{b}\right)
$$

For the second isomorphism, Corollary 20.3.1(ii) gives the first of the isomorphisms

$$
\begin{aligned}
J_{G}^{V}\left(p_{!} S_{G / H}\right) & \cong\left(p^{*} J\right)_{G}^{V}\left(S_{G / H}\right) \cong\left[S_{G / H}^{-V}, F_{G / H}\left(S_{G / H}, p^{*} J\right)\right]_{G, G / H} \\
& \cong\left[S_{G / H}^{-V}, p^{*} J\right]_{G, G / H} \cong\left[S^{-V}, r_{*} p^{*} J\right]_{G} .
\end{aligned}
$$

Here we have used a unit isomorphism, the isomorphism $S_{G / H}^{-V} \simeq r^{*} S^{-V}$ and adjunction. The cited results on change of groups give $r_{*} p^{*} J \simeq \iota_{*} J_{b}=F_{H}\left(G_{+}, J_{b}\right)$, and therefore $\left[S^{-V}, r_{*} p^{*} J\right]_{G} \cong \pi_{-V}^{H}\left(J_{b}\right)$.

Just as nonequivariantly, since $S_{G / H} \simeq p^{*} S_{B}$, the counit maps $p_{!} p^{*} S_{B} \longrightarrow S_{B}$ induce maps

$$
J_{*}\left(p_{!} S_{G / H}\right) \longrightarrow J_{*}\left(S_{B}\right) \text { and } J^{*}\left(S_{B}\right) \longrightarrow J^{*}\left(p_{!} S_{G / H}\right)
$$

which can be viewed as comparisons of fiberwise coefficient systems and global coefficient groups.

To explain the coherence of these systems as $p$ varies, recall from tom Dieck $[46,10.7]$ or $[37,1.1]$ that there is an equivariant fundamental groupoid $\Pi_{G} B$. Its objects are the pairs $(G / H, p)$. Its morphisms

$$
(\alpha, \omega):(G / H, p) \longrightarrow(G / K, q)
$$

consist of a $G$-map $\alpha: G / H \longrightarrow G / K$ together with an equivalence class of paths $p(e H) \longrightarrow q \alpha(e H)$ in $B^{H}$. We interpret such a path as a homotopy $h: G / H \times$ $I \longrightarrow B$ from $p$ to $q \circ \alpha$, and we interpret the relevant homotopies $k: h \simeq h^{\prime}$ 
between paths as maps $k: G / H \times D \longrightarrow B$, where $D$ is a disk with two vertices and two boundary semi-circles, as in the proof of Proposition 20.3.2. Composition is induced by composition of maps of orbits and the usual composition of paths, or by composition of homotopies under our reinterpretation. There is an evident projection from $\Pi_{G} B$ to the orbit category $\mathscr{O}_{G}$, and the fiber over $G / H$ is isomorphic to the nonequivariant fundamental groupoid of $B^{H}$. It is important to remember that the name "fundamental groupoid" is a misnomer here, since not all maps in $\Pi_{G} B$ are isomorphisms. However, a morphism $(\alpha, \omega)$ is the composite of the isomorphism (id, $\omega):(G / H, p) \longrightarrow(G / H, q \circ \alpha)$ and the map $(\alpha, c):(G / H, q \circ \alpha) \longrightarrow$ $(G / K, q)$, where $c$ is a constant path. Taking this into account, we see that the proof of Proposition 20.3.2 applies directly to give the following precise analogue.

Proposition 21.3.3. For ex-G-spaces $X$ over $B$, there is a (covariant) functor $C(X)$ from $\Pi_{G} B$ to the homotopy category $H o G \mathscr{K}_{B} / X$ of ex-G-spaces over $B$ with augmentations to $X$ that sends $p: G / H \longrightarrow B$ to $p ! p^{*} X$ with augmentation given by the counit $p_{!} p^{*} X \longrightarrow X$ of the $\left(p_{!}, p^{*}\right)$ adjunction. The corresponding result for $G$-spectra $J$ over $B$ also holds, and $C\left(\Sigma_{B}^{\infty} X\right)$ is naturally isomorphic to the composite functor $\Sigma_{B}^{\infty} C(X)$.

Formulation of a version based strictly on fibers, like that of Corollary 20.3.3, would be awkward at best. It is more convenient to think of the points of $B$ as the spaces $(G / H, p)$ over $B$. Composing $C(J)$ with the functors $\left[S_{B}^{V},-\right]_{G, B}$ as $V$ varies, we obtain a kind of algebraic coefficient system associated to the cohomology theory $J_{G}^{*}$. However, there are several variants of the equivariant fundamental groupoid and of this definition that are necessary to develop a theory adequate to the applications. In fact, a full treatment must take account of versions of "representations" that are defined on $\Pi_{G} B$ and are built out of $G_{b}$-representations for each point $b \in B$. Such parametrized representations are specified and studied in $[37,41]$.

\subsection{Duality theorems and orientations}

As in Proposition 20.5.1, for $G$-spectra $X$ and $J$ over $B$ we have equivalences of $G$-spectra

$$
r_{!}\left(J \wedge_{B} X\right) \simeq J \odot t X \quad \text { and } \quad r_{*} F_{B}(X, J) \simeq X \triangleright J .
$$

We can therefore reinterpret our definitions of homology and cohomology as

$$
J_{*}^{G}(X)=\pi_{*}^{G}(J \odot t X) \quad \text { and } \quad J_{G}^{*}(X)=\pi_{-*}^{G}(X \triangleright J) .
$$

When $(X, Y)$ is a dual pair, $J \odot t Y \simeq X \triangleright J$ and the Costenoble-Waner analogue of Spanier-Whitehead duality applies to our $R O(G)$-graded theories. We restate it equivariantly for emphasis.

Theorem 21.4.3 (Costenoble-Waner duality). Let $X$ be a Costenoble-Waner dualizable $G$-spectrum over B with Costenoble-Waner dual $Y$. Then

$$
J_{*}^{G}(Y) \cong J_{G}^{-*}(X) \text {. }
$$

Here and below, $-(V \ominus W)=W \ominus V$. This allows us to interpret Example 0.0.2 equivariantly. The (reduced) "classical" equivariant parametrized homology and cohomology theories on ex- $G$-spaces or $G$-spectra $X$ over $B$ associated 
to nonparametrized equivariant homology and cohomology theories $k_{*}^{G}$ and $k_{G}^{*}$ are specified by

$$
k_{*}^{G, B}(X)=k_{*}^{G}\left(r_{!} X\right) \quad \text { and } \quad k_{G, B}^{*}(X)=k_{G}^{*}\left(r_{!} X\right) .
$$

If $k_{*}^{G}$ and $k_{G}^{*}$ are represented by an $\Omega$-G-prespectrum $k$, then

$$
k_{*}^{G, B}(X)=\pi_{*}^{G}\left(k \wedge r_{!} X\right) \quad \text { and } \quad k_{G, B}^{*}(X)=\pi_{-*}^{G} F(r ! X, k) .
$$

We have the $\Omega$-G-prespectrum $r^{*} k$ over $B$, and we have the relations

$$
k \wedge r_{!} X \simeq r_{!}\left(r^{*} k \wedge_{B} X\right) \text { and } F\left(r_{!} X, k\right) \simeq r_{*} F_{B}\left(X, r^{*} k\right) .
$$

By Corollary 20.2.7, interpreted equivariantly, we have the reinterpretation

$$
\left(r^{*} k\right)_{*}^{G}(X) \cong k_{*}^{G}\left(r_{!} X\right) \equiv k_{*}^{G, B}(X) \quad \text { and } \quad\left(r^{*} k\right)_{G}^{*}(X) \cong k_{G}^{*}\left(r_{!} X\right) \equiv k_{G, B}^{*}(X) .
$$

Now let $M$ be a smooth closed $G$-manifold. As in the nonequivariant case in $\S 20.3$, Theorem 19.1.5 gives that $k \wedge M_{+} \simeq S_{M} \triangleright\left(k \wedge S^{\tau}\right)$. Taking $X=S_{M}$ and $J=k \wedge S^{\tau}$ in (20.5.1), we obtain equivariant Poincaré duality in $R O(G)$-graded theories.

Theorem 21.4.8 (Poincaré duality - unoriented version). Let $k$ be a $G$-spectrum and $M$ be a smooth closed $G$-manifold. Then

$$
k_{*}^{G}\left(M_{+}\right) \cong\left(k \wedge S^{\tau}\right)_{G}^{-*}\left(S_{M}\right) .
$$

From here, a restricted version of equivariant orientation theory works in exactly the same way as orientation theory in the nonequivariant theory. We review it, and then say a few words about why it is too restrictive. Just as nonequivariantly,

$$
k \wedge X \simeq r^{*} k \wedge_{B} X
$$

for any $G$-space or $G$-spectrum $X$.

Definition 21.4.9. Let $V$ be a representation of $G$. Define a $V$-sphere fibration over $B$ to be an ex-G-fibration $(X, p, s)$ whose fibers $X_{b}$ are $G_{b}$-equivalent to $S^{V}$ (restricted to $G_{b}$ ) for all $b \in B$. The Thom complex of $X$ is $T X=r_{!} X=X / s(B)$. A $V$-manifold is a $G$-manifold whose tangent sphere bundle is a $V$-sphere bundle.

(i) A $k$-trivialization of $X$ is an equivalence

$$
k \wedge X \simeq k \wedge S_{B}^{V}
$$

of $G$-spectra over $B$.

(ii) Let $k$ be a commutative ring $G$-spectrum. A $k$-orientation of $X$ is a cohomology class $\mu \in k_{G}^{V}(T X)$ such that the restriction of $\mu$ to fibers,

$$
\mu_{b} \in k_{G_{b}}^{V}\left(T X_{b}\right) \cong k_{G_{b}}^{V}\left(S^{V}\right) \cong k_{G_{b}}^{0}\left(S^{0}\right)=\pi_{0}^{G_{b}}(k),
$$

is a unit in the ring $\pi_{0}^{G_{b}}(k)$ for each $b \in B$.

(iii) A $k$-trivialization or $k$-orientation of a smooth closed $V$-manifold $M$ is a $k$ trivialization or $k$-orientation of $S^{\tau}$.

As in the nonequivariant case, a $k$-oriented $V$-sphere fibration over $B$ is $k$ equivalent to the trivial $V$-sphere fibration $B \times S^{V}=S_{B}^{V}$ over $B$.

Proposition 21.4.10. A $k$-orientation $\mu$ of a $V$-sphere fibration $X$ induces a $k$-trivialization of $X$. 
For any "degree" $\alpha=W \ominus Z$, we have

$$
\left(k \wedge S_{B}^{V}\right)^{-\alpha}\left(S_{B}\right) \cong\left(r^{*} \Sigma^{V} k\right)^{-\alpha}\left(S_{B}^{0}\right) \cong k^{V-\alpha}\left(B_{+}\right),
$$

where $V-\alpha=(V \oplus Z) \ominus W$. Taking $B=M$ and applying the equivalence $\bar{\mu}$ to the right side of the unoriented version of the Poincaré duality theorem we obtain the restricted oriented version.

Theorem 21.4.11 (Poincaré duality- $V$-oriented version). Let $k$ be a commutative ring $G$-spectrum and $M$ be a $k$-oriented smooth closed $V$-manifold. Then

$$
k_{\alpha}\left(M_{+}\right) \cong k^{V-\alpha}\left(M_{+}\right) .
$$

The homology Thom isomorphism is also induced by the equivalence $\bar{\mu}$, and the cohomology Thom isomorphism is induced by the equivalence $\hat{\mu}$ given in the following dual version of Proposition 21.4.10.

Proposition 21.4.12. A k-orientation $\mu$ of a spherical fibration $X$ induces an equivalence of spectra over $B$

$$
\hat{\mu}: F_{B}\left(S_{B}^{V}, r^{*} k\right) \longrightarrow F_{B}\left(X, r^{*} k\right) .
$$

TheOrem 21.4.13 ( $V$-Thom isomorphism). If $X$ is a k-oriented $V$-sphere fibration over $B$, then there are canonical equivalences

$$
k \wedge T X \simeq k \wedge \Sigma^{n} B_{+} \quad \text { and } \quad F\left(\Sigma^{n} B_{+}, k\right) \simeq F(T X, k)
$$

and therefore canonical isomorphisms

$$
k_{\alpha}^{G}\left(B_{+}\right) \cong k_{\alpha+V}(T X) \quad \text { and } \quad k^{\alpha}\left(B_{+}\right) \cong k^{\alpha+V}(T X) .
$$

Remark 21.4.14. The diagram chase described in Remark 20.5.9 works equivariantly and shows that the cohomology Thom isomorphism sends $x \in k^{\alpha}\left(B_{+}\right)$to the cup product $x \mu \in k^{\alpha+V}(T X)$ induced by the Thom diagonal, and dually in terms of cap products in homology.

Remark 21.4.15. Relative versions of Poincaré duality follow from Theorems 19.1.6 and 19.1.9, exactly as in $\S 20.6$. Aside from the $R O(G)$-grading, the statements are identical, and we will not repeat them.

We regard the results above as giving a restricted version of orientation theory because the restriction to $V$-sphere fibrations (and $V$-manifolds) is unrealistic except under an unrealistically strong connectivity hypothesis, as we now explain.

Definition 21.4.16. A spherical $G$-fibration over $B$ is an ex- $G$-fibration $X$ such that each fiber $X_{b}$ is $G_{b}$-homotopy equivalent to $S^{V_{b}}$ for some $G_{b}$-representation $V_{b}$ (depending on $b$ ).

It is usual to define the dimension of $X$ to be the set of fiber representations $V_{b}$ or, more precisely, the set of $G_{b}$-homotopy types $S^{V_{b}}$. The following condition ensures that this dimension function defined on the points of $B$ is constant, so that $X$ is a $V$-sphere fibration for some $V$.

Definition 21.4.17. A $G$-space $B$ is $G$-connected if each of its fixed point spaces $B^{H}$ is non-empty and path connected.

Lemma 21.4.18. If $B$ is $G$-connected and $X$ is a spherical $G$-fibration over $B$, then $X$ is a $V$-sphere fibration for some $G$-representation $V$. 
Proof. If $c$ is a $G$-fixed point of $B$ and $X_{c}$ is $G$-homotopy equivalent to $S^{V}$, then every fiber $X_{b}$ is $G_{b}$-homotopy equivalent to $S^{V}$.

Therefore, for $G$-connected base spaces, the $V$-orientation theory above is entirely satisfactory. However, interesting $G$-manifolds $M$ are usually not $G$-connected, and then a more elaborate theory is needed in which orientations take all of the fiber representations $V_{b}$ into account. From a concrete geometric point of view, orientations of spherical $G$-fibrations are described in [37] in terms of parametrized representations of the fundamental groupoid $\Pi_{G} B$. However, it is far from obvious how to connect the geometric definition there to cohomology. From the manifold point of view, that question amounts to the problem of simplifying the cohomology side of the unoriented version of Poincaré duality when given an orientation of $S^{\tau}$.

It is natural to first tackle this question for "ordinary" theories, namely those whose $\mathbb{Z}$-graded parts satisfy the dimension axiom. That is the main theme of the work of Costenoble and Waner [41]. A major stumbling block tackled there is that, for infinite compact Lie groups, the represented dual of an ordinary cohomology theory is not ordinary, as was explained briefly in [118, XIII $\S 4]$. The reason is that orbits are not self-dual, as we have seen in the Wirthmüller isomorphism, so that the category of Mackey functors in which the coefficients of ordinary theories must live is not self-dual. Costenoble and Waner use our parametrized spectra to overcome such difficulties. They work out general Thom isomorphism and Poincaré duality theorems for ordinary $R O(G)$-graded cohomology theories, and they manage to do this using descriptions of the represented homology and cohomology theories in terms of chains and cochains.

In general, a $k_{G}^{*}$-orientation of $M$ should be a cohomology class in the Thom space of the tangent bundle that lives in a "degree" that depends on the parametrized dimension function of the tangent bundle. A naive version of such a theory is given in [119]. Much more work will be needed to reach a calculationally useful understanding of such an equivariant cohomological orientation theory.

REMARK 21.4.19. There are particularly interesting theories where orientation theory works well without such elaboration, such as equivariant $K$-theory and equivariant cobordism. The point is that it is possible for a spherical $G$-fibration that is not a $V$-sphere fibration to have a $k$-orientation in essentially the prescribed sense. For example, this often happens in equivariant $K$-theory, where the equivariant version of the Atiyah-Bott-Shapiro orientation [4] gives any equivariant complex $n$-plane $G$-bundle a $K$-orientation of dimension $2 n$, and similarly for spin-bundles and real K-theory; see French [67] for details. The reason this makes sense is that $K_{G}^{V} \cong K_{G}^{2 n}$ for any compact Lie group $G$ and any complex representation $V$ of dimension $n$. Such orientations still have the implications described above.

\subsection{Products and the representability of homology}

Products and slant products in equivariant parametrized homology and cohomology theories work exactly as in $§ 20.7$, and we shall not repeat the discussion; see also [41]. The starting point is that we have the evident pairing

$$
\pi_{V}^{G}(X) \otimes \pi_{W}^{G}(Y) \longrightarrow \pi_{V \oplus W}^{G}(X \wedge Y)
$$

for ordinary $G$-spectra $X$ and $Y$. The products were derived in $\S 20.7$ from the analogous nonequivariant pairing together with maps constructed in our bicategory 
of parametrized spectra and the commutativity isomorphism for the external smash product. Such maps work in exactly the same way equivariantly.

The representability of equivariant homology theories also works in the same way as in the nonequivariant theory. Here again, there are two approaches. First, Neeman's general result Theorem 20.8.1 implies that cohomology theories defined on compact objects, and therefore homology theories defined on compact objects, are representable provided an appropriate countability hypothesis is satisfied. The following equivariant version of Proposition 20.8.5 verifies the required hypothesis.

Proposition 21.5.2. If each $B^{H}$ has countably many path components and each homotopy group of each path component of $B$ is countable, then the category of compact objects in $\mathrm{HoG}_{B}$ is countable.

Proof. By [135, 1.7.27], there are only countably many conjugacy classes of (closed) subgroups of a compact Lie group $G$. We have the generating set of all $S_{H}^{n, b}$ specified in Definition 13.1.1, where $n$ runs over the integers, $b$ runs over the points of $B$, and $H$ runs over the subgroups of $G$. Observe that the $S_{H}^{n, b}$ for subgroups $H$ in the same conjugacy class are $G$-homeomorphic. By Proposition 21.3.3, if $\pi_{*}\left(X_{b}\right)=0$, then $\pi_{*}\left(X_{b^{\prime}}\right)=0$ for all $b^{\prime}$ in the same path component of some $B^{H}$ as $b$. Thus we obtain a countable detecting subset by choosing one $b$ from each path component of $B^{H}$, where $H$ runs through one subgroup in each conjugacy class $(H)$. We have

$$
\left[S_{H}^{m, a}, S_{K}^{n, b}\right]_{B} \cong \pi_{m}\left(\left(S_{K}^{n, b}\right)^{H}\right)
$$

and these homotopy groups of (derived) fibers are countable by the proof of Proposition 20.8.5.

Second, we could give an ad hoc telescope argument that works in general, in analogy with Theorem 20.8.6. We have not tried to work out complete details. The starting point of restriction to a simplicial complex should be replaced by restriction to a colimit of finite dimensional $G$-simplicial complexes, where a $G$-simplicial complex is a $G$-CW complex with simplicial orbit space under the induced triangulation. We can do that by Waner's result $[168,4.3]$ that any $G$-CW complex is homotopy equivalent to a $G$-simplicial complex. However, this is a less convenient starting point for the precise gluing that we described in the sketch proof of Theorem 20.8.6. That proof also relies on the spectral sequence argument of Theorem 20.4.1, but we have already noted that that result goes through with the same proof, when properly interpreted. Note that all that is needed is a spectral sequence for the $\mathbb{Z}$-graded part of our cohomology theory, but we point out the following digressive observation.

REMARK 21.5.3. Spectral sequences in $R O(G)$-graded equivariant cohomology generally appear as $R O(G)$-graded families of spectral sequences, one for each $\alpha=$ $V \ominus W$ such that $V$ and $W$ contain no trivial summands. The values of the original theory in gradings $\alpha+n, n \in \mathbb{Z}$, form a $\mathbb{Z}$-graded theory, and one finds a family of spectral sequences, one for each such $\alpha$, together with suspension isomorphisms relating the spectral sequences as $\alpha$ varies.

\subsection{Fiberwise parametrized homology and cohomology}

The symmetric bicategories $G \mathscr{E} x_{B}$ discussed in Chapter 19 lead to a generalization of our parametrized homology and cohomology theories in which the implicit 
base object $*$ is replaced by a general $G$-space $B$. Costenoble-Waner duality also generalizes. We start with a fixed $G$-space $(K, p)$ over $B$ and replace the base change functors induced by $r: B \longrightarrow *$ used previously with the base change functors induced by $p: K \longrightarrow B$. We omit the fixed group $G$ from the notations, regarding it as implicit in the structure of $K$ as a $G$-space.

Definition 21.6.1. Let $(K, p)$ be a $G$-space over $B$ and let $J$ be a $G$-spectrum over $K$. For $G$-spectra $X$ over $K$ and representations $V$ of $G$, define

$$
J_{V}^{(K, p)}(X)=\left[S_{B}^{V}, p_{!}\left(J \wedge_{K} X\right)\right]_{B}
$$

and

$$
J_{(K, p)}^{V}(X)=\left[S_{B}^{-V}, p_{*} F_{K}(X, J)\right]_{B} .
$$

Using standard commutation relations, we obtain suspension isomorphisms

$$
\sigma^{W}: J_{V}^{(K, p)}(X) \rightarrow J_{V \oplus W}^{(K, p)}\left(\Sigma_{K}^{W} X\right) \text { and } \sigma^{W}: J_{(K, p)}^{V}(X) \rightarrow J_{(K, p)}^{V \oplus W}\left(\Sigma_{K}^{W} X\right)
$$

exactly as in Definition 21.2.2, and we extend the theory to formal differences $V \ominus W$ by

$$
J_{V \ominus W}^{(K, p)}(X)=J_{V}^{(K, p)}\left(\Sigma_{K}^{W} X\right) \quad \text { and } \quad J_{(K, p)}^{V \ominus W}(X)=J_{(K, p)}^{V}\left(\Sigma_{K}^{W} X\right) .
$$

With evident modifications, all of the basic properties of equivariant parametrized homology and cohomology theories carry over. Of course, when $G=e$, we obtain integer graded homology and cohomology theories this way.

The following generalization of Proposition 17.4.2 makes clear how duality works in this context. The result is obtained by specializing Proposition 19.2.8 to cases where two out of the three $G$-spaces over $B$ used there are $B$ itself.

Proposition 21.6.4. Let $(K, p)$ be a $G$-space over $B$, let $X$ and $Y$ be $G$-spectra over $K$ and let $Z$ be a $G$-spectrum over $B$. Thinking of $K$ as $B \times{ }_{B} K$ and $B$ as $B \times{ }_{B} B$, regard $X$ and $Y$ as 1-cells $K-B \rightarrow B$ and regard $Z$ as a 1-cell $B-B \rightarrow B$. Then, as $G$-spectra over $B$,

$$
Y \odot_{B} t X \simeq p_{!}\left(Y \wedge_{K} X\right) \quad \text { and } \quad t Y \triangleleft_{B} t X \simeq p_{*} F_{K}(X, Y) \simeq X \triangleright_{B} Y .
$$

As spectra over $K$,

$$
t X \odot_{B} Z \simeq X \bar{\wedge}_{B} Z, \quad Z \odot_{B} X \simeq Z \bar{\wedge}_{B} X
$$

and

$$
Z \triangleleft_{B} X \simeq F_{K}\left(X, p^{*} Z\right) \simeq t X \triangleright_{B} Z .
$$

As spectra over $K \times_{B} K$,

$$
t Y \odot_{B} X \simeq Y \bar{\wedge}_{B} X
$$

In particular, we can reinterpret our definitions of homology and cohomology in terms of $J \odot_{B} t X$ and $X \triangleright_{B} J$. We call dual pairs in $G \mathscr{E} x_{B}$ "B-dual pairs". When $(X, Y)$ is a $B$-dual pair of $G$-spectra over $K, J \odot_{B} t Y \simeq X \triangleright_{B} J$. The following generalized version of the Costenoble-Waner analogue of Spanier-Whitehead duality follows directly.

THEOREM 21.6.5 (Costenoble-Waner B-duality). Let X be a Costenoble-Waner $B$-dualizable $G$-spectrum over $K$ with Costenoble-Waner B-dual $Y$. Then, for a $G$ spectrum $J$ over $K$,

$$
J_{*}^{(K, p)}(Y) \cong J_{(K, p)}^{-*}(X) .
$$




\subsection{Fiberwise Poincaré duality and orientations}

We now place ourselves in the context of $\S 19.6$. Thus we fix an extension $\Gamma$ of $G$ by $\Pi$ and a $\Pi$-free $\Gamma$-space $P$ with base space $B=P / \Pi$. To focus on the nonequivariant version, the reader should take $G=e$ and thus $\Gamma=\Pi$. Equivariantly, the special case $\Gamma=G \times \Pi$ is of greatest interest. We also fix a smooth closed $\Gamma$-manifold $M$ of dimension $n$ and let $E=P \times_{\Pi} M$, so that we have a $G$-bundle $p: E \longrightarrow B$ with fiber $M$. Then $S_{M}$ is Costenoble-Waner dualizable with (right) dual $T_{M}=\Sigma_{M}^{\infty} \Sigma^{-V} S^{\nu_{M}}$, where $\nu_{M}$ is the normal $\Gamma$-bundle of an embedding of $M$ in a $\Gamma$-representation $V$. We factor $r: E \longrightarrow *$ as $\bar{r} \circ p, \bar{r}: B \longrightarrow *$.

With our definitions, fiberwise homotopical Poincaré duality as given in Theorem 19.6.1 does not compute homology in terms of cohomology; the cohomological side of that result is a $G$-spectrum over $B$ that is given by the functor $S_{p} \triangleright(-)$ on $G$-spectra over $E$, and that does not fit into either our original or our generalized version of parametrized cohomology. In the case $G=e$, all we obtain is the following result.

THEOREM 21.7.1. Let $M$ be a smooth closed П-manifold of dimension $n$ and let $k$ be a spectrum. Then

$$
k_{*}\left(E_{+}\right) \cong \pi_{*}\left(\bar{r}_{!} p_{*}\left(k \wedge \mathbb{P}_{M} S^{\tau}\right)\right) .
$$

If $k$ is a commutative ring spectrum and $\mathbb{P}_{M} S^{\tau}$ is $k$ orientable, then the right side is isomorphic to $\pi_{*}\left(\bar{r}_{!} p_{*}\left(k \wedge S_{E}^{n}\right)\right)$.

The right side is a mixture of the homological $\bar{r}_{\text {! }}$ and the cohomological $p_{*}$. That is perhaps all that can be expected since $B$ can be infinite dimensional. We now specialize to the case when $B$ is a smooth closed manifold. Then, using the fiberwise description of the tangent bundle of $E$ and its suspension spectrum given in (19.6.4) and (19.6.5), Theorems 19.1.5 and 19.6.3 give the following isomorphisms.

THEOREM 21.7.2. Let $M$ be a smooth closed $\Gamma$-manifold and $B$ be a smooth closed $G$-manifold, and let $J$ be a $G$-spectrum over $B$. Then

$$
J_{*}\left((E, p)_{+}\right) \cong\left(p^{*} J \wedge_{E} \mathbb{P}_{M} S^{\tau_{M}} \wedge_{E} p^{*} S^{\tau_{B}}\right)^{-*}\left(S_{E}\right) \cong\left(p^{*} J \wedge_{E} S^{\tau_{E}}\right)^{-*}\left(S_{E}\right) .
$$

When $J=\bar{r}_{*} k=k_{B}$ for a $G$-spectrum $k$, this takes the form

$$
k_{*}^{B}\left(E_{+}\right) \cong\left(k \wedge \mathbb{P}_{M} S^{\tau_{M}} \wedge_{E} p^{*} S^{\tau_{B}}\right)^{-*}\left(S_{E}\right) \cong\left(k \wedge S^{\tau_{E}}\right)^{-*}\left(S_{E}\right)
$$

Of course, with the first isomorphism, our discussions of nonequivariant and equivariant Poincaré duality in $\S 20.4$ and $\S 21.4$ apply directly. We shall focus on the second isomorphism and its use to prove the $k$-orientability needed for a conclusion in terms of nonparametrized cohomology. We focus on the nonequivariant case $G=e$, but, since it is only the $\Pi$-equivariance that matters in the discussion below, the general case would introduce no added difficulty. The starting point is clear.

Proposition 21.7.3. Let $k$ be a commutative ring spectrum. If $B$ and $P_{M} S^{\tau_{M}}$ are $k$-oriented, then $E=P \times_{\Pi} M$ is $k$-oriented and therefore $k_{*}\left(E_{+}\right) \cong k^{n+t-*}\left(E_{+}\right)$, where $n=\operatorname{dim} M$ and $t=\operatorname{dim} B$.

Proof. Recall that $\tau_{E} \cong P_{M} \tau_{M} \oplus p^{*} \tau_{B}$. Since pullbacks and Whitney sums of $k$-oriented vector bundles are $k$-oriented, the conclusion is immediate.

These results focus attention on the problem of determining when $P_{M} S^{\tau_{M}}$ is $k$-orientable or, more generally, when $P_{F} X$ is $k$-orientable, where, with $G=e, F$ 
is a $\Pi$-space and $X$ is a spherical $\Pi$-fibration of dimension $n$ over $F$. Thus we do not require $F$ to be a manifold and do not require $X$ to come from a vector bundle. Note the role of equivariance. It is clearly not enough that $X$ be nonequivariantly $k$-orientable, as the Klein bottle example again shows. We give two solutions, a more calculational one based on a universal example for $P$ and an equivariant one that illustrates conceptual points about the relationship between "genuine" and "naive" П-spectra.

We owe the first to Shmuel Weinberger. Let $f: B \longrightarrow B \Pi$ classify our principal $\Pi$-bundle $P$, so that we have a pullback diagram

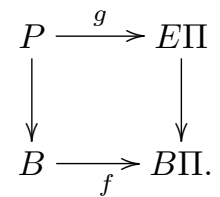

It gives rise to pullback diagrams

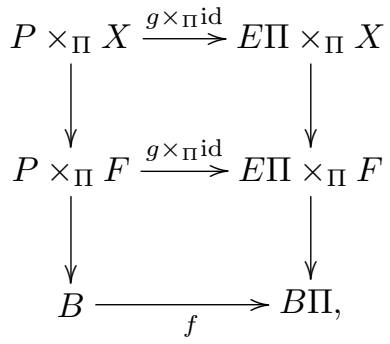

as we see by first checking the lower square and then the upper one. The following result is an immediate consequence.

Proposition 21.7.4. If the Borel construction spherical fibration

$$
E \Pi \times_{\Pi} X \longrightarrow E \Pi \times_{\Pi} F
$$

induced by a spherical $\Pi$-fibration $X \longrightarrow F$ is $k$-orientable, then the spherical fibration $P \times_{\Pi} X \longrightarrow P \times_{\Pi} F$ is k-orientable for every principal $\Pi$-bundle $P$ over any base space $B$.

The Borel construction is relatively calculable, so this is a reasonable criterion. However, a less useful criterion based directly on $X$ may be illuminating. We may regard $k$ as a "naive" $\Pi$-spectrum with trivial $\Pi$-action, "naive" meaning indexed on the ח-trivial universe. Equivariant duality theory does not work in that universe, and equivariant orientation theory works poorly, as we now explain. Working naively, the theory represented by $k$ satisfies $k^{n}(X / \Pi) \cong k_{\Pi}^{n}(X)$ for any $\Pi$ space or $\Pi$-spectrum $X$. We have the Thom $\Pi$-space $T X$. Consider a cohomology class $\nu \in k^{n}((T X) / \Pi) \cong k_{\Pi}^{n}(T X)$. Regard $\nu$ as a map

$$
\nu: \Sigma^{\infty} T X \longrightarrow \Sigma^{n} k
$$

of naive $\Pi$-spectra. Since $T X=r_{!} X, r: F \longrightarrow *, \nu$ has an adjoint map

$$
\tilde{\nu}: \Sigma_{F}^{\infty} X \longrightarrow \Sigma^{n} r^{*} k \simeq k \wedge S_{F}^{n}
$$

of naive $\Pi$-spectra over $F$. Smashing with $k$ and using the product on $k$, we obtain

$$
\bar{\nu}: k \wedge \Sigma_{F}^{\infty} X \longrightarrow k \wedge k \wedge S_{F}^{n} \longrightarrow k \wedge S_{F}^{n} \text {. }
$$


DeFinition 21.7.5. For a commutative ring spectrum $k$, a naive $k$-orientation of a spherical $\Pi$-fibration $X$ of dimension $n$ over $F$ is a class $\nu \in k^{n}(T X / \Pi)$ such that $\bar{\nu}: k \wedge \Sigma_{F}^{\infty} X \longrightarrow k \wedge S_{F}^{n}$ is an equivalence of naive $\Pi$-spectra over $F$.

This definition may seem reasonable from the parametrized point of view, but it is calculationally daunting. A map of $\Pi$-spectra over $F$ is an equivalence if and only if its induced maps of fibers are equivalences. The restriction of $\nu$ to the fiber $X_{f}$ over $f$ is an element of $k_{\Pi}^{n}\left(X_{f}\right) \cong k^{n}\left(X_{f} / \Pi_{f}\right)$. Since the orbit spaces here need not be spheres and we do not have the suspension by representations isomorphisms to transfer our question to one about units in $k^{0}$, it is not clear how to compute from this (orbifold theoretic) definition. However, using the definition $\mathbb{P}_{M}=P_{M} \iota^{*}$, where $\iota^{*}$ is the relevant change of universe, and the commutation relations of Proposition 15.4.4 for suspension spectrum functors, we obtain the following conclusion.

Proposition 21.7.6. If $X \longrightarrow F$ is a naively $k$-orientable spherical $\Pi$-fibration, then $P \times_{\Pi} X \longrightarrow P \times_{\Pi} F$ is a $k$-orientable spherical fibration for any principal $\Pi$ bundle over any base space $B$. 



\section{CHAPTER 22}

\section{Twisted theories and spectral sequences}

\section{Introduction}

This chapter has two goals. We describe certain general types of "twisted" parametrized homology and cohomology theories, and we give further spectral sequences for the calculation of parametrized homology and cohomology.

In particular, we shall locate twisted $K$-theory as a special case of a twisted cohomology theory. There is already a substantial body of work in twisted $K$-theory, and our work is not a contribution to that subject, but rather a contextualization that makes many of its properties transparent. It is well-known that this example belongs to parametrized cohomology theory and that it requires more adequate foundations than were previously available. Our exposition makes folklore precise and is in large part based on Atiyah and Segal [5], especially the sketch at the end of their $\S 3$. In the equivariant case, we rely on details in the general stack-theoretic treatment of $\mathrm{Tu}, \mathrm{Xu}$, and Laurent-Gengoux [164]. We make no claim to originality.

We specialize our general framework to homology and cohomology theories that are represented by bundles of spectra in $\S 22.1$. We give some relevant remarks on automorphism monoids of spectra in $\S 22.2$. In particular, we give some observations about the "unit monoid" $G L_{1}(k)$ for a symmetric or orthogonal ring $\Omega$-spectrum $k$. Especially for commutative ring spectra $k$, there is quite a bit of confusion in the literature about this space. Remarks here and in $\$ 23.6$ help to sort this out. We show how naturally twisted $K$-theory appears as a twisted parametrized cohomology theory in $\S 22.3$. It will be apparent that this is indeed a very special case. There are many analogous examples that await exploration.

In $§ 22.4$, we give a spectral sequence associated to a simplicial parametrized space. Actually, to illustrate ideas, we show how this works in general in any well-grounded topological model category. It is folklore that the spectral sequence applies to Reedy cofibrant simplicial objects in any good model category, but focus on the Reedy model structure is overly restrictive in topological situations. Using base change functors to describe a sheaf theoretic perspective, we specialize this spectral sequence to obtain Čech type local to global, or descent, spectral sequences for the calculation of parametrized homology and cohomology theories in $\S 22.5$.

Douglas [52] used a Rothenberg-Steenrod type spectral sequence to give computations in twisted $K$-theory. We show how to construct such spectral sequences in general in $\S 22.6$. This is another specialization of the simplicial spectral sequence of $\S 22.4$.

The Eilenberg-Moore spectral sequence is a kind of dual to the RothenbergSteenrod spectral sequence. It was viewed by Hodgkin [74] and Smith [153,154] as a Künneth spectral sequence in parametrized homology. In $\S 22.7$, we use parametrized spectra to give a new and generalized version of their construction. It is related 
to theirs in much the same way that versions of the Adams spectral sequence constructed using spectra are related to the original construction using spaces, before the introduction of the stable homotopy category. The new construction raises interesting questions of convergence, but we will not pursue them here. It is also so closely parallel to the construction of the Adams spectral sequence as to suggest a common generalization (perhaps generalizing [102]), but we shall not pursue that idea either.

\subsection{Twisted homology and cohomology theories}

Let $G$ be a compact Lie group. The reader may take $G$ to be the trivial group, but there is no gain in simplicity. We restrict our general bundle theoretic context to the most important special case by taking $\Gamma=G \times \Pi$ (and, in the context of $\S 15.3, F=*$ ). Let $k$ be a $\Gamma$-spectrum indexed on a $\Pi$-trivial $\Gamma$-universe. As we noted in $\$ 15.3$, when working in a $\Pi$-trivial universe there is no reason to restrict $\Pi$ to be a compact Lie group. It can be any topological group, say locally compact and well-based, as always holds in practice. The generality is important in twisted $K$-theory, where the relevant group is an appropriate model for $K(\mathbb{Z}, 2)$, namely the projective unitary group.

We have a theory of principal $(G, \Pi)$-bundles, where the "structural group" is $\Pi$ and the "ambient group" is $G$. Such a bundle is just a proper $(G \times \Pi)$-space, with free action by $\Pi$; see $\S 3.2$ and [91]. Now take $P$ in our bundle construction to be a universal principal $(G, \Pi)$-bundle, denoting it by $E_{G} \Pi$. This means that the fixed point space $\left(E_{G} \Pi\right)^{\Lambda}$ is contractible for all closed subgroups $\Lambda$ of $G \times \Pi$ such that $\Lambda \cap \Pi$ is trivial; such subgroups are all of the form $\Lambda=H_{\rho}=\{(h, \rho(h) \mid h \in H\}$ for some subgroup $H$ of $G$ and homomorphism $\rho: H \longrightarrow \Pi$. Then the orbit $G$ space $B_{G} \Pi=E_{G} \Pi / \Pi$ is a classifying space for principal $(G, \Pi)$-bundles. Of course some models for $E_{G}(\Pi)$ and hence $B_{G}(\Pi)$ may be more convenient than others in particular examples. In the nonequivariant case $G=e$, we are starting with a universal principal $\Pi$-bundle $E \Pi \longrightarrow B \Pi$.

Definition 22.1.1. Define $k_{\Pi}$ to be the $G$-spectrum $E_{G} \Pi \times_{\Pi} k$ over $B_{G} \Pi$. It is a parametrized $G$-spectrum indexed on a complete $G$-universe and represents parametrized (unreduced) $R O(G)$-graded homology and cohomology theories on the homotopy category $\operatorname{Ho} G \mathscr{K} / B_{G} \Pi$ of $G$-spaces over $B_{G} \Pi$. We write $k_{*}(X, p)$ and $k^{*}(X, p)$ for the values of this theory on a $G$-space $(X, p)$ over $B_{G} \Pi$.

We use unreduced theories on spaces over $B$, rather than reduced theories on ex-spaces, to conform with the literature. It seems natural to do so since our focus here is on spaces, in particular manifolds, rather than spectra. However, an unreduced theory determines and is determined by the corresponding reduced theory, and the full panoply of stable techniques is available to us.

Note that $p$ is an actual $G$-map $p: X \longrightarrow B$, not a homotopy class. We may think of $p$ as a principal $(G, \Pi)$-bundle over $X$, namely the pullback of $E_{G} \Pi$ along $p$. If we call the resulting bundle $P$ and write

$$
k_{*}^{P}(X)=k_{*}(X, p) \quad \text { and } \quad k_{P}^{*}(X)=k^{*}(X, p),
$$

then our notations agree with those of Atiyah and Segal [5] (except that they did not consider homology). It is reasonable to think of $p$ as the homotopy class that determines the equivalence class of $P$. This makes sense since the twisted 
$k$-homology or $k$-cohomology of $(X, p)$ depends only on the homotopy class of $p$ (by construction or the weak equivalence axiom).

The altered notations lead to an altered viewpoint. We may view $X$ as a space over itself, and then $p_{!} X=(X, p)$ as a space over $B$. By Proposition 20.2.6(ii), read equivariantly and in the unreduced sense, we then have

$$
k_{*}(X, p) \cong\left(p^{*} k_{\Pi}\right)_{*}(X) \quad \text { and } \quad k^{*}(X, p) \cong\left(p^{*} k_{\Pi}\right)^{*}(X) .
$$

On the right hand sides of these isomorphisms,

$$
p^{*} k_{\Pi} \cong P \times_{\Pi} k
$$

is a bundle of spectra over $X$ twisted by $P$. To conform with (22.1.2), we could denote it alternatively by $k^{P}$ when considering homology or $k_{P}$ when considering cohomology. Unravelling the definitions, we see that $k_{P}^{0}(X)$ is just the set of homotopy classes of sections of the bundle $P \times_{\Pi} k_{0}$ over $X$. Note that from this point of view there is no need to insist on using all maps $X \longrightarrow B_{G} \Pi$. It may be more sensible to restrict attention to a preferred class of principal $(G, \Pi)$-bundles, as seems to be appropriate in the equivariant case of twisted $K$-theory.

We think of our original definition as extrinsic, given by theories represented by a $G$-spectrum over $B_{G} \Pi$ that is independent of $X$. We think of (22.1.3) as giving the intrinsic form of the theory, susceptible of direct geometric analysis starting with $X$. To see the idea, think of classical nonequivariant $K$-theory. It is given extrinsically as a represented theory with values given by homotopy classes of maps into $B U \times \mathbb{Z}$, which is the zeroth space of the $K$-theory spectrum. It is given intrinsically by starting with the Grothendieck group of vector bundles over $X$. We remark that, as in this example, the extrinsic version of homology can be most convenient even when cohomology admits an intrinsic description.

Applying Remark 20.4.2 to (22.1.3), but using the notations here, we obtain the following version of the Atiyah-Hirzebruch spectral sequence for the calculation of these twisted theories in the nonequivariant case.

Proposition 22.1.5. Let $X$ be a $C W$ complex. Then there are spectral sequences

and (with conditional convergence)

$$
E_{p, q}^{2}=H_{p}\left(X ; \mathscr{L}_{q}\left(X, p^{*} k_{\Pi}\right)\right) \Longrightarrow k_{*}(X, p)
$$

$$
E_{2}^{p, q}=H^{p}\left(X ; \mathscr{L}^{q}\left(X, p^{*} k_{\Pi}\right)\right) \Longrightarrow k^{*}(X, p) .
$$

\subsection{Automorphism monoids of spectra and $G L_{1}(k)$}

We change our point of view slightly. We start with a nonparametrized $G$ spectrum $k$ and ask what it means to have an action by $\Pi$ on $k$. As before, $G$ is a compact Lie group but $\Pi$ can be general. We view such an action as a "twisting" of $k$ by $\Pi$. Since we are using naive $\Pi$-spectra, such an action consists of a continuous family of automorphisms of the $G$-spectrum $k$ by elements of $\Pi$. It is given by a homomorphism, usually taken to be a monomorphism, $t: \Pi \longrightarrow \operatorname{Iso}(k)$, where Iso $(k)$ is the topological group of $G$-equivariant automorphisms of $k$.

It is natural to ask how sensitive this is to the choice of one's category of spectra and to the choice of a spectrum within its homotopy type (or weak homotopy type). Since reasonable categories of spectra have a faithful forgetful functor to prespectra and fibrant prespectra are $\Omega$-prespectra, it seems reasonable to work in the category of excellent (nonparametrized) $G$-prespectra of Definition 13.2.2, since those can 
be expected to have the largest automorphism groups. We recall that excellent $G$-spectra are $\Omega$ - $G$-prespectra whose structure maps are $h$-cofibrations and whose spaces are well-based, compactly generated, and of the homotopy types of $G$-CW cmplexes. A weak equivalence between such prespectra is a homotopy equivalence, by Proposition 13.2.5.

There is an evident monoid that is more sensible from a homotopical point of view than the group Iso $(k)$ and is homotopy invariant. In any reasonable topological category with a subcategory of weak equivalences, we can form the topological monoid Aut $(\mathrm{X})$ of weak self-equivalences of any object $X$, which we shall write as $H X$ for the moment. In [113, §3], it is proven in the context of based or unbased spaces that if $f: X \longrightarrow Y$ is a weak equivalence, then there is a natural zigzag

$$
B H X \longrightarrow B H f \longleftarrow B H Y
$$

of weak equivalences, where $H f$ is a certain topological category with two objects constructed from $f$. It follows that $H X, \Omega B H X, \Omega B H Y$, and $H Y$ are weakly equivalent as $A_{\infty}$-spaces and therefore that $H X$ and $H Y$ are weakly equivalent as topological monoids. Inspection of the argument in [113, §3] shows that it applies to quite general topological categories and in particular applies to prove the following result, in which $\operatorname{Aut}(k)$ denotes the monoid of self-equivalences of an excellent $G$ prespectrum $k$.

TheOREM 22.2.1. Let $f: k \longrightarrow \ell$ be a weak equivalence of excellent $G$-prespectra. Then there is a natural zigzag of weak equivalences connecting BAut $(k)$ to BAut $(\ell)$.

Suppose we have a topological group $\Pi$ of the homotopy type of a CW complex, a homomorphism of topological monoids $\Pi \longrightarrow M$ (for example $M=\operatorname{Aut}(k)$ ) and a zigzag of weak equivalences connecting $B M$ to $B N$ for another topological monoid $N$ (for example $N=\operatorname{Aut}(\ell)$ ). Then $B \Pi$ has the homotopy type of a CW complex and, by Whitehead's theorem, we obtain a map $B \Pi \longrightarrow B N$, unique up to homotopy, which is compatible with our zigzag. We cannot conclude that $t$ lifts to a homomorphism of monoids $\Pi \longrightarrow M$, but it is clear that no homotopical information is lost by working with $N$ instead of $M$.

If we have a twisting $t: \Pi \longrightarrow \operatorname{Iso}(k) \subset \operatorname{Aut}(k)$, we can use it to construct twisted homology and cohomology theories as in the previous section. We cannot necessarily lift $t$ to a twisting $\Pi \longrightarrow \operatorname{Iso}(\ell)$ or even to a twisting $\Pi \longrightarrow \operatorname{Aut}(\ell)$ when $k$ is equivalent to $\ell$, but we can lift the classifying map of $t$ to a map $B \Pi \longrightarrow B \operatorname{Aut}(\ell)$.

If we consider general maps $k \longrightarrow k$, not necessarily weak equivalences, we obtain $\operatorname{End}(k)$. It is natural to give it the trivial map as basepoint, rather than the identity map as for $\operatorname{Aut}(k)$. The homotopy group $\pi_{n}(\operatorname{End}(k))$ is the group of homotopy classes of maps of spectra $\Sigma^{n} k \longrightarrow k$ and therefore gives the cohomology operations of degree $-n$ on the cohomology theory represented by $k$. This is clearly invariant with respect to equivalences $k \simeq \ell$.

Now suppose that $k$ is a ring $\Omega$-prespectrum in the classical naive homotopical sense (as in [121, p. 218]). Thus, using sequential indexing for definiteness, we have pairings $k_{m} \wedge k_{n} \longrightarrow k_{m+n}$ and unit maps $S^{n} \longrightarrow k_{n}$ that make the appropriate diagrams commute up to homotopy. In particular, $k_{0}$ is a ring space (in the homotopical sense). 
Definition 22.2.2. The space $G L_{1}(k)$ of unit components of a ring $\Omega$-prespectrum $k$ is the pullback in the diagram

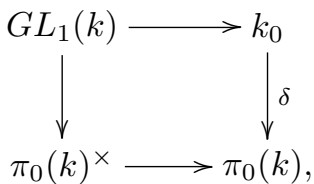

where $\delta$ is the discretization map (which is a fibration). The pairing $k_{0} \wedge k_{0} \longrightarrow k_{0}$ restricts to give a product $G L_{1}(k) \times G L_{1}(k) \longrightarrow G L_{1}(k)$ because, in the ring $\pi_{0}$, the product of units is a unit. The unit $S^{0} \longrightarrow k_{0}$ restricts to give a basepoint $1 \in G L_{1}(k)$. With this product and unit, $G L_{1}(k)$ is a homotopy associative $H$ space. The component $S L_{1}(k)$ of 1 is a sub $H$-space.

With appropriate rigidification, we can think of $G L_{1}(k)$ as a monoid acting on $k$ and thus as a submonoid of $\operatorname{Aut}(k)$. The actions of groups $\Pi$ that are used in practice are thought of as factoring through maps of monoids $\Pi \longrightarrow G L_{1}(k)$. However, the literature on this topic is quite imprecise. We explain some general results that allow this intuition to be made rigorous. The following material should be folklore, but it does not seem to appear in the literature.

We shall recall the definitions of functors with smash product (FSP's) and of their associated ring spectra in the first two sections of the next chapter. We assume here that the reader is familiar with these notions. We can work with either symmetric or orthogonal ring spectra, with their two variant kinds of associated FSP's. For definiteness, we focus on symmetric ring spectra, but orthogonal ring spectra are better suited to the equivariant context. In either case, we work with FSP's with their external products, rather than with the internal products of their associated ring spectra. In the symmetric case, the product structure on $k$ is given by pairings $\mu: k_{m} \wedge k_{n} \longrightarrow k_{m+n}$ and unit maps $\eta: S^{n} \longrightarrow k_{n}$ that that make the appropriate diagrams commute strictly; see Definition 23.1.5.

Proposition 22.2.3. If $k$ is a symmetric or orthogonal ring spectrum and an $\Omega$-prespectrum, then $G L_{1}(k)$ is a topological monoid and is a submonoid of the topological monoid Aut $(k)$ of weak self-equivalences of $k$.

Proof. It is immediate from the commutative diagrams in Definition 23.1.5 that the product on $G L_{1}(k)$ is associative and unital. Fix $x \in R_{0}$. Then $y \mapsto \mu(x, y)$ gives a map $R_{n} \rightarrow R_{n}$ for each $n$. It is again immediate from the given diagrams that these maps specify a map of symmetric spectra, and this map is a weak selfequivalence because, on passage to homotopy groups, it gives multiplication by a unit. Since $\operatorname{Aut}(k)$ is topologized as a subspace of the product of the spaces of maps $k_{n} \longrightarrow k_{n}$, it follows directly that $G L_{1}(k)$ is a topological submonoid of $\operatorname{Aut}(k)$.

The following two remarks are vital to understanding what this result says.

REMARK 22.2.4. The categories of symmetric and orthogonal ring spectra are given Quillen equivalent model structures in [106]. The fibrant objects in these model structures are precisely those ring spectra which are $\Omega$-prespectra. Thus Proposition 22.2.3 applies to a fibrant approximation of any ring spectrum. If $k$ is cofibrant and fibrant as a ring spectrum, then it is cofibrant as a spectrum and its self-equivalences are therefore homotopy equivalences. Moreover, if $k \longrightarrow \ell$ is a weak equivalence of cofibrant and fibrant symmetric or orthogonal ring spectra, 
then its map of zeroth spaces restricts to a map $G L_{1}(k) \rightarrow G L_{1}(\ell)$ of monoids that is a homotopy equivalence in the strong sense that the relevant homotopies are through maps of monoids.

REMARK 22.2.5. The only subtlety is in the commutative case. If $k$ is a commutative symmetric or orthogonal ring spectrum and an $\Omega$-prespectrum, then $G L_{1}(k)$ is a grouplike commutative monoid and is therefore equivalent to a product of Eilenberg-Mac Lane spaces. We conclude that there are no interesting examples of such commutative ring $\Omega$-prespectra. In the previous remark, if we start with a commutative ring spectrum and take a fibrant approximation as a ring spectrum, the result is no longer commutative. The categories of commutative symmetric and orthogonal ring spectra are also given Quillen equivalent model structures in [106], but in those model structures the fibrant objects $k$ are not $\Omega$-prespectra but only positive $\Omega$-prespectra, so that the zeroth spaces $k_{0}$ are no longer well related to the homotopy groups of $k$. In fact, if $k$ is both cofibrant and fibrant as a commutative ring spectrum, then $k_{0}=S^{0}$. As we will recall in $\S 23.6$, we can construct an infinite loop space version of $G L_{1}(k)$ when $k$ is commutative, but no known construction simultaneously gives a submonoid of $\operatorname{Aut}(k)$, and it is the latter structure that is relevant to the theory in this chapter.

REMARK 22.2.6. In the context of Lewis-May spectra (considered in §24.3), the topological monoid $F$ of self-homotopy equivalences of spheres and its identity component $S F$ play a special role. They are exactly $G L_{1}(S)$ and $S L_{1}(S)$, where $S$ is the Lewis-May sphere spectrum with zeroth space $Q S^{0}=\operatorname{colim} \Omega^{n} S^{n}$. LewisMay spectra $k$ are $\Omega$-prespectra in the strong sense that their adjoint structure maps $k_{n} \longrightarrow \Omega k_{n+1}$ are homeomorphisms. The monoids $F$ and $S F$ act from the right on the zeroth space $k_{0}$ of any Lewis-May spectrum $k$ and, when $k$ is a ring spectrum, $F$ acts from the right on the subspace $G L_{1}(k)$ of $k_{0}$ and $S F$ acts on $S L_{1}(k)$. This fact plays a central role in relating highly structured ring spectra to orientation theory; see [98, 112].

\subsection{Twisted $K$-theory}

There are several reasonable models for the spectrum $K$ that represents complex $K$-theory. In any model, the unit space $G L_{1}(K)$ is homotopy equivalent to $\mathbb{Z} / 2 \times K(\mathbb{Z}, 2) \times B S U_{\otimes}$; the third component, and usually the first, are ignored when defining twisted $K$-theory. For the moment, we take $G=e$. There are several quite different group structures on models for $K(\mathbb{Z}, 2)$. For example, as $B S^{1}$, it is a topological Abelian group. However, for purposes of twisted $K$-theory, the relevant models for $K$ see $K(\mathbb{Z}, 2)$ as the projective unitary group, which we denote by $\Pi$ in this section. Note that different topological groups whose underlying spaces are $K(\mathbb{Z}, 2)^{\prime} s$ have bundle theories that look similar homotopically but can be quite different geometrically. While there are several variants, the usual definition of twisted $K$-theory is just our twisted theory represented by the spectrum $K_{\Pi}$ over $B \Pi$ for a suitable twisting $t: \Pi \longrightarrow \operatorname{Aut}(K)$ that factors (at least homotopically) through the second component of $G L_{1}(K)$.

The standard foundational reference is Atiyah and Segal [5], and we shall not repeat their arguments. Rather, we shall say just enough to make it clear that their definition does indeed fit into our framework. They start with an infinite dimensional Hilbert space $\mathscr{H}$ with the norm topology. The space Fred( $\mathscr{H})$ of 
Fredholm operators in $\mathscr{H}$ is a model for $B U \times \mathbb{Z}$, so that

$$
K^{0}(X)=\left[X_{+}, \operatorname{Fred}(\mathscr{H})\right]
$$

The projective unitary group $P U(\mathscr{H})$ with the norm topology clearly acts on $\operatorname{Fred}(\mathscr{H})$. However, it is preferable to take $\Pi$ to be $P U(\mathscr{H})$ with the compact-open topology and to replace $\operatorname{Fred}(\mathscr{H})$ with a homotopy equivalent space $\operatorname{Fred}^{\prime}(\mathscr{H})$ on which $\Pi$ acts, as explained in $[5,3.2]$. With either topology, the unitary group $U(\mathscr{H})$ is contractible and therefore $\Pi$ is a $K(\mathbb{Z}, 2)$.

Let $P$ be a principal $\Pi$-bundle over a space $X$, say a CW complex. Atiyah and Segal use the notation $P$ for the associated bundle with fiber the projective space $\mathbb{P}(\mathscr{H})$, which is more in line with classical $K$-theory but does not affect the details to follow. Under either interpretation, $P$ is classified by a map $\alpha_{P}: X \longrightarrow B \Pi$, which can be viewed as an element of $H^{3}(X ; \mathbb{Z})$. The principal bundle $P$ has an associated bundle $\operatorname{Fred}^{\prime}(P)$ with fiber $\operatorname{Fred}^{\prime}(\mathscr{H})$. Atiyah and Segal define $K_{P}^{0}(X)$ to be the set of homotopy classes of sections of $\operatorname{Fred}^{\prime}(P)$.

The bundle $P$ can be thought of as a cocycle; with other choices of details, it is a cocycle, often denoted $\tau$, and then the notations ${ }^{\tau} K_{*}(X)$ and ${ }^{\tau} K^{*}(X)$ are often used. Whatever the notation, the idea is that $P$ (or $\tau$ ) specifies a twisting that gives rise to a twisted version of $K_{*}(X)$ and $K^{*}(X)$. The intuition is to mimic local systems, and we have seen in our discussion of Poincaré duality that parametrized homotopy theory makes these homotopical. Therefore parametrized homotopy theory provides a natural way to make this intuition precise.

Actually, Atiyah and Segal work more generally with bundles $P$ with an involution, which splits the bundle as $P^{+} \amalg P^{-}$and induces a double cover of $X$. The latter is classified by an element $\xi_{P} \in H^{1}(X ; \mathbb{Z} / 2)$. The set of such bundles $P$ with involution over $X$ is in bijective correspondence with $H^{1}(X, \mathbb{Z} / 2) \times H^{3}(X ; \mathbb{Z})$, but the multiplicative structure is twisted by the Bockstein $\beta: H^{2}(X ; \mathbb{Z} / 2) \longrightarrow H^{3}(X ; \mathbb{Z})$ applied to products of 1 -dimensional classes; see [5, 2.3, 3.3].

For this version, they start with a fixed mod 2 graded Hilbert space $\hat{\mathscr{H}}$ and a modified representing space that they denote $\operatorname{Fred}^{(0)}(\hat{\mathscr{H}})$ and we abbreviate to $K_{0}$, since it is the zeroth space of their model for the $K$-theory spectrum. They also replace their bundle $P$ (not our principal bundle) by $\hat{P}=P \otimes \mathbb{P}(\hat{\mathscr{H}})$ before taking the associated bundle with fiber $K_{0}$. Redefining $K_{P}^{0}(X)$ in these terms facilitates analysis of products and the extension to a periodic $\mathbb{Z}$-graded theory. They explain how to construct $\Pi$-equivariant homotopy equivalences $K_{0} \longrightarrow \Omega^{2 n} K_{0}$ in [5, §4]. Defining the $K$ theory spectrum by letting $K_{2 n}=K_{0}$ and $K_{2 n-1}=\Omega K_{2 n}$, this gives $K$ the $\Pi$-action needed to define $K_{\Pi}=E \Pi \times_{\Pi} K$, and it is then clear that their groups $K_{P}^{i}(X)$ coincide with those given by our general theory. Their spectral sequence of $[5,4.1]$ is the evident specialization of the spectral sequence given in Proposition 22.1.5. It is studied in considerably more detail in their sequel [6] and was already introduced in Rosenberg's early paper [141].

The equivariant generalization is similar, but there are bundle theoretical subtleties. Here we find the alternative treatment of [164] illuminating. We shall not go into detail but just mention some key points. The class of equivariant bundles with Hilbert space fibers that Atiyah and Segal consider seems not to have an obvious classifying $G$-space, but Atiyah and Segal give an ad hoc construction and prove that it is equivalent to $\operatorname{Map}(E G, B \Pi)[5,6.3(\mathrm{iv})]$. Thus the set of equivalence 
classes of such bundles over a $G$-space $X$ is in bijective correspondence with the third Borel cohomology group $H_{G}^{3}(X ; \mathbb{Z})$.

Passage from the bundles they consider to principal $(G, \Pi)$ bundles is subtle and cannot work naively with the norm topology on the structural group $\Pi$, since with that topology the associated nonequivariant principal $\Pi$-bundles do not have continuous actions by $G$. In the relevant specialization of the general theory of [164], the focus is on principal $(G, \Pi)$-bundles rather than on bundles with projective Hilbert space fibers, and in particular they prove the following result.

THEOREM 22.3.1. [164, 2.41] Let $G$ be a Lie group that acts properly on a smooth manifold $M$. Then there is a canonically split surjection from the set of isomorphism classes of principal $(G, \Pi)$-bundles over $M$ to the group $H_{G}^{3}(M, \mathbb{Z})$.

Thus, if we are given a cohomology class $\alpha \in H_{G}^{3}(M, \mathbb{Z})$, we have a canonical principal $(G, \Pi)$-bundle $P_{\alpha}$ that gives rise to it. We can define

$$
K_{\alpha}^{i}(M)=\left(P_{\alpha} \times_{\Pi} K\right)^{*}(M)
$$

as in (22.1.3) and (22.1.4). The discussions in [5, §6] and [164, 3.15-3.17] make clear that this gives the same answer as the definitions in those sources.

REMARK 22.3.3. We note parenthetically that, as explained in [116, Thm 5], passage from principal $(G, \Pi)$-bundles to principal $G$-bundles via the Borel construction is represented homotopically by a canonical map of classifying $G$-spaces

$$
B_{G}(\Pi) \simeq \operatorname{Map}(E G, E \Pi) / \Pi \longrightarrow \operatorname{Map}(E G, B \Pi)
$$

Theorem 22.3.1 suggests that this map has a canonical section when $G$ is a Lie group and $\Pi$ is the projective unitary group.

\subsection{The simplicial spectral sequence}

In this section, we recall the spectral sequences associated to simplicial objects that were first constructed for simplicial spaces by Segal [147]. It is sensible and convenient to work in the generality of a well-grounded model category $\mathscr{C}$. We focus on the based case to fix notations, but the unbased case works the same way. We write $Y \wedge T$ for the tensor of an object $Y$ of $\mathscr{C}$ with a based topological space $T$, and we write $F(T, Y)$ for the cotensor. By a (reduced) homology theory on $\mathscr{C}$, we mean a sequence of covariant functors $J_{q}$ from $\mathscr{C}$ to the category of Abelian groups together with natural suspension isomorphisms $J_{q}(X) \cong J_{q+1}(\Sigma X)$, $\Sigma X=X \wedge S^{1}$, that satisfy the exactness, additivity, and weak equivalence axioms of Definition 20.1.2. In the context of equivariant parametrized spectra, we could just as well work with $R O(G)$-gradings instead of the integer gradings that we use here.

The Reedy model category structure gives the category $s \mathscr{C}$ of simplicial objects in $\mathscr{C}$ a topological model structure. See for example $[75$, Ch. 5] or $[73$, Ch. 15]; although the enrichment in such sources is simplicial, the arguments we quote work equally well with topological enrichment. Using the tensors and cotensors in $\mathscr{C}$, the geometric realization functor

$$
|-|: s \mathscr{C} \longrightarrow \mathscr{C}
$$

is specified by

$$
|X|=\int^{n \in \Delta} X_{n} \wedge \Delta_{+}^{n}
$$


and the singular functor

$$
S: \mathscr{C} \longrightarrow s \mathscr{C}
$$

is specified by

$$
(S Y)_{n}=F\left(\Delta_{+}^{n}, Y\right) .
$$

The pair $(S,|-|)$ is a Quillen adjunction with respect to the given model structure in $\mathscr{C}$ and the Reedy model structure in $s \mathscr{C}$.

REMARK 22.4.1. It follows directly from the definition of Reedy cofibrations that any Quillen adjoint pair between well-grounded model categories lifts naturally to a Quillen adjoint pair on the associated simplicial categories. In particular, in the context of parametrized spaces or spectra, the base change pair $\left(f_{!}, f^{*}\right)$ gives a Quillen adjoint pair on the associated simplicial categories.

For a simplicial object $X$ in $\mathscr{C}$, we have latching maps

$$
\lambda_{n}: L_{n} X \longrightarrow X_{n}
$$

for $n \geq 0$. These should be thought of intuitively as the inclusion of the union of the images of all degeneracy operations $s_{i}: X_{n-1} \longrightarrow X_{n}$ (or the initial map $* \longrightarrow X_{0}$ when $n=0$ ). The formal definition gives the latching object $L_{n} X$ as the colimit over degeneracy operators of the maps under $n$ in the standard simplicial category $\Delta$, the colimit taking account of the relations $s_{i} s_{j}=s_{j+1} s_{i}$ for $i \leq j$; see $[75,5.1 .2]$. The simplicial object $X$ is Reedy cofibrant exactly when each $\lambda_{n}$, $n \geq 0$, is a cofibration in the model structure on $\mathscr{C}$. However, in our topological situation, it is too restrictive to focus only on cofibrant objects in $s \mathscr{C}$ when studying the spectral sequence associated to the skeletal filtration on $|X|$. In well-grounded model categories, every model cofibration is a cyl-cofibration between well-grounded objects in $\mathscr{C}$ and is therefore a bicofibration in the sense of Definitions 5.3.2 and 5.3.3. Bicofibrations suffice to give the gluing lemma and its consequences; see Definitions 5.4.1 and 5.5.4 and Theorem 5.5.1. That is all that is needed to study the spectral sequence. We use the following language adapted from the case of simplicial spaces studied in $[109, \S 11]$.

Definition 22.4.2. An object $X$ of $s \mathscr{C}$ is proper if each $X_{n}$ is well-grounded and each latching map $\lambda_{n}$ is a cyl-cofibration. It follows that each $L_{n} X$ is wellgrounded and each $\lambda_{n}$ is a bicofibration. All Reedy cofibrant objects are proper.

REMARK 22.4.3. If each degeneracy $s_{i}: X_{n-1} \longrightarrow X_{n}$ is a cyl-cofibration, then so is $\lambda_{n}: L_{n} X \longrightarrow X_{n}$. To see that, one uses the description of $L_{n} X$ as a colimit and verifies directly that $\lambda_{n}$ satisfies the required left lifting property.

THEOREM 22.4.4. Let $X$ be a proper simplicial object in $\mathscr{C}$ and let $J_{*}$ and $J^{*}$ be a homology theory and a cohomology theory on $\mathscr{C}$. Then there is a strongly convergent spectral sequence

$$
E_{p, q}^{2}=H_{p}\left(J_{q}(X)\right) \Longrightarrow J_{p+q}(|X|)
$$

and a conditionally convergent spectral sequence

$$
E_{2}^{p, q}=H^{p}\left(J^{q}(X)\right) \Longrightarrow J^{p+q}(|X|)
$$

which converges strongly if the derived $E_{\infty}$ terms $R E_{\infty}$ vanish. 
Before giving the proof, we explain the $E^{2}$ and $E_{2}$ terms. If we apply $J_{q}$ for a fixed $q$ to the objects $X_{p}$, we obtain a simplicial Abelian group $J_{q}(X)$. Taking its associated chain complex and passing to homology, we obtain the homology groups $H_{p}\left(J_{q}(X)\right)$. Similarly, the $J^{q}\left(X_{p}\right)$ give a cosimplicial Abelian group. Taking its cochains and passing to cohomology gives the cohomology groups $H^{p}\left(J^{q}(X)\right)$.

Proof. Our proof is a generalization and clarification of the argument in $[109$, 11.4], where details are given for simplicial spaces. We filter $|X|$ by $F_{p}|X|=\left|\operatorname{sk}_{p} X\right|$. As we shall explain below, since $X$ is proper, each of the canonical maps

$$
F_{p-1}|X| \longrightarrow F_{p}|X|
$$

is a cyl-cofibration and therefore a bicofibration. By the gluing lemma, the cofibers of these maps are therefore equivalent to their quotients

$$
E_{p}|X|=F_{p}|X| / F_{p-1}|X| \text {. }
$$

Since geometric realization commutes with colimits, $|X|$ is the colimit of the $F_{p}|X|$. Following Boardman, [13, Thm. 12.6], we obtain spectral sequences starting with

$$
E_{p, q}^{1}=J_{p+q}\left(E_{p}|X|\right) \quad \text { and } \quad E_{1}^{p, q}=J^{p+q}\left(E_{p}|X|\right)
$$

with the desired convergence properties.

From now on, we focus on the homology case, the cohomological case being similar. To identify the $E^{2}$ term, we fix a $q$ and proceed in two steps. First we define a map of chain complexes

$$
\pi_{*}: J_{q}(X) \longrightarrow E_{q, \bullet}^{1}
$$

and then we show that this chain map is passage to quotients from the unnormalized chains of $J_{q}(X)$ to the normalized chains and is therefore a homology isomorphism.

To define the map, we first use the suspension isomorphism to identify the domain term $J_{q}\left(X_{p}\right)$ with $J_{p+q}\left(X \wedge\left(\Delta_{p} / \partial \Delta_{p}\right)\right)$. Such suspension isomorphisms for $p$ and $p-1$ give the three unlabelled left hand side horizontal arrows in the following big commutative diagram. We note that

$$
F_{m}|X| \cong \int^{n \in \Delta} X_{n} \wedge \mathrm{sk}_{m} \Delta_{n+},
$$

so that we have a natural map

$$
X_{p} \wedge \mathrm{sk}_{m} \Delta_{p_{+}} \longrightarrow F_{m}|X|
$$

for any $m$. For $m=p, p-1, p-2$ we obtain a map

$$
\pi: X_{p} \wedge\left(\Delta_{p}, \partial \Delta_{p}, \partial^{2} \Delta_{p}\right)_{+} \longrightarrow\left(F_{p}(|X|), F_{p-1}(|X|), F_{p-2}(|X|)\right)
$$

of triples. Here $\Delta_{p}=\operatorname{sk}_{p} \Delta_{p}, \partial \Delta_{p}=\operatorname{sk}_{p-1} \Delta_{p}$, and we are writing $\partial^{2} \Delta_{p}$ for $\mathrm{sk}_{p-2} \Delta_{p}$. The map $\pi$ induces the three right hand horizontal arrows in the following diagram, and its upper right rectangle commutes by the naturality of the boundary maps $\partial$ of triples in $J_{p+q}$ homology. The sums in the diagram run over $0 \leq i \leq p$. The map

$$
\delta_{i}: \Delta_{p-1} / \partial \Delta_{p-1} \longrightarrow \partial \Delta_{p} / \Lambda_{p}^{i}
$$

is the $i$-face homeomorphism, where the horn $\Lambda_{p}^{i}$ is the union of all but the $i$ th face of the standard topological simplex $\Delta_{p}$, and the map

$$
\pi_{i}: \partial \Delta_{p} / \partial^{2} \Delta_{p} \longrightarrow \partial \Delta_{p} / \Lambda_{p}^{i}
$$


is the quotient map induced by the inclusion $\partial^{2} \Delta_{p} \subset \Lambda_{p}^{i}$.

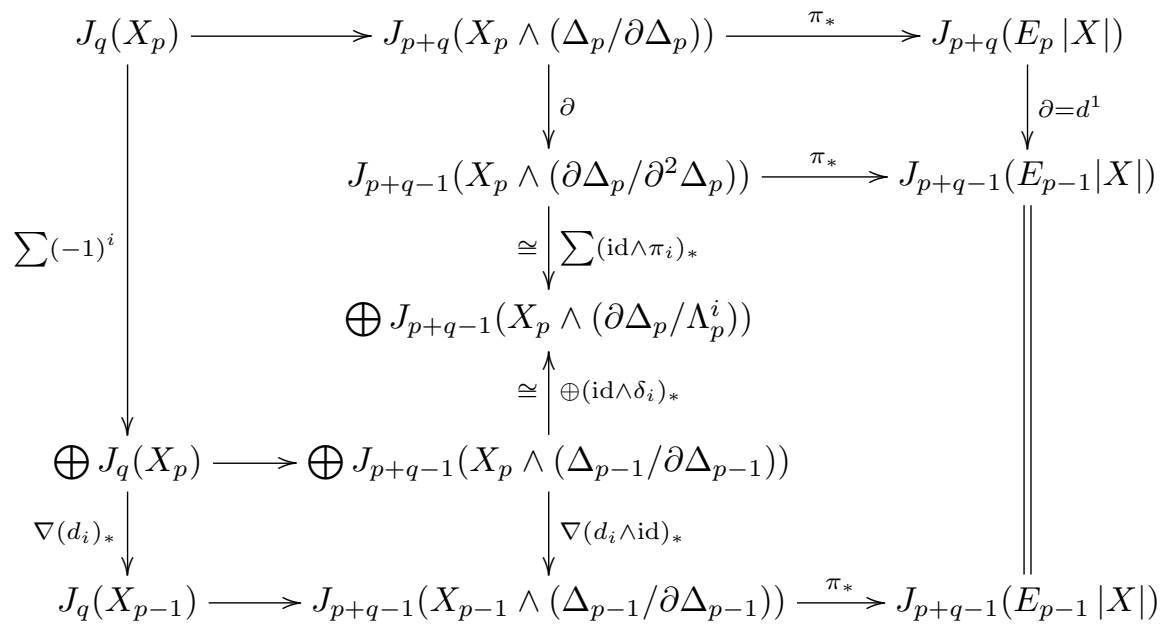

On the left, the top vertical arrow has coordinates $(-1)^{i}$ and the bottom two vertical arrows are the sums of the face maps $\left(d_{i}\right)_{*}$ and $\left(d_{i} \wedge \mathrm{id}\right)_{*}$. The left vertical composite is the differential on $J_{q}\left(X_{p}\right)$. The lower left rectangle is a naturality square for the suspension isomorphism. The lower right rectangle commutes by the face identifications in the coend defining the geometric realization. The commutativity of the upper left diagram is a check of signs. The point is that if we identify $\Delta_{p} / \partial \Delta_{p}$ and $\Delta_{p-1} / \partial \Delta_{p-1}$ with $S^{p}$ and $S^{p-1}$ in the canonical way and inspect faces in the identifications

$$
\partial \Delta_{p} / \partial^{2} \Delta_{p} \cong \bigvee \partial \Delta_{p} / \Lambda_{p}^{i} \cong \bigvee \Delta_{p-1} / \partial \Delta_{p-1}
$$

that are giving the isomorphisms in the middle of our diagram, we see that $\partial$ has coordinates $(-1)^{i}$, this being the geometric reason for the signs in the definition of the differential of simplicial Abelian groups. The commutativity of the diagram shows that the top horizontal composite, again denoted $\pi_{*}$, gives the $p$ th term of a map of chain complexes.

To identify this map, we observe that, up to an isomorphism of the target, the top horizontal composite $\pi_{*}$ coincides with the canonical map

$$
J_{q}\left(X_{p}\right) \longrightarrow J_{q}\left(X_{p} / L_{p} X\right)
$$

Indeed, the definition of the latching objects implies that we have a pushout

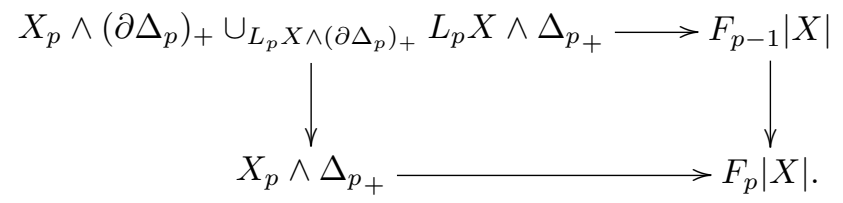

This pushout explains why the assumption that $X$ is proper implies that the inclusions of skeleta are bicofibrations. The quotient of the right vertical map is the 
same as the total quotient of the commutative square

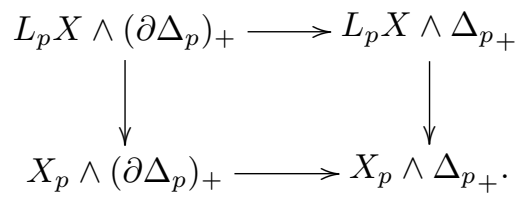

Taking first the vertical quotient, and then the horizontal quotient of the result (or vice versa), we therefore get an isomorphism

$$
\Sigma^{p} X^{p} / L_{p} X \longrightarrow F_{p}|X| / F_{p-1}|X| .
$$

Using the definition of latching objects and the description of $\lambda_{p}$ in terms of the $s_{i}$, it follows as claimed that $J_{q}\left(X_{p} / L_{p} X\right)$ is the $p$ th term of the normalized chain complex of $J_{q}(X)$ and that $(22.4 .5)$ is the canonical quasi-isomorphism relating unnormalized to normalized chains.

\section{5. Čech type spectral sequences}

We apply the simplicial spectral sequence of the previous section to give a Čech type local to global descent spectral sequence, starting from a sheaf theoretic point of view on parametrized homology and cohomology. This approach seems particularly relevant when our parametrized coefficient spectra come from the bundle construction, so that they trivialize over the open sets of a fine enough cover. We described the behavior of parametrized homology and cohomology with respect to base change in Proposition 20.2.6. Using that result, we see that the counits of base change adjunctions give rise to the following comparison maps. They will lead to appropriate restrictions of our theories over open subsets of the base space.

Proposition 22.5.1. Let $f: A \longrightarrow B$ be a map and let $J$ and $X$ be spectra over $B$. Then the counit $f_{!} f^{*} \longrightarrow$ id of the adjunction $\left(f_{!}, f^{*}\right)$ induces natural corestriction and restriction homomorphisms

$$
\left(f^{*} J\right)_{n}\left(f^{*} X\right) \cong\left(f ! f^{*} J\right)_{*}(X) \longrightarrow J_{n}(X)
$$

and

$$
J^{n}(X) \longrightarrow J^{n}\left(f_{!} f^{*} X\right) \cong\left(f^{*} J\right)^{n}\left(f^{*} X\right) .
$$

If $f$ is a q-equivalence, then these maps are isomorphisms.

Proof. The last statement holds since $\left(f_{!}, f^{*}\right)$ is a Quillen equivalence when $f$ is a $q$-equivalence.

Definition 22.5.2. For an open subset $U$ of $B$, let $j_{U}: U \longrightarrow B$ be the inclusion. For spectra $J$ and $X$ over $B$, define

$$
\Gamma\left(U ; J_{*} X\right)=\left(j_{U}^{*} J\right)_{*}\left(j_{U}^{*} X\right) \text { and } \Gamma\left(U ; J^{*} X\right)=\left(j_{U}^{*} J\right)^{*}\left(j_{U}^{*} X\right) .
$$

For $U \subset V$, let $i_{U, V}$ be the inclusion. Since $j_{U}=j_{V} i_{U, V}$, the corestriction and restriction maps give that $\Gamma\left(-; J_{*} X\right)$ is a covariant functor and $\Gamma\left(-; J^{*} X\right)$ is a contravariant functor from the category $\mathscr{O}_{B}$ of open subsets of $B$ to graded Abelian groups. We call these functors the copresheaf of parametrized homology groups and the presheaf of parametrized cohomology groups. The previous result gives compatible families of maps

$$
\Gamma\left(U ; J_{*} X\right) \longrightarrow J_{*}(X) \text { and } J^{*}(X) \longrightarrow \Gamma\left(U ; J^{*} X\right) .
$$


Reverting to spaces over $B$ and unreduced theories, this leads to Cech type spectral sequences for the calculation of $J_{*}(X)$ and $J^{*}(X)$ for a parametrized space $(X, p)$ over $B$. Observe that in this situation

$$
j_{U}^{*} X=\left(p^{-1} U,\left.p\right|_{U}\right) \text { and } j_{U !} j_{U}^{*} X=\left(p^{-1} U,\left.j_{U} \circ p\right|_{U}\right) .
$$

We first recall the Čech complex. Let $B$ be a base space and let $\mathscr{U}$ be an open cover of $B$ indexed on a totally ordered set $I$. Define a simplicial set $\mathscr{U}_{\bullet}$ by letting the set $\mathscr{U}_{n}$ of $n$-simplices be the set of ordered $(n+1)$-tuples $S=\left(U_{i_{0}}, \ldots, U_{i_{n}}\right)$ (possibly with repeats) of sets in $\mathscr{U}$ that have non-empty intersection, denoted $U_{S}$. The $q$ th face operator deletes the $q$ th set, and the $q$ th degeneracy operator repeats the $q$ th set in each $S$. We obtain a simplicial space $\check{C}(\mathscr{U})$ over $B$ by setting

$$
\check{C}(\mathscr{U})_{n}=\coprod_{S \in \mathscr{U}_{n}} U_{S} .
$$

The face and degeneracy maps are induced by $U_{d_{i} S} \subset U_{S}$ and $U_{s_{i} S}=U_{S}$. While it would be unreasonable to introduce cellularity conditions, so that $\check{C}(\mathscr{U})$ is not Reedy cofibrant, it is obviously proper since $L_{n} \check{C}(\mathscr{U})$ is the disjoint union of the subspaces $U_{S}$ that are indexed on ordered sets $S$ with repeated indices in $I$. The inclusions $j_{U_{S}}: U_{S} \subset B$ give a map

$$
j_{\bullet}: \check{C}(\mathscr{U}) \longrightarrow c B
$$

of proper simplicial spaces, where $c B$ is the constant simplicial space at $B$. It is standard that this map induces a homotopy equivalence

$$
j=\left|j_{\bullet}\right|:|\check{C}(\mathscr{U})| \longrightarrow B
$$

on passage to geometric realization; see $[45,147]$.

Now return to our space $(X, p)$ over $B$. Applying the counit of the adjunctions $\left(j_{n !}, j_{n}^{*}\right)$ on the $n$ th-space levels, we obtain a map

$$
\check{C}\left(p^{-1} \mathscr{U}\right) \longrightarrow c X
$$

of proper simplicial spaces over $B$, where properness holds just as in the nonparametrized situation. On total spaces, reusing the notation $j$ for inclusions of open sets, this is just the map $j_{\bullet}$ associated to the numerable open cover $p^{-1} \mathscr{U}$ of $X$ given by the sets $p^{-1}\left(U_{i}\right)$. Its geometric realization $j$ is therefore a homotopy equivalence. We conclude that we have a $q$-equivalence $j:\left|\check{C}\left(p^{-1} \mathscr{U}\right)\right| \longrightarrow X$ of spaces over $B$. Applying the simplicial spectral sequence, we obtain the following Cech type spectral sequences, for which we adopt the notations

$$
\check{H}_{p}\left(\mathscr{U}, \Gamma\left(-; J_{q} X\right)\right)=H_{p}\left(J_{q}\left(\check{C}\left(p^{-1} \mathscr{U}\right)\right)\right)
$$

and

$$
\check{H}^{p}\left(\mathscr{U}, \Gamma\left(-; J^{q} X\right)\right)=H^{p}\left(J^{q}\left(\check{C}\left(p^{-1} \mathscr{U}\right)\right)\right)
$$

TheOREM 22.5.3. Let $X$ be a space over $B$, and let $\mathscr{U}$ be a numerable open cover of $B$. Let $J$ be a spectrum over $B$. Then there are spectral sequences

$$
E_{p, q}^{2}=\check{H}_{p}\left(\mathscr{U}, \Gamma\left(-; J_{q}(X)\right)\right) \Longrightarrow J_{p+q}(X)
$$

and

$$
E_{2}^{p, q}=\check{H}^{p}\left(\mathscr{U}, \Gamma\left(-; J^{q}(X)\right)\right) \Longrightarrow J^{p+q}(X) .
$$

Observe that the space of $n$-simplices of $\check{C}\left(p^{-1} \mathscr{U}\right)$ is a disjoint union of open sets $p^{-1} U_{S}$ and thus the $E_{2}$-terms are computable in terms of local information. 
REMARK 22.5.4. If $X$ is an ex-space or spectrum over $B$, then we can similarly form the simplicial ex-space or spectrum $\check{C}(\mathscr{U} ; X)$ over $B$ by setting $\check{C}(\mathscr{U} ; X)_{n}=$ $j_{n !} j_{n}^{*} X$. However, it is unclear to us whether or not $|\check{C}(\mathscr{U} ; X)|$ is equivalent to $X$.

\subsection{The twisted Rothenberg-Steenrod spectral sequence}

We construct a twisted Rothenberg-Steenrod spectral sequence in this section. A version of it has been used by Douglas [52] to calculate the twisted $K$-theory of simply connected simple Lie groups.

We return to the context of $\S 22.1$, except that we take $G$ to be the trivial group. As there, we let $\Pi$ be any (locally compact and well-based) topological group, and we let $k$ be a $\Pi$-spectrum indexed on a $\Pi$-trivial universe. As in $\S 22.2$, we are thinking of a prespectrum $k$ together with a map of topological monoids $\Pi \longrightarrow \operatorname{Aut}(k)$. We again assume that $k$ is an excellent prespectrum, for definiteness of context. In particular, $k$ is a well-grounded $\Omega$-prespectrum.

Let $\Lambda$ be a (locally compact and well-based) topological group and $\alpha: \Lambda \longrightarrow \Pi$ be a homomorphism of topological groups. Its classifying map $B \alpha: B \Lambda \longrightarrow B \Pi$ will play the role here that $p: X \longrightarrow B \Pi$ played in $\S 22.1$. We are thinking of $\Pi$ as fixed and canonical and $\Lambda$ as varying. We assume familiarity with the bar construction $B(Y, \Lambda, X)$, where $Y$ is a right $\Lambda$-space and $X$ is a left $\Lambda$-space. It is defined and studied in $[111, \S 7]$. We shall only use the case $Y=*$ in this section, and we abbreviate notation to $B(\Lambda, X)$. This space is the geometric realization of a simplicial space $B_{\bullet}(\Lambda, X)$ whose space of $q$-simplices is $\Lambda^{q} \times X$. The last face operator is determined by the action of $\Lambda$ on $X$. We then have

$$
B \Lambda=B(\Lambda, *), \quad E \Lambda=B(\Lambda, \Lambda) \text { and } B(\Lambda, X) \cong E \Lambda \times_{\Lambda} X .
$$

Moreover, by [111, 7.8], we have the following pullback diagram, where $X$ is a left $\Pi$-space regarded by pullback as a left $\Lambda$-space.

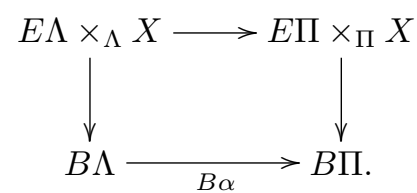

Here the vertical arrows are induced by the projection $X \longrightarrow *$ and the horizontal arrows are induced by $\alpha$.

We may think of $k$ as a $\Lambda$-spectrum by pullback along $\alpha$. We could view $\Lambda^{q} \times k$ as a prespectrum over $\Lambda^{q}$ and build a bar construction using simplicial objects in a category of prespectra over varying base spaces. We shall take analogous (but more elementary) ideas seriously in the next chapter. However, here we prefer instead to consider only a bar construction in the category of prespectra. Applying the bar construction on spaces levelwise, we can construct a bar construction

$$
B(\Lambda, k)=|B \bullet(\Lambda, k)|,
$$

where $B_{q}(\Lambda, k)$ is the prespectrum $\Lambda_{+}^{q} \wedge k$. The last face is determined by the action of $\Lambda$ on $k$ induced by $\alpha$ and the given action of $\Pi$ on $k$. The other faces and the degeneracies are induced by the projection $\Lambda \longrightarrow *$ and the product and unit of $\Lambda$, exactly as on the space level. The discussion of geometric realizations of simplicial spectra in $[61, \S \mathrm{X} .1]$ (in which spectra are understood in the Lewis-May sense) adapts to give similar properties in our category of prespectra. 
Proposition 22.6.1. The bar construction $B(\Lambda, k)$ is isomorphic to the prespectrum $r_{!}(B \alpha)^{*}\left(k_{\Pi}\right) \cong r_{!} k_{\Lambda}$, and the simplicial prespectrum $B \cdot(\Lambda, k)$ is proper.

Proof. Recall that $k_{\Pi}=E \Pi \times_{\Pi} k$. By the pullback diagram above, the pullback of $E \Pi$ along $B \alpha$ is the principal $\Pi$-bundle $E \Lambda \times_{\Lambda} \Pi$ over $B \Lambda$, and

$$
(B \alpha)^{*}\left(k_{\Pi}\right) \cong\left(E \Lambda \times_{\Lambda} \Pi\right) \times_{\Pi} k \cong E \Lambda \times_{\Lambda} k=k_{\Lambda} .
$$

Applying $r_{\text {! }}$ to this, we obtain $(E \Lambda)_{+} \wedge_{\Lambda} k$, which is isomorphic to $B(\Lambda, k)$. For the last statement, each $B_{n}(\Lambda, k)$ is well grounded since $k$ and $\Lambda$ are, the degeneracies are $c y l$-cofibrations since $\Lambda$ is well-based, and Remark 22.4.3 applies to give that the latching maps are $c y l$-cofibrations.

Now assume further that $k$ is a ring spectrum (in the naive sense). Then the products on $k$ and $\Lambda$ induce a ring structure on the unreduced homology $k_{*}(\Lambda)$. We also then have a map (in the homotopy category)

$$
k \wedge \Lambda_{+} \wedge k \longrightarrow k \wedge S^{0} \wedge k \longrightarrow k
$$

given by the projection $\Lambda_{+} \longrightarrow S^{0}$ and the product on $k$, and another map

$$
k \wedge \Lambda_{+} \wedge k \longrightarrow k \wedge k \longrightarrow k
$$

given by the action map $\Lambda_{+} \wedge k \longrightarrow k$. The first gives a right action of $k_{*}(\Lambda)$ on $k_{*}$, and the second gives a left action of $k_{*}(\Lambda)$ on $k_{*}$. We denote by $k_{*}^{\alpha}$ the module $k_{*}$ with the latter action, and we obtain natural maps

$$
k_{*} \otimes_{k_{*}} k_{*}(\Lambda)^{\otimes n} \otimes_{k_{*}} k_{*}^{\alpha} \longrightarrow \pi_{*}\left(B_{n}(\Lambda, k)\right) .
$$

These maps are isomorphisms if $k$ has a general enough Künneth isomorphism.

Theorem 22.6.3 (Twisted Rothenberg-Steenrod spectral sequence). If the maps (22.6.2) are isomorphisms, there is a strongly convergent spectral sequence

$$
E_{p, q}^{2}=\operatorname{Tor}_{p, q}^{k_{*}(\Lambda)}\left(k_{*}, k_{*}^{\alpha}\right) \Longrightarrow k_{p+q}(B \Lambda, B \alpha) .
$$

It is natural with respect to homomorphisms $\alpha: \Lambda \longrightarrow \Pi$.

Proof. By definition and Proposition 22.6.1, the desired target is

$$
\left(k_{\Pi}\right)_{*}(B \Lambda, B \alpha) \cong\left((B \alpha)^{*}\left(k_{\Pi}\right)\right)_{*}(B \Lambda) \cong\left(k_{\Lambda}\right)_{*}\left(S_{B \Lambda}^{0}\right)=\pi_{*}\left(r_{!} k_{\Lambda}\right) \cong \pi_{*}(B(\Lambda, k)) \text {. }
$$

The spectral sequence is obtained by applying the simplicial spectral sequence of Theorem 22.4.4 to the homotopy groups of the proper simplicial spectrum $B \bullet(\Lambda, k)$. That gives

$$
E_{p, q}^{2}=H_{p} \pi_{q}(B \cdot(\Lambda, k)) \Longrightarrow \pi_{p+q}(B(\Lambda, k)) .
$$

When (22.6.2) is an isomorphism, the displayed $E^{2}$-term calculates the desired torsion product since it is the homology of the algebraic bar construction

$$
B\left(k_{*}, k_{*}(\Lambda), k_{*}^{\alpha}\right) \cong B\left(k_{*}, k_{*}(\Lambda), k_{*}(\Lambda)\right) \otimes_{k_{*}(\Lambda)} k_{*}^{\alpha} .
$$

Observe that the spectral sequence depends on the homomorphism $\alpha$ and not just on the map $B \alpha$.

REMARK 22.6.4. Unless $k_{*}(\Lambda)$ is $k_{*}$-flat, the displayed algebraic bar construction computes a relative rather than an absolute torsion product, and we understand Tor in that relative sense; see [59]. 
REMARK 22.6.5. At least when $k$ is a symmetric or orthogonal ring spectrum, the spectral sequence is multiplicative, as we see from the commutation of the bar construction with products; see $[111,7.4]$ or $(23.4 .2)$ below. We omit the details.

REMARK 22.6.6. Let $G$ be a simply connected simple Lie group and let $\Lambda=\Omega G$, regarded as a topological group with product induced pointwise from the product on $G$. Then $B \Lambda$ is homotopy equivalent to $G$, and $H^{2}(\Lambda) \cong \mathbb{Z}$. When a cohomology class is represented by a homomorphism

$$
\alpha: \Lambda \longrightarrow P U(\mathscr{H}) \simeq K(\mathbb{Z}, 2)
$$

of topological groups, the resulting spectral sequence for twisted $K$-theory takes the form

$$
E_{p, q}^{2}=\operatorname{Tor}_{p, q}^{K_{*}(\Omega G)}\left(K_{*}, K_{*}^{\alpha}\right) \Longrightarrow K_{p+q}(G, B \alpha) .
$$

Thus the target is the $K$-theory of $G$ twisted by $B \alpha$. In that form, the spectral sequence agrees with the one described and studied calculationally by Douglas [52]. As pointed out to us by Douglas, one approach to representing cohomology classes by homomorphisms as we require is to view $\Omega G$ as a subgroup of the unbased loop group $L G$ and to use the work of Pressley and Segal [137] to show that $L G$ has appropriate projective representations and therefore group homomorphisms $L G \longrightarrow P U(\mathscr{H})$. We have not checked details.

\subsection{The parametrized Künneth spectral sequence}

We use parametrized spectra to give a quick conceptual construction of Eilenberg-Moore type spectral sequences. We only give the basic formal properties here. Use of parametrized spectra substantially simplifies the geometric (as opposed to chain level) construction of the spectral sequence. Surprisingly, this also has the effects of recasting the convergence and "relevance" questions, which appear in different guises than in some other geometric constructions, and clarifying the identification of $E_{2}$. This approach deserves further study since the literature on the Eilenberg-Moore spectral sequence is still in a quite unsatisfactory state.

Throughout, we fix a map $f: B \longrightarrow A$ of base spaces. The only case we know to be important and useful is that of $f=r: B \longrightarrow *$, but the general case adds no difficulty. We fix a spectrum $k$ over $A$, using the notation $k$ because we have the case $A=*$ in mind, and we let $J=f^{*} k$ be the resulting spectrum over $B$. The essential idea is to use a Künneth theorem for the homology or cohomology theory represented by $k$ on spectra over $A$ to derive a parametrized Künneth theorem for the homology or cohomology theory represented by $J$ on spectra over $B$. To that end, we consider variable spectra $X$ and $Y$ over $B$. For homotopical control, we can work with excellent prespectra or with fibrant and cofibrant spectra. Formally, we are working in one of our two equivalent versions of the parametrized stable homotopy categories of spectra over $A$ and $B$. Recall that this category is triangulated. With these notations, Proposition 20.2.6(ii) and the projection formula (both originally stated with the roles of $A$ and $B$ reversed) give

$$
\begin{gathered}
J_{*}\left(X \wedge_{B} Y\right) \cong k_{*}\left(f_{!}\left(X \wedge_{B} Y\right)\right) \text { and } J^{*}\left(X \wedge_{B} Y\right) \cong k^{*}\left(f_{!}\left(X \wedge_{B} Y\right)\right) \\
J_{*}\left(f^{*} f_{!} X \wedge_{B} Y\right) \cong k_{*}\left(f_{!}\left(f^{*} f_{!} X \wedge_{A} Y\right)\right) \cong k_{*}\left(f_{!} X \wedge_{A} f_{!} Y\right) \\
J^{*}\left(f^{*} f_{!} X \wedge_{B} Y\right) \cong k^{*}\left(f_{!}\left(f^{*} f_{!} X \wedge_{B} Y\right)\right) \cong k^{*}\left(f_{!} X \wedge_{A} f_{!} Y\right)
\end{gathered}
$$


In the following construction, we prefer to use increasing negative indices rather than decreasing positive indices, since that better fits the relevant grading, but the opposite choice works equally well mathematically.

Construction 22.7.4. For $X \in \mathscr{S}_{B}$, construct the tower

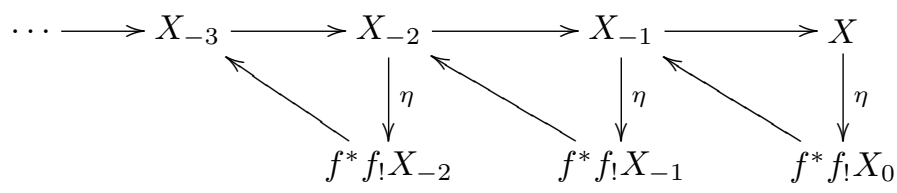

inductively by letting $X_{p}=X$ for $p \geq 0$ and letting $X_{p-1} \longrightarrow X_{p}, p \leq 0$, be the fiber of the unit $\eta: X_{p} \longrightarrow f^{*} f_{!} X_{p}$ of the $\left(f_{!}, f^{*}\right)$ adjunction. The unlabelled diagonal arrows indicate the maps to suspensions over $B$ that are induced by the canonical equivalences of the $C_{B} \eta$ with the $\Sigma_{B}\left(F_{B} \eta\right)$. Thus each triangle is distinguished in the triangulated category $\operatorname{Ho}_{B}$. Now smash over $B$ with $Y$ and apply $J_{*}$ or $J^{*}$. This gives two "unravelled exact couples", in the sense of Boardman $[13, \S 0]$, the second exactly as in his cohomological notations, with $E_{1}^{p, q}=J^{p+q}\left(f^{*} f_{!} X_{p}\right)$ for $p \leq 0$ and $E_{1}^{p, q}=0$ for $p>0$. The first unravelled exact couple is indexed homologically and has $E_{p, q}^{1}=J_{p+q}\left(f^{*} f_{!} X_{p}\right)$ for $p \leq 0$ and $E_{p, q}^{1}=0$ for $p>0$. Following Boardman's details and using (22.7.1)-(22.7.3) to identify terms, there result spectral sequences

$$
E_{p, q}^{1}=k_{p+q}\left(f_{!} X_{p} \wedge_{A} f_{!} Y\right) \Longrightarrow k_{p+q}\left(f_{!}\left(X \wedge_{B} Y\right)\right)
$$

and

$$
E_{1}^{p, q}=k^{p+q}\left(f_{!} X_{p} \wedge_{A} f_{!} Y\right) \Longrightarrow k^{p+q}\left(f_{!}\left(X \wedge_{B} Y\right)\right) .
$$

In Boardman's language, the homology spectral sequence is a (left) half plane spectral sequence with entering differentials [13, §7], and it converges conditionally if

$$
\lim _{p} k_{*}\left(f_{!}\left(X_{p} \wedge_{B} Y\right)\right)=0 \text { and } \lim _{p}^{1} k_{*}\left(f_{!}\left(X_{p} \wedge_{B} Y\right)\right)=0
$$

If in addition its derived $E_{\infty}$ term $R E_{\infty}$ vanishes, then it converges strongly. The cohomology spectral sequence is a (left) half plane spectral sequence with exiting differentials $[13, \S 6]$, and it converges strongly if

$$
\underset{p}{\operatorname{colim}} k^{*}\left(f_{!}\left(X_{p} \wedge_{B} Y\right)\right)=0 .
$$

A priori, what these spectral sequences converge to is not the displayed targets but rather the limits

$$
\underset{p}{\operatorname{colim}} k_{*}\left(f_{!}\left(X_{p} \wedge_{B} Y\right)\right) \text { and } \lim _{p} k^{*}\left(f_{!}\left(X_{p} \wedge_{B} Y\right)\right)
$$

defined in terms of our unravelled exact couples.

The question of relevance is the question of when the canonical maps

$$
\underset{p}{\operatorname{colim}} k_{*}\left(f_{!}\left(X_{p} \wedge_{B} Y\right)\right) \longrightarrow k_{*}\left(f_{!}\left(X \wedge_{B} Y\right)\right)
$$

and

$$
k^{*}\left(f_{!}\left(X \wedge_{B} Y\right)\right) \longrightarrow \lim _{p} k^{*}\left(f_{!}\left(X_{p} \wedge_{B} Y\right)\right)
$$

are isomorphisms. However, this always holds in our context since our limit systems end with their zeroth terms. A good understanding of when our convergence conditions hold is desirable, but we will not consider such issues here. 
REMARK 22.7.5. In some approaches to the Eilenberg-Moore spectral sequence, the convergence problem appears more as a problem of relevance than of convergence. That is, even when the spectral sequence converges strongly, it need not converge to the groups that one wants to compute.

We next identify the $E_{2}$-term in the cohomological spectral sequence. We focus on cohomology only because we do not want to assume familiarity with cotensor products, but it is standard that the identification works more generally in homology. For example, this is where finite type hypotheses enter in ordinary cohomology, although they are not required in ordinary homology. We need a few observations.

LEMMA 22.7.6. Let $k$ be a commutative ring spectrum in $H_{0} \mathscr{S}_{A}$. Then $k^{*}\left(S_{A}\right)$ is a graded ring and $k^{*}$ is a lax monoidal functor from Ho $\mathscr{S}_{A}$ to the category of $k^{*}\left(S_{A}\right)$-modules.

Proof. For spectra $W$ and $Z$ over $A$ we have natural pairings

$$
\left[S_{A}^{n}, W\right]_{A} \otimes\left[S_{A}^{m}, Z\right]_{A} \longrightarrow\left[S_{A}^{n+m}, W \wedge_{A} Z\right]_{A}
$$

and

$$
F_{A}(W, k) \wedge_{A} F_{A}(Z, k) \longrightarrow F_{A}\left(W \wedge_{A} Z, k \wedge_{A} k\right) \longrightarrow F_{A}\left(W \wedge_{A} Z, k\right) .
$$

Since $k^{n}(W)=\left[S_{A}^{-n}, F_{A}(W, k)\right]_{A}$, it follows that $k^{*}$ is lax monoidal when considered as taking values in graded Abelian groups. The statement follows since the objects of Ho $\mathscr{S}_{A}$ are $S_{A}$-modules.

Returning to spectra over $B$ we have the following observation.

Lemma 22.7.7. For a spectrum $X$ over $B, f_{!} X$ is a comodule over the coalgebra $f_{!} S_{B}$. For a commutative ring spectrum $k$ in Ho $\mathscr{S}_{A}$, the $k^{*}\left(S_{A}\right)$-module $k^{*}\left(f_{!} X\right)$ is a module over the $k^{*}\left(S_{A}\right)$-algebra $k^{*}\left(f_{!} S_{B}\right)$.

Proof. This follows from the unit equivalences $X \simeq X \wedge_{B} S_{B}$ together with the facts that $f_{\text {! }}$ is op-lax monoidal (since $f^{*}$ is monoidal) and $k^{*}$ is lax monoidal.

Definition 22.7.8. Let $k$ be a commutative ring spectrum in Ho $\mathscr{S}_{A}$. We say that $(W, Z)$ is a $k$-Künneth pair of spectra over $A$ if the natural map

$$
k^{*}(W) \otimes_{k^{*}\left(S_{A}\right)} k^{*}(Z) \longrightarrow k^{*}\left(W \wedge_{A} Z\right)
$$

is an isomorphism.

We can now identity the $E_{2}$-term.

THEOREM 22.7.9 (The parametrized Künneth spectral sequence). In the context of Construction 22.7.4, assume further that $k$ is a commutative ring spectrum over $A$ and each $\left(f_{!} X_{p}, f_{!} S_{B}\right)$ and $\left(f_{!} X_{p}, f_{!} Y\right)$ is a $k$-Künneth pair. Then

$$
E_{2}^{p, q}=\operatorname{Tor}_{k^{*}\left(f_{!} S_{B}\right)}^{p, q}\left(k^{*}\left(f_{!} X\right), k^{*}\left(f_{!} Y\right)\right) .
$$

Proof. The initial map $\eta$ and the "differentials" of the tower give a sequence $(22.7 .10)$

$$
* \longrightarrow X \longrightarrow f^{*} f_{!} X_{0} \longrightarrow \Sigma_{B} f^{*} f_{!} X_{-1} \longrightarrow \Sigma_{B}^{2} f^{*} f_{!} X_{-2} \longrightarrow \cdots
$$

This is a resolution of $X$ in the sense that the composite of any two maps is null and the sequence is exact on all homology and cohomology theories of the form $J=f^{*} k$. To see the latter, note that one of the triangle identities for the $\left(f !, f^{*}\right)$ adjunction 
shows that the map $f_{!} \eta: f_{!} X_{p} \longrightarrow f_{!} f^{*} f_{!} X_{p}$ (a generalized Thom diagonal) has the left inverse $\epsilon: f_{!} f^{*} f_{!} X_{p} \longrightarrow f_{!} X_{p}$ so that $\epsilon \circ f_{!} \eta=\mathrm{id}_{X_{p}}$. Using (22.7.1), it follows that when we apply $J_{*}$ and $J^{*}$ to the tower, the maps induced by $\eta$ split and the tower breaks up into respective short exact sequences

$$
0 \longrightarrow k_{*}\left(f_{!} X_{p}\right) \longrightarrow k_{*}\left(f_{!} f^{*} f_{!} X_{p}\right) \longrightarrow k_{*-1}\left(f_{!} X_{p+1}\right) \longrightarrow 0
$$

and

$$
0 \longleftarrow k^{*}\left(f_{!} X_{p}\right) \longleftarrow \quad k^{*}\left(f_{!} f^{*} f_{!} X_{p}\right) \longleftarrow \quad k^{*-1}\left(f_{!} X_{p+1}\right) \longleftarrow 0 .
$$

The long exact sequence obtained by applying $k_{*}\left(f_{!}(-)\right)$or $k^{*}\left(f_{!}(-)\right)$to the resolution above is obtained by splicing these together.

Our $k$-Künneth pair assumption gives the first of the two isomorphisms

$$
k^{*}\left(f_{!} X_{p}\right) \otimes_{k^{*}\left(S_{A}\right)} k^{*}\left(f_{!} S_{B}\right) \cong k^{*}\left(f_{!} X_{p} \wedge_{A} f_{!} S_{B}\right) \cong k^{*}\left(f_{!} f^{*} f_{!} X_{p}\right) .
$$

The second holds since the projection formula gives

$$
f_{!} X \wedge_{A} f_{!} S_{B} \simeq f_{!}\left(f^{*} f_{!} X \wedge_{B} S_{B}\right) \simeq f_{!} f^{*} f_{!} X
$$

for any $X$. This shows that $k^{*}\left(f_{!} f^{*} f_{!} X_{p}\right)$ is (relatively) projective over $k^{*}\left(f_{!} S_{B}\right)$. Now our $k$-Künneth pair assumption, together with the above calculation, gives isomorphisms

$$
\begin{aligned}
E_{1}^{p, *} & =k^{*}\left(f_{!} X_{p} \wedge_{A} f_{!} Y\right) \\
& \cong k^{*}\left(f_{!} X_{p}\right) \otimes_{k^{*}\left(S_{A}\right)} k^{*}\left(f_{!} Y\right) \\
& \cong k^{*}\left(f_{!} f^{*} f_{!} X_{p}\right) \otimes_{k^{*}\left(f_{!} S_{B}\right)} k^{*}\left(f_{!} Y\right) .
\end{aligned}
$$

These are compatible with the differentials, and this shows that the homology of $E_{1}^{p, *}$ calculates the desired $E_{2}$-term.

REMARK 22.7.11. The construction we started with is analogous to the canonical resolution used in the construction of the Adams spectral sequence. As in that context, we can develop a general theory of resolutions. To prove that the spectral sequence is multiplicative, we can form the smash products of resolutions and prove that they are again resolutions. We omit the details here, but we recommend Bruner's treatment of the products in the Adams spectral sequence, [27, IV§4], for details that can be adapted to our context. The axiomatization of a compatibly triangulated symmetric monoidal category that we described in $\S 16.7$ and $\S 17.5$, especially (TC3) and (TC4), encodes common features of the two situations.

Remark 22.7.12. Taking $f$ to be $r: B \longrightarrow *, k=H \mathbb{F}$ to be the EilenbergMac Lane spectrum for a field $\mathbb{F}$, and passing to unreduced cohomology, we obtain a version of the classical Eilenberg-Moore spectral sequence. In detail, let $(K, p)$ and $(L, q)$ be spaces over $B$, where $q$, say, is a fibration. Let $(P, \pi)$ be the pullback $K \times{ }_{B} L$ over $B$. Then

$$
(K, p)_{+} \wedge_{B}(L, q)_{+} \cong(P, \pi)_{+}
$$

as ex-spaces over $B$. Applying $\Sigma_{B}^{\infty}$ and using Proposition 13.7.5, this gives

$$
\Sigma_{B}^{\infty}(K, p)_{+} \wedge_{B} \Sigma_{B}^{\infty}(L, q)_{+} \cong \Sigma_{B}^{\infty}(P, \pi)_{+}
$$


as spectra over $B$. Applying $r_{\text {! }}$ to this, we obtain $\Sigma^{\infty} P_{+}$. Assuming that the cohomologies of the given spaces are of finite type, so that we have a Künneth isomorphism in cohomology, our spectral sequence takes the expected form

$$
E_{2}^{p, q}=\operatorname{Tor}_{H^{*}(B, \mathbb{F})}^{p, q}\left(H^{*}(K, \mathbb{F}), H^{*}(L, \mathbb{F})\right) \Longrightarrow H^{*}(P, \mathbb{F}) .
$$

This may appear too strong to be plausible since we have made no connectivity or nilpotence assumptions. With this approach, these can only be relevant to determining when the colimit that enters into our convergence statement is zero. A similar remark applies when $k$ is a Morava $K$-theory spectrum.

We close with a few comments on the literature.

REMARK 22.7.13. The original chain level construction of Eilenberg and Moore [59] (see also [71]) gives a spectral sequence that, in the case of ordinary homology and cohomology, looks formally the same as the ones later constructed more geometrically, but there is no proof in the literature that these different constructions give isomorphic spectral sequences. Each type of construction has its advantages. For example, with geometric constructions it is clear how to put Steenrod operations in the spectral sequence, as was first done by Rector [140]. On the other hand, the chain level construction is essential to the calculational results of $[71,126]$, for example. The proofs of convergence with the various constructions vary considerably. Comparisons among them are needed. Comparison of Remark 22.7.12 with known convergence results for ordinary cohomology under nilpotency conditions suggests that there are real differences. The geometric constructions in the literature focus on cosimplicial spaces, parametrized spaces, or a blend of the two, and there is an extensive literature. An incomplete list would include $[53,54,74,85,140,149,151,153,154,163]$. For non-nilpotent situations and non-connective generalized theories, there are a number of partial results and some illuminating counterexamples, but the complete picture is still quite unclear. 


\section{CHAPTER 23}

\section{Parametrized FSP's and generalized Thom spectra}

\section{Introduction}

Because the passage from spherical fibrations to their Thom complexes is an application of the functor $r$, Thom spaces and Thom spectra are intrinsically part of parametrized homotopy theory. In this chapter, we reinterpret standard constructions in a parametrized way and use the new point of view to construct a variety of new commutative orthogonal ring spectra that are of the same general type as Thom spectra. We recall from [105] that commutative orthogonal ring spectra are equivalent to commutative $S$-algebras in the sense of [61] and therefore to $E_{\infty}$ ring spectra.

The constructions are simple and conceptual. Commutative orthogonal ring spectra are equivalent to commutative $\mathscr{I}$-FSP's, where FSP stands for "functor with smash product". We have already used these in the non-commutative case in $\S 22.2$, but in this chapter we focus on the commutative case. The FSP structure is defined with respect to the external smash product, and its internalization is the product of the associated orthogonal ring spectrum. There is an analogous notion of an $\mathscr{I}$-FCP, where FCP stands for "functor with cartesian product", which is defined in terms of cartesian products rather than smash products. We shall define the new notion of a parametrized $\mathscr{I}$-FSP, to be abbreviated $\mathscr{I}$-PFSP.

In fact, as we will carry further elsewhere, these constructions are the specialization to the domain category $\mathscr{I}$ of diagram objects that can be defined starting from various other domain categories $\mathscr{D}$, notably the domain category $\Sigma$ for symmetric sequences (or collections). Since we shall focus primarily on $\mathscr{I}$, we shall generally omit $\mathscr{I}$ from the notation, writing FCP, FSP, and PFSP instead of $\mathscr{I}$-FCP, $\mathscr{I}$-FSP, or $\mathscr{I}$-PFSP.

Our PFSP's are functors that take values in the symmetric monoidal category $\mathscr{U}_{\mathscr{U}}$ of retracts that we defined in $\S 2.5$. Passage to base spaces gives a forgetful functor from PFSP's to FCP's, and application of $r$ ! gives a "Thom spectrum functor" from PFSP's to FSP's. The definition of a PFSP codifies and generalizes familiar structure that we see in nature. For example, consider the fiberwise one-point compactification of a good model $E O(V) \times{ }_{O(V)} V$ for the universal vector bundle over $B O(V)$, where $O(V)$ is the orthogonal group of an inner product space $V$. This construction gives a functor, $R O$ say, from our category $\mathscr{I}$ of finite dimensional inner product spaces to the cited category of retracts. The direct sum pairing

$$
O(V) \times O(W) \longrightarrow O(V \oplus W)
$$

passes to classifying spaces, where it gives the map

$$
B O(V) \times B O(W) \longrightarrow B O(V \oplus W)
$$


on base spaces of a pairing

$$
\mu: R O(V) \bar{\wedge} R O(W) \longrightarrow R O(V \oplus W)
$$

in the category of retracts. The fibers over the basepoints of the $B O(V)$ give maps $\eta: S^{V} \longrightarrow R O(V)$. The spaces $r_{!} R O(V)$ are the Thom spaces $T O(V)$. Together with the induced external pairing

$$
T O(V) \wedge T O(W) \longrightarrow T O(V \oplus W)
$$

and unit map $S^{V} \longrightarrow T O(V)$, they specify the Thom spectrum $M O$ as an FSP. The internalization of the pairing gives the product $M O \wedge M O \longrightarrow M O$ of the orthogonal ring spectrum $M O$. The triple $(R O, \mu, \eta)$ is an example of a PFSP.

Our generalization of this familiar structure leads to the promised new way of constructing commutative orthogonal ring spectra. In fact, the construction works more generally to construct commutative algebras over a commutative orthogonal ring spectrum $R$. The starting point is then the notion of an $R$-PFSP. With this notion, passage to base spaces still gives an FCP, but application of $r$ ! now gives an $R$-FSP, which is the external equivalent of a commutative $R$-algebra.

We shall use the two-sided bar construction to show how to construct many examples of $R$-PFSP's. Conceptually, the procedure is analogous to the construction of twisted theories in the previous chapter. Instead of starting with a group $\Pi$, we start with a group-valued or monoid-valued FCP П. We replace the bundle construction $E \Pi \times_{\Pi}(-)$ with a parametrized two-sided bar construction $B(-, \Pi,-)$. We abbreviate this to $B(\Pi,-)$ when the first variable is trivial, and we obtain a family of universal principal bundles $E \Pi=B(\Pi, \Pi)$. When $\Pi$ acts on the left on a FCP $Y$, we obtain a family $B(\Pi, Y)=E \Pi \times_{\Pi} Y$ of associated bundles given by the Borel construction. The analogy should be clear.

In the last two sections, we switch gears. In the theory above, we never pass to colimits on the base space level but rather focus on a fixed base FCP. We end the chapter with the bare beginnings of a study of the relationship between parametrized theory and colimits of base spaces. In particular, we show how Thom prespectra of maps $X \longrightarrow B F$ can be interpreted conceptually as a construction in parametrized homotopy theory. This too will be carried further elsewhere, in joint work with Andrew Blumberg.

We work nonequivariantly, for simplicity, but the constructions of this chapter apply verbatim equivariantly. We describe diagram functors with products in full categorical generality in $\S 23.1$, and we specialize topologically to define FCP's, $R$ FSP's, and R-PFSP's in $§ 23.2$. We define group, monoid, and module FCP's in §23.3. As an interesting example, we observe that an FSP $R$ gives rise to an FCP of units $G L_{1}(R)$ and an FCP of special units $S L_{1}(R)$.

We define the two-sided bar construction in the category of FCP's in $\S 23.4$ and show how to construct examples of $R$-FSP's by replacing the righthand variable FCP in $B(-, \Pi,-)$ by an FSP $R$. When $R$ is the sphere FSP, the classical Thom spectra appear as special cases, but now these themselves can sometimes be used in place of $R$, as we show in $\S 23.5$. In particular, we construct iterated Thom spectra such as $M^{q} U$, starting with $M^{0} U=S$ and $M^{1} U=M U ; M^{q} U$ is a commutative $M^{q-1} U$ algebra. This is a tantalizing construction, but we have barely begun the study of these new commutative algebras.

Switching to the consideration of colimits, we recall how $\mathscr{I}$-FCP's give rise to spaces with actions by the linear isometries operad $\mathscr{L}$ and thus to $E_{\infty}$ spaces 
and therefore spectra in $\S 23.6$. We also comment on the analogous infinite loop space theory starting from $\Sigma$-FCP's. We show in $\S 23.7$ that parametrized methods simplify the construction of Thom prespectra of maps. The point is to construct a universal spherical fibration prespectrum $\operatorname{Sph}(F)$ over $B F$. The Thom prespectrum of a map $f: X \longrightarrow B F$ is then just $r ! f^{*} \operatorname{Sph}(F)$. The original definition is due to Mahowald [101], and such Thom spectra were first studied in detail by Lewis [94,98]. We leave the relationship between this construction of Thom prespectra of maps and the theory of $E_{\infty}$ ring spectra to work in progress of Blumberg and the authors.

Some of our constructions are spelled out in more detail but under different names in [112], and a good deal of related work has taken place in the intervening thirty years. We give a dictionary and some historical background in $\S 23.8$.

Aside from basic definitions, this chapter is almost completely independent of everything that has come before in this book. In particular, we make no mention of model structures, and parametrized spectra only appear in $\S 23.7$. Our focus is on the use of parametrized methods to construct new non-parametrized objects, and the passage from the point-set level to homotopy categories is routine.

\section{1. $\mathscr{D}$-functors with products in symmetric monoidal categories}

It seems best to start off in complete generality. The material to follow was first considered categorically by Day [43]. We follow the treatment of [106, $\S \S 1$, $22]$, which gives details in the context of topological diagram spectra. We let $\mathscr{V}$ be a cocomplete symmetric monoidal category and let $\mathscr{C}$ be a $\mathscr{V}$-enriched cocomplete symmetric monoidal category, where cocompleteness and the symmetric monoidal structure are understood in the enriched sense. We write $\otimes, I$, and $\tau$ for the product, unit object, and commutativity isomorphism in both $\mathscr{C}$ and $\mathscr{V}$. In some cases $\mathscr{C}=\mathscr{V}$, but not in the new case that we are most interested in, where $\mathscr{V}=\mathscr{U}$ and $\mathscr{C}=\mathscr{U}_{\mathscr{U}}$. We let $\mathscr{D}$ be a skeletally small symmetric monoidal category enriched over $\mathscr{V}$, and we write $\oplus, 0$, and $\tau$ for its product, unit object, and commutativity isomorphism.

Definition 23.1.1. A $\mathscr{D}$-object is a $\mathscr{V}$-functor $X: \mathscr{D} \longrightarrow \mathscr{C}$. A map of $\mathscr{D}$ objects is a $\mathscr{V}$-natural transformation between them. Let $\mathscr{D}[\mathscr{C}]$ be the category of $\mathscr{D}$-objects in $\mathscr{C}$.

Henceforward, we take the enrichment for granted. When $\mathscr{V}$ is $\mathscr{U}$ or $\mathscr{T}$, this just means that we consider only continuous functors and natural transformations.

Definition 23.1.2. A lax monoidal functor $\mathscr{D} \longrightarrow \mathscr{C}$ is called a $\mathscr{D}$-functor with products, or $\mathscr{D}$-FP. A lax symmetric monoidal functor $\mathscr{D} \longrightarrow \mathscr{C}$ is called a commutative $\mathscr{D}$-functor with products. A strong symmetric monoidal functor is said to be a spherical (or idempotent) $\mathscr{D}$-FP.

Thus, for a $\mathscr{D}$-FP $R$, we are given a unit map $\lambda: I \longrightarrow R(0)$ and product maps $\phi: R(d) \otimes R(e) \longrightarrow R(d \oplus e$ ) such that appropriate diagrams (as in the case $A=R$ and $\eta=$ id of Definition 23.1.5 below) commute. If $R$ is spherical, $\lambda$ and $\phi$ are isomorphisms. Spherical $\mathscr{D}$-FP's are not unique, but there is usually an obvious preferred choice, which will be denoted $S_{\mathscr{D}}$ and made explicit in the examples. Implicitly, the definition above uses an external product of diagram objects. 
Definition 23.1.3. The external product of $\mathscr{D}$-objects $X$ and $Y$ is the evident $\mathscr{D} \times \mathscr{D}$-object $X \bar{\otimes} Y$ given on objects $d$ and $e$ of $\mathscr{D}$ by

$$
(X \bar{\otimes} Y)(d, e)=X(d) \otimes Y(e) .
$$

This product is unital, associative, and commutative in the sense that the symmetric monoidal structure of $\mathscr{C}$ induces coherent natural isomorphisms

$$
\begin{gathered}
X(d) \bar{\otimes} I \cong X(d) \cong I \bar{\otimes} X(d), \\
(X \bar{\otimes} Y) \bar{\otimes} Z \cong X \bar{\otimes}(Y \bar{\otimes} Z),
\end{gathered}
$$

and

$$
X \bar{\otimes} Y \cong Y \bar{\otimes} X,
$$

where the second and third are isomorphisms of $(\mathscr{D} \times \mathscr{D} \times \mathscr{D})$-objects and $(\mathscr{D} \times \mathscr{D})$ objects, respectively.

Conceptually, this gives part of the stucture of a graded symmetric monoidal category on $\amalg \mathscr{D}^{i}[\mathscr{C}]$; here $\mathscr{D}^{0}$ is the trivial category, the unit object $I$ lives in $\mathscr{C}=\mathscr{D}^{0}[\mathscr{C}]$, and the first isomorphism above is one of $\mathscr{D}$-objects.

Since we have assumed that $\mathscr{C}$ is cocomplete, we can use left Kan extension to internalize the product $\bar{\otimes}$ to obtain a product $\otimes$ on $\mathscr{D}[\mathscr{C}]$ such that

$$
\mathscr{D}(X \otimes Y, Z) \cong(\mathscr{D} \times \mathscr{D})[\mathscr{V}](X \bar{\otimes} Y, Z \circ \oplus) .
$$

The category $\mathscr{D}[\mathscr{C}]$ is symmetric monoidal with unit the represented object $0^{*}$ specified by $0^{*}=\mathscr{D}(0,-)$, and the concepts in Definition 23.1.2 internalize as follows.

Proposition 23.1.4. The category of $\mathscr{D}$-FP's is equivalent to the category of monoids in $\mathscr{D}[\mathscr{C}]$. The category of commutative $\mathscr{D}-F P$ 's is equivalent to the category of commutative monoids in $\mathscr{D}[\mathscr{C}]$.

Thinking of a commutative monoid $R$ in a symmetric monoidal category as an analogue of a commutative ring, the analogue of an $R$-algebra is an $R$-monoid $A$, that is, a monoid $A$ together with a central map of monoids $\eta: R \longrightarrow A$. The unit of $A$ is then the composite $\eta \circ \lambda$. We spell out the external equivalent in the following definition.

Definition 23.1.5. Let $R$ be a commutative $\mathscr{D}$-FP. A $\mathscr{D}$-functor with products over $R$, abbreviated $R$ - $\mathscr{D}$-FP, is a $\mathscr{D}$-object $A$ together with a unit map $\eta: R \longrightarrow A$ of $\mathscr{D}$-objects and a product map $\mu: A \bar{\wedge} A \longrightarrow A \circ \oplus$ of $\mathscr{D} \times \mathscr{D}$-objects such that the composite

$$
A(d) \cong A(d) \wedge I \stackrel{\operatorname{id} \wedge \lambda}{\longrightarrow} A(d) \wedge R(0) \stackrel{\operatorname{id} \wedge \eta}{\longrightarrow} A(d) \wedge A(0) \stackrel{\mu}{\longrightarrow} A(d \oplus 0) \cong A(d)
$$

is the identity map, and the following unit, associativity, and centrality of unit diagrams commute:

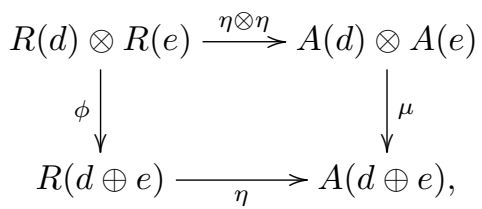




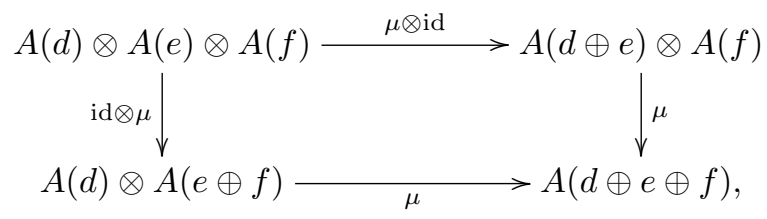

and

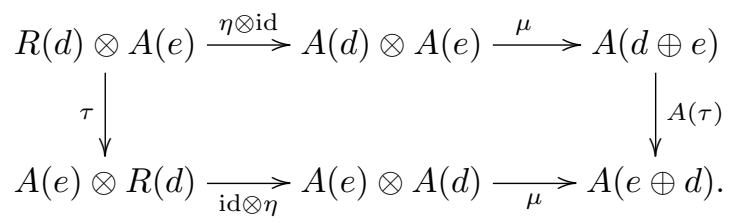

A $\mathscr{D}$-functor with products is commutative if the following diagram commutes, in which case the centrality of unit diagram just given commutes automatically:

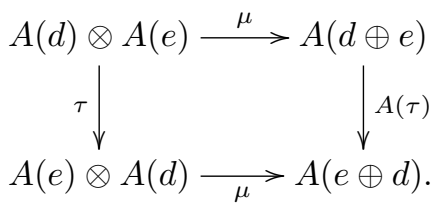

Proposition 23.1.6. The category of $R$-monoids in $\mathscr{D}[\mathscr{C}]$ is equivalent to the category of $\mathscr{D}$-FP's over $R$. The category of commutative $R$-monoids in $\mathscr{D}[\mathscr{C}]$ is equivalent to the category of commutative $\mathscr{D}$-FP's over $R$.

For monoids $R$ and for $R$-monoids $A$ ( $R$ commutative) in symmetric monoidal categories, we have the evident notions of (left or right) $R$-modules and of $A$ modules, and these too have evident external analogues in the case of our diagram categories $\mathscr{D}[\mathscr{C}]$.

\subsection{The specialization of $\mathscr{D}$-FP's to spaces and ex-spaces}

We now let $\mathscr{V}$ be either $\mathscr{U}$ with its cartesian monoidal structure or $\mathscr{T}$ with its monoidal structure under $\wedge$, but otherwise we retain the notations of the previous section. As usual, when our given $\mathscr{D}$ is $\mathscr{U}$-enriched but we are considering functors into a $\mathscr{T}$-enriched target category $\mathscr{C}$, we implicitly add disjoint basepoints to enrich $\mathscr{D}$ over $\mathscr{T}$.

First we take $\mathscr{C}=\mathscr{V}=\mathscr{U}$ and let $*$ be the terminal $\mathscr{D}$-FP, which of course is spherical.

Definition 23.2.1. A $\mathscr{D}$-FCP is a $\mathscr{D}$-FP over $*($ or $*$ - $\mathscr{D}$-FP) in $\mathscr{U}$.

Remark 23.2.2. A $\mathscr{D}$-FCP $R=(R, \omega, \lambda)$ necessarily takes values in $\mathscr{T}$. The basepoint of $R(d)$ is given by the unit map $\lambda: * \longrightarrow R$, and the unit condition says that $\omega(x, *)=x$ in $R(d \oplus 0) \cong R(d)$ for $x$ in $R(d)$ and $*$ in $R(0)$. In fact, a $\mathscr{D}$-FCP is exactly the same structure as a $\mathscr{D}$-FP in $\mathscr{T}$. From the latter point of view, the given basepoints prescribe the unit map $\lambda$.

We now take $\mathscr{C}=\mathscr{V}=\mathscr{T}$ and let $S_{\mathscr{D}}$ be a chosen sphere $\mathscr{D}$-FP associated to $\mathscr{D}$. There is usually a standard choice.

Definition 23.2.3. A $\mathscr{D}$-FSP is a $\mathscr{D}$-FP over $S_{\mathscr{D}}$ (or $S_{\mathscr{D}} \mathscr{D}$-FP) in $\mathscr{T}$. For a commutative $\mathscr{D}$-FSP $R$, a $\mathscr{D}$-FSP over $R$, or $R$ - $\mathscr{D}$-FSP, is a $\mathscr{D}$-FP over $R$ in $\mathscr{T}$. 
Suitable choices of $\mathscr{D}$ for constructing stable categories of spectra are discussed in detail in [106]. The internalized versions of $R$-FSP's are then called $R$-algebras. We focus on $\mathscr{I}$, with its standard sphere FP $S_{\mathscr{I}}$; thus $S_{\mathscr{I}}(V)=S^{V}$. Another important choice is $\Sigma$, the category with objects the natural numbers and morphisms the symmetric groups viewed as automorphism groups $\Sigma_{n}: n \longrightarrow n$; its standard sphere FP $S_{\Sigma}$ has values the $\Sigma_{n}$-spaces $S^{n}$. In these cases, a $\mathscr{D}$-spectrum is defined to be a right $S_{\mathscr{D}}$-module. An $S_{\mathscr{D}}$-algebra $(R, \mu, \eta)$ is a right $S_{\mathscr{D}}$-module via the composite

$$
R \wedge S_{\mathscr{D}} \stackrel{\mathrm{id} \wedge \eta}{\longrightarrow} R \wedge R \stackrel{\mu}{\longrightarrow} R
$$

and these are the orthogonal and symmetric ring spectra.

We can define analogous parametrized orthogonal ring spectra, but, as noted in $\S 14.1$, we would not have homotopical control over that notion. However, allowing varying base spaces and using external smash products (in the sense of allowing products of base spaces), we obtain a useful notion over which we do have homotopical control. To specify this notion, we take $\mathscr{V}=\mathscr{U}$ and we take $\mathscr{C}$ to be the category of retracts $\mathscr{U}_{\mathscr{U}}$, viewed as symmetric monoidal with unit $S_{*}^{0}$ under the external smash product $\bar{\wedge}$. The following definitions work just as well for $\mathscr{D}=\Sigma$ as for $\mathscr{D}=\mathscr{I}$; we delete $\mathscr{D}$ from the notation but allow either choice, continuing to use the letters $d$ and $e$ for typical objects of $\mathscr{D}$. Recall that, for any $B, \mathscr{U}_{B}$ is embedded in $\mathscr{U}_{\mathscr{U}}$. In particular $\mathscr{T}=\mathscr{U}_{*}$ is embedded as a symmetric monoidal subcategory of $\mathscr{U}_{\mathscr{U}}$; we write $\bar{\wedge}$ rather than $\wedge$ for its smash product.

DEFINITION 23.2.4. Let $R$ be a (nonparametrized) commutative FSP. A PFSP $A$ over $R$, or $R$-PFSP, is a $\mathscr{D}$-object $A: \mathscr{D} \longrightarrow \mathscr{U}_{\mathscr{U}}$ together with a unit map $\eta: R \longrightarrow A$ of $\mathscr{D}$-objects, and a product map $\mu: A \bar{\wedge} A \longrightarrow A \circ \oplus$ of $\mathscr{D} \times \mathscr{D}$-objects, that satisfy the evident analogues of the conditions specified in Definition 23.1.5. In detail, writing $\mathrm{A}(\mathrm{d})$ as a retract $B(d) \longrightarrow A(d) \longrightarrow B(d)$ and writing $\omega$ for the map $\mu$ on base spaces, $\eta$ and $\mu$ are given by maps of retracts

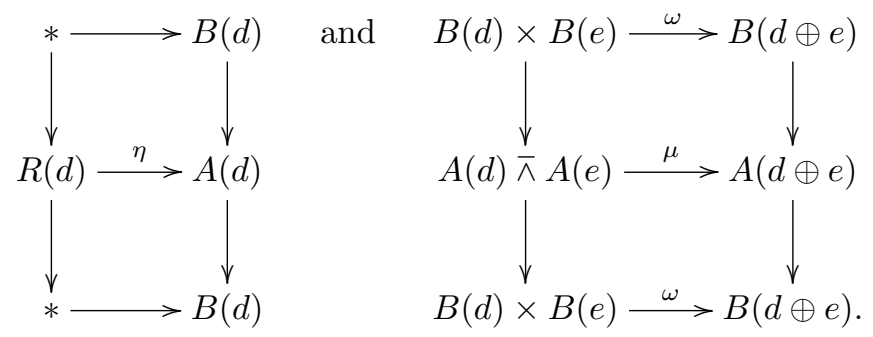

With $R(d)$ and $A(d)$ again understood as objects of $\mathscr{U}_{\mathscr{U}}$, the composite

$$
A(d) \cong A(d) \bar{\wedge} S^{0} \stackrel{\mathrm{id} \bar{\wedge} \lambda}{\longrightarrow} A(d) \bar{\wedge} R(0) \stackrel{\mathrm{id} \bar{\wedge} \eta}{\longrightarrow} A(d) \bar{\wedge} A(0) \stackrel{\mu}{\longrightarrow} A(d \oplus 0) \cong A(d)
$$

is the identity map, and the following unit, associativity, and centrality of unit diagrams commute:

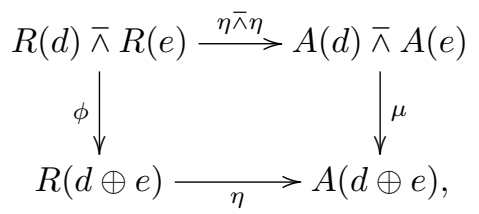




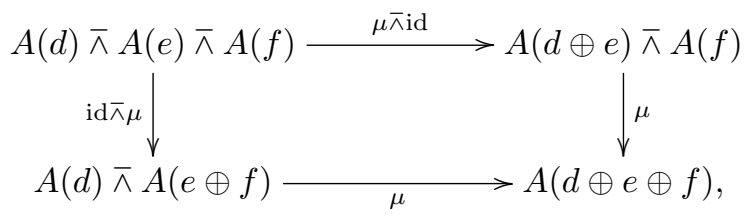

and

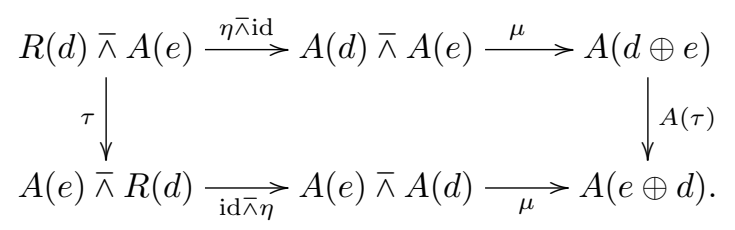

An $R$-PFSP is commutative if the following diagram commutes, in which case the centrality of unit diagram just given commutes automatically:

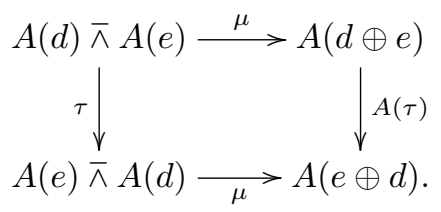

Definition 23.2.5. A parametrized FSP, or PFSP, is an $S$-PFSP in $\mathscr{U} \mathscr{U}$, and similarly for commutative PFSP's.

With the evident morphisms, we have categories of all objects that we have defined, and the following three results are immediate from the definitions.

Proposition 23.2.6. Passage to base spaces defines a forgetful functor from the category of R-PFSP's to the category of FCP's.

Proposition 23.2.7. The fibers $A(d)_{*}$ over the unit basepoints $* \in B(d)$ inherit a structure of FSP from $A$, and $A_{*}$ is an R-FSP via $\eta$. This gives a fiber functor from the category of $R$-PFSP's to the category of $R$-FSP's.

In practice, $\eta: R \longrightarrow A_{*}$ is often an isomorphism.

TheOREM 23.2.8. Application of $r$ ! to all ex-spaces defines a Thom spectrum functor from the category of R-PFSP's to the category of R-FSP's.

Proof. The $\mathscr{D}$-space $r ! A$ is given by $\left(r_{!} A\right)(d)=r_{!}(A(d))=A(d) / s B(d)$. The functoriality on $\mathscr{D}$ is evident. The required unit $\eta$ and product $\mu$ are induced from those of $A$. For $\eta$, notice that $A_{*} \subset r_{!} A$. For $\mu$, notice that

$$
r_{!}(A(d)) \wedge r_{!}(A(e)) \cong(r \times r) !(A(d) \bar{\wedge} A(e))=r_{!}(A(d) \bar{\wedge} A(e)) .
$$

\subsection{Group, monoid, and module FCP's; examples}

Let $F C P[\mathscr{U}]$ denote the category of $\mathscr{I}$-FCP's. We describe groups, monoids, and modules in this category and give various examples. In all of our examples, the underlying FCP is commutative, and we will not reiterate this point. In this section and the next, the basic definitions apply equally well with $\mathscr{I}$ replaced by $\Sigma$, but we focus attention on $\mathscr{I}$ for specificity. 


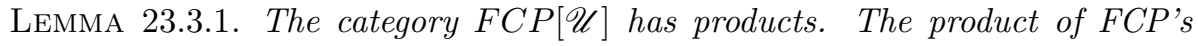
$(B, \nu)$ and $(C, \omega)$ is $(B \times C, \nu \times \omega)$, where $(B \times C)(V)=B(V) \times C(V)$ and where $(\nu \times \omega)(V, W)$ is the composite

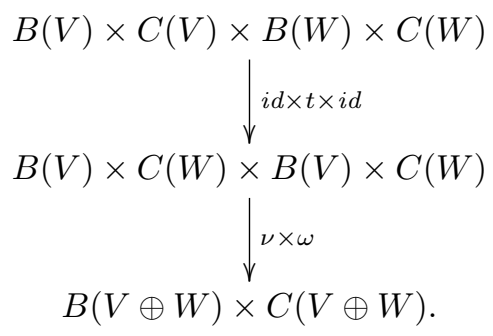

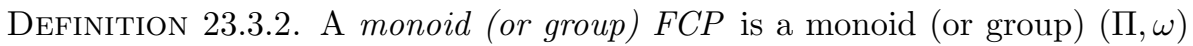
in the cartesian monoidal category $F C P[\mathscr{U}]$. Thus $\Pi$ is a monoid-valued functor such that the following diagrams commute.

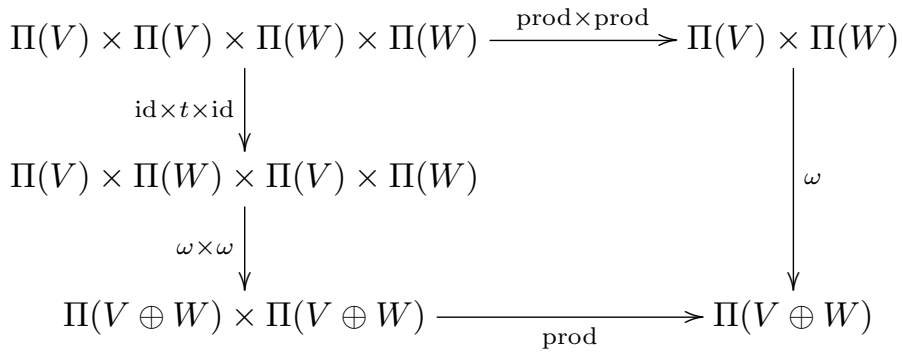

A right $\Pi$-FCP $(D, \nu)$ is a right $\Pi$-module in $F C P[\mathscr{U}]$; this means that there are right actions $D(V) \times \Pi(V) \longrightarrow D(V)$ such that the evident analogue of (23.3.3) commutes. Left П-FCP's are defined similarly.

We recall some examples. For a based space $X$, let $F(X)$ be the monoid of based homotopy equivalences $X \longrightarrow X$, let $S F(X)$ be the submonoid of maps homotopic to the identity map of $X$, let $\operatorname{Top}(X)$ be the subgroup of $F(X)$ consisting of the based homeomorphisms, and let $\operatorname{STop}(X)$ be the subgroup of based homeomorphisms in $S F(X)$. Letting $X$ run through the spheres $S^{V}$, we obtain monoid FCP's $F$ and $S F$ and group FCP's Top and STop. On $F$, the pairing

$$
\omega: F(V) \times F(W) \longrightarrow F(V \oplus W)
$$

is obtained by identifying $S^{V \oplus W}$ with $S^{V} \wedge S^{W}$ and taking the smash product of homotopy equivalences. It restricts to give $\omega$ on $S F$, Top, and STop. Restricting further to linear isometries and linear isometries of determinant 1, we obtain group FCP's $O$ and $S O$. Using complex inner product spaces rather than real ones, we obtain group FCP's $U$ and $S U$. In all cases, we must not confuse these monoidvalued FCP's, which are functors and have external pairings, with the monoids of the same names that are obtained by taking colimits over $V$ and have composition products that make no reference to the external pairings; compare Remark 22.2.6.

REMARK 23.3.4. It seems preferable to think in terms of the theory of "unitary spectra", the reworking of the theory of orthogonal spectra from the starting point of complex inner product spaces, rather than to think in terms of functors defined on real inner product spaces by first complexifying. That is, we think in terms of realification $U(n) \longrightarrow S O(2 n)$ rather than complexification $O(n) \longrightarrow U(n)$. Using 
their underlying real inner products, the complex inner product spaces embed in the real ones. Then "change of universe", as in $\S 14.2$, shows that the two choices give canonically equivalent homotopy categories of spectra.

There are further classical examples Spin and $\operatorname{Spin}^{c}$, and a newer example String, which can be made as precise as we need using the construction in [7]. As explained in $[112, \mathrm{Ch} . \mathrm{I}]$, which gives more details, all of the usual classical homogeneous spaces $\Pi / \Pi^{\prime}$, defined in terms of right cosets, are right $\Pi$-module FCP's.

We end this section by showing how a commutative FSP $R$ gives rise to a unit right $F$-module $G L_{1}(R)$.

Definition 23.3.5. For an $\mathscr{I}$-space $T$, let $\Lambda(T)$ be the induced $\mathscr{I}$-space such that $\Lambda(T)(V)=\Omega^{V} T(V)$. The evaluation maps of the functor $\Lambda(T)$ are the maps

$$
\mathscr{I}(V, W) \times \Omega^{V} T(V) \longrightarrow \Omega^{W} T(W)
$$

that send $(\alpha, f)$ to the composite

$$
S^{W} \stackrel{\alpha^{-1}}{\longrightarrow} S^{V} \stackrel{f}{\longrightarrow} T(V) \stackrel{T(\alpha)}{\longrightarrow} T(W) .
$$

Since $F(V)$ is the space of homotopy equivalences $S^{V} \longrightarrow S^{V}$ and $\Omega^{V} T(V)$ is the space of maps $S^{V} \longrightarrow T(V)$, composition gives a right action of the monoid $F(V)$ on the space $\Lambda(T)(V)$.

To avoid confusion in the following example, recall Remark 22.2.5.

Proposition 23.3.6. Let $R$ be a commutative FSP whose underlying orthogonal spectrum is positive fibrant and satisfies $R_{0}=S^{0}$. Then $\Lambda(R)$ restricts to a right $F$-module $G L_{1}(R)$ of units and a right $S F$-module $S L_{1}(R)$ of special units.

Proof. The homotopy group $\pi_{0}(R)$ is a commutative ring and, since $R$ is positive fibrant, we may use the adjoint structural equivalences to identify $\pi_{0}(R)$ with $\pi_{0}\left(\Omega^{V} R(V)\right)$ for all $V$ of positive dimension. We let $G L_{1}(R)(0)=1$ and let $G L_{1}(R)(V)$ be the subspace of $\Omega^{V} R(V)$ consisting of the components of the units in $\pi_{0}(R)$; we let $S L_{1}(R)(V)$ be the component of the identity element. Since the points of $F(V)$ are homotopy equivalences of $S^{V}$ and the points of $S F(V)$ are homotopic to the identity, our action map restricts to give a right action of $F(V)$ on $G L_{1}(R)(V)$ and a right action of $S F(V)$ on $S L_{1}(R)(V)$. Moreover, the product $\mu$ on $R$ gives rise to pairings

$$
\omega: \Omega^{V} R(V) \times \Omega^{W} R(W) \longrightarrow \Omega^{V \oplus W} R(V \oplus W) .
$$

For $f: S^{V} \longrightarrow R(V)$ and $g: S^{W} \longrightarrow R(W), \omega(f, g)$ is the composite

$$
S^{V \oplus W} \cong S^{V} \wedge S^{W} \stackrel{f \wedge g}{\longrightarrow} R(V) \wedge R(W) \stackrel{\mu}{\longrightarrow} R(V \oplus W) .
$$

Considering homotopy groups, we see that these maps restrict to pairings that give FCP's $\left(G L_{1}(R), \omega\right)$ and $\left(S L_{1}(R), \omega\right)$. The required commutativity of the module analogue of the diagram (23.3.3) is clear by inspection. 


\subsection{The two-sided bar construction on FCP's}

The following old observation, which is [112, I.2.2], is central to the new constructions in this chapter.

Proposition 23.4.1. If $\Pi$ is an FCP, $D$ is a right $\Pi-F C P$, and $E$ is a left $\Pi-F C P$, then $B(D, \Pi, E)$ is a FCP. It is commutative if its inputs $D, \Pi$, and $E$ are all commutative (that is, commutative as FCP's).

The definition and properties of the two-sided bar construction are discussed in $[111, \S 7]$, and we are taking

$$
B(D, \Pi, E)(V)=B(D(V), \Pi(V), E(V)) .
$$

Since the two-sided bar construction preserves products, in the sense that

$$
B(D, \Pi, E) \times B\left(D^{\prime}, \Pi^{\prime}, E^{\prime}\right) \cong B\left(D \times D^{\prime}, \Pi \times \Pi^{\prime}, E \times E^{\prime}\right)
$$

for monoids $\Pi$ and $\Pi^{\prime}$ acting from the right on $D$ and $D^{\prime}$ and from the left on $E$ and $E^{\prime}$, it is clear that the space $B(D, \Pi, E)$ inherits a product $\omega$ from those of $D$, $\Pi$, and $E$.

Notations 23.4.3. When $D=*$ is constant at a point, we abbreviate $B(*, \Pi, E)$ to $B(\Pi, E)$. Similarly, at the price of a little ambiguity, we abbreviate $B(D, \Pi, *)$ to $B(D, \Pi)$ and $B(*, \Pi, *)$ to $B \Pi$.

We see considerable resemblence between FCP's and orthogonal spectra. We now show how to to exploit the comparison. We replace the input $\Pi-F C P E$ of the two-sided bar construction $B(D, \Pi, E)$ with a $\Pi$-FSP $R$ and we obtain as output an $R$-FSP with base FCP $B(D, \Pi)$. We need an observation and a definition before we can implement this idea.

LEMMA 23.4.4. Let $\Lambda$ and $\Pi$ be topological monoids that act through based maps on based spaces $X$ and $Y$. Then the formula

$$
(\alpha, \beta)(x \wedge y)=(\alpha x) \wedge(\beta y)
$$

for $\alpha \in \Lambda, \beta \in \Pi, x \in X$, and $y \in Y$ gives an action of $\Lambda \times \Pi$ on $X \wedge Y$.

We assume from now on in this section that $\Pi$ is a monoid FCP together with a map $\Pi \longrightarrow F$ of monoid FCP's. We say that $\Pi$ is a monoid mapping to $F$. Since $F$ acts from the left on the sphere FCP $S$ via the evaluation maps $F(V) \times S^{V} \longrightarrow S^{V}$, this ensures that $\Pi$ acts on $S$.

Definition 23.4.5. Let $(\Pi, \omega)$ be a monoid FCP mapping to $F$. A left action of $\Pi$ on an orthogonal spectrum $X$, is a left action of $\Pi$ on the underlying $\mathscr{I}$-space $X$ such that the following diagrams commute.

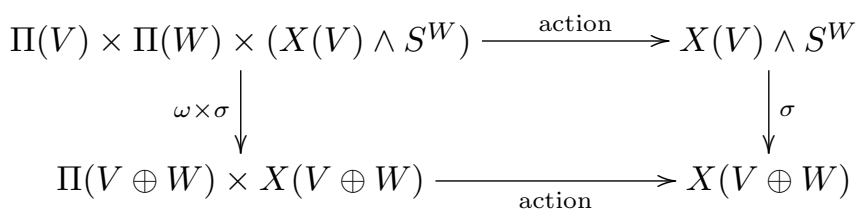


A left action of $\Pi$ on an FSP $R$ is a left action of $\Pi$ on the $\mathscr{I}$-space $R$ such that the following diagrams commute.

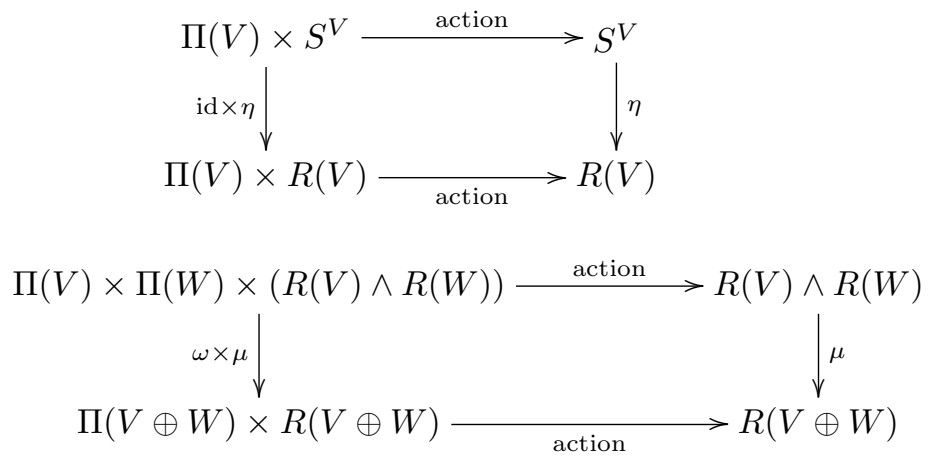

Observe that (23.4.7) and (23.4.8) imply (23.4.6), so that $R$ is a $\Pi$-spectrum with additional structure.

THEOREM 23.4.9. Let $\Pi$ be a monoid FCP mapping to $F$, let $D$ be a right $\Pi-F C P$, and let $R$ be a commutative left $\Pi-F S P$. Assume that $\Pi$ and $D$ are commutative as FCP's. Then $B(D, \Pi, R)$ is a commutative $R$-PFSP with base FCP $B(D, \Pi)$. Therefore $r ! B(D, \Pi, R)$ is a commutative $R$-algebra.

Proof. We define $B(D, \Pi, R)(V)=B(D(V), \Pi(V), R(V))$. As in Proposition 23.4.1, $B(D, \Pi, R)$ is an $\mathscr{I}$-space. The evident maps of $\Pi$-spaces $* \longrightarrow$ $R(V) \longrightarrow *$ induce projections and sections

$$
B(D, \Pi)(V) \longrightarrow B(D, \Pi, R)(V) \longrightarrow B(D, \Pi)(V) .
$$

Thus gives a functor $\mathscr{I} \longrightarrow \mathscr{U}_{\mathscr{U}}$. The basepoint of $D(V)$ gives the basepoint of $B(D(V), \Pi(V))$, in the simplicial zero skeleton, and the fiber over the basepoint is $R(V)$. This gives an identification $\eta$ of $R$ with the fiber $B(D, \Pi, R)_{*}$. Using the identification (23.4.2), we see that the products $\nu$ on $D, \omega$ on $\Pi$ and $\mu$ on $R$ induce pairings

$$
\mu: B(D, \Pi, R)(V) \bar{\wedge} B(D, \Pi, R)(W) \longrightarrow B(D, \Pi, R)(V \oplus W)
$$

over and under the pairing

$$
\omega: B(D, \Pi)(V) \times B(D, \Pi)(W) \longrightarrow B(D, \Pi)(V \oplus W)
$$

induced by the given $\nu$ and $\omega$. The unit, associativity, and commutativity conditions are all inherited from the input pairings. This proves the result, with the last statement following from Theorem 23.2.8.

\subsection{Examples: iterated Thom spectra}

Examples of base FCP's $B(D, \Pi)$ abound. The case $D=*$, written $B(\Pi)$, is already of obvious interest. A particularly interesting non-trivial choice for $D$ is to consider a second FSP $Q$, generally not $R$, and take $D=G L_{1}(Q)$ when $\Pi$ maps to $F$ and $D=S L_{1}(Q)$ when $\Pi$ maps to $S F$; see Example 23.6.5 below. The obvious choice of $R$, which works for any $\Pi$ mapping to $F$, is $R=S$.

Definition 23.5.1. The classical Thom spectra are the commutative ring spectra $M \Pi=r ! B(\Pi, S)$. The generalized Thom spectra are the commutative ring spectra $M(D, \Pi)=r_{!} B(D, \Pi, S)$. 
We use analogous notation in the general case, but remembering $R$.

Notation 23.5.2. For a right $\Pi$ - $\mathscr{I}$-FSP $D$ and a left $\Pi$ - $\mathscr{I}$-FSP $R$, we define

$$
M(D, \Pi, R)=r_{!} B(D, \Pi, R) .
$$

When $D=*$, we omit it from the notation, writing $M(\Pi, R)$.

To give examples, we need to construct actions of $\Pi$ on interesting $R$. We give a simple way to do this. Its starting point is the following observation.

Proposition 23.5.3. Let $\Pi$ be a topological group and $X$ and $Y$ be a right and a left $\Pi$-space. Then $B(X, \Pi, Y)$ is naturally a left $\Pi$-space.

Proof. $B(X, \Pi, Y)$ is the geometric realization of a simplicial space whose space of $q$-simplices is $X \times \Pi^{q} \times Y[111, \mathrm{p} .31]$. Let $\Pi$ act on this space by

$$
\alpha \cdot\left(x, \beta_{1}, \ldots, \beta_{q}, y\right)=\left(x \alpha^{-1}, \alpha \beta_{1} \alpha^{-1}, \ldots, \alpha \beta_{q} \alpha^{-1}, \alpha y\right) .
$$

Inspection shows that the action commutes with faces and degeneracies. Since the geometric realization of a simplicial $\Pi$-space is a $\Pi$-space, this proves the result. More conceptually, we are using that $\Pi$ acts on itself through conjugation and that left and right actions are suitably equivariant.

In the remainder of the section, we assume that $\Pi$ is a group FCP with a map to $F$. It could be $O, S O, U, S U$, Top or STop, for example. The notions of commutative $R$-FSP and commutative $R$-algebra are interchangeable, and we prefer to use the latter term for clarity. We assume that all given FCP's and FSP's are commutative in the following results.

THEOREM 23.5.4. For a right П-FCP $D$ and a left $\Pi-F S P R, M(D, \Pi, R)$ is both a П-FSP and a commutative R-algebra.

Proof. We have $M(D, \Pi, R)(V)=r_{!} B(D(V), \Pi(V), R(V))$, where $r$ ! collapses the section $s B(D(V), \Pi(V))$ to a point. Since $\Pi(V)$ acts trivially on the basepoint of $R(V)$, the section is a sub- $\Pi(V)$-space of the $\Pi(V)$-space $B(D(V), \Pi(V), R(V))$. Therefore we have an induced action of $\Pi(V)$ on the quotient. Using the diagrams (23.3.3) for $\Pi$, their analogues for $D$, and diagrams (23.4.7) and (23.4.8) for $R$, we see that diagrams (23.4.7) and (23.4.8) for $M(D, \Pi, R)$ commute. This gives that $M(D, \Pi, R)$ is a $\Pi$-FSP, and it is a commutative $R$-algebra by Theorem 23.4.9.

Corollary 23.5.5. For any two right $\Pi$-FCP's $C$ and $D$ and any left $\Pi$ FSP $R, M(C, \Pi, M(D, \Pi, R))$ is defined and is both a $\Pi$-FSP and a commutative $M(D, \Pi, R)$-algebra.

Taking $C$ and $D$ to be trivial and removing them from the notation, we obtain $M(\Pi, R)$-algebras $M(\Pi, M(\Pi, R))$. This allows the following definition of iterated Thom spectra. The case $\Pi=U$ should be of particular interest.

Definition 23.5.6. Define $M^{0} \Pi=S$ and $M^{1} \Pi=M \Pi$. Assume inductively that we have constructed a $\Pi$-FSP and commutative $M^{q-1} \Pi$-algebra $M^{q} \Pi$. Define $M^{q+1} \Pi=M\left(\Pi, M^{q} \Pi\right)$. It is a $\Pi$-FSP and a commutative $M^{q} \Pi$-algebra. 


\section{6. $\mathscr{I}_{c}$-FCP's and $\mathscr{L}$-spaces}

Changing focus, we here recall from $[112$, I§1] the relationship between $\mathscr{I}$ FCP's and infinite loop spaces. At the end of the section we briefly describe the analogous theory that starts with $\Sigma$-FCP's instead of $\mathscr{I}$-FCP's.

Let $\mathscr{I}_{c}$ be the symmetric monoidal category of finite or countably infinite dimensional real inner product spaces and linear isometries, not necessarily isomorphisms. We write $U=\mathbb{R}^{\infty}$. In the equivariant case, we would take $U$ to be a complete $G$-universe, so the notation may serve as a reminder that everything can be done equivariantly. We can take the domain category $\mathscr{D}$ to be $\mathscr{I}_{c}$ in our definition of an FCP, but it is sensible to add some point-set topological restrictions.

Definition 23.6.1. We define $\mathscr{I}_{c}$-FCP's exactly as we defined $\mathscr{I}$-FCP's, but with the additional requirement that their underlying $\mathscr{I}_{c}$-spaces $T$ satisfy the following two properties.

(i) The map $T(V) \longrightarrow T(W)$ induced by an isometry $V \longrightarrow W$ is a homeomorphism onto a closed subset.

(ii) Each $F(W)$ is the colimit of the $F(V)$, where $V$ runs over the finite dimensional subspaces of $W$ and the colimit system runs over the inclusions $V \subset V^{\prime}$.

We emphasize that the colimit runs only over the inclusions, not over all linear isometries. With this modified definition, we have the following result from [112, 1.9]. Observe that the inclusion $\mathscr{I} \longrightarrow \mathscr{I}_{c}$ induces a forgetful functor from the category $\mathscr{I}_{c}$-FCP $[\mathscr{U}]$ of $\mathscr{I}_{c}$-FCP's to the category $\mathscr{I}$-FCP $[\mathscr{U}]$ of $\mathscr{I}$-FCP's.

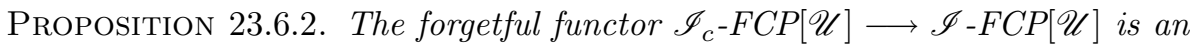
equivalence of categories that restricts to give equivalences between the respective subcategories of structured FCP's (monoids, groups, right П-modules, etc).

Proof. As explained in detail in $[112,1.9]$, an $\mathscr{I}$-FCP $(T, \omega)$ extends to an $\mathscr{I}_{c}$-FCP, uniquely up to isomorphism. For a linear isometry $f: V \longrightarrow W$, where $V$ is finite dimensional, $W$ is the sum of $f(V)$ and its orthogonal complement $f(V)^{\perp}$. When $W$ is also finite dimensional, we define $T(f): T(V) \longrightarrow T(W)$ by composing $T(f): T(V) \longrightarrow T(f(V))$ with the map $T(f(V)) \longrightarrow T(W)$ that sends $x$ to $\omega(x, *)$, where $*$ is the basepoint in $T\left(f(V)^{\perp}\right)$. When $W$ is infinite dimensional, we can and must define $T(W)$ to be the colimit of the $T(V)$ for finite dimensional $V \subset W$. The passage to colimits preserves all structure in sight.

We have an operad $\mathscr{L}$, called the linear isometries operad, whose $j$ th space is $\mathscr{L}(j)=\mathscr{I}_{c}\left(U^{j}, U\right)$. It is an $E_{\infty}$ operad since each $\mathscr{L}(j)$ is contractible [112, I.1.3]. The following observation is $[112,1.6]$.

Proposition 23.6.3. Passage from $T$ to $T(U)$ gives a functor from $\mathscr{I}_{c}-F C P[\mathscr{U}]$ to the category $\mathscr{L}[\mathscr{U}]$ of $\mathscr{L}$-spaces.

Proof. The action maps $\mathscr{L}(j) \times T(U)^{j} \longrightarrow T(U)$ are defined by first using $\omega$ to map $T(U)^{j}$ to $T\left(U^{j}\right)$ and then using the evaluation map of the functor $T$.

Notation 23.6.4. We sometimes abbreviate $T(U)$ to $T$, relying on context to determine whether $T$ is intended to mean an $\mathscr{I}_{c}$-FCP or an $\mathscr{L}$-space.

The previous two results show how to pass from $\mathscr{I}$-FCP's to $\mathscr{L}$-spaces and therefore, by an infinite loop space machine, to spectra. As explained in [114, $\S 5 \S 6]$ and [105, I.8.3], the output of May's machine actually takes values in orthogonal 
spectra. In fact, it gives a functor $E$ from $\mathscr{L}$-spaces $X$ to orthogonal $\Omega$-spectra $E X$ such that the zeroth space $(E X)(0)$ is a group completion of $X$. By composition, we obtain a functor $E$ that starts with $\mathscr{I}$-FCP's and ends with orthogonal spectra.

ExAmple 23.6.5. Passing to the associated $\mathscr{L}$-spaces from Proposition 23.3.6, we obtain unit infinite loop spaces $G L_{1}(R)$ and $S L_{1}(R)$ with right actions by the monoid $\mathscr{L}$-spaces $F$ and $S F$. Because the right actions come from maps of FCP's, the action maps are maps of $\mathscr{L}$-spaces. Taking $D=G L_{1}(Q)$ in Definition 23.5.1, the associated $\mathscr{L}$-space $B\left(G L_{1}(Q), \Pi\right)$ classifies $Q$-oriented spherical fibrations [112, Ch. 3] and is denoted $B(\Pi ; Q)$; similarly we denote the associated Thom spectrum by $M(\Pi ; Q)$. Work of Sullivan [161] (see also $[100,112]$ ) shows that, when localized away from $2, B(S F ; k o)$ is equivalent to $B$ STop and $M(S F ; k o)$ is equivalent to MSTop.

For results like this, it seems essential to consider the Lewis-May $E_{\infty}$ ring spectrum $\mathbb{M} R$ that we obtain by passage to colimits from the orthogonal spectrum $R$, as explained in detail in [105, $\S$ I.7]. With that construction, $G L_{1}(R)$ is exactly the unit space $G L_{1}(\mathbb{M} R)$, as specified in Definition 22.2.2, and there is a general result that often applies to compute localizations of the infinite loop space $S L_{1}(R)$ in terms of more directly accessible data $[115,8.7]$ (or [112, VII $\S 5]$ ). As explained in Remark 22.2.5, it is intrinsically impossible to have commutative symmetric or orthogonal $\Omega$-spectra whose actual unit spaces have analogous properties.

Finally, we consider analogues that start from $\Sigma$-FCP's rather than from $\mathscr{I}$ FCP's. We shall just sketch the ideas. Some details are just like those in the case of $\mathscr{I}$-FCP's, and the rest are given in Schlichtkrull's paper [142, §2.3 and §5].

REMARK 23.6.6. Let $T$ be a commutative $\Sigma$-FCP. There are two ways to construct associated infinite loop spaces. We can apply a prolongation functor as in [106] to construct $\mathscr{I}$-FCP's from $\Sigma$-FCP's, and we can then use the constructions that we have already described.

Alternatively, we can use the category $\mathcal{I}$ of finite (unbased) sets and injections as a kind of analogue of the category $\mathscr{I}_{c}$ that we used above. (In [142], our $\mathcal{I}$ FCP's are called $\mathcal{I}$-monoids, which is reasonable in a context where monoid-valued $\mathcal{I}$-FCP's are not considered). The category $\mathcal{I}$ contains $\Sigma$, and the forgetful functor from $\mathcal{I}$-FCP's to $\Sigma$-FCP's has a left adjoint extension functor, in precise analogy with Proposition 23.6.2.

For an analogue of Proposition 23.6.3, we consider the homotopy colimit, expressed in the notations of $[111, \S 12]$ as the categorical bar construction $T_{h \mathcal{I}}=$ $B(*, \mathcal{I}, T)$ for an $\mathcal{I}$-FCP $T$. The identification

$$
B(*, \mathcal{I}, T) \times B(*, \mathcal{I}, T) \cong B(*, \mathcal{I} \times \mathcal{I}, T \times T)
$$

and the product pairings of $T$ give $T_{h \mathcal{I}}$ a structure of topological monoid. Even when the $\mathcal{I}$-FCP $T$ is commutative, this monoid is non-commutative. Under convergence hypotheses, its homotopy type is identified by a lemma of Bökstedt ([15] or [142, 2.1]). For example, for a convergent commutative symmetric ring spectrum $R$, one might redefine $G L_{1}(R)$ to be the homotopy colimit of the unit $\mathcal{I}$-FCP obtained from the unit $\Sigma$-FCP denoted $G L_{1}(R)$ in Proposition 23.3.6 (taking $R$ there to be symmetric rather than orthogonal). Note that this monoid is not contained in $\operatorname{Aut}(R)$.

Finally, as explained in $[142, \S 5]$, if $T$ is a commutative $\mathcal{I}$-FCP, then we can construct a $\Gamma$-space from it and apply Segal's infinite loop space machine [147] 
to construct an associated infinite loop space. We have not checked details, but it seems to us that the methods of May and Thomason [127] can be adapted to compare these two passages from commutative $\Sigma$-FCP's to infinite loop spaces.

\subsection{Universal spherical fibration spectra}

Let $A$ be an $R$-PFSP over the FCP $B$. In Theorem 23.2 .8 we defined a Thom spectrum functor that associates an $R$-FSP $r ! A$ to $A$ by applying $r$ ! to the ex-spaces that comprise $A$. It would be desirable to perform an equivalent construction in two steps. The first would associate a parametrized $R$-spectrum $U(A)$ to $A$, and the second would apply $r$. The base space of $U(A)$, which we denote $\bar{B}$, should be obtained from $B$ by passing to the associated colimit $\mathscr{L}$-space as in the previous section. Such a two step approach would allow us to define Thom spectra of maps $f: X \longrightarrow \bar{B}$ as follows.

Definition 23.7.1. The Thom spectrum associated to the $R$-PFSP $A$ and the map $f: X \longrightarrow \bar{B}$ is

$$
r_{!} f^{*} U(A) \cong r_{!} f_{!} f^{*} U(A)
$$

The map $r$ on the left maps $X$ to $*$, that on the rightmaps $\bar{B}$ to $*$. The unit of the adjunction $\left(f_{!}, f^{*}\right)$ induces a canonical map $r_{!} f_{!} f^{*} U(A) \longrightarrow r_{!} U(A)$.

There are several difficulties in carrying out this idea. First of all, we have not defined parametrized ring spectra over a fixed base space, for reasons explained in $\S 14.1$. But in fact it is not even clear how to construct $U(A)$ as a parametrized orthogonal spectrum. This stems from the discrepancy of point of view between the passage to $\mathscr{L}$-spaces in $\S 23.6$ and the use of diagram structures everywhere else. Looking back at $\S 23.6$, one's first thought is to pass to colimits, starting for example from the $\mathscr{I}$-indexed diagram of ex-spaces $B(\Pi, S)$ over the $\mathscr{I}$-space $B \Pi$. Conceptually, this would be analogous to constructing $\mathscr{I}_{c}$-spaces from $\mathscr{I}_{\text {-spaces }}$ by passage to colimits. Since $B(\Pi, S)$ is an $\mathscr{I}$-PFSP with base $\mathscr{I}$-FCP $B \Pi$, this would seem to lead to a highly structured spectrum over the space $B \Pi$. This idea is very close to the original definition and construction of $E_{\infty}$ ring spectra in [112]. It seems to lead inexorably to a parametrized version of the kinds of highly structured spectra developed in $[61,98]$. We shall say a little about that approach to parametrized spectra in the next chapter, but it is not the right approach in our context of parametrized orthogonal spectra.

We shall not resolve these issues here, but we intend to treat them elsewhere. We can however give a quick construction of $U(A)$ as a prespectrum.

Construction 23.7.2. Let $A$ be a PFSP over the FCP $B$. We have a map

$$
A(V) \bar{\wedge} S^{W} \stackrel{\operatorname{id} \bar{\wedge} \eta}{\longrightarrow} A(V) \bar{\wedge} A(W) \stackrel{\mu}{\longrightarrow} A(V \oplus W)
$$

in $\mathscr{U}_{\mathscr{U}}$ whose map on base spaces is

$$
B(V) \cong B(V) \times * \stackrel{\operatorname{id} \times \eta}{\longrightarrow} B(V) \times B(W) \stackrel{\omega}{\longrightarrow} B(V \oplus W) .
$$

We denote the map on base spaces by $j(V, W)$ and obtain a map

$$
j(V, W) !\left(A(V) \wedge S^{W}\right) \longrightarrow A(V \oplus W)
$$


over $B(V \oplus W)$. Let $\bar{B}$ be the colimit of the $B(V)$, as in $\S 23.6$. There are compatible maps $i(V): B(V) \longrightarrow \bar{B}$ such that $i(V)=i(V \oplus W) \circ j(V, W)$. We define

$$
U(A)(V)=i(V) ! A(V) .
$$

The structure maps

$$
i(V) ! A(V) \wedge S^{W} \longrightarrow i(V \oplus W) ! A(V \oplus W)
$$

are obtained by applying $i(V \oplus W)$ ! to (23.7.3). It is not possible to retain functorality in the indexing spaces $V$ with this construction, so we only obtain a prespectrum.

Observe that $\bar{B}$ is an $\mathscr{L}$-space, although the definition does not make use of that structure. These ideas are of particular interest for the $R$-PFSP's $B(D, \Pi, R)$ constructed in Theorem 23.4.9. Specializing to $R=S$, we write $\operatorname{Sph}(D, \Pi)$ for $U(B(D, \Pi, S))$ and call it the universal spherical fibration prespectrum over $B(D, \Pi)$. We state this case separately for emphasis.

Definition 23.7.4. The Thom spectrum $M f$ associated to a map $f: X \longrightarrow$ $B(D, \Pi)$ is

$$
r_{!} f^{*} \operatorname{Sph}(D, \Pi) \cong r_{!} f_{!} f^{*} \operatorname{Sph}(D, \Pi),
$$

where $\operatorname{Sph}(D, \Pi)$ is the universal spherical fibration spectrum over $B(D, \Pi)$. With $M(D, \Pi)$ defined to be $r ! \operatorname{Sph}(D, \Pi)$, the unit $\varepsilon: f_{!} f^{*} \longrightarrow$ Id of the adjunction $\left(f_{!}, f^{*}\right)$ induces a canonical map $M f \longrightarrow M(D, \Pi)$.

Thus $M f$ is obtained by first pulling back the universal spherical fibration spectrum over $B \Pi$ to a spherical fibration spectrum over $X$ and then pushing forward along $r$ to obtain an ordinary spectrum. With Blumberg, we intend to study the multiplicative properties of spectra constructed in this fashion and to relate them to $\mathrm{THH}$ in a later paper.

We conclude this section by showing how $\operatorname{Sph}(D, \Pi)$ can be constructed as an excellent prespectrum. When $\Pi$ is a monoid FCP, the projections in $\operatorname{Sph}(D, \Pi)$ are only quasi-fibrations. It is sensible to replace them by (Hurewicz) fibrations, while preserving sections and structure maps, using the approximation functor $P$ of $\S 13.3$. The prespectrum $\operatorname{Sph}(\Pi)$ over $B \Pi$ is well-structured and $\Sigma$-cofibrant, in the sense of Definition 13.2.1, and the level ex-fibrant prespectrum $P \operatorname{Sph}(\Pi)$ over $B \Pi$ inherits these properties by Theorem 13.3.8. When $\Pi$ is a group FCP, the projections in $\mathrm{Sph}(\Pi)$ are already bundles and we can use it as it stands. It is technically desirable to go further and apply the functor $T=K E P$ of Theorem 13.5.1, or $K E$ in the bundle case, to $\operatorname{Sph}(\Pi)$, so as to preserve the cited properties while obtaining an excellent $\Omega$-prespectrum over $B \Pi$, in the sense of Definition 13.2.2; it can be viewed as giving a well-behaved fibrant replacement of $\mathrm{Sph}(\Pi)$ in the stable model structure of spectra over $B \Pi$. The spectrum $T \operatorname{Sph}(\Pi)$ is our universal spherical fibration prespectrum $\operatorname{Sph}(\Pi)$ over $B \Pi$, and $r ! \operatorname{Sph}(\Pi)$ gives a model for the classical Thom spectrum $M \Pi$. In practice, however, to retain algebraic structure, it is sometimes better to replace $f$ by an equivalent fibration, use $\operatorname{Sph}(\Pi)$ directly, and only then pass to $\Omega$-prespectra.

\subsection{Some historical background}

The framework for our construction of iterated Thom spectra is quite close to the framework developed in [112], where $E_{\infty}$ ring spaces and $E_{\infty}$ ring spectra were

first introduced. The use of the category $\mathscr{I}$ and the operad $\mathscr{L}$ (under the name 
PROP) goes back to the still earlier work of Boardman and Vogt [14]. Both $\mathscr{I}$ FCP's and $\mathscr{I}$-FSP's were defined in [112], but with different names. Since we have referred to that source for details, we give a dictionary in Remark 23.8.1 below. The classical Thom spectra were described as $\mathscr{I}$-FSP's in [112, IV $\S 2$, and the two-sided bar construction on $\mathscr{I}$-FCP's was defined in [112, I.2.2].

The notions of $\mathscr{I}$-FCP and $\mathscr{I}$-FSP are obviously related, but it has never been clear precisely how. Our work explains and exploits the connection, but only by virtue of recent progress in our understanding of the foundations of stable homotopy theory. Orthogonal spectra were not defined until the early 1980's [114, §5], under a different name, and they were only recognized as a foundation for stable homotopy theory in the late 1990's [106]. Their use greatly clarifies the original concepts and their interrelationships.

The acronym FSP comes from Bökstedt's well-known but unpublished work [15] on topological Hochschild homology (THH), and the acronym FCP is a suitable companion. The understanding of the comparison between internal and external versions of diagram ring spectra is due to Jeff Smith and was developed by Hovey, Shipley, and Smith [77] in the context of symmetric spectra. As we have seen, commutative $\mathscr{I}$-FCP's lead to $E_{\infty}$ spaces and hence to spectra, whereas commutative $\mathscr{I}$-FSP's lead to $E_{\infty}$ ring spectra. This is also true with $\mathscr{I}$ replaced by $\Sigma$, but the mechanism for passing from $\Sigma$-FCP's to spectra is different, as we observed in Remark 23.6.6. The noncommutative case leads to $A_{\infty}$ spaces and $A_{\infty}$ ring spectra.

REMARK 23.8.1. We give a dictionary of nomenclature.

(i) In $[112$, I $\S 1]$, our $\mathscr{I}$ was denoted $\mathscr{I}_{*}$ and our $\mathscr{I}_{c}$ was denoted $\mathscr{I}$. For these $\mathscr{D}$, our $\mathscr{D}$-FCP's were there called $\mathscr{D}$-functors. The new term seems preferable.

(ii) Our FSP's, or $\mathscr{I}$-FSP's, were defined in [112, IV.2.1], where they were called $\mathscr{I}_{*}$-prefunctors. Bökstedt's later term FSP is much to be preferred.

(iii) In [112, p.54], where they were first introduced and proven to be infinite loop spaces, our $G L_{1}(R)$ and $S L_{1}(R)$ were called $F R$ and $S F R$ (with $E$ used instead of $R$ ). Waldhausen later introduced versions of $G L_{n}(R)$ for $n \geq 1$ [167], leading to the currently standard notations.

(iv) In [112], $\Sigma^{\infty}$ and $\Omega^{\infty}$ had different meanings than are now standard; there, $\Sigma^{\infty}$ was the suspension prespectrum functor and $\Omega^{\infty}$ was the spectrification functor, now called $L$; its role will be recalled in Chapter 24 .

(v) In $[114, \S \S 5,6]$, our orthogonal spectra were called $\mathscr{I}_{*}$-prespectra. 



\section{CHAPTER 24}

\section{Epilogue: cellular philosophy and alternative approaches}

\section{Introduction}

So far in this book, CW objects, as opposed to more general cellular objects, have rarely been mentioned. Even classically, there is a clear distinction between CW theory and the model theoretic cellular theory that we have focused on. It is a general feature of model category theory that it does not know about CW complexes, and the current emphasis on model category theory can obscure the arguably more basic and certainly more calculationally relevant CW theory. Model theoretic cell complexes $X$ come with a filtration whose terms bear no relationship to the dimensions of cells.

For example, in the Quillen model category of spaces, CW complexes are cell complexes such that cells are attached only to cells of lower dimension. When this holds, one can superimpose the skeletal filtration, but that is not possible in general. In the nonparametrized world, one can circumvent the difficulty, up to homotopy, by using the cellular approximation theorem. That result ensures that any cell complex is homotopy equivalent to a $\mathrm{CW}$ complex. This also works for $G$-CW complexes, provided that one works with spheres with trivial $G$-action. There are algebraic model categories leading to derived categories in which cell theory works but the cellular approximation theorem fails hopelessly [90, III§2]. Topologically, the distinction is far more important in the parametrized setting than in the classical setting. We say what we can about CW spaces over $B$ in $\S 24.1$, where we obtain partial versions of the Whitehead and cellular approximation theorems.

We discuss the divergence of the theory of CW objects from the theory of cellular objects in stable situations in $\S 24.2$, heading towards discussion of alternative constructions of the stable homotopy category of parametrized spectra. Stably, the equivariant theory raises new distinctions that are not present unstably. These highlight philosophical differences between the alternative approaches to model categories of spectra mentioned in the introduction to Part III. We have focused on the "diagram spectrum" approach to the stable homotopy category. In the nonparametrized theory, there is an alternative "structured spectrum" approach that offers a closer parallel to the calculational world of CW complexes than is obtainable from the diagram spectrum approach $[61,98,105,108]$. A leisurely intuitive summary of this approach, which focuses on CW theory to the exclusion of model category theory, is given in [118, Ch. 12]. We describe how this theory fits into the framework of well-grounded model categories in $\S 24.3$. This works startlingly well in the EKMM [61] context, where the ground structure can be ignored: the EKMM category of $S_{G}$-modules is better behaved than any category of spaces. 
We return to the parametrized setting in $\S 24.4$. As we noted earlier, a model theoretic approach to parametrized spectra based on structured spectra seems to present more difficulties than one based on diagram spectra. An approach based directly on $\mathrm{CW}$ spectra is still more problematic. Nevertheless, there would be advantages to such alternative foundations. In $\S 24.4$, we start towards such a theory by giving a construction of the parametrized equivariant stable homotopy category based on structured $G$-spectra. Rather than solve the model categorical or CW problems, we just build on the work that we have already done with parametrized $G$-prespectra. We point out the beginnings of an EKMM type elaboration of this parametrized theory in $\S 24.5$, but we shall not pursue the details here.

\subsection{CW spaces over $B$}

It is usual in axiomatic homology and cohomology theory to express the alternative cellular axioms in terms of CW objects rather than the more general cellular objects that we used in Chapter 20. However, as we shall explain here, we have not yet worked out enough of the theory of CW spaces over $B$ or of CW ex-spaces over $B$ to allow such an axiomatization. Nevertheless, up to a point, we can mimic the theory of CW complexes as developed, for example, in [121]. We sketch what is involved in order to emphasize the fundamental points of difference. It seems likely that this theory can be developed further by more elaborate techniques.

One point of difference from the classical case is that we have both the kinds of cells dictated by the $q$-model structure and the kinds of cells dictated by the more useful $q f$-model structure. Recall that a main point of the $q f$-model structure is to ensure that cell complexes of ex-spaces are well-grounded. We surely want CW complexes of ex-spaces to have that property. However, the importance of this distinction is diminished by the fact that we do not yet know how to prove much about $\mathrm{CW}$ ex-spaces over $B$, as opposed to $\mathrm{CW}$ spaces over $B$. Moreover, it is technically and conceptually helpful to realize that much of what we can prove goes through using the more general $q$-cells, but specializes to results valid upon restriction to $\mathrm{CW}$ complexes defined in terms of $q f$-cells. Thus we have both a $q$-CW theory and a $q f$-CW theory. We write $\mathrm{CW}$ complex ambiguously for results that work equally well with either definition, but when we consider homology we always have $q f$-CW complexes in mind.

The definition of CW complexes $X$ over $B$ works exactly like the definition of CW complexes. We take $X^{0}$ to be a disjoint union of points over $B$ and construct $X^{n}$ from $X^{n-1}$ by attaching cells $D^{n}$ over $B$ along attaching maps over $B$ defined on their boundary spheres $S^{n-1}$ over $B$. For ex-spaces, we take $X^{0}$ to be a wedge over $B$ of "ex-points" $* \amalg B$ over $B$ and construct $X^{n}$ from $X^{n-1}$ by attaching cells $D^{n} \amalg B$ over $B$ along attaching ex-maps defined on $S^{n-1} \amalg B$. Relative CW complexes are defined similarly. Some of the treatment in [121, Ch. 10] carries over verbatim. The treatment there is organized around the Homotopy Extension and Lifting Property. Unfortunately, we do not know how to prove an ex-space analogue of this result, hence we focus on spaces over $B$. Although we are interested in CW complexes, the notion of dimension makes sense for cell complexes and HELP applies to them as well.

THEOREM 24.1.1 (HELP). Let $(X, W)$ be a relative $q$-cell complex over $B$ of dimension $\leq n$ and let $e: Y \longrightarrow Z$ be a map over $B$ that is an $n$-equivalence, where $Y$ and $Z$ are $q$-fibrant (or just $q f$-fibrant if $(X, W)$ is a relative $q f$-cell complex). 
Then, given maps $f: X \longrightarrow Z, g: W \longrightarrow Y$, and $h: W \times I \longrightarrow Z$ over $B$ such that $f \mid W=h \circ i_{0}$ and $e \circ g=h \circ i_{1}$ in the following diagram, there are maps $\tilde{g}$ and $\tilde{h}$ over $B$ that make the entire diagram commute.

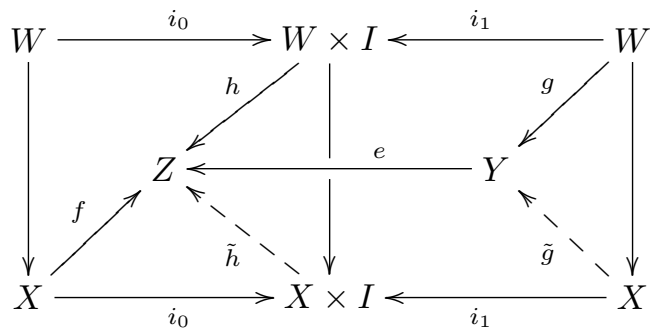

Proof. By the nonparametrized version [121, p. 73], there are maps $\bar{g}$ and $\bar{h}$ that make the diagram commute but that may not be maps over $B$. We use the assumption that the projections $p_{Y}$ and $p_{Z}$ are $q$-fibrations to homotope $\bar{g}$ and $\bar{h}$ to maps $\tilde{g}$ and $\tilde{h}$ that make the diagram commute and are maps over $B$.

Before proceeding to the proof, recall that for any homotopies $h$ and $h^{\prime}$, the inverse and sum are defined by $h^{-1}(x, s)=h(x, 1-s)$ and

$$
\left(h+h^{\prime}\right)(x, s)= \begin{cases}h(x, 2 s) & \text { if } s \leq 1 / 2 \\ h(x, 2 s-1) & \text { if } s \geq 1 / 2 .\end{cases}
$$

Recall too that there is a standard homotopy $\ell$ from $h+h^{-1}$ to the constant homotopy at $h_{0}$ specified by

$$
\ell(x, s, t)= \begin{cases}h(x, 2 s) & \text { if } 0 \leq s \leq(1-t) / 2 \\ h(x, 1-t) & \text { if }(1-t) / 2 \leq s \leq(1+t) / 2 \\ h(x, 2-2 s) & \text { if }(1+t) / 2 \leq s \leq 1\end{cases}
$$

Observe that the homotopies $\ell(-, 0,-)$ and $\ell(-, 1,-)$ are also constant at $h_{0}$.

Write $i$ for the inclusion $W \longrightarrow X$. To homotope $\bar{g}$ to a map $\tilde{g}$ over $B$, we construct the following diagram.

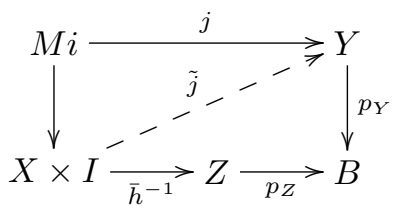

Here $M i$ is the mapping cylinder $X \times\{0\} \cup W \times I \subset X \times I$, and the left vertical arrow is an acyclic $q$-cofibration since $i$ is a $q$-cofibration. Let

$$
j(x, 0)=\bar{g}(x) \text { for } x \in X \text { and } j(w, t)=g(w) \text { for } w \in W .
$$

The rectangle commutes by inspection. Since $p_{Y}$ is a $q$-fibration, there is a lift $\tilde{j}$. Define $\tilde{g}(x)=\tilde{j}(x, 1)$. Then $\tilde{g}$ is a map over $B$ that restricts to $g$ on $W$.

To homotope $\bar{h}$ to a homotopy $\tilde{h}$ over $B$, let $\ell: X \times I \times I \longrightarrow B$ be the homotopy from $p_{Z} \bar{h}+p_{Z} \bar{h}^{-1}$ to the constant homotopy at $p_{Z} \bar{h}_{0}=p_{X}$ obtained by specializing the general construction above. Observe that $\ell(w, s, t)=p_{W}(w)$ if $w \in W$. We 
construct a diagram as follows.

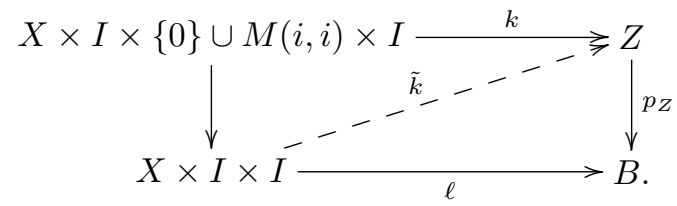

Here $M(i, i)$ is the double mapping cyclinder $M(i, i)=X \times\{0\} \cup W \times I \cup X \times\{1\}$. The canonical inclusion $j: M(i, i) \longrightarrow X \times I$ is a $q$-cofibration. The left vertical arrow in the diagram above is the inclusion of the mapping cylinder of $j$ in $X \times I \times I$ and is therefore an acyclic $q$-cofibration. The map $k$ is defined by

$$
\begin{aligned}
k(x, s, 0)= & \begin{cases}\bar{h}(x, 2 s) & \text { if } s \leq 1 / 2 \\
e \tilde{j}(x, 2 s-1) & \text { if } s \geq 1 / 2\end{cases} \\
& k(x, 0, t)=\bar{h}(x, 0) \\
& k(x, 1, t)=e \tilde{j}(x, 1)
\end{aligned}
$$

and, for $w \in W$,

$$
k(w, s, t)= \begin{cases}h(w,(2-t) s) & \text { if } s \leq 1 /(2-t) \\ e g(w) & \text { if } s \geq 1 /(2-t) .\end{cases}
$$

The rectangle commutes by inspection. Since $p_{Z}$ is a $q$-fibration, there exists a lift $\tilde{k}$. Define $\tilde{h}(x, s)=\tilde{k}(x, s, 1)$. Then $\tilde{h}$ is a map over $B$ such that the required diagram commutes.

A second point of difference is that we cannot expect a result such as HELP to hold without fibrancy conditions. This means, for example, that the usual immediate consequence that an $n$-equivalence between $\mathrm{CW}$ complexes of dimension less than $n$ is a homotopy equivalence is no longer valid in general. The following version of the Whitehead theorem, which refers to the homotopy category Ho $\mathscr{K} / B$ does follow directly.

Theorem 24.1.2 (Whitehead). If $X$ is a $C W$ complex over $B$ and $e: Y \longrightarrow Z$ is an n-equivalence over $B$, then $e_{*}:[X, Y]_{B} \longrightarrow[X, Z]_{B}$ is a bijection if $\operatorname{dim}(X)<$ $n$ and a surjection if $\operatorname{dim}(X)=n$.

The point is that we can compute morphisms in the homotopy category by first applying $q$-fibrant approximation to $e: Y \longrightarrow Z$ and then passing to homotopy classes of maps. The requirement of fibrancy in the Whitehead theorem is a serious drawback, as the following proposition and question make clear.

Proposition 24.1.3. The following $C W$ approximation statements hold.

(i) For any space $X$ over $B$, there is a weak equivalence $\Gamma X \longrightarrow X$ over $B$, where $\Gamma X$ is a $C W$ complex over $B$.

(ii) For any pair $(X, W)$ of spaces over $B$, there is a weak equivalence $\gamma:(\Gamma X, \Gamma W) \longrightarrow(X, W)$, where $(\Gamma X, \Gamma W)$ is a $C W$ pair over $B$.

(iii) For any excisive triad $(X ; W, V)$ of spaces over $B$, there is a $C W$ triad $(\Gamma X ; \Gamma W, \Gamma V)$ and a map of triads $\gamma:(\Gamma X ; \Gamma W, \Gamma V) \longrightarrow(X ; W, V)$ such that $\gamma: \Gamma W \cap \Gamma V \longrightarrow W \cap V, \gamma: \Gamma W \longrightarrow W, \gamma: \Gamma V \longrightarrow V$, and $\gamma: \Gamma X \longrightarrow X$ are all weak equivalences. 
On homotopy categories, $\Gamma$ is the object function of a functor such that $\gamma$ is natural, and $\Gamma X,(\Gamma X, \Gamma W)$, and $(\Gamma X ; \Gamma W, \Gamma V)$ are unique up to isomorphism in the respective homotopy categories.

Proof. For (i), we build $\Gamma X$ by successive cell attachment as in $[121, \S 10.5]$. The essential point is that we are considering the homotopy groups of the total spaces and can work in the nonparametrized context. We give the cells that we attach the projections induced from that of $X$, and we then use Lemmas 6.3 .1 and 6.3.2 to replace the constructed $q$-cells over $B$ by $q f$-cells over $B$. For (ii), we start from a CW approximation $\gamma: \Gamma W \longrightarrow W$ and attach cells to $\Gamma W$ to construct $\Gamma X$ together with a weak equivalence $\gamma: \Gamma X \longrightarrow X$ that extends the given $\gamma$ on $\Gamma W$, as in [121, $\S 10.6]$. For (iii), let $M=W \cap V$, construct a weak equivalence $\gamma: \Gamma M \longrightarrow M$ as in (i), and construct weak equivalences $\gamma:(\Gamma W, \Gamma M) \longrightarrow(W, M)$ and $\gamma:(\Gamma V, \Gamma M) \longrightarrow(V, M)$ as in (ii). Then let $\Gamma X=\Gamma W \cup_{\Gamma M} \Gamma V$ and let $\gamma: \Gamma X \longrightarrow X$ be obtained by passage to pushouts; it is a weak equivalence by $[121$, $\S 10.7]$. In the last sentence, we are just applying the Whitehead theorem formally.

One problem with this result is that it too does not go over to ex-spaces. There is no problem building projections starting from the given ones, but we cannot also build up sections that way. Another problem is that, due to the fibrancy condition in HELP, we cannot use the Whitehead theorem to deduce that our CW approximations are functorial up to homotopy (rather than just functorial on homotopy categories) and that $\Gamma$ is unique up to homotopy equivalence, as was done in [121, $\S 10.5-10.7]$. This raises the following question.

QUESTION 24.1.4. Is every space $X$ over $B$ weakly equivalent to a fibrant $\mathrm{CW}$ complex over $B$ ?

Conceptually, a great virtue of cofibrantly generated model categories is that they allow fibrant approximation by cell complexes. However, since fibrations are determined by the RLP with respect just to the generating acyclic cofibrations, fibrancy conditions are blind to the refinement of cell complexes to CW complexes. Of course, this problem is not significant in the classical examples, where every object is fibrant. The conclusion is that, in the parametrized context, we can apply CW approximation to objects but not, in general, to maps. For example, we only have the following version of the cellular approximation theorem.

Theorem 24.1.5 (Cellular Approximation). Any map $f:(X, W) \longrightarrow(Y, V)$ between relative $C W$ complexes over $B$ such that $Y$ and its skeleta are fibrant is homotopic relative to $W$ to a cellular map over $B$.

Proof. The inclusion $i_{n}: Y^{n} \longrightarrow Y$ is an $n$-equivalence. We apply HELP to the pair $\left(X^{0}, W\right)$ to start an inductive construction and proceed cell by cell at later stages, as in $[121, \S 10.4]$.

\subsection{CW spectra and stable homotopy categories}

Nonequivariantly, CW spectra work more or less in the same way as CW complexes and can be developed in any reasonable model category of spectra. However, the existing literature of diagram spectra does not consider CW spectra, and there is a quirk that we shall explain shortly. Equivariantly, the situation is different. 
Whether or not one can restrict model categorical $G$-cell spectra to obtain a good theory of $G$-CW spectra based on spheres $G / H_{+} \wedge S^{n}$ depends on the choice of the model category of $G$-spectra, as we shall explain shortly.

Even nonequivariantly, there is a subtle discrepancy between cell spectra and CW spectra, quite apart from the obvious dimensional restriction on attaching maps. In all categories of diagram spectra in the literature, both simplicial and topological, cell objects are constructed from generating sets $F_{d} S_{+}^{q} \longrightarrow F_{d} D_{+}^{q}$, where $F_{d}$ is left adjoint to evaluation at $d$ for an object $d$ of the domain category. This construction of cell spectra has as its underlying space level model the based spaces that are cell complexes in the unbased sense. On the space level, using the $S_{+}^{q}$ as domains of attaching maps is clearly sensible since the disjoint basepoint must go to the basepoint and one is only looking at an unbased map defined on $S^{q}$. The corresponding definition of based Serre fibrations is similar: they are maps of based spaces that satisfy the RLP with respect to the maps $i_{0}: D_{+}^{q} \longrightarrow\left(D^{q} \times I\right)_{+}$; that is, they are based maps that are Serre fibrations in the unbased sense.

Stably, thinking about CW spectra, that approach does not give the calculationally right notion of a cell complex. In any stable category, we have

$$
F_{n} S_{+}^{q} \simeq \Sigma^{-1} F_{n} \Sigma S_{+}^{q} \simeq \Sigma^{-1} F_{n}\left(S^{q+1} \vee S^{1}\right) \simeq F_{n} S^{q} \vee F_{n} S^{0}
$$

Thus, homotopically, the attaching maps of cells are given by a pair of homotopy classes, not just one. Looking in terms of the adjoints relating spectra, based spaces, and unbased spaces, the point is that for a loop space $X$, the set of unbased homotopy classes of unbased maps $S^{q} \longrightarrow X$ is in bijective correspondence with $\pi_{q}(X) \times \pi_{0}(X)$. Clearly, a pair of attaching maps for each cell does not give the notion of a cell spectrum that a working homotopy theorist has in mind.

This is related to the distinction in Definition 7.5.5 between detecting sets and generating sets. It would seem more natural in the based context to use the based spheres $S^{n}$ as the domains of attaching maps for based CW complexes, but then the only based CW complexes that we could construct would be the disjoint unions of a set of points with a connected based CW complex.

With Lewis-May spectra and EKMM S-modules [61,98], this discrepancy does not appear. The attaching maps of cells are defined on good models of sphere spectra, with no disjoint basepoints in sight. The relevant space level precursor is given by based cell complexes, which are defined in terms of the based generating cofibrations $S^{q} \longrightarrow C S^{q}$ and have based attaching maps. Since the model structure on Lewis-May spectra does not require these cell complexes to be the cell complexes of a model structure on based spaces, there is no problem.

One might try to reconcile the diagram spectrum setting by reworking the diagram category model theory in terms of such cell complexes, and one can certainly superimpose a treatment of CW-spectra by directly mimicking [98, §I.5], read nonequivariantly. However, no such approach works in the parametrized setting, for the simple reason that while spheres can be given basepoints, spheres over a given base space cannot be given base sections. Moreover, such an approach would not address the deeper equivariant issues, which we now explain. A leisurely explanation devoid of model category theory is given in [118, Ch. XII]. However, these issues are interwoven conceptually with the question of whether or not all objects in one's model category are fibrant. 
Of course, in a topological model category in which not all objects are fibrant, cellular objects are not generally fibrant. This is why the most naive cellular objects may not be sufficient to capture the entire homotopy category. Here we must carefully distinguish between a minimal set of generating cofibrations for a stable topological model category $\mathscr{C}$ and a minimal set of generating objects (or, equivalently, detecting objects) for its derived triangulated homotopy category Ho $\mathscr{C}$. The cofibers of a minimal set of generating cofibrations for $\mathscr{C}$ give a generating set of objects for $\mathrm{Ho}_{\mathscr{C}}$, but this generating set of objects need not be not minimal.

A naive approach to a stable cellular theory is to take objects in a well chosen minimal generating set for $\mathrm{Ho}_{\mathscr{C}}$ as the allowed domains of attaching maps for cells of the form $i: T \longrightarrow C T$, where $C T$ is the cone on $T$. Thus a naive cell complex is the colimit of a sequence $X_{i}$, where $X_{0}=*$ and $X_{i+1}$ is the pushout of a coproduct of cells $T \longrightarrow C T$ along attaching maps $T \longrightarrow X_{i}$. When the objects $T$ have dimensions, we define the $n$-skeleton $X^{n}$ of a cell complex $X$ to be the union of the cells of dimension at most $n$, and we define a CW complex to be a cell complex whose attaching maps with domain of dimension $n$ land in the relevant $n$-skeleton $\left(X_{i}\right)^{n}$. Note that such complexes have two filtrations, the sequential one, $\left\{X_{i}\right\}$, and the skeletal one, $\left\{X^{n}\right\}$. That is essential to an adequate theory in the presence of negative dimensions. When the cellular approximation theorem applies to show that any map $T \longrightarrow X_{i}$ is homotopic to a map with image in the subcomplex $\left(X_{i}\right)^{n}$, where $n=\operatorname{dim}(T)$, any cell complex is equivalent to a $\mathrm{CW}$ complex.

In the Lewis-May or EKMM equivariant stable categories, this naive theory gives exactly the right theory of $G$-CW spectra. The well-chosen objects $T$ are suitable cofibrant $G$-spheres $S_{H}^{n}=G / H_{+} \wedge S^{n}, n \in \mathbb{Z}$. All of the formal properties work exactly as for $G$-spaces, the cellular approximation theorem works in exactly the same way as it does nonequivariantly, and every $G$-spectrum is weakly equivalent to a $G$-CW spectrum [98, $§$ I.5]. Model theoretically, the cofibrations are the retracts of the naive relative cell complexes.

In the nonequivariant stable categories of symmetric or orthogonal spectra, CW theory can be developed similarly, except that, as we have seen, the basic cells $T$ should be the cofibers rather than the domains of the generating cofibrations. However, this fails to work in the equivariant stable categories of symmetric or orthogonal $G$-spectra. Restricting to orthogonal $G$-spectra for simplicity, the $G$-spheres $S_{H}^{n}$ still detect weak equivalences, but they cannot be the domains (or cofibers) of the generating cofibrations of an appropriate model structure. Cell spectra constructed from these spheres give rise only to naive orthogonal $G$-spectra, not to the genuine $G$-spectra that represent $R O(G)$-graded cohomology theories. Model theoretically, these spheres only "see" the right homotopy groups after fibrant approximation, and one cannot use them to construct such approximations.

It is the fact that all objects are fibrant in the Lewis-May and EKMM categories that makes equivariant $G$-CW theory work there with integer spheres. The point is that homotopical information about non-trivial representations of $G$ is packaged into the sphere spectra that are the domains of attaching maps. Explicitly, the Lewis-May sphere $G$-spectrum $S_{G}$ has 0 th $G$-space $Q S^{0}$, which is the colimit over all representations $V$ of the equivariant loop $G$-spaces $\Omega^{V} S^{V}$. This allows one to restrict to "trivial" $G$-sphere spectra $G / H_{+} \wedge S^{n}$ for $n \in \mathbb{Z}$, rather than using $G$-sphere spectra $G / H_{+} \wedge S^{\alpha}$ for $\alpha \in R O(G)$, as domains of attaching maps. 
These issues are discussed further in [105, IV $\S 1]$, where the reader can find a discussion of the Quillen equivalences that nevertheless relate all of the relevant model categories. Even nonequivariantly, it is advantageous to have fibrant CW objects, and of course it is essential to have $\mathrm{CW}$-spectra such that each cell has a single attaching map. In diagram categories, the cell objects with dimensionally appropriate attaching maps do not have these properties, and we conclude that the theory of CW spectra there is more divergent from the model theoretic theory of cell spectra and less convenient than in the Lewis-May category.

Rather than think about these issues in the context of diagram spectra, we can exploit the web of Quillen equivalences of model categories that has been developed in $[105,106,145]$. It is effortless and automatic to take information developed in the Lewis-May and EKMM context and apply it in the diagram category context, and vice versa. Thus, implicitly, we already have a fully developed theory of diagram $G$-CW spectra, just by moving back and forth between Quillen equivalent model categories. It is harder work setting up the Lewis-May and EKMM foundations, but they package useful information that is difficult or impossible to obtain directly in the diagram spectrum setting, especially in their relationship to infinite loop spaces and to equivariant stable homotopy theory.

This discussion applies with comparable force in the parametrized setting, but here fibrancy is more critical and we have not obtained a satisfactorily complete theory based on structured (or "genuine") parametrized spectra.

\subsection{Structured spectra and well-grounded model categories}

We first compare the definitions of model categories of diagram spectra and model categories of structured spectra and then describe how the latter theory fits in with our theory of well-grounded model categories. The discussion will pinpoint the problems involved in obtaining a parametrized generalization of the theory of structured spectra.

In the theory of diagram spectra, one bootstraps one's way up to the appropriate model structure, using the level model structure on categories of diagram spectra as a pivotal intermediary between the model structure on spaces and the stable model structure on spectra. From the point of view of [61,98], diagram spectra are themselves intermediate objects between spaces and spectra. They are of course diagrams of spaces, and their levelwise constituent spaces are taken more seriously than are the constituent spaces of the spectra and $S$-modules of $[61,98]$. In either approach, the basic philosophy is that spectra are the objects of intrinsic interest in their own right, and their underlying families of spaces are generally to be ignored when doing stable homotopy theory. This philosophy is carried further in the structured spectrum approach. The key value of the underlying spaces of structured spectra is that they are infinite loop spaces, on the nose, which is not true for even the fibrant objects in any category of diagram spectra and is usually not relevant when focusing only on stable homotopy theory.

This difference in philosophy plays out in major technical differences. In its naive sequential form, as first defined in [111], a Lewis-May spectrum $E$ is just a sequence of based spaces $E_{i}$ and based homeomorphisms $E_{i} \longrightarrow \Omega E_{i+1}$. We recall and emphasize that this notion of spectrum is incompatible with the structure incorporated by diagram spectra. If $E$ admits a structure of symmetric spectrum, for example, then $E$ is the trivial spectrum: $E_{i}$ cannot be a $\Sigma_{i}$-space homeomorphic 
as a $\Sigma_{i} \times \Sigma_{2}$-space to $\Omega^{2} E_{i+2}$ unless it is a point. In the coordinate-free setting, the same contradiction applies to orthogonal spectra.

There is an adjoint pair of functors $(L, \ell)$ relating prespectra, which for the moment we think of as sequences of based spaces $T_{i}$ and based maps $T_{i} \longrightarrow \Omega T_{i+1}$, to spectra. The category $\mathscr{P}$ of prespectra is clearly topologically bicomplete, with colimits and tensors constructed levelwise. The category $\mathscr{S}$ of spectra is so as well, but colimits and tensors are constructed by first applying the forgetful functor $\ell$, then applying the prespectrum level construction, and finally applying $L$. For example, $E \wedge K=L(\ell E \wedge K)$. Homotopies and $C y l$-cofibrations are defined in terms of $E \wedge I_{+}$. If a map $T \longrightarrow T^{\prime}$ of prespectra is a $C y l$-cofibration, then it is obvious that each $T_{i} \longrightarrow T_{i}^{\prime}$ is a $C y l$-cofibration of based spaces. In sharp contrast, it is not known that if a map $E \longrightarrow E^{\prime}$ is a $C y l$-cofibration, then each $E_{i} \longrightarrow E_{i}^{\prime}$ is a Cyl-cofibration. These maps are closed inclusions [98, A.3.9], but even that is not at all obvious. Therefore, spacewise $h$-cofibrations have no useful role to play in this category of spectra.

In our parametrized setting, the key technical result is Theorem 12.4.2, which gives the long exact sequences of fiberwise homotopy groups associated to a cofiber sequence of orthogonal spectra over $B$. The theory leading to that was based on implementing a boot strap argument that is based ultimately on the space level gluing lemma, in its implied levelwise version. For that, it was crucial that Cyl-cofibrations are bicofibrations, and our axiomatization of the theory of wellgrounded model categories is a careful conceptualization of how such arguments might go in a variety of contexts. Since spacewise $h$-cofibrations are no longer useful in a Lewis-May approach, we need a different way of getting at such long exact sequences, and the axiomatization is not directly relevant. Cofibers of parametrized spectra are not nicely related to levelwise cofibers of total spaces, and it is not clear to us how to set up the parametrized theory this way.

In the nonparametrized setting, there is no distinction between the level and stable model structures on Lewis-May or EKMM spectra [61, VII§5] since a map of spectra (or, more generally, of $\Omega$-prespectra) is a $\pi_{*}$-isomorphism if and only if it is a level weak equivalence. Nonequivariantly, this is true tautologically. Equivariantly, it is a non-trivial theorem; see [98, I.7.12] or [105, III.3.4]. Because the level and stable model structures coincide, the acyclicity of relative cell complexes built up from the generating acyclic cofibrations is straightforward, as is clear from comparison of Remark 4.5.12 with the proof of [61, VII.5.6]. There is no direct analogue of this in the parametrized version of the Lewis-May setting. That is one reason that the over and under Quillen model structure on ex-spaces is an insufficient starting point: we cannot prove the relevant acyclicity starting from the candidates for generating acyclic cofibrations to which that model structure leads us. This may work starting from the $q f$-model structure, but we do not have a proof.

Despite this discussion, it is reasonable to ask whether or not the non-parametrized Lewis-May and EKMM categories of spectra are well-grounded topological model categories. For the Lewis-May spectra, the answer seems to be no. For the EKMM $\mathbb{L}$-spectra and $S$-modules, the answer is yes, and in a surprisingly simple way, as we will explain shortly. An indication of the difference was pointed out in [105, IV.2.10]. The Lewis-May category of spectra, which we denote $\mathscr{S}$ in this chapter, is not known to be left proper under its stable model structure, whereas 
the EKMM categories are left proper. Of course, our axioms force well-grounded model categories to be proper. The theory of "tame" spectra developed in EKMM gets around this problem in $\mathscr{S}$ by giving a large class of spectra for which cofiber sequences have long exact sequences of homotopy groups [61, I.3.4]. Precisely, a spectrum is tame if it is homotopy equivalent to one of the form $L T$, where the structure maps of $T$ are cofibrations of based spaces.

In contrast, by a kind of technical miracle [61, I.6.4], all cofiber sequences of $\mathbb{L}$-spectra have long exact sequences of homotopy groups. We have the following strange result, the gist of which is that EKMM $\mathbb{L}$-spectra, or its even better behaved Quillen equivalent full subcategory of $S$-modules, is better behaved than any known category of based spaces. We focus on $S$-modules and generalize to the equivariant context, where the sphere $G$-spectrum is denoted $S_{G}$.

Theorem 24.3.1. For any compact Lie group $G$, the category $\mathscr{M}_{G}$ of $S_{G^{-}}$ modules is a well-grounded $G$-topological model category with its stable model structure.

PROOF. In the contexts of [98] and [61], all spaces are compactly generated, and we retain that convention. We then decree that every $S_{G}$-module is well-grounded and every map of $S_{G}$-modules is a ground cofibration. The closure properties required of subcategories of well-grounded objects and of cofibrations hold trivially, and the $c y l$-cofibrations coincide with the bicofibrations. Thus we take our ground structure to be the obvious one present in any topologically bicomplete category.

We must show that the weak equivalences, which are the $\pi_{*}$-isomorphisms, are well-grounded in the sense of Definition 5.4.1. We emphasize that the homotopy groups are defined directly on the spectrum level, without use of prespectra or colimits. That is, $\pi_{n}^{H}(M)$ is the set of homotopy classes of maps $G / H_{+} \wedge S^{n} \longrightarrow$ $M$ of $S_{G}$-modules, where $S^{n}$ is the canonical cofibrant $n$-sphere $S_{G \text {-module; see }}$ $\left[61\right.$, p. 33] and $[105$, p. 63$]$. Certainly a homotopy equivalence is a $\pi_{*}$-isomorphism. Any cofiber sequence of $S_{G}$-modules has a long exact sequence of homotopy groups for each subgroup $H$, by the equivariant analogue of [61, I.6.4]. It follows by use of split cofiber sequences that finite wedges of $\pi_{*}$-isomorphisms are $\pi_{*}$-isomorphisms. Since sphere $S_{G}$-modules are compact [61, p. 53], the conclusion for arbitrary wedges follows by passage to colimits. Pushouts of $\pi_{*}$-isomorphisms along $c y l$-cofibrations are $\pi_{*}$-isomorphisms by [61, I.6.5], and the classical mapping cylinder construction factors any map as the composite of a cyl-cofibration and a homotopy equivalence. Therefore the gluing lemma holds by Lemma 5.4.3. The homotopy groups of a colimit of a sequence of cyl-cofibrations are the colimits of the homotopy groups of the terms, so the colimit lemma holds. Finally, the pushout product condition of Definition 5.4.1(v), with $K$ and $L$ restricted to be CW complexes, holds by Lemma 5.4.5 and the proof of [61, I.6.6].

\subsection{The stable category of parametrized spectra}

While an alternative approach to parametrized spectra that is modelled on $[61,98]$ is desirable, we have not worked one out in detail. We sketch what we know in the rest of this chapter. A first step in this direction was taken by $\mathrm{Po} \mathrm{Hu}$ [78], but from the flawed starting point of the over and under Quillen model structure.

As emphasized at the start of Chapter 12, everything there applies verbatim to the category $G \mathscr{P}_{B}$ of $G$-prespectra over $B$, where $G$ is a compact Lie group 
and $B$ is a compactly generated $G$-space. We modify the discussion there slightly by restricting attention to indexing inner product spaces contained in a fixed $G$ universe $U$. We ignore orthogonal $G$-spectra in this section, freeing us to use notations duplicatively.

Definition 24.4.1. A $G$-spectrum over $B$ is a $G$-prespectrum $X$ over $B$ such that each adjoint structure map $\tilde{\sigma}: X(V) \longrightarrow \Omega_{B}^{W-V} X(W)$ is a homeomorphism (isomorphism in $G \mathscr{K}_{B}$ ). Let $\mathscr{S}_{G, B}$ and $G \mathscr{S}_{B}$ denote the full subcategories of $G$ spectra over $B$ in $\mathscr{P}_{G, B}$ and $G \mathscr{P}_{B}$.

Theorem 24.4.2. Let $\ell: G \mathscr{S}_{B} \longrightarrow G \mathscr{P}_{B}$ be the inclusion functor. Then $\ell$ has a left adjoint $L$.

Proof. This is proven exactly as in [98, App $\S 1]$. The functor $L$ factors through the full subcategory $G \mathscr{Q}_{B}$ of inclusion spectra, whose adjoint structure maps $\tilde{\sigma}$ are inclusions. The left adjoint $G \mathscr{P}_{B} \longrightarrow G \mathscr{Q}_{P}$ is obtained by Freyd's adjoint functor theorem or an explicit (and fairly unilluminating) transfinite induction. The left adjoint $G \mathscr{Q}_{P} \longrightarrow G \mathscr{S}_{P}$ is obtained by an evident passage to colimits over inclusions induced by the $\tilde{\sigma}$, so that $(L X)(V)=\operatorname{colim}_{W \supset V} \Omega^{W-V} X(W)$.

Proposition 24.4.3. The category G $\mathscr{S}_{B}$ is topologically bicomplete.

Proof. This is proven exactly as in the nonparametrized case. Limits and cotensors are created in the category $G \mathscr{P}_{B}$, and colimits and tensors are constructed by first applying $\ell$, then the relevant construction, and finally $L$.

For a map $f: A \longrightarrow B$, we have base change functors $f^{*}, f_{*}$, and $f_{*}$ as in $\S 2.1$. The pushout used to construct $f$ ! must be carried out using the adjunction $(L, \ell)$, but $f^{*}$ and $f_{*}$ are constructed levelwise. Similarly, all of the other formal point-set level structure developed in Chapter 2 carries over directly to these categories of equivariant parametrized spectra.

There is also a shift desuspension functor $\Sigma_{V}^{\infty}$ from ex- $G$-spaces over $B$ to $G$ spectra over $B$ that is left adjoint to the $V$ th ex- $G$-space over $B$ functor. It is constructed from our prespectrum level analogue by use of the adjunction $(L, \ell)$.

REMARK 24.4.4. Let $L F I_{B}^{f}$ and $L F J_{B}^{f}$ be the sets of maps of $G$-spectra over $B$ obtained by applying the functor $L$ to the sets specified in Definition 12.1.6. A standard argument $[73,11.3 .2]$ shows that if $\ell$ takes relative $L F J_{B}^{f}$-cell complexes to weak equivalences, then these sets give the generating cofibrations and generating acyclic cofibrations for a compactly generated level model structure on $G \mathscr{S}_{B}$ such that $(L, \ell)$ is a Quillen adjunction. Here a map $f$ in $G \mathscr{S}_{B}$ would be a weak equivalence or level $q f$-fibration if and only if $\ell f$ were a level weak equivalence or level $q f$-fibration. Since the weak equivalences between fibrant objects would be the fiberwise weak equivalences and since $\ell$ would take level fibrations to $s$-fibrations, by Proposition 12.5.6, it would follow that $(L, \ell)$ induces an equivalence between the resulting homotopy category $\operatorname{Ho} G \mathscr{S}_{B}$ and the stable homotopy category $\mathrm{Ho} G \mathscr{P}_{B}$. However, we have not been able to prove the required levelwise acyclicity of total spaces of relative $L F J_{B}^{f}$-cell complexes. Of course, since the level total spaces of a spectrum over $B$ do not form an nonparametrized spectrum, we cannot just reduce to the spectrum level analogue.

Nevertheless, we can use $G$-spectra over $B$ to obtain a model for the parametrized stable homotopy category. 
Definition 24.4.5. A $G$-spectrum $X$ is $h$-fibrant if each $X(V)$ is an $h$-fibrant ex- $G$-space; $q$-fibrant, $q f$-fibrant, and quasi-fibrant $G$-spectra are defined similarly. A map $f: X \longrightarrow X^{\prime}$ of quasi-fibrant $G$-spectra is a weak equivalence if its restriction $f_{b}: X_{b} \longrightarrow X_{b}^{\prime}$ is a weak equivalence of $G_{b}$-spectra for each $b \in B$. Let $G \mathscr{S}_{B}^{f}$ denote the category of $h$-fibrant $G$-spectra over $B$ and let HoG $\mathscr{S}_{B}^{f}$ be the homotopy category obtained by formally inverting the weak equivalences.

The definition works equally well if we replace $h$-fibrant by $q$ or $q f$-fibrant in our definition of the objects of $\operatorname{HoG} \mathscr{S}_{B}^{f}$. With any choice, we have the following result. As indicated at the end of the proof, we skimp on a few easily filled in details.

TheOREM 24.4.6. The category HoG $\mathscr{S}_{B}^{f}$ is equivalent to the category HoG $\mathscr{P}_{B}$.

Proof. A map $f: X \longrightarrow X^{\prime}$ is a weak equivalence if and only if $\ell f$ is a weak equivalence, and $\ell$ takes $q f$-fibrant $G$-spectra to fibrant $G$-prespectra. Using that the functor $L$ preserves homotopy equivalences because it preserves cylinders and that homotopy equivalences are weak equivalences, it is easy to see that any morphism $X \longrightarrow Y$ in $\operatorname{HoG} \mathscr{S}_{B}^{f}(X, Y)$ is represented by a diagram $X \longleftarrow L Q \ell X \longrightarrow Y$ of $G$-spectra, where $Q$ is $s$-cofibrant approximation in $G \mathscr{P}_{B}$. In particular, we have a well-defined hom set $\operatorname{Ho} G \mathscr{S}_{B}^{f}(X, Y)$ between any pair of $h$ (or $q$, or $q f$ )-fibrant $G$-spectra $X$ and $Y$. Either using that $L$ is homotopy preserving or arguing formally from the adjunction, we find that $\ell$ induces a bijection of hom sets between any pair of objects. Thus $\ell$ induces a full and faithful functor on homotopy categories, and it suffices to show that it is essentially surjective, meaning that every $G$-prespectrum $Y$ is isomorphic in $\operatorname{Ho} G \mathscr{P}_{G}$ to $\ell X$ for some $h$-fibrant $G$-spectrum $X$. Certainly, using the excellent approximation functor $T, Y$ is isomorphic in Ho $G \mathscr{P}_{G}$ to an excellent $G$-prespectrum and so may be assumed to be excellent. Recall from Proposition 8.2.7 that each $\Omega_{B}^{W-V} Y(W)$ is an ex-fibration over $B$. Since the structure maps $\sigma$ are $f$-cofibrations, their adjoints are closed inclusions, as in the proof of $[94$, A.8.3]. In fact, with a minor additional condition in our definition of excellent $G$-prespectra, these adjoints are $f$-cofibrations. Precisely, we must require that $B$ and all total spaces $Y(V)$ of excellent $G$-prespectra $Y$ be $G$-LEC (locally equiconnected), which just means that their diagonal maps are $h$-cofibrations. Then the proofs given by Lewis in [95] apply to prove the claim; compare $[61, \S \mathrm{X} .4]$. This additional requirement causes no difficulty in the theory of Chapter 13. Now $(L Y)(V)$ is the colimit of a sequence of $f$-cofibrations that are $f p$-equivalences, by Lemma 13.2.3, and Proposition 8.2.1(iv) gives that each $L Y(V)$ is an ex-fibration. Therefore $L Y$ is an $h$-fibrant $G$-spectrum and the natural map $Y \longrightarrow \ell L Y$ is a weak equivalence, giving the conclusion.

REMARK 24.4.7. Returning to the discussion of CW-spectra in $\S 24.2$, one is tempted to consider the naive approach sketched there, starting from the spectrum over $B$ version of the detecting set $\mathscr{D}_{B}=\left\{S_{H}^{n, b}\right\}$ specified in Definition 13.1.1. As explained in Theorem 13.1.14 and Remark 13.1.16, the localizing subcategory of the triangulated category $\operatorname{Ho} G \mathscr{S}_{B}$ generated by $\mathscr{D}_{B}$ is all of $\operatorname{Ho} G \mathscr{S}_{B}$. However, on the level of spectra over $B$, the resulting cell spectra all consist of fibers glued together trivially, just as in Remark 13.1.16, and these cannot satisfy any reasonable levelwise fibrancy condition. In particular, one cannot hope to prove the factorization axioms by use of the small object argument. 


\subsection{Towards parametrized $S_{G}$-modules}

The construction of smash products and the theory of $S_{G}$-modules of [61] depends on the twisted half-smash product functor. This construction is used to internalize external smash products and is thus the analogue in $[61,98]$ of internalization via left Kan extension in the theory of diagram spectra. It is considerably more useful calculationally, but is less categorically conceptual. The treatment of twisted half-smash product functors developed by Michael Cole, nonequivariantly in [61, App] and equivariantly in [118, Ch. XXII], carries over directly to the parametrized setting, as was noted by $\mathrm{Po} \mathrm{Hu}[78$, pp 12-19]. We give a brief sketch.

Let $U$ and $U^{\prime}$ be $G$-universes and let $\mathscr{I}\left(U, U^{\prime}\right)$ be the $G$-space of linear isometries $U \longrightarrow U^{\prime}$, with $G$ acting by conjugation. Working with $G$-spaces and ex- $G$ spaces over $B$ where Cole uses unbased and based $G$-spaces and with $G$-spectra over $B$ where Cole uses $G$-spectra, there are no substantive changes in the parallel nonequivariant and equivariant constructions of [61, App] and [118, Ch. XXII].

Following Cole, we define a category $G \mathscr{S}_{B}\left(U^{\prime} ; U\right)$ whose objects $\mathscr{E}$ consist of families of $G$-spectra $\mathscr{E}_{V} \in G \mathscr{S}_{B} U^{\prime}$ over $B$ indexed on $U^{\prime}$, one for each indexing $G$-space $V \subset U$, together with isomorphisms $\Sigma_{B}^{W-V} \mathscr{E}_{W} \longrightarrow \mathscr{E}_{V}$ whenever $V \subset W$. For each ex- $G$-space $K$ over $B$, we define an object $\mathscr{E}(K) \in G \mathscr{S}_{B}(U ; U)$ such that $\mathscr{E}(K)_{V}=\Sigma_{V}^{\infty}(K)$. More generally, for a $G$-linear isometry $f: U \longrightarrow U^{\prime}$ we define an object $\mathscr{E}_{f}(K) \in G \mathscr{S}_{B}\left(U^{\prime} ; U\right)$ such that $\mathscr{E}_{f}(K)_{V}=\Sigma_{f(V)}^{\infty}(K)$. There are smash product and function spectrum functors

$\wedge: G \mathscr{S}_{B}\left(U^{\prime} ; U\right) \times G \mathscr{S}_{B} U \longrightarrow G \mathscr{S}_{B} U^{\prime}$ and $F: G \mathscr{S}_{B}\left(U^{\prime} ; U\right)^{\mathrm{op}} \times G \mathscr{S}_{B} U^{\prime} \longrightarrow G \mathscr{S}_{B} U$ such that

$$
G \mathscr{S}_{B} U^{\prime}\left(\mathscr{E} \wedge X, X^{\prime}\right) \cong G \mathscr{S}_{B} U\left(X, F\left(\mathscr{E}, X^{\prime}\right)\right)
$$

where $X$ and $X^{\prime}$ are $G$-spectra over $B$ indexed on $U$ and $U^{\prime}$. The smash product is constructed in such a fashion that

$$
\mathscr{E} \wedge \Sigma_{V}^{\infty}(K) \cong \mathscr{E}_{V} \wedge_{B} K
$$

Now let $A$ be a $G$-space over $B$ together with a $G$-map $\alpha: A \longrightarrow B \times \mathscr{I}\left(U, U^{\prime}\right)$ over $B$; note that $\alpha$ is determined by its second coordinate $A \longrightarrow \mathscr{I}\left(U, U^{\prime}\right)$, and let $\alpha_{b}: A_{b} \longrightarrow \mathscr{I}\left(U, U^{\prime}\right)$ be the restriction of $\alpha$ to the fiber over $b$; it is a $G_{b}$-map. One uses Thom spaces defined as in the original construction of twisted half-smash products in [98] to construct a Thom object $\mathscr{M}(\alpha)$ in $G \mathscr{S}_{B}\left(U^{\prime} ; U\right)$. Its zeroth spectrum over $B,(\mathscr{M} \alpha)_{0}$, is isomorphic to $\Sigma_{B}^{\infty}(A \amalg B)$, where the disjoint copy of $B$ gives the section. If $V^{\prime} \subset U^{\prime}$ is $G$-isomorphic to $V \subset U$, there is an untwisting isomorphism $\mathscr{M}(\alpha)_{V} \cong \Sigma_{V^{\prime}}^{\infty}(A \amalg B)$.

With these preliminaries, the twisted half smash product and twisted function spectra functors over $B$ are defined by

$$
\alpha \ltimes X=\mathscr{M}(\alpha) \wedge X \text { and } F\left[\alpha, X^{\prime}\right)=F\left(\alpha, X^{\prime}\right) .
$$

Here $X \in G \mathscr{S}_{B} U$ and $X^{\prime} \in G \mathscr{S}_{B} U^{\prime}$. The adjunction (24.5.1) specializes to

$$
G \mathscr{S}_{B} U^{\prime}\left(\alpha \wedge X, X^{\prime}\right) \cong G \mathscr{S}_{B} U\left(X, F\left(\alpha, X^{\prime}\right)\right) .
$$

The crucial property of the parametrized construction is that, on fibers,

$$
(\alpha \ltimes X)_{b} \cong \alpha_{b} \ltimes X_{b} \text { and } F\left[\alpha, X^{\prime}\right)_{b} \cong F\left[\alpha_{b}, X_{b}^{\prime}\right) .
$$

When $A=B$ and $\alpha$ is constant at a $G$-linear isometry $f: U \longrightarrow U^{\prime}$, we write $\alpha \ltimes X=f_{*} X$. For $f: U^{2} \longrightarrow U$, such change of universe functors can be used 
to internalize the external smash product which takes $G$-spectra $X$ and $X^{\prime}$ over $B$ and $B^{\prime}$, both indexed on $U$, to a $G$-spectrum $X \bar{\wedge} X^{\prime}$ over $B \times B^{\prime}$ indexed on $U^{2}=U \oplus U$. When $B=B^{\prime}$, pulling back along the diagonal of $B$ gives the internal smash product $X \wedge_{B} X^{\prime}$ in this context. Since the problems with base change along the diagonal are intrinsic, the limitations of the internal smash product and the use of Brown representability to construct internal function spectra would remain as in the approach using orthogonal spectra.

Using the linear isometries operad $\mathscr{L}$, we can operadically parametrize the internalization (over cartesian products) of external smash products. This leads to parametrized versions of the basic definitions of EKMM [61], but we leave further development along these lines to the interested reader. 


\section{Bibliography}

[1] J. F. Adams, A variant of E. H. Brown's representability theorem, Topology 10 (1971), $185-198$.

[2] Stable homotopy and generalised homology, University of Chicago Press, Chicago, Ill., 1974. Chicago Lectures in Mathematics.

[3] - Prerequisites (on equivariant stable homotopy) for Carlsson's lecture, Algebraic topology, Aarhus 1982 (Aarhus, 1982), Lecture Notes in Math., vol. 1051, Springer, Berlin, 1984, pp. 483-532.

[4] M. F. Atiyah, R. Bott, and A. Shapiro, Clifford modules, Topology 3 (1964), no. suppl. 1, $3-38$.

[5] M. Atiyah and G. Segal, Twisted K-theory, Ukr. Mat. Visn. 1 (2004), no. 3, 287-330; English transl., Ukr. Math. Bull. 1 (2004), no. 3, 291-334.

[6] , Twisted $K$-theory and cohomology (2005), preprint, available at arXiv:math.KT/ 0510674

[7] J. C. Baez, A. S. Crans, D. Stevenson, and U. Schreiber, From loop groups to 2-groups (2005), preprint, available at arXiv:math.QA/0504123.

[8] H. J. Baues, Algebraic homotopy, Cambridge Studies in Advanced Mathematics, vol. 15, Cambridge University Press, Cambridge, 1989.

[9] J. C. Becker and D. H. Gottlieb, The transfer map and fiber bundles, Topology 14 (1975), $1-12$.

[10] , Transfer maps for fibrations and duality, Compositio Math. 33 (1976), no. 2, 107133.

[11] J. Bénabou, Introduction to bicategories, Reports of the Midwest Category Seminar, Springer, Berlin, 1967, pp. 1-77.

[12] H. Biller, Characterizations of proper actions, Math. Proc. Cambridge Philos. Soc. 136 (2004), no. 2, 429-439.

[13] J. M. Boardman, Conditionally convergent spectral sequences, Homotopy invariant algebraic structures (Baltimore, MD, 1998), Contemp. Math., vol. 239, Amer. Math. Soc., Providence, RI, 1999, pp. 49-84.

[14] J. M. Boardman and R. M. Vogt, Homotopy-everything H-spaces, Bull. Amer. Math. Soc. 74 (1968), 1117-1122.

[15] M. Bökstedt, Topological Hochschild homology (1990), preprint.

[16] P. I. Booth, The exponential law of maps. I, Proc. London Math. Soc. (3) 20 (1970), 179192.

[17] P. I. Booth, The section problem and the lifting problem, Math. Z. 121 (1971), 273-287.

[18] _ The exponential law of maps. II, Math. Z. 121 (1971), 311-319.

[19] Local to global properties in the theory of fibrations, Cahiers Topologie Géom. Différentielle Catég. 34 (1993), no. 2, 127-151 (English, with French summary).

[20] Librations and classifying spaces: overview and the classical examples, Cahiers Topologie Géom. Différentielle Catég. 41 (2000), no. 3, 162-206 (English, with French summary).

[21] P. I. Booth and R. Brown, Spaces of partial maps, fibred mapping spaces and the compactopen topology, General Topology and Appl. 8 (1978), no. 2, 181-195.

[22] - On the application of fibred mapping spaces to exponential laws for bundles, exspaces and other categories of maps, General Topology and Appl. 8 (1978), no. 2, 165-179.

[23] F. Borceux, Handbook of categorical algebra. 2, Encyclopedia of Mathematics and its Applications, vol. 51, Cambridge University Press, Cambridge, 1994. Categories and structures. 
[24] N. Bourbaki, Éléments de mathématique. Première partie. (Fascicule III.) Livre III; Topologie générale. Chap. 3: Groupes topologiques. Chap. 4: Nombres réels, Troisième édition revue et augmentée, Actualités Sci. Indust., No. 1143. Hermann, Paris, 1960 (French).

[25] E. H. Brown Jr., Cohomology theories, Ann. of Math. (2) 75 (1962), 467-484.

[26] Abstract homotopy theory, Trans. Amer. Math. Soc. 119 (1965), 79-85.

[27] R. R. Bruner, J. P. May, J. E. McClure, and M. Steinberger, $H_{\infty}$ ring spectra and their applications, Lecture Notes in Mathematics, vol. 1176, Springer-Verlag, Berlin, 1986.

[28] A. Casson and D. H. Gottlieb, Fibrations with compact fibres, Amer. J. Math. 99 (1977), no. $1,159-189$.

[29] R. Cauty, Sur les ouverts des CW-complexes et les fibrés de Serre, Colloq. Math. 63 (1992), no. 1, 1-7 (French).

[30] C. Chevalley, Theory of Lie Groups. I, Princeton Mathematical Series, vol. 8, Princeton University Press, Princeton, N. J., 1946.

[31] M. Clapp, Duality and transfer for parametrized spectra, Arch. Math. (Basel) 37 (1981), no. $5,462-472$.

[32] M. Clapp and D. Puppe, The homotopy category of parametrized spectra, Manuscripta Math. 45 (1984), no. 3, 219-247.

[33] A. Clark, Quasi-topology and compactly generated spaces, Brown University (ca. 1963), Mimeographed notes.

[34] M. Cole, The homotopy category of chain complexes is a homotopy category (1999), preprint.

[35] M. Cole, Many homotopy categories are homotopy categories, Topology Appl. 153 (2006), no. $7,1084-1099$

[36] _ Mixing model structures, Topology Appl. 153 (2006), no. 7, 1016-1032.

[37] S. R. Costenoble, J. P. May, and S. Waner, Equivariant orientation theory, Homology Homotopy Appl. 3 (2001), no. 2, 265-339 (electronic). Equivariant stable homotopy theory and related areas (Stanford, CA, 2000).

[38] S. R. Costenoble and S. Waner, The equivariant Thom isomorphism theorem, Pacific J. Math. 152 (1992), no. 1, 21-39.

[39] Equivariant Poincaré duality, Michigan Math. J. 39 (1992), no. 2, 325-351.

[40] Equivariant simple Poincaré duality, Michigan Math. J. 40 (1993), no. 3, 577-604.

[41] , Equivariant ordinary homology and cohomology (2003), preprint, available at arXiv:math.AT/0310237.

[42] M. Crabb and I. James, Fibrewise homotopy theory, Springer Monographs in Mathematics, Springer-Verlag London Ltd., London, 1998.

[43] B. Day, On closed categories of functors, Reports of the Midwest Category Seminar, IV, Lecture Notes in Mathematics, Vol. 137, Springer, Berlin, 1970, pp. 1-38.

[44] B. Day and R. Street, Monoidal bicategories and Hopf algebroids, Adv. Math. 129 (1997), no. 1, 99-157.

[45] T. tom Dieck, Partitions of unity in homotopy theory, Composito Math. 23 (1971), 159-167.

[46] - Transformation groups, de Gruyter Studies in Mathematics, vol. 8, Walter de Gruyter \& Co., Berlin, 1987.

[47] , Topologie, de Gruyter Lehrbuch. [de Gruyter Textbook], Walter de Gruyter \& Co., Berlin, 1991 (German).

[48] A. Dold, Partitions of unity in the theory of fibrations, Proc. Internat. Congr. Mathematicians (Stockholm, 1962), Inst. Mittag-Leffler, Djursholm, 1963, pp. 459-461.

[49] Chern classes in general cohomology, Symposia Mathematica, Vol. V (INDAM, Rome, 1969/70), Academic Press, London, 1971, pp. 385-410.

[50] — The fixed point transfer of fibre-preserving maps, Math. Z. 148 (1976), no. 3, 215244

[51] A. Dold and D. Puppe, Duality, trace, and transfer, (Warsaw, 1978), PWN, Warsaw, 1980, pp. 81-102.

[52] C. Douglas, On the Twisted K-Homology of Simple Lie Groups (2004), preprint, available at arXiv:math.AT/0402082.

[53] W. G. Dwyer, Strong convergence of the Eilenberg-Moore spectral sequence, Topology 13 (1974), 255-265.

[54] W. G. Dwyer, Exotic convergence of the Eilenberg-Moore spectral sequence, Illinois J. Math. 19 (1975), no. 4, 607-617. 
[55] W. G. Dwyer and D. M. Kan, Function complexes in homotopical algebra, Topology 19 (1980), no. 4, 427-440.

[56] W. G. Dwyer and J. Spaliński, Homotopy theories and model categories, Handbook of algebraic topology, North-Holland, Amsterdam, 1995, pp. 73-126.

[57] M. H. Eggar, The piecing comparison theorem, Nederl. Akad. Wetensch. Proc. Ser. A $\mathbf{7 6}=$ Indag. Math. 35 (1973), 320-330.

[58] S. Eilenberg and G. M. Kelly, Closed categories, Proc. Conf. Categorical Algebra (La Jolla, Calif., 1965), Springer, New York, 1966, pp. 421-562.

[59] S. Eilenberg and J. C. Moore, Foundations of relative homological algebra, Mem. Amer. Math. Soc. No. 55 (1965), 39.

[60] S. Eilenberg and J. C. Moore, Homology and fibrations. I. Coalgebras, cotensor product and its derived functors, Comment. Math. Helv. 40 (1966), 199-236.

[61] A. D. Elmendorf, I. Kriz, M. A. Mandell, and J. P. May, Rings, modules, and algebras in stable homotopy theory, Mathematical Surveys and Monographs, vol. 47, American Mathematical Society, Providence, RI, 1997. With an appendix by M. Cole.

[62] H. Fausk, P. Hu, and J. P. May, Isomorphisms between left and right adjoints, Theory Appl. Categ. 11 (2003), No. 4, 107-131 (electronic).

[63] H. Fausk, L. G. Lewis Jr., and J. P. May, The Picard group of equivariant stable homotopy theory, Adv. Math. 163 (2001), no. 1, 17-33.

[64] D. S. Freed, M. J. Hopkins, and C. Teleman, Twisted equivariant K-theory with complex coefficients (2002), preprint, available at arXiv:math.AT/0206257.

[65] - Twisted K-theory and loop group representations (2003), preprint, available at arXiv: math. AT/0312155.

[66] Loop groups and twisted K-theory II (2005), preprint, available at arXiv:math.AT/ 0511232.

[67] C. French, The equivariant J-homomorphism, Homology Homotopy Appl. 5 (2003), no. 1, 161-212 (electronic).

[68] R. Gordon, A. J. Power, and R. Street, Coherence for tricategories, Mem. Amer. Math. Soc. 117 (1995), no. 558, vi+81.

[69] M. Grandis and R. Pare, Limits in double categories, Cahiers Topologie Géom. Différentielle Catég. 40 (1999), no. 3, 162-220 (English, with French summary).

[70] J. W. Gray, Coherence for the tensor product of 2-categories, and braid groups, Algebra, topology, and category theory (a collection of papers in honor of Samuel Eilenberg), Academic Press, New York, 1976, pp. 63-76.

[71] V. K. A. M. Gugenheim and J. P. May, On the theory and applications of differential torsion products, American Mathematical Society, Providence, R.I., 1974. Memoirs of the American Mathematical Society, No. 142.

[72] I. M. Hall, The generalized Whitney sum, Quart. J. Math. Oxford Ser. (2) 16 (1965), 360384.

[73] P. S. Hirschhorn, Model categories and their localizations, Mathematical Surveys and Monographs, vol. 99, American Mathematical Society, Providence, RI, 2003.

[74] L. H. Hodgkin and V. P. Snaith, Topics in K-theory, Springer-Verlag, Berlin, 1975. Lecture Notes in Mathematics, Vol. 496.

[75] M. Hovey, Model categories, Mathematical Surveys and Monographs, vol. 63, American Mathematical Society, Providence, RI, 1999.

[76] M. Hovey, J. H. Palmieri, and N. P. Strickland, Axiomatic stable homotopy theory, Mem. Amer. Math. Soc. 128 (1997), no. 610, x+114.

[77] M. Hovey, B. Shipley, and J. Smith, Symmetric spectra, J. Amer. Math. Soc. 13 (2000), no. 1, 149-208.

[78] P. Hu, Duality for smooth families in equivariant stable homotopy theory, Astérisque (2003), no. 285, v+108 (English, with English and French summaries).

[79] D. Husemoller, Fibre bundles, 2nd ed., Springer-Verlag, New York, 1975. Graduate Texts in Mathematics, No. 20.

[80] S. Illman, Existence and uniqueness of equivariant triangulations of smooth proper Gmanifolds with some applications to equivariant Whitehead torsion, J. Reine Angew. Math. $\mathbf{5 2 4}$ (2000), 129-183.

[81] M. Intermont and M. W. Johnson, Model structures on the category of ex-spaces, Topology Appl. 119 (2002), no. 3, 325-353. 
[82] I. M. James, Ex-homotopy theory. I, Illinois J. Math. 15 (1971), 324-337.

[83] _ Fibrewise topology, Cambridge Tracts in Mathematics, vol. 91, Cambridge University Press, Cambridge, 1989.

[84] I. M. James, Fibrewise compactly-generated spaces, Publ. Res. Inst. Math. Sci. 31 (1995), no. $1,45-61$.

[85] A. Jeanneret and A. Osse, The Eilenberg-Moore spectral sequence in K-theory, Topology 38 (1999), no. 5, 1049-1073.

[86] B. Keller and A. Neeman, The connection between May's axioms for a triangulated tensor product and Happel's description of the derived category of the quiver $D_{4}$, Doc. Math. 7 (2002), 535-560 (electronic).

[87] G. M. Kelly, Basic concepts of enriched category theory, London Mathematical Society Lecture Note Series, vol. 64, Cambridge University Press, Cambridge, 1982.

[88] G. M. Kelly and S. MacLane, Closed coherence for a natural transformation, Coherence in categories, Springer, Berlin, 1972, pp. 1-28. Lecture Notes in Math., Vol. 281.

[89] J. Koslowski, Beyond the Chu-construction, Appl. Categ. Structures 9 (2001), no. 2, 153171.

[90] I. Kř́̌ž and J. P. May, Operads, algebras, modules and motives, Astérisque (1995), no. 233, iv+145pp (English, with English and French summaries).

[91] R. K. Lashof, Equivariant bundles, Illinois J. Math. 26 (1982), no. 2, 257-271.

[92] R. K. Lashof and J. P. May, Generalized equivariant bundles, Bull. Soc. Math. Belg. Sér. A 38 (1986), 265-271.

[93] T. Leinster, Basic bicategories (1989), preprint, available at arXiv:math.CT/9810017.

[94] L. G. Lewis Jr., The stable category and generalized Thom spectra, The University of Chicago, 1978, PhD Thesis.

[95] When is the natural map $X \rightarrow \Omega \Sigma X$ a cofibration?, Trans. Amer. Math. Soc. 273 (1982), no. 1, 147-155.

[96] Open maps, colimits, and a convenient category of fibre spaces, Topology Appl. 19 (1985), no. 1, 75-89.

[97] E-mail to P. Hu; cc to J.P.C. Greenlees and J.P. May, March 8, 2000.

[98] L. G. Lewis Jr., J. P. May, M. Steinberger, and J. E. McClure, Equivariant stable homotopy theory, Lecture Notes in Mathematics, vol. 1213, Springer-Verlag, Berlin, 1986. With contributions by J. E. McClure.

[99] S. Mac Lane, Categories for the working mathematician, 2nd ed., Graduate Texts in Mathematics, vol. 5, Springer-Verlag, New York, 1998

[100] I. Madsen and R. J. Milgram, The classifying spaces for surgery and cobordism of manifolds, Annals of Mathematics Studies, vol. 92, Princeton University Press, Princeton, N.J., 1979.

[101] M. Mahowald, Ring spectra which are Thom complexes, Duke Math. J. 46 (1979), no. 3, $549-559$.

[102] M. Mahowald, D. C. Ravenel, and P. Shick, The Thomified Eilenberg-Moore spectral sequence, Cohomological methods in homotopy theory (Bellaterra, 1998), Progr. Math., vol. 196, Birkhäuser, Basel, 2001, pp. 249-262.

[103] M. Mahowald and N. Ray, A note on the Thom isomorphism, Proc. Amer. Math. Soc. 82 (1981), no. 2, 307-308.

[104] M. A. Mandell, Equivariant symmetric spectra, Homotopy theory: relations with algebraic geometry, group cohomology, and algebraic $K$-theory, Contemp. Math., vol. 346, Amer. Math. Soc., Providence, RI, 2004, pp. 399-452.

[105] M. A. Mandell and J. P. May, Equivariant orthogonal spectra and S-modules, Mem. Amer. Math. Soc. 159 (2002), no. $755, \mathrm{x}+108$.

[106] M. A. Mandell, J. P. May, S. Schwede, and B. Shipley, Model categories of diagram spectra, Proc. London Math. Soc. (3) 82 (2001), no. 2, 441-512.

[107] V. Mathai and I. M. Singer, Twisted K-homology theory, twisted Ext-theory (2003), preprint, available at arXiv:math.hep-th/0012046.

[108] J. P. May, Categories of spectra and infinite loop spaces, Category Theory, Homology Theory and their Applications, III (Battelle Institute Conference, Seattle, Wash., 1968, Vol. Three), Springer, Berlin, 1969, pp. 448-479.

[109] _ The geometry of iterated loop spaces, Springer-Verlag, Berlin, 1972. Lectures Notes in Mathematics, Vol. 271. 
[110] _ $E_{\infty}$ spaces, group completions, and permutative categories, New developments in topology (Proc. Sympos. Algebraic Topology, Oxford, 1972), Cambridge Univ. Press, London, 1974, pp. 61-93. London Math. Soc. Lecture Note Ser., No. 11.

[111] _ Classifying spaces and fibrations, Mem. Amer. Math. Soc. 1 (1975), no. 1, 155, xiii +98 .

[112] — $E_{\infty}$ ring spaces and $E_{\infty}$ ring spectra, Springer-Verlag, Berlin, 1977. With contributions by Frank Quinn, Nigel Ray, and Jørgen Tornehave; Lecture Notes in Mathematics, Vol. 577.

[113] _ Fibrewise localization and completion, Trans. Amer. Math. Soc. 258 (1980), no. 1, $127-146$.

[114] _ Pairings of categories and spectra, J. Pure Appl. Algebra 19 (1980), 299-346.

[115] _ Multiplicative infinite loop space theory, J. Pure Appl. Algebra 26 (1982), no. 1, $1-69$.

[116] _ Some remarks on equivariant bundles and classifying spaces, Astérisque (1990), no. 191, 7, 239-253. International Conference on Homotopy Theory (Marseille-Luminy, 1988).

[117] Weak equivalences and quasifibrations, Groups of self-equivalences and related topics (Montreal, PQ, 1988), Lecture Notes in Math., vol. 1425, Springer, Berlin, 1990, pp. 91101.

[118] _ Equivariant homotopy and cohomology theory, CBMS Regional Conference Series in Mathematics, vol. 91, Published for the Conference Board of the Mathematical Sciences, Washington, DC, 1996. With contributions by M. Cole, G. Comezaña, S. Costenoble, A. D. Elmendorf, J. P. C. Greenlees, L. G. Lewis, Jr., R. J. Piacenza, G. Triantafillou, and S. Waner.

[119] _ Equivariant orientations and Thom isomorphisms, Tel Aviv Topology Conference: Rothenberg Festschrift (1998), Contemp. Math., vol. 231, Amer. Math. Soc., Providence, RI, 1999, pp. 227-243.

[120] _ Stable algebraic topology, 1945-1966, History of topology, North-Holland, Amsterdam, 1999, pp. 665-723.

[121] _ A concise course in algebraic topology, Chicago Lectures in Mathematics, University of Chicago Press, Chicago, IL, 1999.

[122] Notes on ex-spaces and towards ex-spectra (2000), Undistributed preprint.

[123] - Picard groups, Grothendieck rings, and Burnside rings of categories, Adv. Math. 163 (2001), no. 1, 1-16.

[124] $34-73$.

[125] , The Wirthmüller isomorphism revisited, Theory Appl. Categ. 11 (2003), No. 5, 132-142 (electronic).

[126] J. P. May and F. Neumann, On the cohomology of generalized homogeneous spaces, Proc. Amer. Math. Soc. 130 (2002), no. 1, 267-270 (electronic).

[127] J. P. May and R. Thomason, The uniqueness of infinite loop space machines, Topology $\mathbf{1 7}$ (1978), no. 3, 205-224.

[128] J. W. Milnor, On spaces having the homotopy type of CW-complex, Trans. Amer. Math. Soc. 90 (1959), 272-280.

[129] J. W. Milnor and J. D. Stasheff, Characteristic classes, Princeton University Press, Princeton, N. J., 1974. Annals of Mathematics Studies, No. 76.

[130] C. Morgan, Characterizations of $\mathscr{F}$-fibrations, Proc. Amer. Math. Soc. 88 (1983), no. 1, 169-172.

[131] A. Neeman, Stable homotopy as a triangulated functor, Invent. Math. 109 (1992), no. 1, $17-40$.

[132] _ , The Grothendieck duality theorem via Bousfield's techniques and Brown representability, J. Amer. Math. Soc. 9 (1996), no. 1, 205-236.

[133] _ On a theorem of Brown and Adams, Topology 36 (1997), no. 3, 619-645.

[134] _ Triangulated categories, Annals of Mathematics Studies, vol. 148, Princeton University Press, Princeton, NJ, 2001.

[135] R. S. Palais, The classification of G-spaces, Mem. Amer. Math. Soc. No. 36, 1960.

[136] - On the existence of slices for actions of non-compact Lie groups, Ann. of Math. (2) 73 (1961), 295-323. 
[137] A. Pressley and G. Segal, Loop groups, Oxford Mathematical Monographs, The Clarendon Press Oxford University Press, New York, 1986. Oxford Science Publications.

[138] D. Puppe, Homotopiemengen und ihre induzierten Abbildungen. I, Math. Z. 69 (1958), 299-344 (German).

[139] D. G. Quillen, Homotopical algebra, Lecture Notes in Mathematics, No. 43, Springer-Verlag, Berlin, 1967.

[140] D. L. Rector, Steenrod operations in the Eilenberg-Moore spectral sequence, Comment. Math. Helv. 45 (1970), 540-552.

[141] J. Rosenberg, Continuous-trace algebras from the bundle theoretic point of view, J. Austral. Math. Soc. Ser. A 47 (1989), no. 3, 368-381.

[142] C. Schlichtkrull, Units of ring spectra and their traces in algebraic K-theory, Geom. Topol. 8 (2004), 645-673 (electronic).

[143] R. Schön, Fibrations over a CWh-base, Proc. Amer. Math. Soc. 62 (1976), no. 1, 165-166 (1977).

[144] R. Schwänzl and R. M. Vogt, Strong cofibrations and fibrations in enriched categories, Arch. Math. (Basel) 79 (2002), no. 6, 449-462.

[145] S. Schwede, S-modules and symmetric spectra, Math. Ann. 319 (2001), no. 3, 517-532.

[146] S. Schwede and B. E. Shipley, Algebras and modules in monoidal model categories, Proc. London Math. Soc. (3) 80 (2000), no. 2, 491-511.

[147] G. Segal, Classifying spaces and spectral sequences, Inst. Hautes Études Sci. Publ. Math. (1968), no. 34, 105-112.

[148] _ Categories and cohomology theories, Topology 13 (1974), 293-312.

[149] R. M. Seymour, On the convergence of the Eilenberg-Moore spectral sequence, Proc. London Math. Soc. (3) 36 (1978), no. 1, 141-162.

[150] Some functional constructions on G-spaces, Bull. London Math. Soc. 15 (1983), no. 4, 353-359.

[151] B. E. Shipley, Convergence of the homology spectral sequence of a cosimplicial space, Amer. J. Math. 118 (1996), no. 1, 179-207.

[152] J. Sigurdsson, Parametrized homotopy theory, The University of Chicago, 2004, PhD Thesis.

[153] L. Smith, Lectures on the Eilenberg-Moore spectral sequence, Lecture Notes in Mathematics, Vol. 134, Springer-Verlag, Berlin, 1970.

[154] _ On the Künneth theorem. I. The Eilenberg-Moore spectral sequence, Math. Z. 116 (1970), 94-140.

[155] J. Stasheff, A classification theorem for fibre spaces, Topology 2 (1963), 239-246.

[156] M. Steinberger and J. West, Covering homotopy properties of maps between C.W. complexes or ANRs, Proc. Amer. Math. Soc. 92 (1984), no. 4, 573-577.

[157] R. Street, Functorial calculus in monoidal bicategories, Appl. Categ. Structures 11 (2003), no. 3, 219-227.

[158] A. Strøm, Note on cofibrations, Math. Scand. 19 (1966), 11-14.

[159] , Note on cofibrations. II, Math. Scand. 22 (1968), 130-142 (1969).

[160] The homotopy category is a homotopy category, Arch. Math. (Basel) 23 (1972), 435-441.

[161] D. P. Sullivan, Geometric topology: localization, periodicity and Galois symmetry, KMonographs in Mathematics, vol. 8, Springer, Dordrecht, 2005. The 1970 MIT notes; Edited and with a preface by Andrew Ranicki.

[162] R. M. Switzer, Algebraic topology-homotopy and homology, Springer-Verlag, New York, 1975. Die Grundlehren der mathematischen Wissenschaften, Band 212.

[163] D. Tamaki, A dual Rothenberg-Steenrod spectral sequence, Topology 33 (1994), no. 4, 631662.

[164] J.-L. Tu, P. Xu, and C. Laurent-Gengoux, Twisted K-theory of differentiable stacks, Ann. Sci. École Norm. Sup. (4) 37 (2004), no. 6, 841-910 (English, with English and French summaries).

[165] J. L. Verdier, Catégories dérivées (1977), 262-311. in Séminaire de Géométrie Algébrique du Bois-Marie SGA $4 \frac{1}{2}$; Lecture Notes in Mathematics, Vol. 569.

[166] R. M. Vogt, Convenient categories of topological spaces for homotopy theory, Arch. Math. (Basel) 22 (1971), 545-555. 
[167] F. Waldhausen, Algebraic K-theory of topological spaces. I, Algebraic and geometric topology (Proc. Sympos. Pure Math., Stanford Univ., Stanford, Calif., 1976), Part 1, Proc. Sympos. Pure Math., XXXII, Amer. Math. Soc., Providence, R.I., 1978, pp. 35-60.

[168] S. Waner, Equivariant homotopy theory and Milnor's theorem, Trans. Amer. Math. Soc. 258 (1980), no. 2, 351-368.

[169] _ Equivariant fibrations and transfer, Trans. Amer. Math. Soc. 258 (1980), no. 2, 369-384.

[170] _ Equivariant classifying spaces and fibrations, Trans. Amer. Math. Soc. 258 (1980), no. 2, 385-405.

[171] K. Wirthmüller, Equivariant homology and duality, Manuscripta Math. 11 (1974), 373-390.

[172] _ Equivariant S-duality, Arch. Math. (Basel) 26 (1975), no. 4, 427-431.

[173] P. J. Witbooi, Excisive triads and double mapping cylinders, Topology Appl. 95 (1999), no. $2,169-172$.

[174] O. Wyler, Convenient categories for topology, General Topology and Appl. 3 (1973), 225242 . 



\section{Index}

Čech spectral sequence, 385

2-category, 248

acyclicity condition, 73, 91

Adams isomorphism, 326

algebraic stable homotopy category, 267

approximation

ex-fibrant -, 133

excellent prespectrum —, 207

$h$-fibrant -, 132

$\Omega$-prespectrum - 205

$\Sigma$-cofibrant -, 205

well-sectioned -, 132

Atiyah-Hirzebruch spectral sequence, 347, 375

base change

spectrum, 273

duality of,- 277

bicategory, 248

biequivalence of $-\mathrm{s}, 249$

closed - 252

of bimodules, 250

of parametrized spectra, 270

opposite —, 249

symmetric - 250

triangulated -, 262

Brown representability, 123, 125, 197

of parametrized cohomology, 342

of parametrized homology, 353

bundle

construction, 48,241

pseudo-functor, 319

principal - 38,47

category

algebraic stable homotopy -, 267

based bicomplete -, 17

based topologically bicomplete,- 17

classical homotopy - 62

derived homotopy - 63

G- - 22

$G$-topological

bicomplete over $B, 153$

closed symmetric monoidal over $B, 154$

over $B, 153$

of $R$-module spectra, 216 of based spaces, 16

of compactly generated spaces, 16

of ex-objects, 18,78

of ex-spaces, 19

of ex- $G$-spaces, 22

of $G$-spaces, 22

of $k$-spaces, 16

of objects over $B, 17,78$

of parametrized spectra, 164

of retracts, 40, 81

of spaces, 16

over $B, 19$

of weak Hausdorff spaces, 16

sub- of cofibrations, 73

sub- of weak equivalences, 73

sub- of well-grounded objects, 85

topological

bicomplete - 16,151

bicomplete over $B, 152$

closed symmetric monoidal over $B, 153$

over $B, 152$

triangulated -, 261

unital stable homotopy —, 267

cell complex

relative,- 72

cellular approximation theorem, 415

CHP, 64

relative,- 64

classical Thom spectra, 403

closed

bicategory, 252

$\odot-$ - bicategory, 252

cocylinder object, 64

coefficient

groups, 344

system, 344

cofiber, 262

sequence lemma, 92

cofibrant

$\Sigma$ - - approximation, 205

$\Sigma$ - - prespectrum, 200

cofibration

bi-, 86

cyl- - $, 85,176$

$f--, 80$

$f p--, 81$ 
g- -, 85

generating $-\mathrm{s}, 72$

for $q$-model structure on $\mathscr{K} / B$ and $\mathscr{K}_{B}, 98$

for $q$-model structure on spaces, 74

for $q f$-model structure, 101

for $R$-modules, 217

for level $q f$-model structure, 177

for $q f(\mathscr{C})$-model structure, 112

for stable model structure, 188

in $\mathscr{C} / B, 79$

in $G \mathscr{K}, G \mathscr{K} / B$ and $G \mathscr{K}_{B}, 111$

ground $-\mathrm{s}, 85$

h- - 64,80

Hurewicz - 64

I- - 72

mixed - 63

$q f--, 101$

$r-\longrightarrow, 82$

$s-\longrightarrow, 176$

strong Hurewicz —, 64

subcategory of $-\mathrm{s}, 73$

cohomology theory

$R O(G)$-graded parametrized - 359

classical —, 341

fiberwise parametrized - 367

ordinary parametrized -, 344

parametrized —, 338

reduced parametrized - 339

represented - , 341, 360

coinduction, 35, 170

Cole's hypothesis, 69

colimit lemma, 87

commutative

object in a bicategory, 250

compact

object, 122

$\triangleright$ - - 1-cell, 267

set of maps, 72

spectrum, 196

compactly

closed space, 16

generated

model structure, 72

space, 16

compatibility

condition, 73,91

relations, 32

composition

horizontal - 248

condition

acyclicity — $, 73,91$

compatibility —, 73, 91

construction

bundle - , 48, 241

copresheaf of parametrized homology groups, 384

Costenoble-Waner duality, 347 cotensor

for ex-spaces, 20

for spaces over $B, 20$

of $\mathscr{I}_{G}$-spaces over $B, 161$

with based spaces, 17

with spaces, 16

covering homotopy property, 64

relative - 64

cylinder object, 64

$\mathscr{D}$-FCP, 397

$\mathscr{D}$-FP, 395

$\mathscr{D}$-FSP, 397

$\mathscr{D}$-functor

with cartesian products, 397

with products, 395

with smash products, 397

$\mathscr{D}$-object, 395

detecting set, 123, 124, 198

disk

$f--, 101$

lifting property, 101

dual pair, 255

duality

Atiyah —, 296, 300

composition of - 259

Costenoble-Waner — , 287, 347

fiberwise - theorem, 234

fiberwise Poincaré —, 369

homotopical Poincaré —, 313

of base change spectra, 277

Poincaré — , 347, 364

relative Poincaré —, 350

$V$-duality, 291

dualizable

1-cell, 256

Eilenberg-Moore spectral sequence, 391 equivalence

$f$ - -, 80

$\mathscr{F}--, 110$

$f p--, 81$

$h--, 64,80$

internal - 248

$\pi_{*}$-isomorphism, 181

$r-\longrightarrow, 81$

$s--, 181$

stable - 183

well-grounded

level $q-\longrightarrow$ s, 176

of $R$-modules, 216

stable $-\mathrm{s}, 185$

Euclidean neighborhood retract (ENR), 235

Euler characteristic, 236

evaluation functor, 167

ex-fibrant

approximation, 133

ex-fibration, 83, 128

ex-quasifibration, 135 
ordinary parametrized -, 344

parametrized - 338

reduced parametrized,- 339

representability of - 353

represented - 341,360

homomorphism

of bicategories, 248

homotopy

coequalizer, 262

colimit, 262

extension and lifting property, 412

extension property, 64

relative,- 64

groups

of parametrized spectra, 181

$\mathscr{I}_{c}$-FCP, 405

induction, 35, 170

internal equivalence, 248

involution

on a bicategory, 249

$k$-orientation, 348,364

$k$-trivialization, 348,364

$k$-ification functor, 16

$k$-space, 16

$\mathscr{L}$-space, 405

ladder lemma, 87

latching maps, 381

lax functor, 248

Lefschetz constant, 236

left lifting property, 64

lemma

cofiber sequence -, 92

colimit - 87

gluing,- 87

ladder,- 87

$\lim ^{1}$ - 94

wedge -, 93

level model structure, 177

lifting property, 155

relative $f$-disk - 101

$\lim ^{1}$ lemma, 94

linear isometries operad, 405

LLP, 64

long exact sequence of homotopy groups, 184

mapping

cylinder, 64

path fibration, 64

space

external - 40

of ex-spaces, 21

of spaces over $B, 21$

model category

$G$-topological —, 155

model structure compactly generated

on $\mathscr{C}_{B}, 79$

compactly generated,- 72

$f$ - -

on $\mathscr{C} / B$ and $\mathscr{C}_{B}, 80$

on $\mathscr{K} / B, \mathscr{U} / B$ and $\mathscr{U}_{B}, 84$

fp- -

on $\mathscr{C}_{B}, 81$

general philosophy, 62

generic $h$-structures, 66

h- -

on $\mathscr{C}, 69$

on $\mathscr{C} / B$ and $\mathscr{C}_{B}, 80$

on spaces, 70

level $q f$ - - 177

mixed,- 63

on $R$-modules, 217

over and under,- 79

positive,- 178

$q-$

on $G \mathscr{K} / B$ and $G \mathscr{K}_{B}, 111$

on spaces, 74

$q f-$ -

on $\mathscr{K} / B$ and $\mathscr{K}_{B}, 101$

on $G \mathscr{K} / B$ and $G \mathscr{K}_{B}, 113$

Reedy - 380

stable —, 183

well-grounded —, 91

modification, 249

module of units, 401

monoid

FCP, 400

of based homotopy equivalences, 400

Moore

mapping path fibration, 132

paths, 131

morphism

of bicategories, 248

natural transformation

lax - 249

NDR, 83

neighborhood deformation retract

fiberwise -, 83

numerable cover, 44

operad

linear isometries -, 405

oplax functor, 248

opposite

bicategory, 249

orbit functor

of spaces, 37

of spectra, 226

$\pi_{*}$-isomorphism, 181

parametrized functor with protucts, 398

parametrized Künneth spectral sequence, 390 
partial map classifier, 20

path-lifting function, 128

PFSP, 398

Poincaré duality, 347, 364

relative,- 350

presheaf of parametrized cohomology groups, 384

principal

bundle, 38,47

space, 38

projection formula, 34

projective unitary group, 378

proper simplicial object, 381

proper space, action or map, 44

pseudo-functor, 248

quasifibration, 54

$R$-module spectrum, 216

Reedy model structure, 380

representation

generalized homotopy,- 235

stable homotopy -, 235

restriction, 218

of groups functors, 223

of universe, 220

right lifting property, 64

RLP, 64

Serre spectral sequence, 345

shift desuspension functor, 167

simplicial spectral sequence, 381

slice, 44

small object argument, 72

smash product

external - 40

external - of spectra, 162

handicrafted - 210

non-associative in $\mathscr{T}_{o p}, 26$

space

compactly closed,- 16

compactly generated -, 16

ex- - 19

external function,- 162

$\mathscr{F}-\ldots, 110$

finitely dominated,- 235

$G$-connected, 365

$\mathscr{I}_{G^{-}}$- 160

$\mathscr{J}_{G, B^{-}}-, 166$

$k--, 16$

$\mathscr{L}-\ldots, 405$

principal - 38

weak Hausdorff —, 16

well-based or nondegenerately based 82

well-grounded - over $B, 86$

spectral sequence

Čech —, 385

Atiyah-Hirzebruch - , 347, 375
Eilenberg-Moore —, 391

parametrized Künneth —, 390

Serre - 345

simplicial —, 381

Twisted Rothenberg-Steenrod - 387

spectrification functor, 421

spectrum

$R$-module,- 216

base change $-\mathrm{a}, 273$

category of parametrized -a, 164

compact —, 196

Costenoble-Waner dual -, 287

duality of base change - a, 277

dualizable -, 235

EKMM - a, 416

excellent pre-, 200

excellent pre- approximation, 207

homotopy groups of -a, 181

invertible - 235

level type of -, 176

Lewis-May - a, 416

$\Omega--, 182$

$\Omega$-pre- approximation, 205

over $B, 163$

$\Pi$-free -, 243

pre- over $B, 165$

$\Sigma$-cofibrant pre-, 200

sphere - 163

suspension -, 163

Thom - of a map, 407

universal spherical fibration -, 407

weak map of pre $-\mathrm{a}, 203$

well-grounded -, 176

well-sectioned -, 176

well-structured pre-, 200

spherical fibration, 347

$k$-oriented - 348,364

spherical $G$-fibration, 365

$V-\longrightarrow, 364$

stable

equivalence, 183

model structure, 183

strict functor, 248

structure group, 47

symmetric

bicategory, 250

telescope, 94

tensor

for ex-spaces, 20

for spaces over $B, 20$

of $\mathscr{I}_{G}$-spaces over $B, 161$

with based spaces, 17

with spaces, 16

theorem

cellular approximation,- 415

Costenoble-Waner duality —, 295 
fiberwise Costenoble-Waner duality 320

fiberwise duality —, 234

fiberwise parametrized Poincaré duality, 369

homotopical Poincaré duality, 313

for bundles, 325

of Brown, 123, 125, 197

of Dold, 53

of Milnor, Waner, 52

of Palais, 46

of Stasheff, Schön, 53

of Steinberger-West, Cauty, 54

of Strøm, 70

of Whitehead, 414

pairing - of Schwänzl and Vogt, 67

parametrized Atiyah duality — , 296, 300

Poincaré duality, 347, 364

relative Poincaré duality, 350

Thom isomrophism —, 349

triangulation - of Illman, 51

Wirthmüller isomorphism, 312

Thom

complex, 31

diagonal, 31

isomorphism, 349

object, 423

spectrum

classical - a, 403

generalized - a, 403

of a map, 407

topologically bicomplete category, 16

trace, 236

additivity of $-\mathrm{s}, 237$

transfer

fiberwise - for bundles, 244

map, 236

of fibrations, 239

triangle

distinguished,- 261

triangulated category, 261

tube, 44

twisted

function spectrum functor, 423

half smash product, 423

twisted $K$-theory, 378

twisted Rothenberg-Steenrod spectral sequence, 387

unital stable homotopy category, 267

weak

compatibility, 263

Hausdorff space, 16

Hausdorffication functor, 16

map of spectra, 203

weak equivalence

mixed - 63

subcategory of - s, 73 well-grounded - s, 87

in $G \mathscr{K}, 90$

in $G \mathscr{K} / B$ and $G \mathscr{K}_{B}, 90$

wedge lemma, 93

well-fibered

ex-space, 128

well-grounded

ex-space, 86

level $q$-equivalences, 176

model structure, 91

object, 85

$R$-modules, 216

$S$-modules, 420

space, 85

space over $B, 86$

spectrum, 176

stable equivalences, 185

weak equivalences, 87

well-sectioned

approximation, 132

ex-space, 83, 128

spectrum, 176

well-structured

prespectrum, 200

whiskering functor, 131

Wirthmüller

isomorphism, 312

Wirthmüller

context, 32 


\title{
Index of notation
}

\author{
/, 351 \\ $\backslash, 351$ \\ $\cup, 351$ \\ $\triangleleft, 253,270$ \\ $\odot, 248,270$ \\ $\triangleright, 253,270$ \\ $\wedge, 351$ \\ $*_{B}, 80$ \\ $A \times{ }_{c} B, 25$ \\ $\operatorname{Aut}(k), 376$ \\ $\operatorname{Aut}(X), 376$ \\ $\alpha \ltimes X, 423$ \\ $\tilde{b}, 31$ \\ $B(D, \Pi, E), 402$ \\ $B(\Pi ; \Gamma), 243$ \\ $\mathscr{B}_{R}, 250$ \\ $\mathscr{C}_{B}, 18,78,153$ \\ $\mathscr{C}_{\mathscr{B}}, 40,81$ \\ $\mathscr{C} / B, 17,78$ \\ $C f, 262$ \\ $C(f, g), 262$ \\ $\chi(f), 236$ \\ $\mathscr{C}^{\square}(i, p), 66$ \\ $\operatorname{Cocyl}(X), 64$ \\ $\mathscr{C}(X, Y), 16$ \\ $\operatorname{Cyl}(X), 64$ \\ $\mathscr{D}[\mathscr{C}], 395$ \\ $\mathscr{D}_{B}, 124,196$ \\ $D_{B}^{C W} X, 287$ \\ $D_{l}(X), 258$ \\ $D^{n}, 100$ \\ $D_{r}(X), 258$ \\ E, 205 \\ $\operatorname{End}(k), 376$ \\ $E(\Pi ; \Gamma), 243$ \\ $\epsilon^{*}, 37$ \\ $\eta_{\#}, 256$ \\ $\varepsilon_{\#}, 256$ \\ $\mathrm{Ev}_{V}, 167$ \\ $\mathscr{E} x, 270$ \\ $\mathscr{E} x_{B}, 315$ \\ F, 378
}

\author{
$S F, 378$ \\ $F(X), 400$ \\ $F[\alpha, X), 423$ \\ $F_{B}(K, X), 20,152$ \\ $F_{B}(X, Y), 21$ \\ $\bar{F}_{B}(Y, Z), 162$ \\ $F C P[\mathscr{U}], 399$ \\ $F I_{B}^{f}, 177$ \\ $F\left(I_{+}, X\right), 63$ \\ $F J_{B}^{f}, 177$ \\ $F(K, X), 17$ \\ $f_{!} X, 30,168$ \\ $f^{*} Y, 30,168$ \\ $f_{*} X, 30,168$ \\ Fred $(\mathscr{H}), 378$ \\ $\mathbb{F}_{R} F I_{B}^{f}, 217$ \\ $\mathbb{F}_{R} F J_{B}^{f}, 217$ \\ $\mathbb{F}_{R} F K_{B}^{f}, 217$ \\ $F_{R}(N, L), 218$ \\ $F_{V}, 167$ \\ $F(X, Y), 16$ \\ $\bar{F}(Y, Z), 40$ \\ $\mathscr{G}, 110$ \\ $\Gamma\left(U ; J_{*} X\right), 384$ \\ $G \mathscr{C}, 153$ \\ $G \mathscr{E}_{B}, 200$ \\ $G \mathscr{K}, 22$ \\ $G \mathscr{K}_{B}, 22$ \\ $G L_{1}(R), 401,406$ \\ $G L_{1}(k), 377,378$ \\ $G \mathscr{P}_{B}, 165$ \\ $G R \mathscr{M}_{B}, 216$ \\ $G \mathscr{S}_{B}, 163$ \\ $G \mathscr{S}_{B}\left(U^{\prime} ; U\right), 423$ \\ $G \mathscr{V}_{B}, 138$ \\ $G \mathscr{W}_{B}, 138$ \\ $h \mathscr{C}, 62$ \\ $h G \mathscr{W}_{B}, 138$ \\ Ho $\mathscr{C}, 63$ \\ hocolim $X_{i}, 262$ \\ $H X, 376$ \\ $I, 111$ \\ $I_{B}, 98,111$
}




\begin{tabular}{|c|c|}
\hline & \\
\hline$I_{B}^{f}, 101$ & $\operatorname{Map}(I, X), 63$ \\
\hline$i \square_{B} j, 156$ & $\operatorname{Map}(K, X), 16$ \\
\hline $\mathscr{I}_{c}, 405$ & $\operatorname{Map}(X, Y), 16$ \\
\hline $\mathscr{I}_{c}-\mathrm{FCP}[\mathscr{U}], 405$ & $M f, 64$ \\
\hline$I_{B}^{f}(\mathscr{C}), 112$ & $M \wedge_{R} N, 218$ \\
\hline $\begin{array}{l}\left(\mathscr{I}_{G}, G \mathscr{I}\right), 159 \\
\left(\mathscr{I}_{C} \mathscr{K}_{B}, G \mathscr{I} \mathscr{K}_{B}\right), 161\end{array}$ & $N f, 64$ \\
\hline $\begin{array}{l}\left(\mathscr{I}_{G} \mathscr{K}_{B}, G \mathscr{I}_{B}\right), 161 \\
\iota^{*}, 35,170\end{array}$ & $(-)^{N}, 37,226$ \\
\hline$\iota !, 35$ & $(-) / N, 37,226$ \\
\hline$\iota_{*}, 35$ & $\mathscr{O}_{*}(B), 24$ \\
\hline $\operatorname{Iso}(k), 375$ & $\mathscr{O}(B), 24$ \\
\hline$J, 111$ & $\mathscr{O}_{G}, 112$ \\
\hline$J_{B}, 98,111$ & $\Omega_{B}^{\infty}, 163$ \\
\hline$J_{B}^{f}, 101$ & $\operatorname{Orb}(P), 38$ \\
\hline$J_{B}^{f}(\mathscr{C}), 113$ & $\Omega_{B}^{V} X, 163$ \\
\hline $\mathscr{J}_{G, B}, 166$ & $P, 133$ \\
\hline$J_{V}^{(K, p)}(X), 368$ & $\mathbb{P}, 319$ \\
\hline$J_{(K, p)}^{V}(X), 368$ & $\mathbb{P}, 179$ \\
\hline$J_{*}, 338$ & $P_{B}^{\square}(i, p), 155$ \\
\hline$J^{*}, 338$ & $P_{B}(X, Y), 161$ \\
\hline$J_{G}^{*}, 359$ & $P_{B}(X, Y), 152$ \\
\hline$J_{*}^{G}, 358$ & $P_{F}, 48$ \\
\hline$\tilde{J}_{*}, 339$ & $\mathbb{P}_{F}, 240$ \\
\hline$\tilde{J}^{*}, 339$ & $\begin{array}{l}\mathscr{P}_{G, B}, 165 \\
\pi(X, Y), 92\end{array}$ \\
\hline$K, 205$ & $P U(\mathscr{H}), 379$ \\
\hline $\mathscr{K}, 16$ & $P \times_{\Pi} F, 47$ \\
\hline $\mathscr{K} / B, 19$ & \\
\hline $\mathscr{K}_{B}, 19$ & $Q S^{\circ}, 378$ \\
\hline$k_{*}^{B}, 341$ & $\mathbb{R}_{+}^{n}, 100$ \\
\hline$K^{b}, 31,36$ & $\mathscr{R} O(G), 358$ \\
\hline${ }^{b} K, 31,36$ & \\
\hline$k_{B}^{*}, 341$ & $S_{B}, 163$ \\
\hline $\mathscr{K}_{G}, 22$ & Sec, 31 \\
\hline $\mathscr{K}_{G, B}(X, Y), 22$ & $S_{f}, 273$ \\
\hline$k_{P}^{*}(X), 374$ & $S F(X), 400$ \\
\hline$k_{*}^{P}(X), 374$ & $\mathscr{S}_{G, B}, 163$ \\
\hline$k_{\Pi}, 374$ & $S G L_{1}(R), 401$ \\
\hline $\mathscr{K}_{*}, 16$ & $S_{H}^{n, b}, 124$ \\
\hline$k_{*}(X, p), 374$ & $\Sigma_{B}^{\infty}, 163$ \\
\hline$k^{*}(X, p), 374$ & $S L_{1}(R), 406$ \\
\hline & $S L_{1}(k), 377,378$ \\
\hline $\begin{array}{l}\mathrm{L}, 421 \\
\mathscr{L}, 405\end{array}$ & $S_{ \pm}^{n-1}, 100$ \\
\hline $\mathscr{L}, 405$ & $S_{H}^{n}, 196$ \\
\hline $\mathscr{L}_{*}(J), 344$ & $\operatorname{STop}(X), 400$ \\
\hline$\Lambda(T), 401$ & $\Sigma_{B}^{V} X, 163$ \\
\hline $\mathscr{L}_{*}(X, J), 344$ & \\
\hline $\mathscr{L}^{*}(X, J), 344$ & $T, 207$ \\
\hline $\mathscr{L}[\mathscr{U}], 405$ & $\mathscr{T}, 16$ \\
\hline$\lambda_{B}^{V, W}, 187$ & $t, 249,272$ \\
\hline$\Lambda(R), 401$ & $\tau(f), 236$ \\
\hline$\Lambda B, 131$ & Tel $X, 94$ \\
\hline$L X, 132$ & $\mathscr{T}$ op, 16 \\
\hline & $\operatorname{Top}(X), 400$ \\
\hline $\mathscr{M}(\alpha), 423$ & $t S_{f}, 273$ \\
\hline $\operatorname{Map}_{B}(K, X), 20$ & \\
\hline $\operatorname{Map}_{B}(X, Y), 21$ & $U, 78$ \\
\hline
\end{tabular}




$\mathscr{U}, 16$
$\mathscr{U} / B, 19$
$\mathscr{U}_{B}, 19$
$\mathbb{U}, 179$
$\mathscr{U}_{*}, 16$
$V, 78$
$V^{*}, 167$
$W X, 131$
$X \wedge_{B} K, 20,152$
$X{ }_{b}, 31,36$
$X \wedge_{B} Y, 162$
$X \wedge{ }_{B} Y, 20$
$X \times I, 63$
$X \wedge I_{+}, 63$
$X \times K, 16$
$X \wedge K, 17$
$X \bar{\otimes} Y, 396$
$X \times{ }_{B} K, 20$
$X \times{ }_{B} Y, 20$
$[X, Y], 92$
$X \bar{\wedge} Y, 40$
$\tilde{Y}, 20$

